

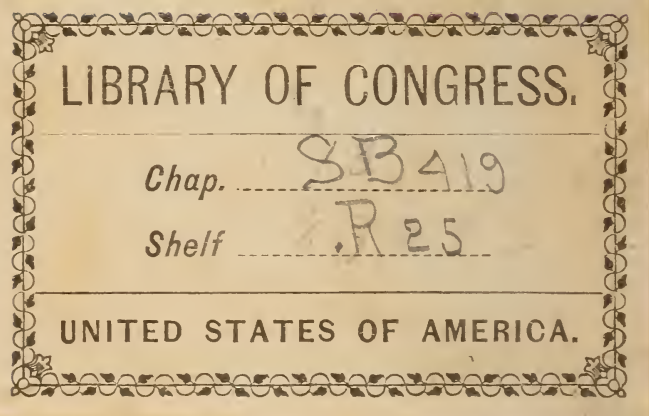





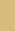




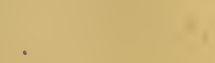

.

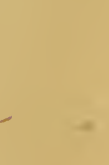

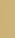





\title{
SRithlitider Sathgeber
}

$f \mathfrak{i r}$

Stubengartuer,

bey

2uzlwabl Der fobonjten (Genádbje uno beren

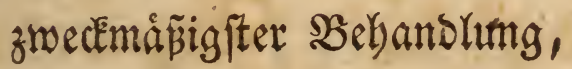

\author{
grổ̇tentbeils
}

nad) eigenen Erfaf̨rungen bearbeitet

ton

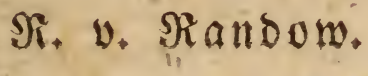

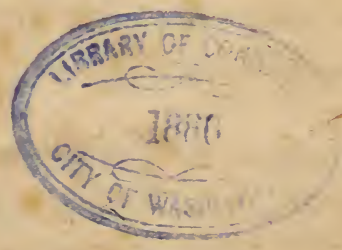

$$
\{\subset i p z i g,
$$

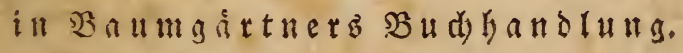

1828. 


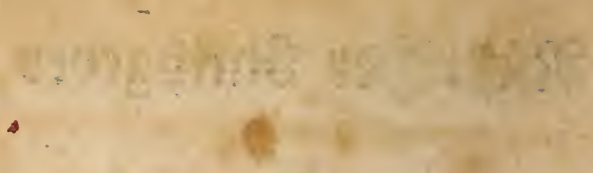

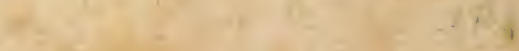

8. 3 की

$$
\text { 15x: In: } 5 B^{\times 19} R^{25}
$$$$
\text { dinguechis }
$$

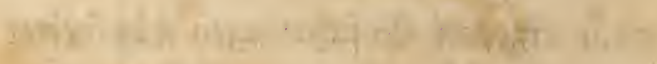

$$
\begin{aligned}
& \text { Whasin } \\
& =-3^{2}
\end{aligned}
$$

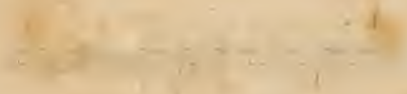

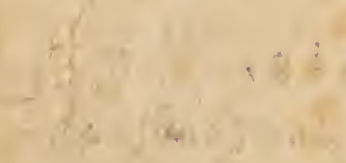

a 


\section{$\mathfrak{B} \cup \mathfrak{x} \mathfrak{w} \circ \mathfrak{c}$}

Die 2 maahl ber (sartenfdyriften, und namentlidy bie

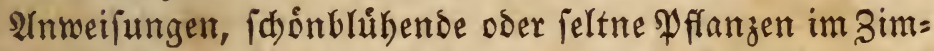
neer zu ziehen, hat fich in neuerer Beit fo vermehrt, da

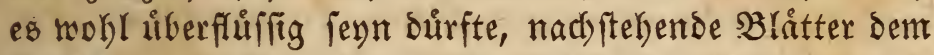
Publictur zu kibergeben. StGer vielleidft findet dennod) ber angehende Błumenfreund einzelne ßemerétungen und WSinfe barin enthalten, bie ihm nidbt ganz werthlos fdeinen, uno ihm bey Erjiehung feiner rffanzen will= fommen (eyn dưrften.

Feinesmeges will id) jebod bas hier (siefagte als fes

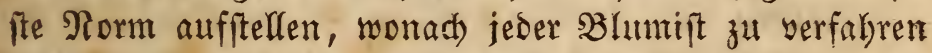
hat. - Nur meine Beobadjtungen uno Errfabrungen theile idh mit, und fehr leidst fann ein Snorer allf

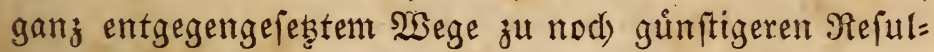
taten gelangt feyn, ja idi felfit merbe vieffeidst in ber

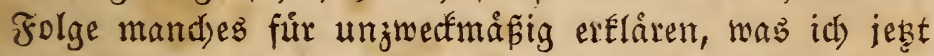
alz gut anerfenne. Alber geht és denn irgend einer Rumpt oder $\mathfrak{S i f f e n f d a f t , ~ b i e ~ f i d ) ~ e i n z i g ~ a u f ~ ( E r f a h r u n = ~}$ gen grůndet, anders? - Darum bitte auth id um gí =

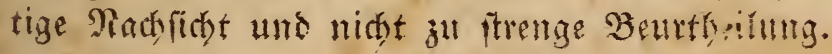




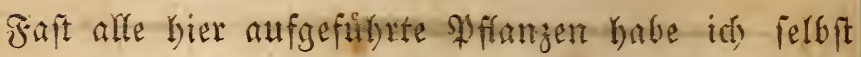

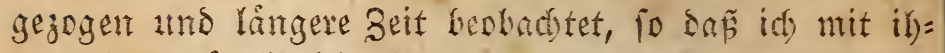

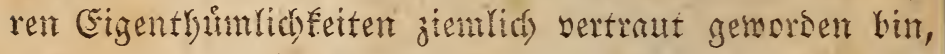

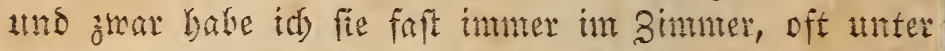

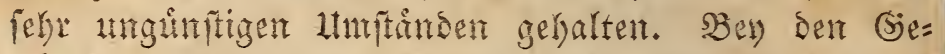

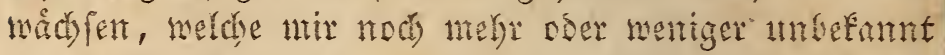

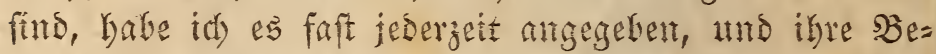

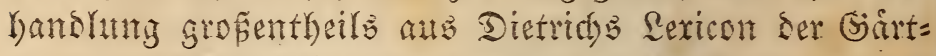
nerey) und Botanif entumututet, jum Theif auts andern bouthen, uns basjenige besgefigt, was its surd pon= ftige Mittheilungen meip. - Scin und mieber hätte ids

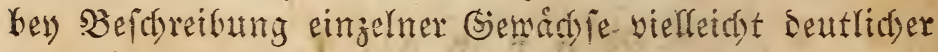

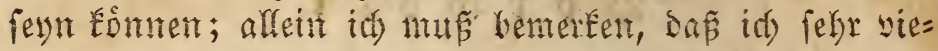

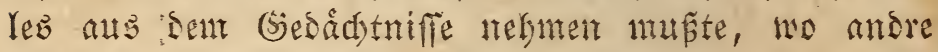

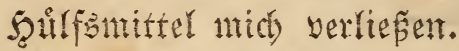

Sebr leidst hatte id bie Babl ber hier aufgefubr= ten Pflangen vermebren fonmen, bratte ith midy bantit

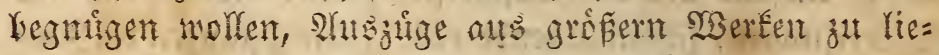
fern; allein siejes war nidst ber swef meiner shrueit.

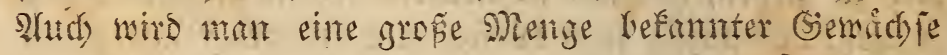
(mamentitid) viele Commer = uno Strutengemáchle) ver= miffen; aber theild mtipte ids es thut, unt nitht obne

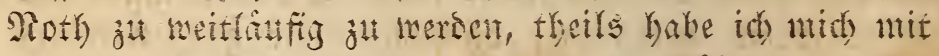

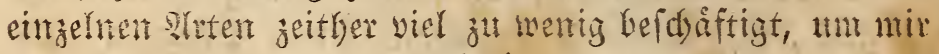

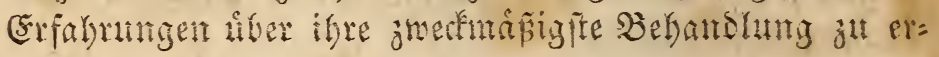
merben.'

Colltent diefe Bratter nidht ganj obne Benfall brei= ben, fo theile idh Dent Publicum vielleidyt nad) einigen Sahreat meine fpotem Beobarbtungen mit.

(5iefdriefen in Dctofer 1827.

Der 2̉erfaffer. 


\section{E゙ $\mathfrak{i} \mathfrak{n} \mathfrak{l} \mathfrak{i} \mathfrak{i} \mathfrak{u} \mathfrak{n} \mathfrak{g}$.}

Dogleid die Blumenlieblaberey in Ëแtropa fidon feit vie=

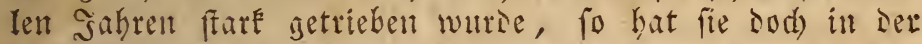
leşten Bett berentens zugenomment no wiro mit jeden Sabre Surd) Die bertidhen nenen Pflangen gefreigert, wel=

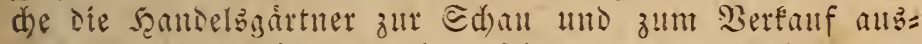
ftetren. SBer frither somit zufriesen war, eill Drangen=

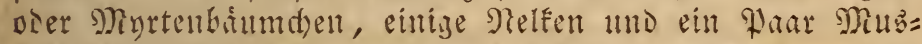
fatflócteden auf feine jenfter zu feelfen, ier jierte yor 15

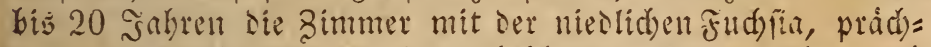
tigen 5artenfia und Der inmerblithenten Bufe. atber auds defe find im sanfe ser zeit bennafy verieffen worden, thto baben mumebr ien pelatgonien, Pltpentofen uns Eamels lien weidsent miffen. - So medjelt siefe siebhaberes mit

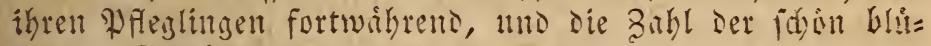

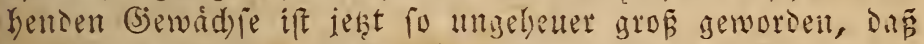

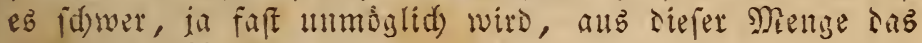
Befte zll wíblent.

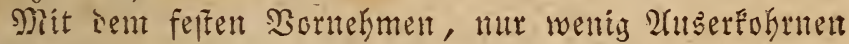

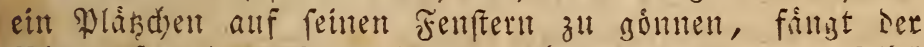
Blumenfreutio zul fammeln an. Stber ohne da will, waddft ihre Zahl, er weis niddt mehr, woher Raum nefmen, fie alle unterzubringen, er will sie faled tepten Eaden wieser entferten, und getáth in nete Berlegengeit.

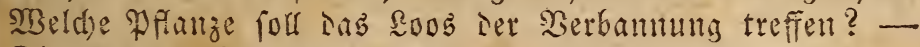

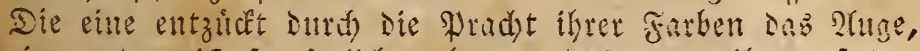

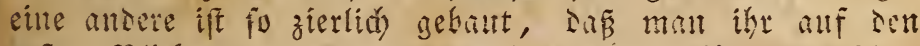

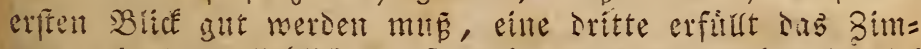

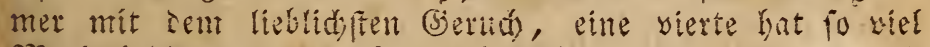

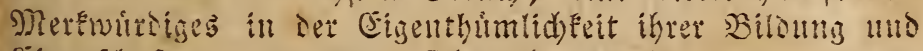

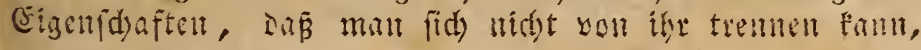


uno cine fünfte follte jeht, eben jeḩt yerftopen werden, da nad) iahrlanger pflege fie fo weit gefradid worben ift, end = lid) einmal mit shlumen die auf fie gementete grilhe zu beloknen? Co hat jedes (sewaids reine befondern Borzuge, weldye den Entfdlli wankend machen, es zu entfertten, und es fdeint, als mitfe dab (s)anze verlieren, fobald man einen Topf yon Den dibrigen trennen will.

2rber deffen ungead)tet fobranfe man bie zahl ber Ett= benpflanzen, went nid)t befonders gunfitige Umftånde es ges

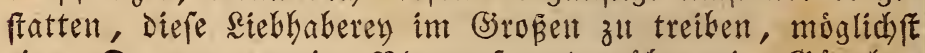
ein. Da nur wenige Blumenfreunde liber ein (j)årtd)en

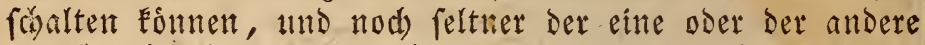
ein (5̈ewadbshaub zum Heberwintern feiner sieblinge benu=

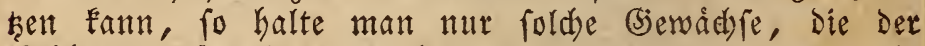
Muke des Erziehens werth find, indem fie entweder fehr viele fdoune oder woklried)ende Blumen bringen, oder bas

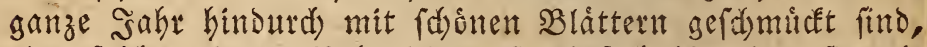

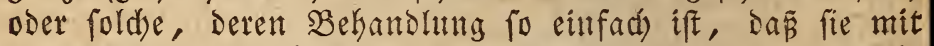
jeser \$flege yorlieb nehmen. Sereintigt eine Pfanze meks= rere biefer eigenfdaften, und bringt fie wohl gar $B$ lu= men zu einer Sabregzeit, wo diefelben felten find, Dann freilidy verdient fie einen der erfen Plähęe.

Nie mus man ferner mehr Blumen balten, alz man, ohne fie felbft zu fehr zat beengen, auf feinen Fenfern, ooer bod bidst hinter benfelben auffetfen Eann. Denn, wenn gleidy einzelne Pfanzen waifrent Des SWinters wents sidht und Feine Sonne bedurfen, um am seben erbalten zu werden, fo iftes sod) refre gut, wenn man ignen beides geben fann; ia im Frublinge if then, ofne 2ubnabme,

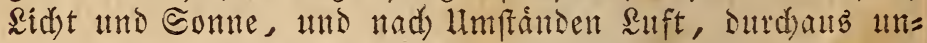
entbehrlids. SMur man fie um diefe Beit fitefmutterlid bes Gandeln, fo leiten fie oft in wenig Sỉodjen mehr, als eitt ganzer Eommer verbefiern fann, mo man ift nidist felten um alle feine Shoffintungen tund Erwartungen betrogen.

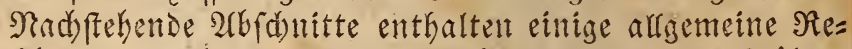

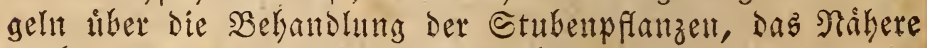
ift bey jeber einzelnen art angegeben.

\section{Heber Den Gtanbort Der- Pflanzen.}

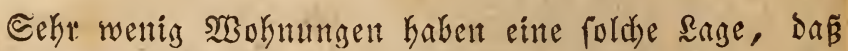
mant fie gưnftig für bie Błumenzud)t nennen fann. - $2(\mathfrak{m}$

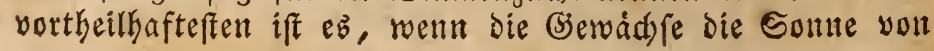




\section{Ans

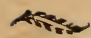

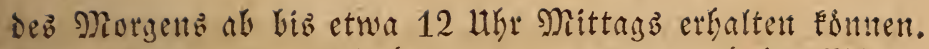

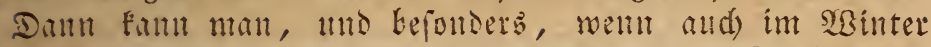
Die Eomne einige Etmusent Lang Die Jenfer trifft, faft alle

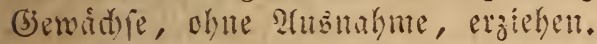

Die volfe Mrittassoune, etwa von $10 \mathrm{Hbr}$ ab bis शrad)mittans um 4 llbe, vertragent in frever suft mut we=

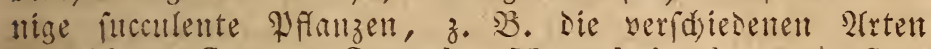
yon Aloë, Cactus, Crassula, Mesembrianthemumi, Stapelia, fo wie sie verwanten afrem, nebfit sentenigen, wel=

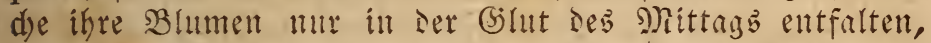
wie Arctotis, Gorteria 11. a. - Dagegen geveífent binter

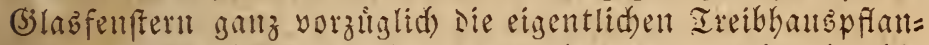

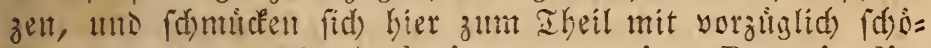
neen $\mathfrak{B}$ tumen, z. B. Asclepias curassavica, Begonia discolor, Caladium bicolor, Clerodendron fragrans, $\mathrm{Cy}-$ rilla pulchella, Gloxinia speciosa, Gardenia florida, Hibiscus rosa sinensis, Jasminum sambac, Ixora coccinea, arfe 2frten Lantana, Mimosa pudica, Musa paradisiana, Nerium (affe 2frten, mit 2tusnabme seb gemeinen einfad)ent rothen), Rhapis flabelliformis, Ruellia formosa, Salvia splendens, Schollia crassifolia, Tradescanthia discolor, Vinca rosea 1. f. w. - Mniffen antere (jesviddje an fold) einem Etantorte ill freyer sufft ferhen, fo gebe iman ihnent

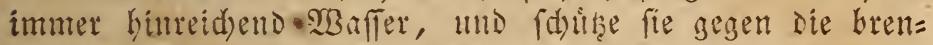
nente Somte Dutd) einen @djum yon Seinemand. - Bum

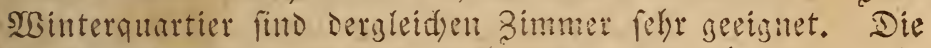

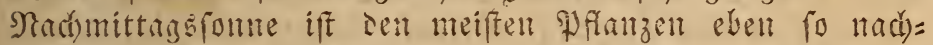

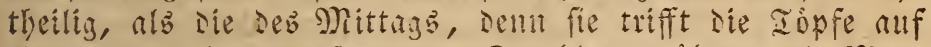
cinmar mit ihren veriengenden Etrahlen, wihterno die Mor=

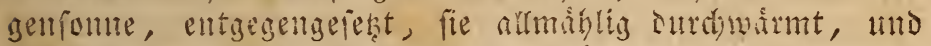

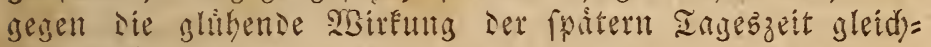
fam vorbereitet.

siegent Die Fenfer cinter SBobmung gegen Mittemadist,

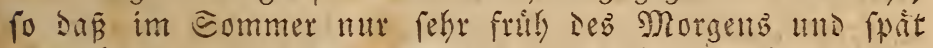
am NGentid Die Etrahlen Der Eonme an than vorbengleiten; fo befommen folgense biewdidje bier einen \$lan: Aucuba japonica, mefrere Gilockenblumen, Canna, Cestrum parqui, Chelone barbata, Corchorus japonicus, Cupressus sempervirens, Cyclamen europaeum, Daphne laureola min cneorum, Dracocephalum virginicuin, Eugenia australis, Fuchsia coccinea, Hemerocallis, Hibiscus syriacus, Hortensia, Jypericum, Ilex, Jasminum officinale tno odoratissimum, sovbeet, Lobelia, Lonicera semper- 
virens, Lychnis, Mimulus, Mrtte, eillfadset gemeitter sleanter, viele Pelargonien, Phlox, Rhododendron ponticum, arfe Rofenarten, Scabiosa caucasica, Tagetes lucida, Tradescantia virginica, Verbena tryphylla, Viburnum tinus, Vinca major $4 n$ o minor 4.6 .6$.

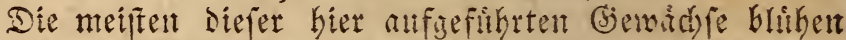

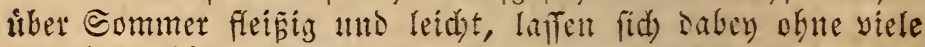

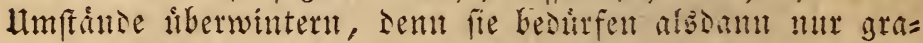

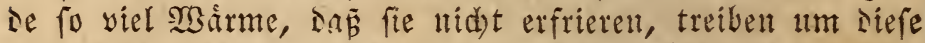

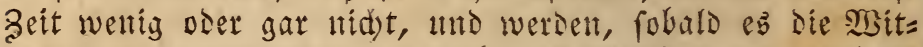
terung mux einigerma B̃en erlaubt, im striblinge fogleid in die freve suft gebrad)t.

Da in Ien Gtabten die yor sen Fenfern febenten

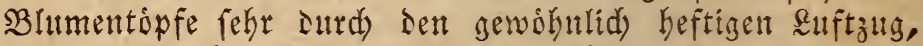
und Die Durd) Den SBiserfdeill verfarten Eonnenftralent

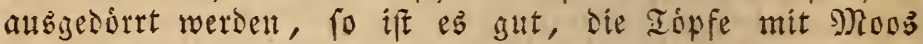
z" umfittern, weldses man an beifen Iasen zumeilen an= fend)tet. - Merben Blumen in einem (s)artent anfigeftelt,

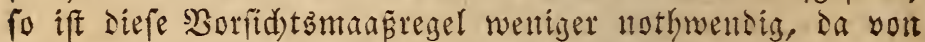
ifnen in der siegel ier Mind mebr abgehalten wirt.

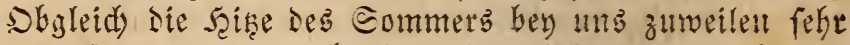
grof ift, fo vertragen Dod) die meiften Pranzen Der beip̈en Done Eeineswegse einen Ctand in fiener suft, indent Diefe zu trodell ift, Hub die Sweige und Blatter nid)t, wie it

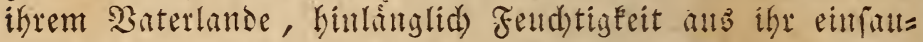
gen fonmen. Daker mun man you ifnen jeben beftigent

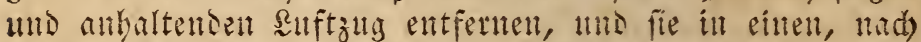

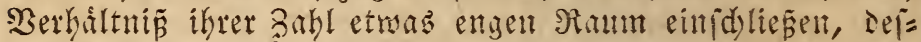
fen suft yon ben, ans ber cise anffeigenten Dumfen

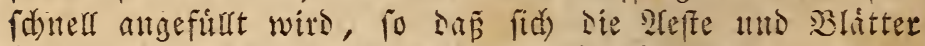
beftinsig in einter fendsten 2ltmofphare befinsen. - Seier= zu eignen fid am beften sie fogennmten Blumenfenfiter,

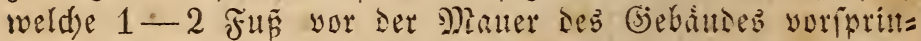
gen, yout, aud vielfeid)t zut Eeite 14 d oben, mit (5) lass=

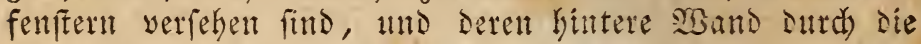
Etubenfenfter felbft gebildet wird. Sill fold eitum gian=

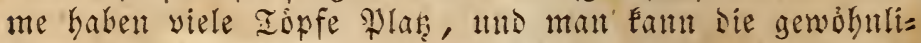
d)en tropifden \$flanzen (verfegt fid) nur riejentgen, wel= d) Eeine besentense şoke erreid)en, umb fid) im Sommer mit Blumen fdmutckell) forf fümmtlid) ebell fo gut zum

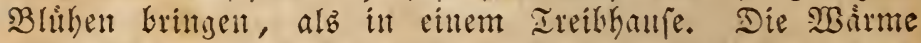
freigt in semfelben ies Mrttags bey Sommenfdein, wem die Fenfter alle gefdloffen find, oft bib nabe an 40 Gitio 


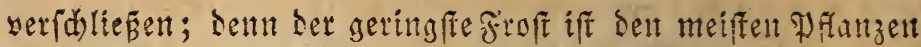
im Frabling fehr nadytheilio, felbit sentenigen, die im frenen sande biferwintern.

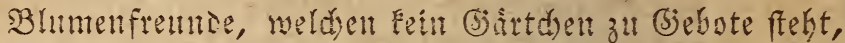
frefen ihre Pfanzen entweser in Die frene suft alif solu=

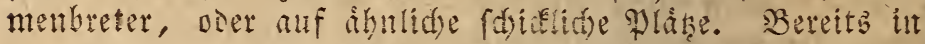

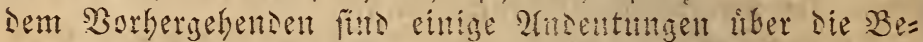
bandung gegebe!t worden, welfye Dic Blument alshann ver= langen, mo bey Befdretbung iet einzelnen Pflanzest fommt

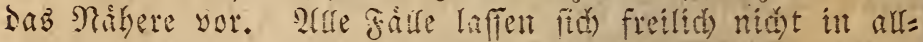

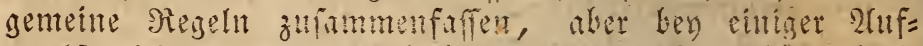

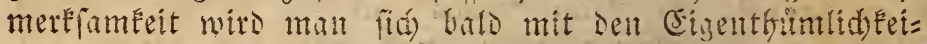

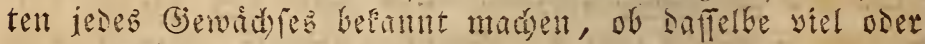
wenig sidit niti suft bearf.

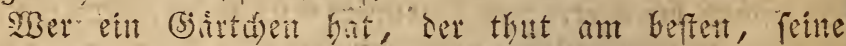
Pflangen dell Eommer diber in saffelbe zub bringen, went

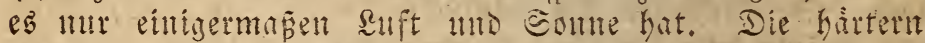

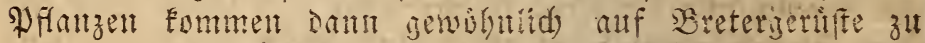
ftehen, welde man bey befinem Sonnenfiden, Begenwet= ter, oder um die Blumen lange blifhend zu erfalten, Durd) seinwaniforme fidsert. Sift man genothist, ste Iopfe auf

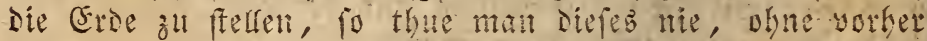
einen Biegel ober ein Etuck Bret Huterjulegen, intem fid)

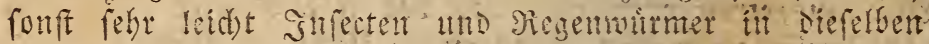

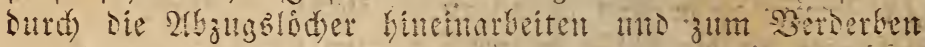
Der Gewidje yiel beytragen. Eelbft, went mat. Pfanjen

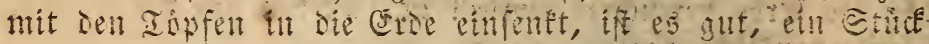
Biegel unterzulegen, Dent die Regenwitmer thstepontere

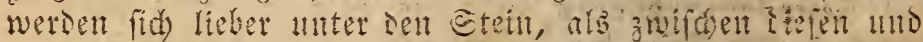

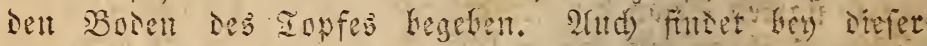

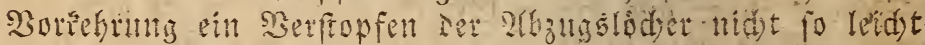
Ctatt, mo sie pifanzen werien gefmo erbaltelt?

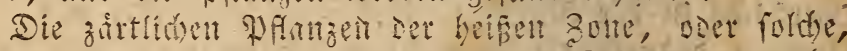
beten Wad)

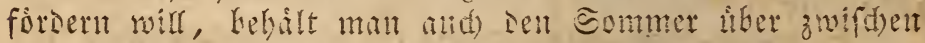
Doppelfenfern oied in sen Bhmenfenfern, tho giebt ib =

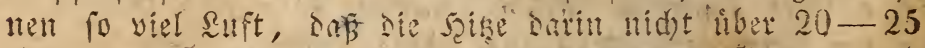

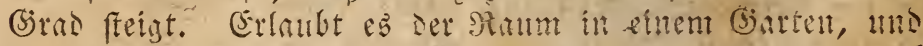
bat man Eonne genuis, fo fant man fidh all stefen Bwect einen befontern Ireib = odet Eommureffen, ifylid einem

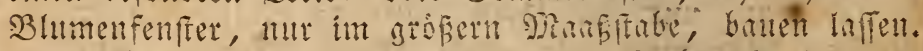
Die Mande madit man entweder you fadjwere ver vou Bretern, 6-10 3oll Dicf, auferbalb mit sefm belegt. 


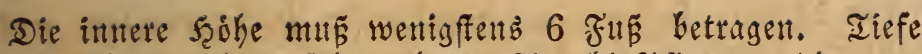
uno Ránze liddten fid nad) Der 2 Hzahl Pflanzen, Die man

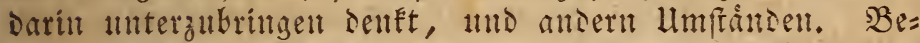
trågt Die Thefe nidd mehr, als $3-4$ Fus, fo rid)tet man Die yorsent Fenfter fo ein, saj fie bequem geofftet, unto Durd) Diefelben Die Iopfe in Den intern Raum geftert wer=

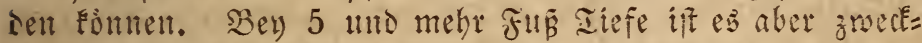

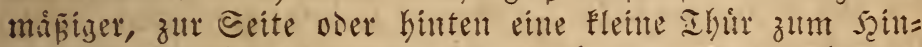

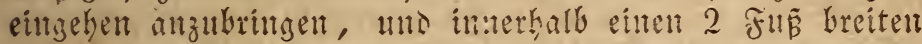
(5)anty zu laffen, fo son man leid)t zu jesem einzelnen (5)e=

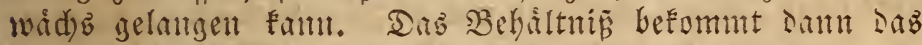
2urfehn einco fleinen Ireibhaufes. Die Fenfer ftehen ge= meitiglidi) unter einem Minfel you 60 (j)ras geneigt. BeF=

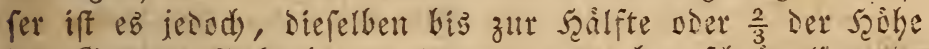

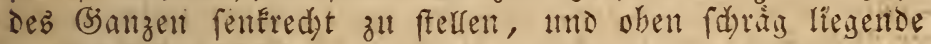
Fenfter allubringen. Dasurd) gewinnt man an Faum und

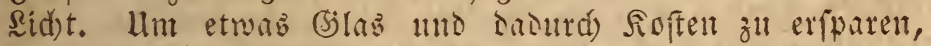
Fant man and) fold) eilten Ireibfaften $1-2$ Fun tief in

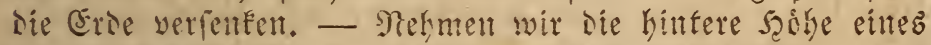

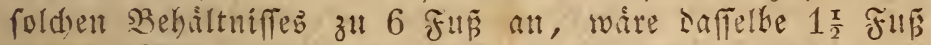

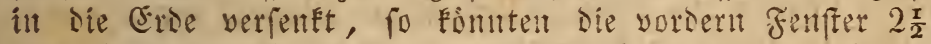
Fus hod) gemadit werden, who bie obern Fenfer warden

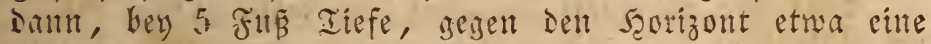
Neigunis ron 20 (3irno erbalten. - 2(ud) Die fdumatan Sei=

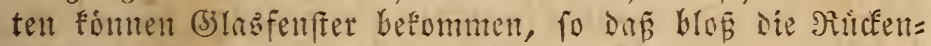
want yon Szolz orer Manterwere ift. Die itbrinen Details

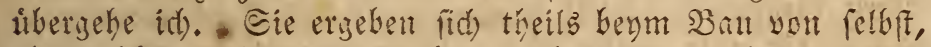
theils baingen fie you llmfturnen ab. Natr nod) fo viel iff z" bemerfen, saf die obern Fenfer gegorig mafferdidt gemad)t werden miffen. Diefes gejajeht am beften, went man sie Sdjeiben Dadziegelformis tiber einander legt, fo

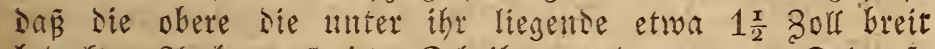
beseckt. Iruch muf jese Edjeibe an der untern Seite fo gejd)nitten jeyn, Dar fie in ser gritte cine yoriprittgende

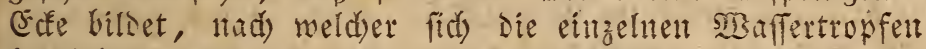

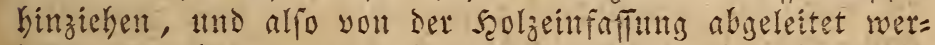

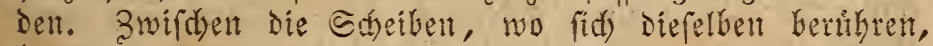

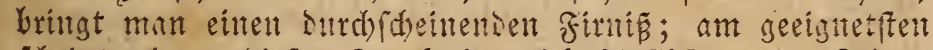

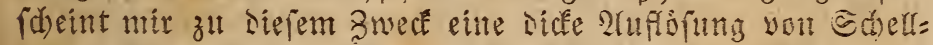

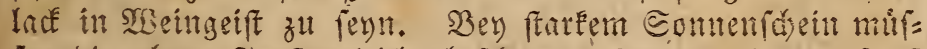
fen bie obern Fenfer leid)t befoattet werben, indem fonfi fefgr leidyt die feinern (Siendidje verbremten. Dbgleidy eq gut ift, Den Pflatzen, meldy int freyen 


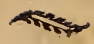

ftehen bưrfen, fo lange als mỏglid) im Serbft die suft zu gủnnen, Damit fie fidt) geforis abfarten, tmo baun um fo leidster surdswintern laffen, fo fen man sod) yon Mitte

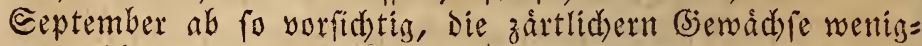
ftenz liber Nad)t in ein Bebáltuiż zu ftellen, wo fie gesen

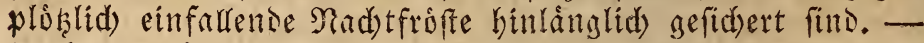
2hld) Die härtern Eachen bringt man gegen (Ende Septem= ber ins SWinterquartier, giebt irnen aber fo lange suft, alz moglids. Shur ganz harte Pfanzen, yon Denen man itberzeligt ift, da

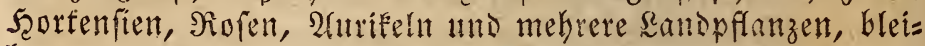

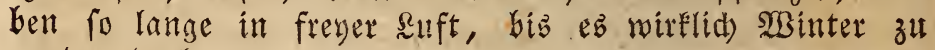
werden brobt.

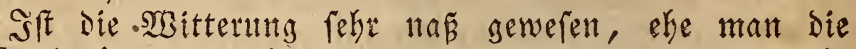

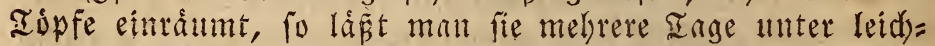
ter Bedad)unis ftehen, samit die sllft die (stoe gehorig alls=

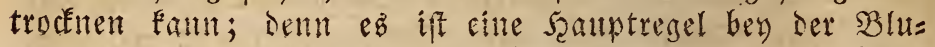

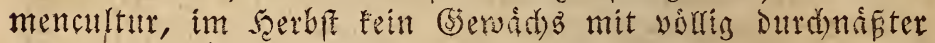
Erde ins Winterquartier z"l nefmen, weil siefeb die erfte

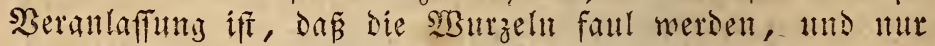
felten ift man um Diefe Sahereszeit im Stande, ihnell Durd)

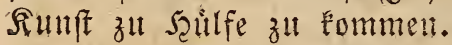

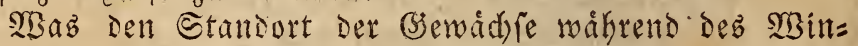
ters anbelangt, fo rid)tet fidi Derfelbe ebenfalls nad) Den

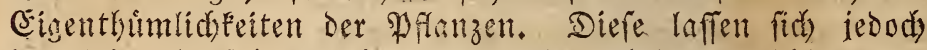

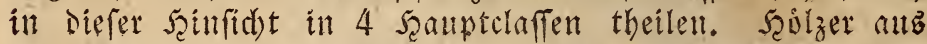

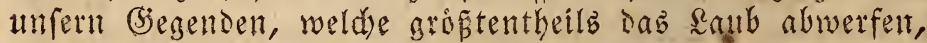
maden bie erfe (Elaffe aus. Mlan bringt fie am befren ill einem falten Bimmer surth, weld)es nut Bann ein we= nig gebeist wird, wenn SBaffer zu frieren anfängt. Dent obgleid) fie in Der Regel eitren ziemlid harten Froft alls= halten finmen, fo wirtt Derfelbe Dody nidyt feiten nad)thei=

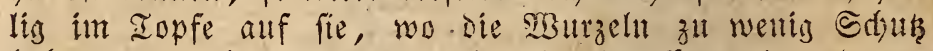
baben, uno bat man viefreidet einzelue Exemplare Davon im Fribling zuvor im Miifbeet getrieben, fo fund siefe les: tern nod) weit empfindidier geworien. - 2llpo it einer

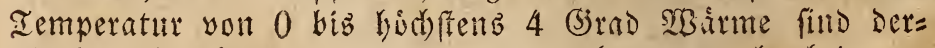

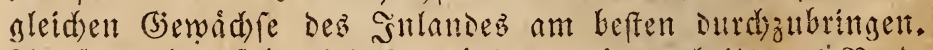

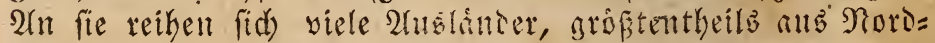
america uno dem mittlern 2ffien an. Jot) nenne bier mur: Amygdalus pumila, Andromeda, Aucuba, Azalea, Corchorus, Fuchsia, Hibiscus syriacus, Hortensia, einige 2rten Saß̊min, Ilex, Kalmia, Magnolia, Punica grana- 

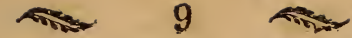

tum, Pyrus japon., Rhododendron, bie immetgrunen Ro= fen, vicle Feisen, Verbena, mo bie meiften Etandenge=

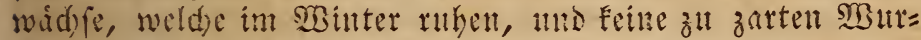
zeln baben. Dicfe leştern fommen fosan gróptentheils in bie yorderfen Reihen, sa fie wentis sidjt ranben, Da= binter sie immerintumen Strảuder, uno ganz nad hinten Dic entlanten Barumden. Bam December bis 2lnfang

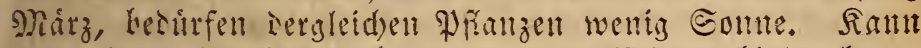

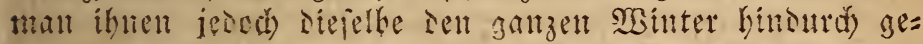

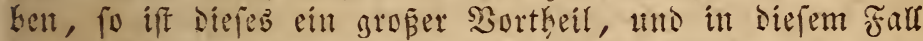
if es meniger mad)theilig, wenn ald sie sisarme zuweilen etwab hober fetigt, als angezeben woroen ift.

8at ser 2 ten Çaffe gebiren Die fogentanten Eappflan=

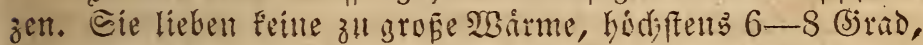
vertragen aber audi) nidd ben gerimgften Froft. Daher ift

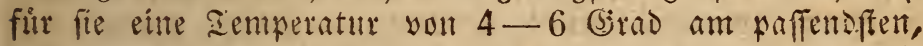

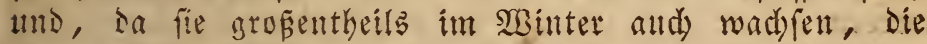
Comme felye nothmendis.

Die Pfanzen ber 3ten CElaffe, eigentlide Ireibrauts= gerwad) fe, Jalten in ser Regel bey ben ser 2. Slaffe alta.

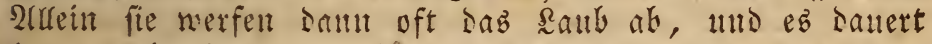
lange, ehe fie im grriblinge wieser zu wadjen anfangen,

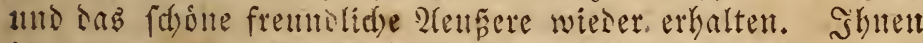
ift sie gemobnlidse Etnbentemperatur yon $10-15$ Sirad

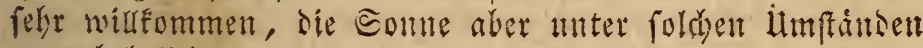
unentbebrlidy.

Die 4. CElaffe bilden nut menige finolfent = unt 3 wies belgewaidse, welde im Sisinter yollig rubent, unts wents voer gar nidst gezoffen erhalten. Die gewofhnliden ent=

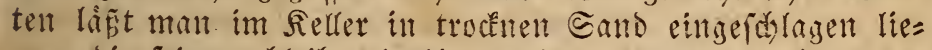
gen; sie feinern bleiben in ifren Iopfen in einem mápigmar=

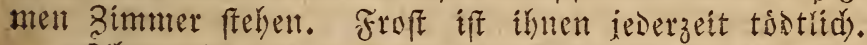

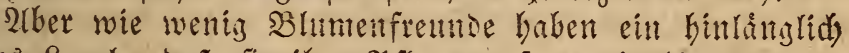

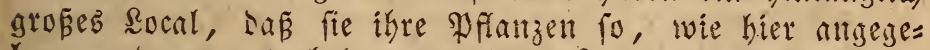

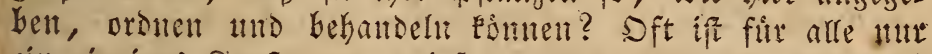
eilt einziges Fenfter angewiefen, und an diefem forten fids

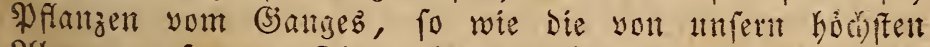
2llpen, gefalten. Diefes ift fieilid) etwab viel yerratugt, Dod) es geft surd)zufulfrent. Ein getúlmiges Dopnelfenfter

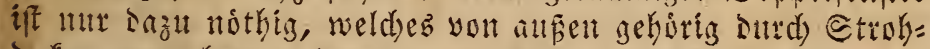
secken verwakgt werben Fann. Siefes theilt mant surd ein

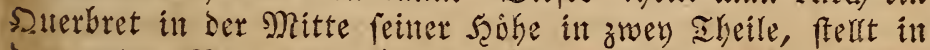

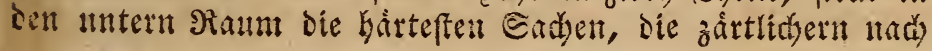


oben, und behált biejenigen, für weldye man am meiften

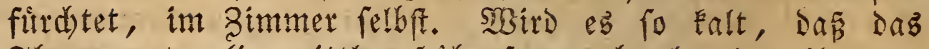
IGermometer (in mittler Frohe fo sugebrad)t, sa ber Sonne noă) Bugluft trifft) etma allf $2-3$ (5rad 3 á:= me zeigt, fo offinet man die innern obern Fenfer etwab,

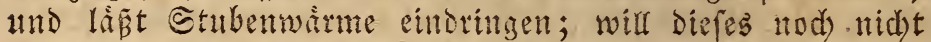
Ginteiden, fo mad)t mant fie ganz auf, tho offint aud im Mothifar sie minterfen. Des Nadhts ift es an Falten Iagen am beften, jedesmat alle Fenfter gattz zu sffinen,

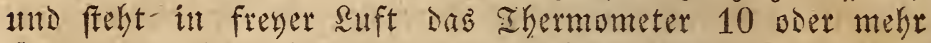
(S) rad unter bem (Siefrierpunct, fo táume man ja alfe zart=

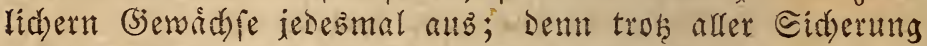
Durd) Deden 11. Dgl. Iringt Der Froft Dennod) oft fehr plobs= lid) eill, befonders in Die B̉lumenfenfer. - (s)ópere Bảl=

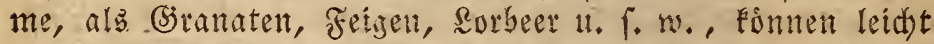
in jesem troutnen, nidst zu warmen Selfer hberwintert werien.

Seat man keille Doppelfenfer, oder fant man befels

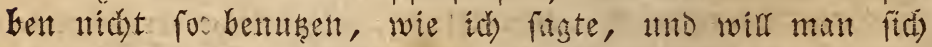
Denndd) eimige Blumen Galten, fo if ber befte Rath, mur

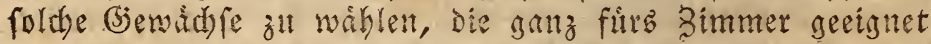
fino, atno mit jeser tum einigermapen für fie paftenden Befandhng vorlteb negmen. Untev antern nente id): Aloë, Aucuba japonica, Cactus, Calla aethiopica, Canna, Clerodendron fragrans, Cyclamen europaeum, Jasminum odoratissimum, Lantana camara, Laurus nobilis, Nerium oleander, Physalis peruviana, Plectranthus fruticosus, Prunus laurocerasus, Rosa semperflorens, Sempervivum arboreum, Schollia crassifolia, utid yon ben Gewaidjen, wel= che Den Winter úber ruben, Begonia discolor, Cyrilla pulchella, Commelina coelestis, Gloxinia speciosa. IUte Diefe genamten Pfianzen balten in gemobnlider ভtubentem= peratur gut alb, wenn fie sid)t am Fenfer fethen founen. SBBie bereits erwahnt watse, im Serbft gebe man pei=

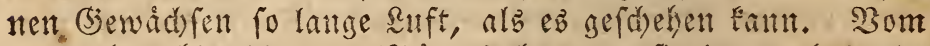
December bis 2rnfang Mintz fude mant fie im Putheftande

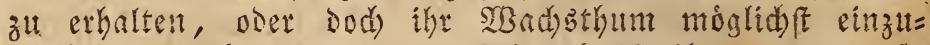
fidränen. Prber yon lester seit ab tft thnen, wenn fie

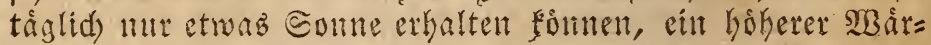
megrad fehr willemmen.

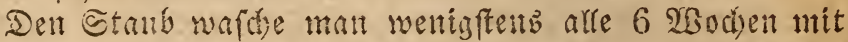

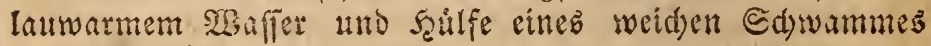
yorfidstig ab. 
Fangen bie \$fangen im sribling an zat tieiben, fo

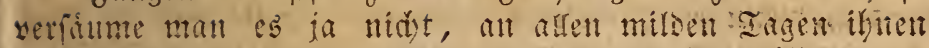
ctwas Ruft z"l gebent. Eie werdent Basurd) geftatist, uno

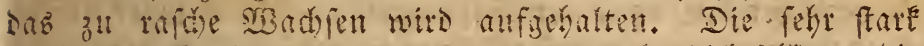
treibenten Eadyen ferte man alssann red)t Didyt Ginter bie Feniter, und zieke dieienigen, welde nod tukent uno keine Snospen baben, cinftweilen zurick. Beigen fid Sinospen, fo fude man niemals surdy vermebrte sBarme séefelbent zwingen z" wolfen, fid friber z"l entfalten, alz es fonft gefdehen witres; felten glicet es, fehrof oft aber wird man

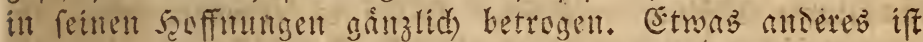

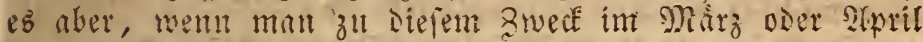
eit Mnifbeet bentsen fann; Dod fen man and in biefer

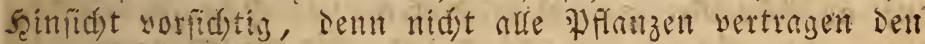
fendi)ten Dunft terfe!ben.

Sebr Garte Gewaidje, mo yorziglid santpflanzent

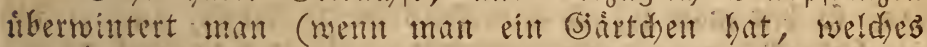

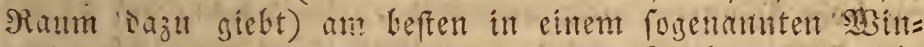
terkaften, Der im 2flgemeinen wie ein oniftbest eingerid)= tet wirt, nur muв Der Raum zwifden Der Eroe und ben Genfterit an bet nedrigfen Seffe wenigften 1 fup betra=

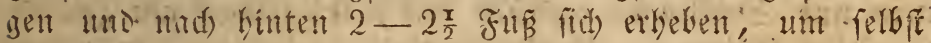

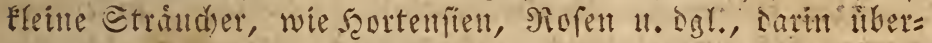

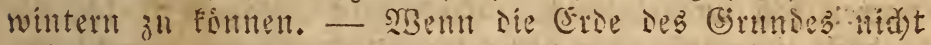

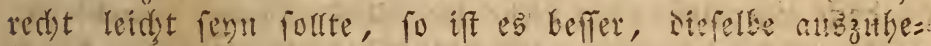

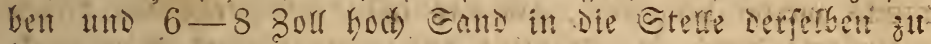
bringen, Dantit man Ite Iopfe vequem Samit ntmiteten faim. So lange es nidit freet oser regnet, Gleibeit bie

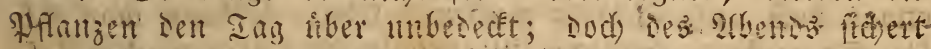

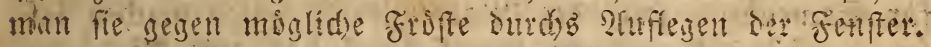
SWennt es aber etmiflid falt zut wersen anfougt; fo tegt man ring

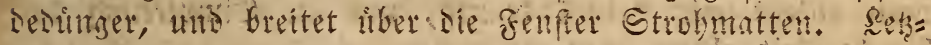
tere miffen, went die Eonthe fideint, abgettonmen wer= Den. atud gebe man fo oft als moglid, suff, samit bie

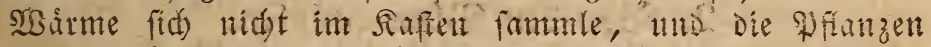
zum Ireiben anretze. Fortht Ednee, fo bleibe serfetbe nutgig auf sen Etrokmatten liegen, uno bey ftarfem froft bringt man nod) fo viel saranf, ba liegt. Co wie ahanwetter eintritt, mus man than fogleid) entfernen. Bey trocinem froft vermelye mant die sagen Der Strobmatten, Itto legt, Damit Der Mind fie nidit fo leidt berabweht, Breter Datiber. Biele Bartentbefiber th= 


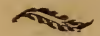

then, Bambrottet 1-2 Fun bod lither bie Fenfer zu Greiten, went $e^{b}$ zu frieren anfingt. Iheils bilt es aber oft fdwer, fids biefelben in foldyer snaffe zu verfdaffen; oft entfitbrt bas sanb Ier 2 Bind grofentheils wieder; theils

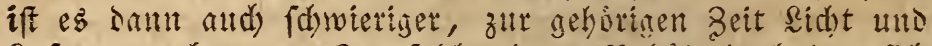
suft zu geben. - Sil fold einem Behaltnis halten fid

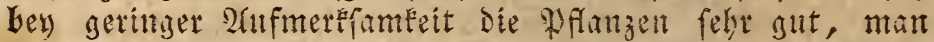
fann iesen Zutgenblict Iopfe Garntintehmen, wie man fie

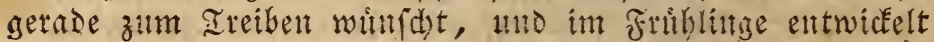

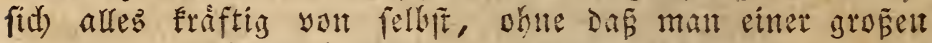

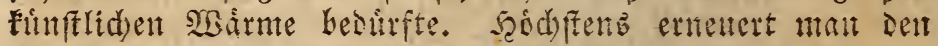
Miffumfdlas surd) eine thenc fratke sage.

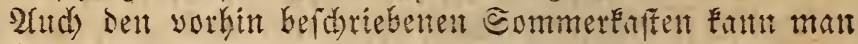

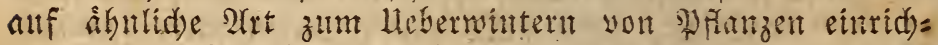
telt, and obgletds Dergleidgen bohe Bebiltnifle weit methe

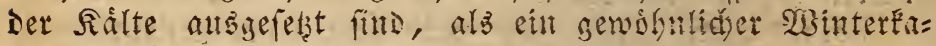

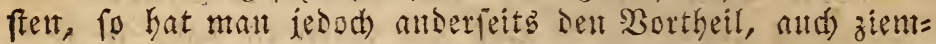

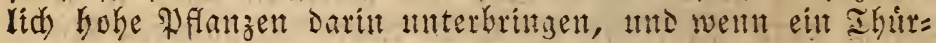

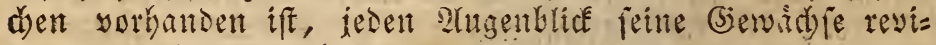
biren zu Eommen, ohute viel zu froren.

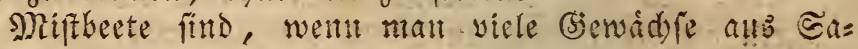

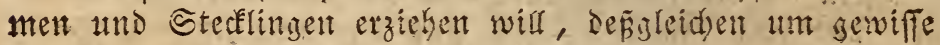

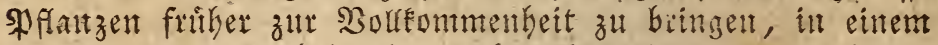

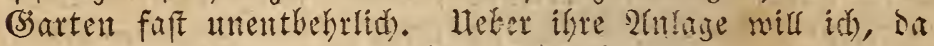
fie bod) eigentlid) nid)t mehr zur @tubengatuturey gebiren,

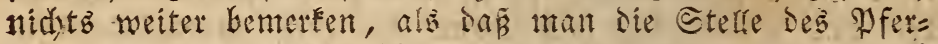

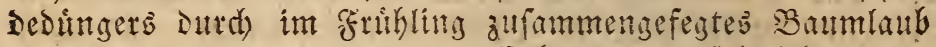

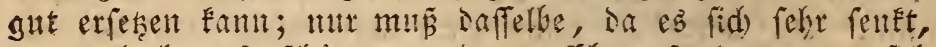

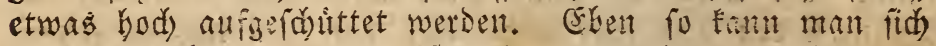
fir Camenbeete ftatt Der (S)labfenfter Rahmen mad)en laf́= fen, weldse riber Frenz von 6 zil 6 zoll nit Binsfaten befpant, uno mit Edjreibapier beflebt wersen, weldhez man zulest mit seinol trinet, um Die Eimwirfung Der Feuditigeit abzumehren.

\section{Bon Den Blumentopfen.}

Die beften Blumentopfe fint sie yon getwónlidem

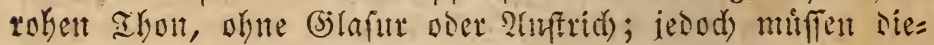

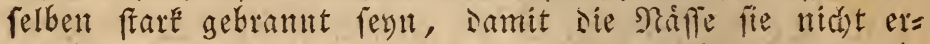
weid)t. Die Iopfe yout gorzerfan fitto nidyt viel werth,

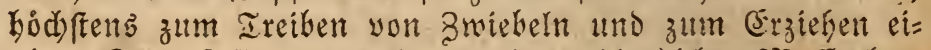

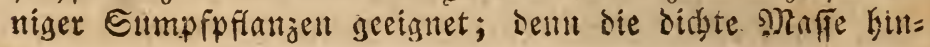




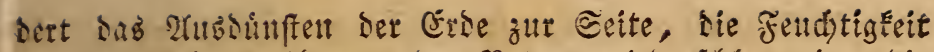

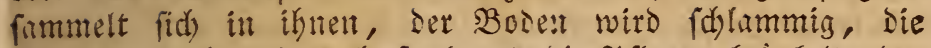

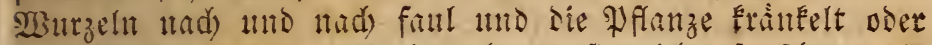
geht wohl gat eitr. 2(ud) nefment fie nidyt fo fdutll bie 28atme alts ber suft an, und theilent fie Der Erte mit, wab zun Geseiken ier Pfanzen fo nothwendis ift. Die glafirten Iopfe, Defgleidjent bie mit Rack ocer Delfarbe tibert=

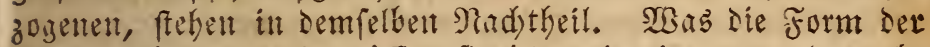
Iopfe anbelangt, fo milfent fie jeserzeit einem geraben $a b=$ gefurzten Jegel gleid)en, ltho zwar obent etwaz weiter fern, alb unten. Ferner muह íne innere flád)e vólfig glatt und eben jeyn, uno weder Bertiefungen, nod yoripringense Ibeile

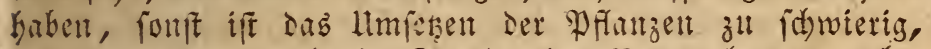
tmo mant ift oft nid)t im Etante, den Batlen berans za bes

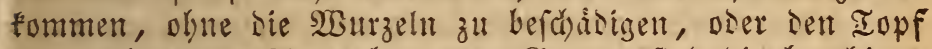

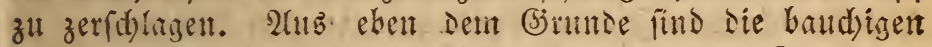
oder als \$2ajen geformten Iopfe gatz zat verwerfen. Der Booen mú in ter Mitte am tieffen fenn. 2(uperbalb et=

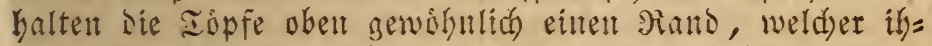
nen mebr Fefrigfeit giebt; zit weit sarf jebod Diefer Rant nid)t uberzelogen feyn, oder vorfpringen, fonft nefmen fie

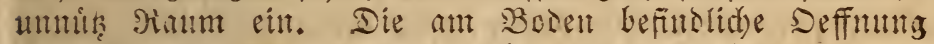

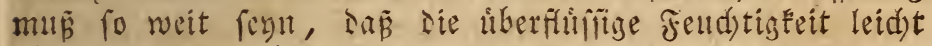

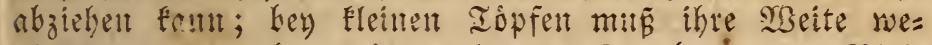
nisjens $\frac{x}{2}$ 8ur, bey grópern $1-1 \frac{x}{2}$ zolf betrigen. Biele Iopfer madjen 3-5 Heitte sobder in ien Boden jesez Iopfes; Diefes tomgt aber nid)ts, Dent Diefe geringen Deff= numgen verfropfen fid leidst, mo ihr awed geft verlorent.

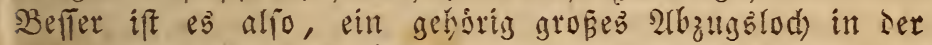
Mitte Des Bodens anzuluringen. Beym (j)ebrand) Der Iopfe

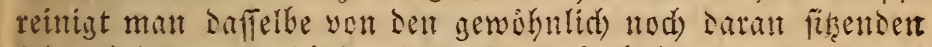

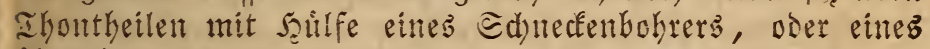
åhnlid)en eifenten Sinftruments, womit man zusletch bie Seffnungen nad) Belieben erweitern Fann. Damit Lie Eis=

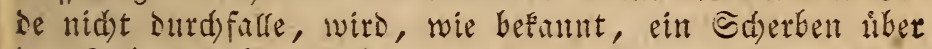
bas Rod) gevedt. Redder in ien Seiten Ler Iipfe anju=

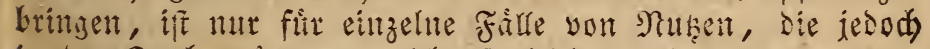
in ber Etlibengatrtnerey nid)t fo leid)t yortomment.

Wab Die Gróse Der Bltmentipfe anbelangt, fo Eant

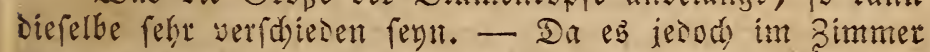
meiftens an アaum fehlt, io laffe man Die Bhumentopfe lieber etwas bỏher, als zat breit madjent. Die meiften (s)e= wád) fe fut Iamit zufrieden, und den wenigen, weidse hierin 


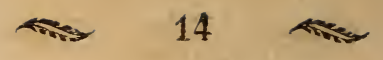

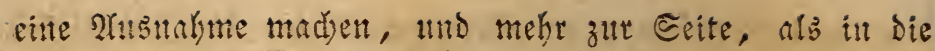

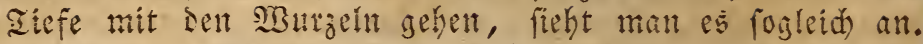
Nati, went Dffanzen fehr bod wad) fen, mo vieleidyt (d)were fronen befommen, fo muffen siejelben mberingt geforig breite Iopfe erbalten, Damit fie feft fiefen, und

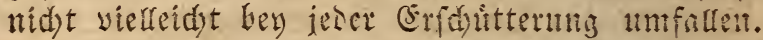

Meine Blumentipfe Gaben sewohnlid' miserbalb nad) theinluntifidem Mara folgente 2lbmeffungen:

a) Eleine Sorten; 1) $2 \frac{\pi}{2}$ zoll hod unt oben eben fo breit; 2) 4 3olf bod uno 3 zolf breit; 3) 4 3olf bot) uno $4 \frac{x}{2}$ soll breit.

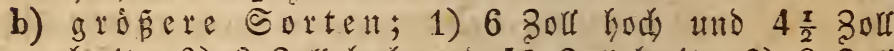
Groit; 2) 8 zolf hod) uno $5 \frac{\pi}{2}$ zolf breit; 3) 8 zoll bod) uno 7 - 8 soll breit.

2fuser Diefen gentantent fann man fid yon Sen Kleits= ften Corten nod) einige Epalttopfe za Den 2rblegern ferti= gent raffen.

Da bie Sopfer felten ganz gentu thad) der Zorfdrift arbeiten, fo erbalt man gewobnlid ieresmal cine-alenge Bwifdenfortent.

Die Unterfę̧uåpfe ridben fid und ser form Ler Błu= mentopfe, fo saj biejelben Darin yolriy feft ftehen fỏnen

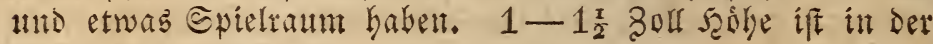
Regel binteiddent.

\section{Szon Den Eronten.}

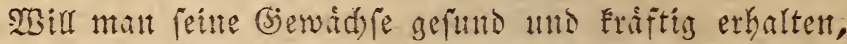

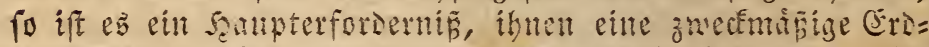
mifáuni zall geben, fo wie lese alrt siejelbe befonoers liebt. Bé eittiget llebung wird man es ber Pfanze balo anfeben, ob fie fandigen, foweren, freitigen ober Enmpflosen ver=

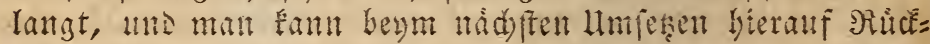
fid)t nefintent.

Die Erdarten, welde man yorzhiglid brandst, firo: sauberde, sehm, Iorf, Sand und Kies.

saub = ooer selolorie erbalt man am befen, went man auf Whasen, wo feit vielen Solnten şolz gefleint ooer bearbeitet worsen iff, Die gut gefanlte (srie ser sherfitid) zufammenfdanfeln, Durd) ein Drabtoitter werfen, uns fo=

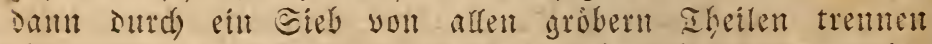

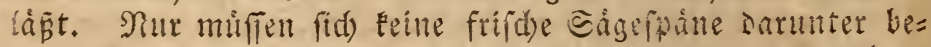

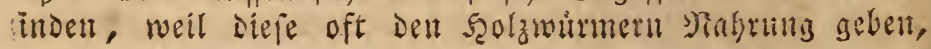




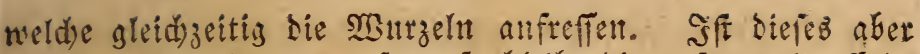
nicht zu yermeiten gewejen, fo bleibt die gefommelte Grde

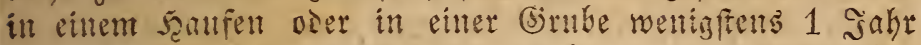

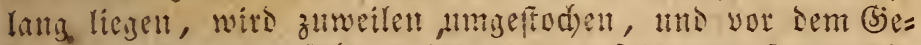
brand) nodymals gefiebt. Szat man auf Dem sofe oser is:

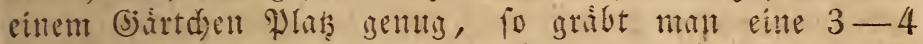
fus tiefe Grube, nud fürt riefelbe mit saub, Etroh, lln=

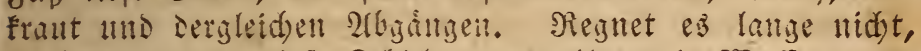

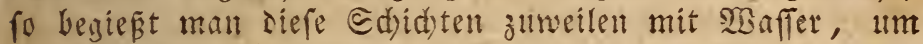
die Făbluis zu befortern. Etwa alle $3-4$ Monate fticht

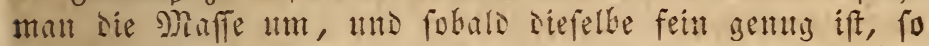
mird fie gefiebt, und sie groben Ifeile netrerding zwifden

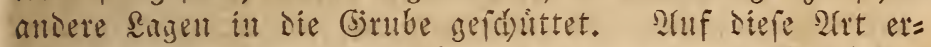

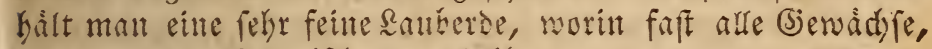
ofne weitere Beymifidung geseiben.

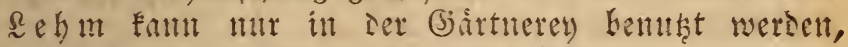
wenn er geboris vermittert ift. Ien befen bierzu tamgli=

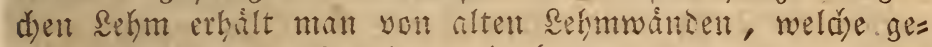
wofnlid) ght mit Etrob surdorbeitet gemeren fins. Sat

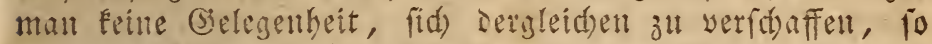
mus Der frifd gegrabene sebm, mit etwas હtrob vermengt, $1-2$ sont an ser suft liegen bleftern, und ofters umge= ftodin wersen. Iann wird er zerflopft umo gefiebt.

Mit sem Iorf yerfithrt man auf åbulidbe Metje, tut

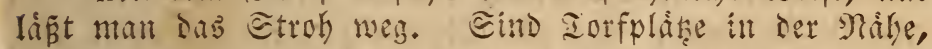
fo fincet man cajilbf oft sanze saanfen, die aus vetwits

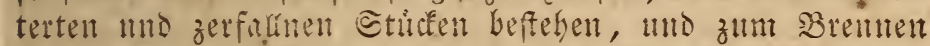
nid)t mebi tanglid) fins. Se weniger sijentheile ser Iorf enthilt, um io beffer ift ex fur Die (sartneres; Dod) mifde man lie za viel unter sie Crise.

Der befte $\varsigma$ and if Derienige, welden man ans Flüs=

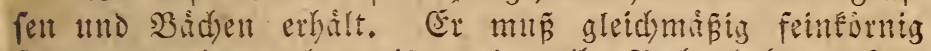
fenn, und eine mehr weife, alb gelbe Farbe baben. Sesh= teres ift ein Beiden von beygemijidem (Eifenoxys. N⿴andhe Cantiguben enthalten ebenfals enten febr reinen Eand.

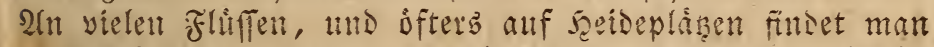
einen febr feinen, mit etwas sefm gemenisten Sans (હ)lide),

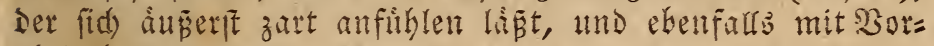
theil benust werden fanm.

Aieb wird gleidfarla in und an Bathen gefunden. Da man thn jeodd in ser Stubengúrtnever feltner bentht,

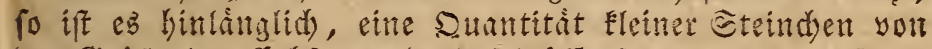
Der Sroffe der Erbjen und Safelmiffe fammeln zu laffen. 


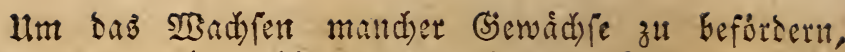
mengt mat aud wohl etmab yerfaulten Noift unter die

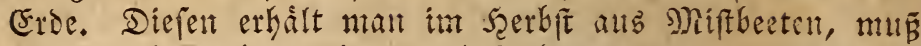
ifn jedod) nod) mentigftens 1 sabr in Szanfen liegen Iaf

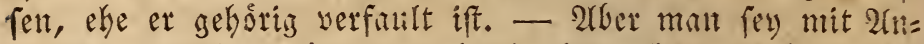
wenoung Diefes Dintungsmittels ia red)t vorfidhtig; es fidabet oft mefre, als eb mitht, und viele pflanzent, nament= lid) Die feinern Cappflatzen, Die Neubouther, die Camel

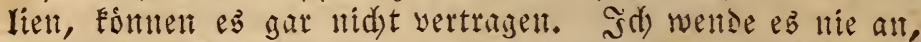
allser bey ganz ordinaten હad)en, z. B. Feisenbáumen uno einigen ansern.

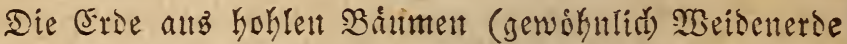

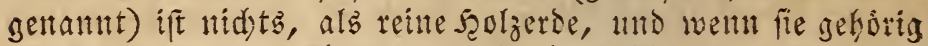
gefault ift, gut ju bentsent. Dod enthált fie midjt feltent

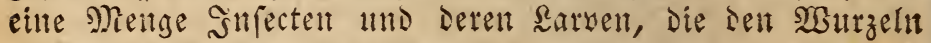
nadyetheilig werden fỏnthen.

Die sauberse tift es, wie bereits obent gefingt worsen, weldye yorzliglid) zum Erzieken yon planzen bentst wirs. Se nadjem die Erise des Drtes war, wo fie gefammelt wurbe, nadbem Eant fie aud (d)on mit Santo ooer mi:

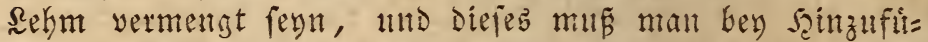
gung ser andern Beftantheile nid)t auser 2ld t laffen. Bey allen Nifdunten ift befonders Darauf zu balten, Das nie

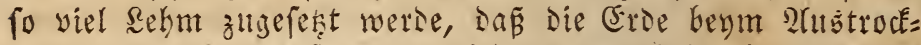
men zu feft wirs; fie reist albsant gemeintylid) auf, uno

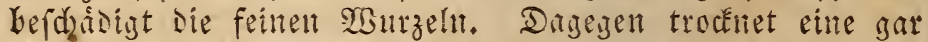

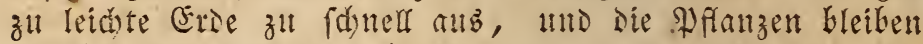

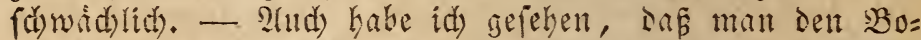
Denfar beym Faffeefoden, oder sent pogenannten Raffees grutb, fammelte uno unter bie Blumenterie mift)te. SBent man Denfelben einige Beit fanlen láft, fo erbălt man als Yerdings eine fehr feine (Erbe, die nuperordentlid) Dazu ges eignet iff, idweren Booen ju verbeffert. Sids felbif babe ifn fefr anwenton gefutien.

\section{Heber ons Degiefien, ben Bebraud ber Hnter fetrinfe uno einiget Düngungsmittel.}

2utgemein anzugebelt, want umb wie oft eine Pfanze

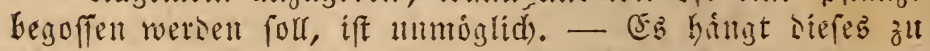

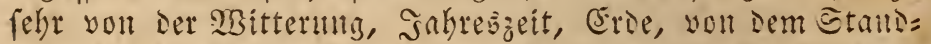
ort ber (Serwadefe, ano woll bem gefunden ober Franeen Bu=

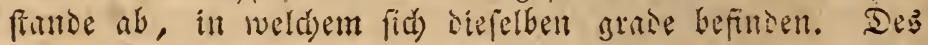


Die Erse yother fehr troden und vielleid) aufgeriffen wat. Daut linft Das waffer ab, ohne Die Erde zu Durdborin: gen. $\mathfrak{H m}$ biejem zu begegnen, besient man fids ber $\mathfrak{l n}=$ terfehnapfe. Diefe find in Gommer fur bie meiften Dflan=

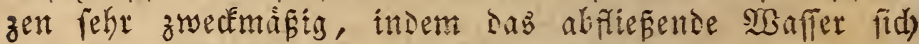
saril fammeln fant, uno nadjer mieder eingefangt wird. Fit Fettpflanzen find fie jeiod nid)t zu empfehlen, und

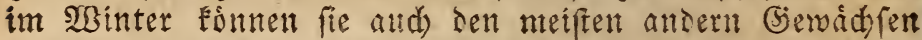
nad)theilig werden, fobald Waffer Darin länger als einen

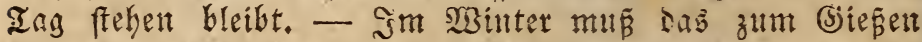
beftimmte Waffer wenigftens verfdulagen, oder etwa 10

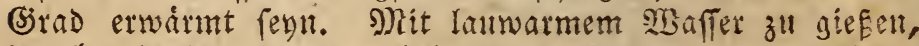

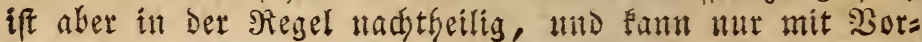
fidt bentibt wersen.

(Sejunde Pfanzen bedurfent melyr Sarfer, als foldye,

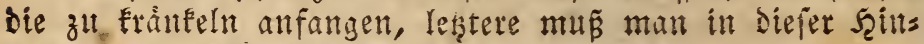
(iid) fehr in 2ldit neimen.

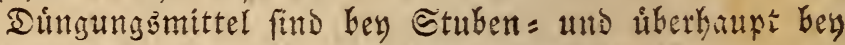
Topfpflanzen, nur mit áuferfter $\mathfrak{B o r f i d}$ anzumenten. Einigez ift beretis bey sen Erdarten bieriber gefingt wor= Den; Nefreres Fonmt bey Befdreibung Der Pfanzen, na= mentlid) unter dem 2(kfid)nitt, Citrus, yor. Ceines Der

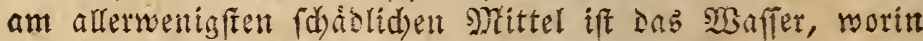
Fleifd) gewaiffert worden ift, aber aud biefes benthe man

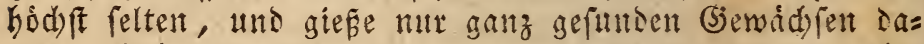
mit. Fráneltide Pflanzen vertragen ez ganz und gar nidjt, und man glaube niemals, ignen Dadurds belfen zal wollen; fie befommen mur reines Maffer.

Dás ถas $\mathfrak{F l u}=$ m

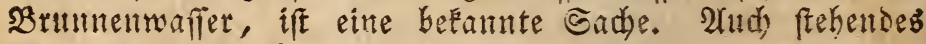
Waffer aus (Sruben und Iltmpelt ift gut, wenn es nut nid)t zul unrein unt ftinend ift. Sn biefem Falle eignet ez fid) zwar fúr Den (s)arten, befonders zum Begieñen Der

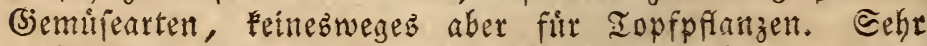
wohlthuend ift den Pfianzen im Fruhlinge, befonders Dent F̧olzern, weldse obne Blätter freben, ehe die neut Iriebe

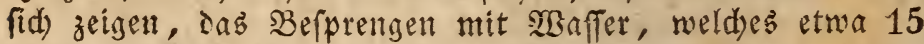

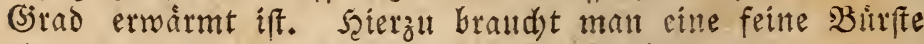

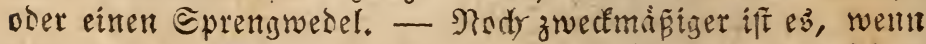

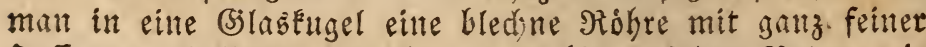

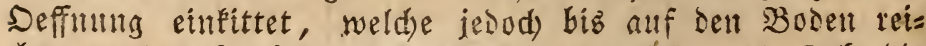

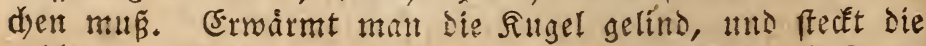

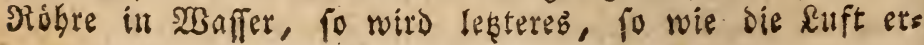


Paltet, in dic Singel bineinfteigen. Nun biaf man fo stel suft hintein, als mojglids ift, und biefe wird bann einen

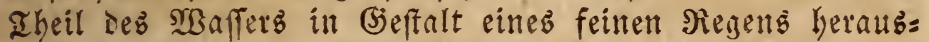
treiben, Sen man nad) (Siefalten auf Die Pflanzen leiten fant. Beffer ift eb nod), die ßóbre fo mit einer Sdyrabe von Binn zu verfehen, oa fie auf bie fingel luftoid)t auf=

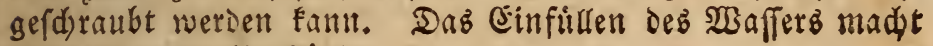
Dann weniger llmftánde.

\section{Ueber bas Berfergen.}

Wenn eine Pflanze einige Beit in einem Topf geftan= ben hat, to baben die Wurzeln derfelben die Erde zu fegr

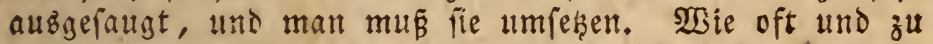

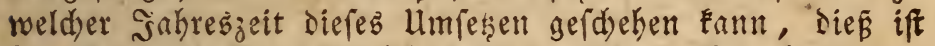
bey jebem einzelnen (sewád) gefagt worden. Manche Pflan: zen find in siefer frinficht fo wenis zártlid, da umperen gar nidut fiórt, uno bey andern, z. B. bey meks= rern Eactusarten, darf es nur fehr felten gefdefen. Eino die Wurzelu einer Nflanze nid)t zu ftare oder fleifdig, fo wers

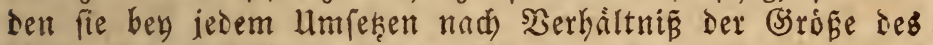
Batlens etwas befdnitten. Man gewinnt hierdudd den Sortheil, lange zeit Eleine Iopfe benthen zu Ẻonnen. Flei=

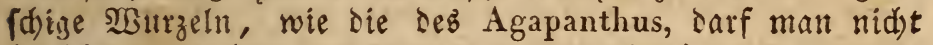
befdádigen; eben fo vertragen die Nentsokututer dab ftarke Befdnetien Der $\mathfrak{B u t}_{2}$ eln nid)t gut, befonders pobald fie fdon eine anjefnlide (5iríge erreid)t Gaben.

Nad) Dem Umeesen bălt man in Der Regel bie Pflan: zen einige zeit fdattig uno feudst, bis fie zu wadjen an=

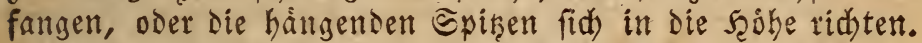
Fleifdige (semád) fé fonnen fogleid) in die Eonne geftert werden, denn auf fie bat bie Berainderung Des @tandez Der Wurzeln Feinen bedeuteno nadhtheiligen Einfur s, ja im (B)egentheil faulen fie leidyt, wenn man fie lange im હd)at:

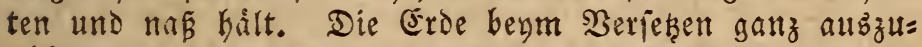

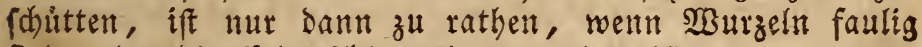
fino, oder die Erde (d)lammig geworden ift.

\section{Heber Das Beftueioen, 2ubinden und 2ufs ftellen Der Gtubenpfianzen.}

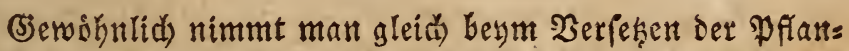

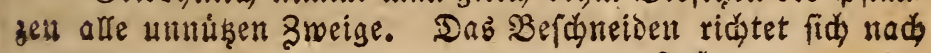


Der 2rbfide, weldye man mit ber Pflanze bat. Soll alts eitrem Strand) ein Brumben gezogen werden, fo nimmt matt de unterfen aweige ab, und lifigt elitweser mut einen

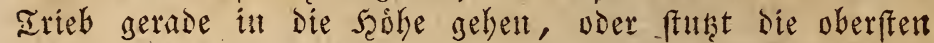
Sweige ein, bamit fith cine frone bildet. Bieft mant eis

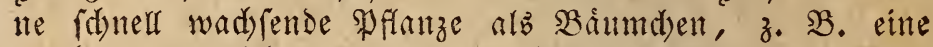
Berbena, fo fehe man Darauf, Dá̉ Der Etamm ganz ge=

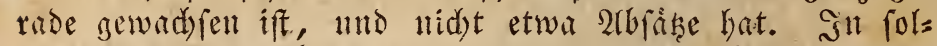
d)em Falle ift ez beffer, Dell Gtamm 1 zoll liber ber MBar=

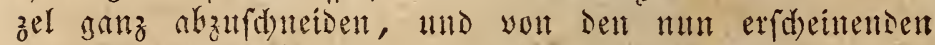
Eproffen Den kriftibfen gehen zu laffen, and thn gleidy anzubinsen, samit er nidit frumm wadje. SBifl man ei= net Wfanze eine beftimmte Form geben, $j$. B. Die einez

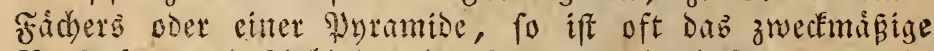
Serftecten und 2rnbinden Der afefte vortbeitbafter, als eit

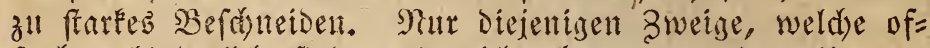
fenbar Ginderlid) find uno nidst bentset werben fonnen, (d) ueibet man fort.

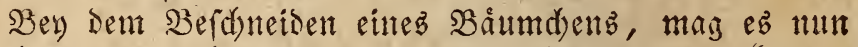
gefdeben, zul weldem Sweet eb will, thut man am beften, Daffelbe gleid) nad) Der Bhithezeit yorzunebmen, indem mant fonft mandide sinftige finospe zerforen witroe. Dant aber

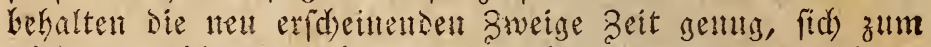

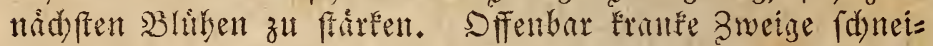
Det man zu jeser Sakyegzeit ab, und zwar entweder sid)t an: Stamm, oder Dodj unterbalb Der Franfent Stelle. Für

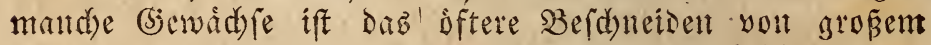
Bortheil, indem nur bie jungern Bweige gut blihen; viele Pflanzen yertragen eb wentiger. - STGit man énzelne Bhăt= ter yon einem (Sjewadds entfertnen, die aber nod) faft am Bneige fithen, fo mits mant siefelben feinešneges gemalt:

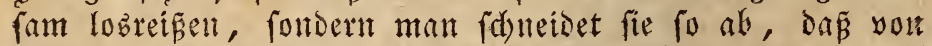
Dem Blattfitel nod) $\frac{x}{4}-\frac{x}{2}$ zod fieken bleibt. Diefes Strid fárut nad) einigen Iagent youn felbit ab.

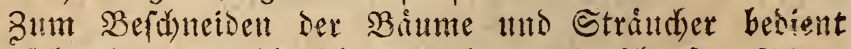
malt fid eilles gewibntliden farken uno fdyarfent Feoer= meffers; nut wenn die zweige bereits fehr ftark fint, nimmt

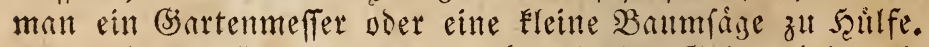

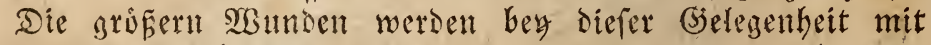
Bammwad)z berecte Damit defe Eterfen nid)t zu fehr aubs= troctith, utto friher mit Minte itberzogen werden. SBentt Pfanzent viel હaft oder Mild enthalten, fo trocknet man

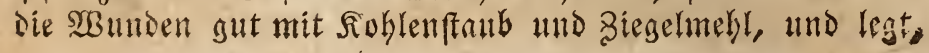




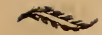

wenn man $c$ fa fir nothwendig hilt, nady eintigen angen Baแmwad) sa Darither.

Die Stócle zum 2rubinten ber Blumen fdutidet man

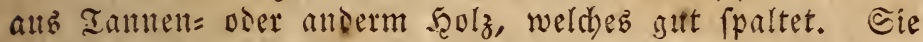

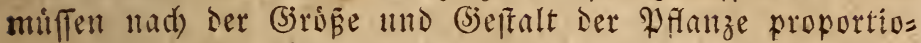
nirt, aljo nid)t ftirfer fern, als nothwendis ifi, sent (J)e

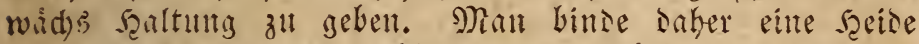

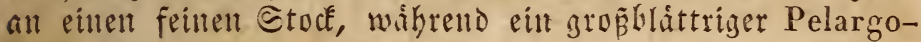
nium eintell weit ftirfern erisalten Eann. Ferner milfen

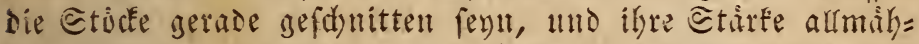

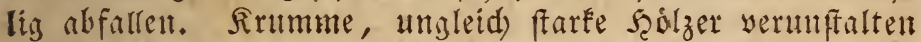

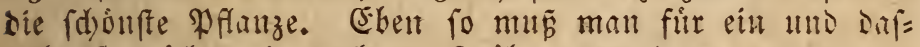
felbe (S)emád)s thie mehrere Stábe anmenten, wenn malt mit eitler eitzigen Eththe altsieid)en fann. Beym 2tubin= sent felbit verfabre man mit geboriger llmfidet, tullo binte

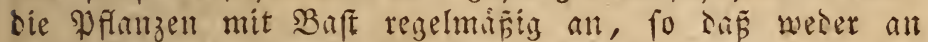

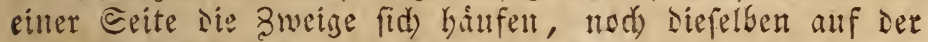
nudern wieser fehlent. Die 2leftyen muffen ferner fo anges bunden mersen, daf ifre Episen frey bleiben, nud nirs gent weder ein Preffen, nod) eine Reibung @tatt findet.

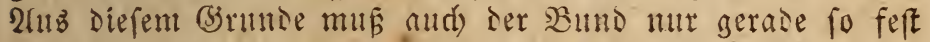
gemad)t wersen, als es nothmentig ift. Snan nehme nie

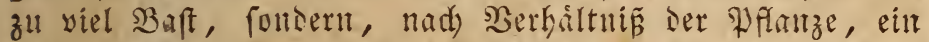
fd) males Streifichen, widkle diefeg 1-2mal um, Enipfe

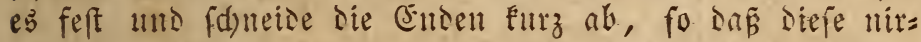
gent herumbängen." Sif man gentothigt, an eillem fthrtern Etamme eille frumme Etelle gerade zu ziehell, fo iftes it biefem galfe freilid) nothwentig, ein ftarfes Band ans zumensen; Dod) mun aud basurd) nid)t die gefärlige Form Der Pflanze verioren gehent. Den Baft made man yor Dem (Jjebraud) jesesmal nas, bamit er gefd)meidig wird. Zwirn, Bindfacen u. Digl. zum 2trbinten Der Pffanzent zu Gent=

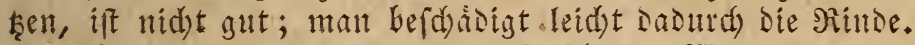

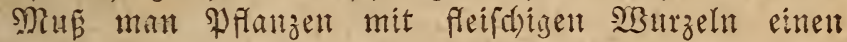
Etocf geben, z. B. Agapanthus, Alstroemeria u. a., fo thute malt Diefes entweder gleid) benm Umfescent, oder fies cle mentigfenz eittent ganz furzent Etock, Det nur etwa 2

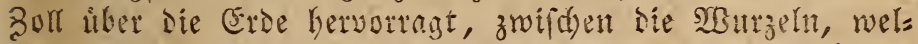
d)ent man herausjegt, fobald man eitlen langen Etab all=

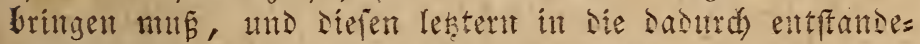

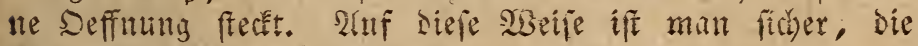

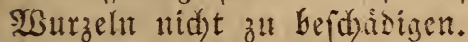

Die Blumenfáde werden oft mit Delfarbe angeftrider, 


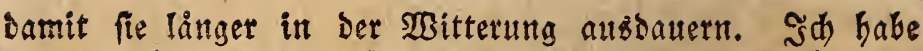
es nie gethan, fondern fie immer im roben Buftande benutht. Wer ez aber thun will, der wáhle dazu eine Duntle, nidid alffallende Farbe, z. B. grau, grůn, bråunlid) u. r. w. - Dft befommen Diefe Etåbe einen red)t grelfen $\mathfrak{A} n=$ frtiä), wobl gat tothe Sispfe tho weike Ringelden; oft fiti Die obern Spisen mit Sugeln, fronen oder niedlis

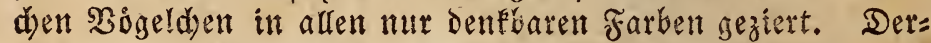
gleidhen verratth jebod einen verdorbenen (Siefchmact, und wird yon Eeinem Menfden, der $2(n$ prud auf teinen Sinn furb mahrhaft Sdjone madyt, nad)geahmt werden. Die Blumenftabe folfen ja nur zum Scalten ber (Semád) pe Dies

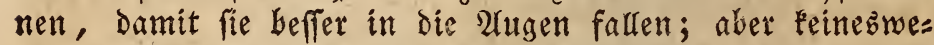

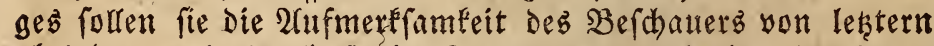
abziehen, roie durd folde Epielerenen gefdiebt, die jeders zeit einen wisrigen Einound mad)en, indem fie bie fdjone Einfadbeit dez Sonanen ftorelt.

Wie viel úbrigenz baz gute und zwedmingige Befefti=

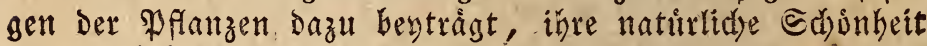
berworzukeben, wird derienige empfinden, der einige 2 htf=

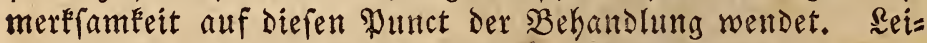

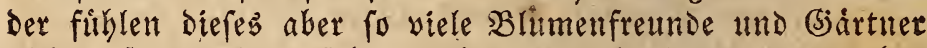
nid)t; fie wundern fid , woher ez wohl fonme, Das bey Defem oder ienem ihrer Freutnde afe Pflanzen fo gut ftehen,

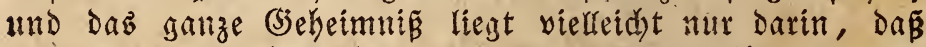

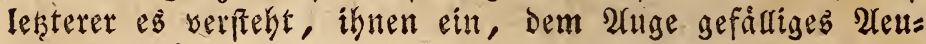
Bere zit gebert.

Daz Nåmlidbe gilt atth von Bufammenffetten ober Gruppiren Der Pfanzen. Wer Die Iopfe obne Wabl und Reegel Durdeinamber ftehen láf̆t, deffen (Bewaidhje werden lans

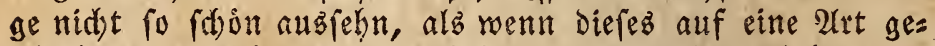

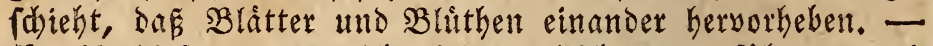

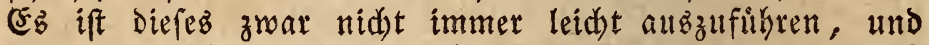
man mus fidi) im Bimmer, bejonders itn Minter, oft nadb \$labs und Umftånoen riduten, allein eine gewiffe Dronung lást fid jederzeit beobač̉ten. Diefelbe Eann aber nidjt ges lehrt, fie muß empfunien werden. - Stedt man Pflan= zen alf einen Iritt, gefthehe diefes im 3immer oder im Fresen, fo werden die niedrigen, Pflanzen in die erften Reiben, und Die bökern nadh binten geftelt: ganz bobe Sa= d)en bringt man am zwedtmáp̂́tgften zur Seite deş Geftel= les an; Eahen, weld e imponiten folfen, z. $\mathfrak{B}$. eine $\mathfrak{F} \mathfrak{a}=$ derpalms, fommen auf bas oberfte Bret su fteken, Damit 


\section{Afro 23 Axer}

fie, wie in ifrem Satetlande, über alfe Umgernungen bit:

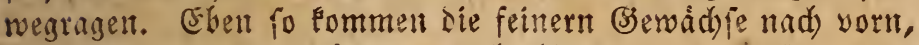
Die mit grósent SBláttern nad) Sinten. - Beffibt man

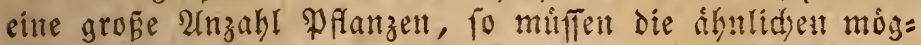

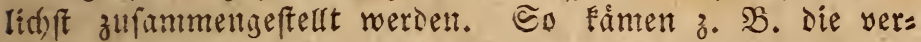
rdiedenten 2rtten Der Erica, Diosma, Melaleuca, Leptospermum, Calothamnus, Phylica, Acacia 1t. f. w. neben

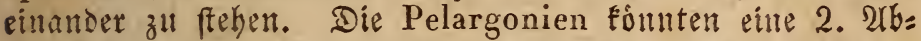
theilung bifben, Cactus, Aloë, Rochea, Stapelia, Crassula und die Mesembrianthem eime 3., Die Camellien, Dillenia, Citrus, Rhododendron, Nerium, Magnolia, Ilex, Ixora, Azalea u. Dgl. eine 4., Agapanthus, Amaryllis, Canna, Eucomis, Hemerocallis, Iris, Lilium, Sisyrinchium, Tradescantia, Veltheimia 11. f. w. eine 5., Die Ropenarten in Serbinoung mit Jasminum, Hypericum, Myrtus, Fuchsia, Punica eine 6., und fo fant man nady

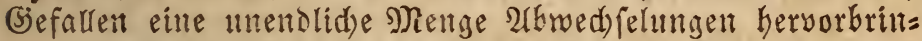
gen, Die surd) ihren (Eontraft einent fdounen Effect mad)ent.

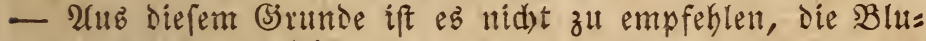

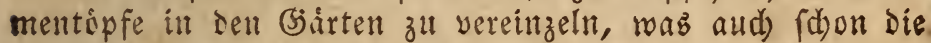

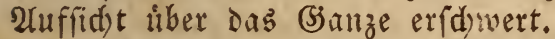

Hebrizens verándere man in diefer argfid)t nicht zut oft Den Etano Der Pflanzen, intem viele Derfelben eb́ Midt vertragen. und oft die- Inoakpen dadurd) leiden.

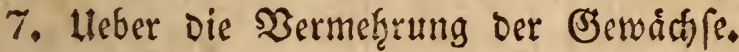

Bey Befdreibung Der Pfanzen ift dab Nothigfte hiet= iber (d) on gefagt roorden; hier nur nod einige Bemer= Funger.

(Siervad) re, weldye man nid)t surd) Samen oder zers theilung vervielfáltigen Eallt, wie die meiften Băume und 6trind)er, vermeknt man Durd) arb fenfer oder Etecflinge. - Die 2lbenfer Funnen zu jeder Subregzeit gemadyt wers Den, benn man ftort Den Fortigang Der 2 egetation nur we= nig. - Man bentht Gierza geroifnlid die unterften 3weige, oder ftehen diefe zul bod), fo bindet man einen Hleinen $B$ lu= mentopf in Der erforderlid en Sroke, zwifden 2 oder 3 bins

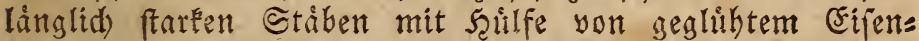
brabt feft, fürt benfeiben mit guter, leid)ter Erde, ziebt den abzulegenden zweig nadj iemrelben bin, sient ihn in bie Eroe 1-2 3oll tief, uno bált ifn Durd holzerne fráts d)en in feinet lage feft. Man giebe fieisis, und bamit 


\section{\& 24 सis}

Sie Eonme Die Erbe nidht zu ftarl allstroche, bebelt mant fie mit Moob oder mit einem @tud verfehrt alfgelegtem

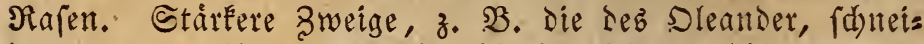
Det man zuweilen etwaz ein, Die ber feinern Şólzer, z. $\mathfrak{B}$. Erica, Diosma 1t. Dgl., werden ohne গ্Beiteres eingelegt.

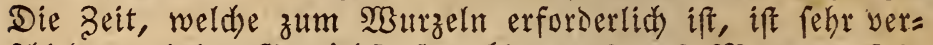
fdieden, einige (Ssewad) fe find binnen 2-3 Monaten feft: gerwad) (ent, wåkreno man bey Camellien, Magnolien u. ogl. oft eben to viel Sahre warten mus. - Die Epalttipfe (weld)e man aud yon Bled) mad)en Fann), um eitten

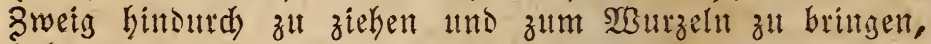
baben mir nie red)t gut gefalten, und verdienen nur in eins zelnen Fallen 2lnwentung. Sann man ez aber moglith mathen, einen zweig nad) obiger 2(rt umzubiegen, fo ift diefes weit beffer, indem er an Der gebogenten Stelle friber

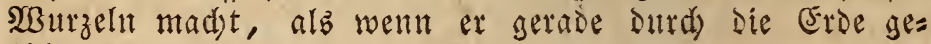
fithert ift.

Die befte Beit, Steclinge zu madjen, ift bey jesem

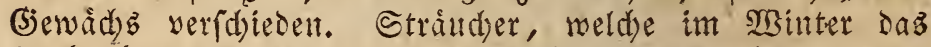
saub abwerfen, laffen fid an leidjteften vermegren, went man bie, Etereflinge mad)t, ehe fid sie neuth Blitter ent= wiffeln. Bey andern pfranzent fdeint die paffentefe zeit

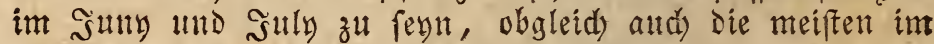
Friblinge annebmen. - Menn es moglidy ift, fo fdneide

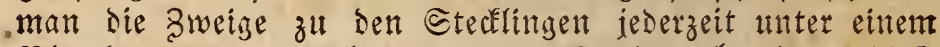
Blattenoten oder Didht am alten sweige ab; Denn diefe

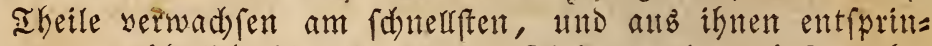
gen gewolknlid) die 2 Burgeln. Diefe Sweige durren aber aud) in ber Regel nidst zu jung fern, fondern miffen et:

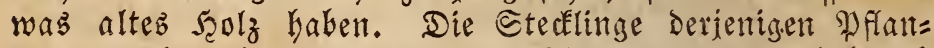
zen, weldse fehr zart fint, oder fdiwer wurzeln, babe ids ftetz am beften fortgebrad)t, wenn idi fie mit einem geráu=

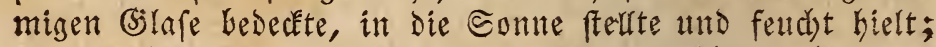
fo wie fie fart zu wadjen anfingen, sewobnte id) fie an Die $\stackrel{\Omega}{\sim}$ ft.

Db man bie bewurzelten Steclinge gleid) in befondere

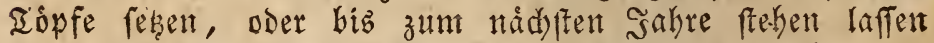
fort, Gángt yon Der Sabrebzeit umb PFanzenart ab. Die

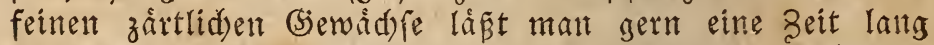
ungeftort, Damit bie $\mathfrak{S}_{3}$ rzeln fid yoúfomment ausbilden. Die Ctectintige Der Pelargonien unto anderer Wfinazen mit etwas faftigem 巨tångel, nebmen fehr leid)t an, went man fie eine zeit lang befdinttet, fouft aber in firyer suft Gált. 
Sann man fie in eitt maisig warmes Miffbeet bringen, fo

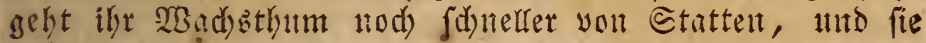

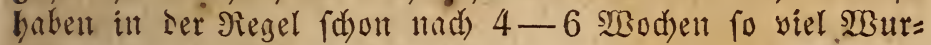
zeln, Dá fie befonders gepfanzt werden Fonnen. - Die Etedflinge ses Sleander werden einige వeit ins $\mathfrak{X a f f e r ~ g e = ~}$

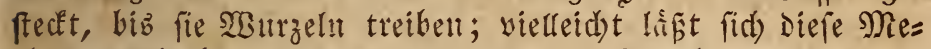

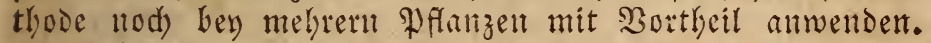
Sd) Gabe aber nod) Eethe Serfude gemadt. - MBenn man

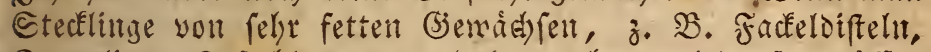
Etapelien, Baferblumen 4. Igl. mad́den will, fo muffen Die sweige, ehe man fie einfekt, einige Iage liegcn bleis ben, Damit fie welken, uno fâhig werden, Den stabrungsz= ftoff aub ber sirse einzufangen und aufauthnen. Sonft

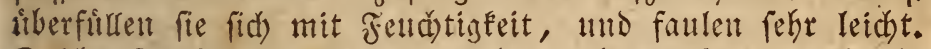
Eoldye Stecklinise mú man Daher mefr trodkelt, als feudst balten; am beften ifteb, fie im sumy und suly zul ma= d)en, und fie an einen fonnigen Plats zu ftellen, wo fie gesent Regen gefithert fint.

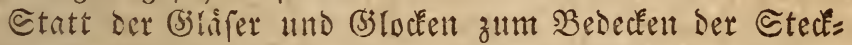
linge besienen fid) viele (s)irtneb glaifertier Irid)ter mit $\frac{x}{4}$ $\frac{x}{2}$ 3oll weiter Seffnutg, welde mit einem Furffoppel ver= d)loffen meroen fointen, ober Durd) weldye man nad) $\mathfrak{B} e=$

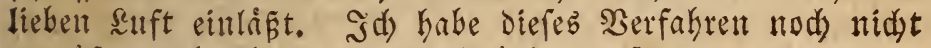

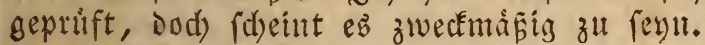

Wab Die \$ुermefrung Duttd) Beredeln anbelangt, bie bey eitrigen (sewadd)en, z. B. Citrus, Ilex, Punica, den $\Re 0=$ fell 1t. a., altgewendet wird, fo habe id bey den Rofen (Eini=

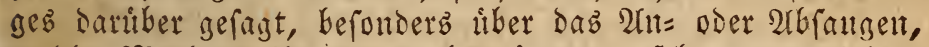
weld)e Methode fúr die Etubengårtnerey Fehr ju empfehlen ift, ba fie fid) gut aubfikgren lispt, uno man fogar ziemlids

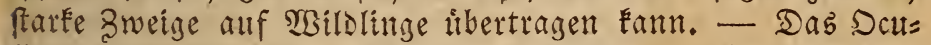
liten und Pfropfen nad) Den verfdiedenen Methoden ift

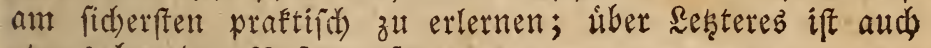
etwab ber den Rofen gefagt.

\section{Bon einigen Feinben Der BSartnerey.}

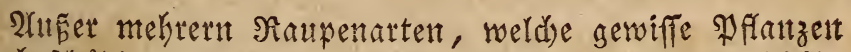

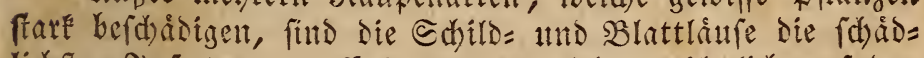
lidffen Sinfectett. - Erftere finden fid gewobnlith auf ben S3åumen utto Stråud)ern ein, weldhe immergrune Blätter Gaben, verberten fid) aber and) nidet felten ibber andere Wflanzen; to Gabe ids fie niddt felten auf Dem indifdren 


\section{6}

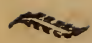

Bfuntentoge, Canna indica, sefunden. Es gift you den Edjiblaulen einige Srten, uno ábulid mit ibr ift sie ro= gentunte Raffeelang, welch) vouguglid alf Raffebåumen als cin wcišs, faft fdimmliges sinfect gefunten wird, tuto aud) auf antere (seweid)fe fid verbreitet. - Durd of fteres 2lbwafd)en Der Blátter uno Rinte mit Şúlfe eines Sd)wam=

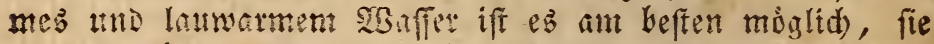

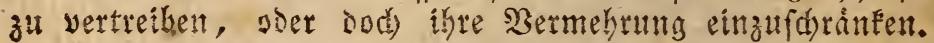
Die Slattluife, bejonders sie grume Sorte, fint Dent Pflanzen nodi) weit verserblider, als sie Edhilshiufe. Durd

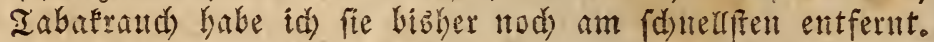
su bem (Ense ftellte id') alfe Blumen, weld)e mit diefem ungeziefer belaftigt waren, zwilden gut fditesende Doppers

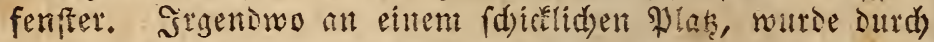
Den Fenfterahmen ein sleines Sod) gebohrt, fo da man

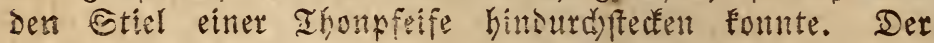
Rayf felbft wurbe etwa $\frac{x}{4}$ mit Iabaf geftopft, ein grof́ç Ertick gut bremnender E ofmamm darauf gelegt, und num Der

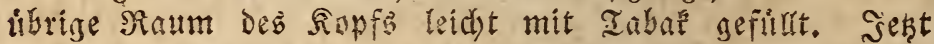

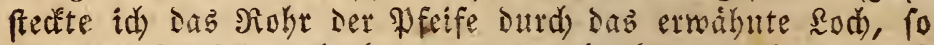

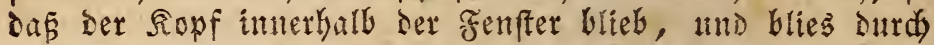

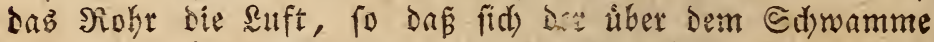

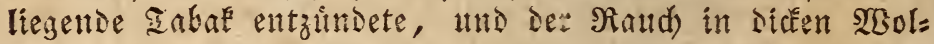
pen anfifien, mo dent innern Raum yỏlig anfülte. Die Fenfier wurben erft nad) 8-12 Ctumben geoffinet, wo Denut bie-metfen Blattláule berabgefallen waren, uth die ábrigeat fid ald bald verloren. Diefes Serfabren ift yon

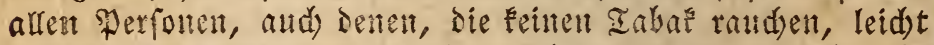

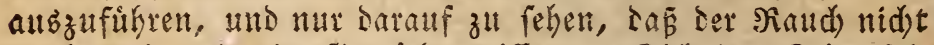

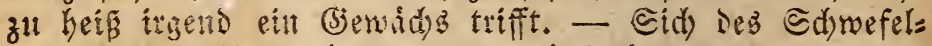
Dunffé zum Bertreiben der Blattlitufe bedienen zu mollen, ift nid)t gut; denn bie sadurd) gebildete Sdbweferfäure (d)a= bet ben meiften Pfanjen.

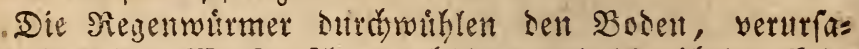
den, Daf Das Waffer fduell abfleft und Die librige Eroe

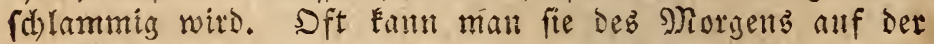

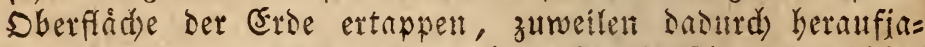
gen, insem man den Topf surd) fanftes 2 (nt fdalagen erfdut= tert. Feált mant sen Iopf einige Iage etwas troffen, uno legt dann ein @tick fentíten Rapen verkehrt auf bie sober= fladbe, fo Estriben fie oft dartuter; ebent fo, ment man einten fiadien Stein einige Beit Daranf gelest hat. - Sa bediene mid, um fie zu faffen, einer Fleiten fdmalen santge, 


\section{N 27 Nit}

wie die Uhrmader fie gebrauden, unt bitt babumd im Etans te, fie alt ibren @d)lupfwinkeln bervorzubolen, wetn ids

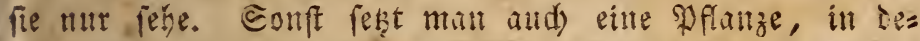
ren Iopf man vid segenwitmer vermuthet, um, wenn

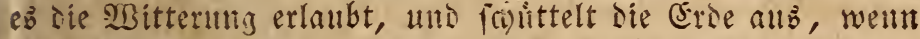

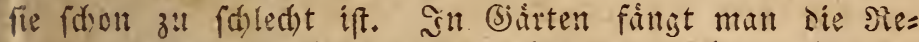
gentutumer an idywullen Eammernbenden am beften bets Der saterne, wo fie ihre sod)er yerhafen mo auf sor (Erde liegen. Dod) mus man baken fehr ftill serfahten, uns leife auftreten.

Die @durecten verwiften oft in Den (siartent viel jut =

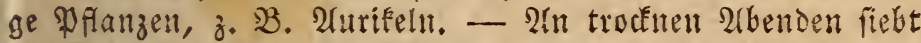
man liber die Etelfen, bie man fidern will, Ufithe, wor= ill fid) ein wemig zerfarner ungelof(u)ter Rale befinset, pods

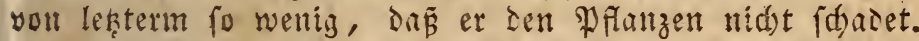

Die (Errfobe folfen fid Dasurd) yertieiben laffen, daz man einige alte jeringe in SBaffer faulen liät, und mit Diefer Dzifdung die jungen Pflanzent begieñt. - Sd) babe eb nod) nid)t verfud)t.

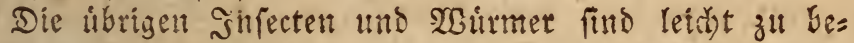
merfen unt zat vertreiben, went man die Etellen unter= (uid)t, wobin fie fid) am Iage verbergen.

9. Erflarung einiger $\mathfrak{B} e z$ cichnungen, und Furge lis. berfidit Des Rinneifchen spflanzenfyftems.

Die bey Befdreibung Der (B̈ewád) fe yorfonmenten Bes zeid)nungen find bie allgemein anerfannten und gebráud)li=

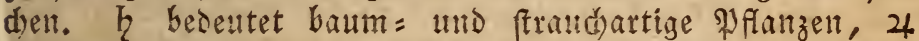

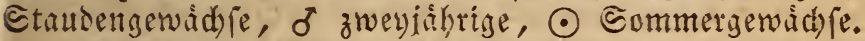

Die bey Den (jattuttgsnamen fehenden romifden $3 a 5=$ len bezeidunen nad) Dem sinneifa)ent Eerualinftem die હlaffe, in welde sie Pflange gebort, rie arabthen Biffern aber bie Dronung riofer Claffe.

Da $e b$ vielfeidyt nidyt unwiakommen feyn sirfte, etwas Nákeres ulber Das sinneifde Syftem zu fagen, fo habe ids (d)lüslid) nod) forgente Furze lleberfid)t seffe!ben bengefugt.

Die (Elaffen werden bis in die 12., nady der Zahl Der Etaubfäcen (Staminae), die úbrigen aber nadh Deren ßers báltniffen unter fidd und ifren 2 (n) eftungen beftimmt.

Die Dromungen grumben fid biz in die 13. Slaffe allf Den (siriffer, oder eigentlid) nod) mekr auf die Narbe, in Der 14. unt 15. Elaffe alf Die Samenfdosther, in Der 16., 17., 18., 20., 21., 22. Claffe auf Die Zahl Der Stamb= fáben, in der 19. Elafpe auf die yerfdedenen Sternngen, 


\section{Nise 28}

alf thren allgemeinen Frudtboden, und endidi in ber 24. Elaffe auf Die Derfdiedenheit ier Eryptogamiften felbft, wie folgt.

I. (5). Mo nandria: Einmånnige, mit einem Stnubfaden. 1. Srin. Monogynia: Eintweibige mit einem Srriffel, oder vielmefr Sarbe.

2. - Dyginia, mit 2 B̈riffeln.

II. Sr. Diandria mit 2 Staubfáden.

1. Sron. Monog. mit 1 S3riffel.

2. - Digynia - 2

3. - Trigynia - 3

III. EI. Triandria mit 3 Stanbfäben.

1. Ston. Monog. mit 1 siriffel.

2: - Digynia - 2

3. - Trigynia - 3 -

IV. Er. Tetrandria mit 4 Staubfäben.

1. Sron. Monogynia mit 1 Siriffel.

2. - Digynia -2 -

3. - Trigynia -3 -

4. - Tetragynia $-4-$

V. Cl. Pentandria mit 5 હtaubfáden.

1. Sron. Monogynia mit 1 Griffel.

2. - Digynia - 2 -

3. - Trigynia - 3 -

4. - Tetragynia - 4 -

5. - Pentagynia - 5 -

6. - Decagynia - 10 -

7. - Polygynia mit vielen (5iriffeltr.

VI. હ̦. Hexa ndria mit 6 Staubfáden.

1. Dron. Monogynia mit 1 siriffel.

2. - Digynia - 2 -

3. - Trigynia -3 -

4. - Hexagynia - 6 -

5. - Polygynia mit vielen B̈riffelt.

'VII. Er. Heptandria mit 7 Staubfådent.

1. Dron. Monogynia mit 1 (Siriffel.

2. - Digynia - 2

3. - Tetragynia - 4

4. - Heptagynia - 7

VIII. Cে. Octandria mit 8 Étaubfídelt.

1. Dron. Monogynia mit 1 Giriffel.

2. - Digynia -2

3. - Trigynia -3

4. - Tetragynia $\div 4$ 


\section{N 29 तs}

IX. El. Ennandria mit 9 Etaubfión.

1. Droll. Monogynia mit 1 Giriffel.

2. - Digynia - 2 -

3. - Hexagynia -6

x. હe. De c a ndria mit 10 હtaubfitber.

1. Drsu. Monogynia mit 1 Griffel.

2. - Digynia $-2=$
3. - Triggnia $-3=$
4. - Pentagynia $-5=$

XI. Cl. Do decandria hat 12-19 Etanbfíden.

1. Drou. Monogynia mit 1 Eiriffel.

2. - Digynia $-2=$
3. - Trigynia $-3=$
4. - Tetragynia $-4=$
5. - Pentagynia $-5=$
6. Dodecagynia $-12=$

XII. હૃ. I c osandria mit mefre als 19 ant Der intuern Ceite dez Feldeb angehefteten Staubfiden.

1. Sron. Monogynia mit 1 Griffel.

2. - Digynia -2

3. - Trigynia - 3

4. - Pentagynia - 5 -

5. - Polygynia mit vielen (s)riffeltr.

XIII. El. Poly a ndria mit mefre alb 19 im $\mathfrak{\text { Blumen }}=$ boden befeftigte Eraubfásen.

1. Sron. Monogynia mit 1 (jriffel.

2. - Digynia $-2=$
3. - Trigynia $-3=$
4. - Tetragynia $-4=$
5. - Pentagynia $-5=$
6. - Polygynia mit vielen 5 Eriffeln.

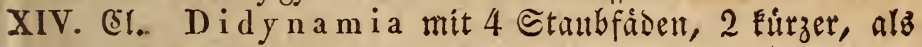
die andern 2.

1. Dron. Gymnospermia mit nacten Samen.

2. - Angyospermia mit behuilten Eament.

XV. El. Tetrady namia mit 6 હtaubfáden, 2 fúrzer, als die andern 4.

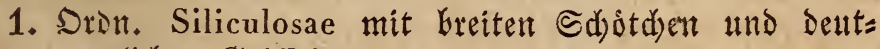
licken (jriffeln.

2. - Siliquosae mit langen @dyoten ofne Griffel. Sin ber 16. biz 20. Elaffe find die Männer unter fïh verwandt; fie bangen entweder mit einem Theile unter fich, Doer mit Dem Stempel zufammen. 
XVI. Cl. Monadelphia: Einbritberige mit in etllem

Eylinder oder Bundel verwadgenen Etaubtrágern.

1. Sron. Triandria mit 3 Etaubfiden.

2. - Pentandria - 5 - -

3. - Heptandria - 7 -

4. - Octandria $-8-10=$

5. - Decandria - Endecandria $-90-$

7. - Dodecandria mit 11-19 હtaubfåden.

8. - Polyandria mit vielen Etaubfádent.

XVII. CI. Diadelphia: వెrenbrúderige mit in 2 Pars tieen vermadjenen Irüjgern.

1. Drsn. Pentandria mit 5 હtalıbåden.

2. - Hexandria - 6 - -

3. - Octandria - 8 -

4. - Decandria - 10

XVIII. CEl. Polyadel phia : Sielbriderige mit in 3 obet mehrere Bumbel verwadsenen Etaubáden.

1. Sron. Decandria mit 10 @taubfẳen

2. - Dodecandria - 12

3. - Icosandria mit vielen im fielde angehefteten Etaubfäcen.

4. - Polyandria mit vielen im Blumenboden einges fenften Ctaubfícen.

XIX. (er. Syngenesia mit in einen (Enlinter verwad)= renen Stanbbenteln, megrere Blimdsen frehen auf einem gemeinfdaftlidjen frudtboden, und wersent you einem gemeinfduaftliden Feldye umgeben.

1. Dron. Polygamia aequalis mit lauter Bwitterblumd)er.

2. - Polygamia superflua mit Broittern uno weib= lid)en unfud, ‘baren Blümd)en.

3. - Polygamia frustranea mit zwittern uno weib= lid)en unfrudtbaren Blumd)en.

4. - Polygamia necessaria mit unfututbaren 3wits tern uno unfudd tbaren weiblichen Blimdent.

5. - Polygamia segregata mit Blimdjen in beion= Dern Relden, in Dem gemeinfdhaftlid)en Feld).

XX. હ2. Gynandria mit veryad) fenen @taubfádent uno Etattbueigett.

1. Sron. Monandria mit 1 Staubfaten.

2. - Diandria $-2=-$
3. Triandria $=3=-$
4. Hexandria $-6=-$ 


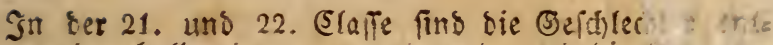
weder balb oser canj getrent, und tie $s+3$ thas gen gründen lid) auf vorbergetyento (Slaffen.

XXI. Cl. Mo no ecia mit getrenten (sejdiledtern at? einer Yflanze.

1. Drst. Monandria mit 1 Etaubfaben.

2. - Diandria - 2 - -

3. - Triandria - 3 -

4. - Tetrandria - 4 -

5. - Pentandria - 5 - -

6. - Hexandria - 6 -

7. - Polyandria mit mekr als 7 Etanbfaisen.

8. - Monadelphia mit verwadjenen Irágern.

9. - Gynandria mit verwadjenen હtaubfícn uno Etaubwergent.

XXII. El. Dioecia mit getrenten (jeid)ledtern attf 2 Pflanzen; alle Prinnjen Diefer Claffe fino ent= reder ganz mánnlid) verer ganz weiblid.

1. Orin. Monandria mit 1 Etaubraden.

2. - Diandria - 2 -

3. - Triandria - 3 -

4. - Tetrandria - 4 - -

5. - Pentandria - 5 - -

6. - Hexandria - 6 -

7. - Octandria - 8 - Enneandria - 9

9. - Decandria - 10 - -

10. - Dodecandria mit 11-19 巨taubfábett.

11. - Icosandria mit mehr alb̈ 19 im Seldje befe: ftigten @tanbfícen.

12. - Polyandria mit vielen auf ben Blumentoden befeftigten @taubfáden.

13. - Nonadelphia mit in ein Bundel vermabjenen Etaubfaicen.

14. - Gynandria mit verwadjenten Staubfiben unb હtaubmegen.

XXIII. (Er. Polyg a mi a kat zroitter und getrennte (5es (d) ledter anf einer Pflanze.

1. Sren. Nonoecia, Zwitter uno getrenute (5efibled)ter aut einer \$rinze.

2. - Dioecia, Broitter uno getrennte Befdeledter auf verfobiedenen Planzen. 
XXIV. CI. Cryptogamia mit yerheimlideten હifen und

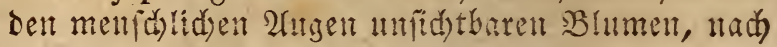
Fet. Prof. SBitr. find 15 Drommien.

1. Gonopterides, Sjewadde mit eittem Glätterlofen, ge=

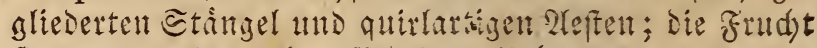
fibt unter cittem hanaformigeit Heberzlts.

2. Stachiopterides, (3)ewhid) fe mit eitrem beblitterten ober nackten @taingel, tho mit fapieln, welde it Slappen auffringen; fie fiten entweder in Blatt= winfern oser in Sebren, man fagt gehrenfarten.

3. Poropterides, (semadj) mit einem saubbratte, wels d)es bey Der Entwidelunt fretsforming gefraltet ift, mit vielfadderigen Fapilu auf ser untern Eeite, welde in soduer alffipritisen.

4. Schismatopterides, (S) ewid) ie mit saubbiattern, bie

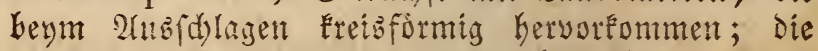
Fructificationen fishen auf Dem saube felbjt, meiftens auf berontion 2lekrent.

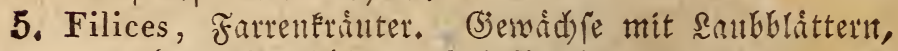

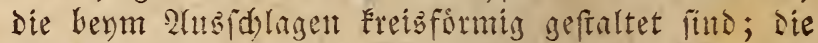

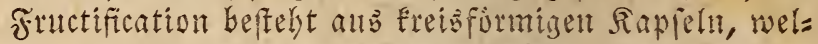
d)e auf Der untern Geite Der Blitter fiben.

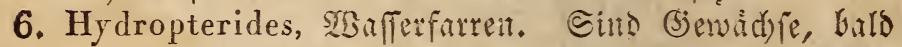
mit Eretsformigen, bald mit ansern Blittern, Deren

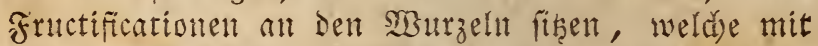
bejonderen Heberzigent berecelt fins. Die meiftent Pflanzen finden fid entweder in oder an SBaffert. 7. Musci, Rnubmoofe, find (jerwadd) (welde bebláttert find.

8. Hepaticae, Sebermoofe.

9. Homallophyllae, fino laubige niedergedructete (5emåd) fe,

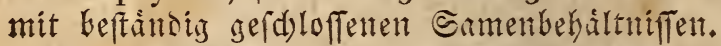

10. Allgae, Meertanger, Deren Fructification unter ify= rer Sberflidae fitit.

11. Lichenes, Fled)tent.

12. Xylomyci, fitto (jerwád) fe, weldye weber Blâtter, nod) Saub, nod eitten Etángel haben, Deten Fugel= formige Fructificationen verfdloffen find.

13. Fungi, Pilze.

14. Gasteromyci, Baudypilze. (5iencid) fe, die weder Blit= ter, nod) saub baben, und Die mit ifrer Fruttifica= tion intuerlidy angefürut fins.

15. Byssi, (3ewadd) mit hautartigen Fructificationen. 


\section{Acacia, 2teacie, XXIII. 1.}

(अ)

in febr reides (siefdrledt. Die meiften 2ruten zeidunent fid) Durd) f(d)ue oder fonberbar gebildete Blatter aus, wels d) bey eitrigen gefiesert finto, z. B. A. decurrens, farnesiana, lophanta H. F. W.; lev antern fins fie einfady lang= (id), z. B. A. dodonaeifolia, floribunda, latifolia, linifolia, longifolia, stricta U. F. W.; zumeilen trígt Das linglidge 8 latt an feiner Epise ein gefiedertes, wie ben A. heterophylla ser fall iff. Mand)e Urten febent mebr

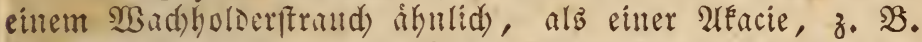
A. juniperina แ⿰tD verticillata; andere bingegen find mit biutigen Blàttern verieben, vie zum sheil an ven 3roei= gen berablatifen, $A$. alata, undulata. Sinige Gorten find mit Dornen bewafflet, antere nid)t. Die Blumen Det

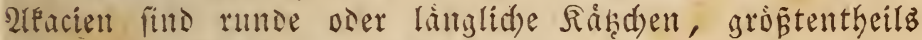
you gelber farbe, oft unbescutend; zumeilen bảngen fie in langen 2lebren orer Elemen lodern Irăbdoen berab.

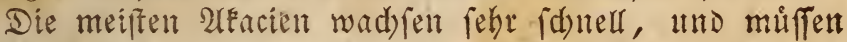
eitre besentente sobbe erreidsen, bevor fie bluben; diefes ift wobl sie saupturfadie, weşbalb mant fie fo felten als Etu= benuflanjen antrift. Jedocí wersen fie in-ibrer sugento jeden Siebbaber frember Siewådje Sergmigen gewaibren, be= fouters, went man fie felbit aus Gamen erziebt; Denu are fiejenigen 2 rten, weldbe in orter feine gefiederten Blátter tragen, baben tergleid)elt dod) in Der erfen Rinobeit, und mui nad) Int nad) fincet fid) Die eigentbumlid)e Form ein.

Jan findet Diefe \$FAnzen in Den beifen und gemá=

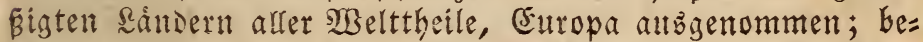
fonders reid) an fdomen Urten ift Neuborrand. Gie lieben Rauberde mit etmas sefm und Cand gemifd, verlangen Des Eommers reidblid) 2 Baffer; im SBinter jebod) (en man mit dem Siefenen vorfichtig. EEben fo bedurfen fie viel sidjt 


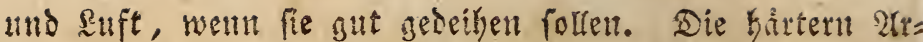
ten bringt man you Der Mitte Nary bis zutn Eeptember inb Frene, und fiberwintert fie bey 4-6 Grad SBarme $^{2}$ nidyt fern yom Fenfer, am beffen in (Seferfid)aft der beis

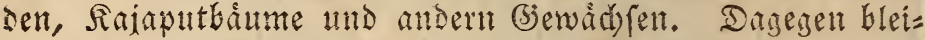
ben bie zartitden arten faft bas ganze Sabj uber binter

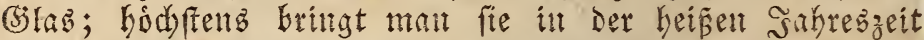

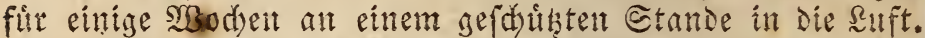
Des SBinters bedirfen diefe 6-10 Grad. Unter den et= fterii befinden fid) jedod) die gefdishteften arten.

Man vermebrt die 2leacien am beften Durch Samen, weldien man im Frtibiabr in leid)te (Erde legt und warm bitt. Sind bie \$flanzen 4-6 3oll bod); fo verpflanzt man fie ill eillzelne Topfe, bey weldyer Gelegenteit man

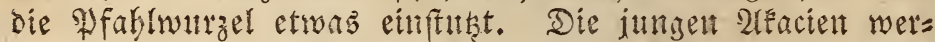

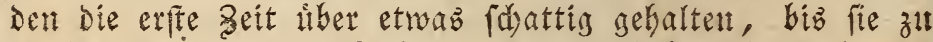

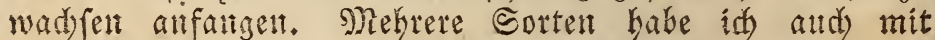

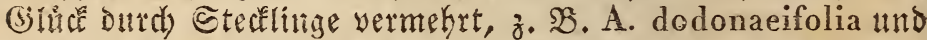

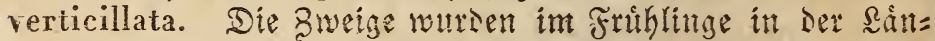
ge von,etwa 230 all abgefdntten, zu $4-6$ in eimen llei= nent Iopf geftedet, mit einem Ginlinglich boben (5lafe be= seckt, und in cilt warmes 2niffbeet geftelt. Sie treiben iil ser Regel fother.

S(t) fithe mut folgende aleacien als Stubenpfialzen an;

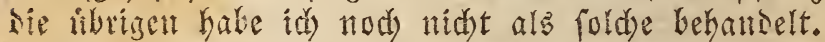

1) A. do donaeifolia, Dodonáentattrige Trácie (mimosa dodonaeifol.), 2 . Neufortant. - Eie but sinfadie lanzettformise, fdumale, etros gebogene Blat=

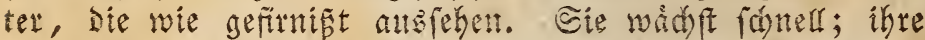
Blument Pente idh nidyt; man yermebrt fie surd Stedituge Ins diberwintert fie leidet bey 4-6 (5) rad.

2) A. longifolia, latiglattrige reacie (mimosa longifolia), h. Menhortums. - Die lanzettfors

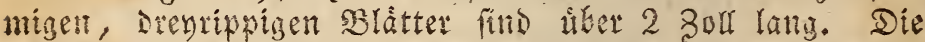

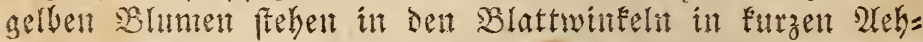

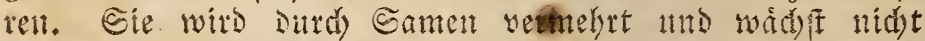
fo fduter, als mandle andere 2irt; wiro tibrigens wie Nr. 1

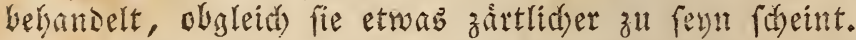

3) A. lophanta, bufdelblutbige ateacie (mimosa distachia), h. Nenthorrant. - Die Doppelt ge= Fiederten Blitter baben 8-12 tho mehr \$aar Fiedern.

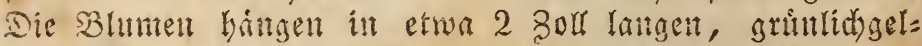
ben Iranben herab, uns tragen latge Edtotent mit 5-S 


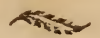

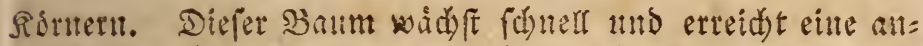
Fehnlidye Srobje. Die Eamen gehen leidyt auf, wenn man

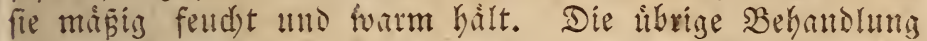
iff sifr. 1 gleid). - A. lophanta speciosa if der

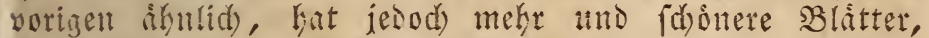
bliht oud) frither uno reidlider. Sie verdient die Aluf=

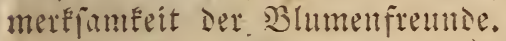

4) A. stricta, feifolatterige affacie, h.

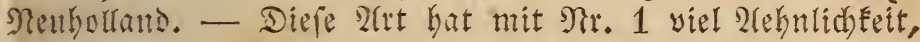
nur find bie Blâtter etwas furtzer, nidht glânzend and

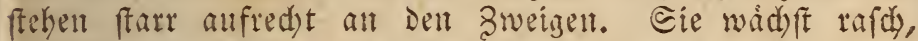
mad)t wentis 2lefe, , and ihre Hleinen, runden Blumden,

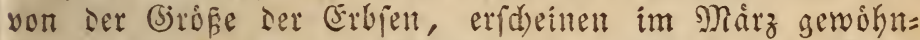
lid) zut zwegen in Dent Blattwinkeln. Man zieht fie alls Eamen.

5) A. verticillata, wittelbiattrige 2akacie (mimosa verticillata), 2. Meuhortand. - Weenn man bies fen Baant ans Sament zieht, fo find Die erfen 2-4

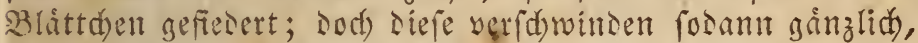

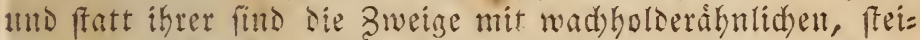
fell, dumfelgrinten Maseln bedeckt, Die zll 6-10 wirtels

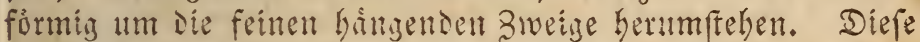

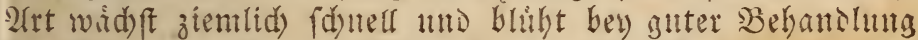

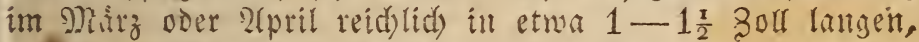

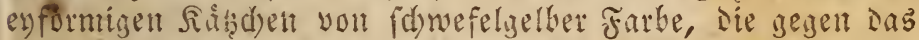
Dunfle (5vin ser Maveln sut abferten. Man vermehrt fie Durd) Eanten unt Etectinge. Seristere werden, wie oben bemerft, bebantelt; man kam fie aud im 2ansula maden,

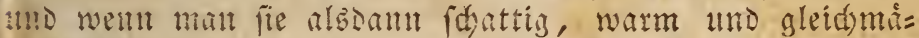

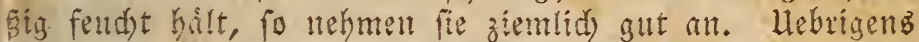

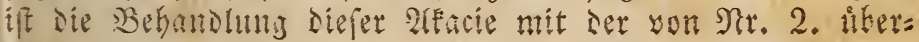
einfinmetts.

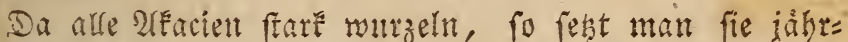

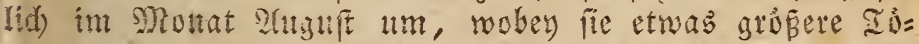

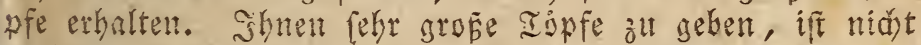

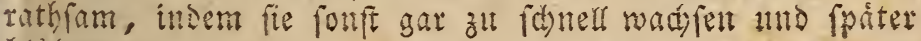
blithen.

A canthus, Båtentalte, XIV. 2.

A. mollis, ed te Barentifaue, 21. Cubctropa.

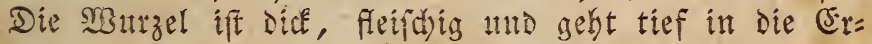
te, Die grofen glânzeno= stinnen Blâtter finto eingeed) tent mo gelappt. Die shlumen fehen auf einem $2-3$ 


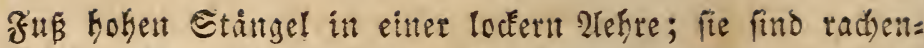

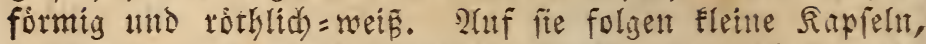
weldye yon Sen"etwas fradliden Felden zum Theil einge= Gulat fint, mo 2 grose Eantent enthaltent. - Man Gailt

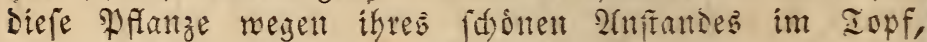
wiemohl fie andf im frenent Rante allsanert. Sie ver=

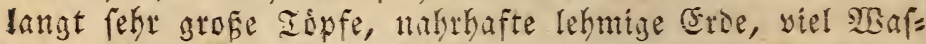

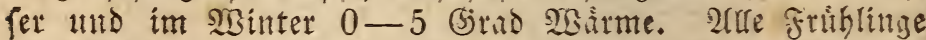

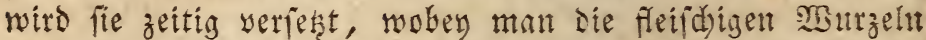

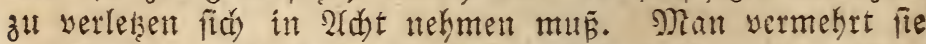

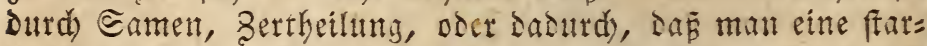
fe. .Q

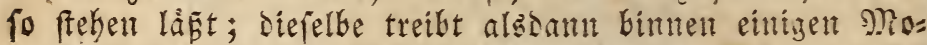

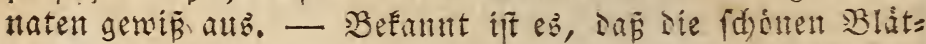

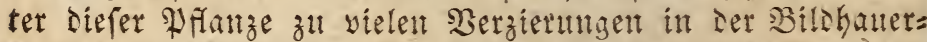

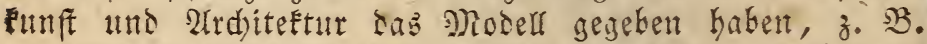

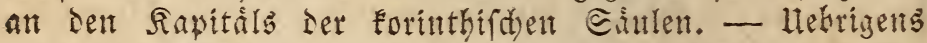

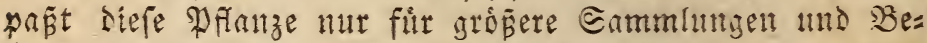

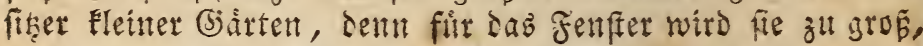
und watrelt zu ftarf́.

\section{Achania, હdampappel, XVI. 8.}

A. malvaviscus, farlarladutothe હdampap= pel (Hibiscus malvaviscus, Malvariscus arboreus, IUt= tenmalve), 万. Samaicar. - Der Etamm ifit aufredte, in mehtere fallanke sweize getheilt, unto trágt herzfórmige, etwab gelappte unt am Rande gefeetbte Balätter, weldhe mit fehr feinen beirdjent besecet fint. Die Blumen, wel=

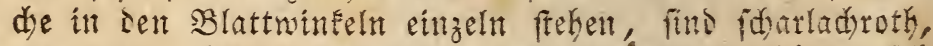

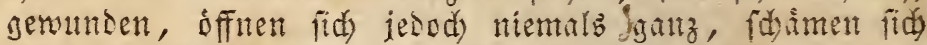

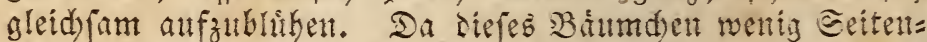
sife madyt, fondern grave in sie hobhe geht, fo nimmt es

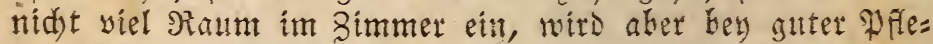

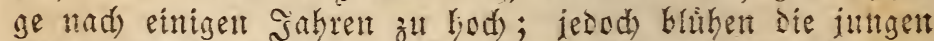

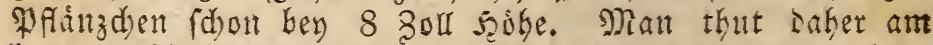

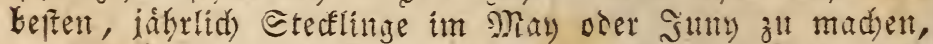
welde, wern mant fie unter (Slas und warm kalt, oft

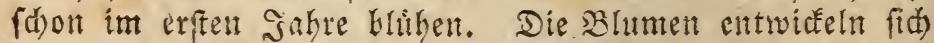
Den yalzen Eommer tiber. Man gibt ser Edjampappel

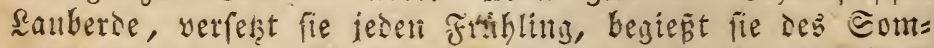

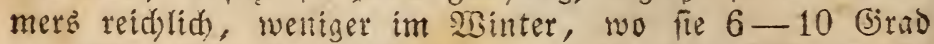
vertangt. Des Evammerz fanin man fie einige Nonate in Die euft fetlent. 


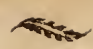

\section{Achillea, (Gjarbe, XIX. 2.}

Uluter ben vielen (sorben zeidunen fid mebrere butä

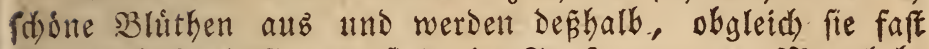
fímmtlid) Santspflanzen fint, im Topf gezogen. Man bebt

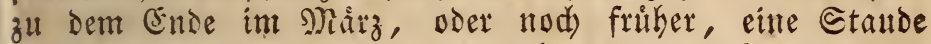
aubs dem sande, ftidht yon Derfelbent fo viel ab, alz man

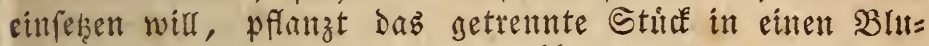
mentopf in leidste Erre, tund grabt ben Iopf im greyen an eitrem fonnigen. Plah eitt, biz fid Die jungen Iriebe zeigen. 2rlşann bringt man ifn in eine más̆ig warme Etube an ein fonnigeb Fenfter, und gibt Der Pfanze reids=

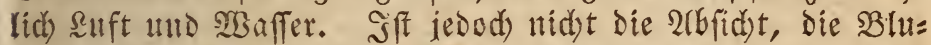

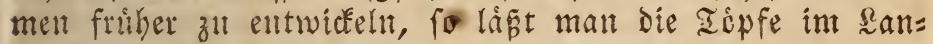

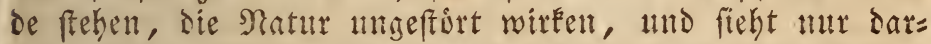

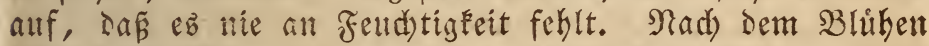
werden die \$flatizen entweder weggeworfen, oder ins sand

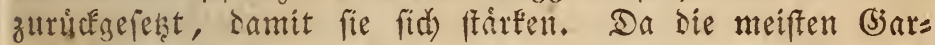
ben fefr wudjern, fo thut man, wenn man ein (5iartd)en bat, am beften, ftetz einige $\mathfrak{P f l a ́ n z}$ chen zum Ireiben yorzu= bereiten, insem man ben Sommer vorher eintige gallz $\mathfrak{k l e i =}$ ne Eproffen abnimmt, und fie alf eift Beet in gute Eroe Fesct. Diefe Eproffen baben fid) bis zum Serbft zu Fraft=

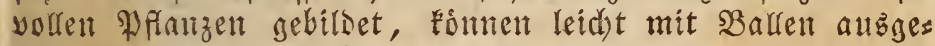
bobent wersen, und bringen fdonere Blumen, als bieieni= gen, die nant erft im Fruhlinge abnimmt. Éin Nerfahrell, weld)eb man mit. Noortheil bey ben meiften Etandengewadd)=

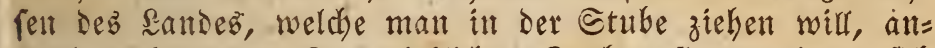
wenden Eant: - Zur wirklidhen Stubenpflanze eigutet fid vorzinglid):

Achillea tomentosa, bie filzige (s)atbe, 21. sanguted oc und Iatarey. - Die Eleinent graugrunen $\mathfrak{B}$ låt: ter find mit feinten Fâjerden befeşt, fo, Daf fie gleid) fam wie tiberfponten zu feyn fdeinen. Die Blumenftangel wet: ben felten hiber 8 sou hod uno tragent goldgelbe Blumen.

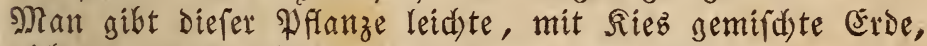
nid)t zal grofe Topfe, Deren Boden man mit Hleinen Stei= nen belegt, Damit bie itberfluffige Feud tigkeit abzieben kann,

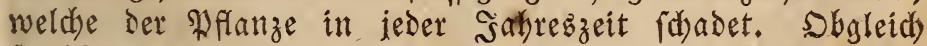
fie ofter' im sande aubdauert, fo thut mant bod am bes

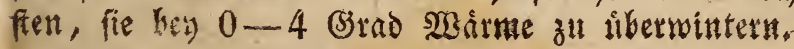




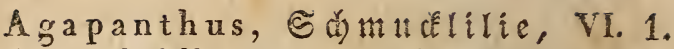

1) A. multiflorus, vielblamige હd) mudi=

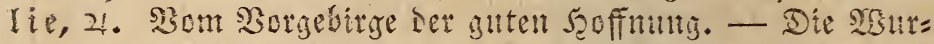

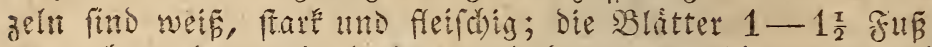

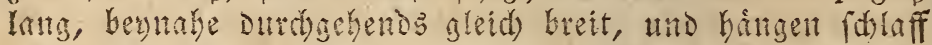
nad) 2 Seiten hifer. Dor Bhmenthaft ift $2-3$ Juß bod,

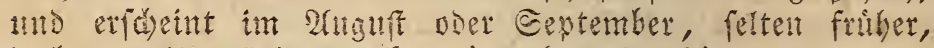

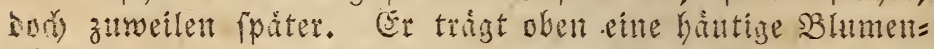
fdreive, aus weldyer fid auf Elizzen Etielen oft $30-50$ gwey Zoll lange, fedjeblattrige, trid)terformige Btument ent= wicerit, beren farbe hellblat, felten weiplid) ift. Dab

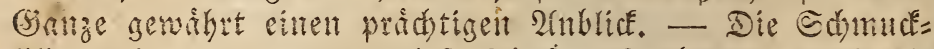
lilie verlangt grofe mo tiefe Iopfe, sauberde, frate mit sebut thio etwas Sant gemengt, uno Den Eommer libet viel SIaffer. SMent Die Iopfe nid)t zu eng find, fo ift

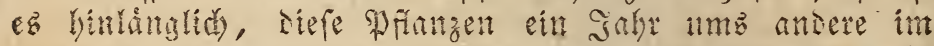
shan in gropere Iipfe jul fehen, wobey die SButzeln nidy befdabigt merden surfen. Berpfangt man diefes (5emides

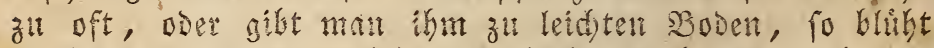

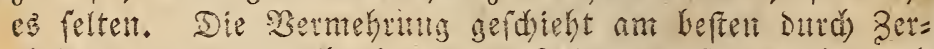

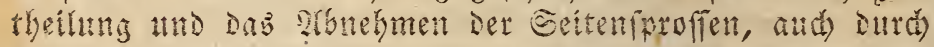
Samen. Dez SBinterg verlangt bie Pfanze einen Plath

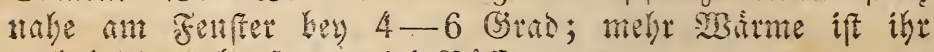

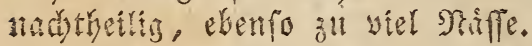

2) A. umbellatus, Doldenbrifthige @ Iilie. Defe 2ut lot mit der youkergehenden in allen

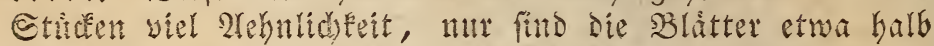
fo breit, unt Der Etingel trajt Fatm 10-12 Bhtment.

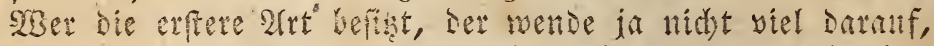

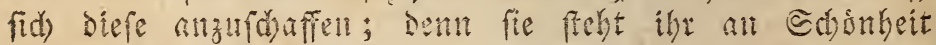
fehr weit nad). - Ess gibt eitte Spirlart mit weisen Blu= men und eitte andere mit gelb gefreeften Blattern. - Ba= terland uno Curtur wie Die vorige. - Mnan findet decfe

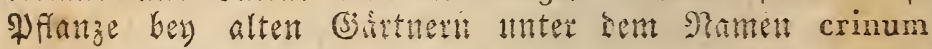
africanum.

\section{Agave, $\mathfrak{H}$ gaye, VI. 1.}

A. americana, americanifale $\mathfrak{x}$ gave, 2 . Eismerica. - Uagemein mnter Dem Nament Der Guthert=

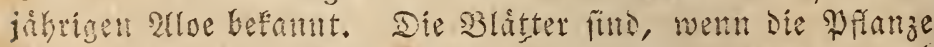

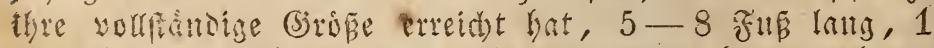

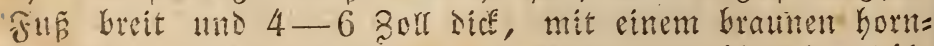

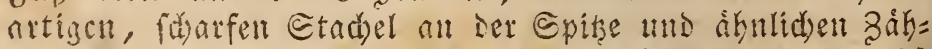
nen am Rande yerfeben. Der Blumenfahaft fteigt 20-30 


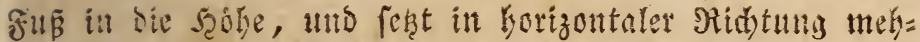

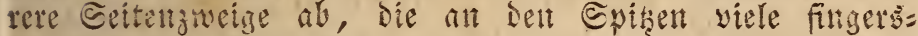
lange, gringelbe, robrige Blument trigen. Die Babl bie=

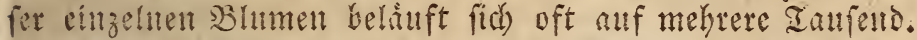

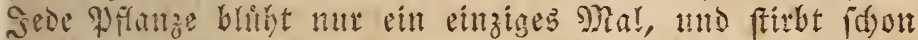
ab, wibrend ier Etangel emporfteigt. Shomerica if ei= gentrich zmar ifre Baterland, Dod iff fie in Stalient mo sem norbliden 2rfica bereits einbeimifa geworsen, wo

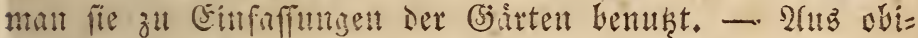
ger Befdreibutig geht berwor, in mant mu int Etande ift, fie in ibret erfen Sugent als Etubenpfanze zu betradoten.

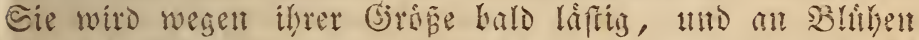
ift gat nid)t zu denfen. Eie nimmt faft mit jober (5nart

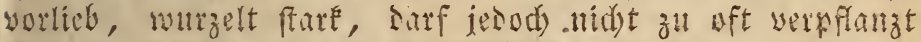

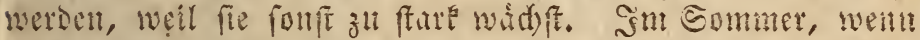
fie im freyen fteht, yertrigt fie zwar ziemlid wiel sonfler,

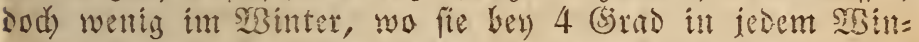

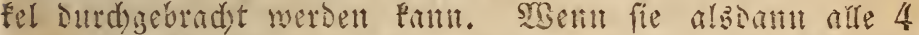

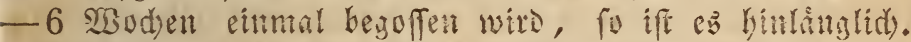
Eie treibt wiel MMnjelipioffen, ans weldben man fie leidjt sermekrt. Ditb Etubenpflanze ift Die Epielart nit gerb

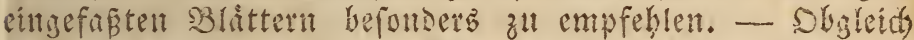

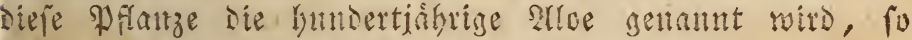
blubt fie dods weit friber, tho es fommt bierbey viel aur Die Dabrung nno Behantumg an.

\section{Agrostema, $\Re a b e, X .5$.}

Agrostema coronaria, (s)artentade, Eaut= ntetnele, 24. Sdumeiz. - Die gewobnlide Sammetnelfs

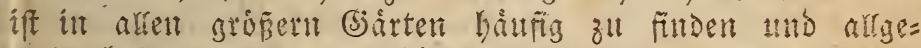
mein befannt. Die am bäufigften yortemmenten Esielat: telt, anfer Der gemeinen buneltotgen, find: bie weife, und bie weife mit rotbet feldseinfaftung, weldye zuweilen gans

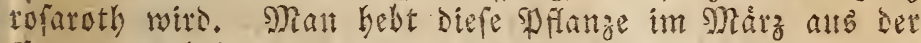
Eroe, und behandelt fie wie bey Achillea gefagt mortent ift. Eite rerlangen magern Boten. Die gefirte Epielart ziebt mat gewobnlidi) im Iopf, gibt ibr lefmige (Eroe, weldse ftare mit Ries and Sand gemifd wird tho liberwintert

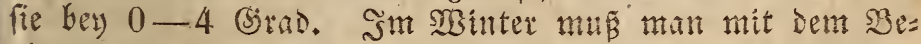
giefen sorfidstig fent. Cie wirb im 2uguf outd Berthei=

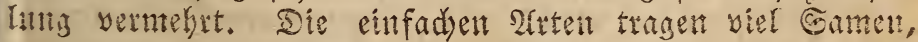

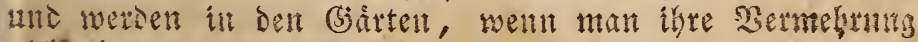

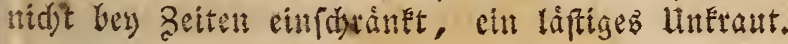




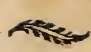

\section{Aloë, Hloe, VI. 1.}

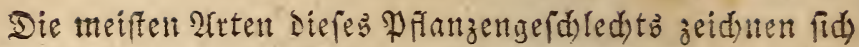

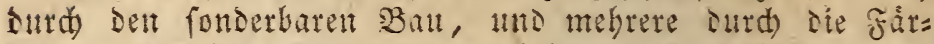

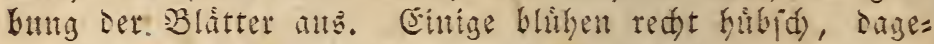
gen find siejentigen, weldye yon SBiloenow Apicra genannt

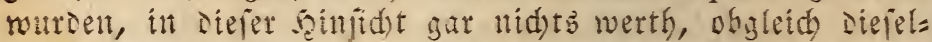

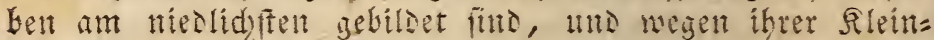

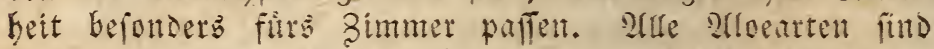

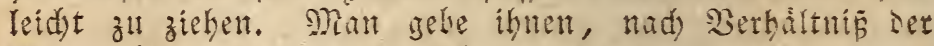

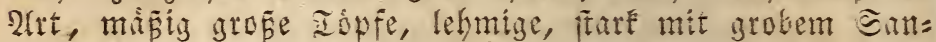

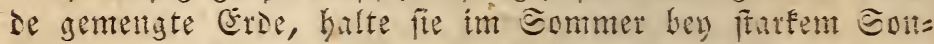

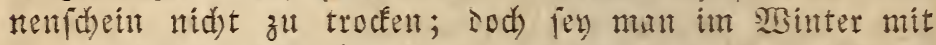

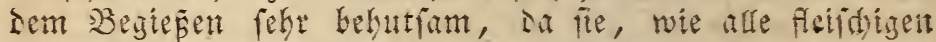

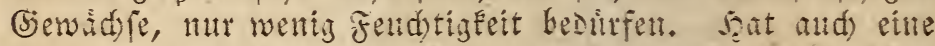

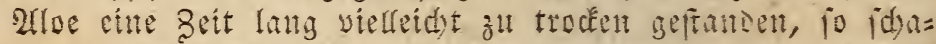

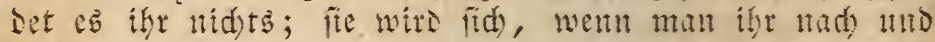
nad) etwas 2 Baffer gibt, fdon weder erholen; Dangegen if

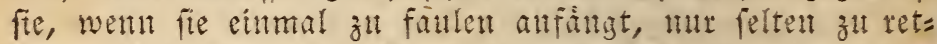
ten. Dod) Eant man fid) in lesterm Falle dift sadurd hels

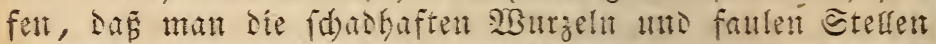

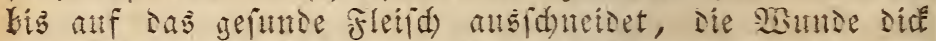
mit Roblenpulver beftrent, tho die \$fanze 8-14 Taige

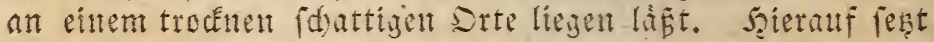
man fie in eitten fehr Eleiten Iopf mit fandiger Froe, uns

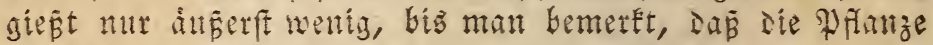
zat wadblent anfátigt.

Des ङommers gebe man sen 2lloearten viel suft, ment

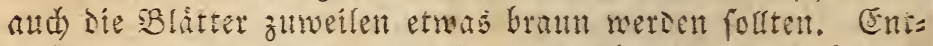
zieft man fie ser suft zu fefre, fo treiben fie fiarte, befom=

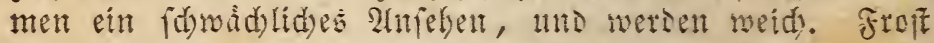
Fonnent fie Dajegen gar nid)t vertragen. - Die ßermeb= rung gefdieht ourd) 2(bnehmen Der Eeitenfproffen, weldye alle 2lloes reidjid treiben, bejonters, went man fie mut

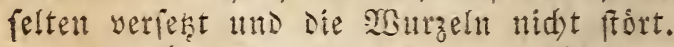

Die Gaumartigen Sorten wadjen meifteng zu fod),

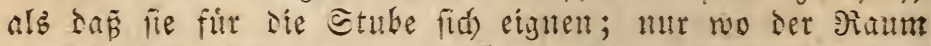
es geftattet, fant man veriud)en, Die oser jene arte zum

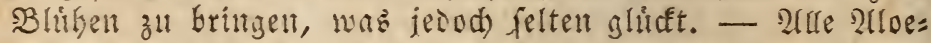
arten wadjen in 2rfrica auf surren, fietnigen Plaken. Des Winter balte man fie nid) zu marm, hiberbaupt, weln fie entfernt yom genfter fieben muffen, fonft treiben

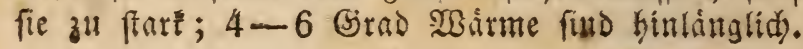


1) A. arborescens, balmartige atoe, Mit beyuahe gleid) breiten, $1-1_{2}^{x} \mathfrak{F} u \tilde{B}$, langen, grangrth= nen, Hnjeflecten Blatte:n, die am Rantie gejahlit fitto. Der Etanm treibt viele Eeitenafife. Sol) Gabe fie fdon úber 6 Jus bodj gefelen; bod) batten Diefe (semplate nods nidht geblíbt.

2) A. atrovirens (Apicra), fá)

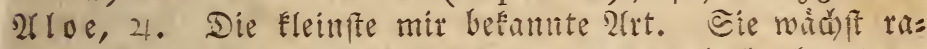
fenartig, in fleinen Rofetten, etma 1 zorr bod, bat din=

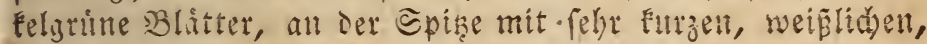
borfentartigen, sveidyen Etadeln befebt.

3) A. brevifolia, equblatterige Slloe, Sie hat, went fie ălter wiro, ethen furzen Stamm, wel= d)er oben eine Piofette you glatten, feifdisen, Glangrimen Blittern trảgt, Die an Dem Rante lino unten einte Reike

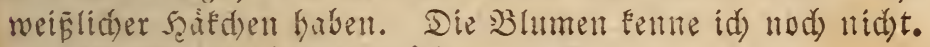

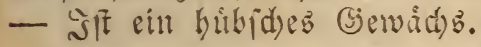

4) A. cymbaefolia (Apicra), fahnformige aldoe, 24. Niedris, mit belligumen, oben etwab dickern,

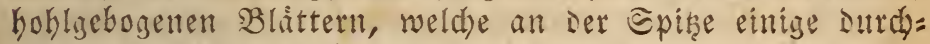
fdeinente Fledent babent.

5) A. echinata, fad) lige 2 loe, 21 . Srat yiel 2lehulid)éeit mit 9 r. 3., ift jebod) ohne Etamm, hat fpis

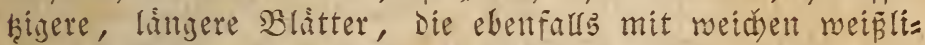

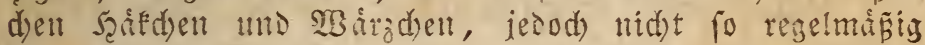
befest fitt. Die Blument find red)t (d)one, getbrothlidje,

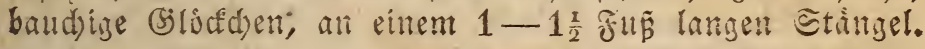

6) A. lingua, จ̊ษ

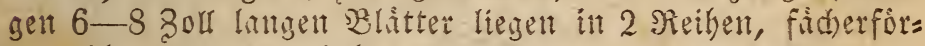
mig liber einamier, baben einen fibarfen Sano unt unsents

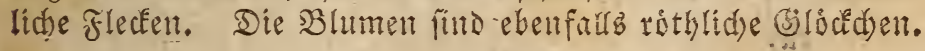

7) A. maculata, geflecte Ulfoe, 2. Beynabe wie Nr. 1., dod) find die gelblid)grunen Blitter nur etwa balb fo lang, laufen fpits zu uno baben am Rambe (á)arfe

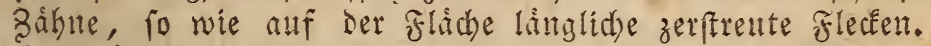
Eie ródjft langjam und wito nidjt fo hod). Die Blumen find mir unbefannt.

8) A. margaritifera (Apicra), Perlaloe, $2 \%$ Mit fd)warggrinen, rundliden, fpis zulaufenden 2 lattern,

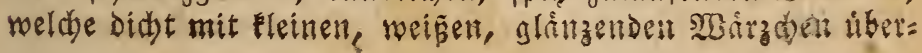




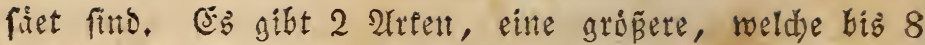
Soll hod) wird, wäfrend die Rofetten ser antern mur 3 -4 Boll siofe erreidjen. Die lestere vermehrt fid weit

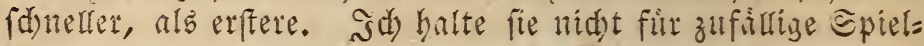
arten.

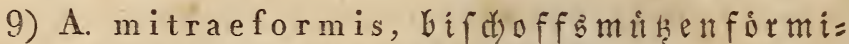

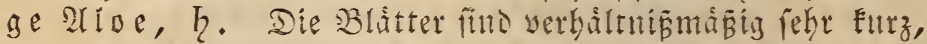

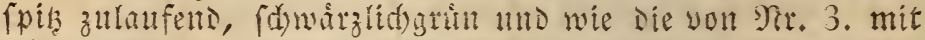

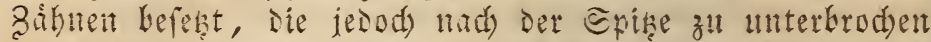

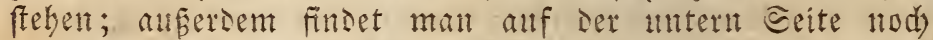

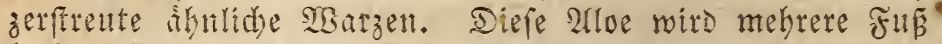

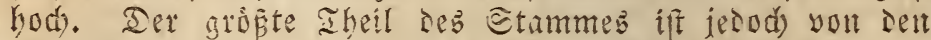

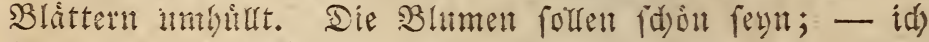
Eettite fie nod nidit.

10) A. pentagona (Apicra), filltectige 2troe, 7. Cie wad fit faulenformig 6-10 3oll hod), unt ifre

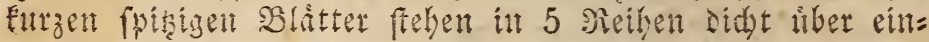
alider.

11) A. retusa (Apicra), abgeft th te 2 toe, 4. Die biten fleifintgen silatter feben als, als wiren fie $1 \frac{x}{2}$

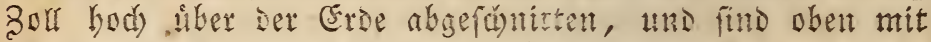

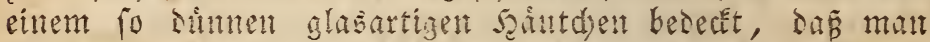

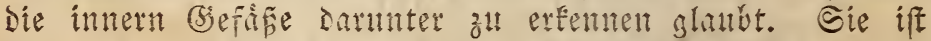

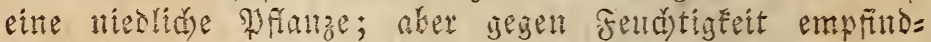
lid), obgleid man fie in Eommer nidit gut trocfen balten Iarf.

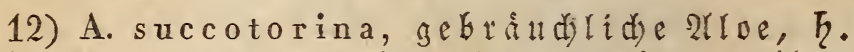

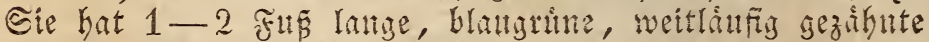

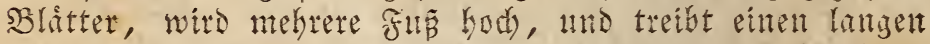

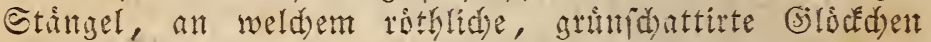
bången. Die Blátter fins leid)t gerbred)lid) und mit pintem grinliden, fehr bittern હaft gefulut, Ier Dab befantute stloes barz liefert.

13) A. spiralis (A. imbricata. - Apicra), ite

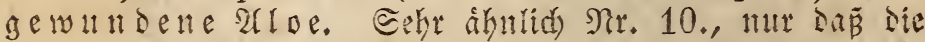

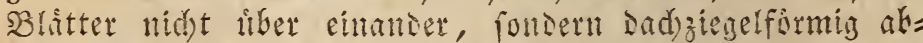

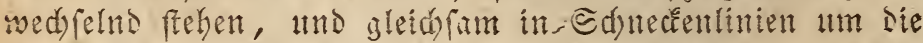

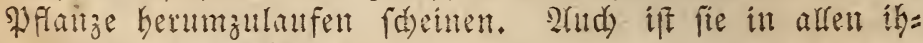
ren Ifeilen fidwidher, als iente.

14) A. variegata, bute atoe, Iigera!oe, 2 .

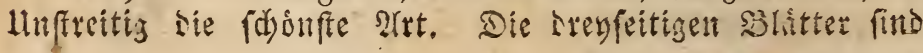


4-6 3oll Ians, sunfelgrut, mit feingejåhntem weipem Rante, mo weinen lángliden Flecten beoecte, sie lau= ter Binter birsen, meldhe um ias Blatt herumlanfent. Die Blumen fino rothlid) mit grith, fie erfdeinen zeitis

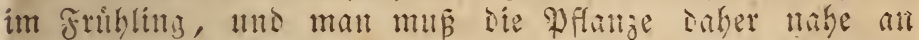
einem fomilgen Fenfer mild nidst ju warm kalten, neif

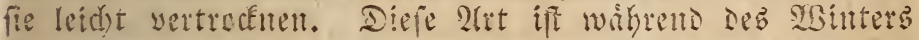
leid)t tem fonien nusgejebt, und man befande fie nus diefem Gramoe etwas yorfátiger, als andre girtelt. 2lue

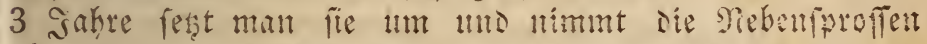
ab. Sluf Den Boden des Sopfes lent man Fleme Etaine, and rebt die phanze jesesmal fo tief, ba b bie unter=

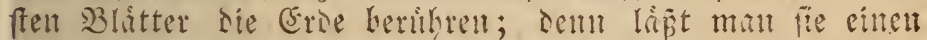
futzen Stamm treiben, was fie im Zalter gewibnitid thut,

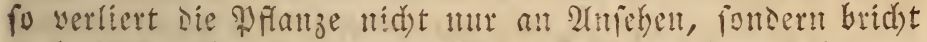

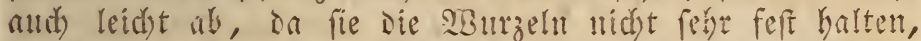

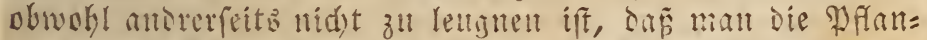
ze, went fie eitten flitzen Etamm bat, amb die SMUtzeln faul werben folten, leiderter beilen tann.

15) A. verrucosa, warjige afloe, oft mit A.

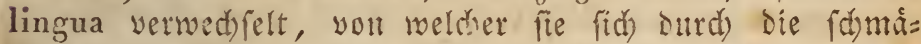

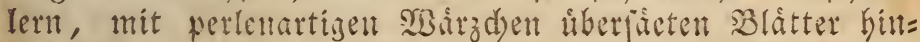
langlid) unterideidet.

16) A. viscosa (Apicra), flebrige rtoe,

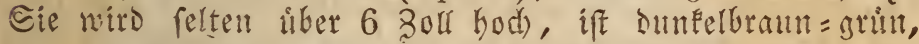

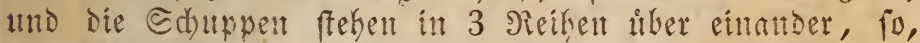

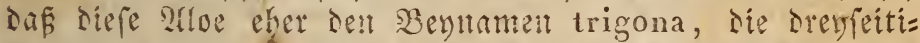
se, versiente, ab obige, Dent Das Rlebrige Des Blument=

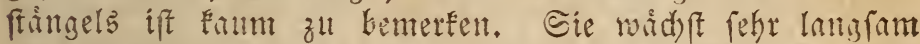

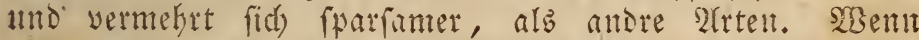
nan fie alfe 4-6 Saht einmal umfeht, fo if es bin= långlid

17) A. vulgaris, gemeine arloe, দ. Cehreakn=

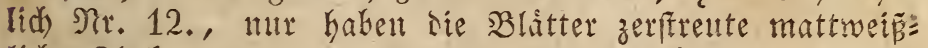
liche Flecten, und die Blument folfen gelb fent. siefert ebenfarte Uloeharz.

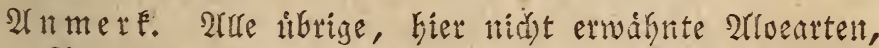
ftimmen mebr oder weniger mit ben genamen hiber= ein. Diee feltenfen 2urten fino: A. dichotoma (aud) A ramosa, plicatilis uno Rhipidodendron distichum genannt) Der fischerbalim, uno A. panniculata. Beide babe id nody nidjt behambelt. 


\section{Alstroemeria, Xlftioneria, VI. 1.}

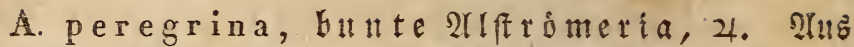

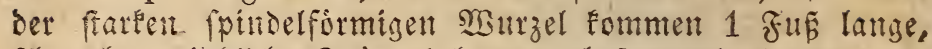

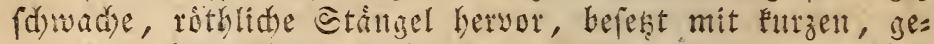

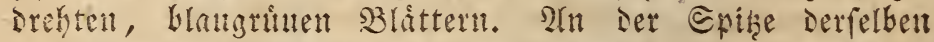
etrdeinten wifheno ber Sonmermonate $1-3$ lilinartige,

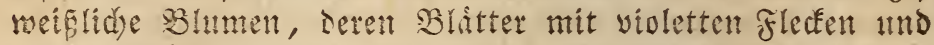

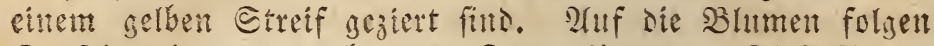
Sapich mit runden, bramnen Samentoutm. Diefe Dflan= ze verlangt fehr tiefe Iopfe, leid)te Erse und miñige Feud)= tigfeit. Mnan githt iff eitten Stand nahe am Fenfer und ftert fie im Sommer an cinen gejduthten Plat ins Freye. snon vermehrt fie surdi) Eamen, wetden man im frub= ling alsifuent, ober sutd) Berthetlung im serbft, wenn die Eamen reif geworsen futi. Des Minters bringt man

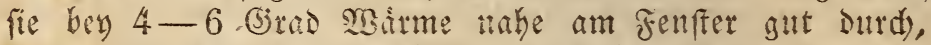

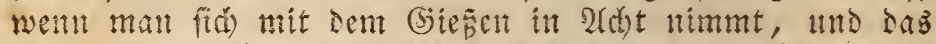

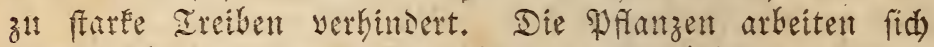
fets nad) Dem Rande Der iopfe, und fdeinen gleidjfam ơt mantern. Das Bateriand ift Dett.

\section{Amaryllis, armaryllis, VI. 1.}

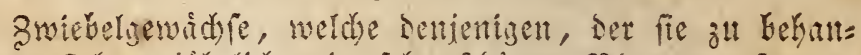
beln serfegt, jithelid mit fegr fdobten Blumen etfrenen.

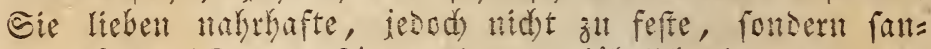

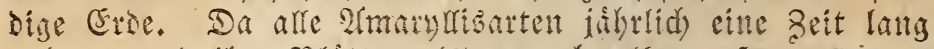
ruthen, thto ihte Blitter alsonn abwelfen, fo mus man

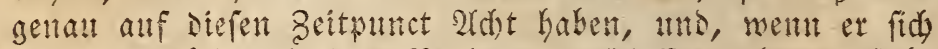
zeigt, allmålig mit Dent Begieñen nad)laffen, whte daffelbe jecod) ganz und gat eitzuffelten, wiewohl einjelne 2frten ez alsosun vertragett, altserbalb ser Erise gatt troden auf=

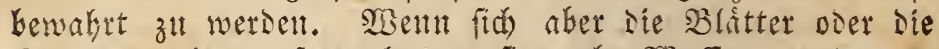
Knospen zeisen, fo verlangent fie nebr Wafter, uno man wird fie, bis zut sem Puncte, wo bie Blumen fid siffuen, nid)t leidyt sutd) (siepent verserben. Freilid) mus malt bie Fraft Der Swiebel, then Ctandort mo bie SBitterung

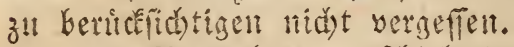

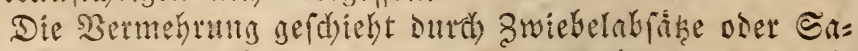
men. Die erfern loft man zur Zeit Der Ruhe, wo Dent die

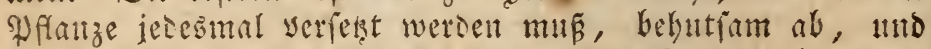

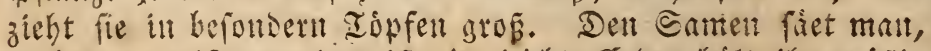

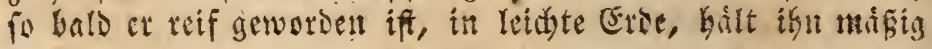




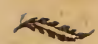

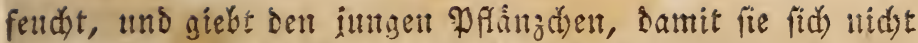
uberwadjen, viel sidjt uto suft. Eollten aud) uad) eint= ger Beit die Blitter gelb werden, fo laffe mant fid Dadurth nidht foren, fondern vermintre das sief̃en, bis in ser Folige nelle fid zeigen. Etehent die Bwiebeln nidit etrua

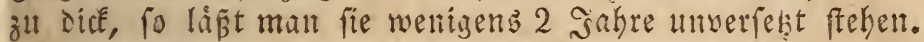
Dann wersen fie wie Die alten Pflanzen befanielt. Sie rodjlen langfam, und es vergeben bey mandin Eorten wobl 10 sabre, éfe fie blibbar werden.

1) A. belladonna, Die fdone Frau, grof e

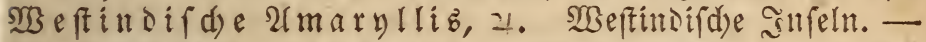
Die Broiebel ift ziemlid) grof, herbraun, und Die trofke (cd)ale ziebt fid in feine, weife, feidenartige faiden, went man etwas sayon abreiśt. Die Beit Der Sebetation ier Brobel ift rer Minter. Die rinnenformigen liberbảngen= Den Blitter find etwa 1 Fuf lang nnd ${ }_{2}^{\frac{1}{2}}$ Boll breit; im Jay welken fie $a b$. Dann verpfanzt man die 3robel, und lápt fie all einem yor Regen gefiderten Drte bernabe ganz trocken fteben. Stm 2ulngft bringt man fie ins Freve, gieft the ofters und lä́t die Natur wirken. Die Rnospe erfdeint vor den SBláttern, uno mant thut gut, um die= felbe gegen Bufårigkeiten zu fdusthen, die Swiebel, fo wie iene herworbridt, zwifden Doppelfenfter, oder wentigfents

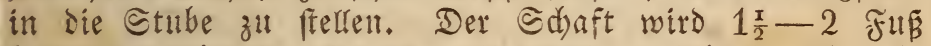
bod), unD triggt $3-6$ grofe, lilienartige, rotblid)e, (d)wad)= geftreifte Blumen, weld)e fehr anzenebm riedsent. Die Blảt= ter entwicfeln fid) nad) Den Blumen. Sm NBinter bålt mant fie bey 4-6 (siad in ser Nabe des Jenfters, gieft ifr jedod nur fo viel, als gerase nothig ift. Diefe fdine 2lmarbls, liz vermebrt fid) ziemlid gut ourd) 2lbjübe, tràgt aud Samen, und ift alfen Blumenfieunden zu empferlen. (E) foll aud eine fleinere Epielart geben, weldye idh jedoch nod) nidst gefeben babe.

2) A. crispa, fraule âmaryllib, 21. গুom Eap. - Die şwiebelt find nut etwa fo grop als Flinten=

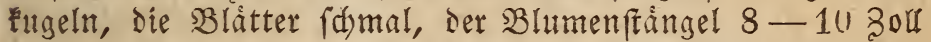
lang, fdroad luto an feiner Epise fteben mehrere Eleine ro=

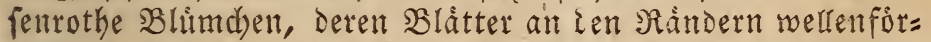
mig gebogen fint. Cie wird ziemlich wie die vorbergebende behandelt, jeood) braudst man fie nur alle $2-3$ sabre ein= mal zu verfetien; Denn je wentiger die Broiebeln geftort wer= ben, defto haufiger blithen fie. Sie vermebren fid far durdy Nebenzwieteln, aud bilien fid zuweilen sergleident 
obent ain Ctängel, went bie Blumen abgefarten fint. Dab Einzige, was diefe ztrt einigerma panen empfiehlt, ift Der lamfand, Daf fie im December blubt, wo andere Bll= men felten fino.

3) A. formosissima, fdo ơnte 2 tmanyllib. Die betanntefte, aber and eine der fdjonfen 2(marylusar=

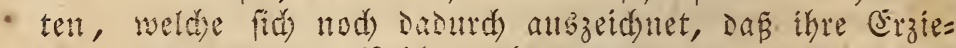
Gung mit fo mentis Intige yerbunsen ift. Bery den alten (5) irtnern paffitte fie unter Dem Ramen Iris suetica, aud Sacobslilie. - Die blutbrothen fammetartigen Bhmen be= fereben ans 6 Blattern, welderetwa 3 zoll lang find; die oberfen biegen firì) beynabe gattz zurhef, weniger die 3 un= terffen, weldye lestere am Reld Die Bengungaorgane zum Iheil umfaffet. Şn Dev Eonne glänzt Die Blume, als wàre fie mit feinem (Soldfanb beftrent. Die Blitter find faum $\frac{x}{2}$ 8oll breit uno gegen 1 sur lang. - Shr $\mathfrak{2}$ ater= land it Êtoamerica.

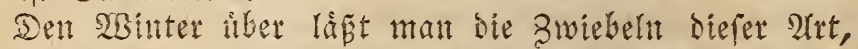
nadbem man fie yon den alten SMurzeln uno Blittern ge= reinigt hat, auper Der Eroe auf Dem Schrant oder Derglet= d)ent itt Der warmen Stube liegen, büngt alth Dieienigen, melate frith bluthen follen, an einem Bintfaren Ginter Dem geheizten Sfen auf, weldyem fie bis auf etwa 12 goll ge= nábert werden founen, obne dá es ser Pflanze fajadet. Die Snospe erfadeint yor sen Bláttern und ift Daran Eents lich, bos fie blaptoth erfatint, und alt ber Spişe cine Narbe bat. 2(ud) brid)t fie niemals aus Der Mitte, fons Dern immer etwas feitwátts beraub. Die jungen Blatter find zwar and robthlid), Dod gemeinislid) fidon mit etwas

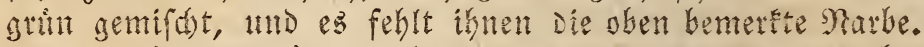
Zeigen fid) Die Blatter, ehe etwab yon Der Snobpe zat be= metken iff, fo sarf man fich mur feten nod in sem num=

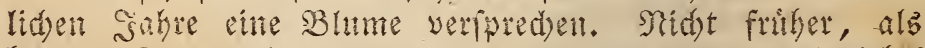

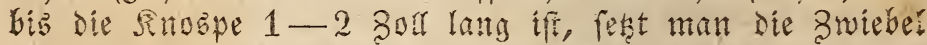

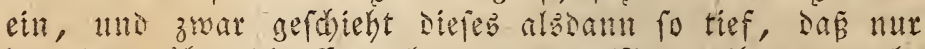
Der Sals liber die Erve Fervorragt. Man sibt nutmegr

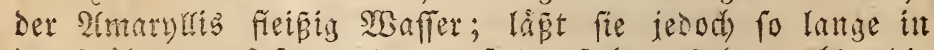

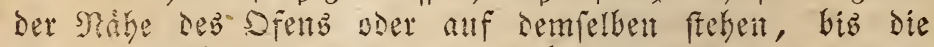
Bitnme fich beynabe offuen will, woranf man fie anz gen=

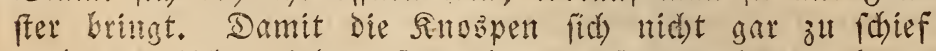
thad) Dem seid) te zieken, fo dreht matt fie zumeilen nad's Der entgegengejebten Seite, oder binoet fie in Der lehten zeit an eiment foinen Stock. 
Nad̆) Sem Stbbliken wadjen die Blåtter, zumeiten fommt allf ser altoen Eeite ald wohl nod) eine zweyte

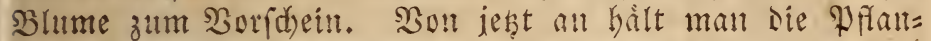

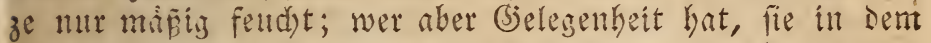
nidyfen Nan ins freye sant zu feben, ser thue es ia; sem im Garten ftarken fid bie zwiebeln auserordentlid), tutto mant Eann fie in Der Regel jeren Fruhling treiben,

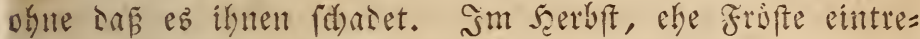
ten, gribt man die Bwiebeln wieder aus, lisét fie an ei= nem luftigen, fdhattigen Sot trocken, reinigt fie yon Den Slittern uno nimmt die Mebenzwiebeln ab, wo dépes ohne

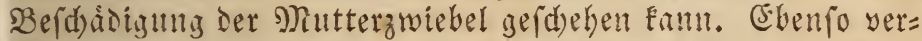
fährt man mit senten, weld)e den Eommer liber im Iopf haben ftehen miffen. Siele Blumenfreunde behalten die 2tmarylliz tas sahe tiber im Iopf, balten fie mur wath= rend beb Sertbfteb ant SBinterb ganz trocken. Cie folltent

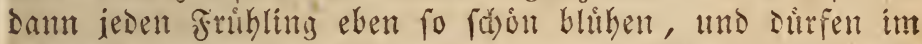

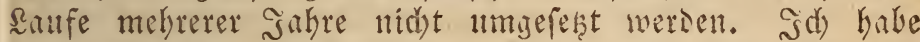
Diefes Berfabren nod) nidat erprobt, glanbe aber, da man eb. albsann nid)t fo gut in feinter (Sjewalt hat, Die Blume zil einer beftimmten Beit beryorzulocken, als wenn man yer= fihtert, wie zuterf befdrieben worden. Denn man Faun

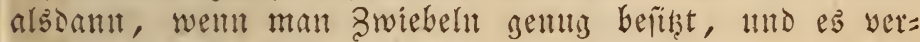
fteft, biefelben, ie nadjoem man die Saarme mehr oder weniger allf fie wirken láp̃t, gehorig zul treiben, yom Sa=

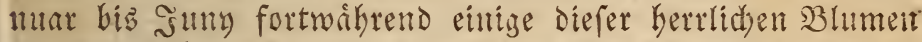
in Flor baben, die jederzeit Durdy inge feltente Farbe ein

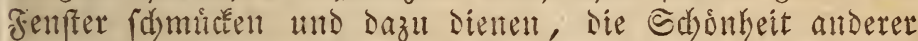
um siefe Beit blitbenser Pflanzen hervorzukeben.

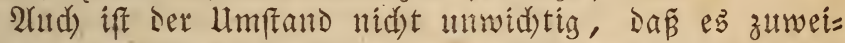

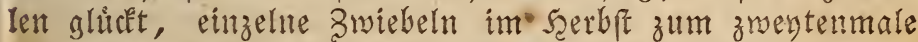

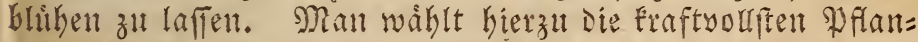
zen, die (d)on im Samuar abgeblitht haben, jedods nui ei= ne SBlume bradsten, gibt der Brwiebel einen suten Stano am Genfter, und wenn es die Sistterung erlaute, ofters suft, Damit fie nidyt zut fehr treiben. Sm Maal bort man

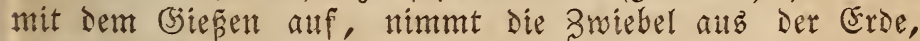
trodénet unto reinigt fie, uno lápt fie an einem warment (d)attigen Drte liegen. (Semeiniglids) zeigen fid im 2luguft bie Sinospen, worauf mat dir smiebeln wieder einlegt, unt wie tefannt behandelt. - Bltia) fente iá) einige

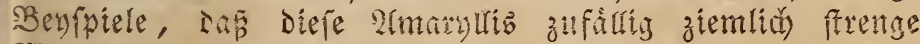
sointer int freyen sante aubgefalten bat; tod) wolte es 
mir nie grtuten, wenn id einige diefer Pflanzen abfithtlid

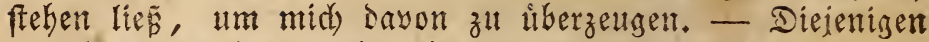
zwiebeln, weldhe zu (d)wad) find, um sinmen zu bringen, diberwintert man in einem frofffreyen Bebáltniê, uno pfianzt fie den Sommer buber inb freye sand, am beften in fdimarze mit Sand gomifd te Eroe.

4) A. sarniensis, iapantibe 2 maryllis. Sapan. - Die Blátter find blêtulidgruin, uto fa grof́,

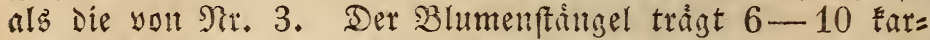
minrothe SBlumen mittler 3itố, uno erfdeint im Som= mer ohne Blátter. Die Swiebel ift weiślid) und yoll mitt=

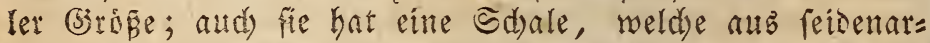
tigen faiden befteht, wie die erfere 2lat, mit weld)er fie gleid) behamselt wird.

5) A. undulata, wellenblattrige $2 \mathfrak{m a r y} l=$ Iis. Bom Eap. - Eie gleidst Der vorigen in vieler f̧in= fidt; iesod) find alle Iheile fáterer und die Blumen find orangeroth. Eie ift eitre fdone Pfanze, die ierod) wegen

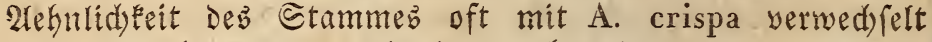
wird, welde lestere mit jener aber in gar Eeinen $\mathfrak{B} e r=$ gletd) zul ftellen ift.

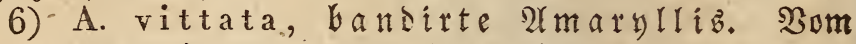
Eap. - Die SBlatter siefer art find flach, $1 \frac{x}{2} \mathfrak{F} \amalg \tilde{B}$ lang und

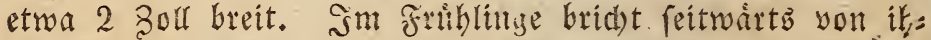
nen bie Snospe herans. Der B̉lumenfdaft wird ungefahr $2 \mathfrak{F} u \tilde{B}$ bod), iff ziemlich fark, hohl und traigt an reiner Epise 3-6 grofe lilienartige Blumen, deren Blàtter et:

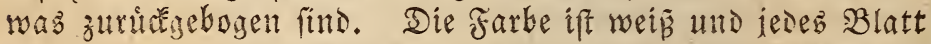
bat 2 Earminrothe Streifen, weldhe an den Blattpisen in

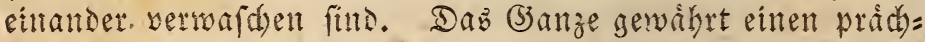
tigen 2tnblict. Bmweilen fringt eine Bwiebel, wenn fie ftart

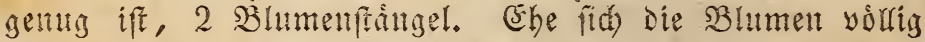
iffnen, riedsen fie wider!ids; Dod) wird Der (jernd in Der Folge angenthmer. Sie hinterlafen rundlide Samencap=

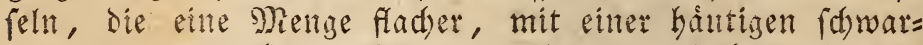
zen Sant nmigbener Sorner enthalten. Sobald fie reif find, fatet man fie in leid) te Erde und ferlt den 'Iopf an eineit warmen fidattigen Drt. Die Pflinzden zeigen fich gewihnlid) bald unt werden máfing fend)t tiberwintert. Sie rad) fen feht langfam, dod bleibt diefe Sietmehrungsart bey=

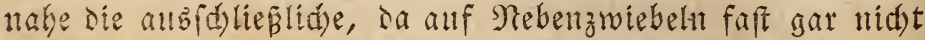
zu rechuten ift. Sm Monat Suny tritt Diefe 2(marylliz in ifren Ruhefand; man läbt fie trodknen, verfeht fie dann, 


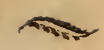

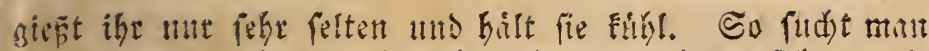

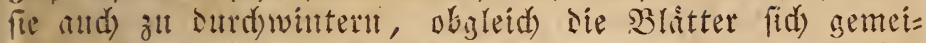

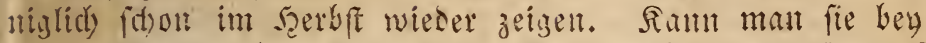
2-5 (sino nidit weit yom Jenfer surdsoringen, fo Darf

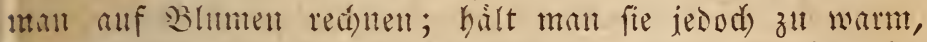

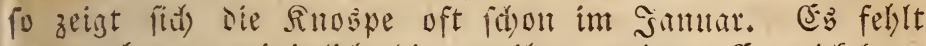
Bann aber gemeiniglid) bie za ister weitern Entwidelung

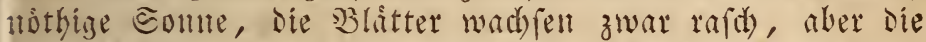
Fnospe bleibt gewobnlid frehen, und ponmt and fpiter

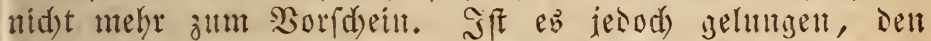
Irieb fo lange als moglid jutrictzubalten, fo ftelle man

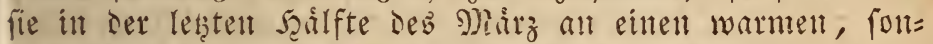
nentreid)en Drt, am beften in eill frifdes, nid)t zul niesti=

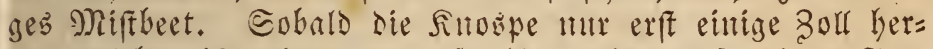
yorigetrieben ift, fam man fie binter jesem fomnigen gen=

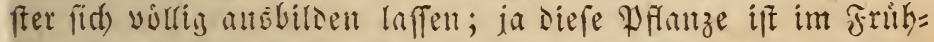

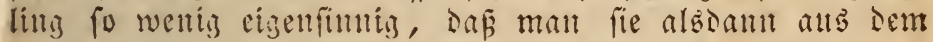
Iopfe nehmen, verfensent tmo wieder eintlanzen fann, fie

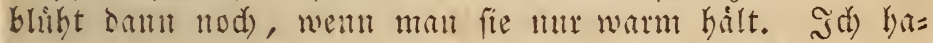
be im Sabe 1823 eine foldhe 3wiebel im Mntrz yoll Dell (Sebridern Boutdsé alts Berlin erbalten, welde mit eilier

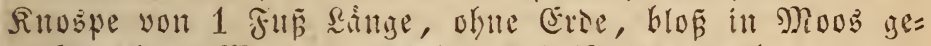

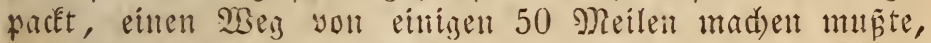

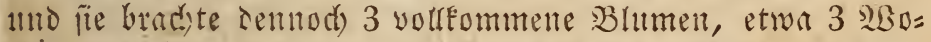
c)en, nad)om id) fie eingefest batte. - $\mathfrak{W a b}_{\text {rae }} 2(\mathrm{ma}=$

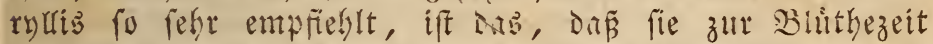

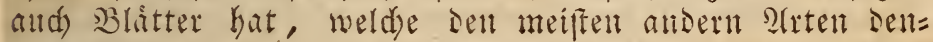
nod) fiflen.

Al mer felbit gezogett.

\section{Amomum, șigwer, I. 1.}

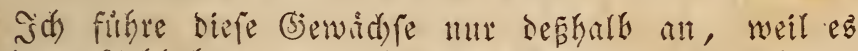

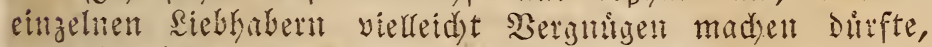
auth (Sewåd) ze zulltiviten, weld)e in mercantilifder Sein= fidet merfwildotig find.

1) A. zerumbet, $\mathfrak{B} \mathfrak{l}$ odfinguer, 24. Dftindien. -

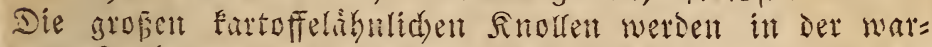
tmen Etube in trodfitit Eroe diberwintert.

Sobald fid) im Fribling die fieime zeiget, fringt

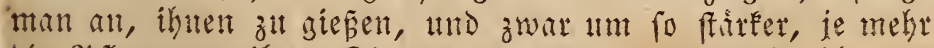
Die NHanze treibt. Eie verlangt Dab ganze Sabr ther ei= nen Geipen Etand binter fenfern, mb Die robrartigen, mit 
grof́en bellgrůnen Blåttern befekten Etångel werien albs Dautn 4-5 Fus bod. Die Blumenftangel zeigen fich im Ferbft, fie bred)en neben Den Blattfängeln aus Der $\mathfrak{B u r}=$ jel hervor, tragen einen etrzen (d)uppigen Folben mit weis = lichen Blumdsen. - Diefer Singwer verlangt sauberde, ftark mit verwittertem Torf und Sand gemengt; grofe Iopfe find ifm ebenfalls nothwendis, fonft fprengen die finollen Sie Eleinen bey Beiten aubeinanier. Ende Detober idnei=

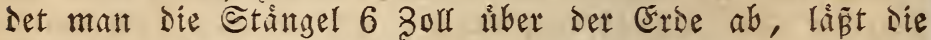
Pflanze trodken, fortirt Die SWurzeln, indem man sie Elé= nen weisen zun nàdffen Sahre in befondere Iopfe legt,

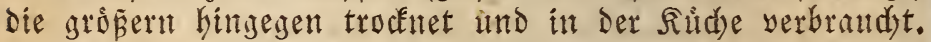
- Db biefer singwer in Der @tube zum Blihen gebrad)t wersen fann, bezweifle id) bennake, weil man nidjt im

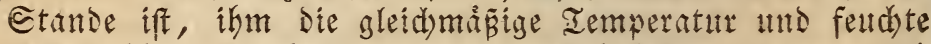

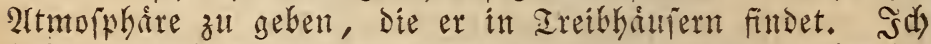
babe die Stångel miemals Gober als bis zu 2 Fu gebrad)t.

2) A. zingiber,ed) ter sing wer, 21. Dfritndent.-

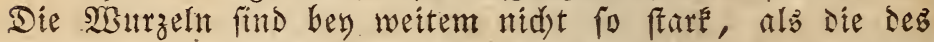

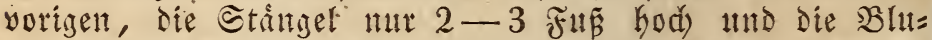
men Eleiner. Die fomalen Blátter babent in der Singend einent (d) wad)en angentermen (jertd), befonders, wenn man

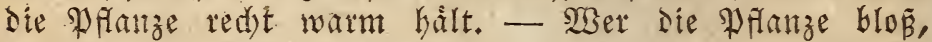
lim fie za beftisen, cultivirt, der gebe ifyr einen nidjt zat

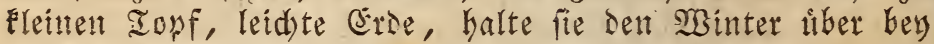
8-10 (Sirad nidut weit yom Fenfer, und ftelle fie Des Eommers mit andern (Siendidfent an die suft. Sie if nid)t

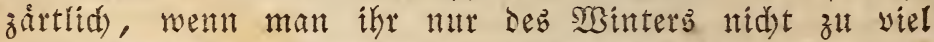
gieft, und delt Froft abmefrt. Blumen babe ide bey sies fer Befandung freilid) nie erbaltent.

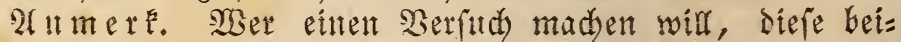
Een Singmerarten im Gropen zit zieken, der lepe die

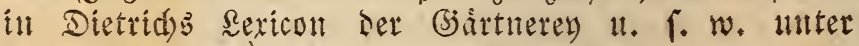
Amomum zingiber enthaltente altmetints.

Amygdalus, 代atdel, XII. 1.

1) A. pumila, niestige Nandel, gefillt blủhende zwergmandel, fer Etraud) im freyen sambe albbattert, fo verdient er sod methe, als mand)e antere \$flanze, einen Stand im Bimmer. Die feinen 3weige fint mit einer rothlichen Rinte besedt, แnd Die Bhatter gleiden den deś gemeinen Pflaumbaumes. Die Eleinen blaprothen gefiuten $B$ lumen erfheinen mit ben 


\section{^ 51}

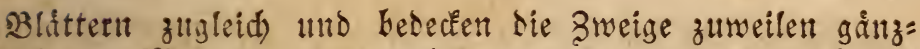

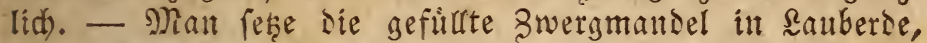
meld)e ftark mit sefhm gemengt ift, laffe fie úber Sommer an einem nidst zu fonnenteidsen Drte fehen, ober grabe fie, wettn mat Bselesenheit bat, thber Sommer im freneat

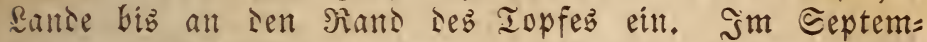

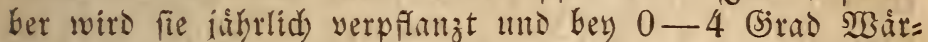
me. úberwintert. Sim Mårz bringt man fie in Die હtu= be an ein fornigeb Genfer und befprengt sie sweine

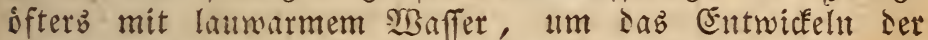

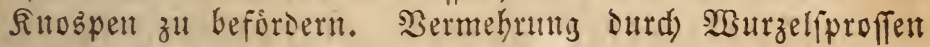
unt Dculiren auf Die folgende 2(rt.

2) A. nana, zwerzmandel, h. 2tus હibirient. Die mattgrunten Blítter find lanzettförmis, am Rante ges záhnt. Die Eleinen einfuden, rothen Blümden erideinen in Nenge im 2lpril, uno feşen aud zuweilent Frủdte an, weld)e fehr bittre Rerne enthulten. MRan Fann siefe Rret nie sie vorige behandeln; Dodh pañt fie beffer fưtra frene

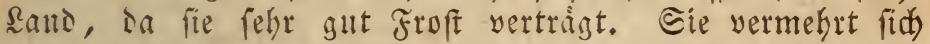

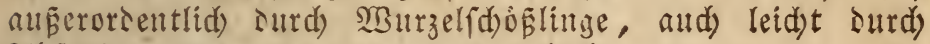

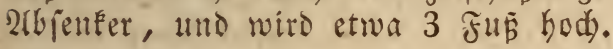

$$
\text { Anagallis, (j)andbeil, V. } 1 .
$$

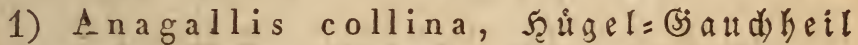
(A. fruticosa), h. - Mardcco. Eine Eleine Pfanze, Die felten tiber einen Fus hod wird. Die vierectigen

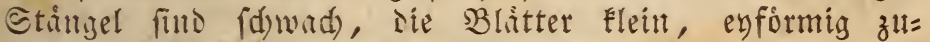
geppişt, auf Der untern Seite fein braun punctirt, uno frehen ohne Êtiele zu $2-3 \mathfrak{u m}$ die sweige. Die 5 blät= terigen B̂tumen finto zimnoberroth, um Die હtanbfáden vio= lett, Etaubbentel gelb. Die Blumen erideinen ien gan=

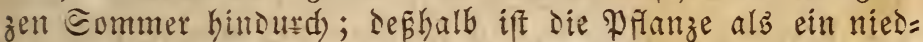

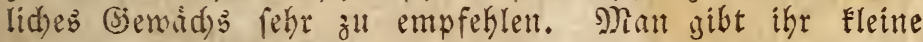
Tópfe, sauberse mit sefhm unt etwab હand gemengt, úber

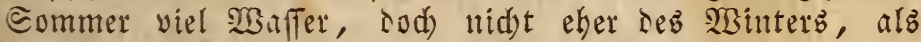

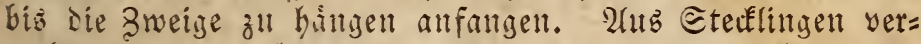

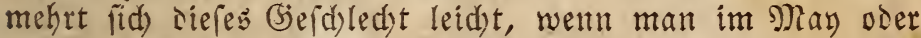
Siunty zollange zrweige abjduneivet, mit einem Gilaje bes Deckt, und fie Der Sonne alsbient. 2(ber ebent fo leidht ges

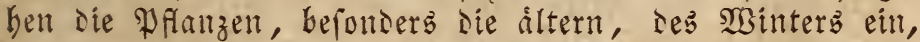
twent man fie zul warm hålt, ofhne ihnen binlảnglida suft geben zu f́ónnen, oder went malt es mit Dem (j)ésen ver=

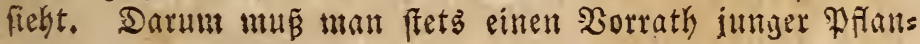



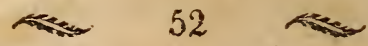

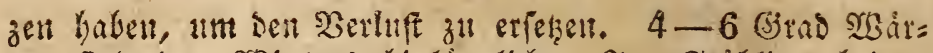

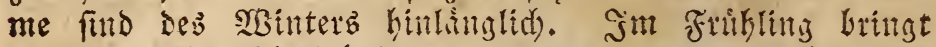

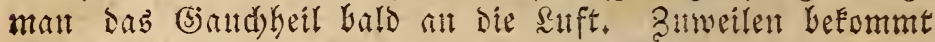
man and Enmen.

2) A. monelli, aufredi) teb (b) तn d) heil (A. fru-

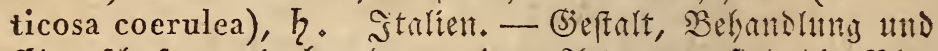
Eigenfidaften wie bey Der yorigen Alrt; mtr fitto die Blu=

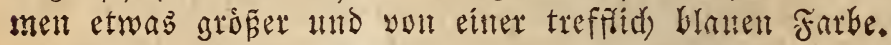

\section{Andromeda, alndtomede, X. 1.}

A. polifolia, poleyblattrige at ntome De, ط. Sorbentopa. - Ein Eleiner Etrantí), weldyer in

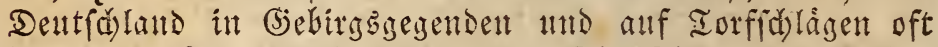
in grofer Menge gefunden wird. Die feinen smeige find

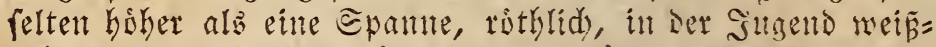

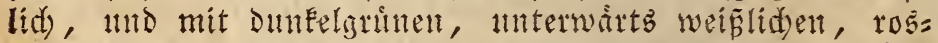
marinartigen Blattern befest. 2lat Der Spise Der sweige

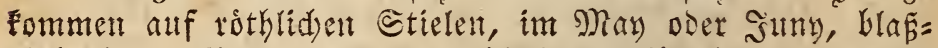

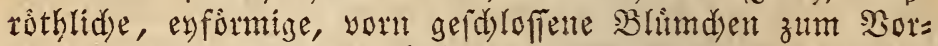
(a)ein, weldhe etwas Gerabkingent. Nhan gibt Diefer Pfan=

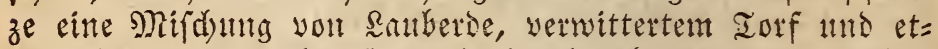

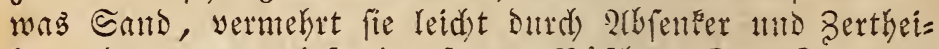
lung Der etwas tief eingefestent Bardae. Des Commerz

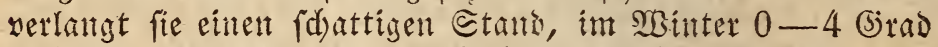

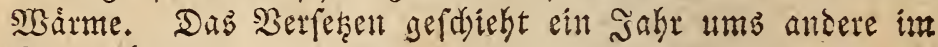
Eeptember.

Anemona, Iftemolie, XIII. 5.

1) A. hepatica (Hepatica nobilis), seber=

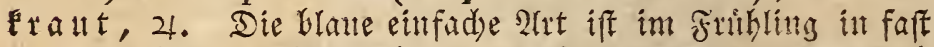

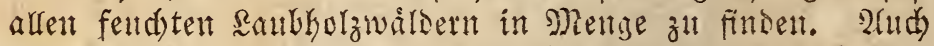

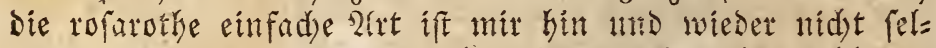
ten vorisefummen, namentlid) alf hem nòrdident 2(bhange

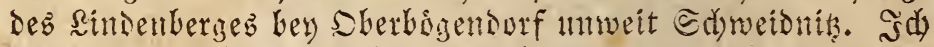

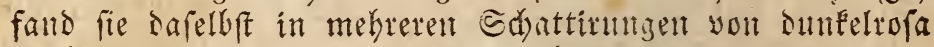
ab bis faft rein weis ; altd) bie blatten gewibntlichen was

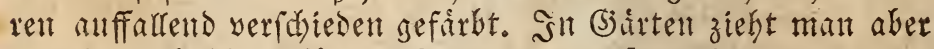
gemeiniglid) bie gefuldten arten yon rofentrotber, putputro= ther, Glauer oder weiper Farbe. Die Blatter find dreylap= pig. Die sBlument erideinen im 2lpril, vor sen Blittern.

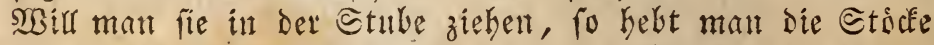
im 2luguft aus der Eride, felst fie int nid)t zut enze, etwas tiefe Iopfe, lábt fie an cillem (d)attigen sorte fethen, 
unto bringt fie erft, went es anfungt febr falt zu wet=

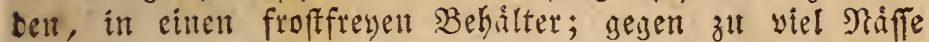
mus mat jerody die Iopfe ebenfars fidsert. Sim Februar ftellt man fie ant ein fommiges genfer, wo bey 4-6 (5)rad fid) Die SBlunten rebi bald entwidelt, aber and) fdnell ver: gebell, went mant fie waimer balt. - Sif man gentothigt, die seberblumen immer in Iopfen zu behalten, fo muffen siefe, wie gefayt, nidjt zul eng feyn, weil bie Pflanzen ftare wurzeli. Sim Eommer verlangen fie viel Waffer Ind einen fdattigen હtand. N)an verpflangt und zertbeilt die Eticfe an beften in shingf, Hild (d) neidet im December die alten brimten Blatter $a b$. Fur Eleine Siarten eignet fid) wergen thres frithen szlibens diefe Pfanze febr, befun=

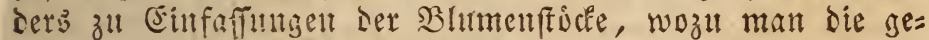
meine, wilde fefr gut bennben fann.

\section{11110}

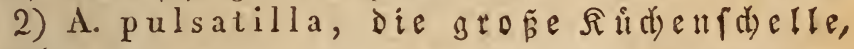

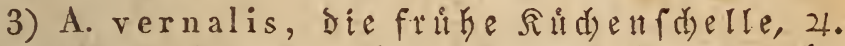

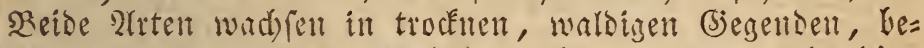

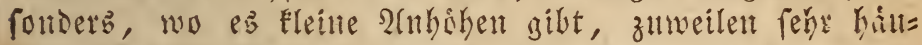

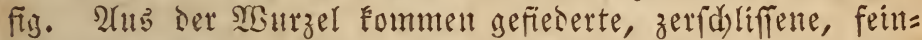
baarige Blatter, "nto zmifden Diefen etwa 6 Bou bohe

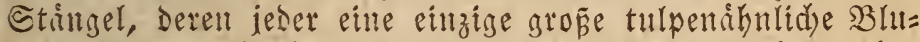
me traist, weldse bey der erftell 2(tt fdon violettblan, ben ber zweyten rothlidwei⿱⺈, auperfalb etwas purpurfarbig and baarin iff; bod) gibt es im Betreff det Farben mebrete

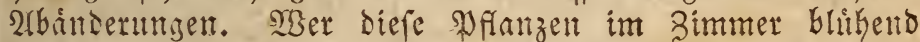

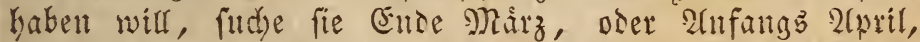
wenn die Subspen berborbredsen, an ifrem naturlidsen Etansort auf, bebe fie mit dem Barren nus und pflanze fie in die nimlidse Eroe, worth fie geftanden. Man balt

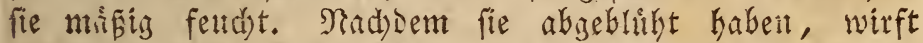
mat die Sudjenfibellen fort, benn fie gemolnen fidy mut feltent ant den Iopf odet an einent Stand im Sarten. Sur, wo diefe (joewaidje felten find, yerfud man es zumeilen; sann gift man ifnen einen moglidif mit iftem naturlis don Standort dibereinftimmenden Mlas.

4) A. a lpina, 2I lpenanemone, 2\%. Cie gleid)t dent sorigen beisen; if aker weit fleiner, und bie Blume ganz

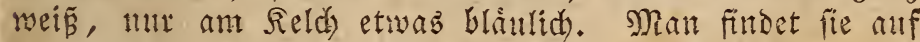

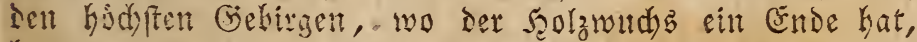

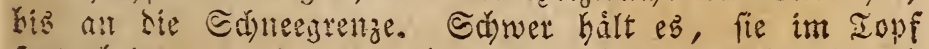
furtubringen, und auser ifret Eeltentseit empfiehlt fie fids nidit befonders. 


\section{Anthemis, chamille, XIX. 2.}

\section{A. artemis a efolium, beyfuß́blattrige} (Ehamille, 24. Ehina. - Eine Febr befannte Pflanze, mel= dhe yom Sctober bis nach Meihnadten Die Fallptzierde ber (Sewåd)b̆aufer ift. Die bolzigen Etångel werden $2-4$

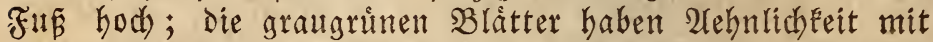
Denen deb $\mathfrak{B}$ eyfunez (Artemisia vulgaris). Die Blumen gleiden einigermapen bell affern, fino jedod) himfidts'ber (s)róne, Bildung der Blatter und Garbe fehr verichieden.

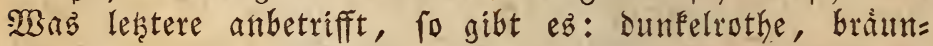
lidi) orangerothe, ocfergelbe, citronengelke, weise, rofarothe und bunte. Stre diefe Farben findet man in unzábligen 2üfufungen, bie aber nid)t alle bleibend find, fondern grỏs $=$ tentheilz yon der Eultur und Dem Buftande Der PFanze ab= bången. Gehr hubid) ift zuweilen Die bunte. Shbre Sraupt= farbe ift weís, bald mit rothen હtreifen, bald mit rofas

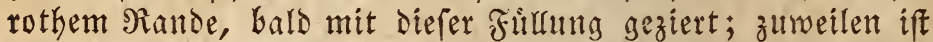
Die eine b̧ảlfte der Blume weís, die antere rofa; mand)mal find mebrere diefer Mifdungen auf einer Pfanze zu finden. Die Blumenblätter find entweder offen, oder robrig (fistulosae). Cinige Blumen find flein, ranunkelartig sebaut, alt= Dere fehr grof und offen. Die eirfachen Sorten find feltner

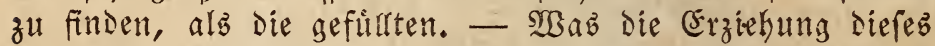
(S)ewaid) fes anbelangt, fo merfe man Folgendes: im 2 pril oder 2 Unfang $\mathfrak{M a y}$ yerpflanzt man bie alten હtócé ; yon ben vielen 2 Burzeliproffen nimmt man to viele $a b$, als man eitrethen will, bie itbrigen werden weggeworfen. Die bes

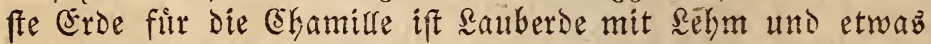
gut gefaultem Mift gemengt. Sedez Pflínzdenen erbált ei= nen Eleinen Iopf, wird, to wie eb bie suft geftattet, in Diefelbe gebradt, und bleibt bis 2 nfang suly Darin ftehen; bann fest man fie in wenigftenz $6-8$ 3oll hoke und eben fo weite Iopfe. Man erlangt bierourd) Den Mortheil, Da man das allzu ftarke Waadjen anfänglith binoert, und ben

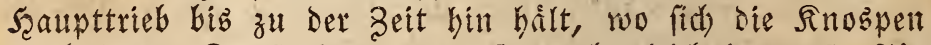

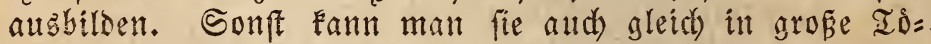
pfe reşen, wenn diefe fo gerôming find, bis zum Şerbft den pfanjen Ginlánglida) Nahrung zli geben. - Den Eommer hiber läpt man fie an einem Plake frehen, wo fie suft uno Regen erhalten, Dod) gegent Die brennensen Sonnenftrablen gefduht find; denn is die Ebamilfen fehr viel Maffer be=

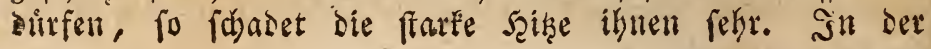


Mitte September goint man ifnen jebod) bie volle Sons me, Reşt Die mit Rnospen verfehenen Stråduer inz Zim= mer binter Genfter, und gibt ifunen taiglid) suft und po viel Sigaffer, alz fie bedurfen. Diejenigen, welde nod feine Rnobpen angejest baben, bleiben in Der suft feken. Befibt man einen Ireibéaften, fo fann man, fo balo man bie erfen Snobpen bemerte, einzelne Iopfe Gineinftellen,

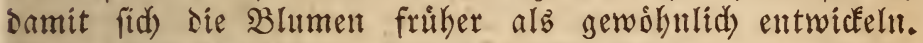
Die fribefte 2(rt ift, nad) meinen $\mathfrak{B e o b a d}$ tungen, alba fistulosa. NBill man bie EGhamillenflor lange gentesen, fo laffe man die blikgenden Iopfe in einem Fuhlen Bimmer in Der Nåke Des Fenfterz ftehen, uno gebe ihnen suft. Ein

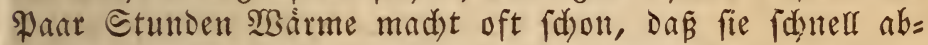
bliken. - Nadjocm die Blumen verwelet find, idhetoet man die Etängel 4 3oll hiber ber Eroe ab, mo hiberwin=

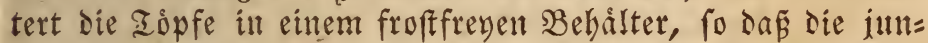

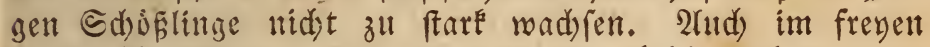
Rande uberwintern siefe Pflanzen oft, blikben aber felten, insem ser froft fie gemeiniglid) úbereilt. Biele bjärtner und Blumenfreunde fenten die Iopfe mit ihren EGamillen úber Sommer in Die (Eroe. Dod babe id immer bemert,

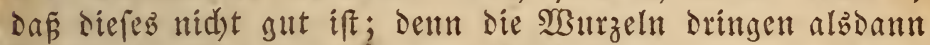

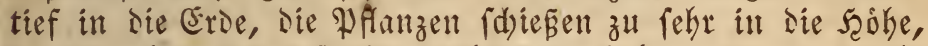
trauern aber, ment fie im Şerbft aubgehoben, und Die burd)=

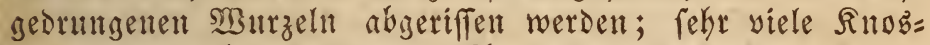
pen geben sabey verloren. Esen fo ift es nid)t rathfam,

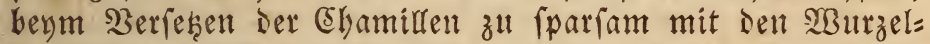
fproffen umzugehen. Nie pflanze man mehr in einen Topf,

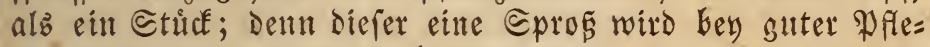
ge gewíz mehr Blumen bringen, alş ein ganzer Buld, weld)em es an Nahrung fehlt. Befitit man eine feltene 2lrt Chamitren, to Eann man Diefe leidst Durdd Etedelinge vermekren, indem man im Nay die Epise Der Pfianze $a \mathfrak{b}=$ (d) neiset unt einftedt; im Nifteet, anfănglid fdattig ges balten, madst ein fold)er Etecting fdnell Burzeln. Die aus Dem Nutterftamm getriebenti Eeitenfproffen werden fpaiter altd) abgenommen und als @tectlinge behandelt. \$flegt mant fie gltt, fo bluhket die biz zum suly gemad)te Sermebrung nod im nåmlid)en Sabre. - Heberhanpt Fann

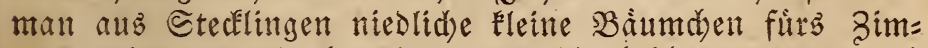

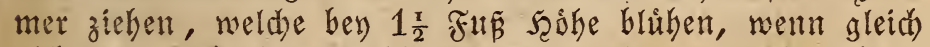

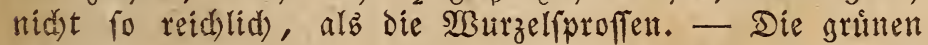

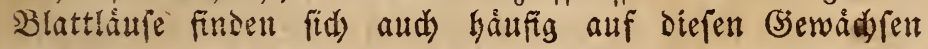




\section{人e⿱乛龰 56}

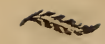

eit, Gejonbers, went man ihnen nid)t genug suft geben

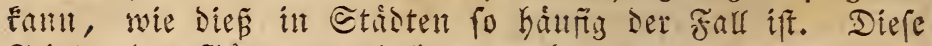
Feinde ser (5iartnerey fudbe mant bers zeiten zu entfermen,

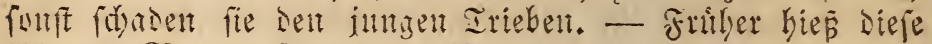
Tianze Chrysanthemum indicum.

\section{Arctolis, Bifenofr, XIX. 4.}

1) A. cineraria, afd farbiges bånenofr,

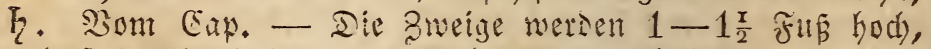

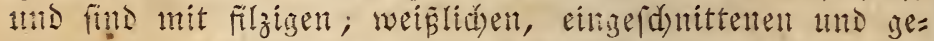
lappten Blittern bejeşt. Die Grünlid orangerothen Blu= ment erifheinen Den Sommer uber, gleid)en Den Ringelblu=

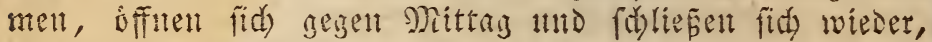
To Salo die Contue fie verhifít.

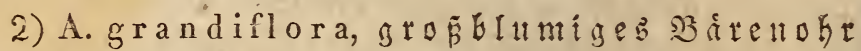
h. Faft wie sas yorige, mut fitto sie Blitter feitter, faft

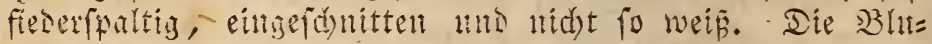

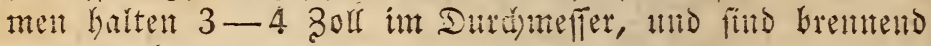
orangetoth.

3) A. rosea, rofentothe $\mathfrak{B}$ ábenobr Dem vorigen afulids, Gat iebod) fleittere, fidmuthis rofaro= the $\$ 3$ himell.

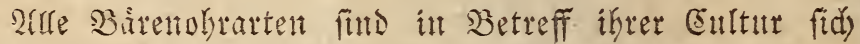

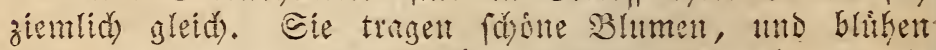
faft ben ganzen Commer liber reidylid. Serodi find fie im Ximmer nidyt ganz leidyt zul liberwintern; senn ent=

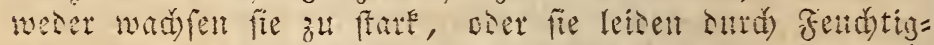

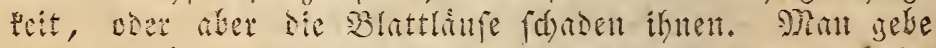

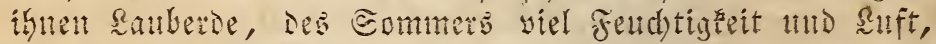

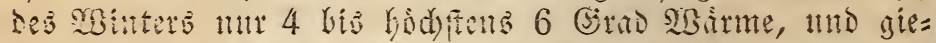

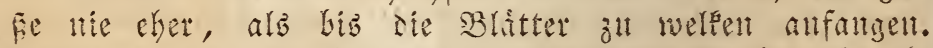

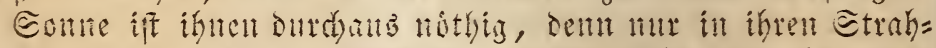

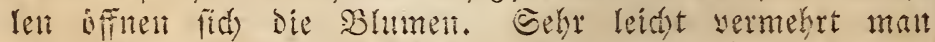

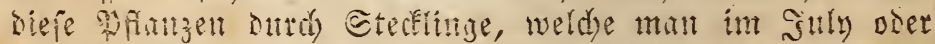

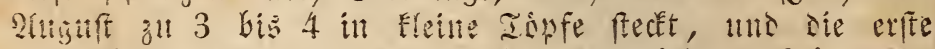

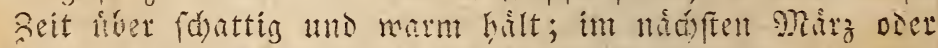

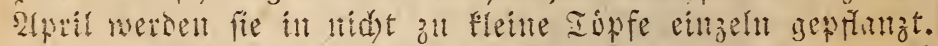

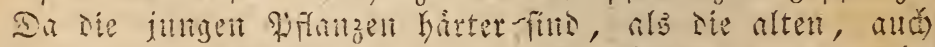

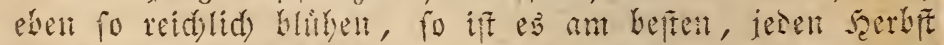

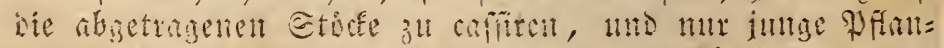

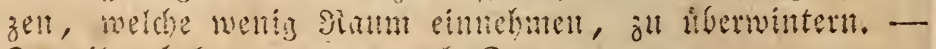
Bumeiten befonmit mant atch Eament. 


\section{Aristolochia, Oferluzey, XX. 4.}

A. sempervirens, immergtultie oferlu= zey, $\zeta$. Ereta. - Die fdlanfen Etaingel wad) fen mehrere Jup hod) mis balten fid) mit seilfe ser gedrehten Blatt= fiele ser beandhe pfelformigen blitter an anoere Pflan=

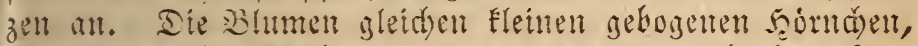

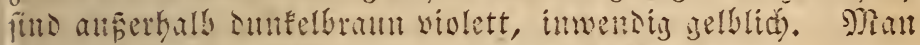

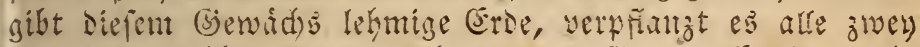

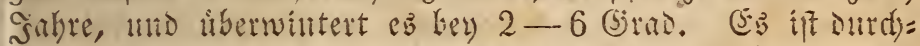

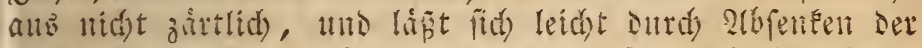

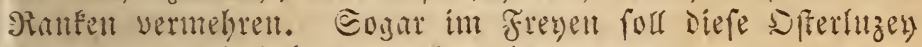
altsonttern; id Fabe es nod) nid) verfutht.

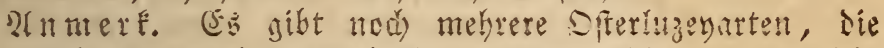

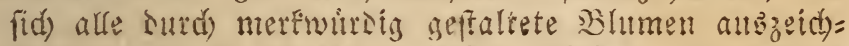
nen; z. 23. A. glauca, triloba, Sipho. Die leţtere

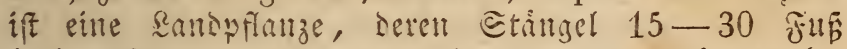
hod) meferigen, mit aropen berzformigen B!dittert be=

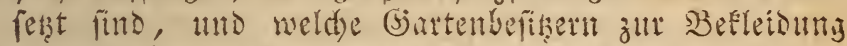

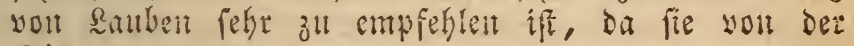
Sîlte wentis leiset.

\section{Arum, כehrmurz, XXI. 7.}

A. divaricatum, finfellde 8 ebrmutr, 21.

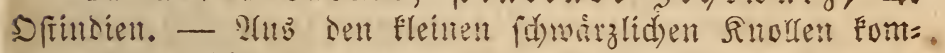
men 3-6 fojldformige, anf einige zoll langen @tielen

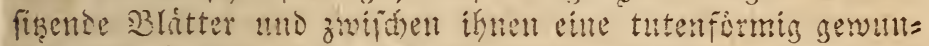

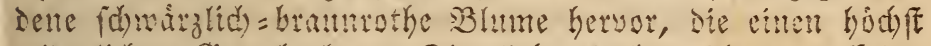

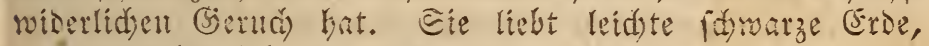

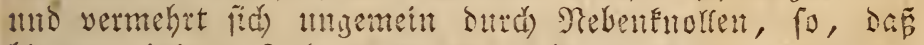

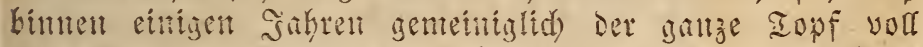

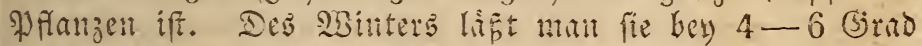
gatr troben frehen; int Eommer verlangt fie jerod) viel

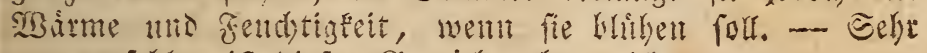

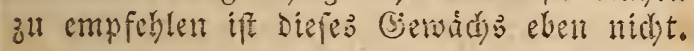

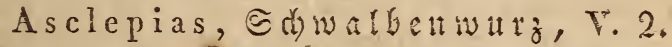

1) A. angustifolia, fdomalb ôttig

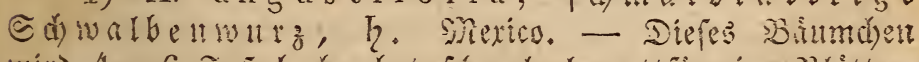
wiro 4-6 Jur hodi), hat fímale lanzettfurmige shitter,

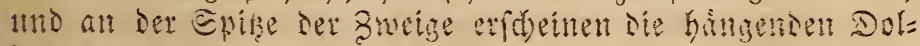

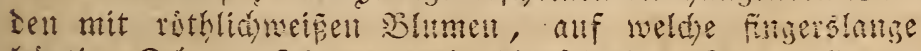

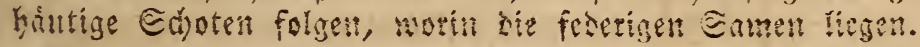




\section{$\pi \quad 58 \leadsto$}

Sie liebt gute, nahrbafte Erbe, wurzelt ftarl, unb roill alliábrlín im Mårz verpflanzt fenn. Des Winters bringt man fie bey 4-6 (Sirad gut Durd), went man fie niddt zul feud)t bălt. Bermehrung Durd) Samen und Etecflinge.

2) A. curassavica, orangerothe Sd walben= $\mathfrak{w} \mathfrak{u} \mathfrak{z}, \quad$. Euraffad - Diefes (J)ewád) bat breitere, lanzettformige Blritter yon Dunkelgrủter Farbe, die einen

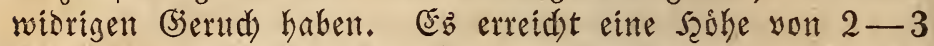

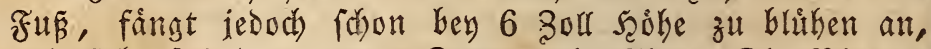
und fielyt faft Den ganzen Sommer in Flor. Die Blumen find lebbaft orangeroth, und b̧interlaffen ábnlidye Sdyoten,

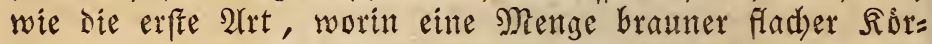
ner liegen, wodurd) man bie Pflanze vermehrt. Da fie

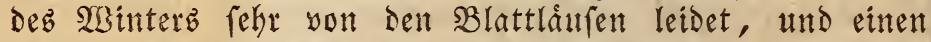
fonneneid)en warmen Ctand zum Durdywintern verlangt, fo thut man am Beften, jábrlid) eintige pflanzen aus હamen zll zieken, ben man bey jedem Şandelg̈gåntuer befommt. Man fịet Denfelben im Márz oder 2tpril, wenn man (S̈ele= genheit Gat, ins Miftbeet, verpflanzt Die Pflanzdhen, wenn

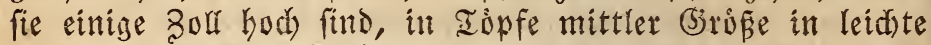
(Erde, und balt fie beftảndig binter (Slas. Sie lieben ziem=

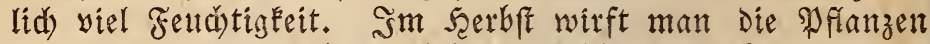
fort, uno erfpart fid Dadurd) bie Mibe des Heberwinterns, mas Dod) felten glicét.

3) A. fruticosa, fraudartige Sdrualben=

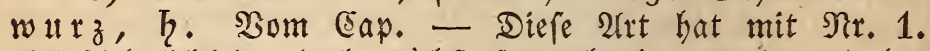

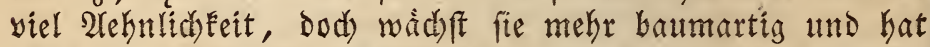
ganz weífe Blumen. Behanolung: wie bey iener angegeben.

4) A. carnosa f. Schollia crassifolia.

Aster, Sternblume, XIX. 2.

A. angustifolius, fdumalblåtrige ভtern= blume, . ' Bom Eap. - Diefer Etrand) mit bunnen

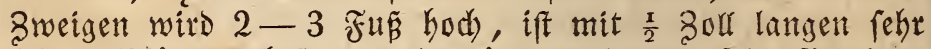
feinen Bláttern befesct, uno trágt an lanigen fadenförnigen Stielen blapblaue Strahlenblimáden. Snan sibt diefer

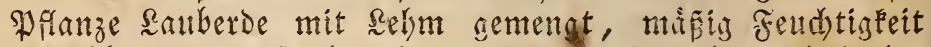
und hiberwintert fie bey 4-6 Grat. Sie läpt fid leidst, und bennake zut jeser zeit, Dureh Stectinge vernebrent.

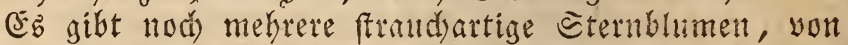
benen jedod) viele nist) felten find. Sid Eenne mur A. fruticulosus und filifolius. Beite babe id znar nod nidat felbft gebaut, dudj wollen fie eben fo wie A. angusiofolius 


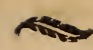

behantelt fenn. - A. carolinianus hat ein guter Frento woul mir mebrere Sabre gezogen; fie wurbe thber 5 Fus hod), Gat aber nie, weder im Iopfe, nod itts sand ge=

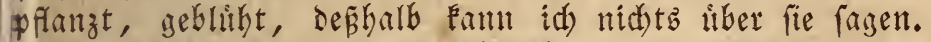

SBif man you den gewobnliden perentirenden sants= aftern eithe oder bie andere Sorte im Iopf zieken, fo ver= fáfrt man, wie z. $\mathfrak{B}$. bey Achillea gefagt worden ift. Nan lápt wo mogglich die Iopfe im sante fieken, bis die Fnospen fid zeigen. Cie bedirfen, viel Maffer.

\section{Atragene, $\mathfrak{A} \mathfrak{t} \mathfrak{a} \mathfrak{g} \mathfrak{e} \mathfrak{e}$, XIII. 13.}

A. balearica, balearifde $\mathfrak{a t r a g e n e ~ ( c l e m a t i s ~}$ balearica), $\zeta$. Balearifde Snfeln. - Eine હd) lingpflanze, weldse aud im Iopf $3-5$ Fus hod), luto Goher wird. Die zerid)liffenten lebhaft grimen Blitter falfen im saufe des Sommers gróstentheils ab, bred)en aber im Serbft wieder Gervor. Die gropent vierblattrigen, offten Blumen

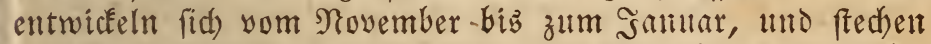

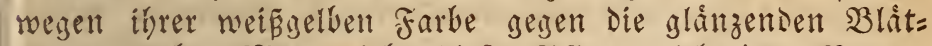
ter gut ab. Man giebt diefer Pflanze lebmigen Boden,

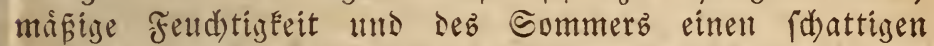
Stand. Sm SBinter mus man fie bey $4-6$ (S)rad in Der

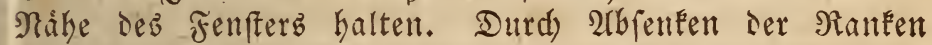
uno 2rbnefmen ber Eeitenfproffen im Monat Eeptember, bey (j)elegenheit dez jä́brlid)en Berfeşens, fann man fie leid)t vermegren.

\section{A ucuba, $\mathscr{A} u c \mathfrak{b} a$, XXI. 4.}

A. japonica, japanifdje $\mathfrak{2} \mathfrak{u} \mathfrak{u} \mathfrak{b} a, h$. Sapan. - Ein ziemlid) boher Etraud), welder wegen (eitter gro=

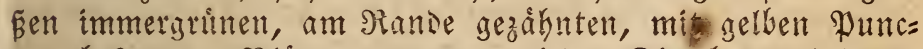
ten beftrenten Blåtter gezogen wirs. Die braunvioletten

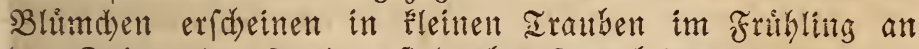
den Episen Der Sweige, find aber fo unbedeutent, Dá fie oft nid)t bemertet mersen. Diefer Straud) liebt (d)were leh=

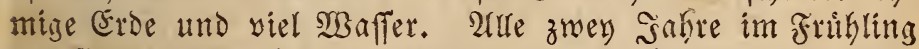

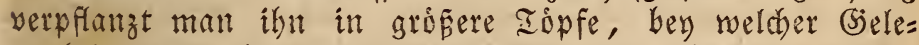

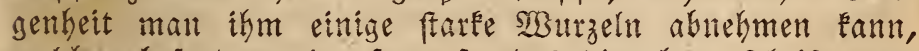
weld)e, befonverz eingefeht, fo, daṕ die obern Ibeile etwa 1 Boul ther bie croe vorragen, nibst felten austreiben.

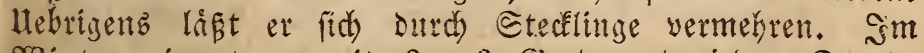
Sinter nimmt ex mit 2-6 Grad und jesem Etande yorlieb, aud) foil er im frewen sande uberwintern. - Eol= 


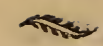

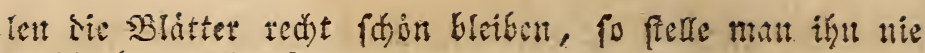
in sie bremento Eonnt.

\section{Azalea, Felfenftaud), V. 1.}

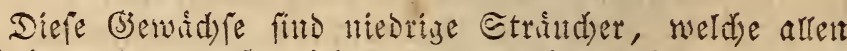

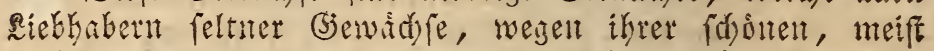

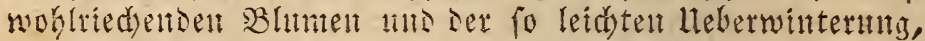
zu empfeglen fint. Nan gibt ifnen sauberse nit etwas Dorf und grofent Sand gentent, mápig Fendotigét und

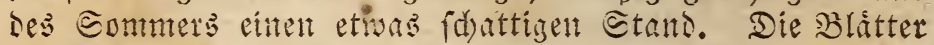

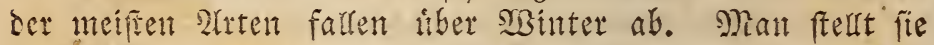
Dantit itl eitl froffreyes Gersobe, oder in eill 3immer,

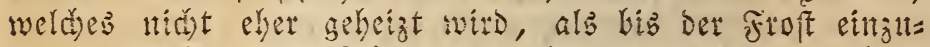

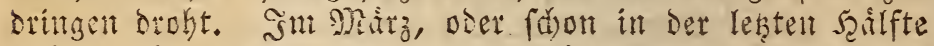

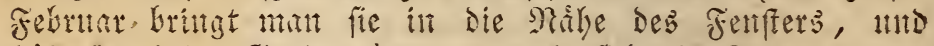

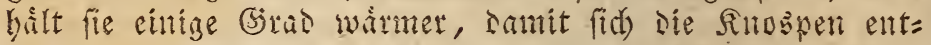

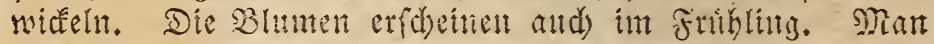

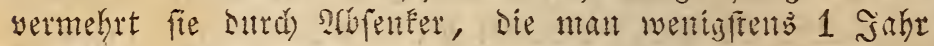

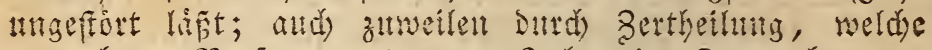
mant beyn Berfesen affe gwey Sinte im Eeptember ver=

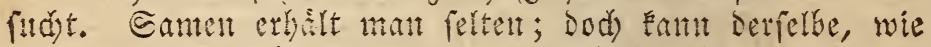
es weiter unten bey Rhododendron bejariben ift, began=

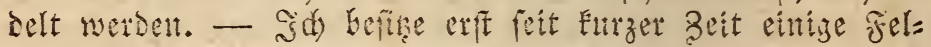

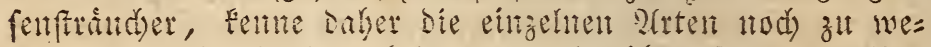

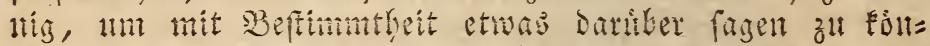

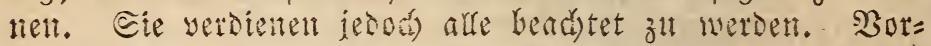

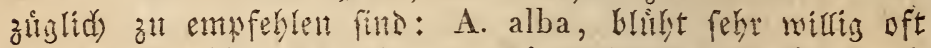
bey 6 zoll sobe weis; A. aurantia, mit orangerothen, und

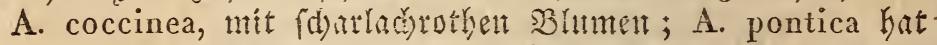

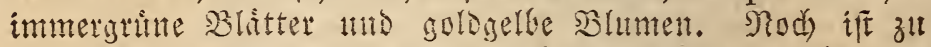

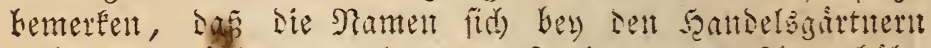

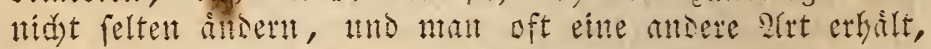
als man erwartet batte. Sie frammen gróstentbeils aus Rordamerica.

\section{Begonia, @dicfolatt, XxI. \%.}

B. discolor (B. Ewansiana), buttes Edulefblatt, 4.

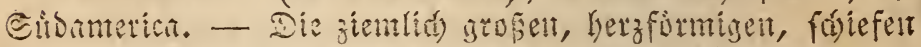

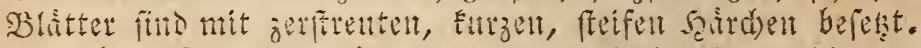

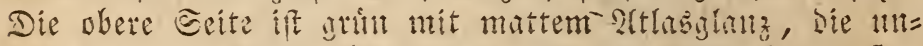

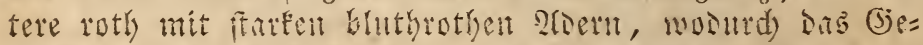

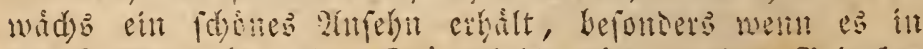

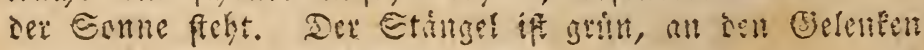


ebenfutro roth gefarbt, aufredt, thailt fid in mântere Eei=

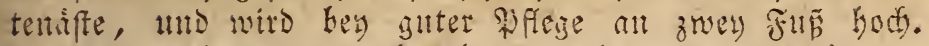

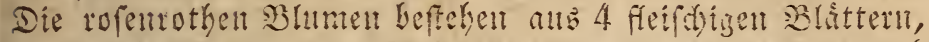

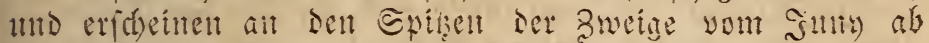
bis ill sell Sctober. Buerft fommen lanter minnlide \$lu=

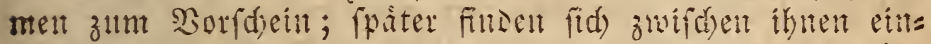

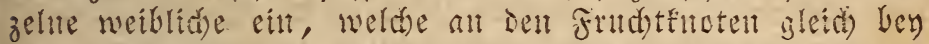

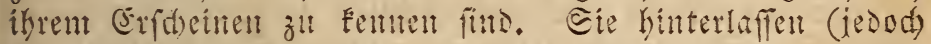
mur nath Eutuflider Befudstung, indent man eine abge=

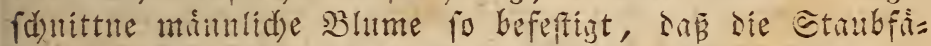

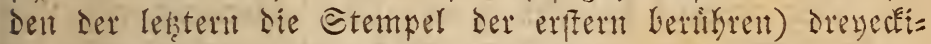

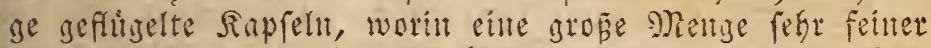

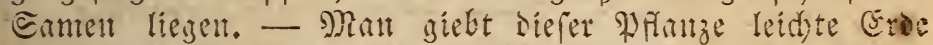

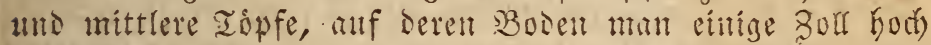

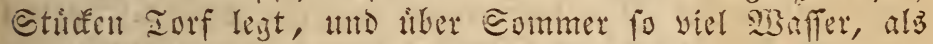

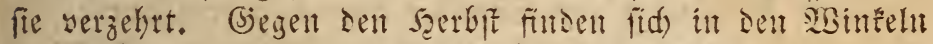

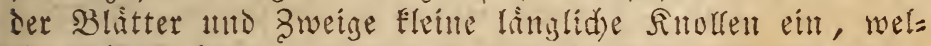
d)e fpater abfalfen, lund gefammelt werben, went man bie Dffanze vermefren will. Die Blitter, sweige mo હtin=

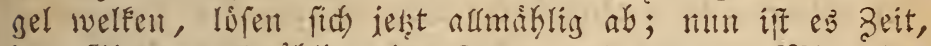

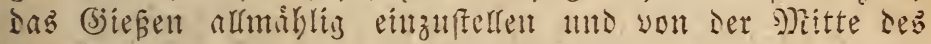
Sctobers ab faft ganz samit aufzuborent. Dent Iopf mit

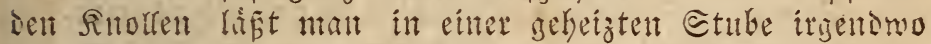

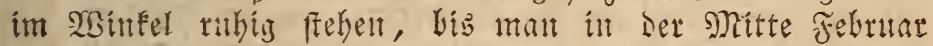
sie 93 mzeln in frifde eroe legt, mumegr alfe 8 oser 14 sage eitl wenis giest, mo wenn Iriebe fid zeisen, Die Dranze ang Ridjt bringt. Die oben erwähnten fleinen Fnothen bebandelt man eben fo. Sftud surd) Eteclinige

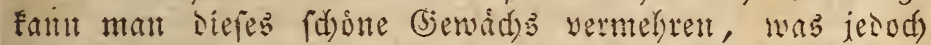
nid) nothwentis ift, ba man had) einem Sahte idon mehr alz zu viel \$flanzen erbalten Eann, ment mant alfe

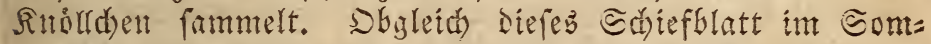

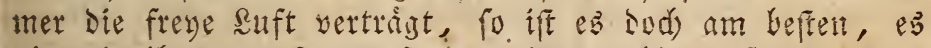

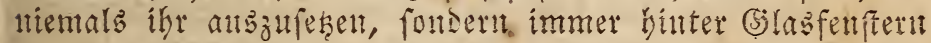

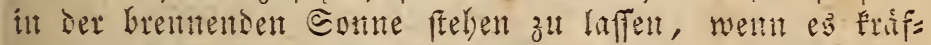

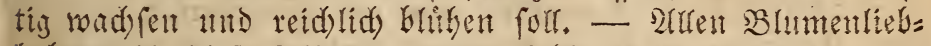
babern if sieje Wfanze zu empfeblent, ba fie im somer bennabe gar feitter Pflege berarf.

\section{Buddleja, Batela, IV. 1.}

B. globosa, Fugetragente Bzudeia, দ. Chilt. Sin Banm, Iet ill feinem Baterlanse genis eine besent=

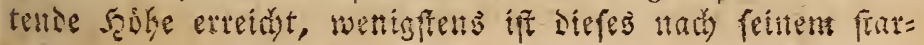




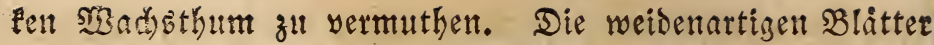
ftehen paarmeife, find weişlid'), ftark geasert. Șm Früb= ling erfotheinen bie Blumen an sen Episen ber Bweige in

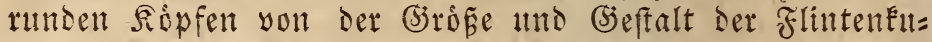

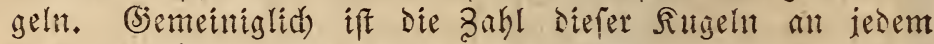

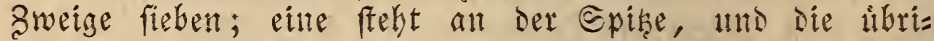
gen parmeife liber Jirltz. SGre Farbe ift lebhaft oran=

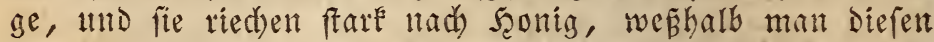
Etrand) auth Den frontigbaum nennt. Die Budleja ver= langt fark mit Refm gemifd) Ere Ero, und will ben Som= mer uber reid)lid) begoffen fenn. Nan uberwintert fie bey 4-6 (stad, tulto gibt ihr, fobalo Die Fnospen fid zei=

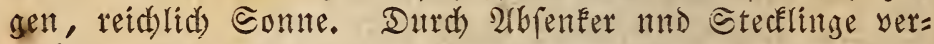
mehrt fie fiù) gutt, nur miffen lestere, bis fie zul wad) fen anfangen, unter (slab und warm gehalten werien. SBent man Die Båumchen Den Sommer tuber aub Dent Topfe ber=

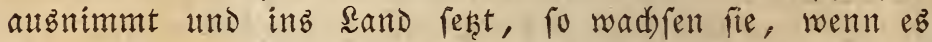
thenen nie an Geudstigeeit fehlt, fo ftare, Daf man fie im Ferbf Faum wieder in die Etuke zuridtnebmen Fann. Dar= um vermetie man eime folde Bebandung.

Bryophyllum, Bryophyllum, VIII. 4.

B. calycinum, gelblides Bryophyllum, 2lfrica? - Die grof́en fletfdrigen, oyalen Blätter find am Rante gekerbt, ftehen an violetten Stielen in Der sill=

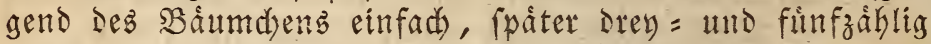
gefiedert. Die Blumen follent gelblidsgrine (s)lodeden mit rothem Rande feyn; idd habe fie nodi nidht gefehen. Die merfwurbigfte (Eigenfd)aft biefer Pflanze ift ibre $\mathfrak{B e r}=$ mehrung. SBent man námlid) ein Blatt abjanetoet, und mit Der untern Geite flad allf bie Errse legt, fo madjt baz

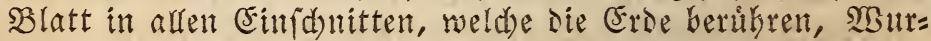

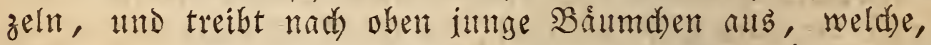
went fie eintige zoll hod fitto, ant dem Blatt Gernisges (d)nitten uno befonders gepflantst wersen Eoumen. Diefez

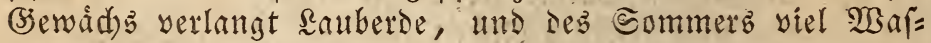

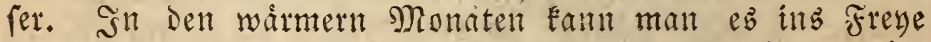

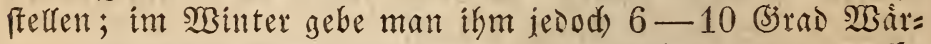
me uns eitten Plats an einem fonnenteid)en Fenfter. (5:

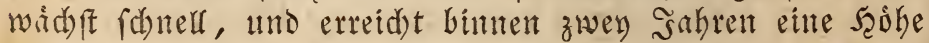

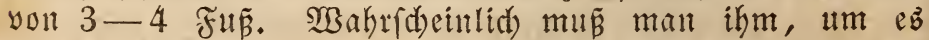
zum Blithen zat bringen, Eleine Iòpfe uno magern Boden gebent. - sfit mit Verea crenata wahrifdeinlid eine pflanze. 
Buxus, $\mathfrak{B} \mathfrak{u} \mathfrak{d} \mathfrak{s} \mathfrak{b} \mathfrak{a} \mathfrak{m}$, XXI. 1 ,

1) B. sempervirens arboreus, hoher $\mathfrak{B}$ แb $b=$ baum, $\quad$. Elideuropa. - (jemeiniglich zieht man in Iopfen die Epielart mit gelb eingefaptem Blatt. Die

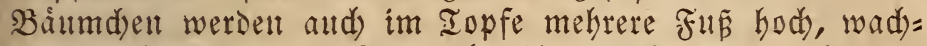
fen jebod) langfam. Nan gibt ibnen fámeren, lehmigen

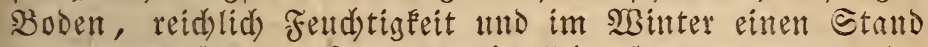
yon $0-4$ (sirad. Es if binlänglid), fie alfe zrwey sabre im 2(pril zu verpflanzen. Die Bermehrung Eam Durd) $\mathfrak{A}(b=$ fenfer uno @teclinge gefdeken.

2) B. sempervirens fruticosus, 8 werg

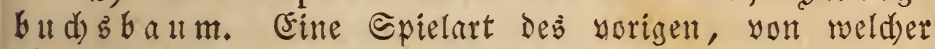
ebenfalls die gelb oder weís gerinderten 2 trten zumeilen im Iopf gezogen werden. Er bleibt niedriger, als ser yorige, uno wird surd) Bertheilung yermehrt, indem man ihn tief einfeht, und im nádbften Sahre die bewurzelten Eproffen abnimmt. Hebrigens Dem vorigen gleid).

Cacalia, Peftwurz, XIX. 1.

1) C. articulata, gegliederte peftwarz, africa. - Die Etingel fitto fleifdis, etwa fo diat wie ein Finger, uno beftehen aนb mehrern ungleid) langen (s)lie= Dern; fie fitto mit einem immergrinen Ihall uberzogen. Sn Der Sittgent tragen fie blangrüne pfeilfỏrmige Blátter auf Eurzen Stielen. Die Blumen, weldse auf einem 4-6 Boll langen Etångel erfdeitten, Der aus Der Epthe Der 3weige im Friflinge Gervorfommt, find Flein, gelblid)= bratu, und baben einen unangentegmen (Serud). ŞBie affe

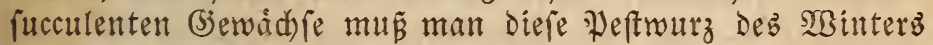
bey 4-6 (3rad ziemlid) trocken balten. Durd) Bertheilung uno Steclinge ift fie fehre leidht zll vermehren, inimmt mit ieder Erdart yorlteb, if aber úbrigens eine pflanze obme befondern 3 Berth.

2) C. Kleinia, canarifde Peftuntr,

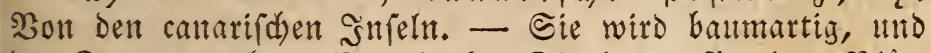
Der Stamm mehrere Zolf ftark. Die lanzettförmigen Blät= ter find 4-5 3olf lang und fdmal, die Blumen grülich. WBir wie bie yorige befandelt und Durd) Stecklinge ver= mehrt. Sie bliht erft im Allter, und verdient Faum gezd= gen zat merden.

3) C. sonchifolia, ritugelblumenblattrige Peftwurz, ๑. Dftindien. - Man füet Den Samen im

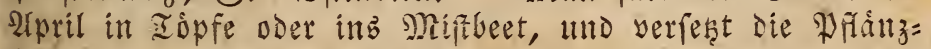
d)en, went fie $2-3$ aoll hod find in Blumentopfe, oder 
ins sano in nahthafte Eros. Eie Whihen fornell, uno ben ganzen Sommer hiber fábin orangeroth.

\section{Cactus, Fadelotifel, XII. 1.}

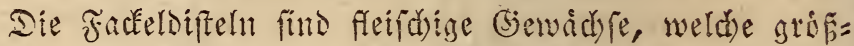
tentheils mur ifyer fonterbaren (seftalt wegen gezogen wer= sen; Denn Die wentigfen Dlten bringt mant im Simmer zur Blitthe; sod belobnen fie alsonan and faft immer bie ge= ringe Muke, weldye man einige Sabre lang auf fie vers wentete. lieber ibre Gultur ift in sifgemeinen Folgendes zu merenen:

Man Kalte die Fackerdiftern fets lieber etwas zat tro=

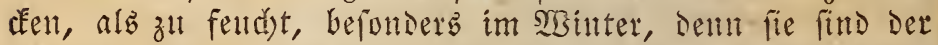

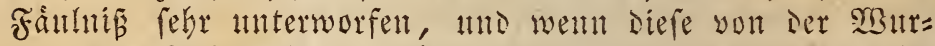
zel an anffieigt, fo ift nidjt felten tie ganze Pflanze verlos ren, ehe man es bemerft. Sonne ift thnen waihrent deb Eommers Durdant nothwerisis; in Minter Darf man fie

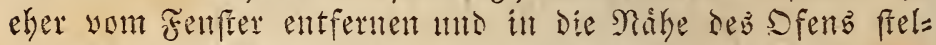
len, wenn bie Riflte febr grof werben follte. Dod), went

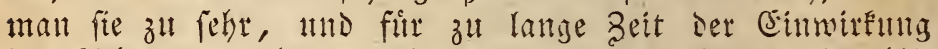

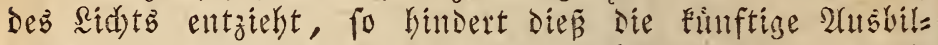
suttg Der Rnospen. Dartnt ifte es beffer, went es bie (belegenheit irgend erlatbt, Den Etrnd der Eactubarten, yon welden man Bhamen za erbalten bofft, nie zat vers andern, und fie fo am Fenfrer anzubrimgen, sas fie felbft

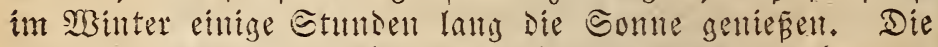

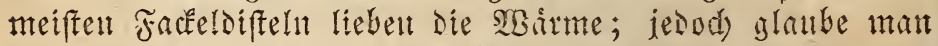

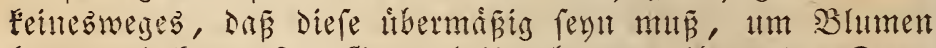

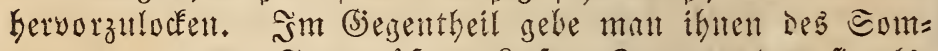
mers ant warmen Iagen ifters Suft. Bmat werden fie als= Dann langfamer wad)fen, aber friftiger wersen. Śn Der gewóhniliden 3immertemperatut yon $10-15$ (3)rad, bringt

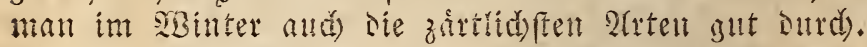

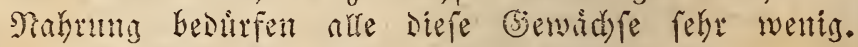
Man gebe ibnen leidste Erise, weldye man mit Eano mo Fleitut Steinen vermengt, theils, damit sie biberfliffitge

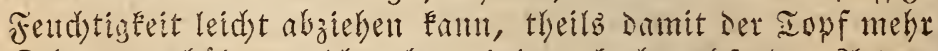

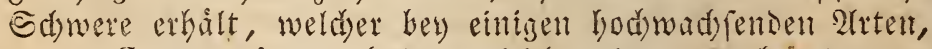
3. B3. C. peruvianus, bald zll leidst wits, mo bey der ge=

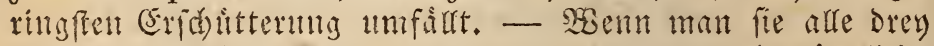
ober yier Sobre eimmal verfegt, to if siefes binlinglid);

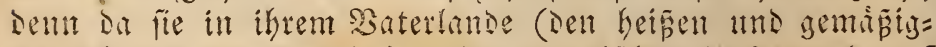

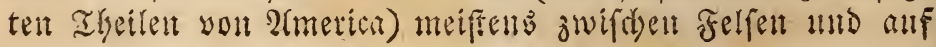


fteinigem 230 ent mad)fell, fo ift ibre Srganifation bou ber

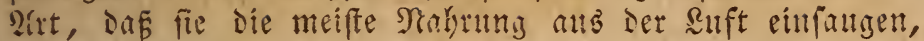

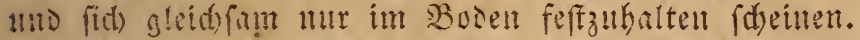

Die Nermelsung gefdicht Durd) Etedinge, Berthei=

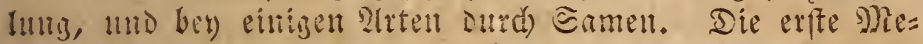

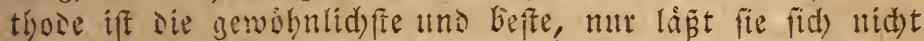
bey alfen 2(rten amwenden. Mgan fdncioet oder bridst zu

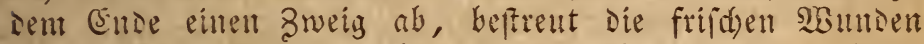
mit foblenpulyer, mnd läst ifn $8-14$ Taje (ie mad)sem

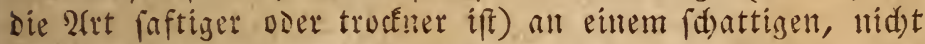
feudstell Drte liezen, fest ibu dann in einen fleiten sopf

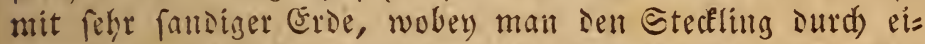
mige Eleitie sojladen in feilier sage zu erhalten fudt. Der Iopf fommt ben zartlideru Olrten binter ein silasfenfer, bey ien bárterll ill die frebe suft zu ftehen, uno man giep̃t nur alşann eill wenig, went man glaubt, die Groe fỏn= ne ganz aubigetroctuet jeyn. Eoute aber wabrend diefer Seit anbaltense trube SBittermis eintreten, fo fen man ia

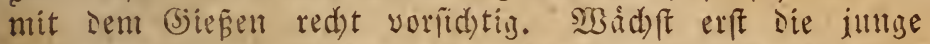
Pflanze, fo besarf es nid)t meist fo groper \$orfid)t. Bumeilen wartet man Sabre lang verigebens, baş eine Fa= dfeldiftel Eeitenafte treibe. Es bleibt fodann fein anderes

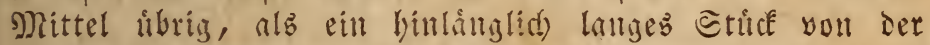
Epibe abzurdíneiren, und Diefes gleid) einem Etecflinge zu bebandeln. Der untere Eturz treibt nad) einiger Beit oben am Ednitt 2lefte alts, weldse man, fobalo als fie die

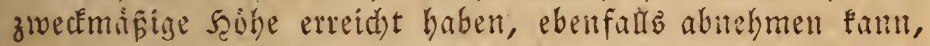
sa fie ftets ourds neue Eproffen erfest werden. - Die Zer= theilung gefdieft bey einigen 2lrten im Frublinge, wenn man sie Etrádier umpflanzt.

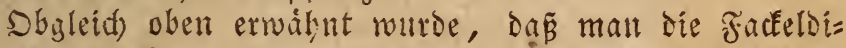
fteln eher trocken, als zu fendst balten folle, fo mus man

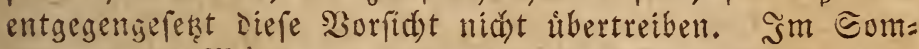
mer, wenn Warme und suft ftaut auf fie wirken, Eann man ifuen ofter giešll, fo raf die Erde nie ganz uno gar austrodfut; mur nebme man die in frever suft febenden ben anbalteniem Regenwetter in 2(d)t, und bringe fie alsisant rogleid) unter eine Beoadung. Froft fonnen fie surdoub nid)t vertragen.

Dab lintphanzen der Facfeldifteln fann, wenn man Eeine Bluthen erwartet, im Frubling gefdebn, fonft läpt mait es altfebelt, bis alle slumen abgewelfet find. Um

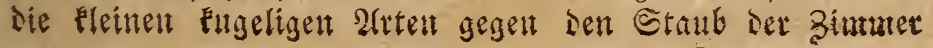


zu verwabrelt, ift eb gut, fie beftindis mit geraiumigen

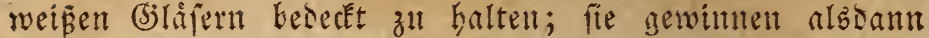

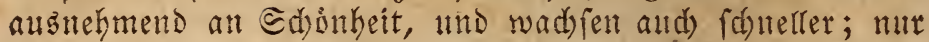
fer mait mit sem Begies̄en in siefem Fall nid)t zH freyge= big, ia die Pfanzent und sie Erse wegen set Beseckung nidit fo fart allsoluften, alb wenn fie frey fteben.

Hnter sen vielen 2ltten versienen folgende mir betannte im Bimmer gezogen zu werden.

1) C. alatus, geflitgelte Fafeldifter. Die zungenformigen, $1-2$ Bolf breitell und $8-15$ Boll lan= gen, am siande eingekerbten Blátter find obne Stadbeln, nur zurveilen baben fie in ser sugend einige fteife sardjen. Die fingerslangen berfarmintrothen shlumen fommen im Fribling กub Den Sinfdnitten Der Blitter berbor, und ge= waibren im $\mathfrak{D e r e i n e}$ mit ien helgrünen Blittern einen rei= zenien 2nblick. Man balte Diefe 2lrt, wenn fie bluben foll, Durdaub ridut zu warm, gebe ifre fleiğig suft, und

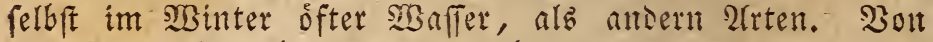
Der Mitte Nay bis in Den Serbft, wenn es Ealt zu wer= ben broft, bleibt diefe 2ut in frever suft fteben. Ner= mehrung Durd) Steclinge uno Bertheilung. Des Winters 4-6 (5irad, nidyt fern yom Fenfer. Sebr oft blibt diefe

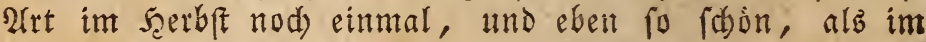
Fruflitiog.

2) C. brasiliensis, brafilianifde fadel= diftel. Die Blatter baben etwas Zlehnticheit mit denen des C. opuntia, befoniers fo lange die DFanze jung iff,

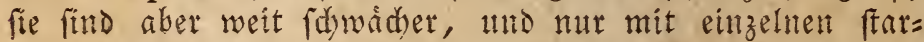
Een Dornen verfeben. Disto die Pflange áter, fo befommt fie altmíblig einen bolzigen, fä́runien, mebrete fur ho= ben Stamm, Der oben eine fdone, áftige, blátterreidue

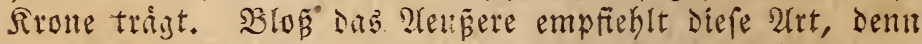
id babe nod) nidst gebort, Iañ fie in Deutfdland ge= blubt batte. Eie liebt viel Nabrung, in Eommer ziem= lidb viel 2 baffer, und es ift gut, fie immer binter (Silas zu befalten, insem sie Srone bam groper mo fdoner wird. seidyt vermebrt man fie surd) eingeftecte Blátter.

3) C. compressus, z"fammengedrufte $\mathfrak{F} a=$ deloiftel. Diefe nieslidye 2rut balt gleidfarm sas Mittel zwifden C. mamillaris und stellatus. Die Marzent feben

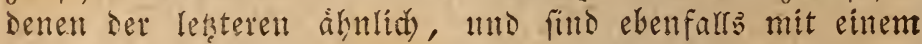

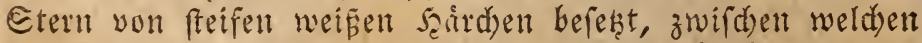
4-5 kerfbrantue Etacisent ftehen. Dagegen bleibt bie Form 
des forpers mekr tuns, und nut fparfam treibt et Ceitet= fproffen, wointd) man ifn vermefrt. Blumen wno Frubiste babe ids nod nidst gejeken. Man behanielt ifn wie C. mamillaris; er (d)eint ziemlid) rafd) zu wad)(en, iod) fant id) nidt fagen, ob er groz wird, insem er nod) ni.jt feit gat lange befamt ift.

4) C. flagelliformis, peitidenfirmige $\mathfrak{F} a=$ feldiftel. Gine rebl befannte art. Die langen, fingerb= diden, runden, bangenten B.veige find uber und ủber mit Fleiten, meis̆lid)en Etad)eln besedft. Die Blumen fom= men aus den áltern 3weigen, gleiduen den yon Nr. 1., mit weldsem aud) die hbrige Bebanslung libereinftimmt;

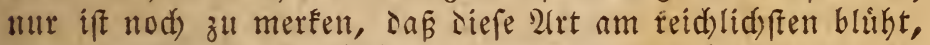
wenn die Broige berabbanigen fonnen; fonft bindet man fie an ein Eleines Epalier. (Es foll and eine Eleinere Sarie: tât geben, die jerod) mir nod) nid)t vorgefommen ift.

5) C. grandiflorus, grof́blumige $\mathfrak{F}$ a dél $\mathfrak{d} i=$ ftel (Nonigin ier Nad)t). Die Sweige find bodsfens 1 Bolf didf, mebrfeitio und mit Birfdeln gelblid)er Etadbeln befest. Man bintet ihn, Ia Die Sweige feine eigene Shaltung Gaben, an eill (sitter. Diefe 2rit liebt eine unmuterbrod)ene SBärme und einen berren, fonnigen હtant. Bey guter Pfle: ge bringt fie in iell Sommermonaten febr fdjone Blumen an langen Stielen, beynabe von tex Siröse uno (sieftalt ser fogenannten weifen Eeerofen (Nymphaea alba); jeood) find Der Blátter mebr, und fie find fdomáler. Die áuzern

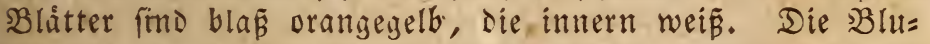

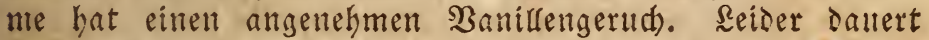
die Pradt Derfelben nur wenige Etunden. (siewobnlid off= nen fie fid) res 2lbends und. verbluben am folgenten Mor= gen. Sm Winter $10-15$ (Sirad und wenig Feudtigkeit. 6) C. hexagonus, fed) bुeitige Fackeldiftel. Der Etamm diefer art wad)ft grade in die Jobe, und folf

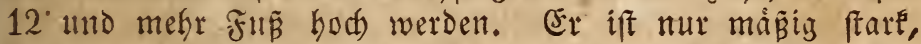
hat 5-10 Ecfen, meld) didht mit fleinen Burdjeln gelb= lid)er Ctad)eln befeşt find, die keinešmeges die Rảnge und Stàté Der Etadbeln Des C. peruvianus haben, yon wel= d)em er fid liberbaupt surdh geringere Ståte, viel weni= ger vorfpringende Eafen und eine mattere Farbe des હtam= mes binlinglid) unterideiset, obgleid) oft eine Nameneyer= wedjelung yorfommt. - Die Blumen babe id) nod) nidst gefeben, und fenne fie blof aus den XInnalen Der Rlumiftes

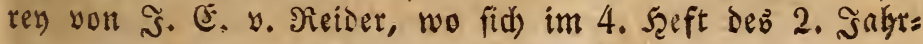




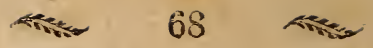

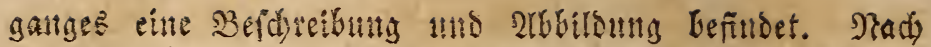
jener Befdretbuth fino Die slumen $4-5$ 3oll grop̃ ; die än Feldbblátter gläzenogrin, mit braumrotgen Episen, uno

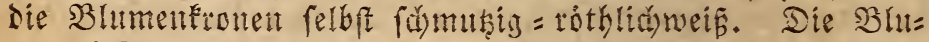
ment iffnen fid) gewobnlid am 2lkent, und find am nad)= ften Nittage verblifft. Eie erfdeinen im 2atguf, mo ift ein Exemplar mur erfit Glibbar, fo entwickeln fids ill jedem Eommer mebuere Bhumen. Diefe Factelsiftel wirb wie C. peruvianus behandelt, D. h. mant ilberwintert fie bey mi=

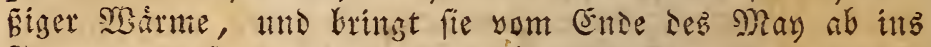
Freze, wo fie yom regent nicít getroffen wirt. - Da Diefe 2lrt exft in einem 2ater you 15-20 Jahren bliben foll, uno saber febr bod) wirs, fo eignet fie fidf weniger

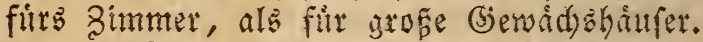

7) C. mamillaris, warzentragende facel= Diftel (Jugeldifel), eine niediche, faft Eugelrunde, im

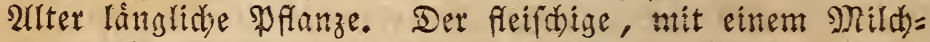
faft gefurte Forper if mit grunen Warzen bedect, an ie= ren Ende eit Stern yon barten braumrothen Etadyeln frebt. Die fleinen SBlumen erfdeinen zwifden Den SBarzen, und ragen nidbt tiber befelben Gervor; fie find gelblicbreif, und binterlaffen länglid) Beeren yon bodrother Jarbe, bie

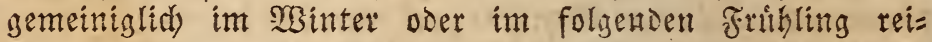

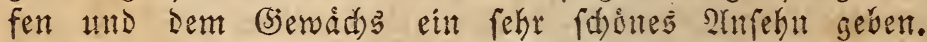

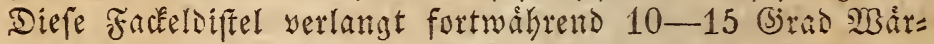
me, Sonne, wenig Feudtigét, und wird nie in. Die suft

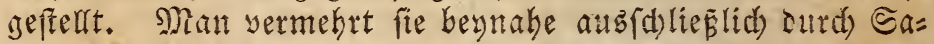
metr. - Dns Berfabrent, weld)es id) immer mit (situce alt= gewentet babe, ift folgendeg: Die reifen Beeren werden zwi= fden Den Singern im Maffer. zerdrutut, wo Dant der feine Same zu Boden fortt, uns leidht gefammelt werden famm. Diefe Roundben lápt man ein Paar Etunden auf Ródapa= pier liegen, Damit fie trodknen, und ftrent fie Dam in ei= nen fleinen flad)en Blumentopf mit feiner, fandiger $\mathbb{R} a u b=$ erde; Gierauf befprengt man fie mit einigen Iropfen 2 ấ fer, und feckt eill Mhrglaz, das genaul in Dell Iopf pas̄t,

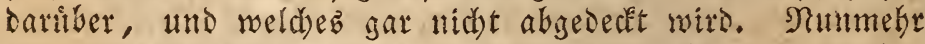
ftellt man Den Iopf in Die હonne, nlib fieht nur yon Beit jut 3 eit nady, obez Ier (Frde nid)t ant Feudstigfeit fehlt, wel=

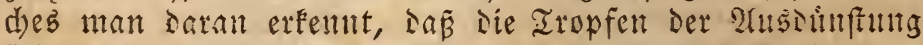
fid) nur farfam an dem (silaje anfescen. Bemerft mant

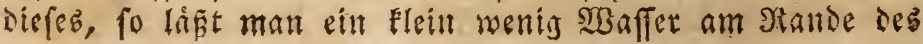


Iopfos mter bas Glas lanfen, ohne lehtetes in bie brobc

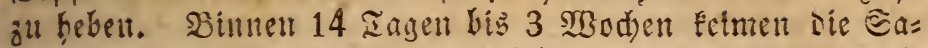
men, und bie jungen Pflangent fehen ans, wie Fleite meis

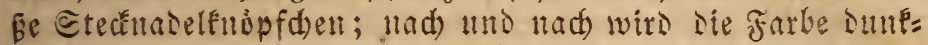
ler, und die jarten @tadbeln bedbent alt Der Mitte ber=

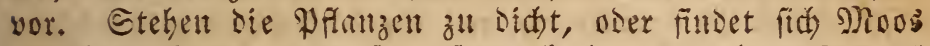
zwifden ifnen, ooer fins fie nad) $1-2$ Sakren fo grof

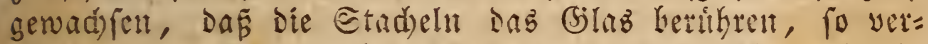

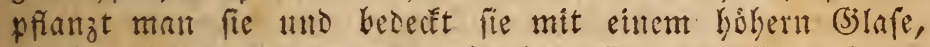
weldes in ser folge ein niestiges bierglas feylt fant.

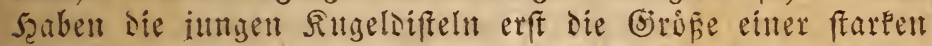

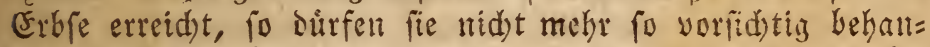
selt werden, oówohl ca immer gut ift, fie mit Gilas be:

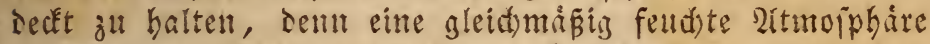
fdeint ifrem $83 a d$ sathume imuter beforderlidy zu feut ; oft

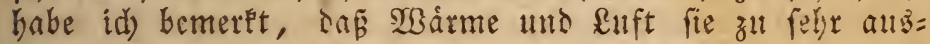

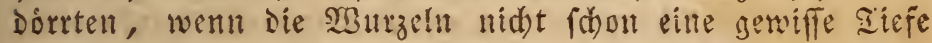
erreidyt hatten. Bey guter \$flege find fie in 5-6 jah= ren tragbar. - SGenn mall ein ziemlid) altes Exemplar im

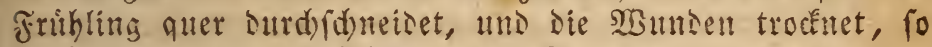
treibt das untere Etid zuveilen Eproffen, ite man, wenn

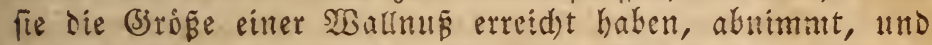
wie Stectlinge bebandelt. Die alten \$flanzen verfest man

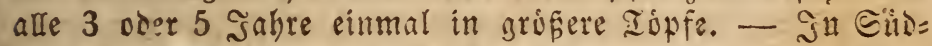
america follen bie willen Dferde in ier dutren Jabrebjeit, wem alle Duellen verfiegt, uno die grunen Pfianzelt ver:

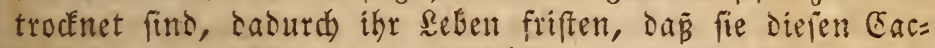

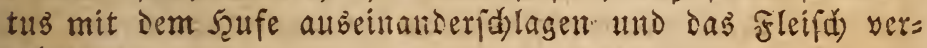
zefren.

S) C. mamillaris spinis flavis (C. flavispinis), warzentragende zacteldiftel mit gelbent Etad)eln. 2lufer den Herlgelben Etadbeln unterfdeidet

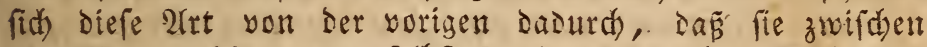
Den $23 a r z e n$ ofters yon. Felbit ąweige anstreibt, surdy wel= d)e man fie leidster, alb bie vorige vermegren Eant. Die

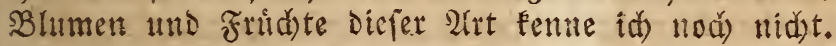

9) C. melocactus, melonenartige Facel= diftel. Bon biefer nod) feltnen Fackelsifel fimb unter be= fondern Nament bereits an 20 2lrten betannt, sie fid alle

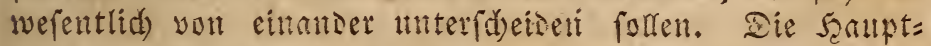
form des Gorners ift Elugelig, mit vielen berablanfenden

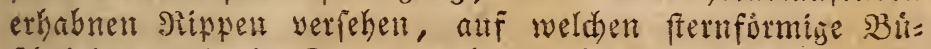

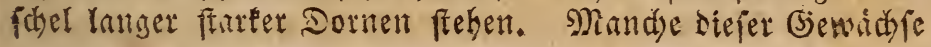


werden bis 18 zod hod), unb baber oft mekr, als eben fo Dicf. Die Blumen follen roth fern, und fisten all eitler furs zen cylinderformigen, filzigen Erbóntmi, -weld)e erfe dann, wenn sie Pflanze eine betrid tlidye (jrópe erreid)t hat, aus Der Nitte Dez Ropfez Geryortritt. - Heber Die Bebandlung biefer Fackeldifteln fann id) atb eigner (Erfabrung nur we= nis fagen, da id) erft (eit nid)t gar lantyer Beit won drey Sorten fleine Exemplare bejitie. Sd behande, ite wie C. mamillaris; uno obgleid) man mit Iem Begiesen ebenfalla fefre vorfid)tig feyn mus, fo (d)eint ez mir Dod), als vertru= gen Die Pflangen es nid)t, weln man fie zu fehr austrocfnen

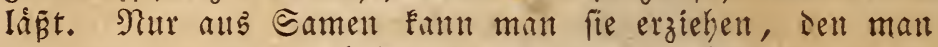
wie den yon Nr. 7. behantelt; fie wadjen binnen den er= ften Sahren ebenfarts rebr langfam.

10) C. opuntia, gemeitue Fafeldiftel, gemei= niglid) Die indianifde oder fpanifde feige genannt, wie: wobl diefer Name eigentlid) einer ansern 2(rt Jufommt. - Die eyformigen fladjen Blåtter wadjen eintes aus bem andern, uno find ftelfenweife mit Bufdeln Faum erkenn= Garer Etadjeln befest, welde ben Der leifeften Berifyrung in ber seant fiken bleiben und ein heftiges sutcken erresen. Die Blumen entipringen aus sen oberfen $B$ lättern im Nay uno sumn, aud) fpáter, fino rofenartig, fdoon gologelb, uno hinterlaffen rothe genieb̈bare frudde, Nan behandelt fie mie গlr. 1., nur bålt man fie etrab trodfuer. Die Spuntia if jest im fürliden (Europa einheimifd geworden, und for fogat fdon in ber italienifden Edyeiz gefunoen

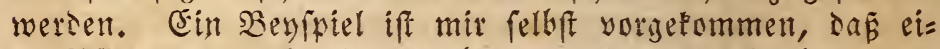
ne Pilange, weldye im Ferbft aus dem Topfe heraltbe = worfen wurde, unter dem Sd)nee ohne andere Bedectunis fid) erhalten, und im nádulfen Fribling neue Blâtter ges mad)t hat. Diefe Pflanze erfror leodh im folgenden Winter. 11) C. pendulus, há ngende- Fackeloffel. Die unbewehrten, fdwadien, binfenartigen, glatten 3 wei= ge baingen, wenn fie nid)t befeftigt find, Galb berab, und treiben, oft bennabe red)twintlig, einige Eeitenåte. Die Blumen follen hibfid) roth fern. Bebanslung wie C. alatus, uno vermefyrt fid auté) leid)t Durd) Etecklinge.

12) C. peruvianus, pernvianifde Fadeloi= fiel (C. hexagonus, die 6 Geitige Facfeldiftel). Diefe ânt fort in ifrem 3aterlanie 25-30 Fus hod rerden. Der aufred) 4-6 3oul ftarke Stamm bat 5-10 હefen, wel= the mit fternformigen Bufdeln nadelformiger, Grauter, 
fiatfer Etad)elt yerfeben finto. Die Blumen Pentue id nodh nidft; er wadd)f ziemlid) fdjuer, treibt im 2llter zuweilen Nebentaffe, wirt Den Eommer viber inz Freye geftellt, unto im 2 Binter bey 4-6 (sirad ibernintert, wabrend welder Beit er hód)fens affe 3 SBodsen einmal ein wentig SBaffer

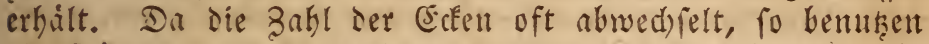
die Bjärtner zuneilen biefen Umifano, und geben die ab= weidjendent Sproffen fúr neue 2 (teten aus; $;$. B. C. pentagonus, Die 5jeitige, C. heptagonus, Die 7peitige Fackelsifer. șd) will zwat nid)t in 2 (brede fellen, daf vielleid)t wirts= lid) Felbeffitindige Natent unter Diefen Namen gehen, iedods fint fie mir nod) nidst vorgefommen, fontern alfe, bie ids bisher gejehen, mareni $2($ bweid)ungen yon C. peruvianus.

13) C. prolifer, faroffente gadeldifer (C. mamillaris prolifera). Diefe fleitte fingelige, warzentra= gente 2(rt treibt ófters Eeitentife, weld)e man abnehmen und Dadurd) die Pfanze vermehren fann. Die Etadheln fehen an Der Episte der Eleinen SBarzen, find fdjwarz=

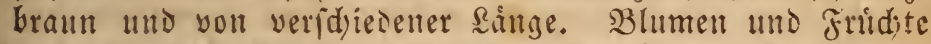
Fenne id) nod) niddt. Beflyantlung wie C. mamillaris; yat Fo, wie siefer, imterhalb einen Mitd) jaft gleid) Den Emphor: bien, bod ofue હdátre.

14) C. Royeni, weinffadylige Fadeldiftel.

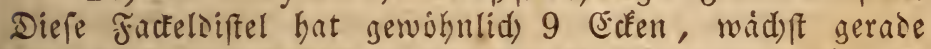
aufrecht, ziemelid) hod), und ift mit kérrzern und lángern

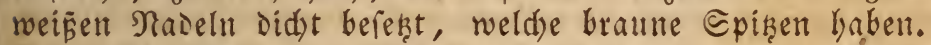

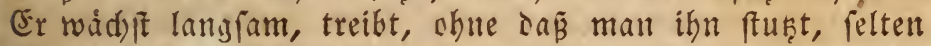

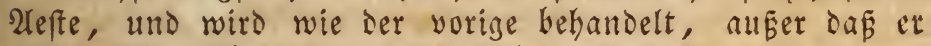

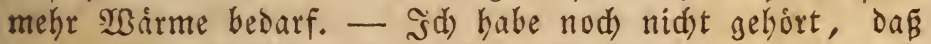
er irgentwo, in einem Ireibbaule geblúht bat. Şeíst aud) zuweilen C. peruvianus.

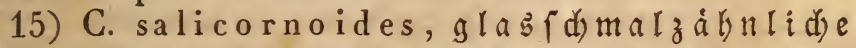
Fadeloiftel. Deefe 2rit würde man faum allf Den er=

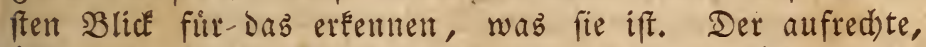

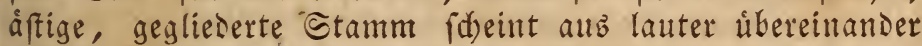

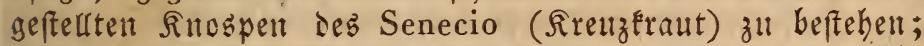

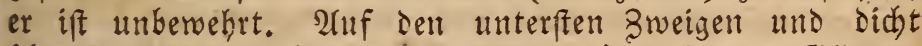

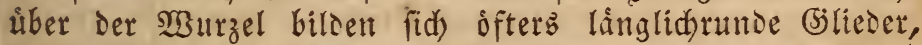

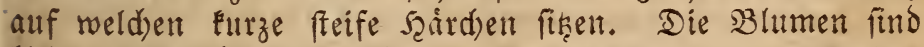
Elein, gologelb, und feehen einzeln oder zu zweyen ant Dent Epişen der obern 3weige. Sdd habe fie zut verfdhiedenen Beiten erfdeinen Fehen; mein Exemplar blúhte im gewóbn=

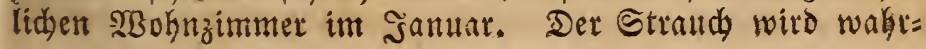




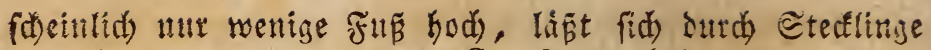
vermelgran, und fann wie C. alatus behandelt werben; (d)eint jesod) etrab jâatlid

16) C. speciosus, prad dige fadelofifel.

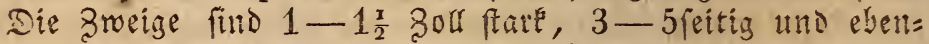
farts mit Stadieln befest. Sie Faben ziemlich viel eigene Sealtung und dirfen mut an einet Etode befefint werden, ivent fie zu famad) find. Die Blumen fint bennabe fo

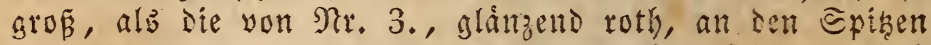
faft lila, und bauern mehrere Iange. Er whidft zientid

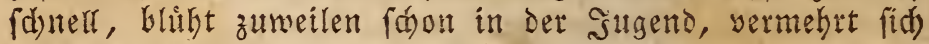
Dutd) Etecflinge tub wird wie গtr. 1. behandelt.

16) C. stellatus, gefternte Fadeldifel. Fleit unt fugelig wie $\mathfrak{N r}$. 4.; aber treibt feht millin sei= tenfproffen, die oft, went er zu warm und fidattig freht,

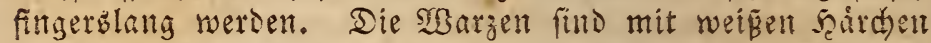
Ino Etadeln verfehn, Die Bhtumen Flein und de Beeren

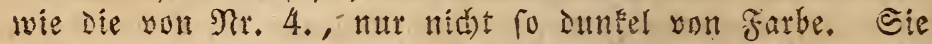
vermegrt fid leidst Iurd Eproffen; Begen fencitigfeit if fie empindich.

18) C truncatus, abgeftute Faceldifiel.

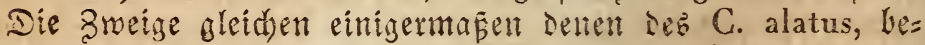
freben jesoch aus lauter an einanter gefesten $1-1^{\frac{x}{3}}$ zod langen und balb fo breiten (s)liesern, Die an den Eeiten einige weidse Bakndien baben und oben abiefutht fino; an diefen Etelfen fommen die netten (s)leder zu 1 oier 2 zum

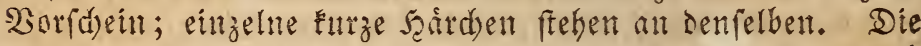
Blumen folfen fehr fdon roth fern. Sermehrung Durds

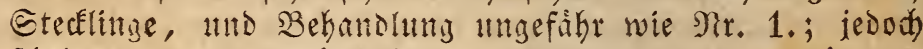

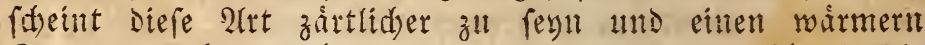
Etand zul lieben. Sbez gut iff, fie Den Eommer uber in Die

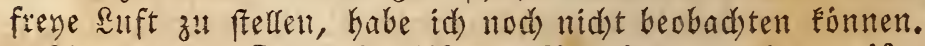

$\mathfrak{A} n \mathfrak{m} \mathfrak{e} \mathfrak{k}$. C. coccinellifer, cylindricus (and) parvifolius) decumanus, ficus indica, monacanthos, perescia, spinosissimus, tetragonus, triangularis (yout wel= them es ald eine mir nod unbefannte Epielart mit bunten 3weigen gibt) und tuna eignen fid́) mur firt

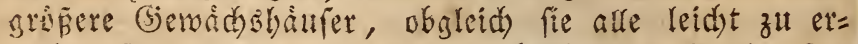
ziehen fins, tho wenig ilfege bedurfen. Zatuein faft feine einjige Diefer atren bliht in Bimmer. - C. phyllanthos wird wie C. alatus befandelt; iedod)

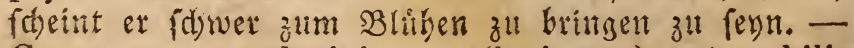
C. monstrosus, laniginosus (lanigerus) und nobilis 
(multangularis) fitto nod) Felten titt binfidets ber $\mathfrak{B} e=$ banshumg tem C. Royeni áfulid). C. coronatus unb scopa fino beise zu empfehten, jesoch) cúenfatra nodh fehr feltent wabride eintián laffen fie fid wie C. mamillaris behantelt. - Won Den hubigen Soitet siefez rei=

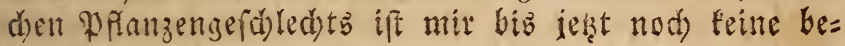
faunt gewordell.

$$
\text { Caladium, Calabiunt, XXI. } 7 .
$$

C. bicolor, zweyfarbigeb Ealadum (Arum pictum), 4. Elidamerica. - 2tus Der brauten, mit $\mathfrak{F} a=$ fern befenten Enolrigen șurzel etheben fid alt $6-12$ Zoll langen હtångeln Die fátilo = pfeilformigen Blitter. Cie fino, ie nadyem die Pfanze behandelt norsen ift, $2-6$ zorf lanis ntmo $1 \frac{x}{2}-4$ zoll breit, hefrgran, uno baben in ber Mitte cittell gropen Earmimrothen Fled, weld)er fid mit fei= tent 2riern in Den grüten Rand yerläuft. Die Blumen er= fdeinen im ștny oder suly jivifden ten Blåttern; ragen Eaum liber diefe reg, find weís und tutenförmig geroun= Den. Die Fnolfen, welche währent der Sisintermonate gar nid)t besoffen- wersen, nimmt man Ende Febrma: sus ber Erbe, reinigt fie volt Den Burzeln, und nimmt die jun= gen Futren, welche fid leidit ablofen laffen, ab. Die

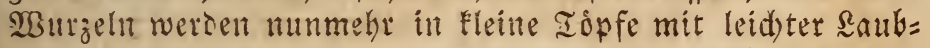
erde gelegh, und mit feinem 1 Bafferfande umfittert. $\bigodot_{0}$

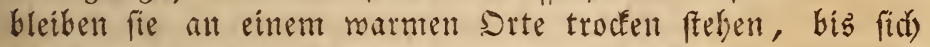
die Epişell Der Reime jeigell, morall man ihnen etmas

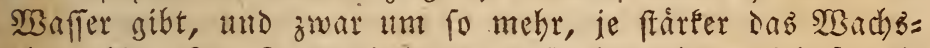
thum ift. Sim Juny fann man fie in grofere Iopfe mit dem Ballent pflanzen. Se mehr SBarme nan diefer (d)ónen Pflanze geben faum, Defto beffer gedeigt fie; deşhalb ift ihr ein હtand zwifhen Doppelfenftert, uno wenn auds

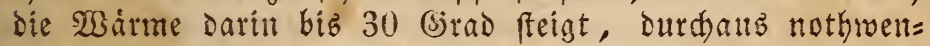
big. sm Miftbeet, wenn biefes nidht bod gentig ift, facint

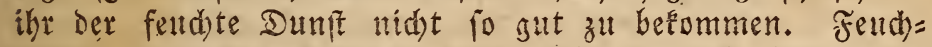
tigleit Darf Dem Ea?noium nie fehlen; Dod balte man

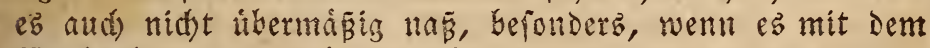

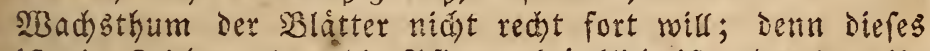

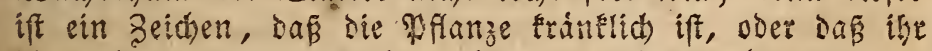
Die Behandlung forif nidis: gefádt. Sm Eeptember oder DC=

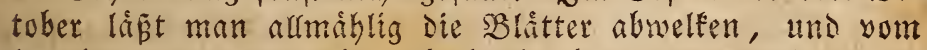
Sctober oder Noyember ab bleibt bey $8-12$ Grad dic Rnoure ganz troden fteben. - Diefe Pflamze yerdient sie Blufmerfameit alfer Brumenfremide. 
Calla, Ediangenfuat, XX. 9.

1) C. aethiopica, ittiopt fdes $\subseteq$ d) lattgent

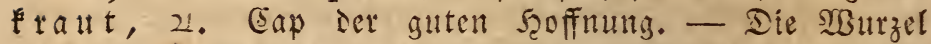
ift Enollig lno ftark; als ihr erbeben fid allf $2-4$ Jus langen glatten Etielen Die grof́nen fdiloformigen Blàtter,

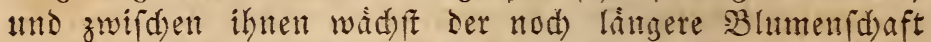
bervor, weldyer eitte grose, blendenoweine, tutenformige Blume tringt; ber folben, an meldem oben bie gelbent Etrubzefáfe frehen, freigt mitten in Der Bhtume fingerbs= lang hervor. Die Blume hat einen fhwad)en, angenehment (5erud, uno ifre Blithzeit if yom Marz bis ill Die Mitte Deb Eammers. Dab Edalangenfraut verlangt nahrbafte,

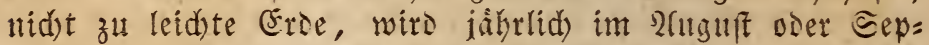
tember yerpflanzt; wohen man siejenigen Eprofien, melde fidi) leid)t toblofen, fortnimmt. - Dbgleid) Das હd)langen= fraut frans wurzelt, fo gebe man ifm im Bimmer beffen Ingeaditet nidut zu grofe Iopfe, aber ben Sommer riber einen Unteriestapf, weld)er immer mit $\mathfrak{B}$ affer gefült wird, fobalo diefes verzehrt iff. Suft iff Diefer pfanze nidst no this, man fant fie das ganze Sabr hinter Jenfern ftehent laffen; rod) fdadet isr ein @tand im Freven vom Ente Ray bis itt. Den September gar nid)t. Rlud Die Sonne fann fie lange Beit entbehren, und aub all Diefen Girindent eignet fie fid ganz befonters zur Etubenpflanze, vorzing=

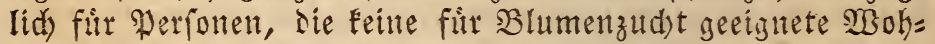
mung haben, uno sod) gern zu jerer Sahtebzeit etwas (s) rú nes auf ihren Fentern fehen woulen. Freilid) blibt dies Felbe, ment fie cinen on fdiledten Stand hat, reltner. Sm Sinter verlangt fie $6-12$ (j)ad Márme. Froft ift îf bajegent immer tódlid). - Dab Sdjlangenfraut trágt zumeilen fleine Folben gelblid)er Beeren, worin bie Rór=

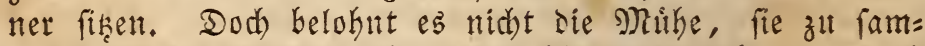

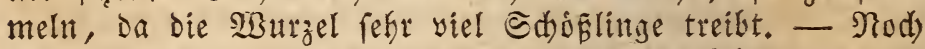

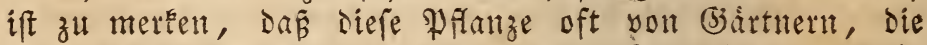
Eeine Botanif verfteben, mit Arum colocassium verwed)= felt uno colocassia genannt wirt.

2) C. palustris, Sumpffdyangentratt, 4 . Nordlides Europa. - Sbgleich biefe Pflanze wenig Werth bat, uno wo fie einmal wadbit, aud) in grofister Nens ge gefunsen wirt, fo weís id) sod), sas Botanifer fid oft viele Mathe gezeben baben, siefe pfianze furr thre Ser= barten blifhend zll befonmen. Datum will id ige ein 
Paar Beilen wismen. Cie gleidt im Plfrgemeinen ber yo= rigell, wiro aber nur $4-6$ soll hod), bie Blåtter fino mehr berzformig, uno Die Blumen flad); ' lektere Ginterlaf:

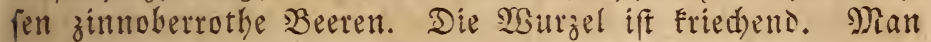
fincet fie in Sumpfen und frehenden (Siemáffern nid)t weit yom Rande. SBill man jie im Iopf ziehell, fo kebt man die $23 u r z e l n ~ G e r a u s$, legt fie in einen geriumigen Blumen= topf, ien man mit fdammiger Erde fillt, uno in eitten $2-3$ zor tiefen Unterfah ferrt, Der, wenigftens uber Som= mer, ftetz mit Waffer angefüut fern muß́. Sm NBinter bált man fie ben $0-4$ sirad mefr trocén.

\section{Calluna, Ealluna, VIII. 1.}

Calluna vulgaris, gemeine Calluma (Erica vulgaris, gemeine 5eide), $h$. Europa. - Don Diefem

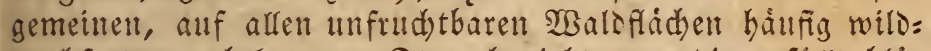
wad) fenden, befannten Etrauld zieht man sie gefitht blis= hende Epielart båuing in (jadrten. Man behandelt fie gantz fo, wie reiter unten bey Den freiden gefagt werben wird, vermehrt fie surd) 2rbleger, und hbermintert fie bey $0-6$ (5ras. - 2lber and die gemeine einfade freite, yon wel= d)er eв, auser einer herf = und Dunkellilafarbigen, aud) eine weisblikgende Spielart gibt, welde nid)t ganz felten ift, ift eine redyt nieslid) blithende Pflanze, uno yersient robl, im Iopfe gezogen zu wersen, befoncers von Blumenfreun= ben, weld)e nid)t viel auf frembe (Siewadbje wenden fon= nen. Bul Dem Bweck fud)t man auf Den Şeideplázen jun= ge Eamentplanzen auf, bie nod) nid)t liber 2 zoll hod find. Bey einiger 2 fufmerffampeit wird es nidjt (d)wer werden, an den Etellen, wo die aiten Bulfde aufhoren, viele junge Pflảnzden zu finden. Diefe fitd)t man mit Şülfe

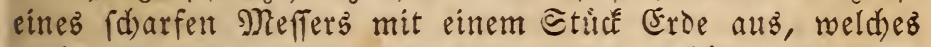
nad) allen Dimenfionen etwa $1 \frac{1}{2}-3$ zolf bålt, uno febt iedes Exemplar, ehe es wele wird, in einell f́leinen, mit fandiger Sauberde gefiututen Iopf. 2Unfănglid) Gălt man

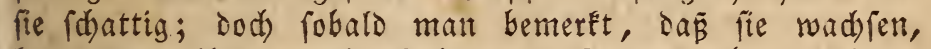
Fann man ifnen megr sidjt und Eonne gebent. Findet man jedod) eine befonderb (d)in gefartbte, z. B. weíse Epielart, sann ift es am beften, sen ganzen @traud mit einem redht grofen Ballen aus Dem sante zu heben, und befen in einen Blumentopf ober bolzernen fiaften zu pflat= zen. Nan hålt Denfelbent fo lange als móglidi) an einem fdattigen Srte und úberwintert ign bey $0-4$ Grat. Da 


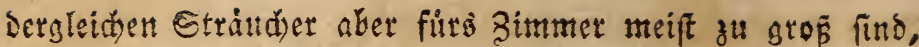
fo madis man fogleid) fo viel 2 ibjenter, als man tann, uno bebt Diejelsen nadh einem Sabre mit Ballen ats. Sann

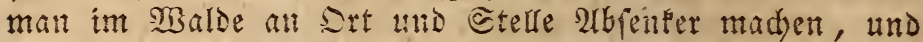
ift mant diberzengt, da fie bafelbft nid)t vernichtet wercen,

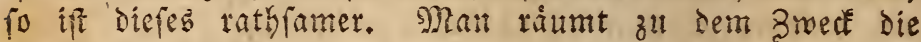
nebenfekenden Geftrippe res, und fente die 3roeige we= nigftens 4 soll tief in bie (rite. S(lld) tift es gut, tum

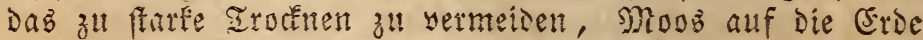
zu Decketh. Sin Etádten wird bie gemeine seide nut felten in Ghitsen gezogen wersen fonnen; Denn eg fehlt ihr bort

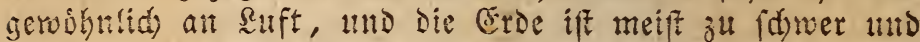
fhlammig. Sonft Fann man fie zu nioblidjen Einfaffun=

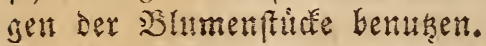

\section{Calycanthus, Feldy flume, XII.}

C. floridus, floribanifde Reldbitme (Ses

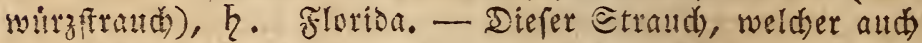
im freyen sanse fortfommt, tund gut wád), wenn ibm Se: Boven zufagt, wird wegen ber idjunte, faft fernfor:

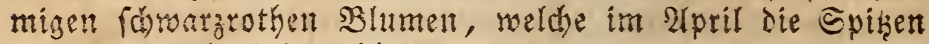
Der sweige fdumiaten, baung in Iopfen gezogen. Die paer=

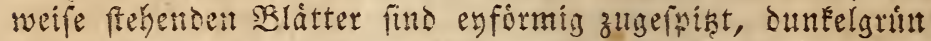

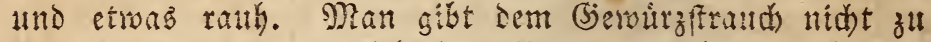
enge Topfe mit gutem lef migen Boden, weid)er jesods ftan

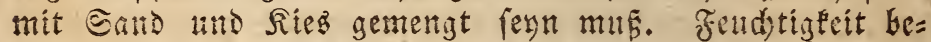

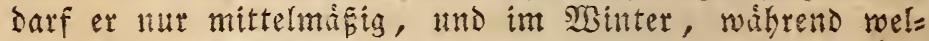
dher zeit er entlaub́t fteht, nu位 man befonders yorifidtig mit dem (siesen fent. (Er braild)t zum Hebermintern, gleich

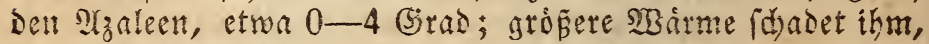
indem die finozpen fid zu frith entfalten tuto verberben.

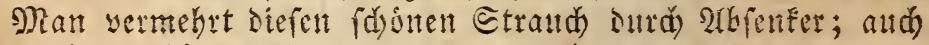

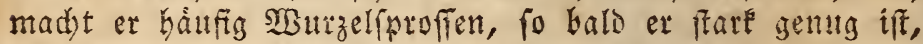

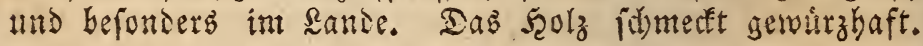

Camellia, Camellia, XVII. 8.

C. japonica, iapanifde Eamellia, F. Sapan unt GGina. - Die Eamellien Gaben in neuerer zeit in ber

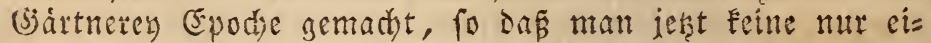
migermafen bedelitende Eammtung fintet, in welder nidst wentigfens einige Diefer Bäumben angetroffen witrden. Intd verdienen fie weit efher einen \$lah im 3immer, als mand

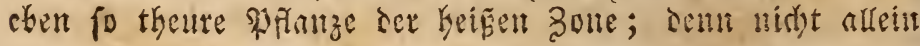




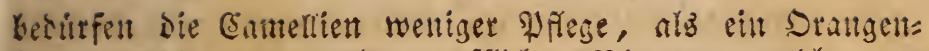
baum, fondern auper ben trefflid)ent BInten, weld)e yout Februar bis shary fid entwicfelt, zieren fie aud) dab zallze

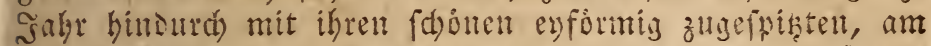

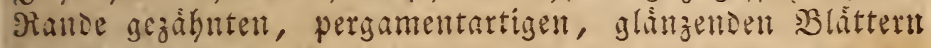

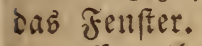

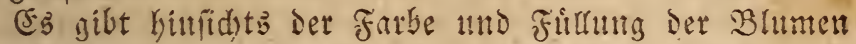
eine Nenge Epielarten, fo Dá man jest in Deutidhland

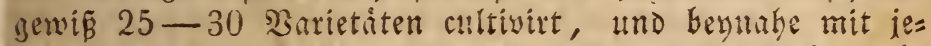
Dem Sabre fendet Englano netue Sorten. Sh felbft babe biakger wenige in den Bluthen gefehen, wage daher feill all= gemeines llttheil; ieood) fateint es mir, als wiren die llt=

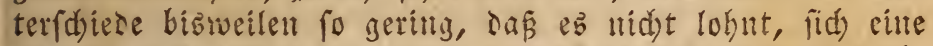

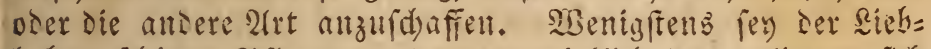
baber fdỏner Pflanzell, dent eb wirflid) Daran liegt, fids Ead)en zu balten, meldye ourd) ifre Mammigfaltigket das

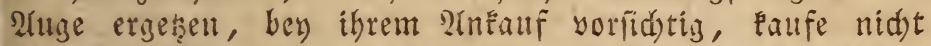
iese Eorte, die cinen neut Namen fibrt, fondern liber= zelige fid) zuvor mit eignen 2latgen, ob wohl fie es aud)

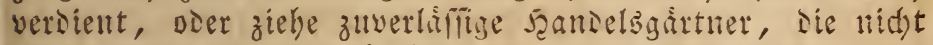

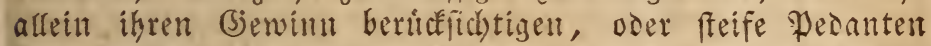

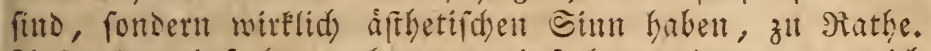

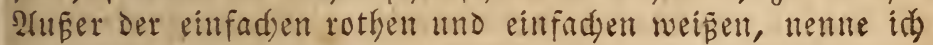
nod) folgende mir befannte Eorten:

C. japonica alba plena, unftreitio eilte iet (d)onften 2laten, mit febr grofen, regelmas̄igen, beynabe ramunfelurtis gebanten, flad)en Blumen. Sie i, von den andern 2lrten Dutrd Dab ftark gezabute Blatt binlánglid unterfdiesen, was bey der einfadj weī̄en mur nod etwas

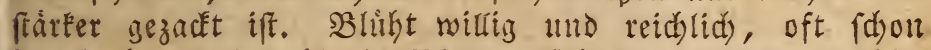
im Serbft, wiewohl die Blumen lid) Daut felten fo fisou ausbilden, als im Frubling.

C. jap. anemonaeflora (and) Warrata ge=

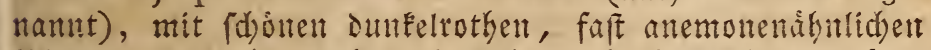
Blumen, Die id jedod) nod) nid)t nad) ifher volfigen (Ent= wicelung gefehen habe. Die Blätter meilles Exemplars

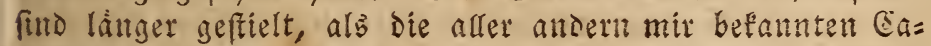

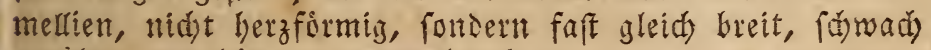
gezahtit und hángen etwas herab.

C. ja p. coronata. Die Blumen folfen fefre fobon Godroth fenn, unto an Grópe die Der weipen gefiuten ủber= treffen. Nein (Exemplar hat nod) nicht gevlihgt.

C. jap. flarescens (aud) Poff). Der weipen ges 


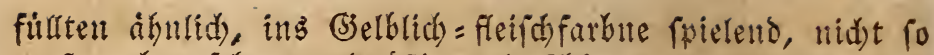

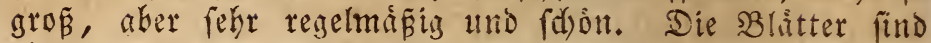
ebenfalla etwas fda árfer gezahnt, als die der meiften úbri= gen હorten.

C. ja p. paeoniflora, hellroth gefurtt, fleişig bluheno. (Wentig yon thr follen unterfateden feyn: carnea, mittelmisd's uno Pinc; Iod) babe id) nod' nidjt affe biefe Eorten fo genall beobadtet).

C. jap. pomponic a alba. Die Blument fitt gut gebaut unt gefitut, gewóbulid) weis, zแneilen an dem nàms lid)en 'Etraud rofa geftrefft, oder faft ganz incurnat.

C. ja p. rubra pl., ratit grofen bod)rothen $\mathfrak{B l}$ lu= men, faft yon der Geftalt bes Hibiscus rosa sinensis $\mathrm{pl}$. C. ja p. rubricaulis, bodroth gefiut, bubidh. sd) babe zeither nur ganz fleine Exemplare bliken fehen, Deren Blumen nod) nidht ibre ganze Boulfommenbeit fouten erreid)t haben.

C. ja p. variegata, roth mit weis̃ geftreift, eine ziemlid) grope, fdone Blume; reid) blihend. (Ẻ foll eine Urt geben, meldhe wenige meise @treifen hat, worant sieb= haber aufmertam fenn mogen. Sid felbfi habe yor meh= rern Sabren ein. Exemplar unter biefem Namen gefehen, an Dem die Blume nut ein einziges weis geftreiftes Blatt batte.

Bon alfent thrigen 2trten Eente id) Die Blumen gat nid)t, obgleid) id nod) einige Eorten belise. Empfoblen werden yon Den (S)artnetn: C. jap. atrorubens (aud) atropurpurea), conchiflora, Lady Admiral Camphel, myrtifolia, paeoniflora alba, W elbankiana. - C. j. longifolia forf, wie sie gemeine rothe, einfad)e, blühen; ihre Blát= ter find nur etwas lẳnger, alz bey diefer.

Die Bebandung Der (Eamellien iff ganz einfad). Cie erbalten sauberse mit etwas sefm uno @and vermifdt,

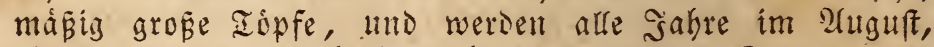

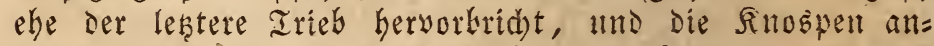
fesen, oder gleid) nad) Der Blithe im May umgepfanzt.

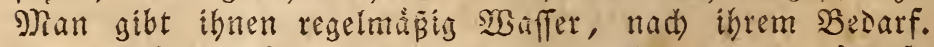
Sn Der Mitte May ftert man fie ins Freve, uno lápt fie Dafelbit bis gegen Enie Eeptember ftehen. Şat Das Báum= d)en jedod im frithling nod) Fnospen, fo behált mall ez lieber einije Sodten länger im Zimmer, denn in ber suft fhadet, befonders der Regen, ien Blumen fehr. Blt viel Eonne ift den Camellien im Sommer eher fobadid, als 
vortheilfaft; sarum weife man ifnen wo moglid einen

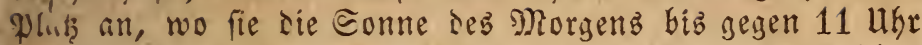
be en, sod) pater gegen ifre beftigen Etrablen gefdust finv. Des Minters fielt man fie nabe ans fenfter, und hălt fie bey etwa 4 (jiras. Som Februar ab yertragent fie einen bobern Wärmegrad, weil Dann die fnospen und jun= gen Irtebe fid) zu entroickeln anfangen; alssann fann mait fie in ein gewobnlidbes Sobuzimmer you $10-15$ (Girad গ্Batme bringen, wenn siejes die geborige Conme bat; ift jedod) leşteres nidyt der Fall, to treibe man die Camertien in nid)t z"l fart, fondern laffe fie lieber nod $4-6$ MJO= d)en lánger in ifrem Winterquartier. - Da Die Camelfien nitht wie anoere aubländifàe Báume fortwäbreno madjen, foncern mur 2 bis $3 \mathrm{mal}$ im Sabre fdnelf einen Irieb yon einigen Boll sănge mad)en, uno wäbreno der librigen Beit ruben, fo beruffidatige man dieje Gigenfdaft berm Begies fent borzhglid). - Niemals gebe man ien Eamelten, in Der Neimung, den Irieb zu befordern, Nitt, wenn aud) (d)on Galb verfault, unter die erde; er fdadet ifnen immer.

$\mathfrak{B a b}$ die Bermefrung Diejer beliebten Etrăuder anbe= langt, fo fann id) aus eiguen Erfabrungen nod nidjtz biertber angeben. Nebrere gröere Merte llber (5)årtnerey fpred)en fid) weitläufig luber siefen Punct aub, uno geben veridjedene Methoden an, die Eamellien alls Etectlingen

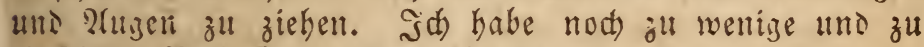
einfeitige Serjud)e anfterren fonnen, von ienen Eein einzi= ger bibher geglitet ift, nitd uberhaupt wird es wobl nie

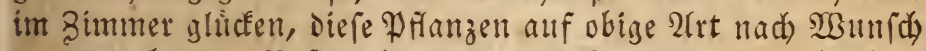
zu vermefren. Befint jemand eine Camelle, weld) bin= lánglid) bezweigt ift, und derfelbe wrinfat, fein Exemplar zu verbielfältigen, fo rathe id), 2(bjenter in Eefoniere Elei= ne Topfe zu madben, die Zmeige eill flein wenig einzuidnei= den, und nun rubig abzuwarten, bis man uberzengt iff,

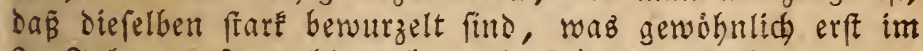
2. Sabre, oft wohl aud nods pater gefdieft. - Sat

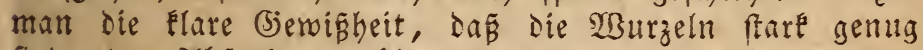
find, den ubjenfer ernábren zu fonnen, fo fdoneise man ifn nidst auf einmal, fondern alfmálig los, insem man etwa alle 8 Tage etwas solz yon dem (Ende abidinetdet, an weld)em er mit ier Nutterpfanze zufammenbangt. jod) geftebe ebs, diefe Bermebrung art if langfam, aber ziemlid) gewifs, wenn man nur (s)esuld hat und die Deit

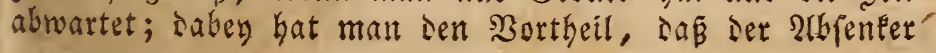




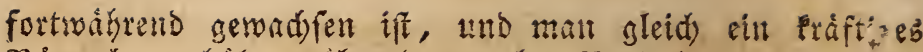

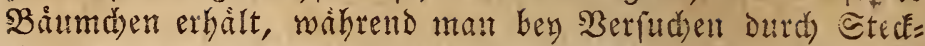
linge oft Die ganze Mutterpflange vertorben bat, obne alth nur eithen einzigen 3weis zim Ireiten zu bringen. Lebri= gens will ith binzlifingen, Daf wenn auth ein Etedeling

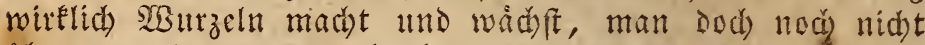
liberzellgt ift, da relbe im naidfiten Sinter wieder ein, wenn man ing zu fend) oder gar zu trocfen hált. Seltene Eorten vermehrt man auld Durd Seredeln allf Die gemeitte cinfad)e, weldhe lebtere 2 (rt fid) nebft der paeoniflora und den yerwandten

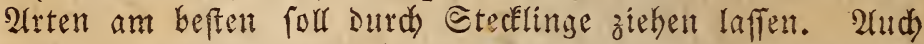
weis id), Da管 Die einfad)e (Eamellie in Dentid)land હamen getragen bat.

Hebrigens find die Gamellien wábrend Der leştern Sath= re fehr im Preife gefunken, ein zeiden, Das jeşt fdon viele (S) irtner mit der Funf vertraut find, fie zu vermeh= ren. - Êhr gefunde (Exemplare zu ziemlíd) birfigen Prei= fen vertanft Der Feandelögatutner (E) haupt in Brealau, weiden idh ald wegen feiner librigen fobonen (berwadde uno reeten Bedienung empfehlen Eant. Camellia sansangua alba. Die Blitter die fer 2frt fint flein, wentig glinzent und fdyad gezafint. Sie faeint ftat zu wad)fen, und hat dimne ruthenformige Brweige. Die Blimen forten mbedeutend und weís fern,

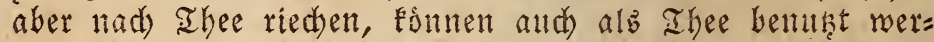

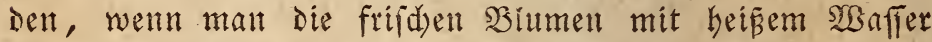
ubergiest. Mein Exemplar brachte zwar eine Bzlume; da

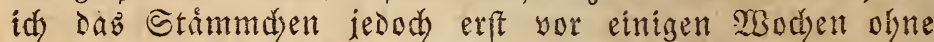
Iopf betommen batte, fo fielen bie Blumenblatter gleid)

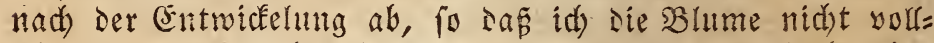
frándig gefehen babe, fie glidh eintgermaşen einer balbgefutls= ten Rirfdblitthe. Diefe planze wird wie C. japon. be=

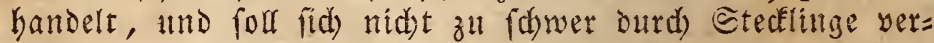
mefren laffen; id habe nods Eeine Berfude gemacht. Sun= ge Pfanzen yon $6,-12$ zoll şobe foften bey frn. Mohn= Hallpt 1 Ihlr. - Sie brist aud) I Iee= (5 a mellie.

C. sansangua rosea foll eine febr nieslide Bhts

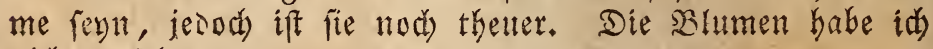
litiolt geregen.

\section{Campanula, Gு lodéchrame, V. 1.}

Dir meiften (Silderenblumen find sandpflanzen, yon des 
nen jesod) mifrere all sas bimmer jieten. Da sic $\mathfrak{B} e=$

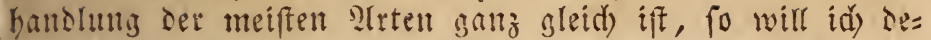

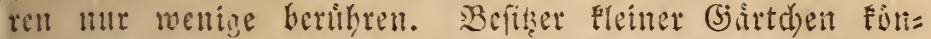
llut mit geringen Foften fid. cinte Nenge Sorten bey ie= Dem frandelsgatuer anfd)affen, fie obne grof́e Nrube ver=

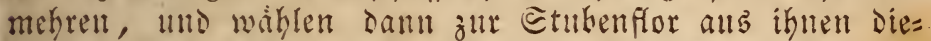
jenigen alls, weldye am beften gefallett. Nall gibt delt

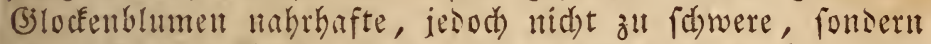
etwab fansige Croc, pFangt fie entweder im frerbft, oser zeitig im frubling mit Ballen in Topfe nnto bebandelt fie wie die 2fdilleen. Sm erftern falle liberwintert man fie bey $0-2$ Grad, und ift ser SButer gelind, fo bleiben fie be= ftinsis in ber freyen suft fteben; nur fdube mant fie als: sam geizell zul viel Fend)tigfeit, weld)e ifnen, went Der

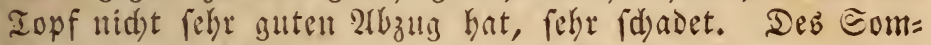
mers bingegen besurfen fie viel $\mathfrak{M a f f e r}$.

Sid) will bier eille slethode anjebell, weldse id im= mer alb rebr zweckmiñ gefunsen babe, diefe und äbnli= d) Pflanjen fo vorzubereiten, Daß̧ man fie im Fribling aแb sem sande heben Fant, ofne die Samzeln febr zu

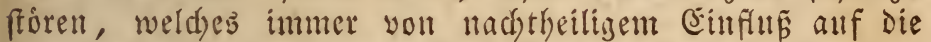

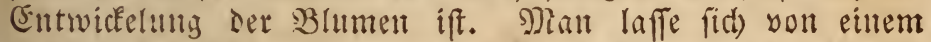
Iopfer eille 2luzahl Blumentupfe mad)en, welde jesod) Eei= nen Boden babell. Dieje Ringe you $6-8$ 3oll Szoke und verbåltnismápinger Weite werden gut gebrannt. Man grábt fie im Trthbling auf einem Beet bis an Den Rand eit,

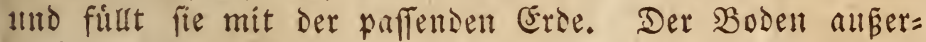
balb des Iupfes wird um renjelben feftgedruckt. Sierallf wird eine junge Pflange (z. B. von C. persicifolia ein Sieim, yon C. medium eitre Eamenpflanze u. f. w.) eint= gefesct, und man befummert fich num daż ganze Sabr nidut weiter um fie, als dấ man iflten, fo oft es mothis ift, giest, und fie yom unfraut reinigt, furz, fie fo bebandelt,

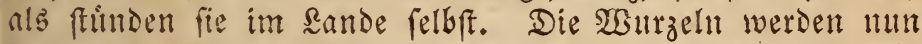
den Saum inmerbalb ses appes ausfillen; baben fie nitat mebi Plas, fo wadsfen fie unten Durd), und breiten fid in Dem Beete nad) Wilfubr aus. Du viel Feudrigfeit fann den 2 Zurjeln nid)t fdraden, denn da die Topfe Eeinen Boden baben, fo fammelt fie fid) nid)t Darin, was bey ge=

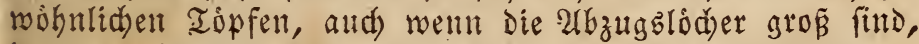
immer mebr oder wentger Statt findet. Sm Fribling, roenn man in bie Erde Pann, wafhlt nall unter feinen Pflanzen diejenigen aแb, weldye einem fitr ben 2lugenblick am be= 
ften gefalfen, befrent die Iopfe you ier fie umgebenden (Et= De, und (d) teidet mit einem idarfen Neffer die unten surd)=

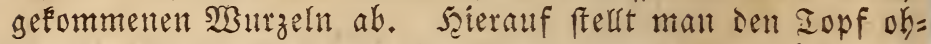
ne Boden in einen paffenten Unterfestapf, uno behandelt

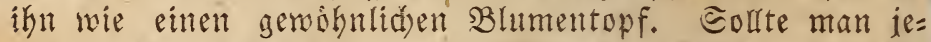

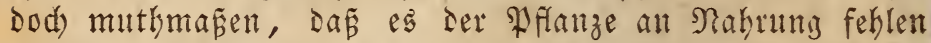
Durfte, fo lispt man Den galtzen Ballen alls Dem Ringe

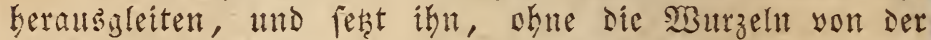
Eise zu entblópen, in einen Blumentopf, wie man ifn

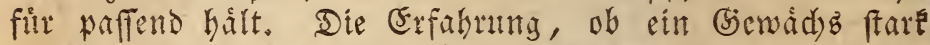
mutzelt oder nidyt, wird bald beftimmen, wie grof́ bie Ringe fern milfen, bie man fiur biefes oder jenes (jewadds anwenden mur. 2(nd) bey vielen andern sandptranzen fdeint

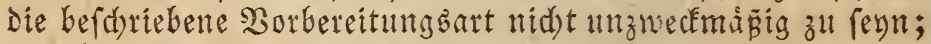
nur bey Giendiden, weldse lange, fleifdise Pfablwurzeln baben, modste fie nidjt amwentbar reyn, da man die Srampt= wutrzeln beym Serrub̆beben befdadotgen, uno die Pflaltze Duburd) frántelid madsen witrde.

1) C. aurea, gold farbene çlodentitme, Nadera. - Sie Gat lanzettformige, gezäbnte, 4-6 zoll lange, lebhaft grume Blátter, und treibt einen etwa 12 Boll langen Etăngel mit fdumuhîg gelben Blumen; ba fie zârtlidser ift, alz andere 2luten, fo mus mant lie bey 46 (Sirad úberwintern, und ifgr nidst zat viel Fendstigkeit ges ben. Sie miro Durd) Eamen und Eteclinge sermehrt; id finde jeood) nid)t das 2 (t) Serfaffer you (sartenbidbern fo febr erbeben.

2) C. carpatica, Earpatbifd)e (3) lodentulu= me, 21. - Diefe 2frt miro boddfenb einen gun hod), hat sunfelblaue, ztemlid) grof́e Blumen unt beynake Gerzfors mige Blätter. Man zithe fie alts ভaman; fie blitht etfit im zwenten sabre.

3) C. medium, Die Marienglocke, o 24. Edrweiz

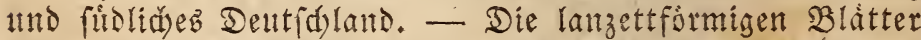
find rauh, ber હdjaft wirb 3-4 Jus boch, uno trågt

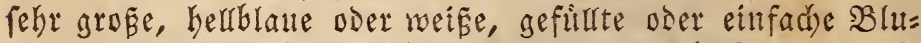
men. Die einfadse 2 rat yermehrt man Dutd Samen, die gefirte surd Bertheilung. Seshtere mus im SiBinter keron=

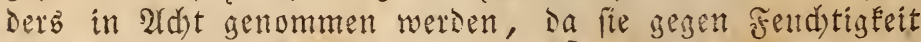
(ehr empfindidy ift, obgleid) auds bie einfad)e nid)t felten iber Winter ganz ausfault. Die Mariengloce ift eine fefr fdione ziterpflanze.

4) C. pendula, båmgente (3) rodenblume, 2. 
Die Blumen ftehen einzeln anf 1 Fñ langen Etielen. Eie fint suntelblan, Flad), mo feken redyt nieslidy aus,

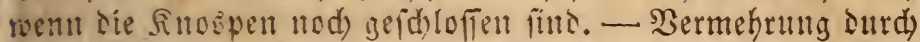

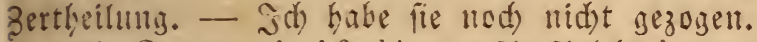

5) C. persicifolia, firfidb bisterige (5) $10=$ de nblume, 24. Deutidlant. - Dieje 2lrt fintet man

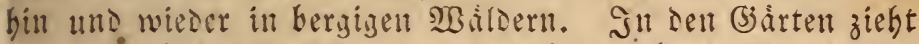
man iefod) gewoohnlid) nur Die gefilute, blate ntho weis̆e.

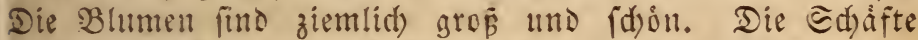
werten im sante 3-4 FuE hod), uno blukgen fehr reid = lid); bleiben dagezen im Iopfe gerwibnlid viel niesriger, da fie ftare wurzeln uns reid)lider Nabrung besurfen. Sie ift cine der dauterhafteften (sjlockenblumen, und vermebrt fich unzemein ftark Durd) Eproffen und 2 Burzelfeime.

6) C. pyramidalis, pyramidenformige (j) lo=

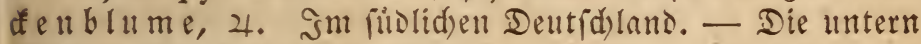
Blitter find beynabe berzformig, geferbt, und ftehen auf 5-6 3oulangen Etielen. Der Blumenfd aft erreid)t im Iopf oft eine 5robe you $5-6$ Fun, if mit furzen, aufs red)t fehenten 3weigen befest, die fid nad) Der Epibe zu verlieren. 2rn diefen Bweigen ftehen eine grofe शnenge bell=

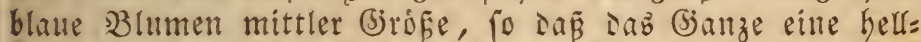

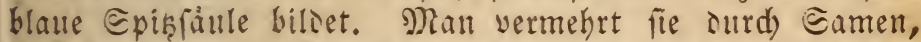
Etectinge and Bertheilung. Die Samempflanzen blihen erit im zweyten, oft im oritten Sabre. Nan gibt ifr lehmigen, fiefigen Booen, uno liberwintert fie her 4-6 (sirat; sent obgleid) fie oft glut im freyen sance alts= dautert, fo fann man fidh dod) nidd feft Darauf verlaffen, befonierz, menn der $\mathfrak{B o d e n}$, in weldem fie fegt, fehe (d) wer ift.

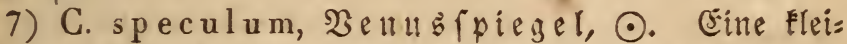
ne Pflanze mit faft fried)endem Etángel, weld)e man im mittlern Dentid)land bälfis in den sietreidefeldern finiet. Eie trẳit Det: ganzen Eommer über flad)e, sumelviolette,

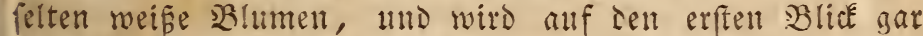
nid)t fir eine (sjlockenblume gehalten. Nan fäet sen હa= men im frubling in Iopfe, und zieht Die úberfúfifigen Pflanzen beralls. Sm sante vermebrt fie fids, went ihr Der Boren zufaist, yon felbfr.

8) C. versicolor, bunte (5) lodentume, Ilus (5)riechenlant. - Eine figr fdone Pflanze, Die idt) nod) nid)t felbft cultivirt, tho nut ein einziges Mal faled let bliben gefeben habe. Sie nábert fid in igrer (Seftalt Nr. 6. 


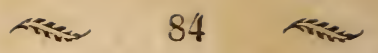

Der SBlumenfibuft wirb etwa 2 gun bod unt trigt gró= Eere Brumell, weldye Gerblau oder lila, itl Der Mitte weí,

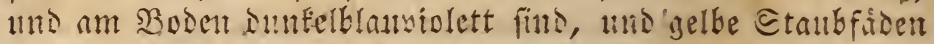

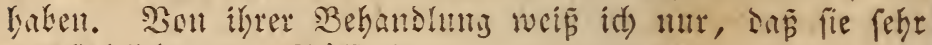

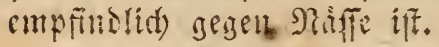

\section{Canna, BInmentofyr, I. 1.}

1) C. angustifolia, (d) malblittriges $\mathfrak{B} \mathfrak{l}=$

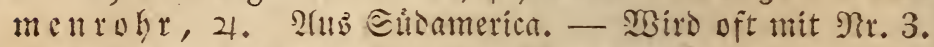

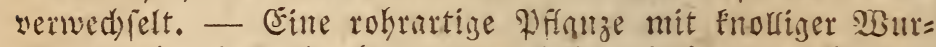
zel, weld)e einen $1 \frac{1}{2}$ bis 2 Jnt bohen Etringel treibt, an weldem obest sie gelb ant roth gefirbten and roth punc= tirten Bhtmen fefhen. Sie Ginterlaffen tunse, Dreutheilis ge, warzige Sapieln, worin sie runden, glatzentofluwat= zen, beintarten Eamen liegen, welde bernabe sie Grópe

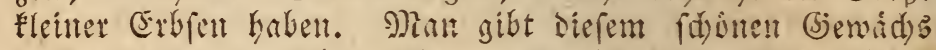
fdyarze, etwas fdanmige, mit Eand gemifde (Froe,

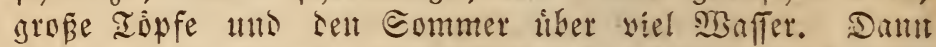
fellt mall es entweder in bie suft, ober befalt es im sim= mer, sent es ninmt mit jeder BeGandluth yorlieb, went man ihm im leştern Falle utu zuweilen suft gibt. Sim SBinter lápt man elltweder bent Topf bey 6-10 (Srad

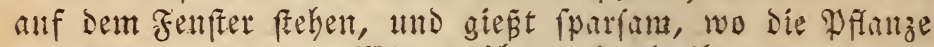

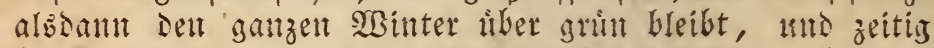
blibt, oder, went es an raum fehlt, umb man befferent

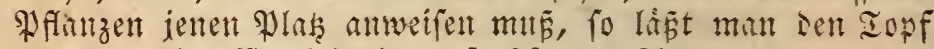

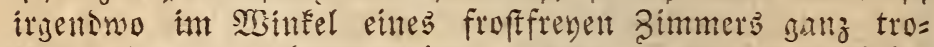

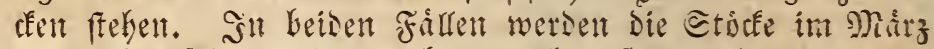
ial frifhe (Eroe gepfianzt, bey weld)er (Jjelegentheit man Die

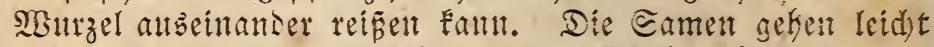

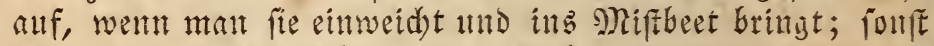
liegen fie zutweilen fegr lange. 2(ud) imt freyen sande fant man sab Blumentohr zieken, went man sie Sinorfen in May alf ein Beet mit gut gedungter (Frte 18 zoll anz= cinamber legt und fleisig begiept. Nan befommt Dann Dent ganzen Commer thber Bhment und Cament die Nenge. Die.Strainder yermegren fid fehr frare, werten Ende @ep= tember aubigeboben, bie Etángel abjeiduitten, tho sie SOHrzelt bidyt neben einander in einen Faftent mit Gand ober trockner Eroe gelegt, fo, Das nutr Die Etángel bervor=

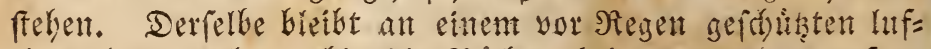
tigen Drte freken, bis sie Niddte falt zh wersen anfan= gett, wortuf man ín in ein froffreyes. Simmer, semolbe, 


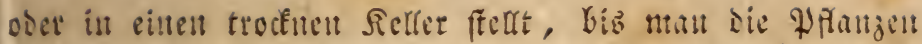
wieder an lie suft bringen fanu, mul man fieht ber Diefer (jielegenteit unt zumeilen nad), ob bie finolfen nid)t viel= leid)t fdimmelut.

Berm nad)ften Seyen ins Sant werten die Mitrzern

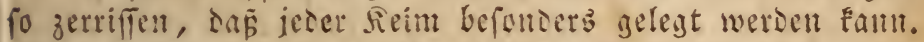

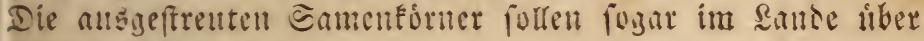
minter fid) baltell.

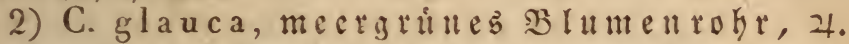
2lus Carvlua. - (5: gleid)t iem vorigen, ift aber in al= len Ibeilen friter, die Blatter blänlid)srun, febr breit, ullo Der Blumenfdaft 4 fun bod), aud) Damber. Ii

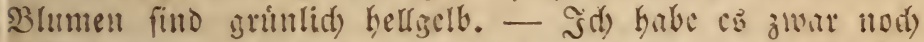

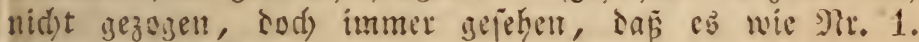
bebandelt murie.

3) C. indica, inbifd)es $\mathfrak{B}\lfloor$ unentofi, 2 .

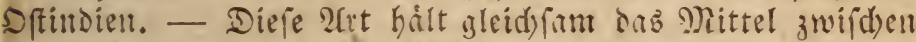
den beisen vorigen. Dic 3!átter find cbenfarks breit, nno die Etingel ctwa 3 Fun hod). Die Btumen find eillfir:

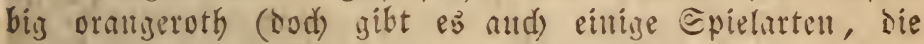
id) icood) nod) nid)t femme), Die Eamentorner grofiper a!s

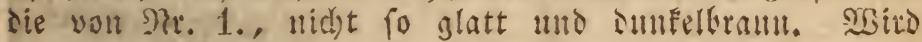
wie Nr. 1. begandelt.

\section{Capsicum, Beiñbeete, V. 1.}

Die Beipberen, you benen ser fogenannte fpanifas 3) feffer (C. annumm) die befantefte atrt ifr, find Ploule Etrituder, mo wersen aubz Eamen gezogen. Sie liebst

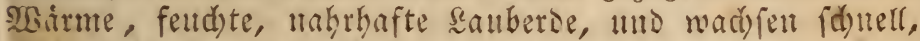

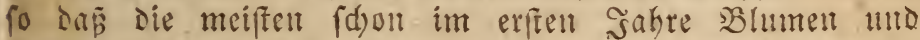
Frudite liefern, Daker zum Iheil als Eommerpflanze be=

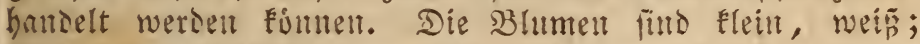
Die Fridite verfdichen geffaltet, grof oder feldr Elein, lamg= lid) ober runo, dumeltoth you Farbe, fduarz oder gelf. 2rue cutbalten einen idsorfent, åmperft bremenden Eaft.

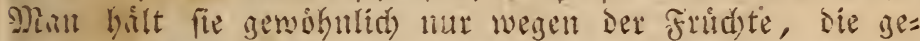

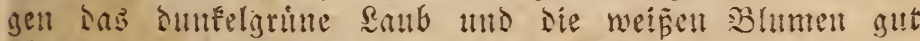

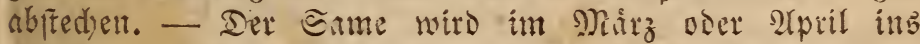
siftbeet gefaet, ntto sie $2-3$ Boll boben Pfling ben in einzelue Iopfe gefest. Șm 3 Binter gibt mant ifm $6-12$ (Sirab Warme. Zu merfen find: C. baccatum, Die beereli= tringente, C. cerasiforme, die firfátragente, C. frutescens, die firuduartige, C. grossum, die grofffrudutige, C. 
nigrum, die fdwarzfridjtige, C. sinense, die gelbfrudytige Beis̄bere.

Cardiospermum, her子łame, VIII. 3.

C. halicacabum, gemeitter f̧erzfame, Şerz= ertie, $\odot$. Dftindien. - Der feitte Elimmente Stintiget if mit zulammengefeșten glatten, långlid)ent, in scipp= d)en getheilten Biatten bejeşt. Die unberentenden wei= pen Blumdhen feken an langen @tielen, ntto hinterlaffent

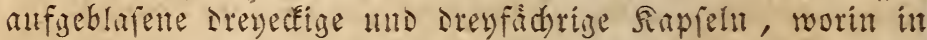
jevem fad)e eit runser (d)wargbrauner Same soll ser (5ito= Ee einer Eleinen Ërbe liegt, der mit einem gelblidweisen, herzformizen Fled geziert ift. Diefes Eommergewidds ver= langt rehmigen, mit Eand gemifden Boden, und wibrend ¿es Sommers jiemlid) viel Fendtigfeit. Man legt im

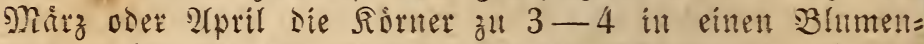

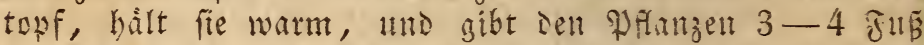
lange Etängel, an Denen fie binanfigeleitot werden. Sie

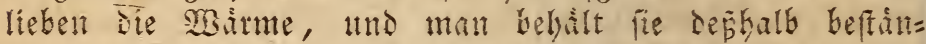
Sig im Bimmer, bis die Eament reif fint. Yluser ien forntern hat die Pflanze nidsts, wab fie befonters em= pifiblt.

\section{Carduus, Diftel, XIX. 1.}

C. c a sabona e, Ca fabong biftel (C. nicus casabonae), $\sigma$. Gitsuropa. - Die 4-6 3or langen, ungetheilten, lanzettförmigen Blitter fino am Banse mit

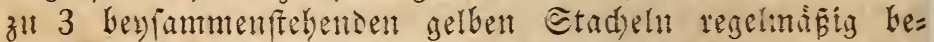
fest, weld)e Der \$flanze in Der Singent ein nettes 2tnefrn geben. Die viulettrótbliden Blumen erfheinen im zwerten Sofre, und find nidyt you Bereutung. SMan vermebrt diefe

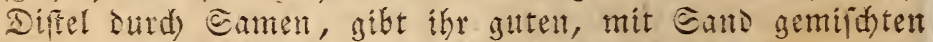
Boden, und bilt fie diber SBinter bey 4-6 (sirad nidst zu feudyt. Sm zweyten sabre Fant man fie im Nay ins

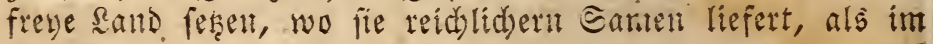
Iopf.

\section{Carlina, बétermurz, XIX. 1.}

C. acaulis, fitngellofe Ebermurz, 2!. Diefe,

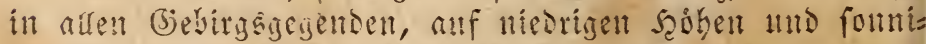

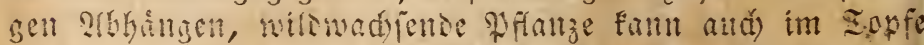
gezogen wersen, went man fid im serbif Eamen fammelt,

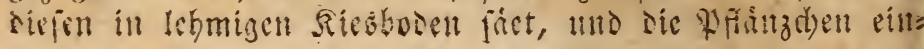


jeln in grop̃e, befonters tiefe Befinfe Pest. Sie verlans

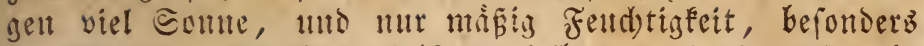
im SBinter. Die SBurzel if Dunfelbraun, ftaré, uno gebt tief in bie Eroe. Die Blatter fint ficcerfpaltig, mit Dor= nen befest, 6-10 Boll lanty, mo liegen flad) an ber Eroe.

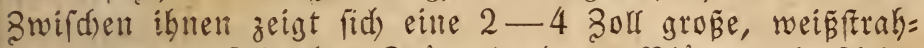
lige Blume, faft ohne @tångel, Derent Blâtter wie 2lthả glänell, mint lange Dattern. - Blithzeit in Den Eommer= monatelt.

\section{Ceratonia, Sofaltiabrod, XXIII. 2.}

C. siliqua, gemeines sobantiabrob,

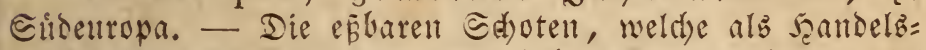
artifel ber jesem Ranfmant zul haben fins, enthalten viel flad), braungrofe Ferne, weld)e, wem man fie legt, leid)t aufgehen. Die lederartigen Blátter fint gefiedert, eyrmo uns fteben anf braunen Etielen. Nan gibt Diefer Pantze

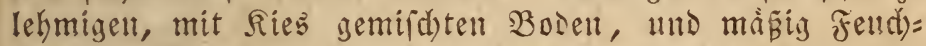
tigéit. Die violettrothlid)en Bhmen erfdeinen in Eleinen Irảubden aนs sen Eeiten Ier Etåmme, tragen jedod, io weit id) fie beobad)ten fonnte, im Bimmer niemals Frididte.

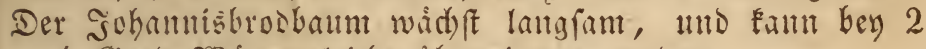
-4 (Sjad SGarme leidst liberwintert werden.

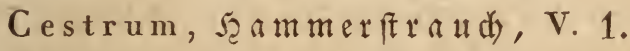

1) C. laurifolium, lorbectáttiger fram merfterad), Blitter find glattrandis, langlids =eyformig und Die Blu= men fefre fleill, selblids. 2ruser dem Blatte babe id Durd)aus nid)ts an Der PFanje gefunden, weldye im SBitt= ter eillen guten Ctand uno 6-12 Grad Wárme verlangt.

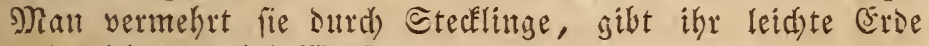
mo nid) zut viel SBaffer.

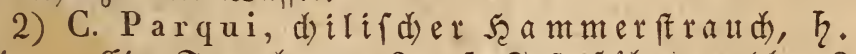

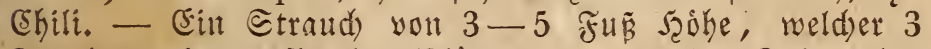

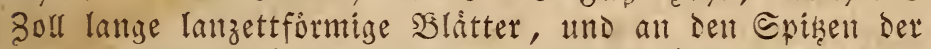
Sweige eine gedrångte 2lebre yon gelblid = bråunlid )ent Blu= men trảizt. Die Blatter Gaben, went man fauft mit der

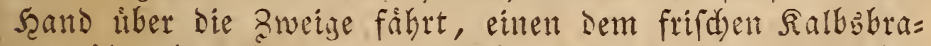

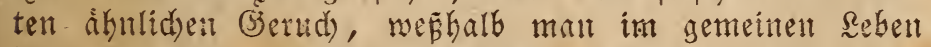
Diefe Pflanze and oft den Salbsbratenbaum nennen bort. - Er nimmt mit iesem Boden yorlieb, wurzelt fark, treibt viele Estroffen, surdh weldhe man ifh int Friflitis 


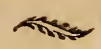

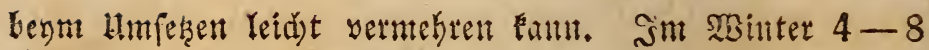
(5)at.

Chelone, 厄dirbbrume, XIV. 2.

C. barbata, bittige Sbilbblume, 21. Me= xíco. - Der Etångel if halbirrandartig, die Blätter fino linglid), blaugriun, und bie Blumenjđadfte merden $1 \frac{\mathrm{T}}{2}$ biz $3 \mathfrak{F}$ ment mit bartiger sippe. Diefe Pflanze liebt Fiefigen sefm=

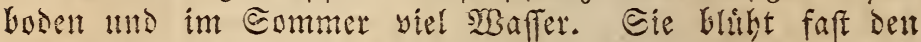

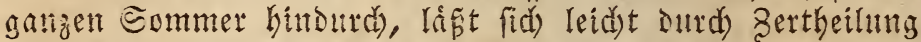

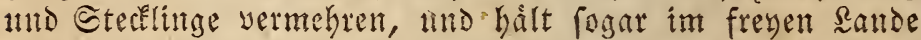
alb. Conft liberwintert man fie im Iopf bey 2-6 (Sirad.

\section{Cineraria, $2($ d d enpflanze, XIX.}

1) C. amelloides, amellenartige $2(f d$ en =

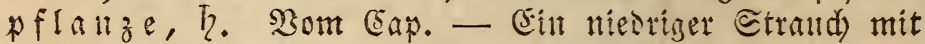

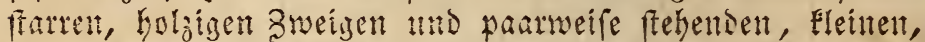

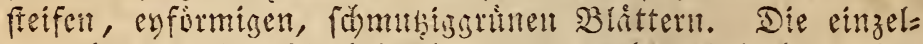
net blatten @etrablenblimáten mit selber Edjeibe ftehen anf langen Gtielen. Niant gibt ine lehmige santberse mit Eatro gemengt, minfig Feuchtigfeit, und im şinter 4-6

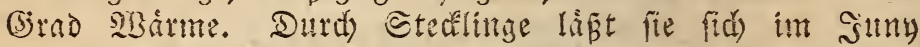

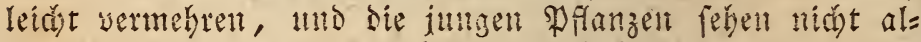

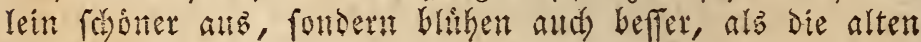
Etukfe.

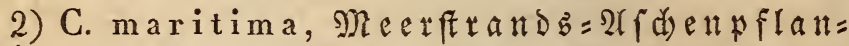
ze, h. 2(1ts bem fitbliden Europa. - Die fiederlappigen

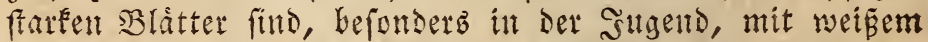

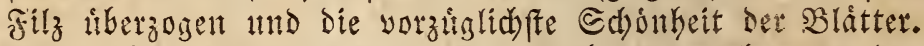
Die unberttenten Blumen find gelb und feeben an bent Episen der Zweige beyjammen. Man gibt ibr gewóbnli=

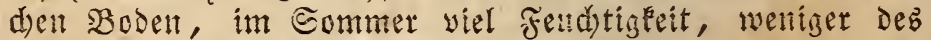
SBinters, vermegrt fie suth Bertheilung mo Etectinge, und if

Cistus, ciftentofe, XIII. 1.

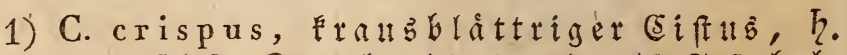
Portugal. - Diefer Etrand) wiro nut $1-1 \frac{1}{2}$ Fun hod, hat cyformige, grangrine, am Rante wellenformige, ge=

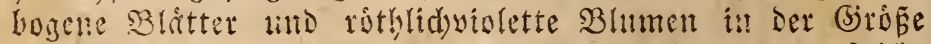
uns Gejalt Ier wilden Nofen. Shan zieht ign am seidd)= 


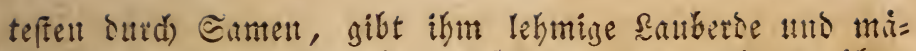

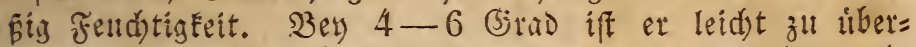
winten, unt blikt beunake den ganzent Eommer hindurd.

2) C. ladaniferus, lansanmtragende CEi= ftentrofe, দ. Epanten. - Diefes Bătmá)en wito 3 -5 fun bod), bat lange Blitter, Die, fo wie die jun= gen Zweige, mit eintem Elebrigen Satz liberzogen fint. Sm

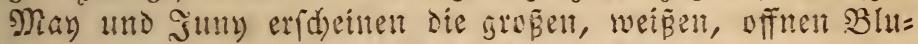
men, yoll Dentelt jeses Blatt ami Felchi einen bintrothen,

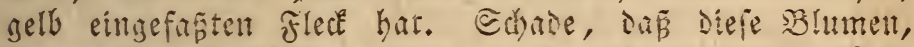
fo wie die alfer Eiftusaten, nutr cinen Iay banern. Shan

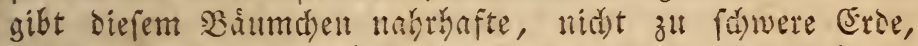

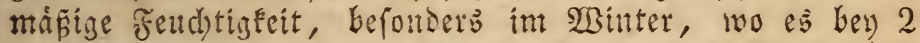

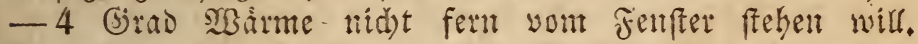

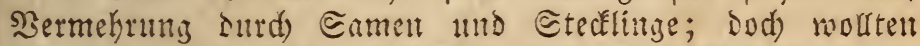
mir leştere niemals red)t leid)t wutrzelit. Sglan matht fie am fiderften im Sumn, fidreiset zwey zoll lange Bweige unter einem finotell ab, berecet fie mit einem Bierglafe,

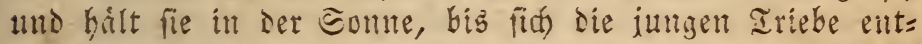

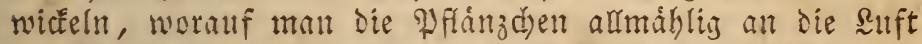
gewobnt, wo fie fidnell wachien. Den Eament fảet mant im Lipril, besedet ign mut leid)t mit (5rse, leagt aber eit

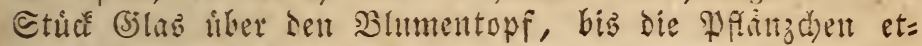
wa $\frac{x}{2}$ golf bod) fins.

3) C. purpureus, furpurothe Eiftertofe, 7. - Die Blitter find Ealbey áhnlid), die grosen Blu= men violettroth mit sunfeln flectent am field. Man be= hantelt ign wie ien vorkergehenten; Dod) (ideint er nod) williger @ament zu tragent.

\section{Citrus, $D$ iange, XVIII. 3.}

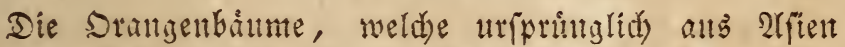

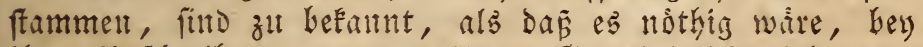
ifrer Befdreibung zu verweilen. (jemeintglich ziegt man

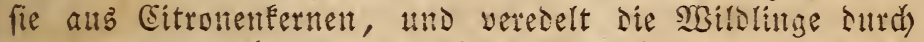
Sculiren oder R(blatiren, weldecs sethtere befonterb bey den: jenigen 2arten gefdehen muf, weld)e fehr Didit ftehente

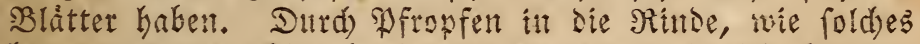
bey Den Sojen natber beidhrieben ift, laffen fie fid ebenfalls

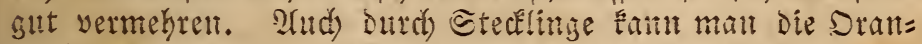

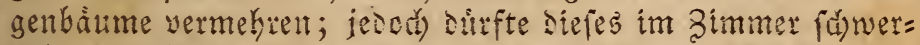

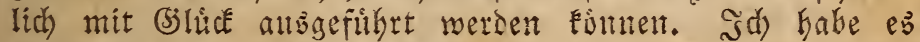

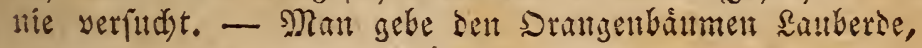


weld)e mit etwas sebnt, Sand und gut verfanltem und ausgefiebtem Dunger (roou man den Pferdemift aub Frubs= beeten benusen fann, wern derfelbe nod) $1-2$ Jabre ge= legen bat) gemifdt feyn mur. orre ein oder zwes sabre

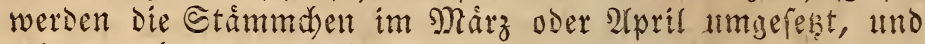
erbalten bey diefer (S)elegenbeit gröere Iopfe. Sirosert Srangenbaumen fann man, wenti fie gefund find, wåkreno Des Commers etwa 2 mal einen (S) Sd)afmift und Blut, weldbes geborig mit $13 a f f e r$ verdinnt fenn mú, geben; fránelt jedod) Det Baum, fo ift ifm Diefes Dingungsmittel eber fdablid), als vortbeilhaft. Da es aber fur Diejenigen Perfonen, weldye feine siarten ba= ben, nidit gut moglid ift, eitte folde Mifdung fids zuzu=

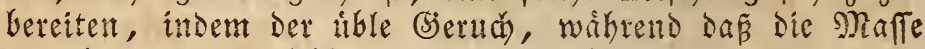

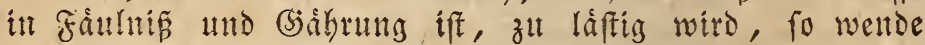

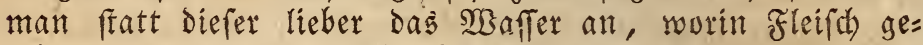
waffert worden ift, dod) ebinfalls nur febr felten. Das

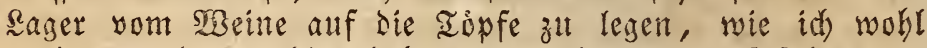
alia) empfeblen gebort babe, ift nad) meinen Erfabrungen (d) àblidb.

Befantitid ftellt man die Drangenbaume um Mitte May ins Frene; und bringt fie Ende September wieder in ibr Winterquartier. Sier gebe man ifnen einen Etano nitht zu feru vom Fenftei, suft fo lange als noglid), uno balte fie mifis feudt. Biel QBárme vertragen fie des Winters nidst, befonders balte man fie in ien monaten, wo es ou raub ift, als daf man iflten suft geben cann, fo fubl ars moglids; 2 - 4 (s)rad find binlanglid). Menn

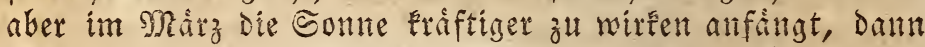
if den Băumiben eine Temperatur vou $6-10$ Sirab dien= lid), Denn die jungen Iriebe und Snospen entwidfeln fid um fo eber. Daber fann mant fetnen Bánmdyen um diefe

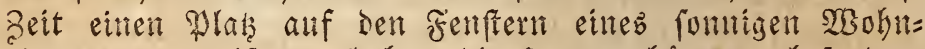
zimmers anweifen. Saben die Sraingenbaume, befonders sie Pommeranzen, jesod bell gallzen Winter liber feht warm geftanden, fo lafien fie nidbt alreit die Blatter gro=

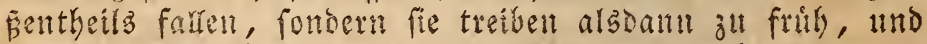
die jungen Iriebe Dorren in Der segel wiener ab. Bemert man, dâj eill Baum anfangt, gelbe Bloteter zut tragen,

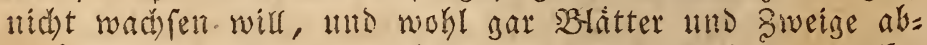

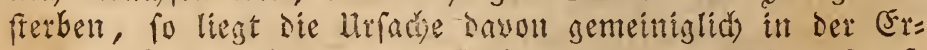
be. - Nan nefnte elliell foldyell Saum aus sem Topf,

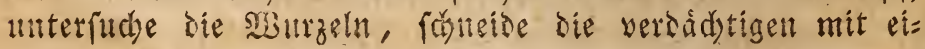




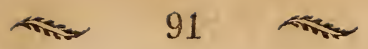

nem fdarfen Meffer ab, fdutttle Die vielteid)t (d)lammige,

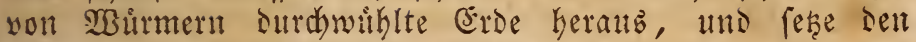
Baum mit leidster, fansiger sauberde in einen Eleinen Iopf. Die franten zweige finge und fineide man ab, uno

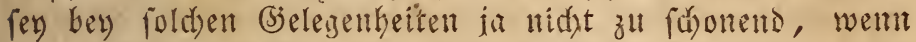
man, aud yelfeidht alfe Breige abnehmen muiste. Die munteli Êtelfen wersen mit Baumwadb bebecét. Dem franfen Băm giese man mit reinem Fluswaffer, ia mit

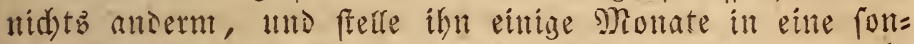

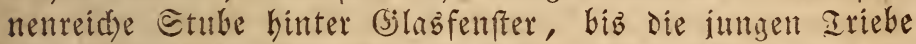
fid) zeinen, welde man affmihlis in ber sufft abhatrtet.

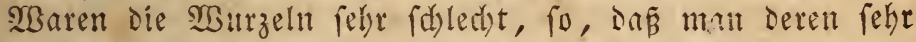
viele abnehmen muste, fo ftubie man jeoenfalls die Srone ein, uno umbitfe sent @tamm, fo wie Die ftíteften Iefte mit Noos, weldes man zumeilen anfendtet, bamit die Rinte zefdemeidig erbalten wird, uno nidst zu fal" aubs trodfnet. - Dft zeigen fid) in Der Rinse bransige Glecten

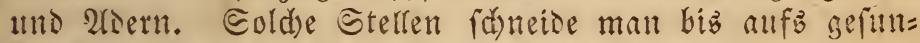
De frolz aub, unt besecfe fie ebenfalls mit Banmwach s und cinem Streifen seinemant. Srat man bey Beiten fo ver= fabten, und ift ser Baum fonft bey Siáften, fo fino nadh

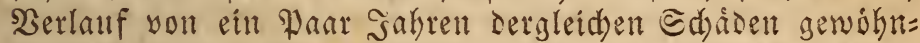
lid) mit junger Rinte berectet.

Sefr: viel leiden die Srangen = mno and andere ábnli=

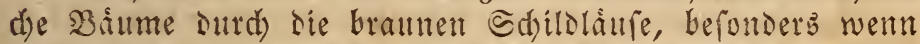
fie Érånfelt, wiemohl aud̆) die gefundeften Bàume oft anges

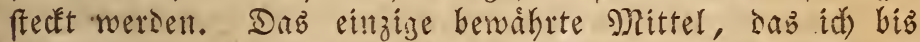

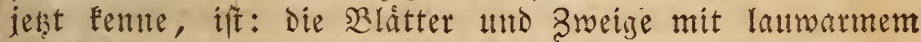
Woffer zumeilen abzumaichen, uno nadjoem diejes zeiche= ben, diefe Iheile abzufriten, indem man den Baum hori=

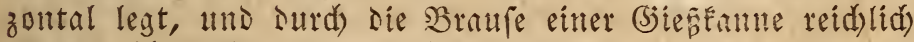

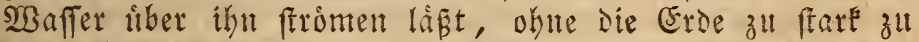
Dutd)uaffen. 2fud wegen Des Staubes, Der in Den Zim=

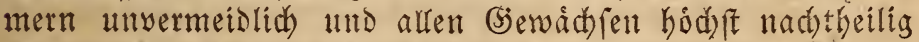

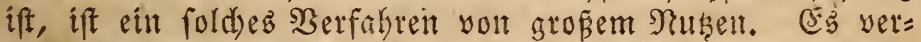
ftebt fich wohl you felleft, dan man fid) bierbey in $2(d) t$ nimmt, bie iungen Iriebe zu befdảdigen. 2(ud) if es dent Båuma)en febr zutraglid, zuweilen die Prinbe mit einem feudten Iudalappen abzuteiben, und fie yon sem Edamu=

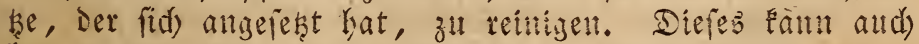
bey folden selegenheiten gefdehen. Huter Den gewöhnli= d)en Drangen eignet fict) Der Pommeranzenbaum (citrus aurantium) am beften zur Etubentultur. E: Kat bas 


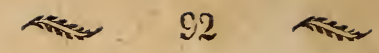

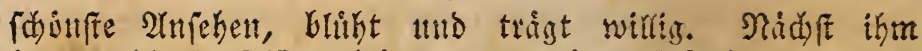
Fommt bie 2lpfelfitte (citrus aurantium dulce). 2(m we= nigften past bierzu Der Gitrontenbaum (citrus media). Son Den feltnern arten fino folgense zu- merfen: Citrum aurantium plenum, \$ommeranze mit gefiulfter Błhme. C. a. salicifolium, Die weidenblittrige \$ommeranze, mit langen (d)maiell, oft weíp geffecten Blättern. C. a. striatum, mit geftreiften frudsten. C. decumana, bie som=

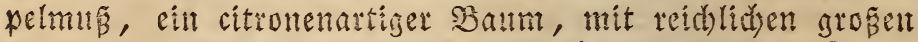
Blumen und gropen, tuntent beflyelben Friditen. C. japonica ntit C. myrtifolia; beibe baben rebr Fletne Blátter,

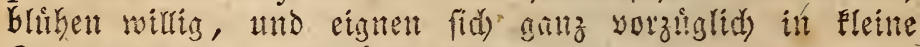
Cammlungen yon Ctubenpflatizen.

Clerodendron, Soośa

C. fragrans, wohltied)enter 200 ba befannteften unter selt Pamen Volkmannia tut Volkameria japonica, 20lismerie, $h$. Sapan. - Diefer Etrand)

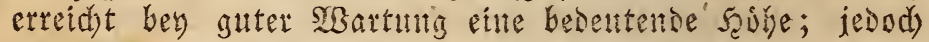
fiefr man ifn felten uber 5-6 Fur hod). - Die paar=

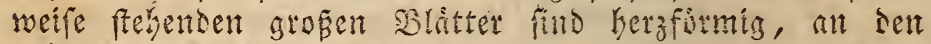

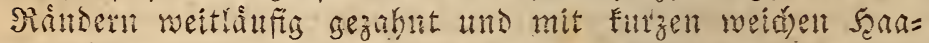

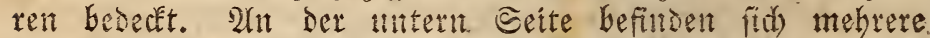
Fleine Drifen, bejonders da, too dab Blatt am Stiel fibt, alls. weldyen bey gefunden, Eraftigen Pfatizen ein bicker, farbentofer, zuderartiger Saft fidwist, Der ait Der suft er=

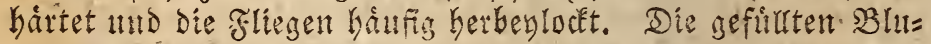

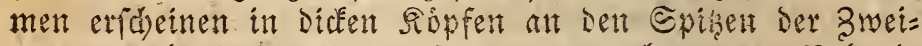
ge zur unbeftimmten Zeit, fo, Dá man bey guter Bebant=

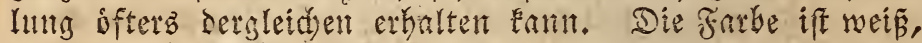

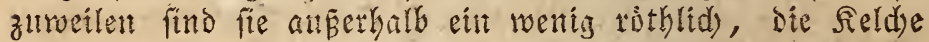
felbfit find siolettbraun. Die Invspen iffinet fid nicht alle alf einmal, fontern gleityfant lagenweife. Sift bie erfe $\Omega a=$ ge abgeblift, fo entwidelut fid die Blumen der zwerten, uno oft treten nod) zum taitten Dal neue findspen hersor,

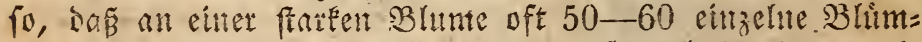

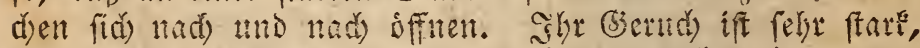
bat viel abnlides mit bem ber iltherofe, sodj dabey etwas nad) bittern Mandelt.

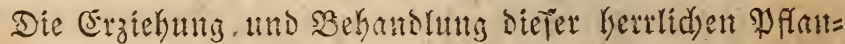

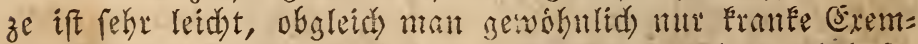

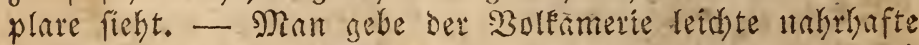

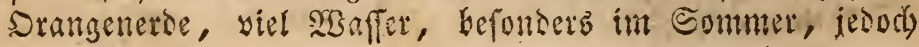




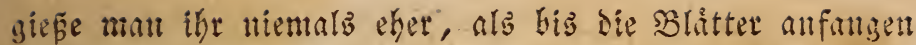

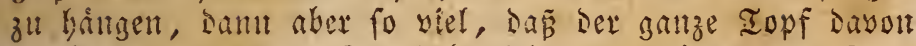

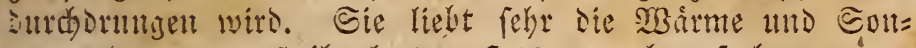

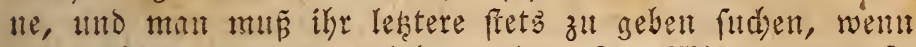

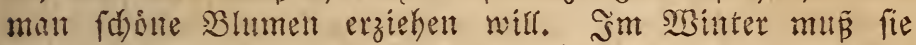
wenigftenb frets $S$ (jordo şarme Gaben, Dod) fint and 12

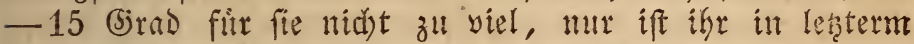
Falfe Eome und eill Nlak nabe am Fenfer nothwentig, weil fie unter folden limftanien ftare widdft. Sim Som= mer babe iă) fie anth fehr warm gehalten, und nie an Die suft gebradst. Dod) fann man fie zuweilen einem mildent

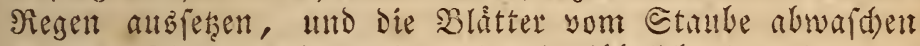

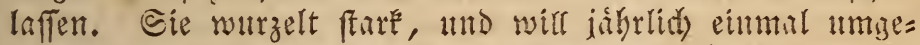

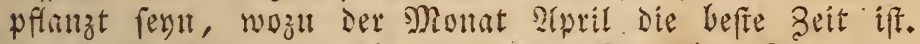
Durd) Etectlinge, weld)e man yom Man bis sitte 2(u= guft maden fant, ift Die \$olfamerie leidjt zu vermehren.

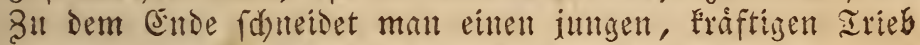

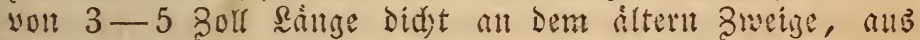
Dem er gefprof

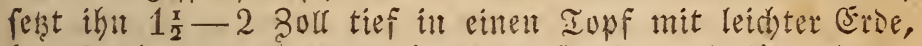

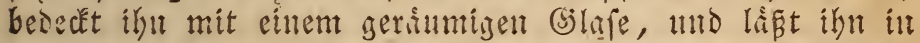

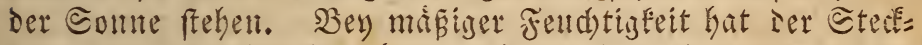

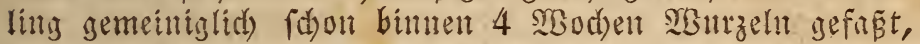

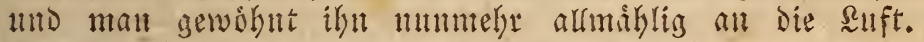

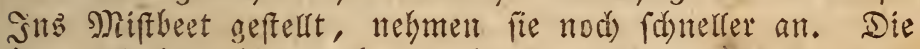

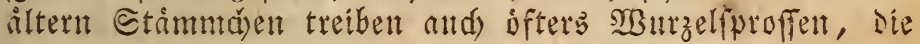
man beym Serpfinazen abzunehment futht; aub Diefen zieht

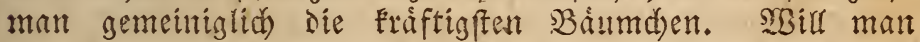

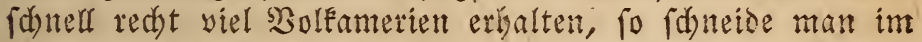

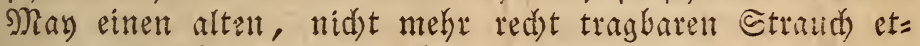
ma 2 zorl riber der Erde ab, und pftanze denfelben unmits

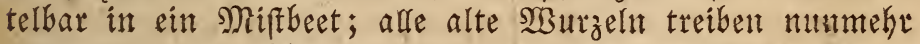

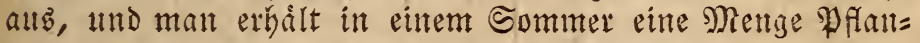

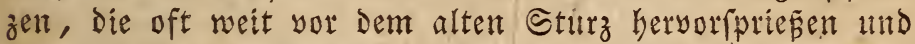

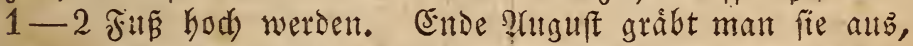
pflanlzt fie einzeln in Iapfe, unto bált fie etwa 8 Iage fd)attig unto warm. 2fudd) 2(bfenfer mad)en, wellit man

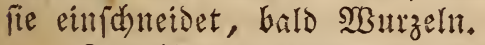

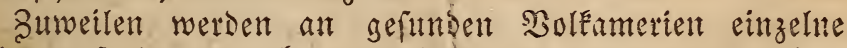

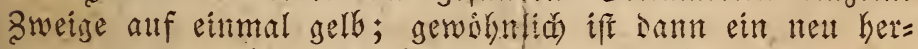

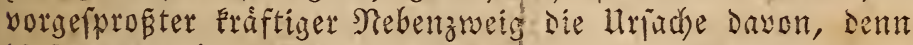
Diefer entzieght dem erfern die Nahrung, uno man fantei=

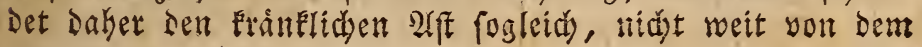




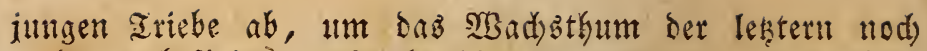
mekr zu befordern. Drobt bie ganze Pflanze einzugehen,

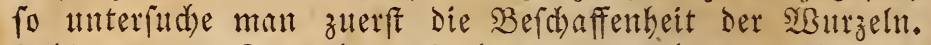
Fehlt es sem Straud) an Nahrung, fo gibt man ihm fri= fhe Erise und einen grofern Iopf; fino fie faultis, fo fdheidet man die fdashaften aus, und fellt die Pflanze in einen fleinen Iopf mit leid)ter Groe, bålt fie rarm,

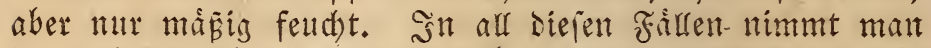
Den gróften Theil Der Bweige ab, uno war bie Pflanze fehr (d)wad), fo wir fie ofine SBeiteres 3 oder 4 zold hiber Der Frde abgefdnitten. Heberhaupt nehme man bey diefem (Ges

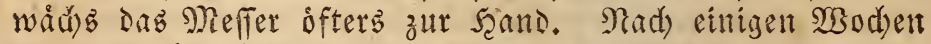

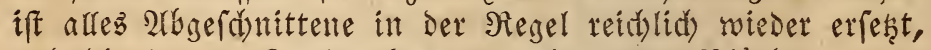
Into die jungen zweige fommen mit nenen Blutthent.

Die grimen Blattláufe werden Durd) 2tbwafden Der Blåtter mittelfi eines @d)wammes am beften vertrieben.

Daz Saterland der Soléamerie foll Sapan fenn; dod) fo weit id die Pfanze beuttheilen Fann, modte te fie wohl alts Den waimern Iheilen yon Ufien ftammen; vielleid)t gar alis Sndien; Denn fie hat feine Beit Der Rube, fondern treibt fortwibrend nette Irtebe and Blumen, wenn man

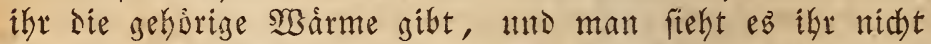

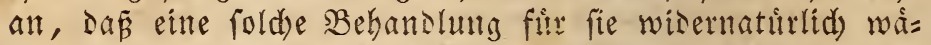
re, fondern fie fheint diefelbe fogar zu verlangent. Sm (S)egentheil, wirl man fie in (s)emeinfd)aft mit andern Pflan= zen, sie aus Dem gemápigten Sapan zu unz gerommen fino, iberwintern, fo gedeibt fie niemals fo gut.

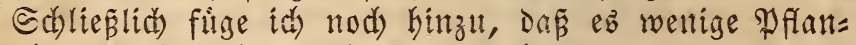
zen gitbt, weldhe fidh fo fehr zur Etubencultur eignen, wie

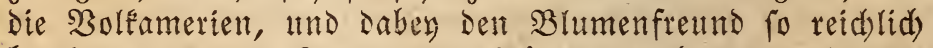
belobnen; nur ift Gonne uno SBarme, verbunoen mit Dem

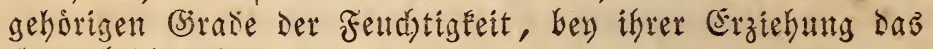

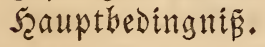

\section{Cneorum, zeiland, III. 1.}

C. tricaccon, oreyfamiger zeitand, Epanien. - Ein Fleiner Straud) mit länglidłen Bláttern, fleinen gelfliden Blumen und rothen Drebectigen Samen= fapfeln, ábnlid) Den beg Euvonimus europaeus (PFaffen=

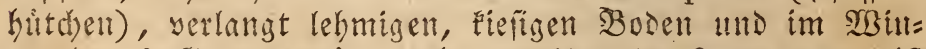

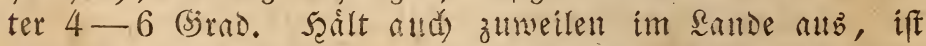

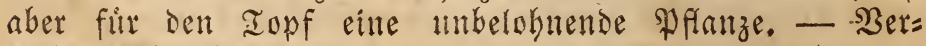
mehrming iurd) Samen. 


\section{Aser

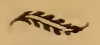 \\ Cobbea, çobbea, V. 1.}

Cobbea scandens, fdingente robbea, Mexico. - Die Blátter diefer Edylingpflanze fino gefiedert,

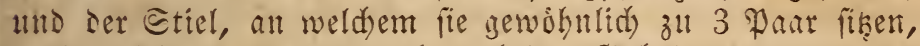
endigt fid in eille vielfaid) getheilte (Gabelrante. 2(n Den

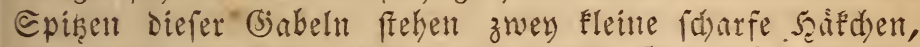
mit weld)en fids die Pfinze an alfen (Siegenfturnen, die fie erreidhen Eann, fefthált; fokalo diefes gefdehen, zieben fid) Die Gabeln fpiralformig zufammen, tho náhern den Smeig auf diefe 2 trt igrem Ethispuncte. Die groşen glo= denförmigen BInmen, weldye Den ganzent Commer tiber et= f(d)einen, fint anfinglid grinliá), verinoen aber fidon am zwerten Iage ifre Farbe ins Blátlide, und werden endida sunfelviolett. Shr (siendd ift wiserlid).

Man gibt ser Cobbea geraitumige Iopfe mit nafukaf=

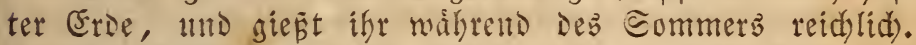
(5) if alsbann am zwedkmişisften, Die langen Rankell an

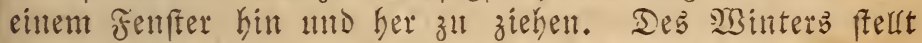
man fie bey 4-8 (sirad nidjt weit yom fenfer ab, gief́t ifr aber nut sann, wenn fie es grade nothis bat. Die

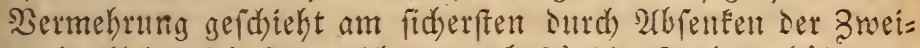

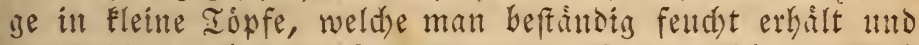

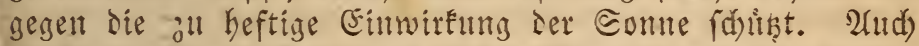
surd) Etedflinge fant man siefe Dflanze vermebren, Die malt aber einige zeit warm balten mus. Der Same wird bey uns felten reif; mur ein einziges Nal babe idj eine

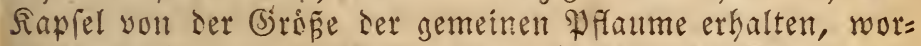

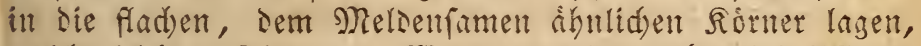

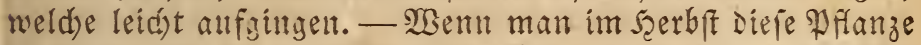

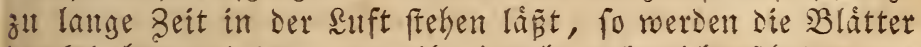

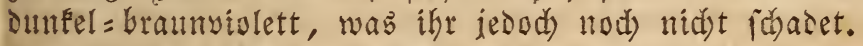

\section{Colutea, $B \mathfrak{Z} \mathfrak{a} \mathfrak{e n f t} \mathfrak{i} \mathfrak{d} d)$, XVII. 5 .}

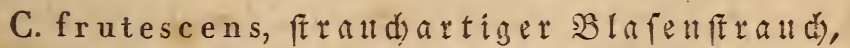
h. Eap. - Ein 3-4 Juj hohes Botuntden mit foinen grautgrituen, gefiederten $B$ làttern. Die fícntetterlngsartigen,

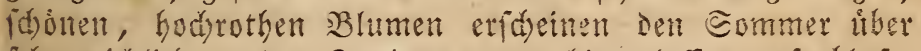
fehr reididid) an ren sweigen, und binterlaffen anfieblape= ne $હ d$ oten mit vielen Floiten, fdwarzen, platten Fornern.

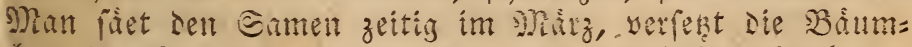
d)en im May einzeln in Iopfe mit nahrhafter Ranberde, und bringt fie an einten gephuhsten હtano ing Frege. Eie 


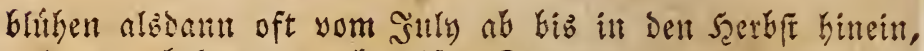
und man Eeépment nod) reifen Sament, fo, saś man dies

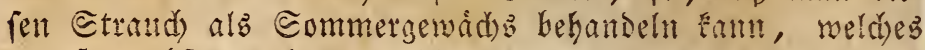
um fo rathfamer iff, Da die Pfanjen, went man ifhen

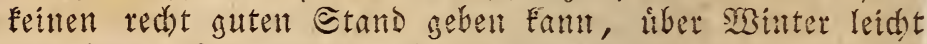

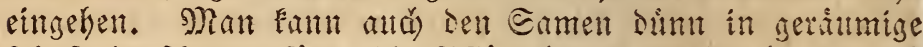

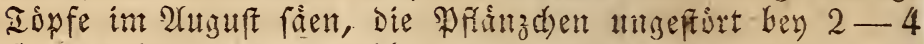
(Gitad nahe am Fenfer uberwintern, insem man thuen nur

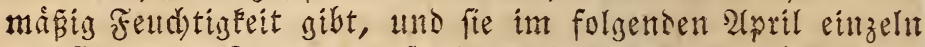
verpflanzen. Fann mant ile in Diefem Farle 3-4 in cinem Miffbeet antreiben, uno gewobnt mant fie im Nay gleid) an die suft, fo bluken fie fegr balo mo reid)= liti). 2litch in freyem salloe, wobit mant fie erft nad) Der

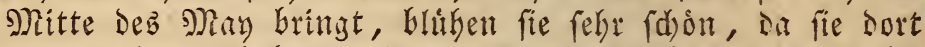
yolfe Nabrung baben. Şierzal eignen fid befonders die át=

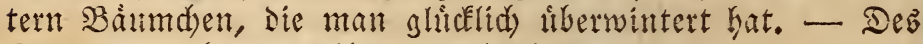

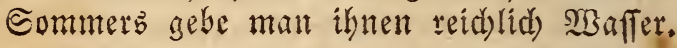

Commelina, Eommeltue, III. 1.

1) C. coelestis, himmlifde Commeline, $2 \%$

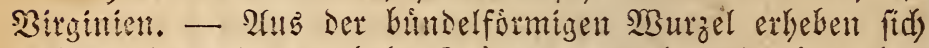

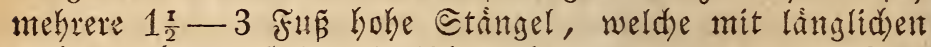
Blittern befeht fins. Bwifhen ifnen Fomnten an ber Epi=

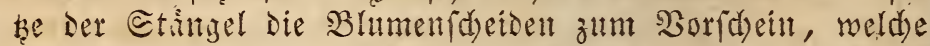
kletue, Dresblättrige Blimd en enthalten, Die youl lebbaft berrblater Farbe find, Dez Morgens fid) offnen, aber ge= gen 11 uhr fidon wieder verblitht find. Dod etfdeinent mit jedem Morgen nelte Bhtmen, Hud zwar Dauert bie Blithzeit. yom Stuny bis it Den Seerbft. Man gibt Der Commeline nabrhafte, leidiste (Erde, ziemlid) viel şaffer mo einen gefduhtent @tand in Freyen, wo fie Die Mor= genfonne genefien Eant. Den Winter viber Gat mant ourd)= alls feine \$lage mit Defer Dffanje, Denn man braud)t nut Die Ctingel auzufdneident, Imo Dell Iopf ganz troden itt

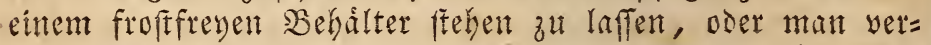

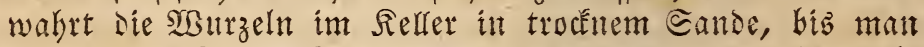
fie in Der Nitte Nary. wieder its sand legt. Wermeh=

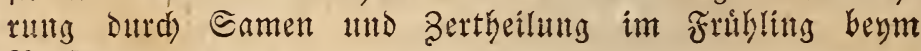
Merfesten.

2) C. tuberosa, die fuollige commeline, 4. șit fdyadder, als die yorige, fonft aber beynake gar nidjt von ifle zul unterfdeident. 
Convallaria, Mayblume, VI. 1.

1) C. japonica, iapanifde Naabb\{ume, 4 . Sapan. - Die fdmalen, glänzenigrủnen, grabartigen Blitter find Dauterno, die fd)mubis weislidhen unbedenten=

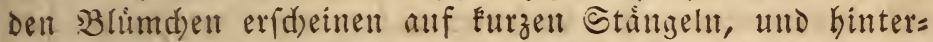
laffen bimmelblatte Beeren. Nan gibt ihr lehmige saub=

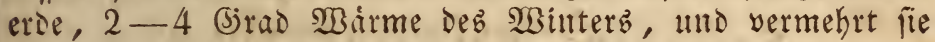
Durd) Bertheilung. Sift ůbrigens eine unbelohnence Pfanje.

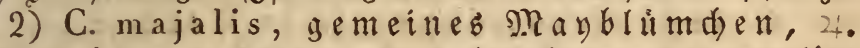
- Diefe befannte Pflanze mit wohlried)enoen Blumen lást

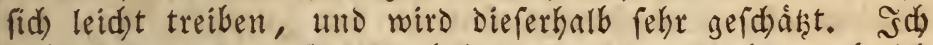
habe fie folgender (seftalt behande!t. Sm Ferbft grub id

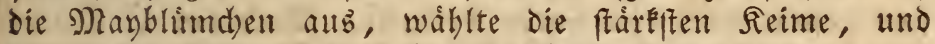
(d) nitt die $\mathfrak{W u r z e l u}$ derfelben fo $a b$, Dá nur etwa $2-3$ N3urzelquirle Daran blieben. Bon diefen Seimen wurben fo viel in einen Iopf gelegt, alb Raum batten, mit nabr:

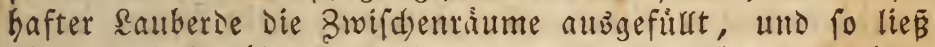

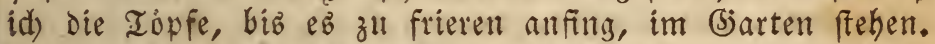

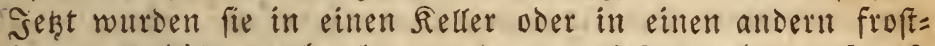
freyen Behálter gebrad)t, uno aub biefem geholt, fo oft id) fie treiben moulte. Dab Iteiben felbft geid)ab in fol= genoer 2trt: Der Iopf wurse in einen Unterjeşapf mit Maffer, und beide allf ben warmen Sfen in eine Iempe= ratur yon etwa 30 (Sirad geftell. Ulm Die feime heryor= zulocfen, Decte id) einent Blumentopf verfehrt liber bie (Er: be, uno waren bie Iriebe 3-4 zolf lang, fo gewoihnte id) fie an suft uno Sonne, wo Dann die Fnospent fid' fdinefl entwidfelten. Die Şauptbesingungen zum Ireiben ber

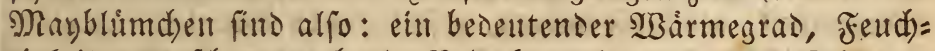
tigkeit und fdattengebente Bedeckung in ber erften Beit. -

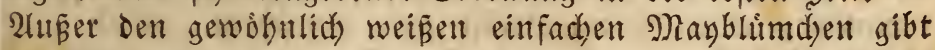
es eine weisgefülte und eitle róthlid)e Epielart. - MBenn man die Manblimden im Serbft allb der Eroe beralls= nimmt, und die ftárften Reime aus̉wáhlt, fo Eann man bie fd'wådhern 4 zolf weit allb einander auf ein Beet les gen, Damit fie fich bis zum folgenden Feerbfte ftärken.

\section{Convolvulus, פinde, v. 1.}

C. cneorum, die હilberwinde, h. Eyrien. - Ein nebriger Etrauld mit feinen Bweigen, weldye mit lanzettfỏrmigen, auf beiden Seiten mit filberweišnen şår=

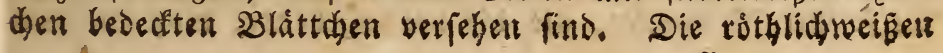




\section{Ant 98 אise}

Blumen gleidjen bernabe Denen bet gemeinen Feldwinde.

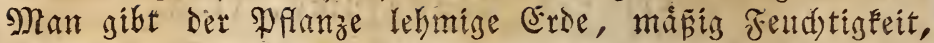
uberwintert fie bey 4-6 Girad, und fegt fie alle sabre im Frubling um. Sie blubt faft den ganzell Sommer bin=

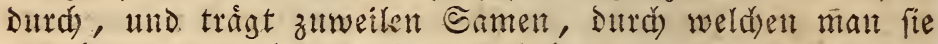

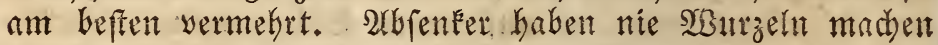
mogen, eher glitekte mit es mit Etedfingen.

\section{Corchorus, Cord or $u b$, XIII. 1.}

C. japonicus, iapanifäer (5 ordi) orus. Die Bhatter find zugefpitht, frark gezahnt, und falfen uber SBinter ab. Die Brocige find in ser șugento grin, Die Blumen, weldi)e zeitiog mit Den Blättern hervorfommen,

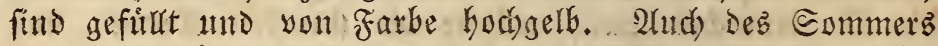

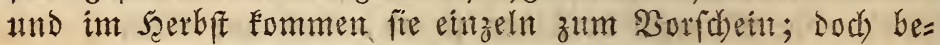
fonderz báufig, wenn man biefen Straudi als sandpflanze

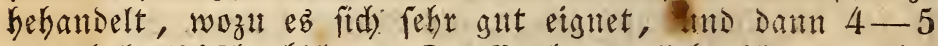

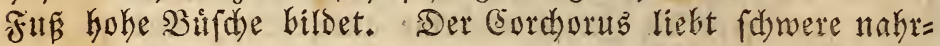
bafte Erde, viel waffer, wurzelt ftare, uno sermehrt fid

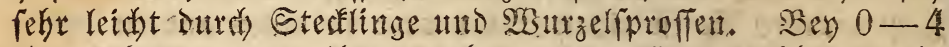
(S) rad bringt man inn am beften Dell SMinter liber Durd), benn zu viel Marme mad)t, bas er fids libertreibt, und (d)led)t blitgt.

\section{Cotyledon, Nabelfuat, X. 5 .}

1) C. coccineum, fdarlad) rothes Nabel= Eraut, 万. Som Eap. - Die farten fieifotigen, lanzett= fờmigen Blâtter find beynabe rinnenformig gebogen, uno

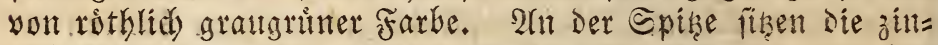
nobertothen, glockenformigen, gefdlofienen-Bhlumen faft ohne Etiel zwifhent dent immer fleiner werienten $B$ lättern, fie

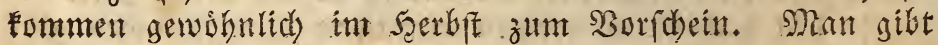
diefem Nabelérant, wie allen. Fettpfanzen, magern Boben,

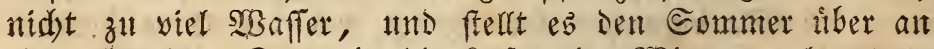
eitter fonnigen @telle in Die Ruft; im SBinter verlangt eb 4-6 (Sirad. \$3ermehrung: Durd) Stecklinge und abge= brocíne, eingepflanzte Blátter.

2) C. orbicularis, Exef

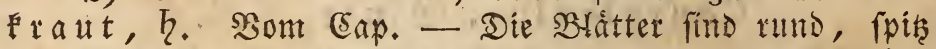
zulaufeno nind Glattgrin. Die Blumen fino robthlid) grain=

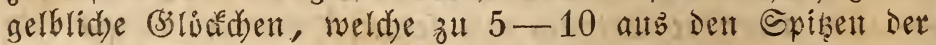
Sweige im jerbft, oder zu Ënoe Sommers berworbred)en. Diefe 2frt ift fdobner, alb. bie vorkergehende, muñ alld) et= 

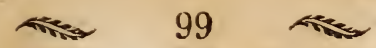

was majet Itno fibl gebalten werben, went fie blúben folf, und wito tibrigens eben fo befandelt. Sald habe ofe

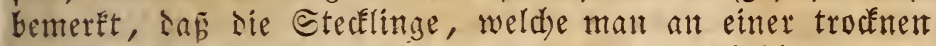
Etelle ins sand geftect' batte, am willigften blubten.

\section{Crassula, Difflatt, V. 5 .}

Unter Sen Didfblittern gibt es einige Eorten, bie fidh surd) Die fonderbare (jeftalt Der Blatter auszeid) nen. 2(ud) fint die $B$ lumen mehrerer nidht libel. - C. coccinea, decussata und einige andre geken jeşt unter Dem Namen Rochea. - C. perfoliata fieht atts, als waiten die Dicken Blătter auf einen Faden gereibt. - C. imbricata uno lactea find Dagegen wenig werth. Cie werden wie die Rabelf́ráuter bebanicelt.

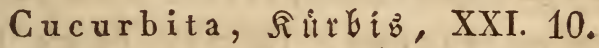

C. lagenaria, ber flafdenturbib, ○. OfE indien. - Diefes (jewwad)s gehort zwar eigentlids nid)t zut Den @tubentianzen, Da man ihm im Iopfe nid)t fo viel Nabrutg geben fann, als die Fridd) zu ihrer 2tubbiloung

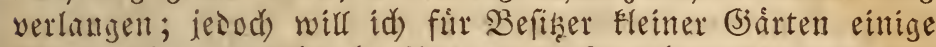
Worte liber Daffelbe benfiigen. - Ea gibt zwen Epielar= ten Flafdhenfurbiffe, den eigentlidhen Flafdhenfurbis, wel= d)er, wenn er red)t fidon ift, nid)t liber 15 zoll lang wirb, tuno eine regelmásige flafdengeftalt hat. Dod) fehr oft ar:

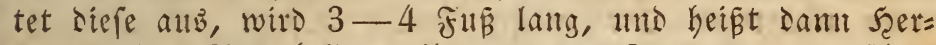
fulebente. Son beiten gibt es zwey Corten yon verfdies senter Farbe; Die eille trágt weislidse, die andere ounkel=

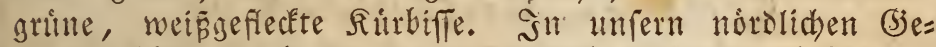
genten bâlt $e \vec{b}$ fdwer, Den eigentlid)en Flafd)enflurbiz zur Reife zu bekommen, ia er gemeiniglids zu faát anfebt,

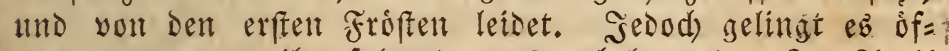
ters, went man ihn folgenterma voer fdyon Endre Miárz legt man jeden Fern eillzeln in ei= nen nid)t zu Hleinen Blumentopf, und fteld die, Niápfe an eill fonnenteides senfer, oser in ein Miftbeet. Sind die Rffanzen aufgegangen, fo bâlt man fie warm, uno fendit, bis man Diefelben in Der Nitte May an eine gegen Mit= tag liegende Mauter mit sem Ballen in Die Eroe feşen Fant, weldye man vorker gut ungegraben, ino mit ges fanltem Mift gedungt hat. Die Ranken werdent an der.

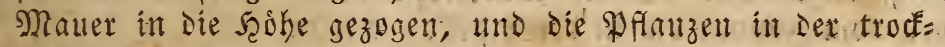
men sahreszeit feudst gehalten. Dod gieñe man thnen ges 
gen ben Şerbft, wenn bie Fruddte zu reifen anfangent, nid)t

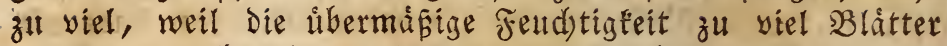

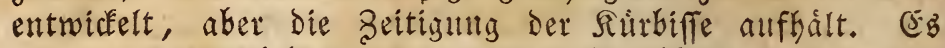
ift gut, Den Frudd)ten, Damit fie nid)t abbred)en, eitte Un= terftithung zu geben. Die reifen Sürbiffe werden, thadjoem man fie abgefdnitten hat, in einem warmen Bimmer allf= gefangen und getrodtuet. 2llbdann erft fdueidet man fie auf, und nimmt die Rerne betalb.

Der Pomeranzenfarbib, C. a urantium, folf firt) wirflid) in der Etube zieken laffen; bod) glaube id)

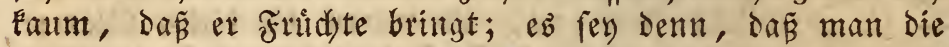
Serne itt einen yor Dem Fenfter angebrachten, gerảumigen Saften mit Erobe legt.

\section{Cupressus, Épteffe, XXI. S.}

C. sempervirens, immergrute Eทptef= fe, h. Sadeuropa. - Ein befannter Baum, ber in fei=

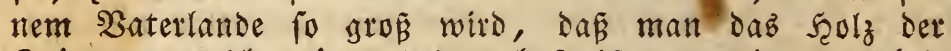

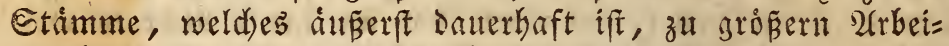
ten bemusen Eann. Nan gibt Der Enpreffe Srantierie = E: De, febt fie alle zwey Sahre in grósere Iopfe unt hiber= wintert fie bey 2-4 Girad. (Siewihnlid) zieft man fie atb Samen, Den man im Nay fatet ulto nitht zu roarm

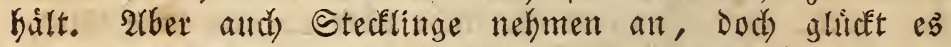
nid)t jebesmal; man madjt fie im sulth und lispt fie alt

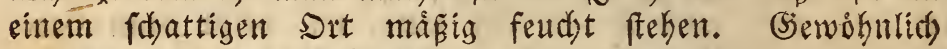
wurzeln fie erfî im nåd)fen Sabre.

$$
\text { Cyclamen, extracibe, V. } 1 .
$$

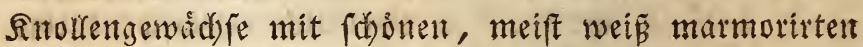

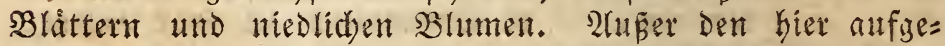
filbrten findet man zumeilen in ben Pflanzenverzeidntiffen nod) mefrere atrtent angegeben, sie jedod) faft fámmtlid́) nur zufátatg alı Samen gezogente Spielarten fino. Darum nebme man fid bey 2(nfdaffling unbefannter. Sorten in 2(d)t.

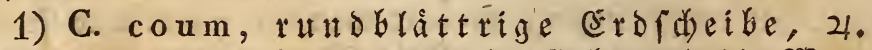

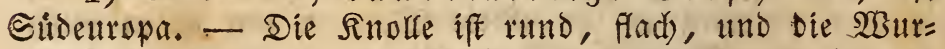
zeln Fommen unten alls berfelben in einem Bitndel Gervor;

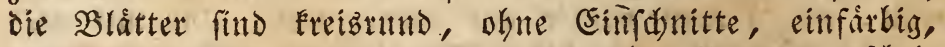
Furzfielig. Die fleinent wépelrofenrothen Blumen erfdei= nen vom samuar biz in ben $2(p r i t$. Befandlung wie Sir. 3 .

2) C. europaeum, europififde Extoftheibe, 


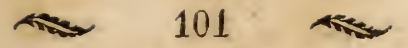

4. Iyrol mis Deftreidy. - Die SBurzeln dicfer alut find nur in Der Sugeno runs, fonft aber bilden fie eine fobwarz= braune, mit Zadfen verfebene Fnolle, an Der die Blátter uno Blumen erfdeinen. Die Blatter find Gerzfórmig, ge= ferbt, oben matt meis, gemolet, unten roth. Die Blit= men erfdeinen auf 5-6 zoll langen Etielen, beynabe Daz ganze Sahr bindurd). Share Jarbe ift ebenfallz dut= Felrofa, uns fie suften rehr altgentehm nad) Deilden. Diefe Eorte wirft, wenn fie gefund ift, bie Blátter nidjt ab, uno diefer Umftant, fo wie, dap fie immerblithend iff, unterfdeiret fie Ginlänglidh yon andern 2lrten. Sie liebt

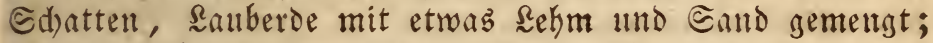
wurzelt nid)t ftatk, und aแb Diefem (5irunde ift $\mathfrak{e z}$ gut, fie utr etwa alle 2 oder 3 Safie einmal umjupflanzen, úberdem, da fie faft iesesmal eine zeit lants tramert, bes fonders, wenn man fie zertheilt hat. Man fehe nur bars

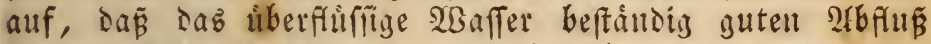
hat. - Daz umpflanzen gefdéleht úbrigens entweder im Nay oder im 2luguft. Die Sermefrung gelingt am beften, intem man die bewurzelten Backen abbrid)t, die Munden mit Soblempulver beftreut, wno die pflanzen an einem fidat=

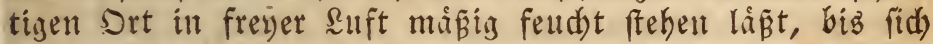
frifde Blatter zeigent. Itautert eine Pflanze, hat fie viel= leidgt die Blätter abgeworfen, fo laffe man fie, wenn man liberzengt ift, die Errde fer gut, ungeftort ftehen, bailt den Regen yon iht ab, unto bedecft fie oben mit Moob. Sie fonmt gewiś, wern man aud länger als ein Sabr war: ten folte. Nur ftore man Die-\$flanze surd)aus nid)t, nody

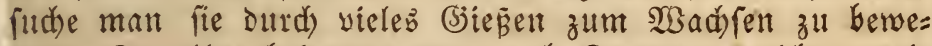
gen. Bumeilen belommt man auth ভamen, weld)er, wie ber yon C. hederaefolium behandelt wird. Mill man aud

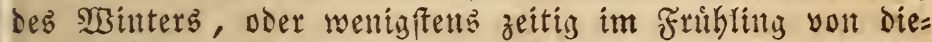
fer (Erofdeibe Blumen erhalten, fo fellt man fie in einem 230 hnzimmer Did)t an ein Fenfter, wo fie tigglids ein Paar Etunden Sonne befommt. Sonft Fann man fie aud bey 0-4 Grad úberwintern, ja fie hallt pogar im frenen san= de aus, ment der Boden nidht zu fdhwer ober fdlammig ift. - Es foll aud eine weişblibende Epielart geben, wel=

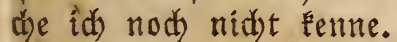

Sdließ̧lid) erlaute id mir nod) folgende Bemertung:

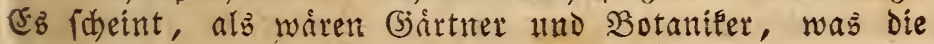
Beftimmung biefer art anbelangt, nod) nidjt ganz im Ret= nen. Eine Erofdacibenart befthe ids, weldhe in ben lumge: 
Gungen yon 2 Bien bånig wild angetroffer werben for. Cie wurde ald C. europ. genannt, wiewohl fie fid) you der obi= gen in folgenten Etideen unterfdeidet: Die Blätter find zwar

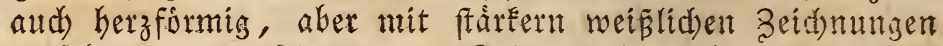
verfehen, und anf Der untern Eeite wentig roth. Die Fnothe treibt keine Backen, fondern bleibt rund, wienobl die eine \$flanze fodon genen 8 sabr alt iff. Die Blumen erjedei= nen nid)t das ganze sahr hiber, fondern nur im serbft, zugleid) mit benen des C. hederaefol. Cie find nidit to (d) don roth, fonsern mebr matt, rechen aud nidjt ganz ro angenefm, tragen aber weit leid)ter Samen, als die

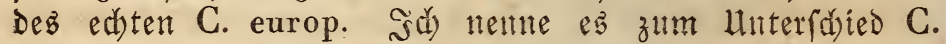
e. austriacum. - Dab wirflithe C. europaeum wutro nett= (idi) yon einem meiner Berwandten in grofer Menge it Dent

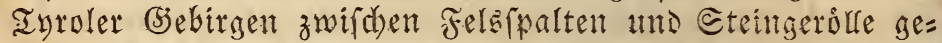

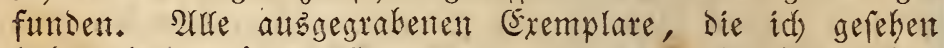

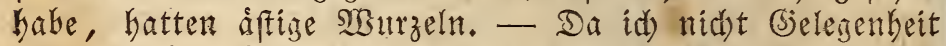
sebabt babe, beise planzen an ihrem natirlichen @tand= orte zul beobadiden, fo laffe id es Dabingeftelft, ob beide

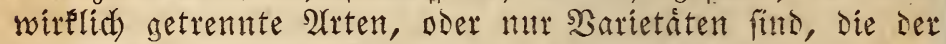
Bufall bat entfekent laffen. Dod) wirte id fur erfereb ftumment. - Eelgr oft verkanfen sie ziwiebelgandler C. hederaefol. fui C. europ.

3) C. hederaefolium, enheublattrige Ert = (d) eibe, 24. Stalien. - Die Finolfen find rumb, flad), in Der Mitte oben etwas eingedridut, unten erbaben. Die Blátter find fehr verfdededen, gewobnlid) gleidjen fie mehr oder weniger benen Des gemeinen Erben; zuweilen find fie jeodd) faft ganz glattrandig. Shbre Farbe if Dunelgrin, mit fdónen regelmåşigen, weisen Beichnungen. Die Blu= men find entweser pfirfid)bluthfarben mit dut Eelrother Ein= faffung Des Sdylundez, Der wie mit weißßen Perlent geziert ift, doer fie find rein weís. Dod) gibt ez in biefer Fein= ficht einige 2lbainderungen. Sie ried)en zumeilen, jedod)

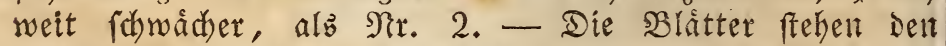

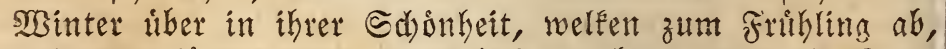

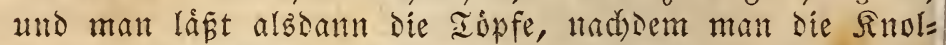
len umgelegt hat, balb trocken an einem yor regen ge=

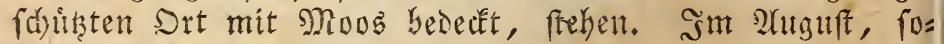

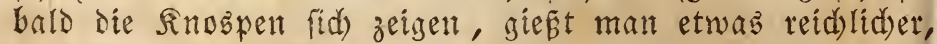
entfernt aud) Die Moosdecfe, und bringt fie Ginter (s)las. (Evft Fummen Die Blumen zum $\mathfrak{B o r f d e i n , ~ u n d ~ m i t ~ D e n ~ l e s s = ~}$ ten zugleid) entwideln fid' aud) Die neten Blátter. Nad, 
sem Blukent ziekent fid) Die Błhmenftanthel (wie bey C. europaeum) fpiralformig zufammen, tho bie ant ifuen fihen= ien Samentapfeln reifen im folgenten Sommer, wákrents

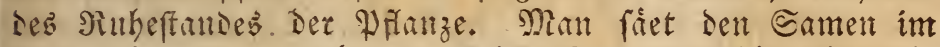

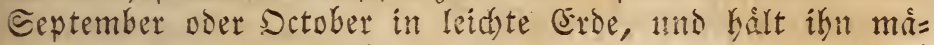

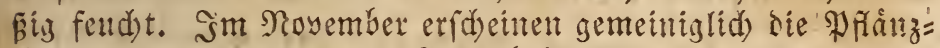
d)en mit einem Blatt. Non behandelt fie mie die alten Pflanzert, legt fie jâhrlid) um, und fie Glithen gemeinigs= lid) in 4. Sabre. Stud) Eann man biefe (Erofdeibe surd)

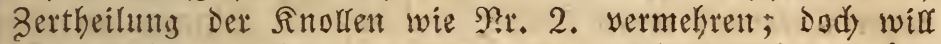

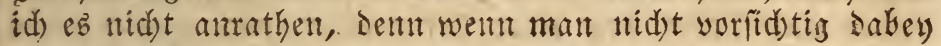
verfährt, fo verliert man zumeilen bie ganze Pflanze. Naant gift diefer Erdidsetbe gute sanberde, und hiberwintert fie ber $0-4$ (sirad; aud fie frált zumeilen im sante aus.

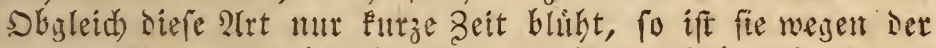

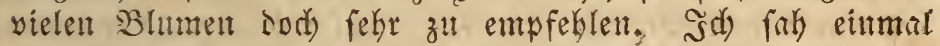

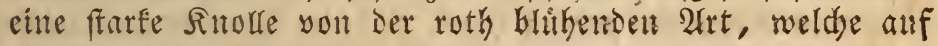
cinmal einige 50 Blument trug.

4) C. persicum, perfifde (E) tof eibe, 4. \$erfien. - Unftreitig sie fdouffe 2trt. Die Fnolle ift eken=

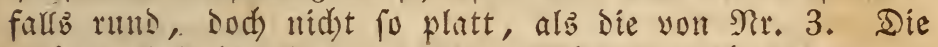
Blitter fitto herzformig, oft irreguliar, unto ebenfatts weís gewolft. Bumeilen werben fie 3-4 3oul lang. Die Blatt= ftiele, fo wie and) bie jungen Blâtter auf der untern @eite, find rothlits. Şom februar ab bis Ende May erfacinen

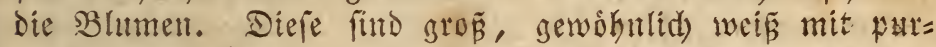
purrotfem Edjlutide, zuweilen gauz weis ntmo mandsmat rofa mit sunfler Énfaffung bes Geldyes. Die Blumen baben einen idwadjen (Siernd), Der aber zuweilent wenig zu bemereent ift. Die Befandtung und Bermekrung ift gantz

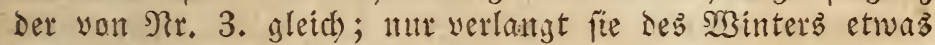

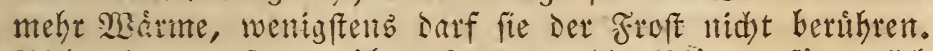
Nidjt immer falfen liber Commer bie Blatter faimmtlidy

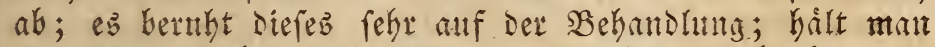
bie शranze liber Sommer etwas fendt, fo bleibent faft immer einige ftehen, und diefe verfdwindent enf im Serbft oder Minter. Diefe Erofdeibe bat Das Eigenthumlidbe, Daw

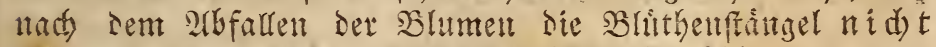

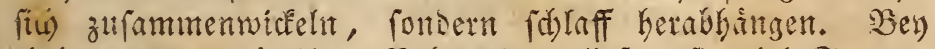
einigermanen gitnfiget Behandhng liefert fie viel Eamen,

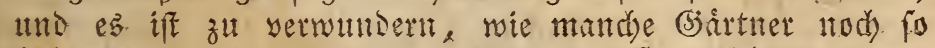

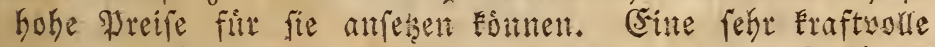

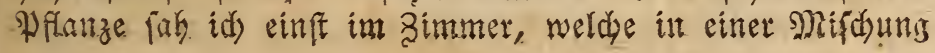




\section{Ant 104 N}

von sauberde und verwittertem Saffeegrunde fand. Diefe batte im saufe yon zwey Nonaten geren 100 ber idjonften Blumen geliefert.

C. aleppicum uno indicum balte idh nur fir Spiels arten Der leb̨tern (Etofdeibe.

$$
\text { Cydonia, Qutite, XII. } 5 .
$$

C. japonica, japanifde $\mathcal{Q u}_{\text {utte, }}$ 2. Sapan. - Bekannt unter Dem Namen Pyrus japonica. Ein Dor= niger @traud), Deffen fenone zimnoberrotbe Blumen im Je= bruar uno Nárz bey eiller Iemperatur yon $6-8$ Girad fid entwicfeln. Hebrigens foll er fehr gut in freyen sante aubgalten, und fann baber bey 0-4 (3rad uberwintert mer= Den. Bermehrung Durd) Murzeliproffen, 2lbenfer und હtect= linge; iedod) habe id) nod) Feine (Erfahrung hieruber, sa id erft feit Furzer zeit ein junges Exemplar befibe. Hebrigens ift diefes Băumden wergen feitrer fhónen Blumen und leid)= ten Befandlung fegr zu empfehlen. (Er fdeint guten $\mathfrak{B D}=$ Den und im હommer einen etwas fichattigen હtand zll lies ben. (E) gibt aud) eine Spielart mit weišen BBhmen.

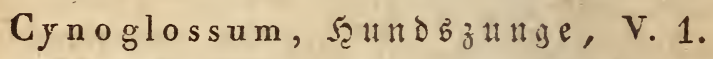

C. omphaloides, Frúbling 2. Sn Portugal auf Bergen. - Siewihnlid beist diefes

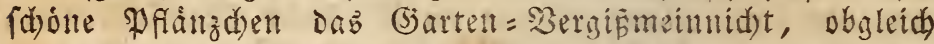
Die Blumen bröper und lebhafter, als die des gemeinen

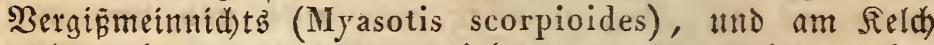
nicht gelb, fondern meís gefárb́t find. Die Blitter Gaben cille eyrunde zugerpiste form. - (Siemoibnlid brantht man

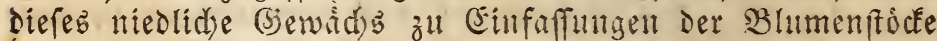
in Eleinen (Sitrten; Dod wiro $e \vec{b}$ wegen feiner friben $B$ lut men, die in eillet aemperatur yon 4-6 (5rad fdon im Márz erfdeinen, fehr bălfing in Iopfen gezogen. Man fann ez, fo wie bey den (5)lowenblumen gefagt ift, behan= Deln, diberwintert jesod) diejenigen Iopfe, weldye man zei= tìg treiben widr, in einem froftfreyen Bebáltniś. Bermebrt fid) Durdy feitle fried)enten Etringel fegr fark.

$$
\text { Cyrilla, eintille, XIV. } 2 .
$$

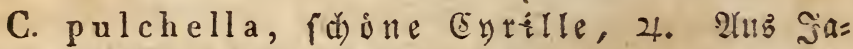
maica. - Ilts der Eleinen fduppigen $\mathfrak{B}_{3}$ urzel sommen fpan= nenlantage tỏthlid)e Stångel Geryor, mit Eleinen gezántent B Blat=

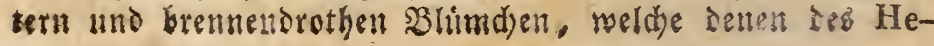




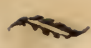

mimeris urticifolia ibntid) fint, und zuweilen bie ganze Pfanze bedecten. Nan befandelt fie ganz wie Begonia discolor, mit weld)er fie int siefer frinfid)t viel 2(ehntlid)=

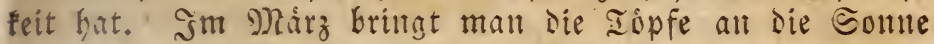
und giest ifnen fparfam, bis die pfandzden hervorfommer, sie Den Eommer thber gern warm uno etwas feldd ftehen.

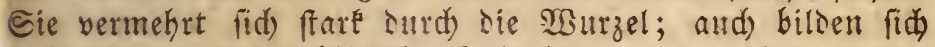
nid)t felten fleine áfulidbe Rnoldden an ien Epişen ber Bnveige, wenn sie Blútbzeit voriber ift. Eine febr zu em= pfeblente Pfinaze.

\section{Daphne, હeibelbaft, VIII. 1.}

1) D. cneorum, bas હteintobden, Ed) weiz und fublide Dentid)lans. - Sin niedriger beynahe liegenter Etrandá, Deffen 3weinge mit (d)malen, länglid)en,

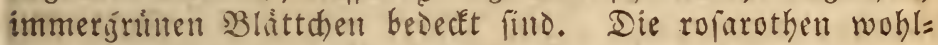
ried)enden Blumen erfdeinen im গxay, zuweilen aud im Serbft in Dolien an Dent Epiţen Der Uefte. Nan gibt siefer fdónen Pflanze nidyt jul id)were sallberse, hiberwill= tert fie bey 2-4 (Grad, und vermehrt fie Durth $2(b$ fenfer oder surd) Sereieln allf D. laureola. - (s's for aud) eine meisblibence Epielart geben. Sie will, wab пав Begies Ben anbelangt, yoriditig behanselt fenn, und fdeint gern etwab idattig, Dod) lufting ftehen zul wollen.

2) D. odora, woblriedender Eeidelbaft, Sapan. - Diejer Eeidelbaft miro ziemlid) hod, uno blitht feltell eher, alö bis er $2-3$ Fus Şóke erreid)t hat. Die bellgrimen lecerartigen Blátter find breit=lanzettformig, ftehen in ziemlid) bidsten Buifdeln an Den Zweigen. Șm

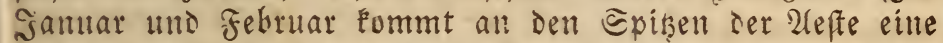
ziemlid) reidse Dolde weifer, fehr angenthm ried)ender Blu=

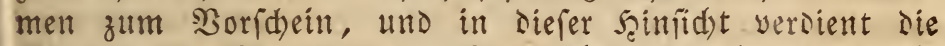
Wfanze alfe Empfehlung. Nan gibt ifr nahrbafte saub= erde, máfig Feudutigkeit und debs Eommers nidyt gar zu viel Eolune; Dagegen ftellt man fie im SBinter nahe ans Fenfter, no in einer Iemperatur volt 4 (S)rad fid die Blu= men fehr leid)t entfalten. Die Bermefrung gefdicht ent= weder Iutd) Eteclinge (weld)e man beynabe za jeder Sab= reszzet mad)t, und nidjt zu feud)t halt; fie traulern oft lange, nefmen aber bod am Ënoe an), 2tbienter, ober Durd) Bereicln, indem man einen Sweig. an ein Stảmm=

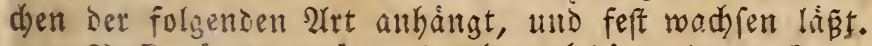
3) D. laureola, lotberblattriger Setoel= 


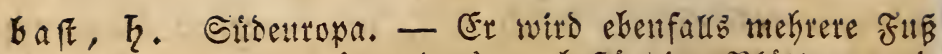
God), Gat lange, glânzendgutute, beftånoige Blåtter, zwi= fden benen in Frubling bie Eleituen grtultiden Blumen er= fdeinen, welde fdwarze Beeren Ginterlaffen. Diefelben

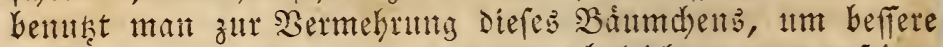
Ortent Darauf veredeln zu foumen, obgleids er wegen feinter fdidnen Blatter, und weil er aud) im freyen sante atts= Gait, oft gezogen wird. NRan gibt ihm lehmigen Boden,

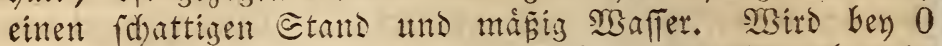
-4 Sirad iberwintert. - Sid) babe diefen Seitelbaft in einem (5artent immer Eaffano nentent borent of er wohl itt

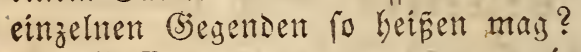

4) D. mezereum, gemeiner Seiselbaft, $\{$.

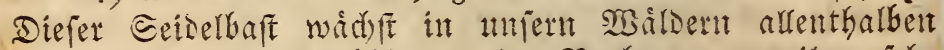
zerftrent, in in sanbGolzern Der Borberge zumeilen Fefr Gánfio, mird jesod felten Goker, als $2-3$ Jin. Die lanzettformigen mattgrituen Blatter fallent in $\mathfrak{S e r b f t} a \mathfrak{b}$,

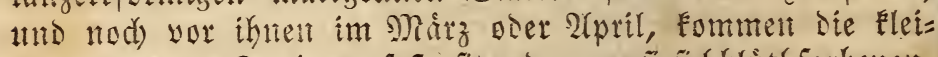

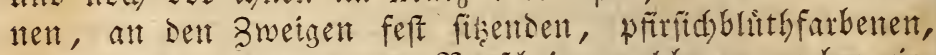

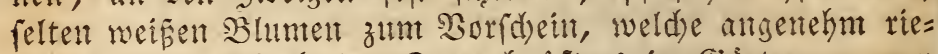

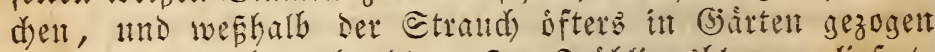
wiro, wo er bennabe die erfen frublingshlumen liefert.

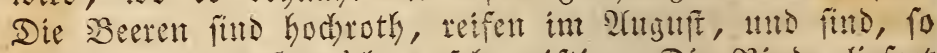
wie Das ganze (sownads, fehr giftis. Die Rainde liefert

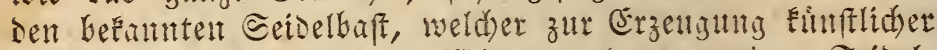

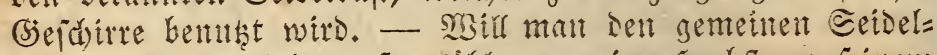
baft im Iopfe ziehen, fo withle man im Serbft an feittent naturliden Etandort eine ntát tiber einen Fus Gobe Pfant= ze, Gebe fie mit Dem Ballen alls, mno pflanze fie itt einent

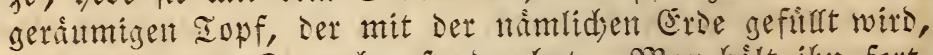
worin man Den Etrand) gefunden Gat. Man bitlt ifn fort=

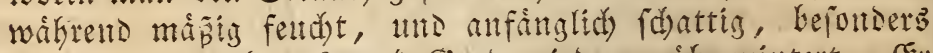
in Eommer; bey $0-4$ Girad witro er ifberwintert. Er

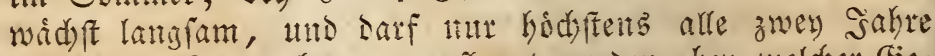

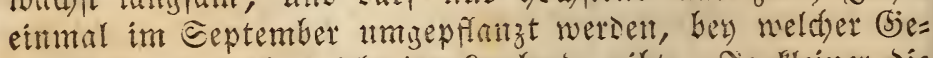
legentheit mant inm lebmige sauberde gibt. Se fleiner bie Pfanzen find, um fo leidter gewobnent fie fid ant bent Iopf, und da fie fason bey 6 zoll sibe blitgen, fo fithrt man am fiderfen, Derigleiden zu wáblen; sem ber gro=

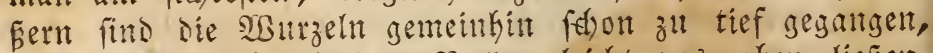

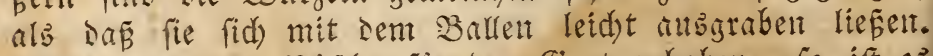

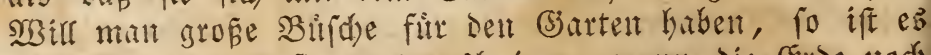
gut, mit Baffen fie zut tratsloctren, wetn die (Evbs nod) feft gefroren iff. 


\section{Datura, Etedapfer, VIII. 1.}

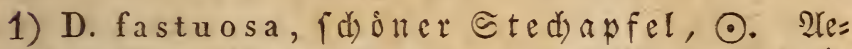
gypten. - Nan fiket Den Eamen in einen Rapf mit leid)= mes Niffteet, ooer sod binter ein fehr founiges Genfer,

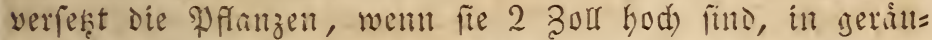
mige Iopfe mit Sanberse gefirtt, unter welde man etwa $\frac{\pi}{3}$ gut gefanlte und gefiebte Miftbeeterse uno etwas Eano

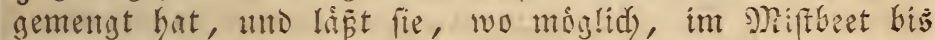
Nitte Sumy ftehen, woralif man biefen Etediafel an bie Suft gewojhnt. Die grosen wohlried)enten Blumen fint gelblid)weís, juweilen violett, einfach, and) gefullt. Nian gebe ifm reidlidy Maffer. BOon Den im Iopf gejagenen Pflatzen babe id) felten reifen Eamen erbalten, benn ent= weder fehlte eb ifhen an Nabrumb, oser die Bhlumen er= fdienen zu fpat. Beffer gludete es, wenn idy einize Pflanzen in ein mit hohem Gaften veriehenes shiftbeet pflangte, you Dem, fo wie die Eptsen Ierfelben die Genfter berifrten, letitere absenommen whrsen. Diefe Pfanzen wursen Dann

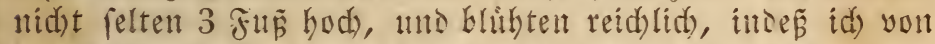
Denten im Iopf, Die hod)fents $12-15$ zold sobe erreid)= ten, felten mehr, als $2-4$ Bhlumen erhielt.

2) I. suaveolens, wohlriedender Sted)= apfel, 7. Merico. - Bernabe allyemein fübrt Diefer Eted)apfel Den Plamen D. arborea; Der wirkliche baumar= tige Eted)apfel, welder in \$eru fid findet, foll jerody you Dem unfern fefr unterfdieden fenn; id) fente ifn mur aub

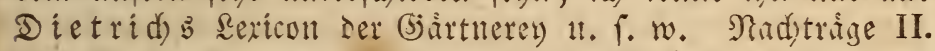
Diefer Baum mit langen, ziemlid) breiten, glatten,

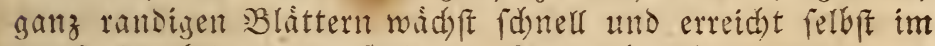
Iopf eille bedeutenie Giróse. Mian gibt ím guten, mit sefm gemifdten Boden, und währeno des Sommers viel Waffer. 2luf ein Blumenbret pä́t Diefer @ted)apfel feinter (5)rỏe

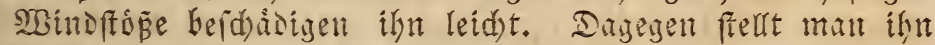

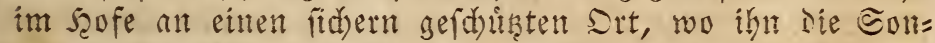
the yon des Morgens bis gegen Mittag trifit, oder nod beffer, man gräbt ifn bis an Den giand Deś Iopfeș in Die Erde ein. Die Blumen etfdectnen im 5etbft, gewöhn= lich) yom 2luguft ab, find 8-10 zod lang, trid)terformis,

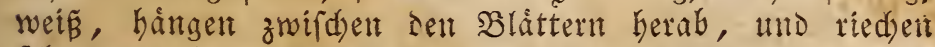

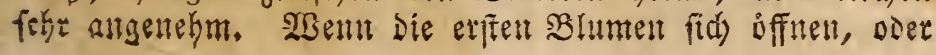


Fuble Nåd) zu furdyten find, weldye den Fnospen fdaden fonnten, fo ftellt man den Baum ins Bimmer, nidst weit vom Fenfter, gibt ihm jedod) Den Iag liber reidlid suft. Nad) Dem Blủhen fey man mit dem (siepenen vorfiditig, uno gebe diefer Pflanze nur fo viel SBaffer, daj die Rinde nidjt welf miro. Die Blatter falfen alşdann zwar gróstentheils

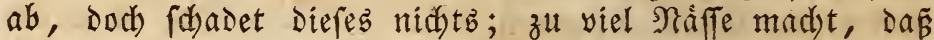
Die SBurzeln faulen. Froft vertrågt fie gar utidt, aber zu grose Warme ift ihr ebenfalls nad)theilig; 4-6 (sirad find Ginretident, fie Durdzubringen, und Fann mant ihr ofters Somie geben, fo ift biefes fehr wohlthuend fitr fie. Sm Fruhjafr vertringt fie aber eine weit hohere Iemperatur, - bib man fie im Nay an bie suft getwohnen Eant. Durd) Stectinge, die man zeitig im Frifling mad)t, if Der baum= artige Stedhapfel fehr leid)t zu vermebren, befonders, wenn man fie ins Niftbeet ftellen mo antreiben fann. Da es jesoch gemeiniglid) an kleinen 2 eften fehlt, die man zu Diefem Brod benusen finnte, fo fdneidet man einen ftar= Fen aff in fingerblange Stuteren, fo, Dás an jedem $2-3$ 2lugen fid) befinden. Sie nehmen (d) nelf an, wadjen ges fdwind, unt will man bie jungen Pflanzen baumartig zies ken, fo werien bie Nebenidsoflinge, robald fie eintige Solf lang find, ebenfalls abgenommen uno als Stedlinge vet: wendet. um Den Trieb Der jungen Baiume zu.befördern, pflanzt man fie Ende May auf ein Beet mit leidyter nabr= hafter Erde, und giefst bey beisen Iagen ihnen fleis̄ig. Sie werden bis zum herbft $1-1 \frac{1}{2} \mathfrak{F} u \tilde{5}$ hod) gerworden

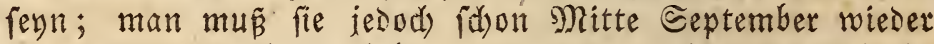
als Dem sande heraubkeben, Damit fie nod einige Beit in Der suft freben, und in den Topfen einwurzeln Eonnen. Sim folgenden Sahre behandelt man fie wieder fo, wo fie bann 3 Fun und Gober werden, aud wohl fdon eillzelne Blumen bringen. Sim britten Frihling pflanzt ntan fie in einen geraumigen Iopf, uno behandelt fie, wie oben gefagt rurde, denn nun eignen fie fid am beften zum Stuben= flor; hingegen werben fie im niddfen Sahte, wenn man fie nid)t eimftust, zu bod, und fürs Fenfter tnpaffend, wiemohl fie, went man fie in Rubel fetien Eann, rebr. reidlid) blithen, und aud albdann eit Bimnter fdmilden.

Delphinium, Ritterfporn, XIII. 3.

D. grandiflorum, gro

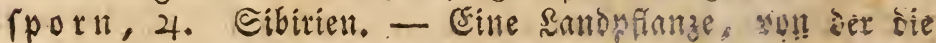


gefurlt blikende Epielart eine wathe Bierde ber Şäten ift, indem Der $2-4$ Fü bohe હtăngel im Sunillz mit gro=

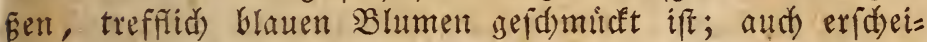
nen fpoter gemeiniglid) fleine' Etängel um den grofien, wel= d)e bis zum September Blumen tragen. - Sad) babe eb erft eimmal verfucht, diefe fdome Pflanze im Iopf zum

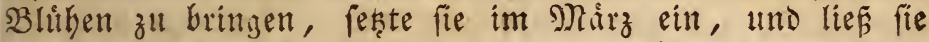
wor dem fenfer in der suft ftehen. Dod) wurbe ser slu= menftingel unt etwa 18 zoll bod), trug zwar viele gut aubgebiliete Blumen, jedod) Dauterten fie faum 6 Iage, Dann fielen fie alle zugleidy ab. Da diefe Pflanze febr ftar wurzelt, fo war ser Iopf, in Dem fie fand, zull Pleit geweren, und ibersem batte ich fie erft im Frubling von einer ältern Pflanze mit Gienalt abgenommen. Seitbem

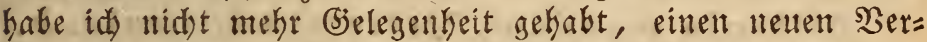
(udi) zu madien. Neines Dafurbaltens wäre es wohl am beften, fie zll bebandeln, wie ber Den (SIlocfenblumen ge= fagt worden ift. Diefer Ritterporn liebt nabrhafte, etmab lefmige (erde, und lifift fid) febr leid)t intd) Butheilumis vermebren, befonders, wenn man eine Stande $2-3$ Jabre ungeftort bat int rande fteben laffen, und im Frubling umfekt. Seder Irieb, Der einige Murzeln bat, faum $a b=$ genommen, ia fogar die altell Etangel, an benen fid 2 flu= gen zeigell, der sảnge nad) gefpalten werden.

Di anthus, Nelé, X. 2.

D. japonicus, japanifde Nelfe, 24. Sapan? Eille niesrige Pflanze, weld)e in affen Ibeilen mit der

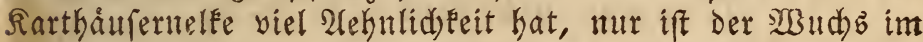
allgemeinen mebr zufammengedraint. Der Blumenfdaft ift nut roenige 3oll hody, und trágt eine grofe Dolde you fidonen carminrotben $\mathfrak{B l u m e n .}$ - Diefe Pflanze ift leidht zll bebandelu. Man gibt ibr Eråftige, leid)te (5rde, bált fie etwas feudst und im Winter bey 4-6 (sirad 9 Barme bid)t am Fenfer; fie vertragt and einen leidten Froft. sim Eommer gift man ifr eillen Stand im Frenen. Eefr leid)t läst fid) Diefe Nelfe Durd) abgebrodune Eeitentproffen, rie fie in Menge treibt, wermefren, yon denen faft ein

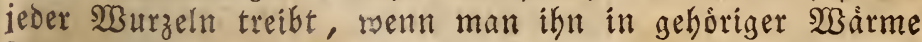
Infangs fdattig und feucht bâlt. - NBegen Deb niesrigen Wudjes iff fie eine fdo one Iopfpflanze. Die grumen Blatt= laufe niften fid aber leid)t zwifden sen Did)tftebenden Blát= tern ein, und múffen fleifig abgefud)t und durd) Iabacts: rauds betrieben werben. 
Digitalis, Fingerfut, XIV. 2.

1) D. Iutea, ber gerbe fingerfit, 24, mits

2) D. purpurea, Der rothe fingerhut, 24,

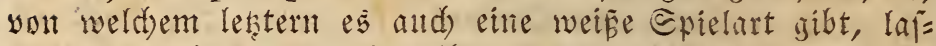
fen fidi) leidst im Iopf treiben, wenn man fie im Mădz mit Balfen ats sem sande bebt und in samberse pflanzt, weld)e ffart mit sebm the Fiesfand gemengt ift, oser mant

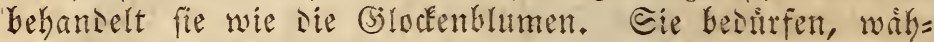

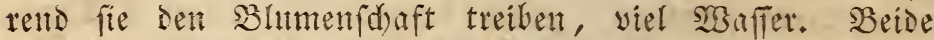
Trten finset man in Berggesenten in Dentfidand jutwei= lelt batufig will madben.

\section{Dillenia, Dillenia, XIII. 6 .}

Dillenia scandens, 任tternde Dillenta,

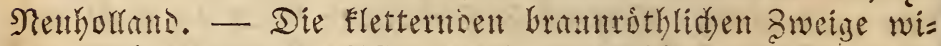
ffeIn fid) ant antern Pflanzen in bie scobe, who find ab=

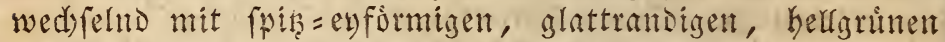

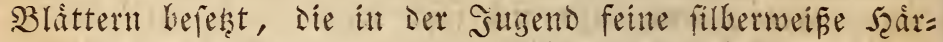
dyen trajen, weldye man jecod forater wenis bentert. Die Blumen fitien auf fehr Eutzen Stielen in dent Blattwinkeln,

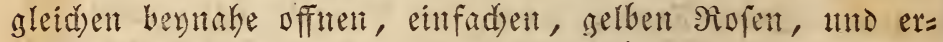
fideinen einzeln ien ganzen Eommer itber. - Die Dillente

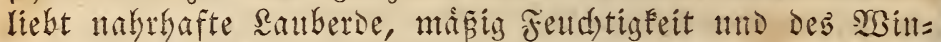

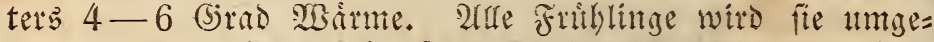

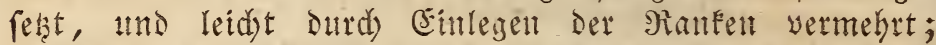
Etedinge nehnen ald

$$
\text { Diosma, (jotterduft, V. } 1 \text {. }
$$

Bon Den Diosmen gitbe es fefre viele 2irten, weld)e

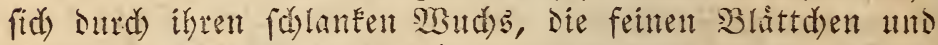

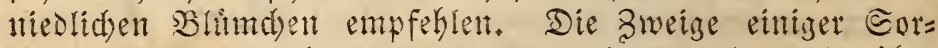
ten Duften angenten, went man mit Der Sand Darber fâkrt. - Sidh babe bisher nut folgente atrt gezogen:

D. alba, weifer (S) itterduft,

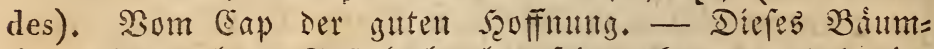
d)en wird mehrere Fur hod), hat feitte, furze, nadelartige Blatter yon eittem angentehmen harzigen (S)erud). Die flei=

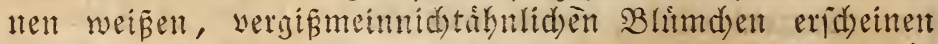

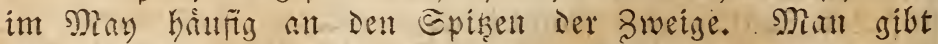

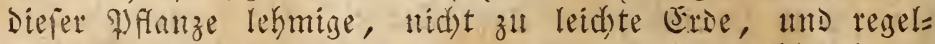
mâfin SGaffer. Sie wutzelt ftare, uno wird iâhrlid im

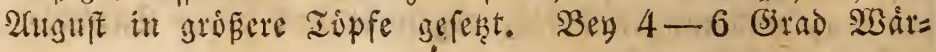


me bált fie fehr gut alts, went fie nut nid)t zut weit yom

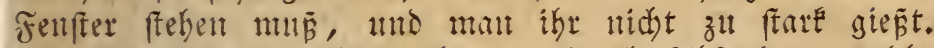

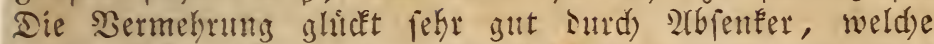
malt you jungen aweigen im Frifyling mad)t, mo nid)t felten fdon beym nidyjen llmiesen abnehmen fün, fonft

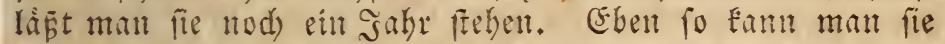
Durd) Etecelinge ziehent. Diefe mad)t man im Nany oder Stury you $1 \frac{7}{2}$ Zoll langen Sweigen, frect Deren $4-6$ it

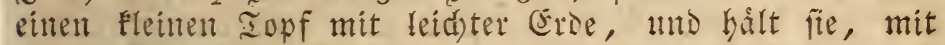

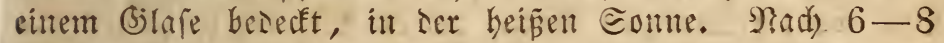
MJodjen bat es fidd int ber Regel entichieden, weldye eintges gangen find oder wadjen wersen.

$21 \mathrm{mer}$. Es gibt anter Den dibrigen Diosmen nod) einige fefr idone 2(rtent, weldie id) jesod) nidit ge=

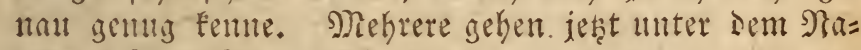
metr Adenandra; z. $\mathfrak{B}$.

Diosma uniflora Geift jeşt Adenandra uniflora,

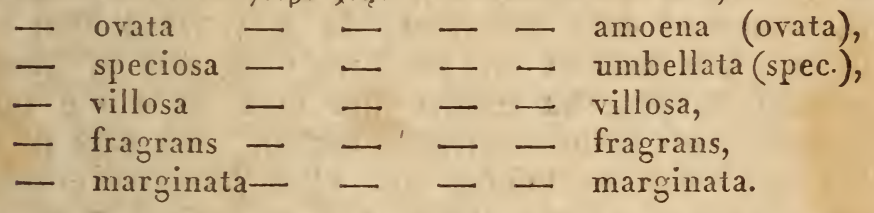

Dodecatheon, sjotterblume, V. 1.

D. meadia, vitginifde (5) otterblume, 2\%.

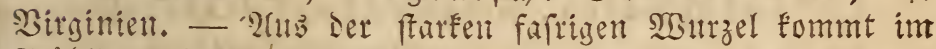
Frithling eill Bufdyel Blatter zum Borfdein, weldye viel

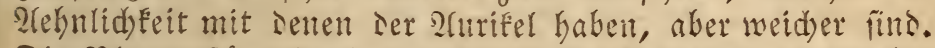
Die Blumenftangel jeigen fid́) mit Ien Blättern zlt gleid)er Beit, werden S-12 3our hoch, uno tragen oben einen Bufd)

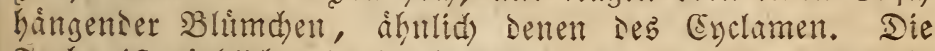

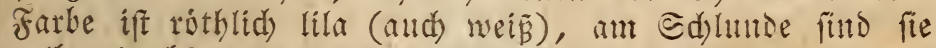
gelb mit bramten Flectent. Diefe niedlide Pflanze bedarf wenig Nfege. Man gibe ifr tiefe Iopfe mit leidster, et= was fansiger sauberse, uno bålt fie itber $\mathfrak{S i n t e r}$ bey $0-4$ (5irad, Damit fie nid)t zal fribh treibt. Ulm Diefe Zeit be=

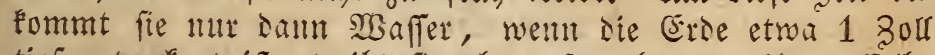
tief getrochet iff; treibt fie aber, fo gibt man ifr Daffelbe reiddlid)er. Die Blätter sattern nidbt lange; fie welfen ge= wobnlid) bald nad) Der $\mathfrak{B l i t h e}$ ab, und mun felft man die Nápfe in Der freyen suft an einen yor Nezen gefduhtent Srt, und gieft ebenfars nur io viel, Daś Die Pflanzen

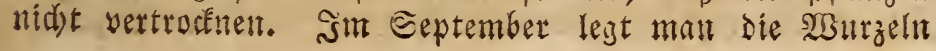


Der (B)ötterblume in frifthe Eroe, bey weldjer Bselegenteit man bie einzelnen Feime zu trenten find, indem gewohn=

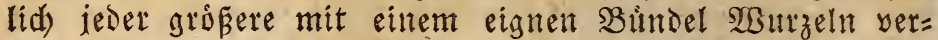
reben ift. 2aid Samen befommt man zumeilen. Die (5sot: terbhtume bålt auferdem auds fehr gut im fresen sande

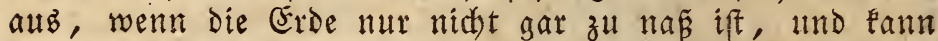
im Fribling zu einer befondern Sierde Fleiner (Jåartchen bienen, wo fie mit 2 turifeln, primeln u. f. w. zu glei=

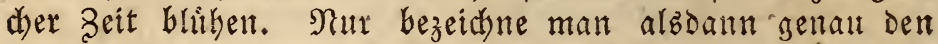

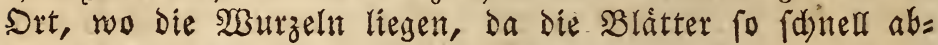
melken.

\section{Dolichos, Bofne, XVII. 4.}

D. lignosus, holzartige Bobne, h. Sftin= dien. - Die fdwadhen Stängel tragen Fleitte Bláttden, und winden fid) in die scoke. Die Dunkeltofarothen Bhlt= men ftehen in $B$ úfheln und fehen red)t hilbid) aus. Samen babe ide tod nidst befommen, fie trágt jesod) eben nidbt felten. Sie verlangt gute nabrbafte Eros, und im Sommer reidlid) WBaffer; im SBinter gibt man ibr 4-6 (3)rad. Sabrlid) wird fie im September verfest. Sid) habe fie bibs

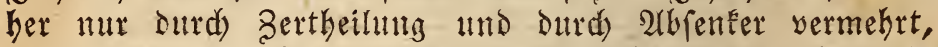

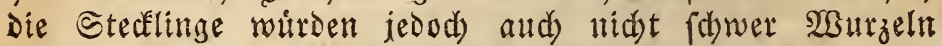
madyen.

Dracocephalus, Dradentopf, XIV. 1.

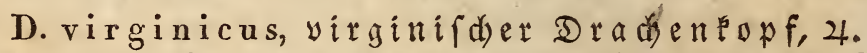
Birginien. - (Eille sandpflanze, bie jedod) aud) im Iopf

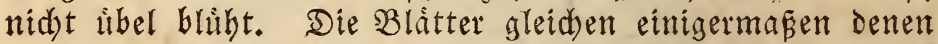
der Campanula persicifol, mit weldber iberbautpt die ganze Behandlung ábereinftimmt. Der Blumenthaft wird int Iopf 3 Fus hod), theilt fid in mehrere aufredst ftehende Rebenzmeige, uno tright yom sulty ab eitte Menge rad)en= formiger, róthlidser Blumen.

A $\mathfrak{n} \mathfrak{m} \mathfrak{i}$. D. canariense, mit ftare nad) Fampher rie= d)enden Blåttern uno rỏthlidben Blumen, verdent nad̆ meiner Neinung Éeine Etelfe im Bimmer.

D. moldavicus, eine Eommerpflanze, bat blau= violette Blumen.

\section{Ericà, Heide, VIII. 1.}

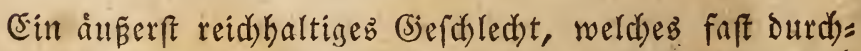

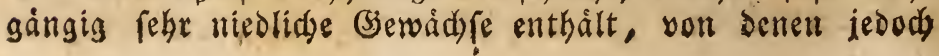


Die meiften nod) immer in jiemlid) Gohen Preifen ftehen. So fehr bicie \$flatzen affigentein gefallen, fo wentig eignen

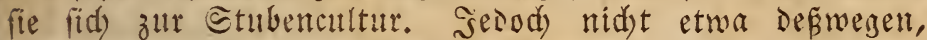

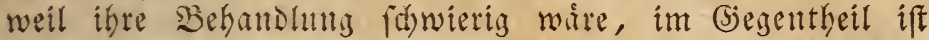

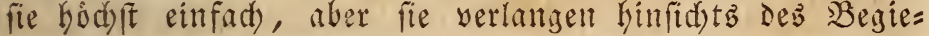
Ëens und Der Iemperatur gewiffe 2lufmertiamfeiten, die man ishent nicht immer wiomen fann, zunt wenigftent ber=

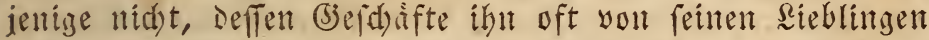
entfernen. Die Seiden wollen furtwáhreno einen gleidhma: Ëigen (5)rad yon Fendligkeit baben; gibt man ihnen des

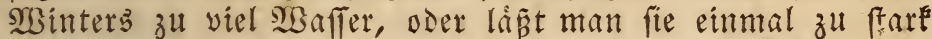

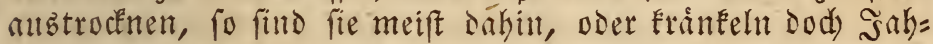
re lang, tuto bringen weder Blumen, nod) erlangent fie

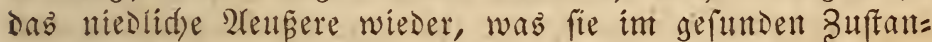
De hattel. Stuberwatme und Staub vertragen fie ganz ullo gar nidst, uno eben fo iff den meiffen 2atten Der Froft

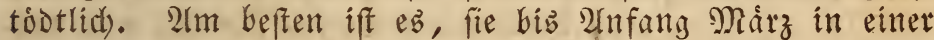
Ienteratur you 2-4 (5jad zu erbalten. 3. $\mathfrak{B}$. zwifhen Doppelfenfern, weld)e, went es zul kalt zul werden drobt, eint wentig geoffnet werden. Sour babe man alsoann ia

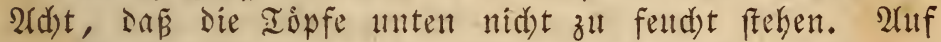
Den Fenftern vertragen bie feisen die zh heftige Conne niăbt; Dent ba bie suft ohnehin in Der Regel Deb હom= mers frart zehrt, fo whirde die Eonne die feinen Whurzeln leid)t ganzlid) austrocknen. Darum ffelfe man fie nur an ein Fenfter, wo fie hodjftenz bis 10 uhr Eonne befonmen. Befinden fid icdod) im frithling die Sreiden nod) Ginter (5las, und find fie_zegen ftarlen suftzug gefdubt, dann Fann man ihnen die volfe Gonne geben, die ihnen des SBin= terz fehr bienlid) ift, indem fie bie Erse allf les Dberflád)e trockitet, mo Den fo nad)theiligen Edjimmel zerfort. -

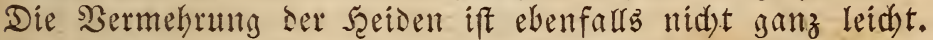

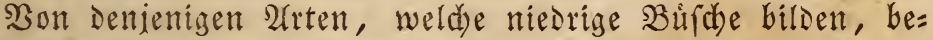
feftigi man junge Sweige im 2(pril ober May mit Shákden 1 zoll tief in bie Eride. Einige arten wurzeln fechon biz

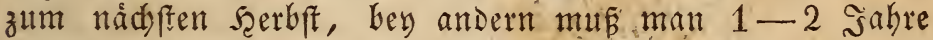
warten, ehe liefes gefdieht. SGhe man die 2rbfenfer log = (d) neidet, unterfudse man $4-6$ rSodsen vorber fie vorfid)= tig, wie es mit. Dem Berwulzeln fteht, uno nehme fie dann

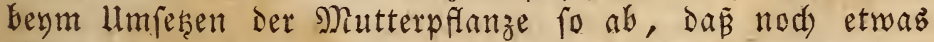
Eride an ben 3meigen bången bleibt. Die jungen Pflállz= d)en bringe man anfánglidb weder in ein Sraub, nod) in Die Conte, fondern laffe fie etma 14 Iage lang int 


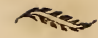

Edhatten fteken, bis fie suff zum SWadjen zeisen. Die

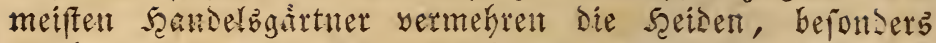
Die baumartigen Eorten, Intud) Etedtinge. Şd) babe biz

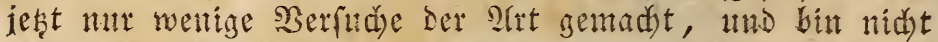

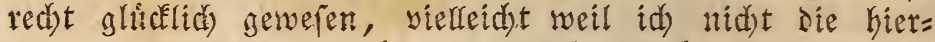

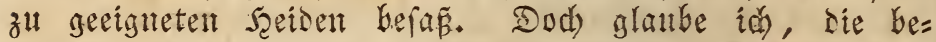
fte Beit, fie zu machen, iff Irnfang Suny, roent bie jun=

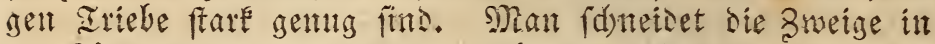

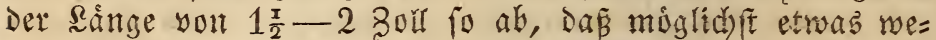
niges volt dem åltern $\mathfrak{h o l z e}$ saran ftehen bleibt, ftecke fie in Eleine Iopfe mit leidster Erode, etwa $\frac{3}{4}-1$ zold tief, bebecke fie mit einem geràumigen Silare, bringe fie in bie

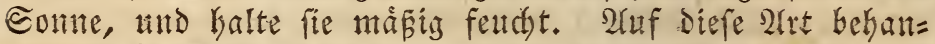
Delt, babe idf bereits yon einigett, z. 2 . E. gracilis und phylicoides, Bermerarung erbalten. - Samen befommt

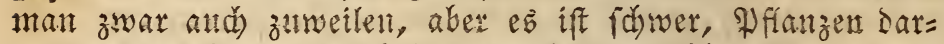
als zu erzieben; man behantelt ifh ungefähr wie delt vout Sen Melaleuta $=$ Plten uni Rlododendron.

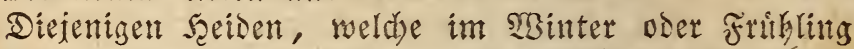
Gliben, wersen im 2huguf ober September, Die andern aber im 2rpril oser Man jâfrlid untgepfanzt. - Die Er:

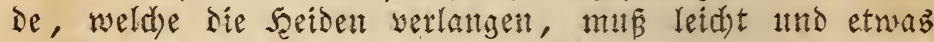

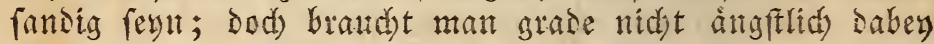

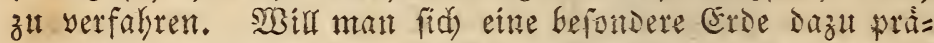
partien, fo fant man folgende Nengung anwenden: 2 Theile sauberse, 1 Iheil yerwitterter sehm, 2 Iheile ver= witterter Iorf und $1-2$ Theile feiner weiner Sallo. 2lls

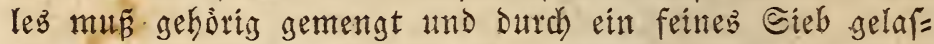

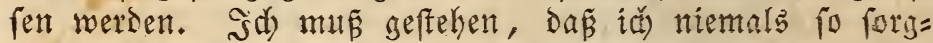

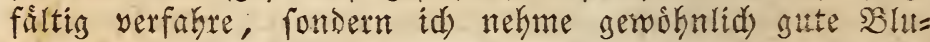
menerde, yerfesce siefe, wenn es nothwendis ift, mit Sans, und babe tife gefunden, Das meine Setsen sadurd gelittent baben. Nur vermeide id) Den gelben eifentaltigen Sant, Der alfen Pflanzen nadjtheilig ift. Die meiften ftamment vom cap. Shut wenige fino in Guropa zu saute, und viele yon diefen vertragent fogar froft ntho fonten im san= be gehalten werden. 3. B. E. carnea, cinerea, multiflora, stricta, tetralix $\mathfrak{u}$. a. - E. carnea (E. herbacea) if eine yon denjentigen Sreiden, weld)e-am meiften zu em= pfehlent fins. Sie wird felten ulber $\frac{\pi}{2}$ gun horh, dent sie feimen smeige neigen fid nady ber eride, babent sumelgrit= ne glänzente Nabeln, tho trajen an ben Episen ge= Drangte rofarothe Blumblen mit fdumarzen Staubfáden. 
Die Snospen ertheinen zwar fhon in September, alletit bie Blument entwickeln fid erfi im Februar oder Mảrz.

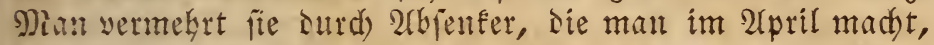
und im scerbft abnimmt; febr leidst bey 2-4 (jorad if fie gut Durd)zubringent.

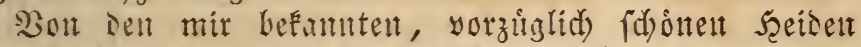
nenne id) folgente: E. aitonia, cerinthoides, conspicua, grandiflera, jasminiflora, mammosa, margaritacea, speciosa, taxifolia, translucens, tubiflora, ventricosa, verticillata, vestita. $\mathfrak{X} \mathfrak{n}$ mehreren Diefer Sorten gibt $\mathfrak{e z}$ Enietarten. - Erica vulgaris, f. Calluna.

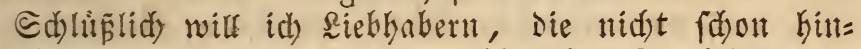
länglid) vertraut mit Behandung ăhnlid)er (Serwád) fe find,

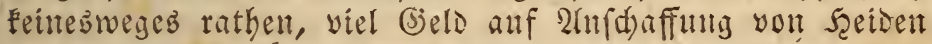
zu verwenten, bent fie whroen ifhen in ber Regel mehr Serdrus, als Freude madjen, indem eill eillziger Iag oft eine mehriábrige Erwatumg yernidutet.

Eucomis, 厄dopf= Syazithe, VI. 1.

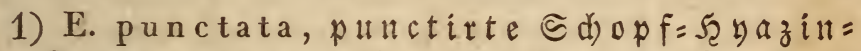

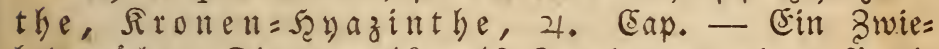
belgewid s. Die etra $10-12$ zoul langen, rinnenfồrmi= gen Blätter find auf Der untern Eeite braun punctirt, Deß $=$ gleid)en ber $1 \frac{1}{2}$ Fus bohe Etångel, weldyer mit fehr vielen Eleinen grinlid)weifen Blumen befest ift, Die lange Dautern, uno mit ser zeit immer Dunfler werden. Rad) obenzu ver= lieren fich Die Brumen, und ftatt ihrer fteht eine frone Fur= zer Blatter Dafelbft. Naul gibt Der-3wiebel, welde man elttweder im Seerbit oder im Fribling umlegt, nidat zul en= ge Iopfe uno fdweren Bosen; Des 2 Binters ftell man fie ber 2-4 (5rad in iriend einen 2 Sinkel, oder in einent troctuen, froffreyent Seffer. Cie vermegrt fid fehr leidst ourd) Nebenzwiebelt, und bliht yom Suly bis in den Ferbft. Die leid)te (Erziehuth, Die eigenthümlidye Form, und Die lange Bluthjeit geben Diefem (jerwadjs einigen Mißerth.

2) E. regia, fdidne 巨d) opf = Fुy

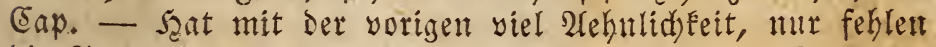
bie Puncte, Into sie Blumen find grunlidjer. Sie wird eben fo wie jene behandelt, ift aber weniger zu empfehlen.

Eugenia, Erigente, XII. 1.

E. a ustralis, fiblid)e Eugenie, দ. - Ein 


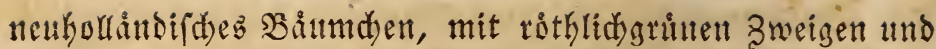
Blättern, weldhe Der gemeinen breitbláttrigen Minte ábns lith) fint. 2lud die $\mathfrak{B l u m e n ,}$ weld)e zu 2-4 auf einem Eurzen Etangel frehen, gleidyen Denen der Minte, und die anf fie folgenden tothbratmen Fridyte Denen Des Mespilus

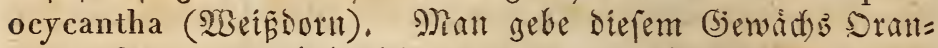
gerte= Erse, mo behandle es ganz wie Marte; Dod) vers

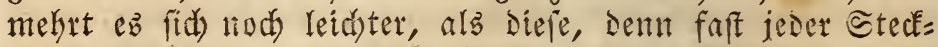
ling nimmt an, wenn man ifn im sinn mad), uno einige

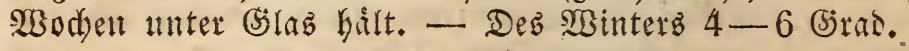

\section{Euphorbia, $\mathscr{B}$ olf şmild, XI. 3.}

Unter ben vielen Solfämildarten, weldye in grósern (5)âten gezogen werden, gibt ez nur fefre wentige, weld)e

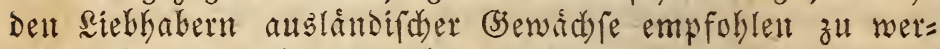
ben verdienen; id nente bier mur:

E. capuit medusae,

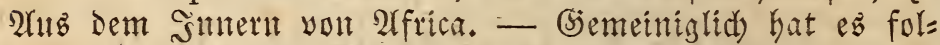
gente (Seftalt: Der Stamm wird nut wenige Boll hod), bildet aber mit zutnebmenden Sabren einen dicfen plattrun=

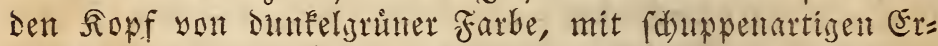
bohungen verfehen. Sin ser Mitte freht allf jeder Edup=

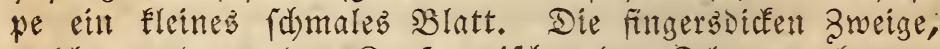
weldye runo um Den Fopf zwifden Den Edyppen Kervor=

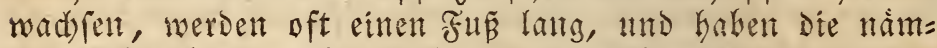

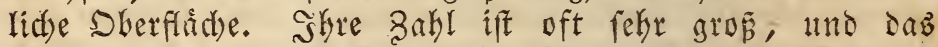
(Sanze bat eine entfernte 2rehnlid) keit mit sem fallangen= Grarigen Fopf Der aus Der Nythologie befanten Medule;

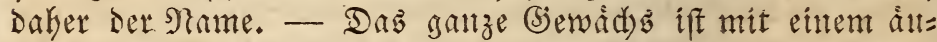
Eerft fdarfen, Garzigen Nildy faft angefurt. Die tleinent

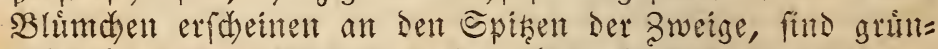
lidgelb, uno riedsen angenterm, bennake wie- Refeda.

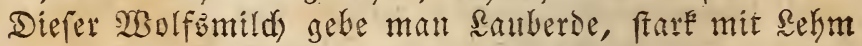
und grobem Cano gemengt, und lege alf sen Boden bes Iopfes einen Finger hod) Eleine Steine, Damit Die úberflif: fige Fendhtigfeit leid)ter abzieken foume, weldye fie, fo wie

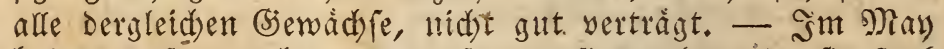
befommt fie, nadjoem man fie verpflanzt bat, da fie fare

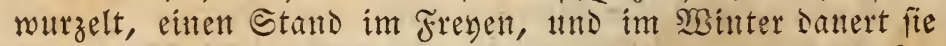
bey 4-6 (sirad fehr gut, wenn man fie etwas trockent

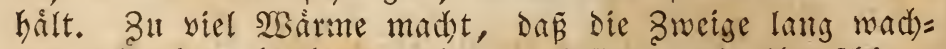
fen und bunn bleiben, Daker die Pflanze oft ífe fdjonez 2rifeken verliert. 


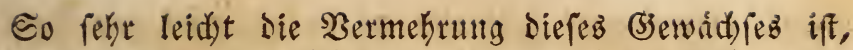

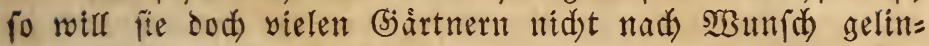
gett. Sdi) verfahre auf folgente 2rt. Sm May oser șitt= Ity) (d) neide id) einen gefunden sweig etwa 6 soll lang ab, trudde ifll gleid) in feintes Foflentulver, beftrene and) bie SWunce am Nutterftod Damit, weil beise fonft zu fat bluten. Diefer 3wein bleibt an einem trodnen fdattigen Drte 14 Iage bis 4 SBodsen lang liegen, wiro Dann in einen Fleinen Iopf mit magerer Eroe gepflanzt, tht in bie freye suft geffelt; $\mathfrak{S B}_{\mathrm{B}}$ ffer befommt er, fo oft bie Erde trocken ift. Ment fid) an Der Epise Dez Brweigez junge Blatter zeigen, fo hat er angenommen. - 2fuf biefe SiBeife erbalt man jesod) nur eitte berwurzelte Sdylange, weldhe in Der Regel erit nad mebrern Sabren einen Sopf bekommt, welden Der (d)wadhe, lantge @tamm nid)t zu ertragen ver: mag, fontern zufammenfint. Um nut eher zut meinem Swed zu gelangen, fdultoe id) Den bewurzelten Stecting in Den lehten Sommermonaten etwa zwer Zoll bod liber

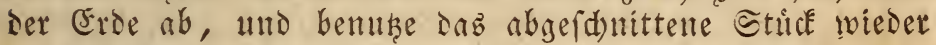

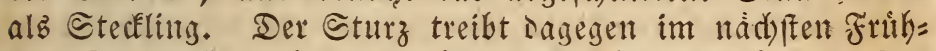
ling (oft aud hod) im Serbft) Geitenafite, bie bey $1 \frac{x}{2}$ Zolf sange fdon Ropfdyen anfeken, fobald fie fark gentug find, abgefdutten, und, wie oben gefagt, eingepflangt werden fóntuten.

Şd) will zugeben, Dá Dieje Methode etwas langmei= lig ift; bod) fente id) feine beffere, fidjerer zum Biele zut gelangen, und, da bie Blumenzud)t im Simmer bod) meis fentheils mur Epielerey ift, fo gerwágrt die Bermehrung defeses (sewrid) fes lange Beit unterhaltung.

Samen babe id nie erfalten; bat man jesod) erft eint=

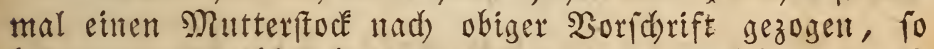

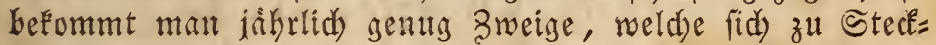
lingen eignen, und defe wadjen fditell, wenn man ifhen nid)t zu enge Iopfe gibt.

Ficus, $\mathfrak{F} e \mathfrak{i g e n t} \mathfrak{b} \mathfrak{u}$, XXIII. 2.

F. carica, gemeiner Feigenbaum, $\zeta$. Sm fitoliden Europa, affien und 2lfrica. - 8u beérnnt, als

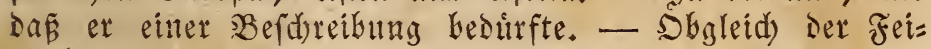

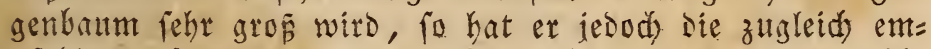

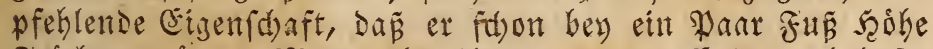
Frid) te tragt. Nan gibt ihm zu Dem Einde nahrbafte

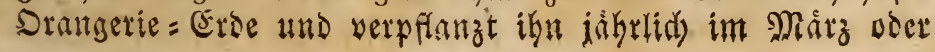




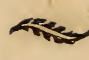

2Tpril. Er bebarf wåhrend feineb WBadjathumb feht viel SBafter, befonsers zat der zeit, wo die Frid)te reifen; aud) ift es gut, sie Blátter zumeilen, fo lanje der Baum ill Der હtube fteht, mit laumarmem $\mathfrak{B a f f e r}$ zu befprengen oder ifn einem milloen Regen ausujeşen. - Dell Eom= mer úber bringt mant Den Feigenbaum an einen Plaţ in

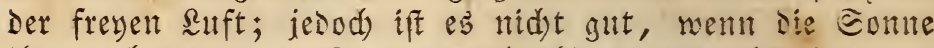

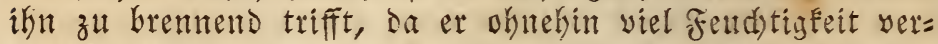
jehrt, uns sie Gridhte nid)t feltent alsoant abgeworfent wers Den. Des Minters überwintert man ifn bey $0-2$ (s)rad

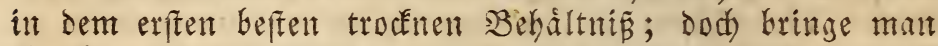
ihn bey gelinder SMitterung oft an bie frene Ruft, Damit

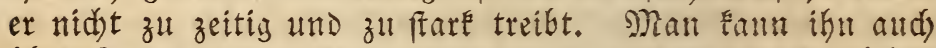

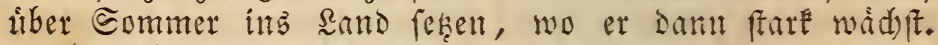
Sim Sctober bebt man ihn entweder wieder alls, oder man

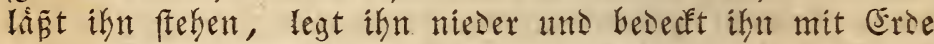
und saltb, oser wenn er fdjon zu grop ift, fo liberbaut

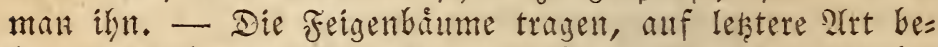
Gandelt, fefr reidlity. Die mit (erde, Strof 11. Dgl. be=

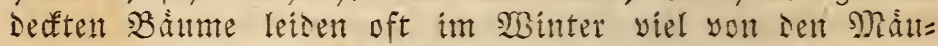

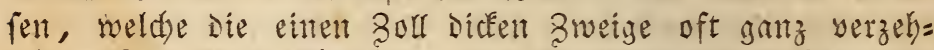

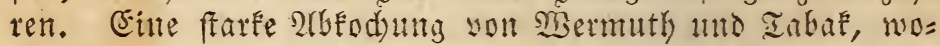
mit man die zweige vor Dem Niederlesen befterdst, for Dieje'geinse am beften abbalten. Nan yermebrt Die Fei= genbáme am befen burd SBurzelperoffen uno grbeneer. Sestere liefern Die beften Bartumben furb Zimmer, weld be fruib tragen. 2ad) Stecklinge wadjen zumeilen, wenn man fie zeitig madist uno ills warme Niffbeet fieckt. - Esz gibt Ginfidyts ser gridjte mehrete Epielarten, weldye bey allen Srandelsgintuern zul finden fint.

\section{Ferraria, Ferraria, XVI. 1.}

F. pavonia, Iigerblume, 2. Nexico. - Utub Der nid)t gropen Bwiebel Eomment mehrere $8-12$ zoll lan=

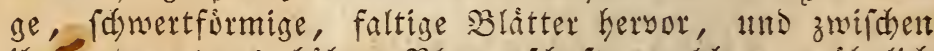
thuen Der etwas Gókere BIntmendaft, welder gewobntid) alf einmal nut eine grope stenblattrige Blume trägt, wel= d)e flad) und von einer brenneno zinnoberrothen Farbe iff. Sie bilsen, wo fie zufammenfopen, eine balbengelformige જ̦ertiefung, weldye gelb mit violettrothen Fleden geziert ift, uno eben fo gefarbt fino sie ster immern eleinen $B$ lu= menbläter. Die Swiebeln Der Iigerblume biberwintert man am beften in einem froffreyen, jesod) nidht zu beisen Bim= 


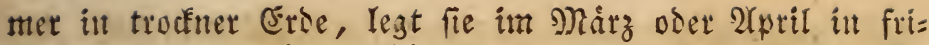
(d)e nabrhafte sauberoe, bailt fie anfangz máfis feudt, mo ftellt fie im @ommer an eitten: gen Mint und zu Keftigen. Regen gefdusht iff. Die fdjo

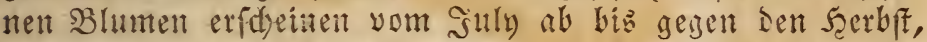
sauern aber unt 4-6 Stmnoen, unb Ginterlaffen lánglide Rapfeln mit vieren brauten Samen. $30 m$ Sctober ffellt

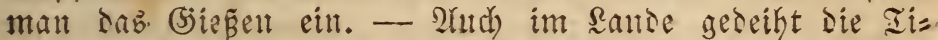

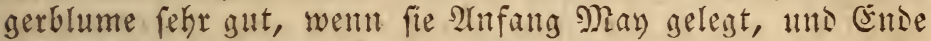
Eeptember berangenommen wird. Sian fant fie alio wie Die Commeline behandeln. - Die Bwiebeln liber Minter auper ber Erde zulaffen, ift nid)t gut, wie idj bemerft babe, Denn menn bas Bimmer zu warm ift, fo trocklten fie zu fare, ino mant fittiet im Fruhling oft nut leve Ed)a len. Die Sermebrung gefidebt fehi leidt Durd. Bwiebel=

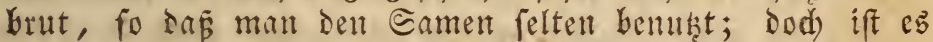

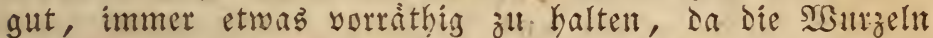
zuweilen fehr leidea, wella man fie nid)t red)t in oldst nimmt. Die Samenpflanzen bluken im zwenten Sahre,

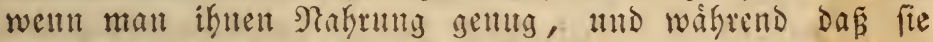
wadjell, Die gehorige Fenditigeit gibt.

Fragaria, Erobeere, XII. 5.

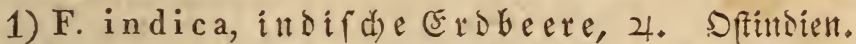

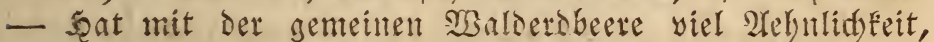
jebod) erfdeitten. Die fleinen Blumen mur einzeln an ient Panen in Dent Blattwineln; fie blibent furge Beit, felten eilten vollen Ias, fint jitroneniselb uno gleiden beynahe

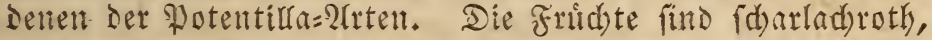
gefdmacklos uno die rothen Samentorner liegen alf ter Sberflade beynabe ganz frey. Nan zieht Diefe Erobere nur zur Bierde, intem man sie langen Ranfen an einem Fenfer oder Stabe in sie sabke leitet, wo die rothen Frudte fid) gut aubnehmen. Cie liebt nahrhaften Bosen, im Com= mer viel Maffer, Les Minters 4-8 (5iad 93 irme, und wird vermehrt, insem man હtude der Ranfen einftectet, fo Daf ier Blattengten in bie (Froe fommt.

2) F. vesca, gemeitre Erdbere, Sibalderd= Geere. - Man zileht Diefe alfgemein betante $\$$ felten ifrer frudste wegen in Iopfen, indem selefelben bey= nafe zu jeder Sahregzeit reifen. Bu bem Ende pflanzt man

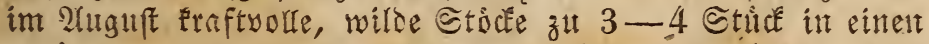
geràumigen Iopf mit nahthafter, fegmiger sauberte, feht 
Denfelben an einen etwas fdattigen Sort, bis man fieft, bas̆

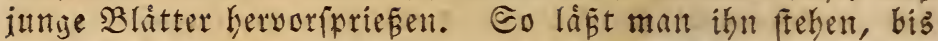
Froft fommt, uno iduht ifn nut gegen anhaltende serbft= rezen, insem sie $\mathfrak{B}_{4}$ zeln in ben Iopfen leidt faul werbent. Wird eb fehr falt, fo ftellt mall fie in eill froftreyes $B_{e}=$ Gáltnis, aber, fo wie eb gelins miro, wieder in die sullt. Sobarb man sie Eonne titglid ein Paar Etunten lanis anf Den Genfern hat, ftellt man feine Eroberpflanzen-sid)t an raffelbe, wo fie in einer Iemperatur yout $6-10$ Girad bals zu treiben wns zubliken anfangen. Fiterber befpen=

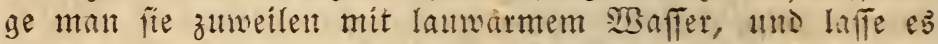
nie an ser gehorigen Fentidigfeit fehlen. Die fadenformi= gen Raneen wersen ftets abgefdntten; Denn ez fehlt ia wohl in Eeiner Segend an Diefer Pflanze. Sant man Dop= pelfenter, fo ftelfe mant fie in siefe, wo fie gegen dent Etaub Der SBofujimmer gefditht funo, und man die Iem= peratur surd) Deffnen uno Berfdliesent Der Fenfter ínen geben fann, wie man will. Sim 2rusult wersen fie umge= prianzt, over man bolt mene @tocke. - Don den in (j)íl: telt gejogenten Erobeeren eignet fid) sie inmertrizense, oder Monatsersbere (F. virginiana) aud) zum Ireiben, แno wird ganz fo wie bie vorige befandelt.

\section{Fuchsia, $\mathfrak{f}$ ud (ia, VIII. 1.}

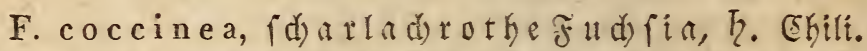

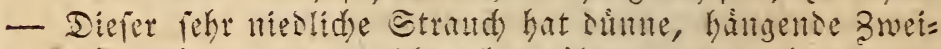

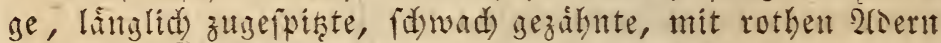
durdzogene Bläter, weldye zu zweyen und an ben Eriftig=

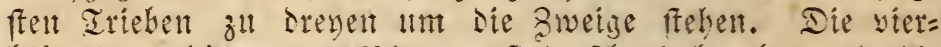
blattrigen bangenten Bhmen fino fdurladyoth, und bie langen rotgen Etanbfiren werben von flethen zufammenge=

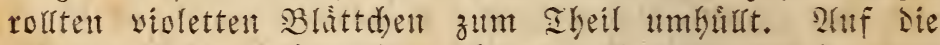
Blumen folgen länglide, fdhwarze Beeren, weld)e viele Fleine Eament enthalten.

Man gebe siefem (5ewad)s nahthafte Srangerie= ertse, unb fetce es, sa saffelbe ftar wurzelt, alle frublinge im 2lpril um. Des Sommer's gibt man ím einen gejduthten Etand im freven, wo eb nidyt zu yiel Eame bat, nut

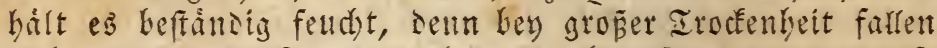
nidft feltent alfe Gnospen ab, mo eben fo, wenn man oft Den Etant Ier Iopfe verintert. Sm Sertif bleibt die Fud fit io lange im Freven, bis Nadjtfrofite eintreten, latto

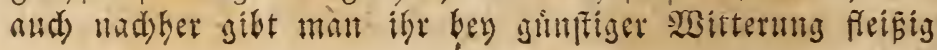




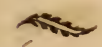

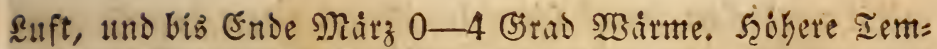
peratur fhadet ifr im Minter fehr, uno oft geht bie ganze

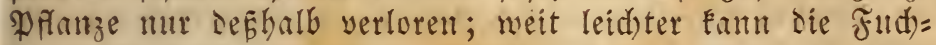
fia einen froft ertragen, ia man bat viele Beypiele, dáB fie bey einer Bereckung im frenen sante alsgehalten hat. Die Blatter fallen grốtentheils im SBinter ab, แnd man gieß̃e wifhrend diefes Ruheftandes nicht zu viel. - Die

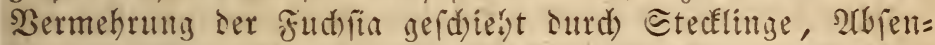
Fer und SBurzelfproffen Fehr leidjt. Stecklinge, Die man sen ganjen Eommer uber madsen fann, wadjen am fdnell= ften, went man fie mit einem (s)laje bedeckt, tho ins grift=

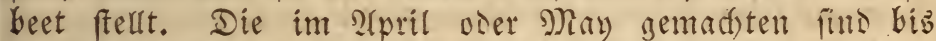
zum Serbft 6-8 soll hohe Pflanzen mit ntehrern 2leftden semorden, und haben oft fdoun (ehr reidhlid) geblibt, bes fonders went malt fie in einem Ireibeaften balt. Nut mus man fie in biefem falle Ende 2 tuguft in bie freve suft bringen, um fie abzubărtell.

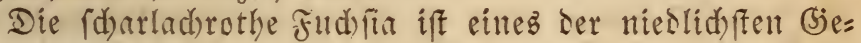
widjle, die ez gibt, und verdient, wegen feiner leid)ten $B_{e}=$ bandhng, alfgemein gezogen zu werden.

\section{Gardenia, (s)ardenie, V. 1.}

G. florida, vielblubende (s) ardenie, h. Sft= indien. - Ein orangeartiges Baiumden mit evfórmigent zugefpişten Bláttern. Die Blumen fint mein, einfad) oder gefiut, uno erfheinent in ben Sommermonaten; ifr (jerud) ift fehr angenehm. - Nan gibt ihr Srangerie= Erose mit Eanto gemengt, fesct fie alle zwey Sahre nadj Der Blithgeit it grópere Iopfe, uno hålt fie må̄̄ig fend)t. Sill Dent wàt= mern Nonaten gibt man ifr einen gejduhten @tano im

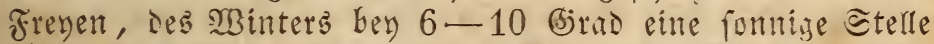
Didi) am Fenfer. Etets pehe man Daranf, Das Das SBaffer gehorin abziehen fant. Nan vermehrt Die Siarsenie durd Etecklinge, die man im May oder Šnty abfdneiset uno warm bailt. Saenn biefer Etraud) Bluthen anfesct, fo iff

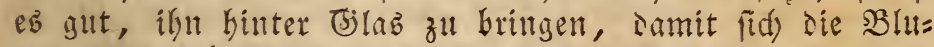
men um fo beffer entwideln.

\section{Georgina, (j)ergitue, XIX. 2.}

G. variabilis, verád Mexico. - Sbgleid) diefeg befannte (Siewdids surd)ang Feine Etubenpflanze ift, fo gibt es Dodh einige niedrige Epielar= ten, weldye zumeilen im Iopf zur Blithe Eommen. Man 
wajlt zu biefem Zweef fdon im Serbft unter bent Eamen= planzen Die Daju geeigneten Eorten aub, nub biberwintert

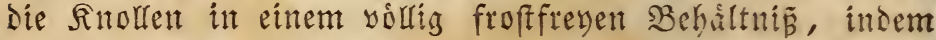
mant fie in trodften @and legt. Sm Nárz orer 2(pril pflangt man fie in fefre geráumige Iơfo mit guter lehmiger

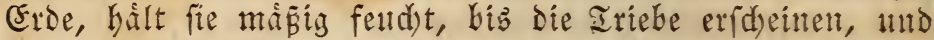

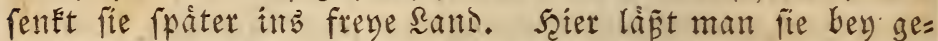

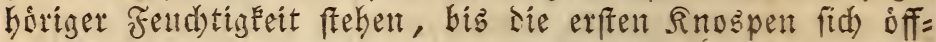
nen; alsbann bringt man sen Iopf an feinent beftimmten

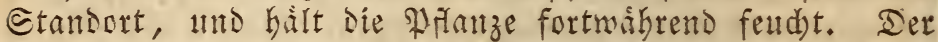

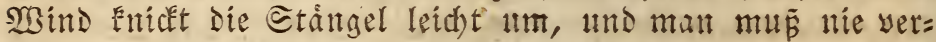
făumen, feitre \$flanzen gehorig anzubirsent.

Da bie gefirltent altent fo fehr beliebt, uno zum Iheit iest trod) felten fins, fo will id bier bemerten, auf weldae 2lit eb oft gelingt, fie ohne Samen fduell zu vermetrent. Nhan lege sie Rnolfen im 2rpril in Iopfe, fo taṕ ser obere

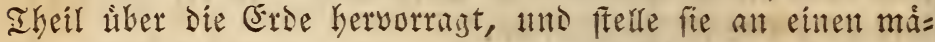

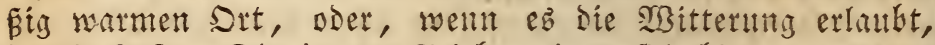
in Die snft. Die iungen Triebe zeigent fid binnen wenigen SOBden, wto mersen, fobald fie $6-8$ zoll lants fint, fo tief abgefdnitten, baf mo moglid nod) etmas yon ser Fnotre felbfe ant ifnent fisen bleibt, nono gleidjam einen

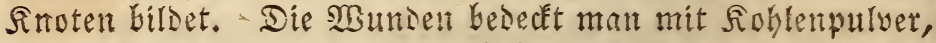
pflanzt jeden aweig in einen Eleinen Iopf mit leid)ter (5r= De, Dealt einen Ginlanglidy Gohen Blumentopf yerfebrt Dar=

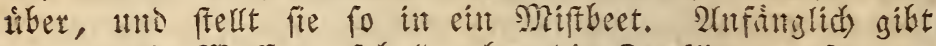
mat wentig SBaffer, fobalo aber sie Etedtinge anfantent, fid) alffuriditen, etwas mebr, aud iect man fie alssann liber Nadst ttito an truben Iagen ab; wadjen fie erft, fo

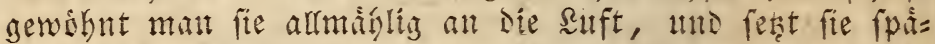
ter ins sand anf gut gedungte Plähe. Neidjt immer glidet

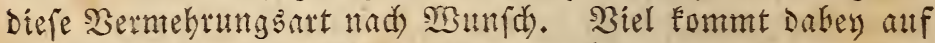

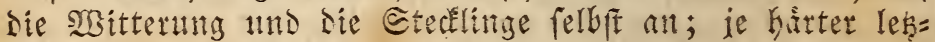
tere waren, Iefto eher watrzeln fie. Daker mus man die

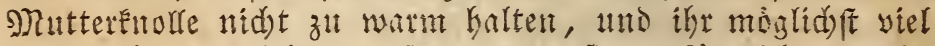

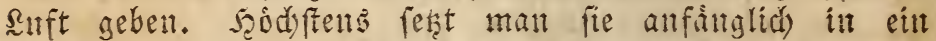

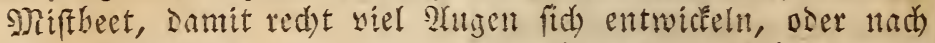

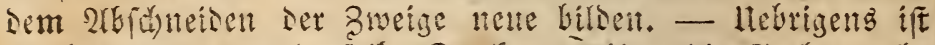

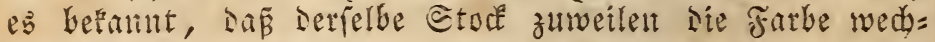
felt, went er in einen andern Bodent fommt; Dafer

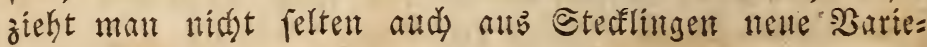
tiaten. 
Geranium, Stord) (d)abel, XVI. 5.

1) G. anemona efol., anemouenblattriget Stord) fdnabel, h. Nnatera. - Der fuzze dicee Etamm tribt auf 8-12 3olf lanzen Êtielen blittzend grime, funf= theilige, zerfdiffene Blitter, uno auf ebenfalls langen ge= theilten Etangelit helfcarmintothe, weişgeparte Bhumen, bie fait Den ganzen Eommer tiber erfdeinent. Man'gibt ifm nabrhafte sauberse, veriest ifn alfe Fritglinge, uno vermebrt ifu au beften Durd) Eanten, Dent mant im 2rpril fiet; zuweilen treiben Die áltern Etamme aud Seitenáfte, Die man als Etecklinge bentht. Des હommers gibt man ifm einten \$lah im Freven, unt Des Minters bringt man ihn bey 4-6 Girad 2 Batrme fegr gut surd).

2) G. macrorhizum, grof (d) nabel, 24. Stalien. - Er gleidht Dem vorigen, ift aber viel fleiter, bie Blatter wentger zerfdliffer, matt= grin und fein behaart. Die Blumen fomment bey einem

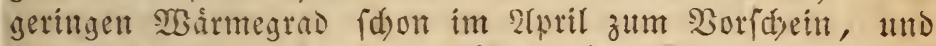
fint violettroth. Da fie in Menge kervorfommen, fo ver= Dient Diefe 2trt einige 2fufmerffamfeit. Eie nimmt mit je= Der Ersart vorlieb, vermehrt fid fehr ftare Durd, Watrzel= fproffen, iarf jecod nid)t zu fehr zertheilt werden, wenn

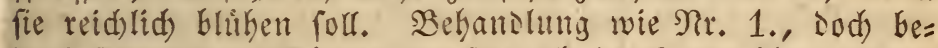
sarf fie roniger WSarme, Da fie altd) im Sande biferwintert.

\section{Gladiolus, હiegwurz, III. 1.}

1) G. cardinalis, (sardinalb= ङtegrourz, 21. (Eap. - 2tus einer crocusantigen Bwiebel Fommen blaugri: ne, fdwertillienáhnlid)e Blâtter. Der Blumenfd́aft wito

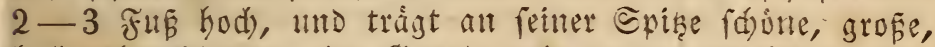

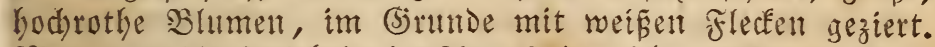
Shan legt Die Bwiebeln im 2tuguft in nidyt zu Eleine Iopfe mit nahrhafter, fandiger Erise, etwa zwey Boll tief, und

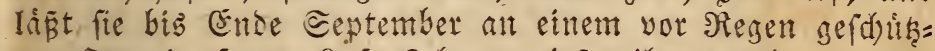

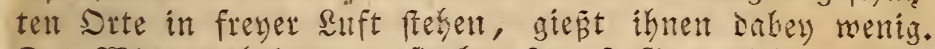
Des Winters befomment fie bey 2-6 (Sirad $\mathfrak{W a r m e ~ e i n e n ~}$

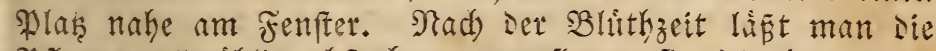

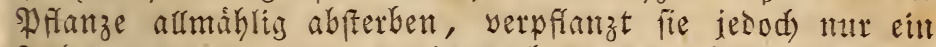
Safhr umb andere, lund nimmt bey Diefer Ejelegenteit bie 3̧wiebelbrut $\mathfrak{a b}$.

2) G. communis, gemeitie ভiegwitz, 24. Diefe 2lrt findet man ill Deutfahland nidut felten in gebir= 

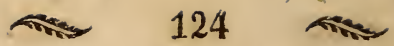

gigen, waldigen Gegenden, und fie wird wegen ifrer fabs= nen Blumen bảufig in (siårten gezogen. Cie lǟt fid) aud) leid)t treibeli, wenn man die Swiebeln zu 3-5 im હep= tember in Topfe pflanzt, und fie bey måziger Feudtigeteit an einem froffreyen Srte uberwintert. Sim Máz bringt man die Iopfe in sie warme @tube ans Fenfer, wo oft (d) on im alpril die Gellcarminotben Blument eridseinen. 2ud fann mant die Brotebeln mit Ballen im Frubling aus Dem sande beben und in sopfe feten. (s) foll auds eine gefurte und eine weifblitbende Epielart gebent.

2lnmer. Son den ubrigen Giegmurarten babe id nod) feine gebant, fie werben aber fammtlid) wie Nir. 1 . bebandelt, und liefern dione Blumen.

Gloxinia, (5) loxinie, XIV. 2.

G. speciosa, (d) ine Gloxinie, 24. Eubames rica. - Die Wurzel siefer fdonen Bietpflanze ift grof, plattrund und you der Farbe einer gewibnliden weífent Kartoffel. 2fus Derfelben treiben furze zweige mit paar= weife ferhenten, $3-4$ zort langen, zungenformigen, am Rande gelerbten, fammetartigen Bláttern. Die Blument erfideinen Den ganzen Sommer hiber eitrzeln allf $4-6$ zon

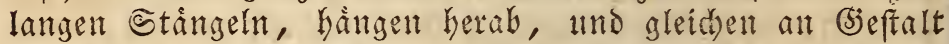
and Sirofáse Denen Des gemeitten Fingerfutt (Digitalis purpurea). Shre Farbe ift fdón blau. Man bebandelt fie gewobnlict) wie Begonia discolor oner Caladium bicolor; Dod) Fann man fie audb, wenn man ben ganzen Minter bindurd) Mittagfonne bat, bey 8-12 (sirad in Der Rábe Des Fenfers grin erbalten; nut gię̧e man alšbant nie eher, als bis bie Blátter anfangen welk za merden, denn

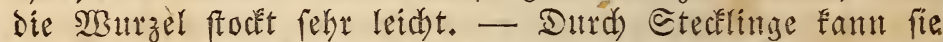
am beften vermekrt werden, aud befommt man zumeilent

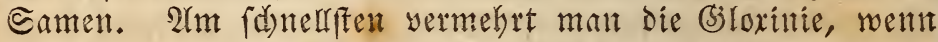

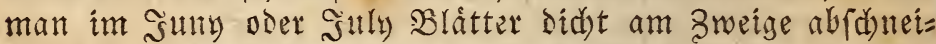

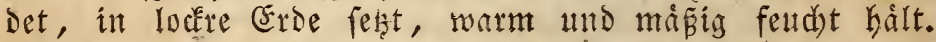

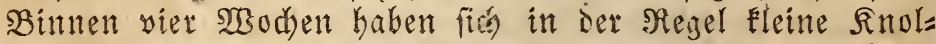
lent gebildet, und felten bleibt eill Blatt aub. - $\$ \mathfrak{b}$ G. speciosa fiore alba eine eignte Sorte ift, oder cilte Epiels art, wial idh nidyt entideioen. Neein Exemplar bat meit

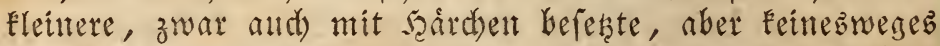
fo fasine fammetartige Blatter, und bie 3meige wath fent

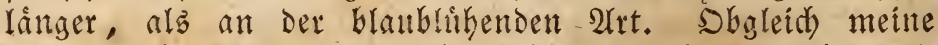

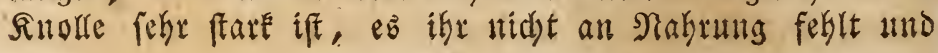


fie viel Iriebe madyte, fo babe id dod) feine BInmen er: balten, wiewohl die blane den ganzen Sommer itber Damit besect war. Daher finn id) nidht mit Beftimmtheit ange=

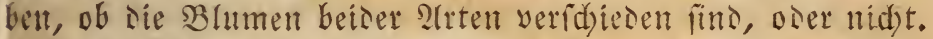
al mere. G. maculata ift nidst viel merth, senn fie blüht ăuferfi felten im Bimmer, und ift laatge nid)t fo fdith, als jene. G. coccinea if wabridheinlidy ei= ne antre Pflanze.

\section{Glycine, (f) lycine, XVII. 5.}

G. rubicunda, ontuelrotbe (s) lycine,

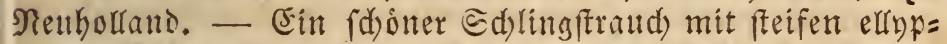
tifduen Blâttern, weldbe immer zu Drewen alf einem Eurzen Etiel an ben fletternden Zweigen fteken. Die ziemlid) gro=

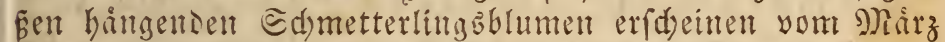
biz gegen dent હommer auf Nebenzmeigen z"l 3-5. Shre Farbe ift sunfelroth, die innern Blättchen beynake fleif(d)= farben. 2Auf fie folgen Sdoten, Durd) Deren Samen man rie Pflanze vermebrt. Cie liebt friftige Erde, einen hel=

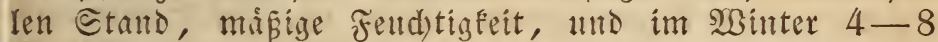
Sirad 2 Barme. - Die Eamentfanzen blihen erit im zwey= ten oder sritten Sabre; vielleidst zmingt man fie eher da=

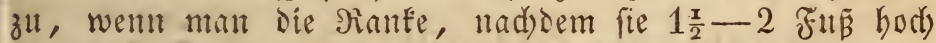
gewad) fen ift, einftust und nothigt, Eeitentriebe zumadien.

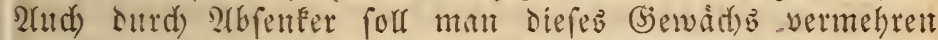
Fonnen; id) babe ez nod) nidht verfud)t; Stecklinge worten bey mir nidjt anmadjent. - (s.s fdeint, als fielen die Sinospen leicit $a b$, fobalo man Den Ctand Der Dfanze oft yeråndert.

\section{Gorteria, (s)orterie, XIX. 3.}

G. rigens, fare csorterie, 21, h. Bom cap. Die fdmalen, langen, fteifen, oft halbgefiederten Blàt= ter fino oben Duntelglänzend grün, unten Dagegen mit ei= nem feinen weíseri Filz überzogen. Die Blumen erfobeinen eiltzeln yom May bis Suly; fie gleidten Denen Der Arctotis grandiflora, find aber nod) brennender, uno jedes Blatt bat am Reldi) einen braunen Fleck, Der wieder in Der Nitte

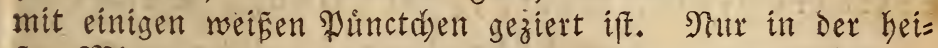

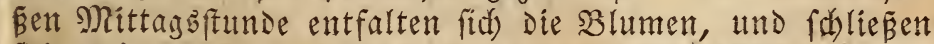
fich fpater mieder. Man thut gut, auf Den Boden der Iopfe fleine Steine zu legen. Die librige Behandlung ift mit der, weldbe die Srectotisarten verlangen, libereinftimmens. 


\section{सises, 126

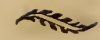 \\ Grevia, (siteyie, XIII. 1.}

G. occidentalis, aficanifale (5)eyie,

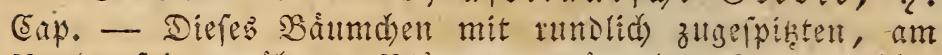
Rande foin gezánten Blảttern, trågt sen Eommer hiber einzelne fternförmige, violett uno rôthliche şlumden. Nhan gibt ifm gltte nahrhafte side, verfeç freat es belt Sommer thber an sie sllft, und im sBinter,

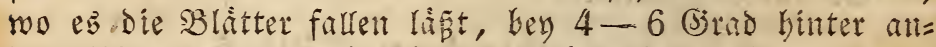

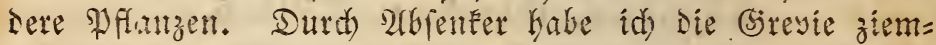
lid) leidst vermefrt; Stedelinge wolten niemals antegmen;

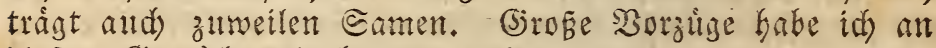

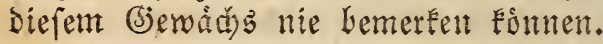

Helianthemum, Sonnenthimden, XIII. 1.

H. la evipes, glattes sonnenblimben, Grantreid). - (Cistus laevipes) eine niebrige Rianze mit feinen Broeigen utto graugtumen nabelartigen Balattd)ent. Die Blument fitto Fleitt, gelblid), unt gleid) senten ber eis

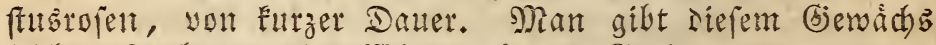

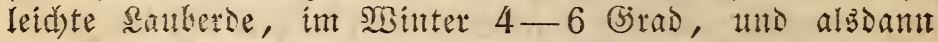

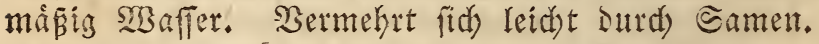

Helianthus, Sonnenblume, XIX. 3.

H. multiflorus, vielgltmige હonnentra= me, 4. Virginien. - Son siefer 4-6 Fun bohen Sonnenblume wird die gefitut blithende, mit grof̧en gold=

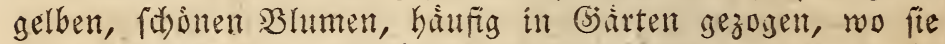
jedod) liber sointer gut bededet werden murs, da fie leid)t

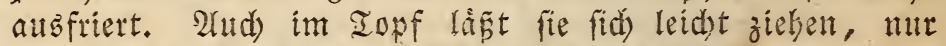
mun siefer recht gerdumis feyn. Sm frithling legt man einen Reim (nie mehrere) in fette tabrhafte Erde, und

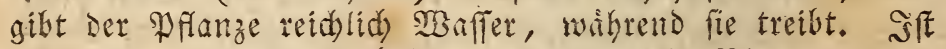
Der Iopf zu eng, fo befommit man wentig Blumen, und Sie Etăngel werden faum $1 \frac{x}{2}$ Jus hod). Sie liebt im Iopf einen fíattigen Etand, weil bie Comte Die Manr= zelit zul letd)t altoborrt.

Heliotropium, Scorpionsfdwanz, Sonnen= wende, V. 1.

H. peruvianum, perayianifder Scotpions=

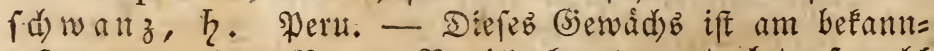
teften unter dem samen Sontlfentraut, uno bedarf woht 


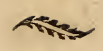

weife, lilienartige, angenebm suftende Blumen tràgt. Sie Glîlyen zwar felten långer, alż einen I Ing; ba aber ofie fid nad) uno nad) offinen, fo bat man die pflanze Dods 14 Iage, Imo bey Eủhler truber SBitterung wohl lảnger it Flor. Diefe Taglilie bált fehr gut über sointer im Frenelt aub; Ioch ift es beffer, the aliann einte Decke yon aub zu geben, Da bie trocknen frofte ifr fdasen. Sim Iopf liebt fie nabrhafte, mit Sand gemengte Erise, nno hiber Som=

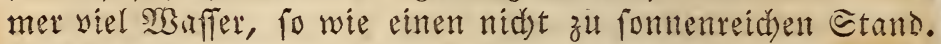
alte friblinge wird fie verjest, und mait fann bey diefer

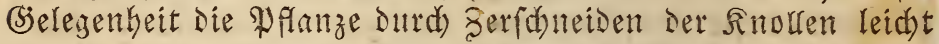
vermegren. Da fie fract wutzelt, fo löst man in einem Iopf mittler (jirófe nicht mehr als $2-3$ Reime. Sm SBinter bålt man fie im Reffer oder in fonft einem froft=

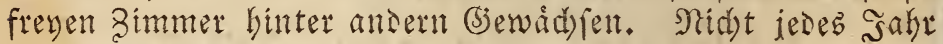
will tie blithen.

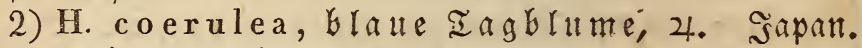
- Die Blâtter gleiduen Denen der vorigen 2 ret, fino aber

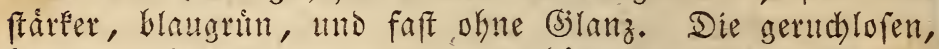
blauen, glockenförmigen Blumen bången an einem $1 \frac{x}{2}-2$ Fuß langen Etãngel. Bebandlung wie bey Nir. 1., Der fie aber an Werth nadyfteht.

Al merf. Beide 2lrten gehen oft unter dem Namen H. japonica, aud) werten fie yon, mefreren Botani= fern Hosta alba und Hosta coerulea genannt.

Hemimeris, Salbblame, XIV. 2.

H. urticifolia, neffelblattrige Halbblume, $\odot$, h. Elidamerica. - Die Blätter Diefeb Bàumdenb,

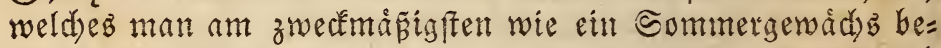
Handelt, gleidyen einigermapen Denen Der Neffel, find jedody

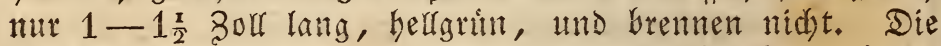
Blimdyent erfdheinent Den ganzen Sommer liber bib fpåt it Den Serbft, fisen in lodern Uebren an Den Episen Der Sweige, und find ounkelorangeroth; auf fie folgen Eleine zwentheilige Sapfeln mit vielen Hleinen હamen. Man fäet Den Samen im 2rpril in Iopfe oder inb Miftbeet, verfeht Die \$flimzden, wenn fie $1-2$ gol bod find, in nid)t zu enge Iopfe mit nabrhafter sauberde, und bringt fie, fos bald es die Witterung geftattet, ins Freve; Dod) mus Der. Etand nidbt zu fehr yon ier Sonne getroffen merden; fie

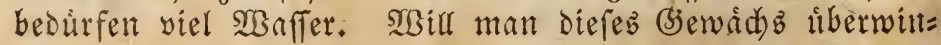

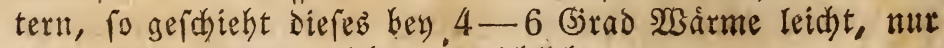

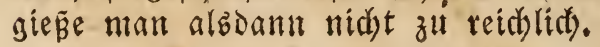




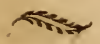

Hesperis, פadidyiole, XV. 2.

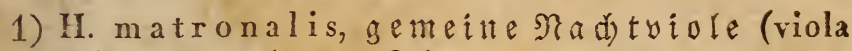
matronalis), 2. Eibirten, Edmeiz, Deutidalano h. F. w.

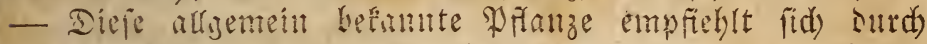
ben berrliden (bertid), weld)en die Bhlumen Des afbents

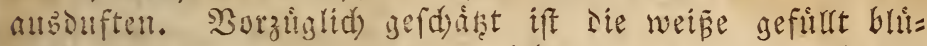
hende 2frt. - Diefe verlanigt lefimige, fette, jesod) mit grobem Eano und Fies gemente Erise, und einen etwab

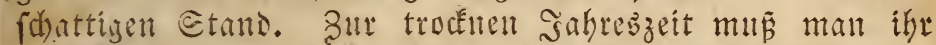
reid)lid) giesen; follft (d)aret ibr sie viele Feudstigkeit. Man vermebut fie im 2fuguf entwerer surd) Zertheilums, oder nun zwingt die Pfanze sasurd), Das man die Bhlu= menfringel bidt tiber ber Groe abfdnetset, eine Menge Eei=

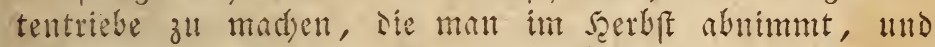

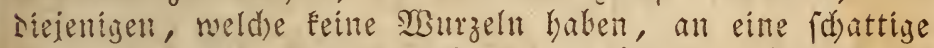

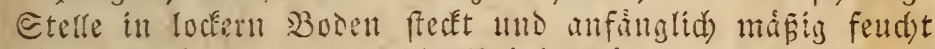
bălt. 2lud) Eam man Die Blithenftangel zu Etedflingen bemthen, wem man fie unter einem Blattéluten abidneioet tmo hiber sempelben nod) ein 2tuge ftehen láât. Nur gebe

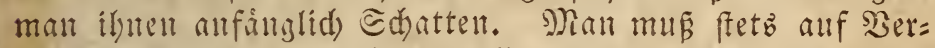

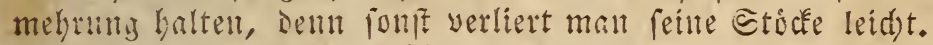

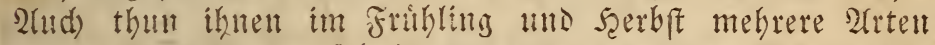

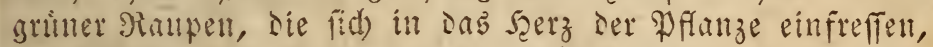
viel @d)aden. - SWirl man Die gefürte Nad)tviole im Iopf ziehen, fo behandelt man lie wie die Birodenblumen; mus man fie jesod) immer im Topf feben lafien, fo forge man

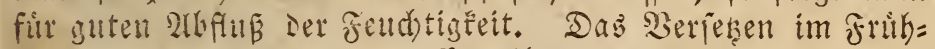
ling ift ifr nad)theilig. - (5s gibt aud) eine lilafarbige ge= fürte 2lrt, bie nod) felten ift. - Die gemeine einfad)e Eorte

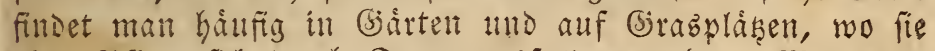
ohne \$flege fid) Durd) Eamenausfaul verntebrt. Wirl man Diefe in Iopf zieben, fo bebt man junge friftige Pfanzen int. 2)ăz mit Ballen ans, mo befandelt fie auf befannte STEeife.

2) H. melancholicus, trantiue Nad)tuiole,

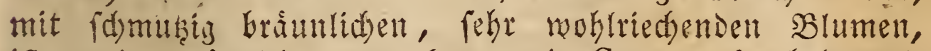
if weniger jádetlit), und fann wie Campanula befandelt werten.

Hibiscus, efifif di, XVI. 8 .

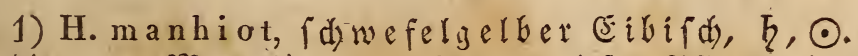

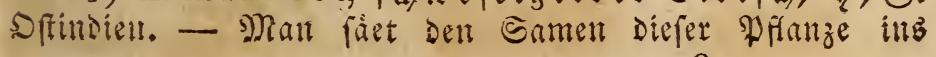


Miftbet, oder in Iospe, Denten man jedody einten warmen Stand gibt. Die Pfonzen wurzeln ziemlid) fente, und merden beynake wie Datura fastuosa behandelt. Die gro=

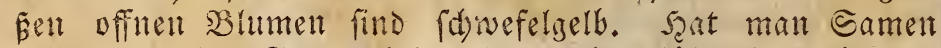

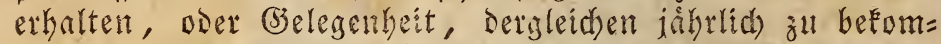
men, fo thut man am befen, fid) mit Dem Heberwintern gav nid)t énzulaffen, denn es glidete felten, und im nádb= fren Conmer molfen Die alten Cticke ohne Ireibefaften oder Miffeet niemals gut wadjen; fonft gibt man ihm Dez sinterg- bey 6-10 (s)rad einen Stano nid) weit yom Fenter, uno nidst zu viel Saiffer.

2) H. rosa sinensis, dinefifde Rofe, Dftindien. - Diefer Baum emreidst, wenn er Rabrung genug und Sadrme hat, fduell eine bedentende Srohe, und febt beynabe dab ganze Sabr liber in Der Bhluthe. Dab saub iff glälzens immergrut, Die Blitter faft herzformig, fumpf gezánt. Sm Berteff der Blumen gibt eb. folgende Spielarten, auser ber gewibnliden einfaden, bodbrothen. a) Die Kefroth gefulte; b) Die Duteltrothe gefulfte;

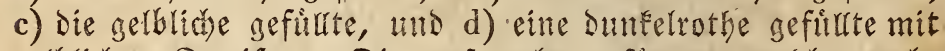
gelbliden Etreifen. Die rofarothe gefurte, weidse mehs= rere (s)artmer alffithren, babe id) nod nirgend gefehen, und fiheint nut in der Einbildung zu exiftiren. Sm Bimmer

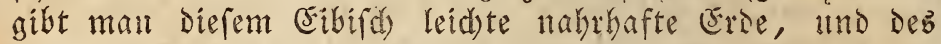

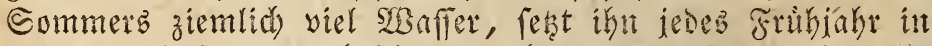
grofere abpfe, uno befdnetdet bey defer Gelegenheit de Swnzeln etwab. Er fommt nie in bie suft, und wird fo warm alb miglid) gehalten. Solf ex audy dez WBinterb blithen, fo mus er bie votre Conne und einen Strmo yon

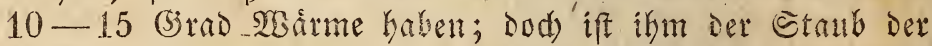
Simmer fehr zuwider, und ex wirft saber faft immer dab

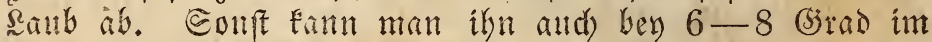

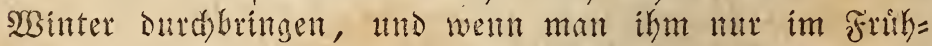
ling viel Eonne und SGarme geben fann, fo erbolt er fiá) alfmiblin wieder. - Man vermehrt Diefe fidone Pflanze am beften Durd) Stectlinge, weldse man im 2(pril oder Nan

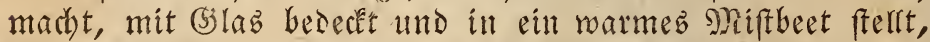
bis fie zul treiben anfangen. Sie blingen gewibnlid) im ers

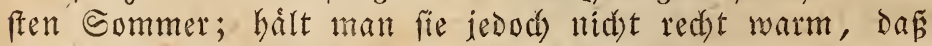
fie fid) gut eintwurzeln fonmen, fo gehen fie im folgenden SWinter leidlt ein.

3) H. syriacus, furifaer (ibifd, h. Sald= europa. - Diefer Baum, welcher fdyon im fubitchent Deutids)= 


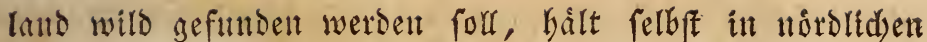
(5)egenten ofterb einelt Winter im Freyen ans. Fr liebe lebmigen Booen, und wird ieden frubling. verfest. Sm SGinter, wo Die Blitter abfarlen, ftellt man ihn in einen Feller, oser ein froftfrenes Bimmer. Seder હtecting nimmt ill ber segel all, uno oft befommt man ald Sament. Die Bảumdjen weroen gros, tno id) habe fogar in Fibeln 4-5 solf farte Etamme gefehent. Nir futd folgende Epielarten bef́ntut: mit einfad)en, weisen oder lilafarbi= gen SBlunten, Deren Blitter am Feld) ein blutrothes 2(uge Gaben; beide arten gefillt; uno buntblättrige mit lilafor= bigen gefiuten Blument. - Sit wegen feiner leidten Eull tut zu empfehlen.

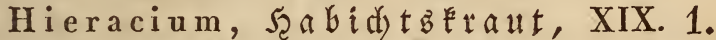

H. a urantiacum, orangetothes $\mathfrak{S} \mathfrak{a} \mathfrak{b} \mathfrak{i}$ d t $\mathfrak{b}=$ Erant, 2. Sil Der Edjweiz, Dentfdland u. r. w. auf Bergen. - Die bennabe zungenformigen Blatter find haa rij, Der ebenfalla mit fararen beferte Stångel wird 1 - $1 \frac{x}{2}$ Fü hod), und tringt an feiner Spibe einen flad)en Strauz fdouner orangefarbenter Blumen. Diefe Ranipflanze fommt faft in iesem Boben fort, utto yermebrt fid ftart ourd)

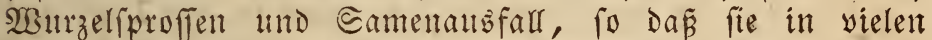

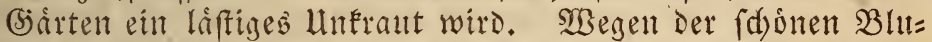
men, weldye faft Den ganzen Sommer liber erfdeinen, zieht man fie gen in Iopfen, mo bebandelt fie, wie bey Den 2ldsilfeen und (sjlodkenblumen gefagt nurde. arm beften ges Deiht fie in fdywerem Boden, und fie verlangt den som= mer liber viel Maffer. Bey $0-4$ (Girad if fie fehr leidst zll dibermintern, und wird im Frubling verpflanzt.

Hortensia, Fortenría, X. 2.

H. in utabilis, veránderlíche footenfía, China und Sapan. - Heber Die Crultur diefer alfgemein befannten Pfanje will idh mir folgentez bemerken: Da Die Shortenfia wahricheinlid in ifrem Baterlande an fenthtent Stelfen madd ft, fo gebe man ihr im Iopf eine Mifdung yon sauberde, verwittertem Torf und etwab Gand. TBáh = reno Der Begetation bedarf fie viel SGaffer; audd wutrzeln fie ziemlid) ftarḱ. Die befte zeit zum Berpflanzen ift Der 2luguft oder Geptember, fobald die Blumen ihre fibune Farbe verloren haben. Alsbann lä́t man Die Iopfe fo lange in frener suft fteben, bis Naddfrofite fommen, wels 
the bie Blitter berabwerfert. Bum lleberwintern ift jebez

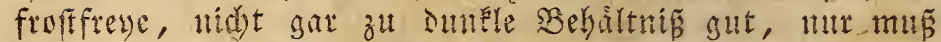
Eeffest Iemperatur biz (Ense Februar nidst liber zwey) (Sirad SBarme ferigen. Beffer if es, fie befommt einen gelinsen

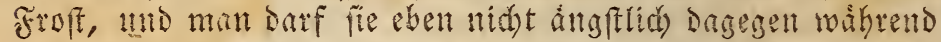

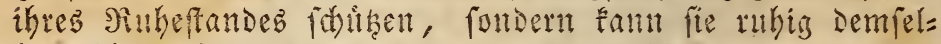
ben liberlaffen. Dagegen ift fie im Fribling, wem erf Die Snospen fich altangen zแ offnen, gegen ftarte Falte fegr empfindlid). sm Mary bringt man fie in Die Nathe bes fenfters an bie Sonne, gibt ihe an milsen Iagen suft,

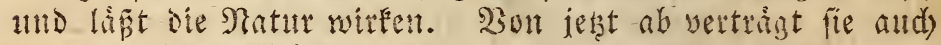

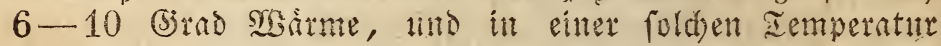
entwideln fid bie jumgen Iriebe fdued und traftig; mat verfăme man alsbann in nidyt dab (3)

Soat man bagegen bie Sertenfien, wie fo oft gefouleht,

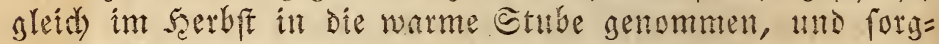
fáltig gepflegt, fo werden die Rnospen zll frith geztunngen,

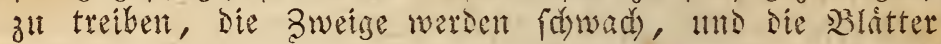
welfen gemeiniglith gegent dent Frthling ab, wo fie gerade

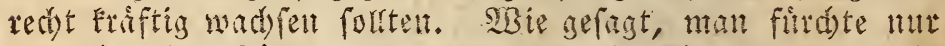
ia nidit, bie Sallte werde der frotenfia fánden; wenn fie gleith) yom Sereft ab saran gewsint wird, fo sant fie viel

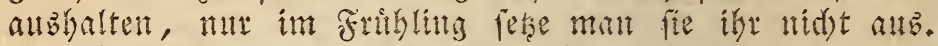
- Obgleid die sortentia im frifling fegr das Eumen=

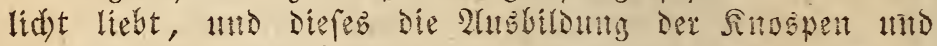
SBlatter ungemein befordert, fo mus man sodi fpater fie

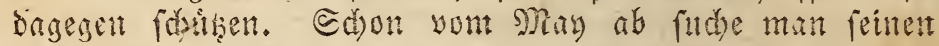
Pfanzent eiment folden Wlats anzumeiren, wo fie bodyftens

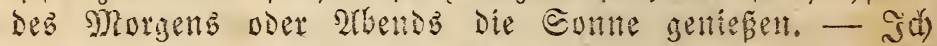
habe Das eitle Nat mehrere Pfanzen Den ganzent Sommer iber im simmer behalten mitfen; fie franoen etwa $3-4$ Fus vom Fenfer ab an einer S⿰氵 shadimittagb you 4-6 ubr Somte, und Deffen ungeaditet. hielten fid) defelben fehr gut, mito fahen vorghtglich gefund aub. Dod fehlte ifnen weser bey Iage, nod bey Nadjt Die suft, und dfterb ftellte id fie ill einen gelinden Re=

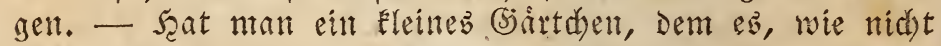
felten in Der Etadt, an Eome feklt, fo eiguten fid' Die - Shortenfien ganz yorguglidi) zur Bierbe Defferbent. Sim Eom= mer ferle man die Pfianzen, weld)e nidjt blikgen, binter

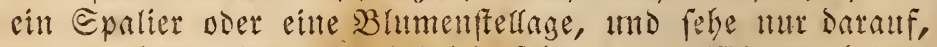

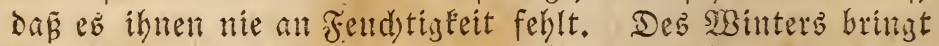
man fie in sen in ber Einleitung befdriebenen SBinter: Enttell. 


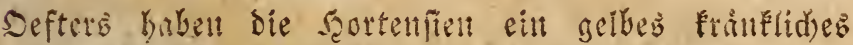

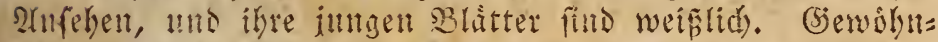
lid) liegt sie Edyuls ant Boden, indem entweder sie (Eroe

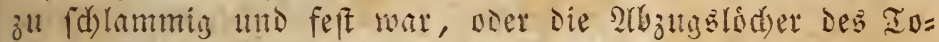
4) Baren yerfopft, Doer ç kaben Die Regenwuitmer Die

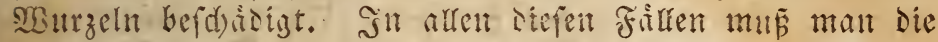
Sertenfien gleiti), went man diefen Bufall bemert, altb

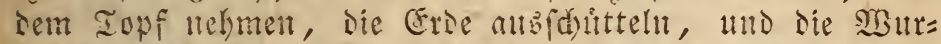

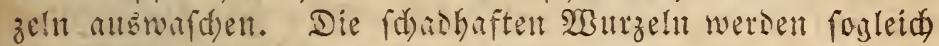
abigefdutten, mo Der Etraud in einent Eleinen Sopf mit

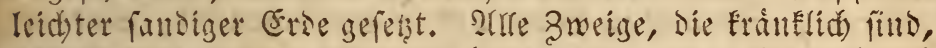

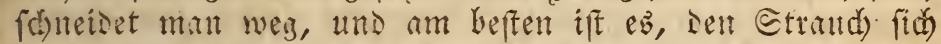

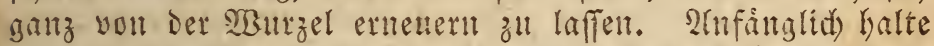

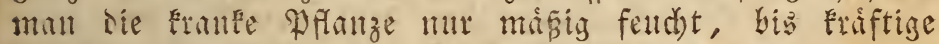
Epróslinge erfdeinen, unb gebe ibr פnorgenfontue.

2lud) im frenen sande fan man sie sartenfien diber= wintern, went man fie im serbfit niederlent, mo nit croe

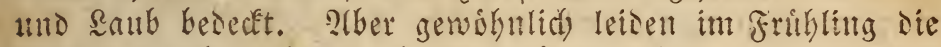
jumgen ariebe fehr surd) sie fraten groffe, fo sa man

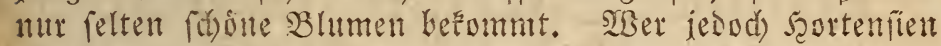
im freyen sante zieken will, Ser becke fie nitht zul fruh

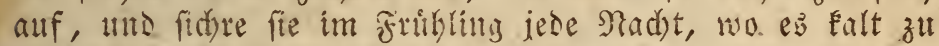

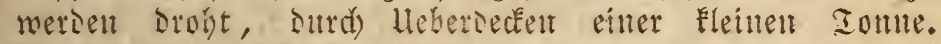

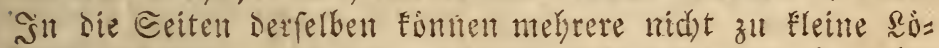
d)er geidnitten werden, weldye man mit Papier tiberflebt, Dab malt mit Rzinol trinft, Damit and an falten Iagen etwab Ridi)t einfarfen Eamit.

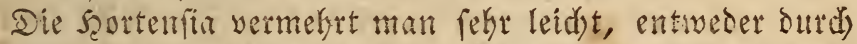

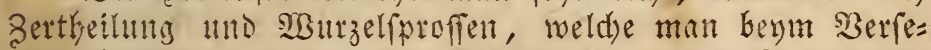
fien abnimmt, over man madyt Stecflinge im Monat su=

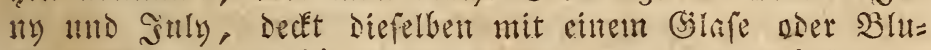
mentopf zu, uns hailt fie warm. Şierzl Dienen befonder

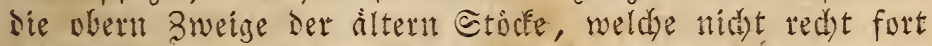
wolfen. 2im fidonfen fino fins Bimmer bie zwey = tho

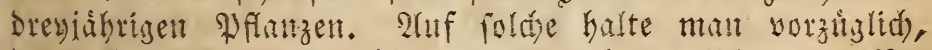

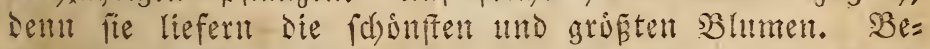

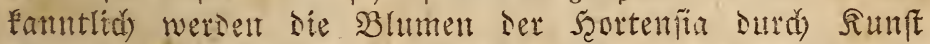

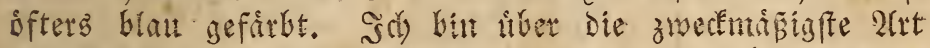
und 2 Beife, wie hiermit ztt verfahren ift, nod im ameis fel, obgleidy id mebrmals tedist fonone blane Blamen et: Galten habe. Eifengaltige (Frten voer Beymifa)ungen Der

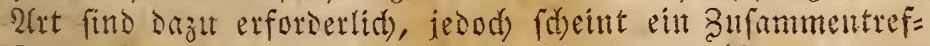

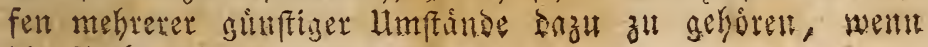
die Farbe rein angfanen foll. 
Houstonia, f̧auftonia, IV. 1.

H. coccinea, (d) arlad)rothe Saltitonie, $h$. Mexico. - Diefez Bátmdent tright anf Eutrzen હtielent grangrute, eyforming zugefpiste, ganz randige Blatter, zu Dregent quirlförmig an ien 3weigen. $2 \mathfrak{n}$ den Episent Der lestern e:fdeinen faft ben ganzen Sommer binourd) flétre

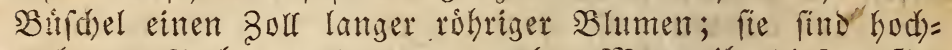
roth you farbe, und etwas tauh. Man gibt diefem (s)e wådb alfe sabre im Frubling eitten grósern Iopf und sauberse, mit etwab sehm und Cand vermifid, bâlt es

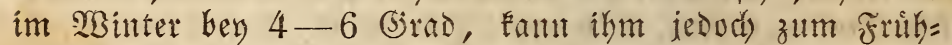
ling etwas mehr SMárme geben, um die jungen Iriebe ber: vorzulocken. Den @ommer iber ftell man sie sallfonie ins Frene. Bum Iheil wirft fie im Winter sie Blatter

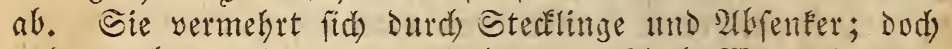
nebmen beide nidbt ganz leidst an. 2(ud) Sagurzelfproffen betommt man yon áltern Pflanzen zuweilen.

Hypericum, Soḅaniźaut, XVIII. 3.

1) H. balearicum, balearifde šobantib=

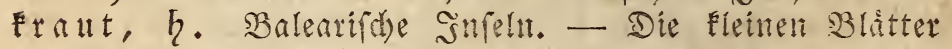
Diefer 1-2 fun bohen Pfanze find am Rambe wellenfors= ming gebogen, uno fo wie sie Etangel mit Fleiten gelben

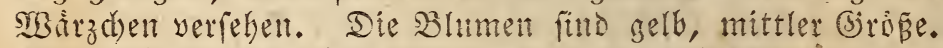
Man gibt ifm sauberie, yemebrt $e$ s Durd) Samen, und liberwintert eb bey 4-6 (5iras.

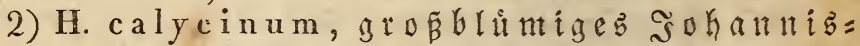
fraut, h. (Şried)entano. - Die Etängel fimb vieredéts,

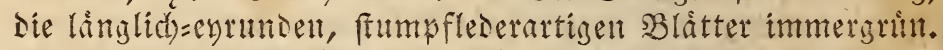
Die Blumen, weldye gropstentheits einzell an Den Episen Der Zweige erfacinen, fino groṕ uno bodgelb, mit fehr

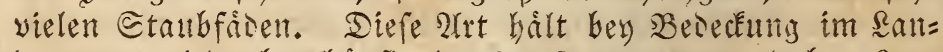
de aub, wird aber bautfin im Iopf gezogen, tuto ber 0 4 (j)ras diberwintert. Sermehrung Dutd) Bertheilung uno Etedelinge.

3) H. monogynum, indifdeb Sogannib=

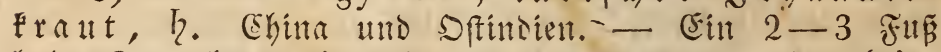
bober Etraud) mit länglid) lanzettförmigen, ftumpfen, Gláu=

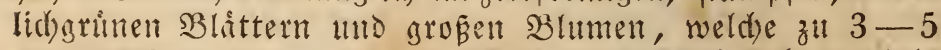
all Den Episen ier sweige fteken. Eie baben braumröth= lid) gefarbte Feldie, fino gelb von Farbe, unt ihre Ctaub= fáden febr lang. Eer verlangt sanberde, Eeinen zu fonnen= 
reiden Etans, im SWinter 4-6 Birab NBáme, unb witb surd) Sertheilmu, 2(bienfer mo Etedelinge vermebrt. Faft das gange sabr hinsuret) freht es in ier Bluthe.

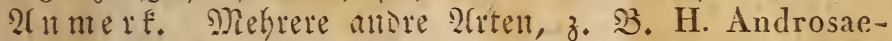
mum, Ascyron, Hircinum, Olympicum, floribundum, Kalmianum, pyramidatum 1t. f. w. Fontutelt allf

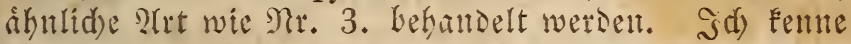
sie bier angefubrten 2roten jesod) faum mebr, als sem Nanten nad), und weis mur, saź fie alle fdosne gelbe Blument trigent.

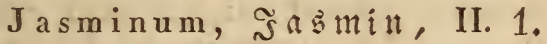

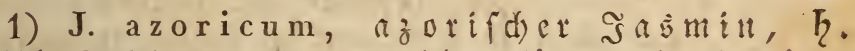
2lzorifd)e Snfelu. - Die runslid)en glenzend = sumfelozrumen Blatter fteben zu stenen beyfammen. Dex Etrands wito

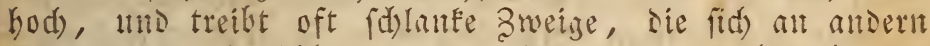

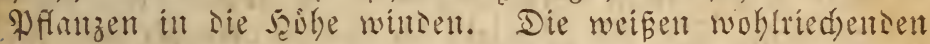
Blumen erfdeinen yom Suln ab in reiden Bufduell. Diefe

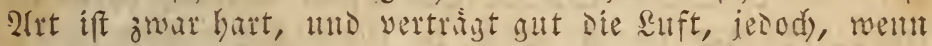

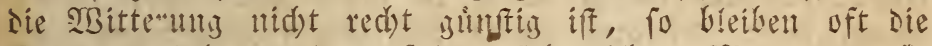
Snospen ftehen und entfalten fid) nidbt. Snan ftelle fie saber alsonatu binter (sjlas, gebe ifr suft ntto bie geborige Feudtigfeit. Eie befommt Dramgeric= Sorde, wird alle

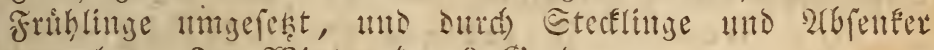
vermebrt. Sin Minter 4-0 (jirid.

2) J. fruticans, ftrauch artiger șa s̀mit, そ. Clibenropa. - Diefe 2lit mit gefiederten Brittern und fleiten gelben, gerud)lojen Blumen versient nid), im Bim= mer gezojen ju werten. Sibsdit fobnell, ball zumeilen im

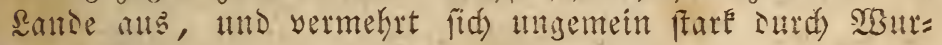
zelfproffen.

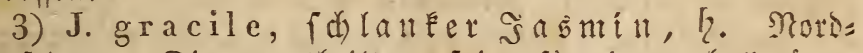
folfinfelu. - Die nugetheilten, fpişeyformigen, berbrument Blatter freben parmeife enander gegentber an sen fawa

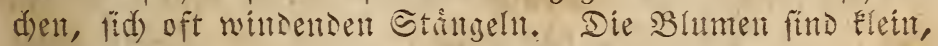

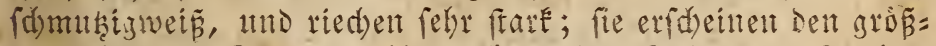
ten Iheil ses Eommers binturd an den Episen ser sweige. NBird tibrigens wie Nr. 1. bebandelt und surd) 2rbfenfer vermebrt.

4) J. grandiflorum, grof́blumiger șa șin, 7. Sftudien. - Die Blatter find gefiesert, matt, glanzent= grü und befindig. Die Blumen erfdeinen grosstentbeils

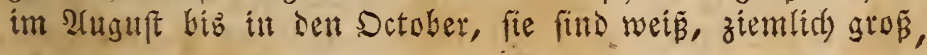




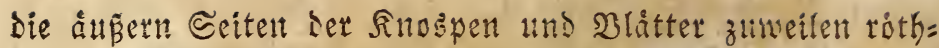

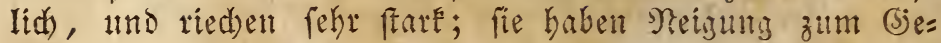
firftwersen, wenigfens finset man die meiften mit mebr, als vier Bhmenblittern. Die Befandung Diejes sasming ftimmt mit Der yon 9R. 1. ganz liberein; auth er liebt

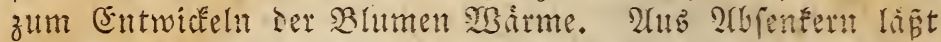

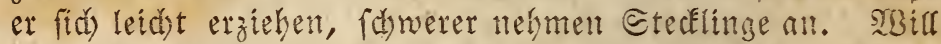
man fidnell red)t yiel jumbe poranzen erhalten, fo fest man einten mit vielen aweigen verfebenen Etrand im Fribling

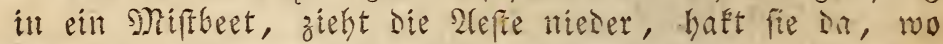
fid ein Blatterpar befindet, in die Erie eill, listet Dab folgende Blitterpart frey, befefitig dab folgende wieder in

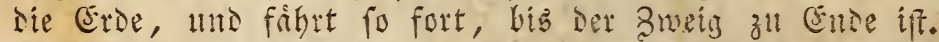

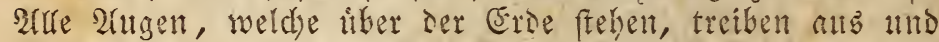
geben bis zum 2tugut 6-10 zorf lange Pfanzen, weidde alsbann abgenommen mo einzeln it Iopfe eingefeht wet=

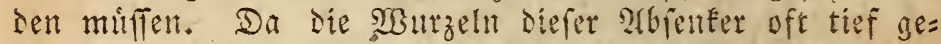
hen, uno beym seranbheben fehr geftort merten, fo ift es am beften, Die 3weige gleid) in fleite Iopfe abzhiften. Man erhatt anf Diefe sbeife von eitrem Etrand mittler

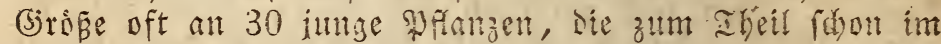

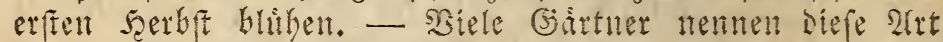
hispanicum ober catalónicum.

5) J. humile, niestiger jasmin, h. Cato= ettropa. - Gre bat mit $\mathfrak{N}$. 1. wiel Rebnlideret, bleibt aber neoriger, bat breitere Blatter uno grésere gerndalofe Bhument. - Er tift faft eben fo hart, mo yermebit fid gut sutth Etedinge.

6) J. odoratissimum, wohltiedenter sas= min, দ. Niarera. - Die grofen, lebhaft grimen, am Rante wetrenformis gebogenen Blatter feben zu 3 und 5 an sen Stielen. Die Elemen gelben Bhtmen erfdeinen saz ganze sabr iber an Ien Episen ter 2tefte, und rieden an= genebm, wenn gleidy weniger farke, als an antern 2raten.

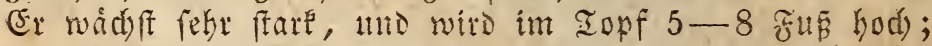
alle Friblinge mur er verfeht wertert, uno man gibt ifm lefmige Erse. Sa et willfy Eament trägt, fo yermefrt

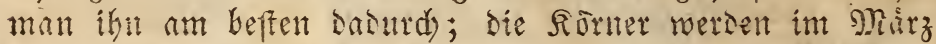
selegt, wam und másig fentigt gebalten. Die jungent Binmogen bliken felten yor tem iritten Sathe. 2tud Etectlinge nefmen an. Diefer sasmin ift mit iesem Stani= ort zufrieden, und eighet fid vorzhiglidi zur Stubenpflanze.

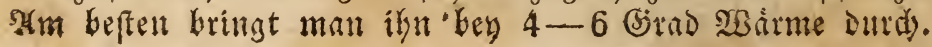


7) J. officinale, bemeiner Sasmill, দ. Ses yante, alld in Der @dweiz. - Diefe 2ret bat mit sir. 4.

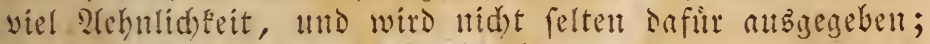

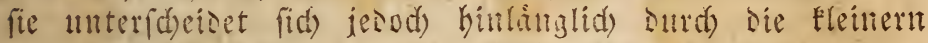

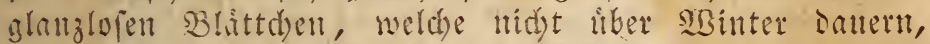

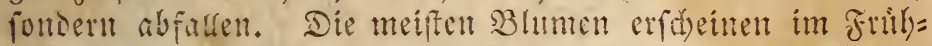
ling, sod) einzelne anch den ganzen Sommer liber, bejon=

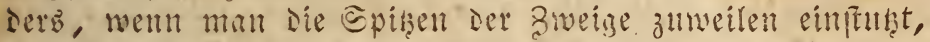

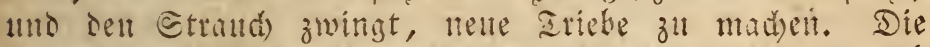

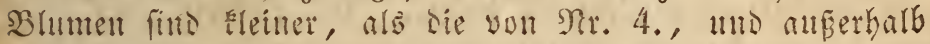

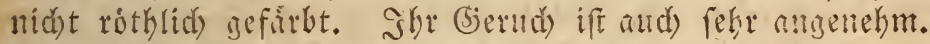

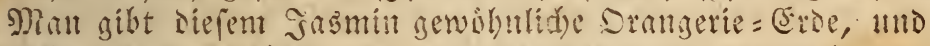

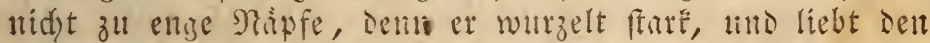
Eommer biber viel waffer. Sm Jribling, ebe sie nenen

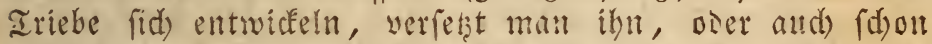
im September. Rrbenfer nno હtecklinge negmen leidit an.

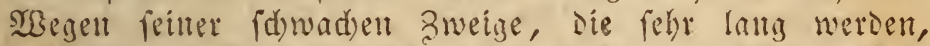

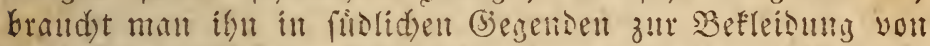

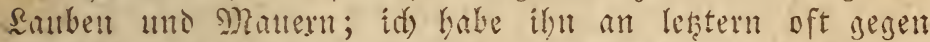

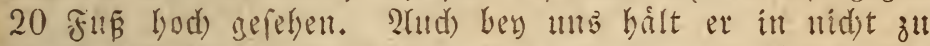

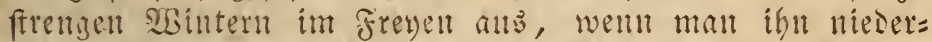

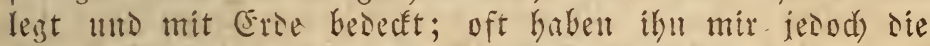

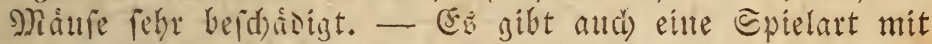
bunten Blittern, die zortlider ift.

8) J. revolutum (J. triumphans), zut ite berollter sabmin. Ere gleidht Nir. 6. , Dod) fitto bie Blitter nicht fo sunferirun, nidst welfenformig gebogen. Die Blumen fino getb uno meit groserer. Sid babe ith

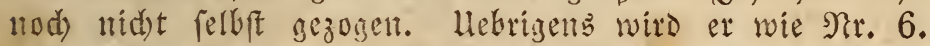
behantelt, lind intid) Etectinge vermehrt.

9) J. sambac (J. arabicum, Nyctantes sambac), niabifder sasmin, দ. Sftindien mo antere war= me benadjarte Rander. - Die parrweife fekenden Blåtter

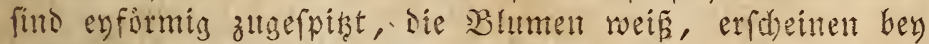
guter phege Das ganze sahr tiber, tho find rehr wohlrie= d)ent. Nian gibt Diefer 2art Sauberde, mo verpfanzt fie

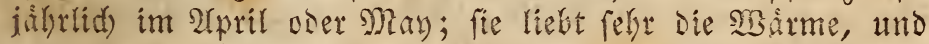
wit: in defer frimfid)t wie Hibiscus rosa sinensis behan= Delt. Er wird aus Etecklingen gezagen, die jedodi int

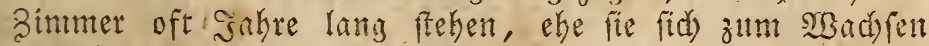
verfefent woulen. - Ess gibt einige @pielarten, you denen bie gefultt blukente febr zu empfehlen ift.

10) J. trifurcatum, dreygabliget sabmin, 


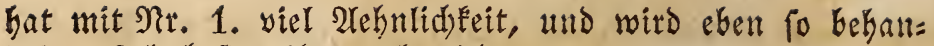
Delt. Sid) befiţe ifn nod) nidt.

\section{Ilex, Stedyarme, IV. 4.}

I. a quifolium, gemeine Sted)palme (Ģille), 7. Sin mebreren (Siegenden yon Dentfdland, in (Siebiris wáldern u. F. w. - Die Blátter Diefes baumartigen Straudso find leberartis, aber sunfelgrin, glälzend, un= tent matt, weislich; ibr Rand ift werrenformig gebogen

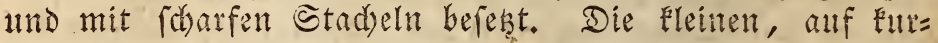

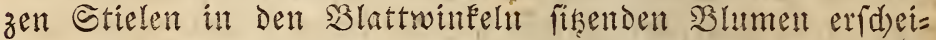

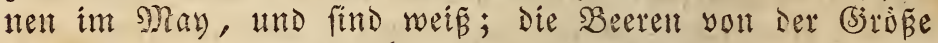
Der Erbfen, und zintoberroth. Die Pflanze nimmt fid

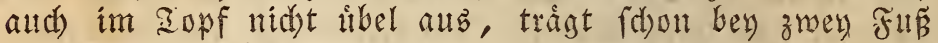
Shobe, Ind waddif langfan. Nalt gibt der Stedjpalme Drangerie = Erie, mit grobem Sande gemengt, uno ver: pflangt fie alle zwey Sabre zeitig im Frihling. Sie Fann Durd) Gamen, 2lbfenerer und Stedelinge yermegrt werden; jeboch) nebmen lehtere niăs immer an, mo ftehen oft lans ge seit, ehe fie alstreiben; man balte fie foltig uno bes

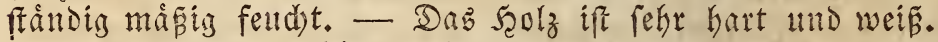
- Diefer Strand bailt and im norblichen Dented land im Freven ans, went er einigen $S_{\text {dut }}$ und paffenden Boden hat. Die grósten Báume Diefer 2trt fah ith in Dem Dorfe Monzen bey 2(ad)en; fie waren getwi tiber 15 Fuf hod), batten 5-6 zoll ftarke Stamme, tund, was mir am mere" mutroigfen war, Die Blatter battent the Stadseln verlowen, uno nur einen an ber Spise, fo bas fie anf bent erftent SBlick mit einem sorbeerbaum feht viel 2rebnlidereit batten. Sid) wate beynabe bewogen worden, biefe Stedpalme fit ei= ne andere 2 rrt zu balten, wenn nich)t an einem Baume untent ein Breig gefeffen batte, welder nod) mit Den gewobnlt= d)en Blatern verfehen wat. - Die Epielarten mit gelb= uno weibunten şattern, fo wie I. echinatum, weldye lestere aud auf ber Sberfád do Der Blätter Eleme/Dornett bat, Gâlt man zur zierse, vermebrt fie entweser Durd Steck

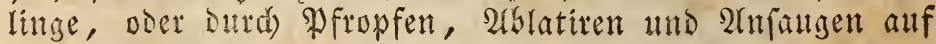
die gemeine 2rrt.

I pomea, Itid terutide, V. 1 .

1) I. coccinea, (d) arlad) tothe Irid)terwith= be, $\odot$. St. Domingo. - Die sBlítter find fpib=berz= formig; bie fleinen fit)arlad) = ober orangerothen $B$ lumen 
entipringen in ben Blattwinteln, und erfdeinen faft Den ganzen Eommer binturd). Nan legt 4-5 Samen im 2(prif in eitten nid)t zu Eleinen Iopf, ftert sie Planzen Mitte Nay in Die Rnft, und leitet fie an BBindfaden oser

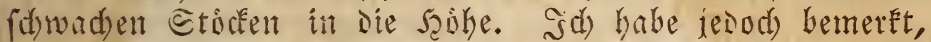
bas fie weit foloner blikben, wenn man fie ins freye sand fest, wo fie hinlenglid) Nobrumg baben.

2) I. quamoclit, befiederte iriderwinde,

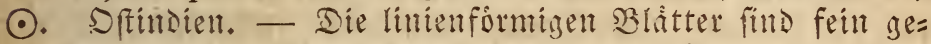
fiesert, und die fleinen niedlichen Błhmen blttroth. Man legt in Der letzten Feálfte des 2lpril Die Fỏner einzeln oder za $2-3$ in Iopfe mit leidster sanberde, uno bălt fie red)t

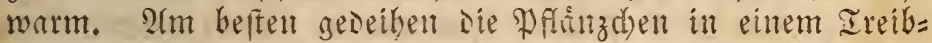
Enften, oder zwifden Doppelfenfern, wo man bie Șárme alf $20-25$ biras treiben Fant. Eie besarf waikrems Des

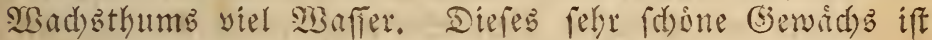
Die Réblingspflanze ser oftindifden Másdien, weldye fie

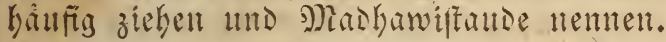

\section{Iris, Sdivertilite, III. 1.}

Dab reidye (joefdled)t Der @edwertitlien enthalt meh=

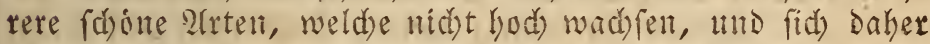
fitr Den Fenterizarten eignen. Sie ftehen faft fämmelid) bey Den Fandelägatmern in jegr nederigen Dreifen. Daher thut man am beften, fid) waibrend ifrer Bhithzeit (im Nay) uno Sinny) Dieientigen aแszumihlen, weld)e eittem gefallen,

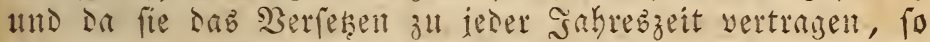

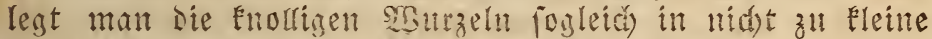

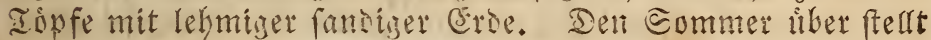

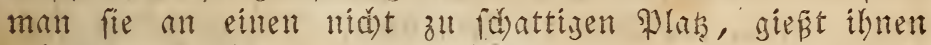
máfin und tiberwintert fie bey $0-4$ (siras. Sm Marz bringt man fie in die worme Etube an eill fonnigez Fen= frer, giept ifnen nad) Besarf, morallf fie bald tweiben und Bhumen bringen. Sigenn Die SMurzeln Den Iopf zu fefr atsfitlen, fo gibt man den Pfanzen nad) Dem \$zlithen einen grófern, oder zertheilt lie. Şon niesrigen Eorten nenne id): I. graminea, pumila (in verfdiedenen Farben), variegata thid verna.

1) Iris persica, perfifde Sduetel, $2 \%$ Derfien. - Die Zwiebeln siefer becannten 2trt wersen im Eeptember oder Setober in Iopfe mit glter fansiger Ranb=

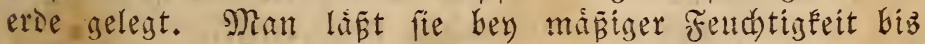
zum Samuar in einem frofffreyen Behailtnís ftehen, bringt 
fie bont int bie warme Etnbe an ein fonniges Fenter, unb gibt ifneat, wemt fie zut treiben altfangen, nehr Fenditg= Eeit. Die Bitmen erfdeinen gewihnlid) yor den B3lattern anf fehr furzen Etielen; fie find berblan ober perfartent mit intelen Edjattirungen, und ried)en angentím. Die

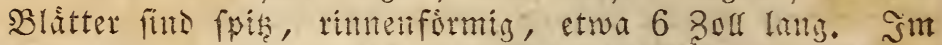
刃any fest man fie, went man (jelesentheit bat, ins sand,

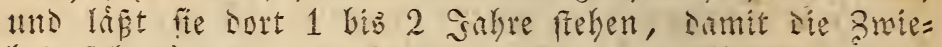

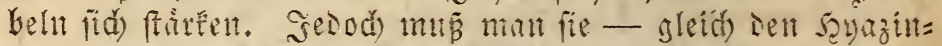
then - mit sanb besecten, werd)es bey gelinter MBttertnis wieder abgenommen wird. Nü man fie im Iopf behal ten, fo giefst man ibnen dtad) Der Bhithzeit mur wentis, lingt fie, fobalo die Blatter gelb werben, ganz troden fes ben, tho legt bie Bwiebelit in serbit in friface (Ever.

2) Iris sussiana,

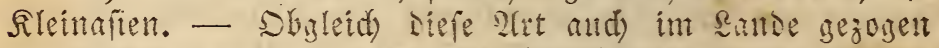
werden fom, fo iff fie iod) weit zintlidyer, als andere strten,

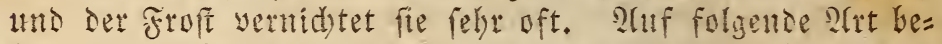
bandelt, babe idh fie immer gut zum Bliben geburats: if

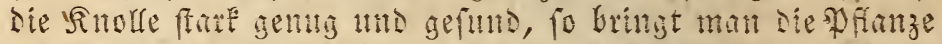
im 2tpril oder May in eitten ireibenften oder zwifhen Dop=

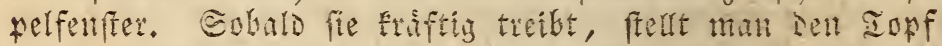

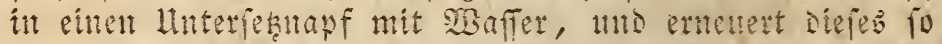

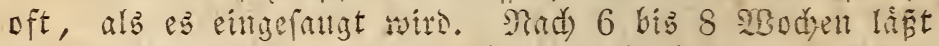
man mit Diefem Irthen olfmihlis ntd), bringt Die Pflans ze alt bie Buft, mas, fobrid die Blitter gelf werden, bort

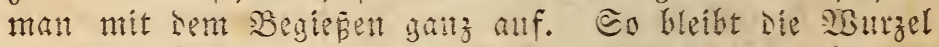

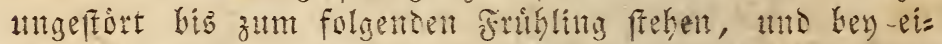
ner zwenten ähnltden Behandung liefert fie beftimmt BBlu= men. Colde ser Iopf zu Elein fern, fo mú fie im 2lu= glif mit Dem Balfen in einen grösern Napf gejeht merden;

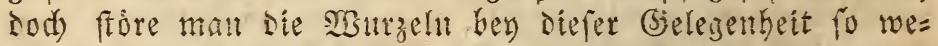
his als mônlidy. Die Bhtme if grof mit vielen fleinent

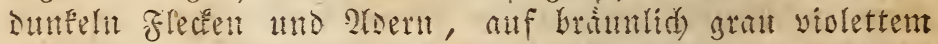

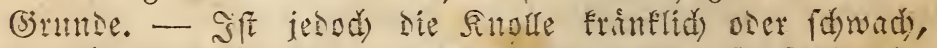
fo gethe man ify nicht zu viel sisarfer, Ient fie fanlt als=

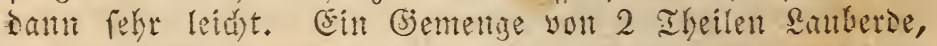

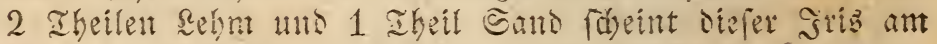
beften zu betommen. Sift es nidht Durdura nothmendis,

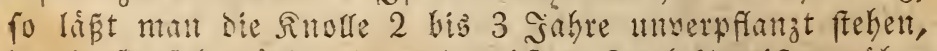

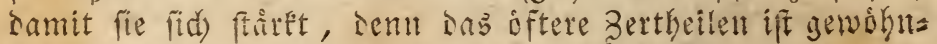

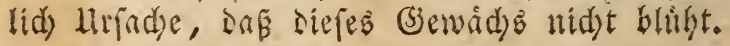




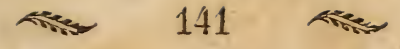

3) Iris xiphium (Iris hispanica), fpani= (d)e डd wertel mo

4) Iris xiphioides (Iris anglica), engli= (d)e હd)wertel. - Beise ?(rten wadjen in Epanten,

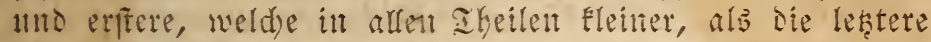
ift, and) in Eibitien. Nan bebanseit fie wie Nr. 1 ; Iod)

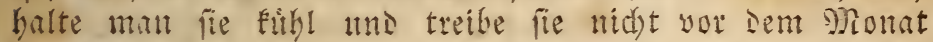

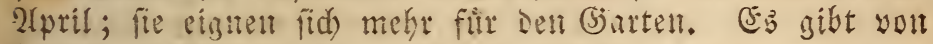

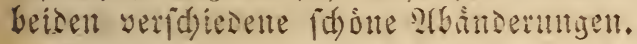

$$
\text { Ixia, Site, III. } 1 .
$$

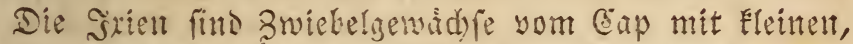

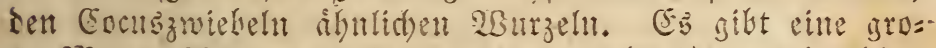

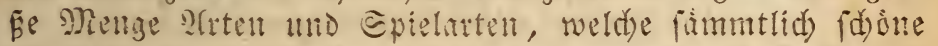
Bltment traselt, turo roie Gladiolus cardinalis befantelt werien. Die befanntefte Eorte ift Ixia crocata, mit beyna=

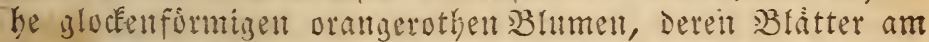

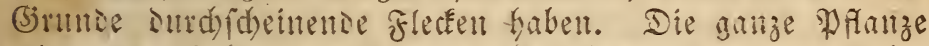
wird etwa 6 bis 8 zoll hod), hat falwertlitentartige Blatt=

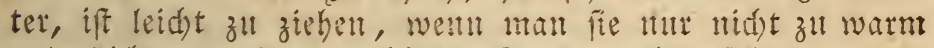
ImD sidjt am Femfer bilt. Eie vermefrt fid unisemein ftare surd) 9 surzelbut, und wird unt ein Sabr umb an= bere verpflanzt.

$$
\text { Ixora, sxore, IV. } 1 .
$$

I. coccinea, fortadtothe store, $\zeta$. Sfindien. - Ein niedriger Etrandid, mit lorberartigen immerguthen Blitterth, Die in Der Sthent, fo wie die juth= gern sweise, gelbrothlid) fins. Die stument, weld)e im Eommer zu unbeftimmten zeiten erdoeinen, Gitsen eitue

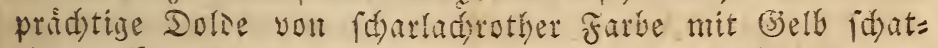
tirt. Es folgen allf Die Blumen orangerothe Beeren. Nan gebe ser Srore nid)t zll fibere, nahrhafte, mit Eant verfehte sauberde, und pfonze fie im 2husut oser

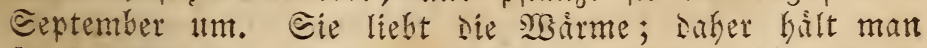
fie im Flibling gern in Ireibeaften, oder zwifdent Dop=

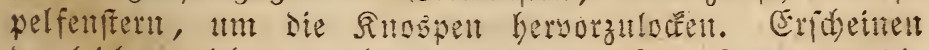
serigleiden nidyt, fo bringt man fie (Enve șunty an sie suft, an einen gefdithten, nid)t gar ju fomizen Plats, Sim SBinter babe id fie mit ien camerfin bey 4 bis 6

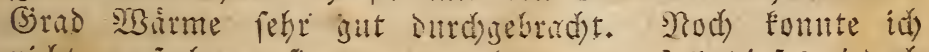

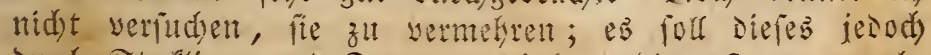
Durd) Etedinge und Eamtut gefdehen; Die erfern wurzeln 

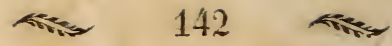

fdwer. Dieje Pflange gehort zmar nod) unter die feltnert; allein fie if fehr zu emfehlen und eben nidjt fdywerio zu bebanielit.

\section{Kalmia, falmie, X. 1.}

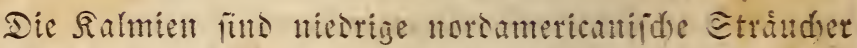
mit intmergrimen Blittern umo nieslidsu, fiaden, glocent= formigen Bzamen you rofentotber farbe nom fomarzen

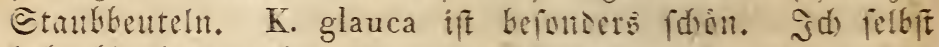
Gabe bis jetst nod) Eeine stejer Rtren gezogen; fie merden jesod) wie sie gelfenftránder (Azalea) oser nepentopen be= banselt.

\section{Lachenalia, sad)enalie, VI. 1.}

L. tricolor, stenfarbige and)enalie, 2. Cேap. - Die swiebeln fino weislid), etwa jo gró wie flinten=

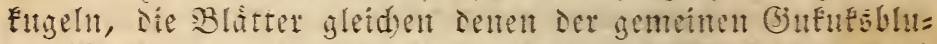
me (Orchis maculata), baten and athnlide Fleffe, Die jered) verfowinten, wenn bie Pfanze zu warm felt. Sie blu= menftangel wersen 4-S soll hod), mo trajen eine Irat= be sou 1 bis $1 \frac{7}{2}$ solf langen robrigen (s)tockden, Ieren

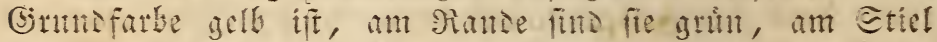
zimoberroth gefurbt. Sie seit ser Blithe if ier glay.

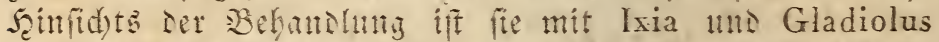

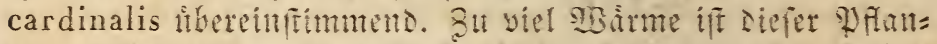

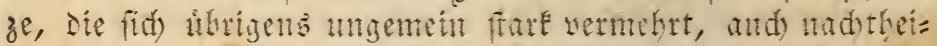
lig. Bey guter Befandung mo émem paffenten fomigen Etano blith dieje swebel oft idjon im februar, mo ge=

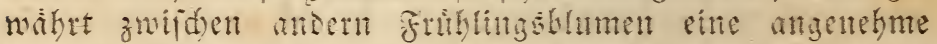

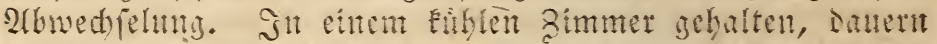

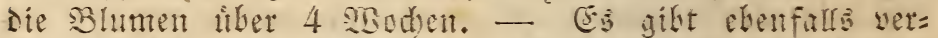

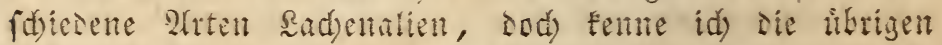
nod) nidit.

\section{Lantana, $\Omega \mathfrak{\Omega}$ attana, XIV. 2.}

1) L. aculeata, fradige ligentana, h. Euts= america. - Die pardveife fehenden, meliffentigen

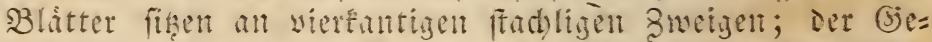

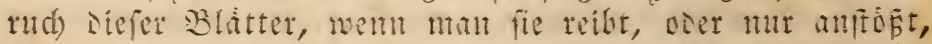
ift manzenefm. Die Blumen, welde zmifhen sen Blit= tern auf 2 bis 4 solf langen Eticlen bervorfommen, be=

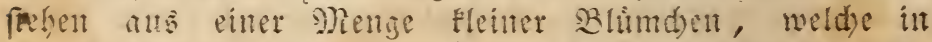
eille flad) balbuthelformige Dolde zufammengedringt futh. 
SBentu fie fid offuen, fo baben fie eine bodigelbe Farbe, tur werien almathlig sunfelorangeroth. 2uf fie folgen fdrmarglate Beeren, fart you ser (jeffalt ser gemeinen Brombere. - Jian gibt ier santana nafrbafte sauberse แnI Ies Eommerb viel SBaffer; entweser lä́t man fie be= ftindis im Bimmer fteben, oder man bringt fie vom Suny

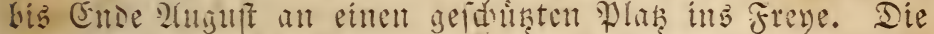

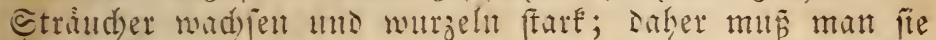

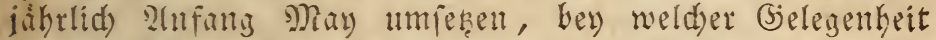

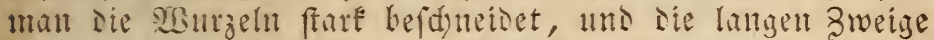
einfutht, leb̧tere werien zu Eteclingen verwentet. Sm

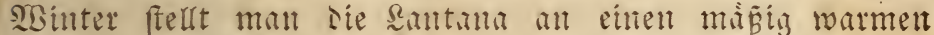
Drt you 4 bis S Sirao, litdit zu weit yom Janfter, wo fie etwas Eontte befonmt. Die Blatter verltert fie ge= meiniglid) wâfrend Des sointers im Bimmer, woyon der

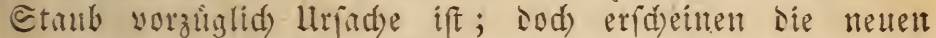

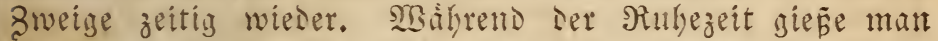
nid)t zu reid)lid). Sont bie santana jedod) nud im NBin= ter eimen red)t fommigen Etant und 10 bis 15 Grad Bar= the, fo bleibt fie nid)t allein grtut, fonsern blifht aud bis

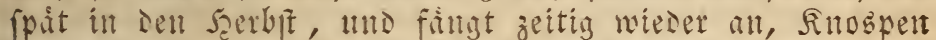
zu treiben. Die Etecklinge nadjen beynthe in jeser Sath= reszeit, uns blithen fdon ber 6 bis 8 zoll sobe ren gan zen Eommer biusurd.

2) L. camara, yeratnserlid)e santana,

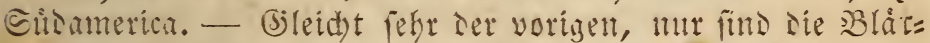
ter uns Blumen etwas Eletner, mo lestete mefr rund. Shre Farbe ifr, went fie fid offnen, citronenge!b, und geht surd) alfe $\widehat{E}$ dattimuselt ins sothe, mo zuweilen bis ths Carmintothe uber; weśbalb siejes sen ganzen Eommer ther blifhente Bămdyen eine fehr fdone Bierptanze ift. Hebrigens mit Nir. 1 gleid).

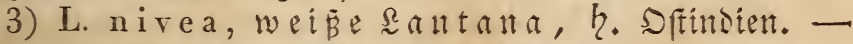
Cie kat febr fark mit Dornen befeste vierfantige ameige, grófere, etwas glătzende Blitter, ntto weife, innerhalb gelb gefarte Blimaden, mit fdwadhem jasminartigen (j)e= rud), Iet jedoch bey ser geringften Beweinnug surd Den wiserliden Gernda der Blatter yerdrangt wird. Die silu= men erfdeinen gemeiniglicí) in Epatherbit, nno entwideln

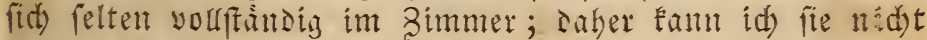

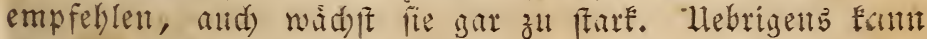
fie wie 9r. 1 befandelt wersen.

4) L. rosea, rofentothe santana. Solf aime 


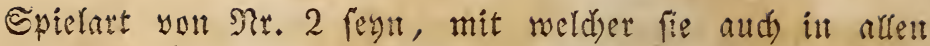
Etucen, bis alf ite Farbe Der Blumen libereinftimmt,

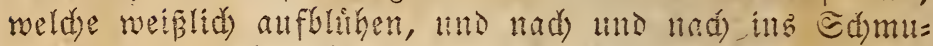
higounfelrofarothe dibergebent. Sid) kemte ald cine aus Eamen gezogne Epielart yon Diefer tmo L. oculeata, in weldyer beise farben vereinigt fint.

5) L. trifoliata, Degblattrige santane, Sorsmerica. - Sie Gat einen nubewehrten, megr frant= artigen Etangel, als die thrigent alten, longere, taube Blátter, die immer zt ireven ftehen. Die Elemen lilafar=

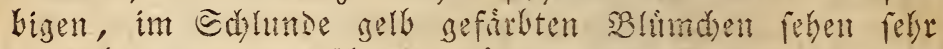
niedid) alls, und erideinen hiultis. Die Beerent find bets violett. Der Same feimt gut, uno da hbrigens die alten Stocfe fich mitr bey einem yorzinglid) guten, fonnenteidsut Etambe Durdwintern laffen, fo mus man immer Daranf besadyt fern, fid einen sorrath yon Sornern anzurdaffen.

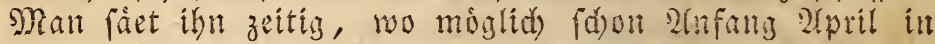
Iopfe, Die man in ein Melonenbeet fellt, damit die Pflinz=

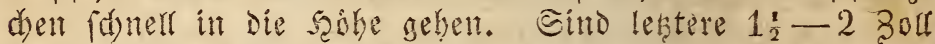

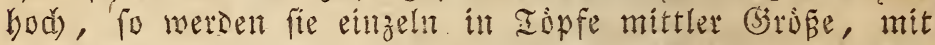
guter sauberde gefulat, gefost, uno went es fern kann, fo range im Miftbet gehalten, bis Snospen erfdeinen, was

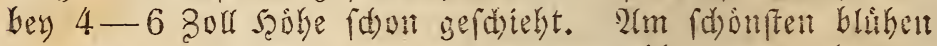

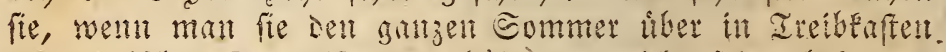
oder zwifan Doppelfenfen batt, und nidat felten befommt mant and bey folder Behandung foun int erfen sahte

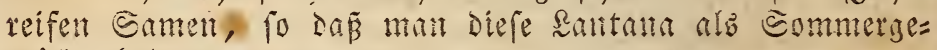
wàdys behandeln famt.

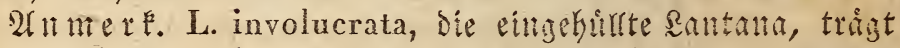

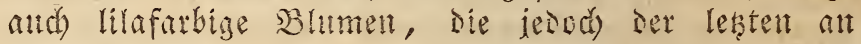

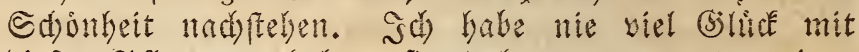
diefer Dfanze gehabt, fie Daber ganz aus métmer Eammlung verbantt.

\section{Laurus, 细tieer, IX. 1.}

L. nobilis, ber gemeine rorber,

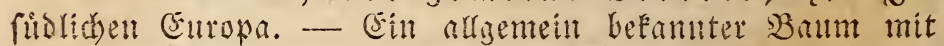

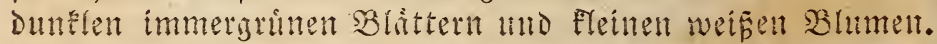
Mran behanoelt ifll wie einen Dringebaum; Dod vertrigt er too ä) mehr sálte, ia er foll fogar, wie alts mebres

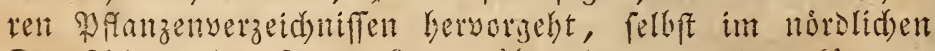
Dentfidand im freyen sanse iberwintert merden fomen. S⿹\zh13一 
Erfahrungen Dariber, bod) bezweiffe idl die unbedingte

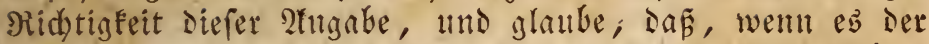

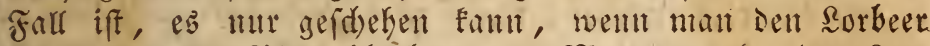

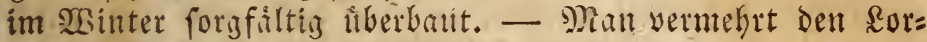
beer leid)t burd) SBgurzerferofferi.

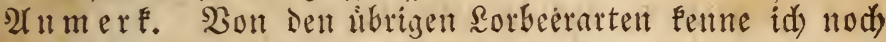
Feine genalt. L: camphora, Der Siampherbaum, unto L. cassia, Der Bimmtbaum; fint beide nod) feltent. L. Benzoë unt L. sassafras fint Lanopflanzent.

Lavendula, sayendel; XIV. 1.

L. multifida, vieltbeilige savendel, $\overline{\text { q }}$.

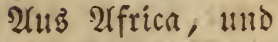

L. pinnata, gefiederte savendel, 2. Ma= dera. - seide 2lrten, befonsers leţtere, fintet man baill fis in Dent Etubett. Eie lieben lefmige, etwas mit Sand

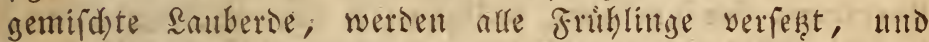

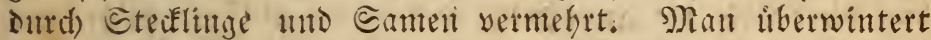
fie bey 4-6 (3rado nidit fern yom Fenfter, indem fie fonft

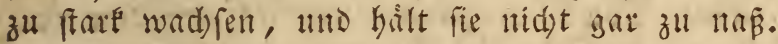

$$
\text { Ledum, Profd), X. } 1 \text {. }
$$

L. palustre, wilser Rosmariti, d)es Dentid)hand. - Diejer Fleine Etrand finset fid in

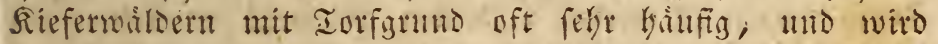
2-4 Jus hod). Die jungen! aweige fund mit toff farbuten

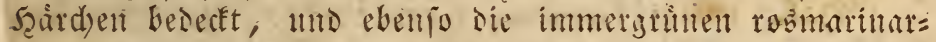
tigen Bhatter anf Der untent Eeite. Die sBhmen Fommen

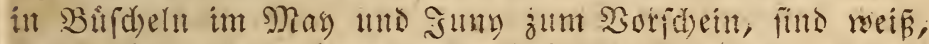

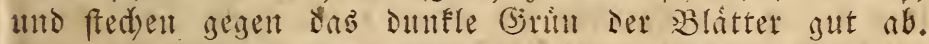

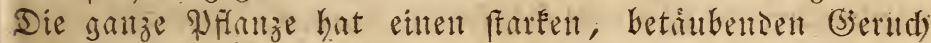
unto giftige Eigenifdaften. - SBill man fie im Iopf jies

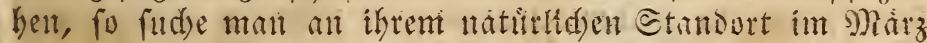
oser Sctober fleitle, 6-12 zoul bohe Pfiatzen; Diefe wer= Den mit Balten beraubigeftodben, mo in getánmige Iopfe

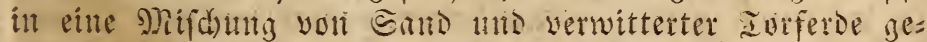
pflangt. Mian gibt ihnen beftansig einen etwas febattigen Etants, reidylids. Fetid)tigfeit, uno liberwintert fie bey 0

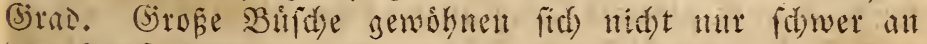
Den Iopf, fonsern fehen mid) nidat fo gut aus. Man

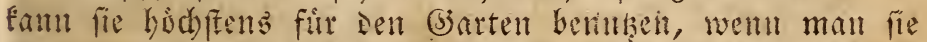
im Februar oder Márz mit Bałten franeportirt, uno bie

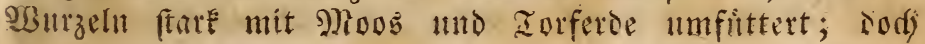


fins audi) in biefem Fatle junge Pflanzen paffender. Den Profd) yermebrt man burd) 2(bjenker. 2(us હamen lást er fidh fdwerer erziefen; diefer mun, wie der yon Rhododendron, befandelt werden. - Sd) babe nod) nie felbft

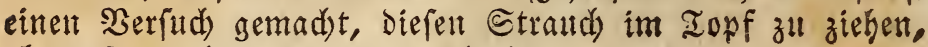
aber oft gefehen, dás ez gefdieht.

\section{Leptospermum, Sud feemyte, XII. 1.}

Diefe Båumden, weld)e fîmmtlid) aub Uluftralien ftammen, baben feine 3weige und myrtenábnlidse Blåtter, oder feine Nabeln. MJebrere wachfen fehr hoch, andere bleiben niedriger. Die. Blumen gleidyen Denen der Mryte, find weí, bey wenigen 2 (rten rothlid). Sie Ginterlaffen Heine Rapfeln mit vielen feinen Samen. Shre Beband= lung ift ganz Der der 2Relaleuca=2(rten gleid); am belten zieht man fie aus Eamen. Sid) enne folgende 2trten: L. flavescens, juniperinum, obliqua (id) habe nod) nie gebórt,

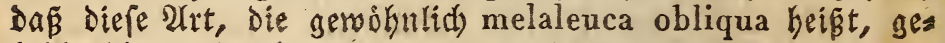
Glübt bătte, fie låjt fich Durd Steckltnge vermebren), scopa-

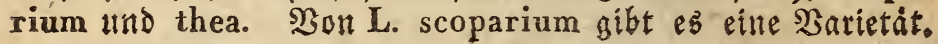
L. s. multiflora, bie fehr viel Blumen tragen folf.

\section{Lilium, silie, VI. 1.}

Unter den silien gibt ez fehr fdone 2rrten, weldje im Iopf gezogent zat werden verdienelt, wiewoht die meiften im sande weit fdoner bluben.

1) L. bulb iferum, Fenerlilie, 2\%. Sibirien, aud) in Dentfdand an vielen Soten. Man hehe Die fdups pigen 3wiebeln im September voer Sctober alls der Errbe, lege $3-5$ Der ftárfften in einen geråumigen Topf, und

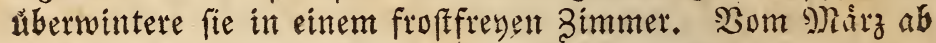

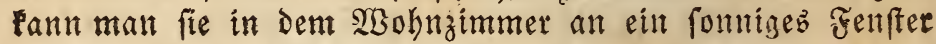
ftelfen, und zu treiten anfangen; fie bedurfent alsbant viel Waffer, und blúben willig. Sift der Iopf nidut zueng; fo lást man fie zwey Sahre darin ftehen, alsonnt bringt mat

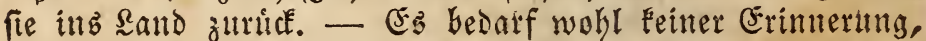

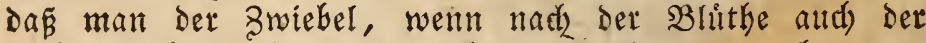
Stangel abgetrodfuet iff, nidyt zul ftark siept; aber maut mú fie aud) niemals ganz aub̆troufnen laffen.

2) L. candidum, die gemeine weífe Rilie, 21. Enrien 1. F. w. - Sie ift im Iopf nid)t leid)t zum SBluken zu bringen, jedod) erreid)te id) ber folgender $\mathfrak{B e b a n d}=$ lung oft meinen 3wed. Śeitig im Frúblittg bob id) eitte 
einzeln ftebente ftarte zwiebel mit bem Ballent alls dem sanse, und ferste fie in cinten gerátumişent Iopf, ber mit

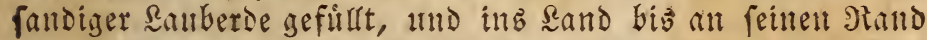
eilngefenter wurse. Dafelbft blieb er ftehen, bis die erfent Strobpen fid bffiten wolten, worauf er eitnen @tand im

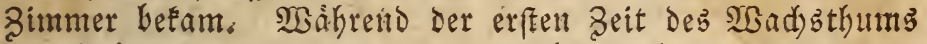
bez Ctângelz mus man ben Iopf bey anthaltendem Regen= metter gejen zu viele Feudjtigkeit (d) itsen, fpảter fdabet biefelbe weniger, Dutd) Sigarme die weipe Rilie zwingen zu wollen, zeitig zu bltihen, mod)te mir niemals glücken,

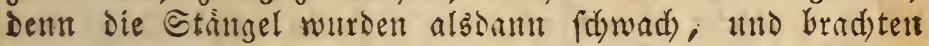
gar Feine, oder nur fdled)te Blument. Nadjoem fie abge= blift haben, feht man die Bwiebeln inz sand zurtef. -

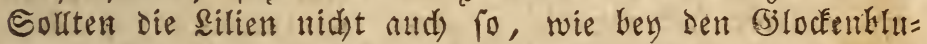
men gefagt wuree, fid in Iopfen ohue Bodedr jum Irei= ben yorbereiten laffen? - Diefe misten jedody tief gennis

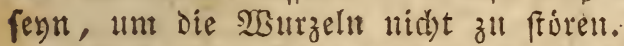

3) L. tigridum, Die Iigetlilie, 24. Sapan.

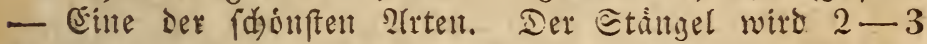
Fus hod), ift mit lánglidjen Bláttern ganz befeht, uno trảgt oben 3-10, aud) wohl nod) mehx, grofe hängende Blumen mit zurictéged)lagenen Blättern. Shre Farbe ift orangeroth, nut die Blätter find mit braurfdyarzen Punc= ten didjt befàet. Sie wirb wie Ni. 1. behandelt, Darf aber im fribling nidst ju warm gehalten werden; felten

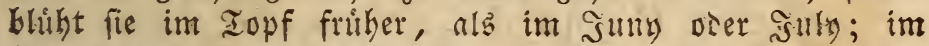

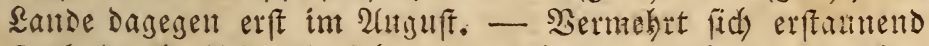

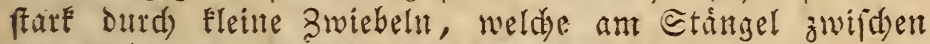
ben Bláttern erf́d)ẹitteti.

If tt ther. L: catesbaec tno L. superbum forfen pridd: tige 2frten feyn, idy Fente fie jedod) nod) nidyt.

\section{Lobelia, ̊obelíe, V. 1.}

1) L. cardinalis, Carditalabtame, 24. 2ir= ginten, Carolina n. . w. - Der Bltmenfdaft nird et=

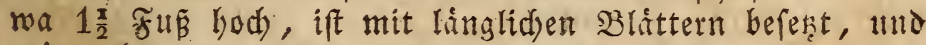
traigt oben eine lockere 2lehre yon buttothen Blument.

2) L. fulgens, feurige sobelie, 24. Nexico. - Der vorigen áfnlid), aber viel fáneer; die Blikter fino mit feinen graumeisen frardsen fammetartisy bedeckt, dic Blumen fino ebenfalls fammetartiy, brenneno carminroth. Beide arten befommen lehmige, mit grobem Eanoe gemengte Erde, nidht zu Eleine Iopfe, ఏes Eommers yiel 
Waffer, und Fosment fey 2-4 Brab Ş3arme uno gerill= ger Fend)tigfeit fefre leidst ourdymintert werden. Sim 2rprif verpflanzt man fie, nimmt die Nebenfroffent ab, und febst Deren nie mehr, als 3-5, in einen Iopf, fouft nehnen fie einander bie Nahrung. Sbgleid) Diepe sobelien zumei= len im seande aubhalten, fo find fie bod) im grubling fegr empfindlid) gegen jeden Froft.

3) L. erinus, fleitle $\stackrel{\Omega}{\sim}$ belie, $\odot, 24$. Cap.Die Stångel find Eriedsend, fpannentanb, mit feinen Blátt= d)en verfehen, unt tragen Den ganzen Eommer luber viele fleine betrblane Blimdsen, Deren Farbe Durd) weíse und Dunfelblaue Puncte geboben wird. Man făet einige Fón= d)en Samen in einen Blumentopf auf feine sauberde, bes becte Diefelbe faft gar nid)t, fprengt fie zumeilen mit $\mathfrak{S B a f}_{3}$

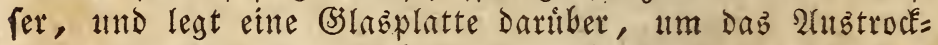
nen zal verhindern. Sobald es gefthehent kann, gewohnt man die Pflinzdyen an die suft, worauf fid Die Blum= d)en bald zeigen. Die feinent Samen liegen in Eleinen faps feln, die man, fobald fie gelblid) werden, mit einer Edheere

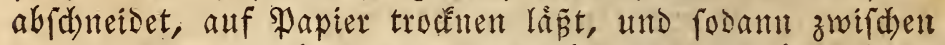
den Fingern zerreibt. S'm September oder Sctober fonei=-

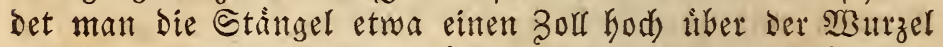

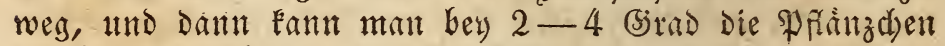
oft bis zum naddjen sabre erbalten.

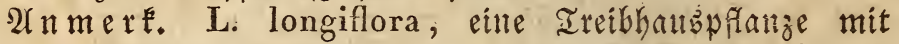
meisent uno L. sylphytica, eine antrpfanze mit blaten Blumen, Fenne id nod) nidyt.

\section{Lonicera, (befbblatt, X. 5 .}

L. sempervirens, $i m \mathfrak{m} e \mathfrak{g} \mathfrak{x} \mathfrak{a} \mathfrak{e} \mathfrak{b}$, (s) 7. Mexico. - Die Blítter gleichen Detten Deb gemeinent

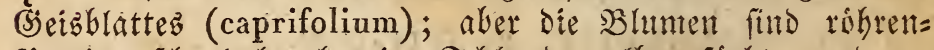

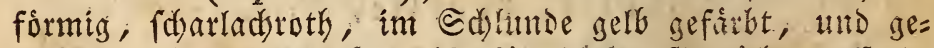

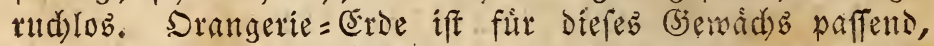
uno es verlangt geraimmige İpfe, to wie den Somnter liber Ginlånglid) Fend)tigfeit. Bey 0 - 4 (5)rad diverwitl= tert man es̉; fobald im Fribling Die itmigen Iriebe bersot= brechen, ftellt man es nabe ans Fenfter, und gibt ism zu= meilen suft. Die Blumen entwidéln firb) f(t) neal, and er= (d)einen bis in Dell Sctober binein fortwährend. Şålt man diefé (Seišblatt dagegen etwas zat warm, fo gehen die et= ften $\mathfrak{B l u m e n}$ gemeiniglidy verloren. Dent Esmmer liber ftelft man es yor die Fenfter; und zieht bie langen Ran= 


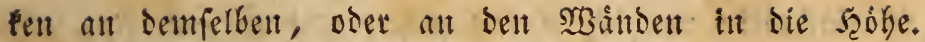

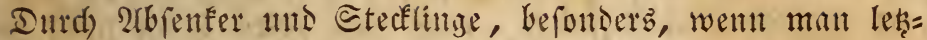

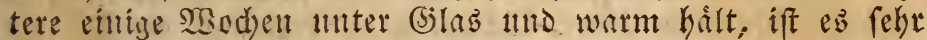

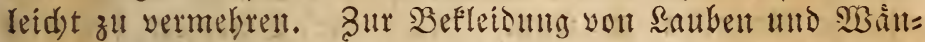
Dell ill Fleitlelt (s)irten ift Diefe 2lat befonders zu empfehlent. Die in Iopfen fehenden Pfranzen werden im Sctober oder

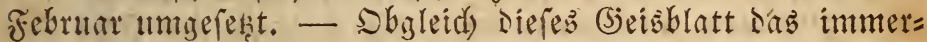

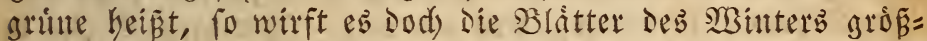
tentheils ab, felbft in Drangeriebătlfern; paffender fheint mir Daker Der. Beyname semperflorens zat fendt, dent es trảgt Den grósten Theil Des Sabres itber Blumen, felbft im SBit=

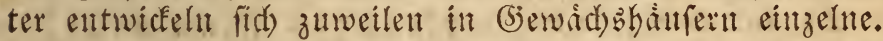

$\mathfrak{A}$ it merf. Die tibrigen Lonicera, z. $\mathfrak{B}$. caprifolium grata, priclymena, tartarica u. f. w. babe idi) nod) nid)t im Iopfe zu ziéren verfud)t. Die leķte 2Urt, eill allfred)ter (traud) mit weisen, rofarothen, and)

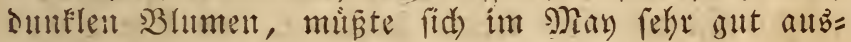
nebmen, went man fie in Iopfe ftellte. Sie wiro altb Etecklingen gezogen, sie mant im Fruthling mad)t.

$$
\text { Lotus, Sctiotentele, XVII. } 5 .
$$

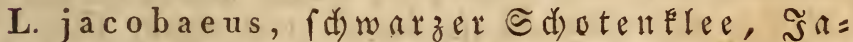
cobselee, 2. Dfturien. - Man finet bent Samen itr Miftbeet, und verfetzt die jungen Pflatzen, went fie zwey Solf hod gewordent fitio, in nidst zu leidste Rauberde. Die

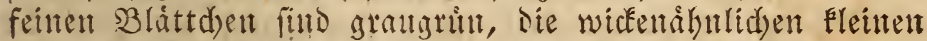
Blumen outufelbraut, fanmetartig. Da fie bereits im er=

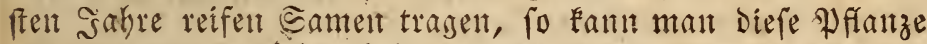
als Eommergewåd) behandeln. SYBill mant fie mehrere sabre erbalten, fo diberwintert man biefelbe bey 4-6 (5iad.

Lychnis, Flammentobden, X. 4.

1) L. chalcedonica, bieltiende riebe, 2.

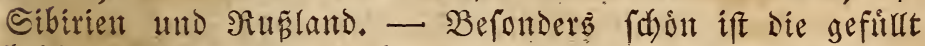
blithende 2rt. Nalt gibt ibr im Iopf lekmige, ftark mit grobem Eante gemifd) sauberde, und belegt Den Bodent Des Iopfes mit kleinen હteinen. Den Winter hiber bålt

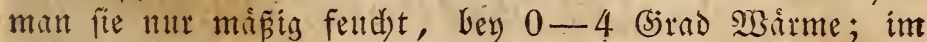
Frithling bringt mall fie zeitig in Die suft, wo bain bie Blumentantrgel bald treiben. Selbige werden $3-4 \mathfrak{F} \Perp \tilde{B}$ God), bod foll eb altd) eine ntestige gefildte Spielart ge= ben, bie idj jesod) nod) nidht Eente. Die Blumen find 
(d)arlad)roth; yon ber eillfadien 2tut gibt es aud eine weis Be und eine fleifd) fatbute, bod) fint beide lange nidst fo

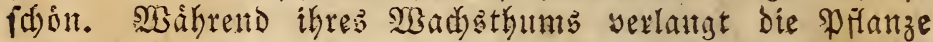

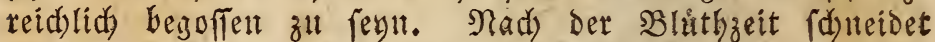
man die Ctångel ab, unb feht die Etaude um, bety wels

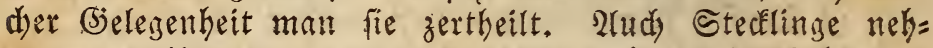
ment zolweilen an, wenn man bie હtångel in fed)s zort Iange Stucke (d)neidet, und an einer fdattigen Stelle in die Erode frect. @o Iange feine froffe eintreten, bleiben bie Iopfe in Der suft ftebent. - sm frenen sande gedeifgt fie beffer, als int iopfe.

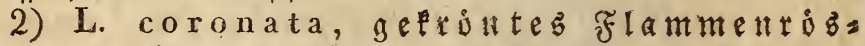
d) elt, 2. CGina und Sapan. - Die Blatter fitto fats=

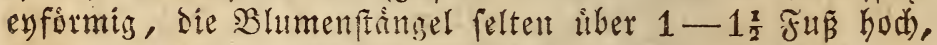
uno tragen ben güizen Eommer liber grope, einzelue oran=

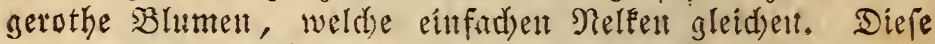
fdune Pflanze liebt leid)te Satberoe, unto ihre Behandints gleidst der von der yorigen alt. Nur iff fie zartlider, mitd verlangt zum Durdininten 2-4 (5ino, uno einten Etand nid)t fern yom Fenfer. 2(uper sutd) Bertheilung fam man fie Dutd) Stecklinge vermelyen, weld)e mant 14 Iage bis 4

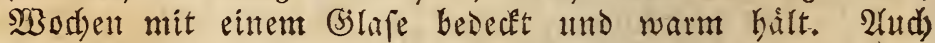

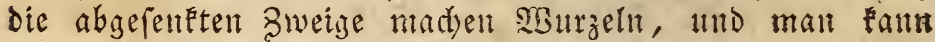
jeden derfelben all 2-4 Eterfen mit Erde bedecten, alfo

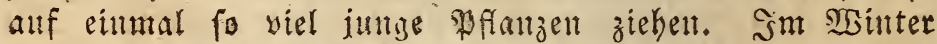
fd)abet ihr bie Feudgrigfeit fehr, fowie aud) oft eine Orrt

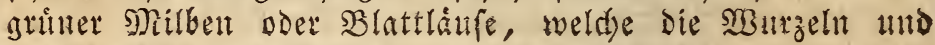
jumbell Broeige zerftorent.

3) I. viscosa fl. pl., gefullte peduelfe. Dentfdiland. - Eie wird wie 9lr. 1. behandelt, und ifre caunturotben Blument mebment fith im Iopf red)t gut ans.

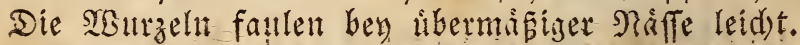

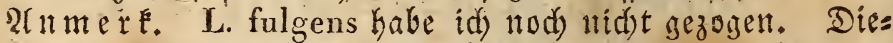

fes Flammentrobdent hat mit Der einfad)en brennenden

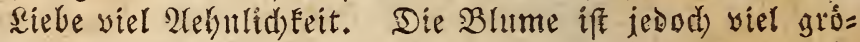
Eer, Der Blumenftringel aber niebriger. - Nat ziegt fie alls Samell.

$$
\text { Ly cium, Bod }
$$

I. jap onicum fl. pl., geflilter, japantfder

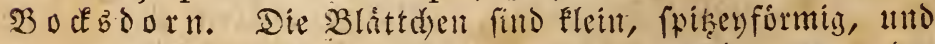
bie Eleitren weisen, gefitrten Blimdsen gleidjen senten ber

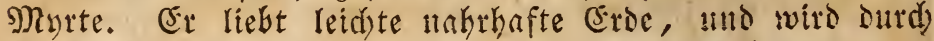

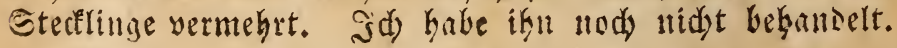


Magnolia, Magnolia, XIII. 6.

M. grandiflora, grof́blumige Magutie, Florisa. - Diejer fdóne Banm foll in feinem Saterlande eine frobe you 80 Fun erreident. Dennod) zieht man ifn it faft alfen (j)înten im Topf. Die grofen, glinzentogri= nen, lederartigen Slatter find linglids)=elliptifd), zugefpilit, bauterns, unto auf Der untern Eeite mit roftbrautem, fei= nen Filz hiberzogetr. Die Blumen erfdeinten an ben Epi=

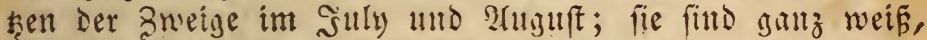
flad), halten etwa 12 zolu im Durdimeffer, mo Duften orangenartig. Bisher habe ids diefe Pflange nodh nidjt felbft zezogen, Gin baker mur im Etande, etwas ärges meines iber ifre Eultur zu fagell. Sie verlangt $\Omega_{2} a l b=$ erde mit $\frac{x}{3}$ verwittertem Torf und etwas Sallo gemifd,

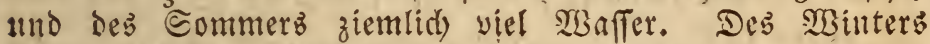
bált man fie in einem belfen Bimmer yoll $0-4$ (Sirad

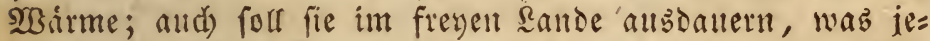
rod) in Den nordidyen (Siegenten nidst anzurathen ift. Man Eennt verfdiesene Epielarten, die wahrid)einlid surds die Eultur entfanden fint. Eintige von biefent bliken fdon in

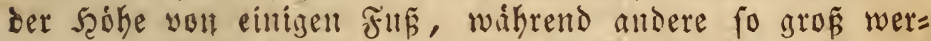

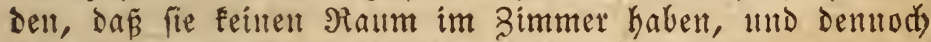

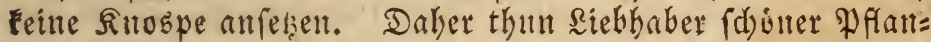
zen wohl, went fie mur you einem zuyerláfitigent frandeläs gertter, welder Die ted) te frubblibente Eorte (M. g. exoniensis oder praecox) befitit, fid) eine Magnolie baufen, uno zahlen lieber ein \$aar IGaler mehr, weil fie fonft leid)t in ifren Erwartungen getiufdet werben burften, uno vielfeid)t nie eine S3lume erbalten. Beponders muE man fid) huten, Eamenpflanzen zu befommen, die oft fehr birlig find, aber (nad) (batesby) daran erfaunt werden, saz der braune Filz Den Blittern fehlt. Ferner faufe matt foldhe Etimmdid, an Denent man 3weige bemerft, Deren Blatts ringe red)t Didgt auf einander ftehen, benn yon biefen fann man am eheften Blumen erwarten, wie man mir verfidjert hat; To lange die Blitter $3-4$ zoll und mefr yon eits=

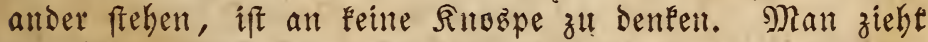
die Magnolien, intem man einent zwẹig Dutd) Das untere sod) eintes Blumentopfes wadjen låấ, und ihn etwas ein= Ferbt, jebdd Dauert es in der Reget zwey Sahr, ehe matr

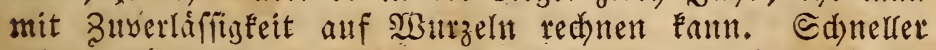
mógen 2 rb fenter annehmen. Die Magnolien wad) fen fdyelf,

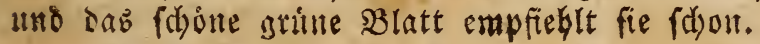


IXmerF. M. c onspicua habe idy in ber Seble yon $2-3$ Fur mit finospen gefehen, bod) die Bltumen felbft fente id) nod) nitdt. M. ob ovata (purpurea) bat Eleinere tulpenartige $\mathfrak{B l u m e n ,}$ als M. grandifol., beren Blatter auferbalb roth gefortbt find; fie foll rehr willig blikgen, und vermehrt fid durd) Stbeneer. Bei= de 2frten Fònnen wie M. grandifol. behandelt werdent. - M. gla uca tullo tripetala finto aldí) zut $\mathrm{em}=$ pfehlen, aber mir nod) wenis befannt. - M. pu-

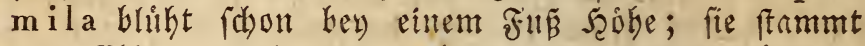

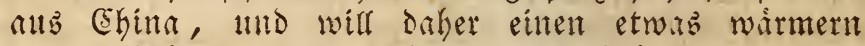
Stant Gaben, als bie hubrigen; idy babe fie gemolnt= (id) int Ireibgăufern feken gefehen.

\section{Iahernia, Mafernie, V. 5.}

1) M. odorata, wohlicidende Raferite, (6ap. - Diefer Stralld midjit $1 \frac{\pi}{2}-2$ Fun hod), hat gelbgrime fämale Blâttd)en, weld)e mit langen einzelnen,

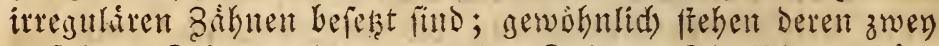

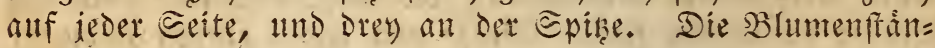
gel fitto $1-1 \frac{\pi}{2} 80$ ll lant, und tragen faft immer zmen funf: blittrige, glocétiformige, hatugende gelbe Blumen. Der (Serud) berfelben ift ziemlid) ftare, insntinartig. Diefe (si=

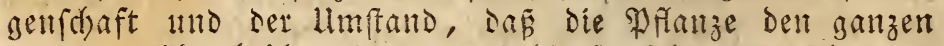
Sommer diber blifhen forf, empfiehlt fie rehr, uno id watl= Dere nuid), das man fie in faft gar Feiltem Pflanzenverzeid)=

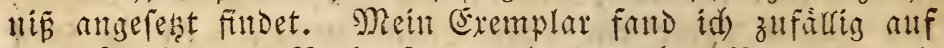

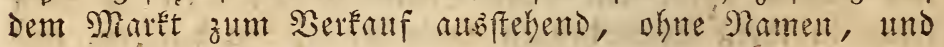

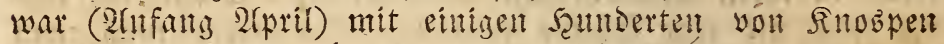

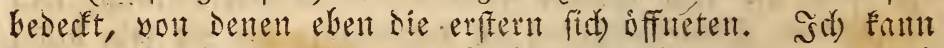

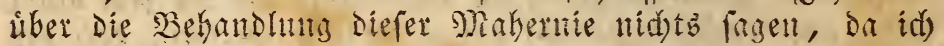

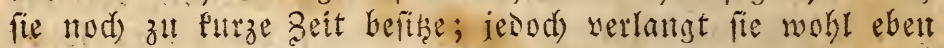
Die Cilltit, wie-die folgente, uno vermegrt fid) surd) Etedelinge. - Soldte fie mobl nod) unter einem zweyten Nament geken?

2) M. pinnata, gefiederte Nabernie, (Eap. - Diefer 6-10 3oll kohe Strauld tritgt feine und vielfad) zertheilte Blitter, weldye fark Den inallzen Sommer diber fleine glockenformige Blimdsen tragen; fie fins rofa= roth, unt jeses Blatt mit einem sunflen Etreifen verfe=

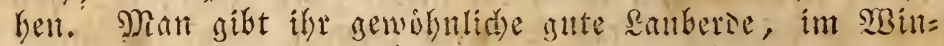

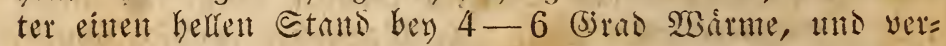
melyrt fie leitht surd) Etedelinge. Sm Eommer will fie et=

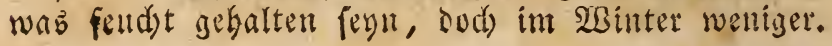


Martynia, Jempenhorn, XIV. 2.

M. annua (M. proboscidea), içhrige (5) em.

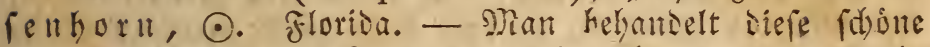
Whanze wie Datura fastuosa, iod) lielit fie etwas mekr

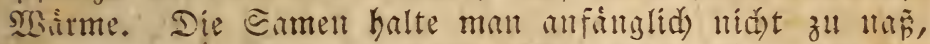
fie verberben fonft leid)t. Die rithliden, gelb getistelten

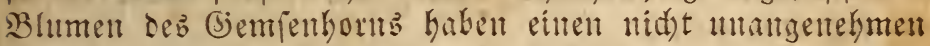

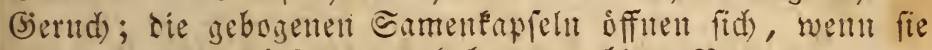
reif fins, zur Scilfte, mo baben zu obiger Benentunts we=

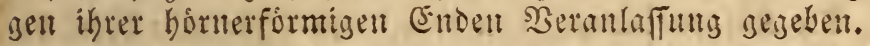

Maurandia, Maurandie, XIV. 2.

1) N. artirrhiniflora, Iómemaulảbnli= d) e Xa arandie, $\zeta$. Nexico. - Eine feine fletternde Pflange mit flethen 2 littden und rad)enformigen blauvio= letten Blumen; man zieht fie alls Sament. Befandlung wie bie folgente.

2) M. semperflorens, immerblubende $x_{R} u=$ ratidie, $h$. Mexico. - Die Blatter fino lang geftielt,

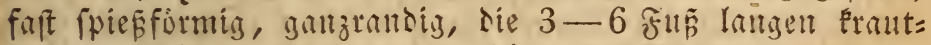
artigen Etininel Elettern mit Şulfe der Blattfitele an an=

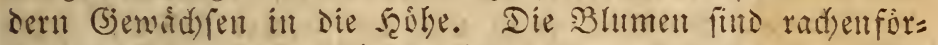
mis, rothlid), und erideinen Dent ganzen Sommer hinturth. Nan zieht die Nantandie ans Eamen, wermehrt fie ald leid)t surd) Etectinge, gibt ihr nabibafte (5rde, mo fellt fie yom 9) Jay bis Eeptember ill Die suff, wo mant fie an einer Maner oder an feinen હtiben, die mit einigen Suer=

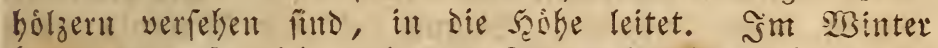

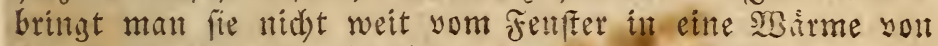

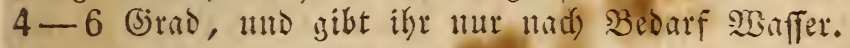

\section{Medicago, Sdluedentere, XVII. 5.}

M. a rborea, baumartiger ङ duefentee,

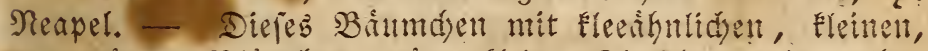
grangranen Blittdyen tringt Eleine Ropfdsen mit gelfen umbereutenten Blumen, auf weld)e gebogene şitien folgent: (Es verdint faum einen What int zimmer, befommt Srans

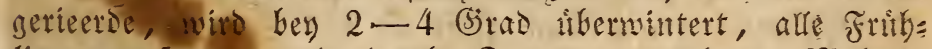
linge verfest, ulto Durd) Eamen vermefrt. Miefrere Medicago $=2$ rrtent fins, weisen ifrer fonterbaren Eament: fapfeln, es werth, als Eommergemadofe gezogen zu werden, Sd) nenne bier utur: II. ciliaris, coronata, murex uni 
nigra. Nhan legt den Samen (weld)er aus nierenformt=

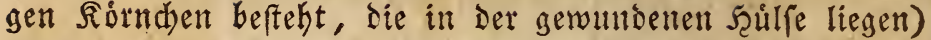
zul $3-5$ in nid)t zut enge, mit guter Erde gefiutte Iopfe, und gibt den Pflanzent, fo bald Eeine Frofte mehr zul furrd)= ten find, einent Etand in Frenen. Sie bedirfen alsdann

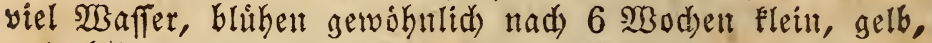
und bringen viel Gamen. 2(ud) im freyen sande ziehen fie fid feht gut.

\section{Melaleuca, 疋iaputbaum, XVIII. 2.}

Die Sajapntbaume, yon benen mun fojon eine betrad $t=$ lidbe Menge ver(d)iesner Grten in Dent fdland befantt find, ftammen faft fämmtlid) aus Neutholfans. Biele erreiden in

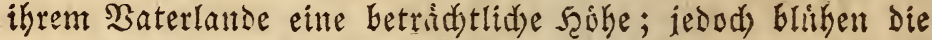
meiften im Topf, wenn fie mur einize Frti body find, ia einige (d)on bey 1 Fus Frobe. Şhre Blätter gleidant benen Der Myrte, Ifymian oder Der heiden, find mit ourd)fid $=$ tigen Puncten wie befäet mb baben einen Fampherartigen (5iefd)mack und (Sierthd, wenn mat fie zerreibt. Die Bhu= menblatter find fehr furz, dagergen die Staubfäden (wel= d)e gemofnlid) im (Sinnde in 5 Buifhel verwad) fen find) febr lans, und bilden, sa fie it Menge erfdeinen, und bie Blument meiftenz rund um die Brweige ftehen, did) te

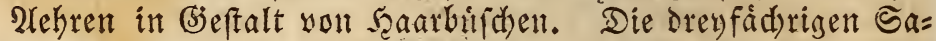
mentapfeln braudsen mehrere Sahre, ehe fie reifen, und man muz fie, fobald mant diefes bemerft, mit (Sewalt abbredsent. Nad)dem fie trocten gewordent fitto, fpringen fie all, und Die fehr feinen Findden fallen heraus. - Die Rajaput= båume liefen Setideerde. WBarnte besurfent fie wents; matt ftellt fie ben હommer viber ins Freye ant einent nidist gar zu fontigen Srt, und ilberwintert fie bey 4-6 (3) rad Särme.

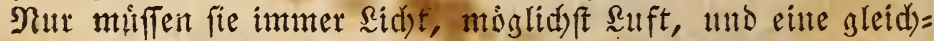
másige Feudtigkeit erbalten. Cie baben alfo mit bent Sretdent viel gentein, itho fint altd wie diefe fin immet yerlorell, went fie zll ftark gegoffen befommell, ober went man fie einmal z"l fath austrocknen lápt. Man fann fie zu Denienigen હtubenpflanzen záhlen, Die wenis Pflege,

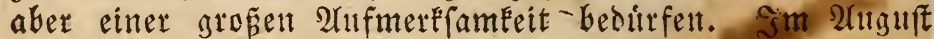
verfegt man fie. Man zieht fie alts Eamen oder Stede= lingen. Die Camenpflanzent blikgen aber int der Rezel fpät,

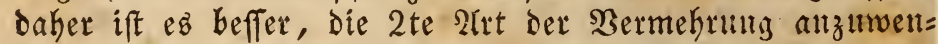
ben. Man finteidet zut diefem zweck entweder im 2upril, oder $\mathfrak{A n f a n g}$ May 2 3oll tange, paffende ziweige ab, ftect 
fie in sauberse, bebeat fie mit eillem Bjlape, unb bảlt fie warm, wo moglid in eittem Miftbeet; over man mad)t biefe Etecklinze 2(uffaitg Suln; Dann fteben fie aber zuweiler: bis zum Mád)fen Fritgling, ehe fie austreiben. Den Sa= men faet man allf torfige Grise, beseclet ign faft gar nidjt, oder mur mit einigen Rornd)en @ant, legt aber eine (sjlabs platte alf ben Iopf, Damit Eomne und suft die jarten Sei= me nid)t zu fefr austrodken. Die jungen pfatuzden miffen etwas fdattio und beftándis maşis felld)t gebalten werden, fonft find fie zumeilen alle auf einmal in eit paar હtuns ben Dabin. SYBenn fie nidjt ju Did)t ftehen, fo pflanjt man fie erft im zwentent Eommer eillzell itl Iopfe. - Sid) will utr folgente, bie mir genauler befannt fint, nảher be: (d) reiben.

1) II. alba (armillaris, linarifolia, ericaefolia), weišer Sajaputbaum. Er hat fdyale, lillenformige Blått= d)en, und treibt im Fruhling aus den ftatfern 2 teften 3 -4 3orr lanise weise Blumenbuifdel. Ër blifht erft in

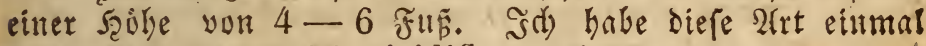
unter bem Namen M. viridiflora erbalten.

2) MI. coronata, fronentagender faiaput= baum, tait (d)malen thymianthnlid)en Blattern, แnb Fleinen, einzeln auf Den zlweigen ftehenden Blimd)en, wel= d)e gleidy fam eine belfearmintothe Frone bilden. Diefe 2 trt

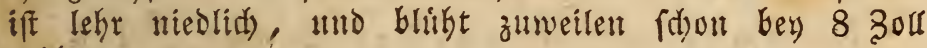
Şóke.

3) AI. hypericifolia, iohanuigerattblat= triger Raiaputbaum. Die efryptifden 3 látter find et= wa $\frac{3}{4}$ Bolf lang und unten mit einer ftarfen Rippe verfeken.

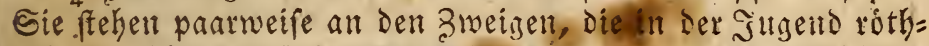
lidh und Gaingend fint. Die Blumen entfpringen im Frabling alls Dem @tanm nthb den ailtern Bweigen, find matt zin= noberroth, und bilden eine fingerslange, etwa 2 zolt farte 2iefre, atts weldser gemeilliglid) ser Bmeis fortwaddft. Eamenpflanzen werden in ber Regel $5-6$ Jun Kọ̆, ehe fie bliken; die alls Stecklingen gezogentent blikgen zu=,

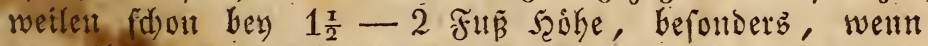

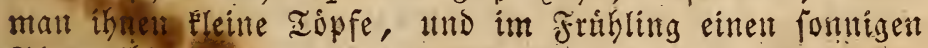
Plat gibt.

4) M. pulchella, nieblider fáaputbaum.

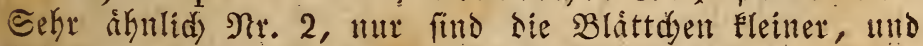
bláulid) grủn. Bhlúht ebenfalts frúl.

5) M. splendens, glátzenter $\mathfrak{\pi} a$ aputban. 


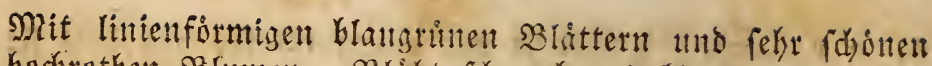

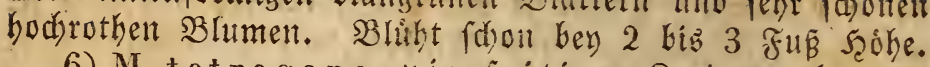

6) M. tetragona, vierfeitiger $\Omega_{a}$ iap $11 \mathrm{t} b \mathrm{a} \| \mathrm{m}$,

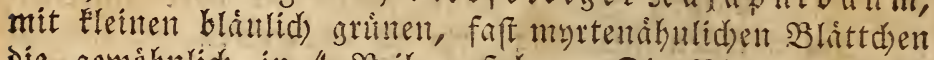
die gewibnlid in 4 Reiben fteben. Die Blument erfdet= nen băfig in Eleinen Uebren, faft an ben Episen Der Bwei= ge unt find rilafletfofarben. Sie blibt fdyon bey 2 3 Fun scoke. - Sd) babe fie alld) unter Dem Samen M. decussata enthalten, renigfens Eonnte id) Feinen gro= Ben Uluterifbied zwifben beisen futien.

M. calycina, densa, foliosa uno tenuissima baben ein (d)ones Silatt; foliosa blift grin in ber sobe von 3 - 4 Fun; M. depressa bat 2rehulid) feit mit M. pulchella, and blabt ebenfarts febr wirrig.

arre ubrigen Fajaputbitme, seren SBlumen ich jesod) bis jest nod) nidst fentre, setionen fdyon, wegen ber feinen nieblid)en Blátter gezogen z" wersen. - M. obliqua, (d) iefblättriger Saiaputbaum, ift ganz gewí̄ Feitte Melaleuca, fonderti ein Leptospermum, Sent die Bläter baben weder bie oben erwifhten Puncte, nod) Sen Sampherge= fd)mace, ber afent dibrigen mir befannten 2rten eigen ift. Blumen babe id) nod) nirisent an ifm gefeben, wiemohl idh felbft ein Exemplar vou bedentender Sjrófe und Státe berab.

Mesembrianthe mum, 3aretblume, XII. 4.

Die Baferblumen geboren zu- Den Fettplanzen. Die meiften 2riten trajen nierlidse Brimditu, Die oft Den affern gleidsen, tuto empfeblen fid basurd und ourd die ver= fdiesene (sieftalt Der Blitter fehr. Die Farbent, weldse bey den $\mathfrak{x}$ lument gewolynlid) youfommen, find gelb, orange, ounfelroth, violett, rofa, fletfd fatb, weiflids; émige futo mebrfarbig, sie meiften meftrifdiglánzend. 2rue. Baferblu= men (berent es weit liber buntert Eorten gibt) ftammen, bib

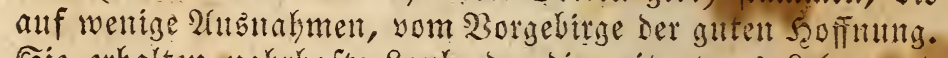
Sie erbalten nabrhafte sanberde, die mit etwas sebm und Eant yermifd wirb. Den Eommer uber, ment Eomte und Ruft frate trocknen, gebe mant ifuen viel sisaffer, Dod) menis bes NBinters, oder bey refr nubaltentert triber Mit= terung. Won Mitte May bis Mitte Eeptentber gebe man ben Zajertulumen einen gerduhten @tand in freyer suft, wo

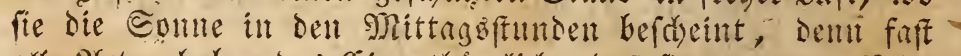

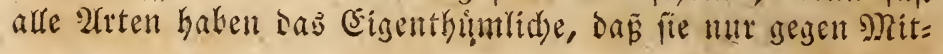


tag bliken, went bie Sontenftrablen mit aller Feftigleit fie treffert. Durd) Etedflinge vermebrt man die Zaferblumen

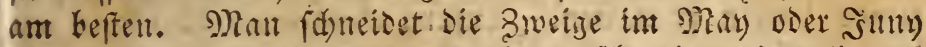
ab, lispt fie eintige Iage alt eittem fdyattigen Sot liegent,

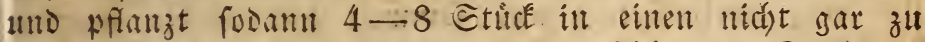

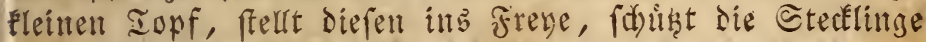

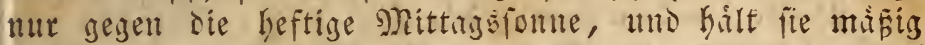

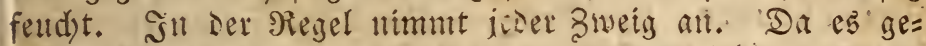
wobulid) wabrent ses WBinters an Raum fehlt, fo laffe

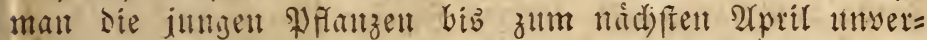
feht fteken, gebe jedod alsonn einer jeden einet befon= dern Iopf, und bittoe Die Sweige an Etochden, indem fie

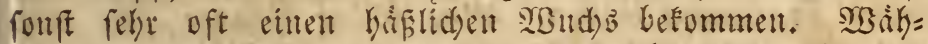
rend des WBinters ftefle man bie Bnjerblumen nake ans Fenfer, wo fie viel sidd), Sonne, uno an ghinfigen $\mathfrak{I} a=$ gen, befonters im Fribling, aud) suft belommen fontmen, zuto gief̃e ifnent mur batu, went fie anfangen wele za

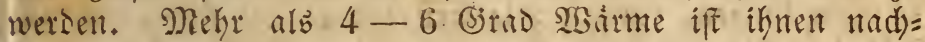
theiliz, bejonterz yom November ab bis Ende Februar.

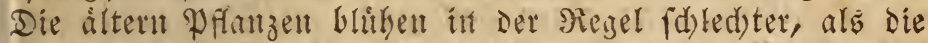

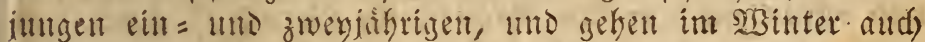

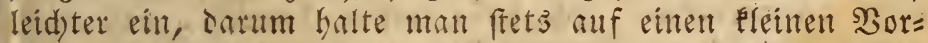

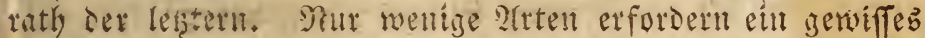
alter, ehe fie blibbar find, ober blithen alsoam reid)= lidfer, unt siefes bentert man fonon im 2 ten Sommer, went fie farte wad)fen, okne bुhment zut treiben, z. $\mathfrak{B}$. I. stipulaceum, maximum, pugioniforme etc. $\mathfrak{B o n}$ fd $0=$ ment mil befantuten 2(rten nenne id): M. acinaciforme, aureum, aurantium, barbatum, humile, bicolor, caninum, deltoides, dolabriforme, felinum, micans, pugioniforme, pulchellum, spectabile, splendens, tenuifolium, tigridum.

Sod) cinter náketh Befdreibung verstenen:

1) M. crystallinum, (5izkaut, Diamant=

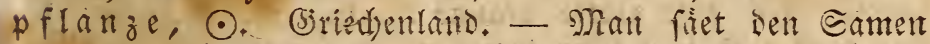

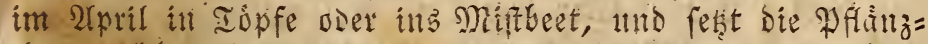

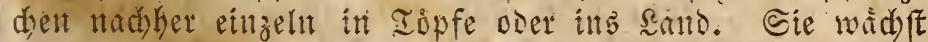

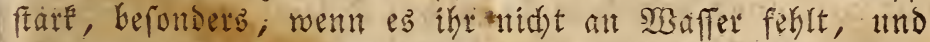
fie guten Bosen hat. Die Etangel liegen auf Der Erde,

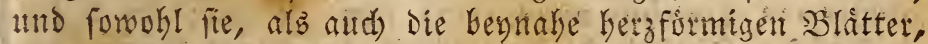
fino mit glabartigen Blásdjent uno fleinen Zadfent bedectet,

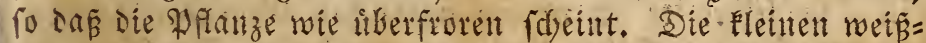

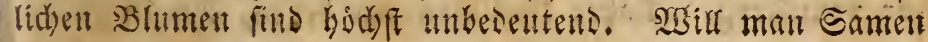


erhalten, fo ift ez gut, cintige Pfianzen in Iopfe mit mas gerer Erorde zat feşen, umb, fobald fie zul blithelt anfangett, etwas trodfen za haltent. 'Tltfang September Gort mant mit

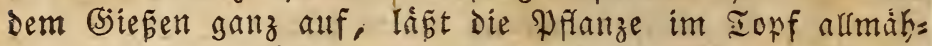
lig ganz vertrocfnen, uno fammelt bierall die Samen= Fapieln.

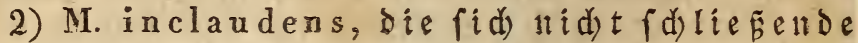
zaferblume, そ. Cap. - Die Blitter find Drenfeitio,

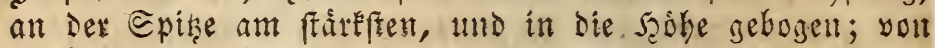
Farbe Dunfelgrin, die jungen Sweige find rothlich and et= wab breit gedritut. Die Blumen find etwa $1 \frac{1}{2}$ Zoll grof́, violettroth, glänzend, Hnd bleiben ben ganzen ĩag offen,

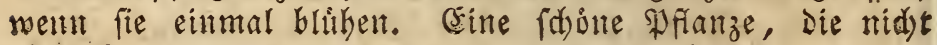

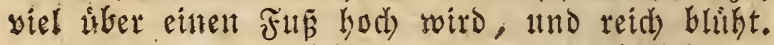

3) II noctiflorum, uad) tblibende 8 afers

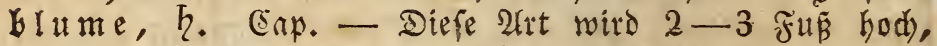
hat aufredste siweige mit weitlånfig ftehenden graugrinen,

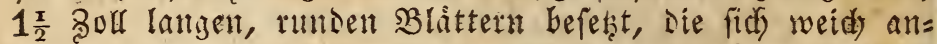
fühlen. Die gefillten Blumen find weip̈gelb, auțerbalb etwab rothlidy, etwa einen zoll grof́, am Iage gefduloffen,

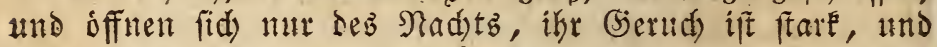

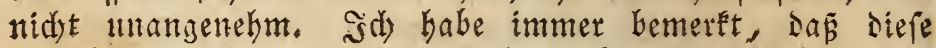

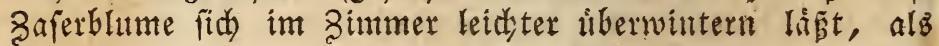
die meiften arderer 2 (rtent.

4) M. pinnatifidum, gefieberte zafertur me, ○. Eap. - Die krautartigen Stängel fitts fturz, liegen anf Der Erse, mo tragen lisuglide, fladje, halbges

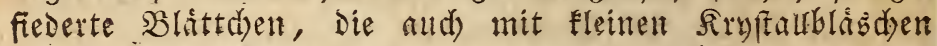
befest find. Diefe 2frt wito wie श?r. 1. bebandelt, uno trigt fleitte gelbe Blimdsen.

\section{Mespilus, Mispel, XII. 4.}

M. pyracantha, ber feuerburd), h. Fratie reidy und Stalien. - Diefer Etrand if fehr iffij, bujdig, dornig, Gat sille glatte braute Rinde, und wird 6-10

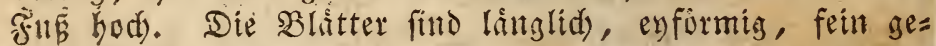
ferbt, glatt, dunfelgrun und ausoaternd. Die Blumen firto weis, mo erfdeinen in areiden. Doldentraulben; fie bin= terlaffen Eleine, runde, fdarladyrothe Fridste, Die Den Eber= efdenbeeren (Sorbus aucuperia) fehr gletden; fie reifert

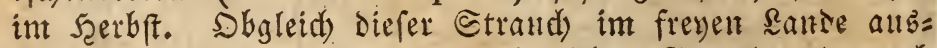

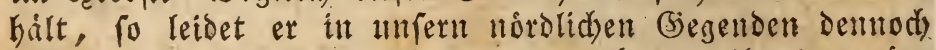
feGr yom Froft. Daber thut man am beftel, ibu in geráll= 
migen Iopfen ober fleitren Ribeln zu zieben, und ex vers

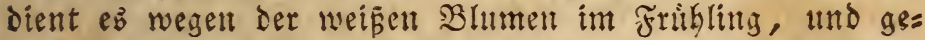
gen den $\mathfrak{W}$ inter wegen feiner fdonen fenerrothen Frudje, weld)e beide gegen dab Dutfle (jirut Der Blátter gut $a b=$

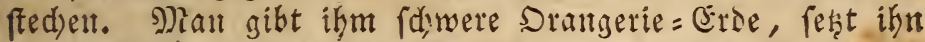
etwa alle ziver Sabr in 2rpril um, und liberwintert if bey $0-4$ (jirnd 2 Sarme. Sbigleid), er fid) gut Durd) Cas men vermefrt, weldyer ein Sabr in der Gise liegt, fo ift

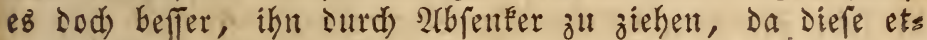
ras fruber bliken. Colrten nidjt vielleid)t alld Stedinge annebmen? - Fit Blumenliebbaber, weldse exine Sjelegens beit haben, Dent Fenterbufd liber Gommer im Scofe, oder in eintem (5atten an einen fiddern, gejduthten Plats zu ftels len, ift er wegen feiner (sirófe nidyt zu empfeblen.

2f $n$ met.. Won Mespilus oxycantha, iem gemeinen

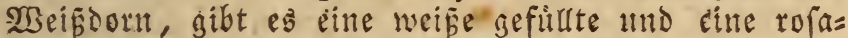
rothe einfadse und sefiute Epielart. Diefe bren find (5jartenbefisern fefr zu empfeflen, ob fie jeods allds im Iopf zum Blihen ju bringen fino, babe ids nods midjt verfudst, fouft founten fie wie M. pyracantha behandelt werden.

Metrosideros, (e) ifenmaá, XII. 1.

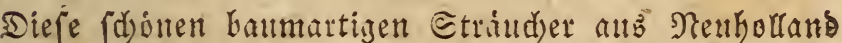

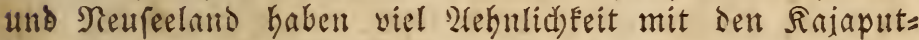
băumen, mur fird die sllitter aller 2laten, die id) Eenne, grofer, und die Blumen entfpringen im Frithling an bent Qinten der Zweige; Dieje futto ebenfars fo geftaltet, als die Der melaleuca alba ofer hypericifolia. '2ud Die Blatter

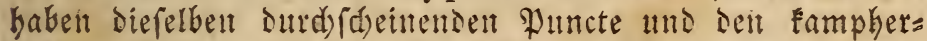
artigen Siefamade. Shie Befandung frimmt ganz mit ies jester salzer itbereit. - Eie unterfdeise!t fid yon ben

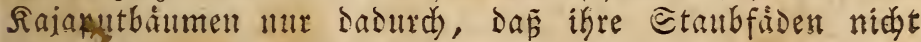
it Bundel veruad) fen fins.

1) M: citrina, orangenblattrigeb eifens maaß̧: Die Blatter gleidjen bennabe denten der grob́bláts trigent Nyrte, fint $1 \frac{\pi}{2}$ Boll lang uno etwa $\frac{x}{2}-\frac{3}{4}$ 80l breit. Die Bltment find bodroth. Whadf ftrattdartig,

2) M. lanceolata, lanzettblattrigez (5) fent

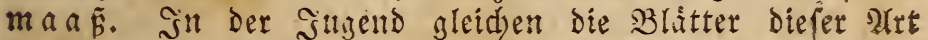
beunabe dettent iet vorigen, jedod) werdent fie im affer ims mer fdumáler, unb find alsbant 3-4 3oll lanis, bey $\frac{x}{4}$ 3oll

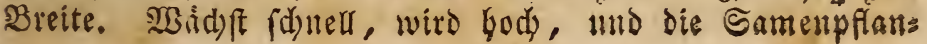


zent blityen felten eber, als bis fie 5-6 fill hod getoor:

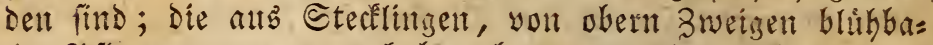
rev Pfanzen gezogen, habent bey mir fdyon Blument ge= bradt, ebe fie einen fHE hod) watent. Die Blumen find blutroth.

3) M. lophanta, bif

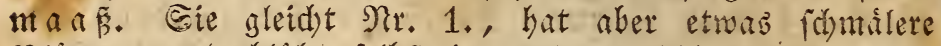

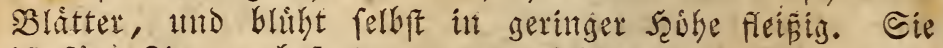
ift furs Simmer befonder zu empfehlett.

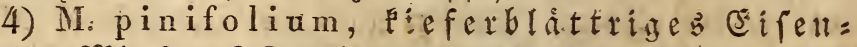
$\mathfrak{m a n}$. Nit $2-3$ soll langen nadelartigen BLáttern und grunliden Bhtumen.

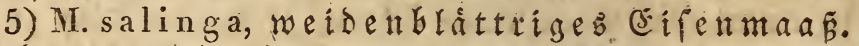

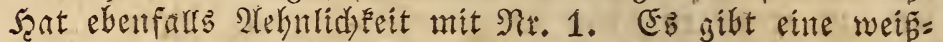
und eitue rothblukende atrt.

6) M. semperflorens, immerblubendes (5i fenma a te, Into tritgt das ganze sabr binourdy einzelne tothe Blumen zmifd)en Den Bzlattern. Sift nods felten; id babe biefe 2lit mut ein einzigez Nal gefehen.

Mimosa, ভinnpflanje, XXIII. 1.

Mi. pudica, fdambafte ভinnpflanze (noli me tangere), そ. Brafilteit. - Die feinjefiederten Blát=

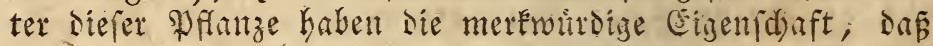
fie fid beg Der leifeften Bervigrung zufammentlesen; beribrt

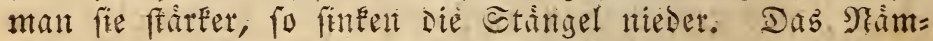
lid)e exfolgt, went man sen Iopf etwas erfiduttert. Nad)

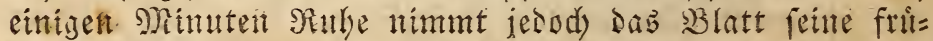

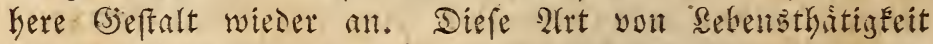
ift utm fis grofer, je wirmer bie Somte fdeint; thto ie

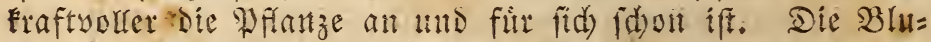

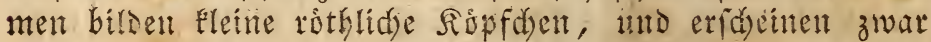
(d)on in erfen Sahre, Dod) gemeitíglids zu fpit, als ba

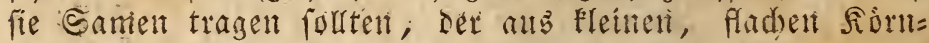

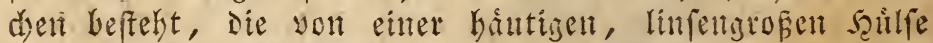

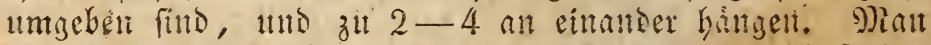

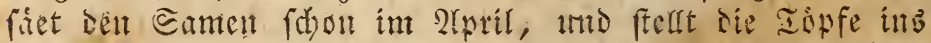

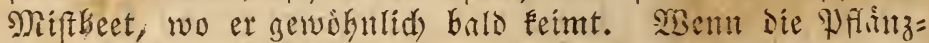
d)en Das britte Blatt baben, fo feht matt fie cinzeln in

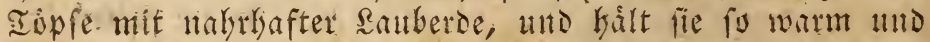

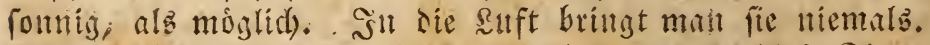

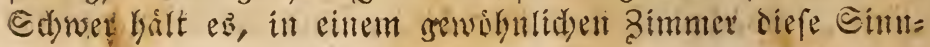




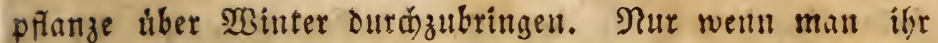

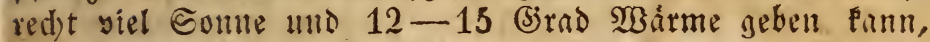
glícft ez zuweilen. S'm zmenten Sabre, ment man Die Pflanzen in ein gutes SRiftbet bringt, treiben fie zume: len $1 \frac{x}{2}-2$ Fur lange liegente dornige Etängel, uno brin= gen Eamen. Dod) ift $\mathfrak{e b}$ weit beffer, fid) jibbrlid) eintige rounden Samen zu faufen und bie Pflanzen als Sommer= gewáchle zu behandeln.

\section{Mimulus, (bjanflerblame, XIV. 2.}

1) Mimulus glutinosus (M. aurantius), riger (5) a fler, h. Mexico. - Diefer Etraud) miro

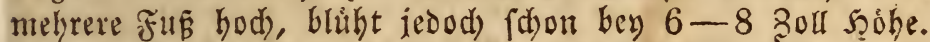
Die lảnglid) zuge ipişten Blåtter, fo wie die iungen Zwet= ge, find flebrig, die Blumen faft radienformig uno offer= gelb. Nan gibt ihm lebmige Sauberse, uno liberwintert ifn bey 4-6 (5irad. Er trágt faft bas gallze Sabr hin= Durd) B̧lumen, wird im 2lpril verpflanzt, uno Durd હa= men und Etedfinge vermefrt.

2) M. guttatus, getupfelte (B)a flerblume, 21. Mexico. - Ein Miedriges, auf ber Erbe fried)endes (5)ewád) mit rundlidsen gezáhnten Bláttern. Sm હom= mer treibt fie fingerb̉ange Stångel, an Denen ziemlid) gro= Bé, faft rad)enfórmige, hod)gelbe, inmerbalb braunroth ges tipfelte Blumen, erfd)einen. Sie vermehrt fid ungemein ftart" Durd) die Fried)enden Etángel, weldye úberall 2 Gurzeln madjen, ift hărter, alb $\mathfrak{N r}$. 1., Dauert fogar im frenen sande, fault jedod) dez Winterb leid)t in den Iopfen, weÉ = halb man fie albsant mebr trodén, alb font balten mus. Ulte Friblinge verpflanzt man Die Etólé.

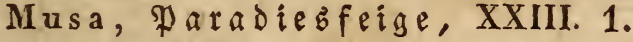

M. paradisiaca, gewóbnlidze Paradiebfei= ge, Pifang, 21. Sftimbien. - Diefe palmenartige Pfanze

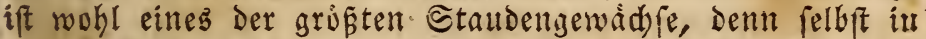
unfern Ircibbåufern wird fie $15-20$ Fu bo bo, und trágt 5-6 Fuß lange und $1 \frac{1}{2}$ F̋ß breite Blátter. 2lber Deffen ungead)tet fann man fie Dod, viele Sabre niedrig erbalten, wenn man ihr Feine zu grofen Iopfe, und nid)t mefre şár= me gibt, als die gewóbulide Bimmertemperatur betraigt. Der Difang yerlangt torfige, leid)te Rauberde, uno Deg

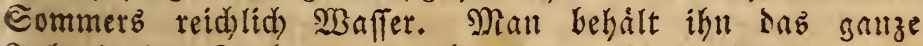
Sabr ill ber Etube, und gibt ifm ald im MBinter einen 
berten fontmigen Etalto bey $12-15$ (3rab Wäme. Da die

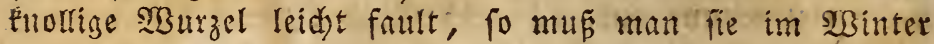

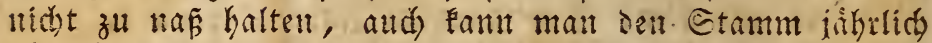
abferben laffen. Er madb ifters Eeitenfprofien, die man im Fribling benm llmpesen afnimmt, ofne jesod die Mut= zel zu fehr zul befdádigen. - 22n Bluthen und frudde

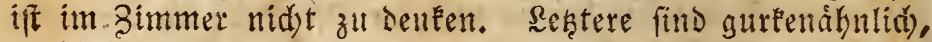
wohlfdmecterto, und bangen in einer gropen Traube.

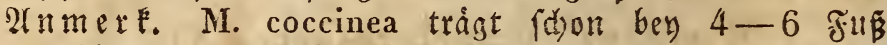
Shike im December biä Februar prád)tige Blumen, mitre fie rohl aber fatm im Bimmer entfalten. Sh Gabe ifn nod nidyt gezogetl.

$$
\text { Myosotis, Máujeobr, v. } 1 .
$$

M. scorpioides, B̧erginneinnidt, 24. Srill man dieje befannte Pflanze in ser Etnbe ziehen, fo bebt man Ente Sctober einige \$flinnzet aliz, legt fie mit etwab @d)lamm in ein (S)lab mit $23 a f f e r$, weldhes man im= mer wieier ergánzt, wenn ez einzejangt wird. Die zrweige

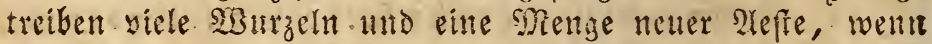
ntan fie bey 2-4 Giras nabe am Genfter bált, tutb blit=

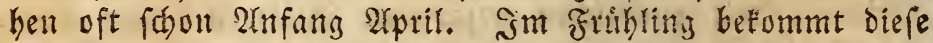
pflanze viel suft. Nan fann fie aud in Blumentopfe $r e=$ Bell, nur milfen biefe immer in einem unterfeşapf mit waffer ftehen.

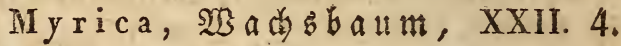

1) M. cerifera, wirefider $\mathfrak{B} a$ d $\mathfrak{b} \mathfrak{b} a \mathfrak{u t}, \mathfrak{h}$. Nordamerica. - Diefez $\mathfrak{B a d u m d}$ en erreid)t bey uns nut

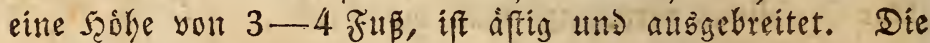
fteifen glånzendogrinen Blåtter find $2-3$ zoll latis, am Rnube eingeid)nitten, uno baben einen aromatifden (sjerudb. Die sBlumen Eente ith nod nidst. Man gibt ihm guten mit Iorferse uno Sant gemifditen Booen, in Eomnter vier Feudtigét, uno im SBinter $0-4$ Grad Márme.

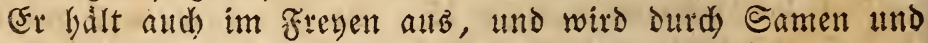
2lbleger vermehrt. seden Frihling fest man ifn um.

2) M. quercifolia, eid enbláttriger $\mathfrak{B} a$ d $b=$ baum, T. Cap der guten froffutuls. - Die fleitten, einen Borl langen, mattgrimen Blátter gleidhen Denen Der

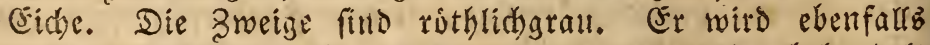
nut wenige fun hod), und Eann wie Der yorige behandelt

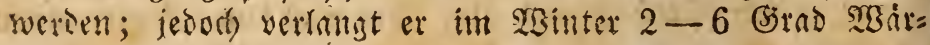


me, da er Eeinen Froft yertrigt. Bermebrt fiú) wabrfbeins

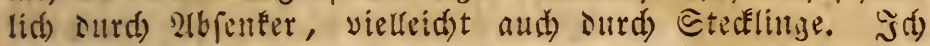
babe nod) Feine Serfudice angeftellt, da id nut ein etra

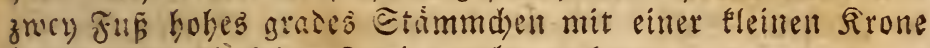
befitie, ser id́) Eeine Brueige nebmen Fonnte.

3) M. serrata ( M. banksiaefolia), gezáb $n=$

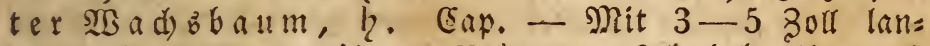
gen, fágeformig gezihnten Blattern. Sob babe ifn nod nid)t gezogen, et wirs jesods wie Nr. 2. bebandelt. arle

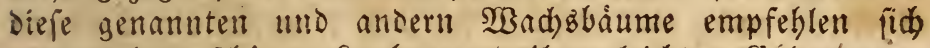
wegen ifres foblien saubez und ifrer leidjen Eultur.

\section{Myrtus, Myrte, XII. 1.}

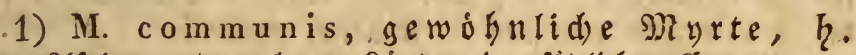

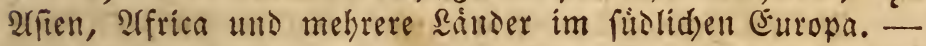
Man unterfdeicet yon ber gemeinen Myrte zwey Saatst: arten: Die grofblattrige (M. c. 'marophylla) uno die fein= Uláttrige (M. c. microphylla). Db beise wirelid) getrenute Irten, oder nur surd) Ëultur entfandene $\mathfrak{B a r i e t a t e n ~ f i n t , ~}$ mỏgen Botanifer entfabiden; das sebtere (d)eint mir nid)t ganz einleud)tent. - Sede biefer 2rbtheilungen fást wieser eine Nentge Epielarten in (id), von denen die vorzủglid): ftert folgende fint :

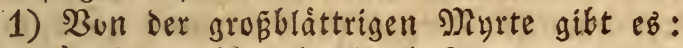

a) Die gewobnlid)e (aud) Subenmyrte genannt, wenn bie Blåtter zut dreyen am 3weige ftehen);

b) Diefelbe mit buttem Blatt;

c) Die mittlere mit nur halb fo grö́en fpişigen Blát: tern.

d) Dieferbe gefullat blihend.

2) Bon Der fleinblättigen Myrte find mir befannt:

a) Die gemeine;

b) bie fdymalblattrige;

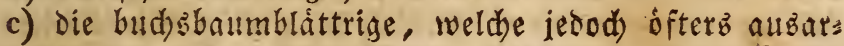
tet und zur gemóbnlichen feinblåttrigen Pryte wird;

d) mit weif̧́cuntem 3 latt ;

e) mit punctirtem Blatt.

(Die feinblattrige, gefiut blufende Mnyte babe ith nod) nie erhalten pismen, iesod) wurde id) (d) on ei= nigemal samit betrogen. Jid) Fann nid)t jagen, ob eb witelid) eine poldye gibt.)

Die Behandlung aller diefer Barietáten ift fíd) gleide. - Mar gibt den Myrten Orangerie = Erbe, verieģt fie alle 11. 
zwey Safte im Grufling, tmo gibt ifllen sam grónere

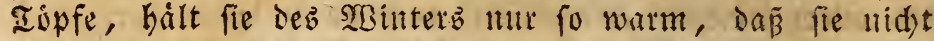
yom Froft leiden (bód)fens 6 (sirao), unto gibt ibnen zeitis im Fribling suft. (Benobnlid) werden Die Murten, in Der Neinung, fie redst zu pflegen, yom suvember bis Ende Februar biel zu warm gebalten. Eie werfen bann nidst felten bie meiffen Blátter ab, treiben (d)wad)e, gelblid)e Bweige, und gehen oft ganz cin. - Fenditigeit besarf die Myrte Des Eommers ziemlid) viel, iedod) im MBinter

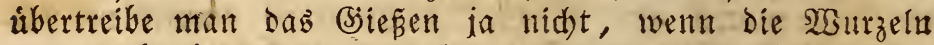
gefund bleiben forfert. Duntrangsmittel fino bey diefen Baamdsen nidst altzurathen, semt fint fie gefunt, fo wadh=

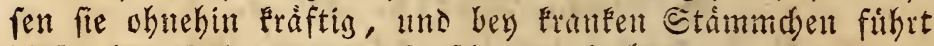
deefez den Tod nutr um jo fdneller herber.

Da bie Bweige der Myrte fehr biegfam fitto, fo Fant man biefe Ctråuder nad) Sisitréthr ziehen, und viele Per= fonen finden ein Bergnigen Daran, fie in S3effalt eines

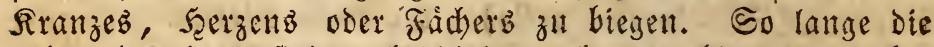
Badumdent jung find, geht diefes nod) etwa bill, wenn aber Der Etraud) gróper wird, und Die unterftent Blitter abfallen,

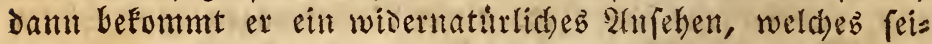
nem Befiter felbfi unangettebm fegn mus. - 2(m beften ifteb, die Murte ftraudartig geken zulaffen, ober burd)

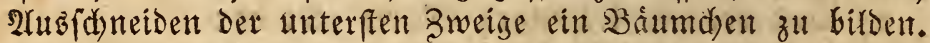

$\mathfrak{Z} \mathfrak{m}$ Dankbarftelt, Ginfid)ts der Blumen, ift die sefült Glithende 2rtt. Eie blikht, mentt fie fingerblang ift, uno 2-4 3oll ftarke Bánme find, ebenfalls iedes Sabr mit

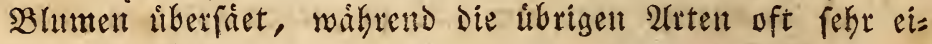
genfinnig fino.

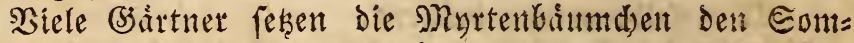

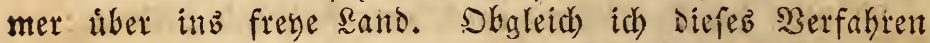

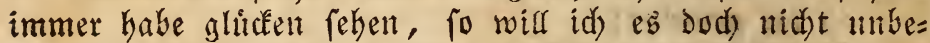
bingt und aflgemein anathen. Thut man es, , fo mus malt

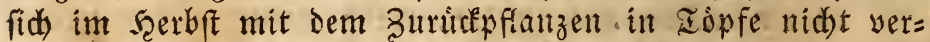
fpiten, Damit bie Pfanzent nod) Beit behalten, fids, fo

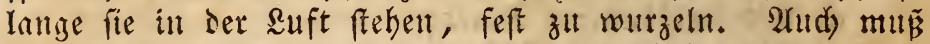
ins 2fubheben móglidsft mit Ballen gefdeken. Die Ber=

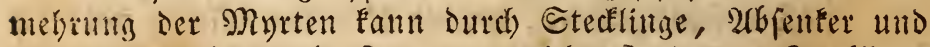
zuneilen aud Durd) Eamen etreid)t werdent. Etedfinge nebmen ant fduelffen an, wenn man. fie im May ooer

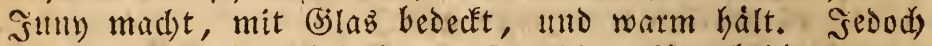

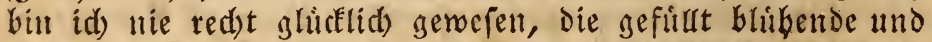
sie punctirte Myrte auf siefe 2 lut zul vermefren, fondern 


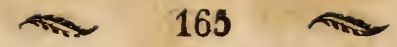

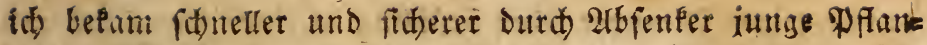
zent. 2afenfer fumten zul jeser zeit gemadjt werden. Die fárfern 3 weige fdneidet man ein wentig ein, die feinen

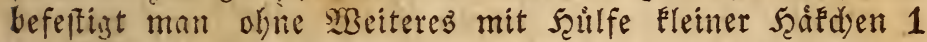
-2 zull tief in die Erote. Souften fie beym nidyften $\mathfrak{B e r =}$ Fetsen nod) nid)t fo bewurzelt fenn, Das man ez wagen Dirfte, fie abzudducicen, fo láşt man fie nod ein Sabr

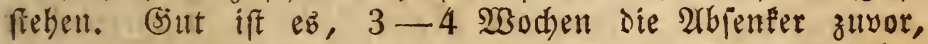
ehe man bie Myrte verpflanzt, zu unterfudben, und baben

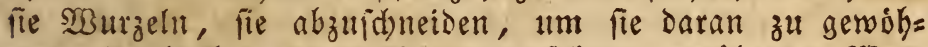

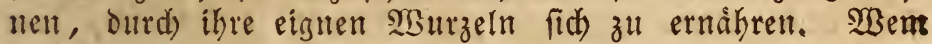
Daraut gelegen ift, Dutd) einen 2 (bfenter zmen Pflanzen zu befommen, Der trente benfelben nidst didst uber der Eroe, fondern laffe you Dem, mit Dem Mutterftod zufammenbåa= zenden Iheil nod) ein $2-3$ zoll langez Stude am 2 bren=

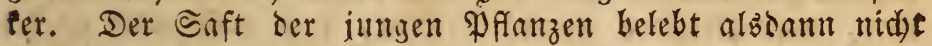
alfein Den leţtern, fondern theilt fidi) aud Dem entgenen=

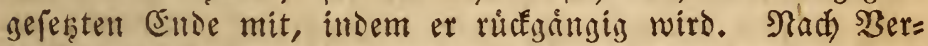

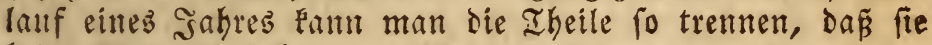

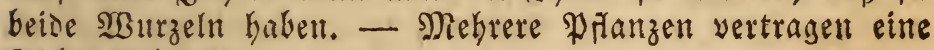
folde Bekandlung.

2) M. zeilanica, zeilanifbe Murte, そ. Zei= loit. (?) - Eie hat grofe zugerpiste, fehr bidht fethende Blitter, uno wiro wie die vorige befandelt, nur ift ibre

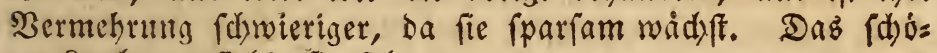
ne samb empifeblt fie fehr.

Nerium, steander, V. 1.

1) N. odorum, woblriedender oleander, Oftindien. - Die fdimalen $3-5$ Bolf langen, immergri: nen Blitter fteben ftets zu dreven an Den Zmeigen. Die Blument erfdeinen alt Den Spiben berfelten, find Kerlgelb= róthlid) (Daher zuweilen gelber Sleander genaunt), und ries d)en angenehm. Die sehandlung ift der bez̉ folgenden gleid), nur verlangt er mebr Wárme, wenn er fojỏn blü= bent rofr.

2) N. ol eander, gemeiner oleanser, そ. -

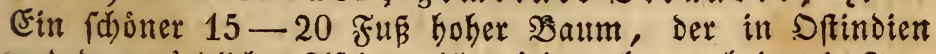
und bem nơrdlidyen affrica witb waddst, aber ald iekt in sta: lien einheimifd geworden ift. - Die Blåtter gleidjen Denen bes yorigen, fino aber etwas kurzer und breiter. Bewobnlid

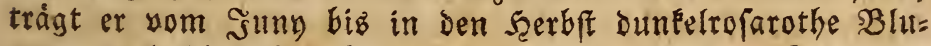

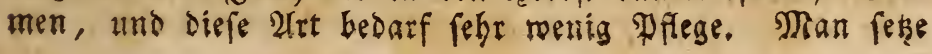


den gemeinen Dleanber jeden Frifhling in einen etwas grogent Iopf, nadjem man die $\mathfrak{B u r} \mathrm{eln}$ ein wenig beidnitten bat, "tno gebe ifm Drangerie = Erde. Bom Man bis Ente Geptember barf er im Freyen fteben, utto mǘ wábrent diefer Beit reidjlid) begoffen werden. Jim sBinter gefdieft Daš Unfeud)ten der Sirde fparfamer. Bey $2-6$ (srad Warme bringt mant ifn leid)t durd, nur gebe man ifsm im Fribling einen fonnigen હtand, und sanu vertraigt er $10-12$ Girad Marme febr gut.

Die rothblithente gefulle und die weis̆e cillfahe aret find beide etwas fdwerer zum Bliben zubringen, sod) gelingt diefes fefri leidt, weltu man fie zur seit, fohald rie frnospen fid) zeigen, binter eill Fenfter fteld, wo fie bie volle Gonne gentesen, uno wenig suft erbalten; vor= ziglid) gut entwideln fid) sie Blumen in einem Ireibera= ften, oder zwifden Doppelfenftert, Itur lafie man eb nie an Waffer fehlen. Bon Den gefiut blibenien Dleamer gibt es eine belrotbe und eine weibgeftreifte Epielart, zu=

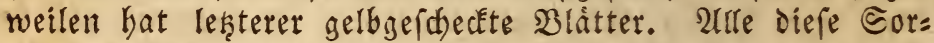
ten gewábren in ber Blithzeit eilien berrliden 2lnblice, ntho Niemantem wird es leis thun, fid siejelben angeid)afft zu baben. Nan vermebrt alle Dleanderarten am fiderften ourd) Urbiener, weld)e man im frubling madit, indem man die Bweige etwas einfduneidet und zwey Boll bod mit Grde besedt. (Semeiniglid) sarf man fie fdon im nàd)ften

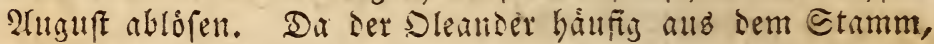

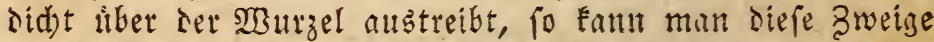
nm beften zu 2rbjenfern bemisen, ofne genothigt zu fern, die frone zu verterben. $2(n d)$ aub Gtectingen Fan man Den Dleanier zieken. Snan fdneidet hierzu im Shay die paffenden, fingerblangen Bmeige didyt unter einem fnoten

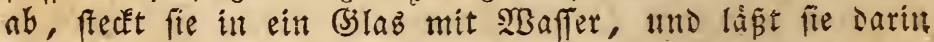
an einem red)t warmen Srte, z. $\mathfrak{B}$. zwijden Doppelfen= ftern ober in eitrem Miftbet, 4-6 Moct)en lang fteben,

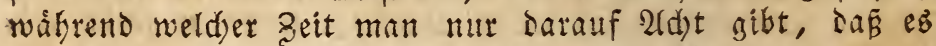
Dell 3meigen nie an Waffer fehlt; aud fdeint es gut zu fenn, wenn man bie Cinmirfung ber Sonnenftrablen ses

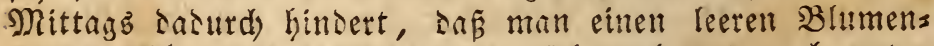
topf sariber fturzt. (5s finsen fid) nad) und nad) unten

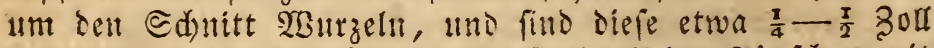
lang gervorien, fo pfianzt man fie in Eleine Iopfden mit leid)ter (side, uno bált fie aufänglid) feudit ulto warm, biz man luberzeugt ift, das fie gebirig Sisurgelu baben, und 
Tie zu treiben anfangen, worauf man fie an bie Ruft ge= wobnt. - Btweilen tragen sie sleanterarten aud Eamen. Diefen füet man in fruhling in Iipfe, uno bălt riefe

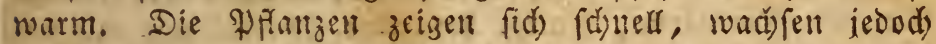
mekrete sabre, the fie blitbell.

Die Dleanderarten entbalten einen grưliden, giftigen

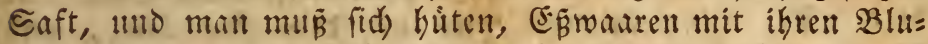

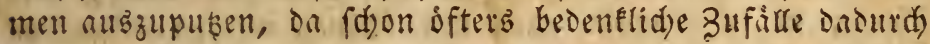
veranla get worten find. - Buweilen, mienobl in Dentid $=$

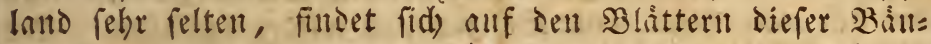
me eine grofe santipe, welde ben fdounen und Foftbaren Dámmerungbfd)metterling, Sphinx nerii, liefert, uno nor= auf Befiner Des Sleanderb alfmerffam fenn énntut.

3) N. splendens, prád) tiger Dleander. ghit gefuluten rofarothen, fehr fdonen, wohlried)enten Blumen; er wird wie Der gemeine gefúlte Dleander behandelt, und ift wohl mur eitte Epielart yon siefem.

Uf nmer F. N. coronarium (nud) Taberne montana coronaria "IIt Jasminum zeilanicum) lyat elliptifde Blátter utro meise fehr wohlried)ente Blumen. Ex liebt Fehr die vermelyen; id Fenne ifn jecods nod nidst genau ges nug.

\section{Oenothera, $\mathfrak{R a}$ di) teege, VIII. 1.}

O. mollissima, weid bláttrige Nad) tectze,

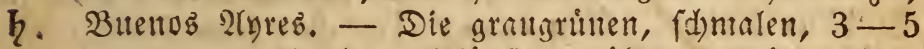
Soll langen, am Bande weitlảufig gezâthnten Blätter fikgen an bitunen Zweigen, weldye fidt) gegen die Eroe neigen, uno an ihren Enten im saufe bes Eommers einzelne fäwefel gelbe suftenise Blumen tragen, die fid) nut Des serbents

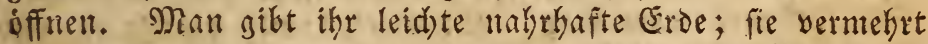
fid) Durd) Eteclinge uno Eamen, und wirb bey 2-6 (Grad leidst liberwintert. Eelten wird fie liber $1 \frac{\tau}{2}$ Fus

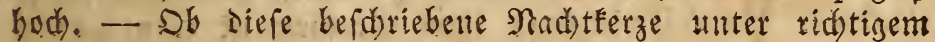
Namen angefest iff, will id nid)t befaupten. Da mir je: Dod) Eeine Urt befannt ift, anf meldye Die Befthreibung ganz paß̈t, to will tă den Namen beybeba!ten, unte. Dem ich) fie erbielt.

Un merk. Die hibrigen Nad)téerzen, als O. grandiflora, rosea, tetraptera etc., merben iåbrlid) auz @amen sezogen. Cie blüben alle dez Radhtz, oder an trủ= ben, regnigen zagen. 
Ornithogalum, 2ogelmild, VI. 1.

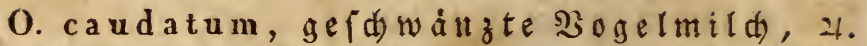
Cap. - Die grofe, glatte, Gellgrine Zwiebel fiht allf ber (Erte, treibt lange, gleidbreite, beynahe rimlenformige slätter, die fid) in eine lange pfriemenfömige Spise en= digt. Der Blumenfángel wird oft Drey Fut lang, und böber; er if benutake ganz mit fleinen weinen $B$ lúmdjen bejeşt, Deren Bláttden einen grimen Etridh baben, uno

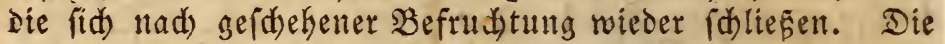
Blumen blifen nad) und nady auf. - Cie nimmt mit ie= Der Eroart vorlieb, wird alfe Friblinge verfest, im Som=

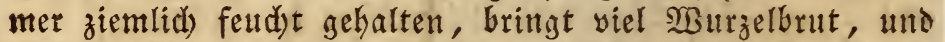

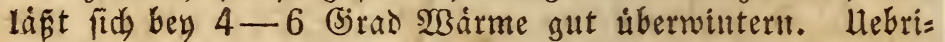
genb versient fie keiner (srmáfnumg, als daś fie oft fálfd)= lid) firr bie addte Meerzmiebel (Scilla maritima) aubgege= ben miro. Die 3wiebel siefer lebtern ift mit einer rotbbrau= nen Edbale umgeben, bie Blitter futo lanzettförmig, fteif, Dunfelgrin, uno erideinen erft, wenn der Blumenftangel

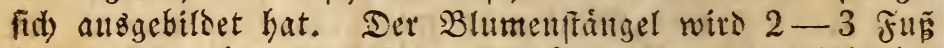
bod, uno trágt eine lange Iraube weiß̈er oder rótglider Blumetr.

\section{Oxalis, Eauerelee, X. 5 .}

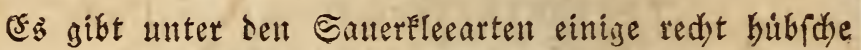
Eorten, welde meiftens zu Ende bes SBinters, ober im Frubling Gliken. O. versicol or hat feine Blatter und weî́ und rothe Blumdien. O. purpurea ift faft ftin= betlos, mit breiten Fleeblättern und carntintotheat Blutmen, áhnlid) Denen ier Mirabilis jalappa, die allf etwa eitren zolf langen Ctielen fizen, uno fid refr gut aubnehment. Eie ftammen yom Gap, uno werden mie die Şxien uno sadhenalien bekandelt. Co lange fie grün fint, gieft man înen regelmás̆g, Dod) ruthen fie einen grof̧en Theil des Sabres. But Entwidelung ifrer Blumen ift Sonne Durd): aub nothig.

\section{Paeonia, Páanie.}

Paeonia arborea, ba unatigé moutan, P. fruticosa), h. Woll biefer Pradtpflanze, welde aus (Shina ftammt, Fannt id aus eigner (Erfabrumis nod) nidht viel fagen, indem id fie erfĩ feit eintem halben jabre befiche, ulto mein Exemplar'zur zeit nod) nidat geblügt Gat. 


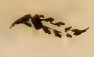

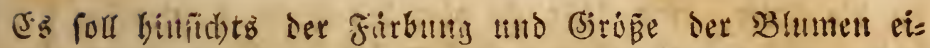

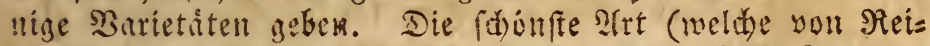
Der in feincu 2 mutalen set 23 llimifteren P. albiflora fragrans uno $P$. arborea sinensis albiflora nennt, alld Davon eitte febr verfleilierte 2(bbilim liefert) fort eine an 10 3oll gro=

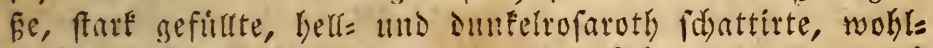

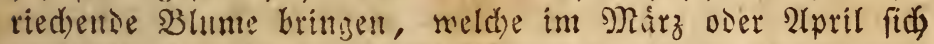
entfaltet. - Die baumartige Wionie verlangt ziemlid gro=

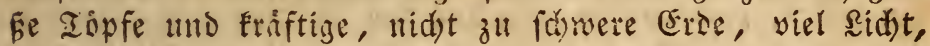
und im Winter 2-6 (j)rad 23 itrme. So lange fie nod

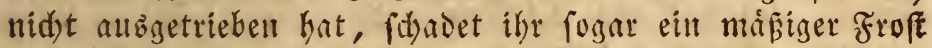
nidht, wenigftens war an meiner Pflanze die Erove ifters burt gefroren, uns semnod) trieb Die Pflanze hiernuf éråf= tig, uno batte nid)t ben geringifen Ed)aben gefitten. Sm Eommer felt man fie an cinten idattigen Plah inb Frene;

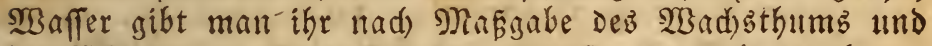
Der Witterung. Zum Minter wirft fie Die Blitter ab. Die Sermebrung foll fowierig fern, ierod) sefdebt fie

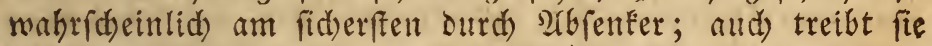
zuweilen bidit am ভtamm alb ber Sisurgel, wo mall benn

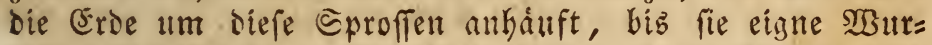

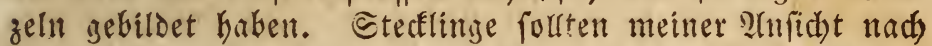
ebenfallz anwad) fen, iedod) erlanbt Die Seltenteit der Pflanze nod) nidjt, viele Berfude Der $2 \mathfrak{r t}$ zu unternebmen. Die

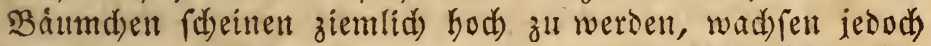
langfam. - Blumenfremben, die etwab auf fdjolte (Bes=

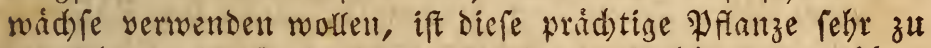

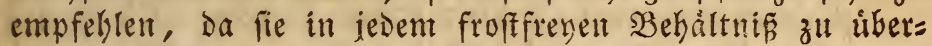
wintern ift, und ănerft danterbaft ju fenn fideint.

\section{Passiflora, Paffionbblume, XVI. 2.}

P. coerulea, blate $\mathfrak{P a f f i o n} \mathfrak{b} \mathfrak{l}$ me, filien. - Die Dimnen Zweige diefez befannten Edylingftrau= d)eb find mit fủnffad) gefingerten Blảttern befeşt, und zwifden ignen erfdueitten yom suly bis in Dett Sherbft die

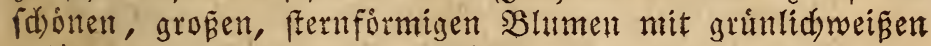
Blättert, auf benen ein bellblatter Fadenfrantz liegt, und Dariber berwdragenden, merfwurdig gebildeten (Sejd)lechtz= theilen. Die Paffintsblunte verlangt nahrhafte \&auberde mit Refm vermengt, uno des Sommers reid)lid) ŞGaffer, weniger aber im 9 Binter, wo fie bey 2-4 (Sirad gebalten wiro, uno alsoann bie Blåtter grós̆tentheils ahwirft. Ses Des Saht im 2rpril verfeht man fie, bringt fie im Mag 


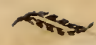

all bie suft, utto lispt fie dort biz Ende Eeptember fte= Ven. Die langen Zweige werden im Fribling etwas ein= geftubt, Debs Sommers aber, cutweser an einer Nauer in Die Srobe geleitet, oser um einen Reif sefúbrt, oocr an lange Etocfe befeftigt. Buweilen will bicfe fdone Pflange Iurd)aus nidht bliben, bejonters die åltern Etóce, wenn mant iften nidt Nafrung gentis geben Eant, oder fie dez NBinterb zu (d)led)t ooer zu warm bålt. Man thut Daber am beften, zumeilen im Fribling yon fraftyolken Nünfen 2 bb= fenfer in befondere Iopfe zll mad)en; bailt man dicfe redht feudt, und hat man die 3meige Da, wo fie in Die Eroe Fommen, etwas verwultoet, fo madsen fie binten $2-3$ Monaten Ginlinglid) Mutrzeln, Fonmen abgenommen mer=

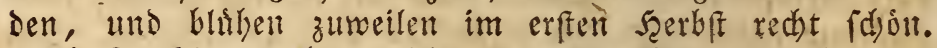

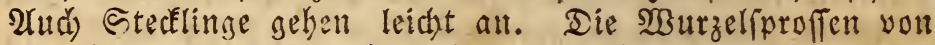
ben åltern Pflanzen abzutehmen, rnthe id nid)t, indem

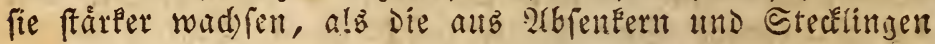
gezogenen, uns in ser Regel fpát bliken. Man Eann aud) Die Paffionsblumen den Sommer thber ins frene sand

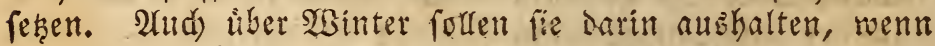

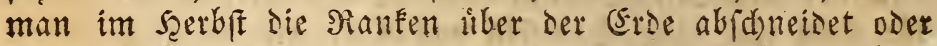
zufammenbindet, nesderlest, und $1-2$ Jü bod) diejelben,

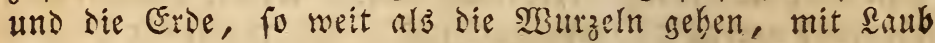
und Iannenzweigen gut bedect. Sidh babe nod Feinen Berfud) Der $2(12 t$ gemad)t. (Ë gift altich eine Epielart mit

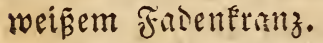

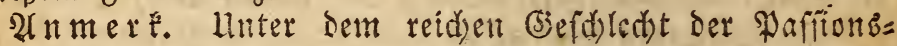
flumen gibt es nod) viele fdóne Sorten. Sb fie fid) aber fur sie Etube eignten, if eine antere Frage. P. lutea mo rubra bate id mekrere sabte gezogen, ofhe Blumen zu befommen. - P. minima bribt in =

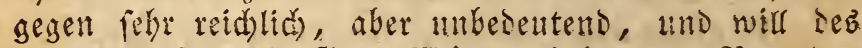
Winterg 6-10 (3tad Máme Kaben. - Bon ben tod) feltuern Pafitonśblumen werden $P$. princeps tht

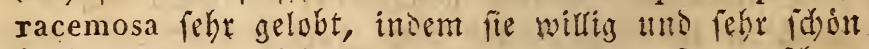
Glúben forfent. Die Blument ier ezfern folfen idjar= lad)roth fien, sie Blatter fint ireylappis, bethruin und leserartig. Sid befitie fie erft feit futrger seit. P. racemosa (aud) P. princeps coerulea) forf violett= roth blithen, ids) Pentie fie nod) weniger. Beise find Ireibhaubpflanzen, und misten im Zimmer Den હom= mer hindulth zmifden Doppeifenfern felen. 
Pavonia, Pasonie, XVI. 8.

P. praemorsa, abgebiffene payonie, Cap. - Mnit faft feilfurmigen, yorn abgerundeten, fumpf

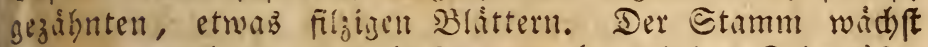

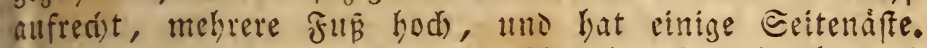
Die fleinen Blumen find malvenálylid), rotblid)gelb, und erfdeinten einzeln in ien Sommermonaten. Man gibt dies

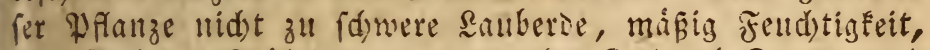
leght fie jesen frubling um, vermehrt fie Durd) Eamen uno Etectlinge, uno úberwintert fie bey $4-6$ (5iras. - Frat eben nid)t yiel Merth.

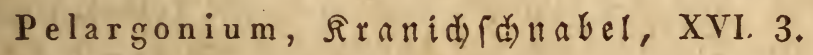

Die Rranid)fdnibel gehoren ieşt zu den Nodeblumen, und Die Zabl Der verfdiedenten 2rten und Epielarten if

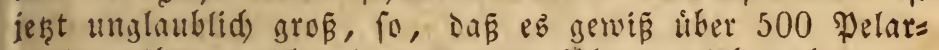
gonien gibt, weldse eighe Namen fihtren. 2rber ebell wes

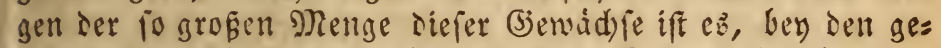
ringen lluteridieden jwifden einanier, faft unmoglid, obne Beidjnungen fie fo zul befdureiben, ia uno beftimment fonnte, uno fíbrt man fort, in allen (joist= ten Blendlinge aub Samen zu ziehen, uno zu felfiftándis sen 2rrten zu erbeben, fo ift eb Eluftis sallz unmoglid, Die urfpringliden Eorten bermbzufinien, und kein Botas nifer fann fid) mit ibrer 2 ufzáblung mehr einlaftẹn, da Die Hebergange zu unmerelid) fino. હ̈ gebt alfo diefent Pfluzen, fowie sen Mofen, Melfen, senazintGen, Iulpen i. F. m., fie wersen finftig bloź yon Gsáttnern, um die

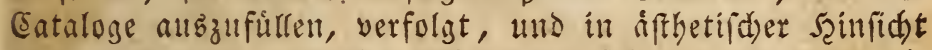
sezogen werden; ein Botanifer fann fid) nur im 2ugemeis

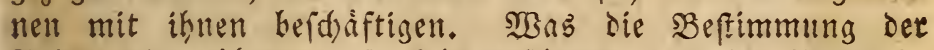
Pelargonien libersem fo (ehr erfd)wert, if ser umftand,

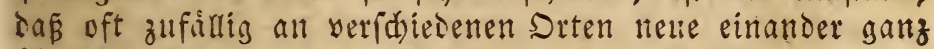
ánlid)e Epielarten gezogen, voll igren Befiţern verfdies sentlid) benant, uns io in bie melt gefdickt werden, des Serftummelnz Ser Nanten surd) gewobhnlid)e (Gartner nid)

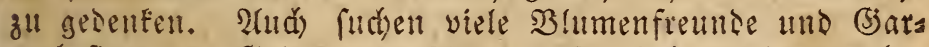
tenbefitier nus (jrifle neue 2rten auf. Sd) wenigftens has be trob alfer alufmerefameit oft feinen Hnterfdied zwis (d)en einzelnen Pelargonien anffinden foimen, oier fie bes tuhten auf ber (Eultur, bem aller und sem gefunden oder Fránfliden Buftande Der Pflanze. Deffen unseadztet ift die 


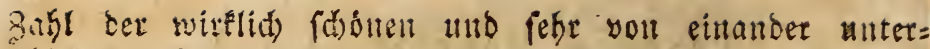

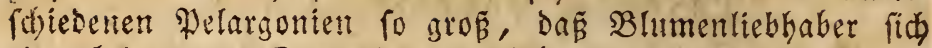
eine besentende Eammlung Derfelben anfd)affen fountir.

Eine arfigemeine Befdreibung ier Pelargonien ju ges

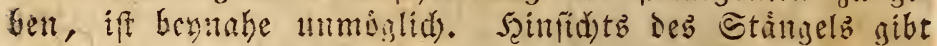
eb fángeflofe; Delarigonien mit traltartigen, fleifoigen uno bolzigen Etingehn. Die Blitter find entweder ungetheilt runs, nieren $=$, berz $=$, fdillo = uno lanzettförnig; voer fie find länglid), gezúbnt, ooer brey $=$, finf = tund fiebenlappis, oter aftig, oder aber fiederfpaltig. Die Blätter einiger $2(r=$ tent finb glatt, von vieicu raub uno baarig, you mand)en mit Flebrigent હdyleint thergogen; ofters find fie fefr wohlrie= d)ent. Buweilen lianft ein brauter Bsurtel (zona) úber Das

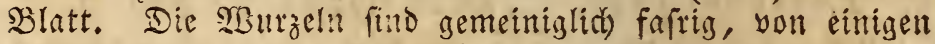
Eorten lnollig uno faft rúbenartig. WBas sie Zabl Der Bhtment anbelangt, fo giebt es einige 2frten, die nur $1-2$ Sblumen anf iedem Etangel tragen; Dagegen bringen andere fehr reidse Dolden you 20-30 Bhlumdent. Die Farbe ift ebenfalts feht verfdieden. es gibt einfarbige (concolores) mit weisen, bellrotben, rofarothen, lilafarbigen, yioletten, blutrotben uno bod)rotber, mit gelblidsen und bráunliden Blumen. Bes eintigent wenigen Sorten bat iedez Blu= menblatt allf bellem Girunde einen bunelen Etreif (bicolores); einige Gaben Drey Farben, weifi, roth uno Dun= felbraun (tricolores); bie metfelt Pelargonienblumen find jebod) bunt (pictae), ঠ. 5. Der (Grund ibrer Blatter ift belffarbis, gemeiniglid weis, betroth, rofa, lifa, violett oder carmintotis, this bie zwery oberften Blattchen find mit

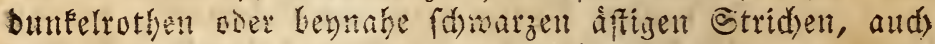
wobl nit fleien geziert; felten baben sie untern Blatter

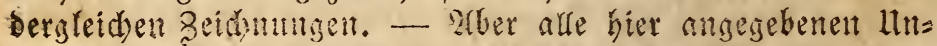

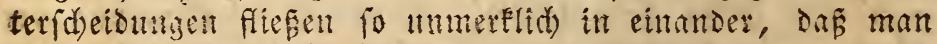
zuneilen ungewís bleibt, wojlt man biefe oder iene Sorte

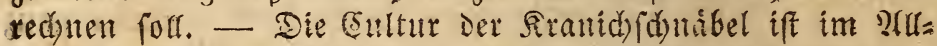
gemeinen leidst. Naan gibt ihiten folgente (Fromiftumg: orey Ibeile sanberde, einen Iheil sefgm uno einen Iheil Sand. Sm Fribling veriest man fie, mo bringt fie yon oer Mitte des Mran ab bis Mitte September in die suft, iebod) an einen gef(r)uthten Etant, - wo fie bie Mittagb" fome nidjt zu fegr drict. Sann man biefer nidjt ver= meiden, fo fichere mant fie wenigftenb burd) eitten Sdjirm yon Seinemant. WSikgreno diejer Zeit bekmmen fie reids=

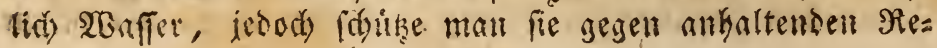




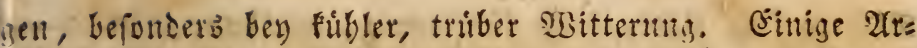
ten fint in siejer frinfid)t bejonsers jưttidh. Froft vers tragen fie gar nidt); befonders fino die mit frantartigem Etángel gleid) Dabin, die bolzartigen treiben zuweilen attz

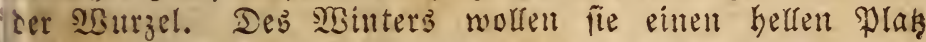
une am jenfter, móglid)fi Sonte, uno etwa 2-6 (sirad S3irme. Mat Dem Bergtefen fey man Dam yorzinglid) yors fid)ti, befonders an tribent Ingen, mo wab die fleifd t= gent utto ftátgetrofen Sorten allbelangt, oder went fie zu tranfeln anfangen. Nur went man bab Bedurfniés an ihs

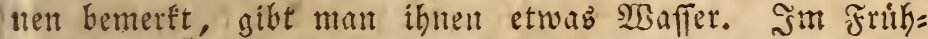
jabr ift ibuen an milden Iagen die suft febr zutráglid, Darum offine man alsbant tinglid) Die Fenfter. Die meiften \$elargonien traisen Eamen, uno, wie bereits gejagt, nan

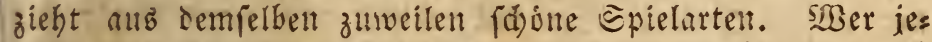
Dod feite Sorten rein erbalten wial, Der vermelyre fie Durd

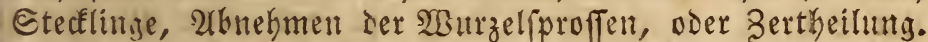
Die Stecflinge mad)t man am beften im Monat Suly; bie 3weige you sen holzartigen Corten babe id) gewoiknlid), be: fonders went mir saran etwas gelesen war, mit Glab bes tedlt, und in die beiffe Sonnte oder in einen Ireiblaften geftert. Son Denen mit faftigen @tångeln fterte id) fie jes bod) in sie frene Ruft an eillent etwas fdattigen Plak, bis mete Blatter fids zeigten. 2(m (d)onften bluthen in der Re=

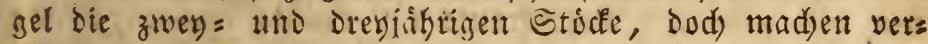
fhiedene Eorten eine 2usnahme, und gewinnen mit bem 2llter an $\mathfrak{X S e r t h} ; 3 . \mathfrak{B}$. bicolor, echinatum, quinque vulnerum u. a. m. - Die abgetragenen Stófe fann man liber Eommer in einen (Barten feşen, wo fie gewóknlidy nod) red)t (d)on bluthen, uno fide oft erbolen. Mandje

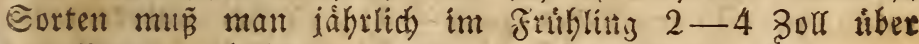

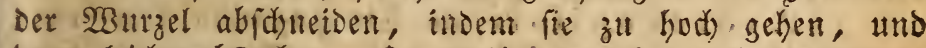
bann leidst abfterben. Eo veriungen fid jeood) oie 3roeige immer von neuem. - Eintige pelargonten ruben Deb̆ Coms mers; satul giefe man ifnell wentig, bis im seerbft neue Blátter exfdeinen, jedod) laffe man fie nie zu rebr nusz= Dorten. Diefe Arten fino befonders gegen Regen zu vers wabren. - 2tue Pelargonien, mit wenigen 2lünahmen, frammen yom Cap. Frúker záflte mant fie zu Den Gỉeras nien.

Folgenbe Rranid)fduábel fente id) als fdoune 2(rten:

1) P. a m plis simum, mit weínen Blumen, beren obere Blatter dunfelpurpurroth affig geftridbelt find. Dis 
Blatter find glatt, halb finflappis, gezábnt, wird $2-4$ Zú bod, mit bolzigem, àftigem Etảngel. 2-6blumig; blibt fleisitg.

2) P. angulosum, mit rauken, faft berzförmigen, fein gezáfnten, etwab faltigen Blattern; Der Stamm wird

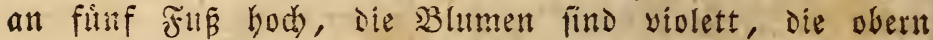
Blätter mit fowwarjothen Etrithen. 4-6blumis.

3) P. betulinum, mit fleituen faft enrunden, ges zåbliten Blattern uno Elétiten Blumen, welde Sir. 1. glei= den. 2-4bhimis.

4) P. bicolor. Ser Stángel, faft Érautartig, ift

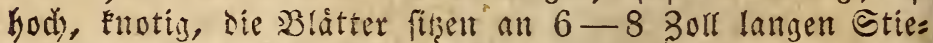
len, fino grof́, finflappis, etwab fralls, und fo wie die Stiele und Stingel kurzhaarig. Die Blumen ftehen in eis ner reid)en Dorse. Sie find weís, uno jedes Blatt bat einen breiten sumelblutrothen હtreif. - Er hat eine Enols lige $W_{3 u r z e l, ~ m u \tilde{~}}$ ifterz eingeftub̧ und etwa alle zwey sabre in leidyte side verjebt wersen. Stecklinge nefmen etrab fáwer all, da die frnoten zu meitláuftig ftében, boch fann man die jungern Zweige bierzu benuben; auth treiben die áltern Pflanzen zuweilen M̉urzeliproffen. Ea= men befam id) niemalz. (Eille idjone Corte, weldbe faft Dab ganze Sahr Ginourd) bliht.

5) P. c a ndidu m. Die åftigen Blátter gleiden, Des nen Dez gemeinen Rofentrautz (P. radula roseum), fino aber weišlids), faft filzis; die Blumen faft reiś, mit fei= nen rotben @tridsen uns pünctden. 4-8blumig.

6) P. citrio d o r u m. Die fleinen Blátter finto 3laps pig, eingefdutten uno gezaknt; fie haben einen febr ans genehmen (Serud), gleidst Dem Der Eitronenmeliffe. Die rothlid)en şlument fehen zu 2-4, und fino unbedeutend.

7) P. co ronopifolium. Die långlidben, fàgefórmig eingefdunittenent Blátter fino graugrủn. Der Straud) wiro

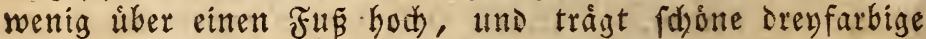
Blumen yon weiser, carmintother uno fdwwarzbrauter Farbe. Er will etwas aufmertfam wie Nir. 28. behandelt fenn. 2 -3blumig.

8) P. da u cifolium, faft ftängellob. Die Mußtrzel if fuolig, bie mofrenartigen Blatter fiederpaltig, etwas rauh. Die Blumen folfen in vielblumigen Doloen fethen, gelblidy unto in ber Nad)t roblried)ento fenn (P. flavum). Er fdoint úber Sommer zu ruben. Sils habe ifn nod nidbt befandelt. 


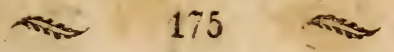

9) P. echi natum. Ier Etanget if faft fleirdois,

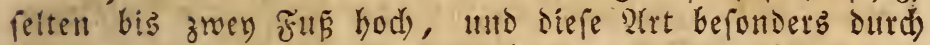
di: bleikenten, faft dornigen, gebogenen Blattanfäbe fennt: lid). Die Blatter freben, went Diefer Pelargonium gut behantelt wirs, auf 4-6 zoll langen Etielen, die am Etamm fidh folbentartig verdicfen. Eie find glatt, feid)t, 3-5lappig, ftumpf geziffut. Die 3lumen exfdeinen fpat=

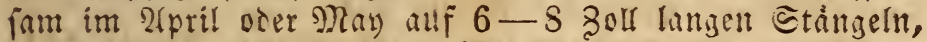
bie fich zuweilen in zrey bio orey Nebenftangel theilen. Die Blumen fieken zu 3-5 an senjelben, fino rein weís, sie obern Blatter blutroth geflect. Nads Der Bluttgzeit, ald fidjon frufher, wirft bieje Pfanze in Der Regel bie Blitter $a b$, unt fteht bis Ente Eeptember Fabl, Dagegen im sinter yoll sant. Die Stedflinge madyt man am bes ften zu (Ense 2(ugut, und bált fie anfinglid) nidat fehr felldt, biz junge Blitthen erfdeinen; vielleid)t treibt die

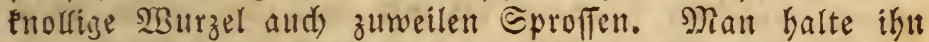

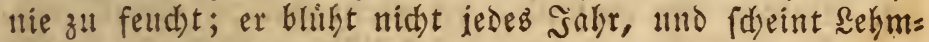
bobell zit verlangen.

10) P. fulgidum, hat viel Hehnlidéteit mit iem vorigen, uno wird aud ebento behandelt. Die Blåtter fino lánglid), gelappt uno gezáhut, etwas rauk, und die Blumen, weld)e im suny bis in sen 2 (uguft binein fidy entwidfelt, einfarbig, brentrent = Dunfel=bod)roth. $1-1 \frac{x}{2}$

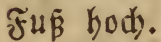

11) P. gibbos um. Der mit vielen runten unt lángs lid)en ftarEen Finoten verfehente allfed)te @traud) ift aftig, und wird 3-6 Fuß hed). Die Blítter find glatt, graus grin, unbeftimmt gelappt und gezáfht; sie Blumen ftehen in mefrblumigen Dolsen, und find bråunlidbgelb. Sm Winter balte man ihn etras trocen. 2lud) er wirft im Commer die meiften $B$ lätter ab.

12) P. gr a ndiflor u m. Die Blâtter uno Bfumen

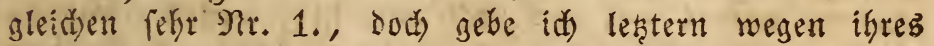

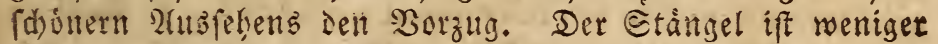

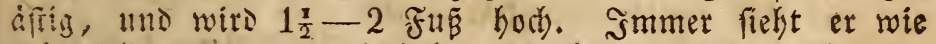

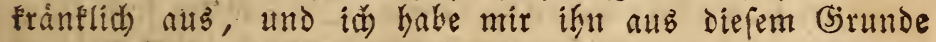
nodi) nidjt angefdafft. Heberbaupt fou er zärtlid) renn. 2 -3blumig.

13) P. hermanifolium, witb $2-3$. Fur bod,

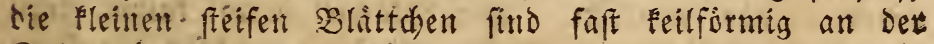
Epise abgeftht uno gezábnt. Die Blumen fehr bleidgroth, ofen fupurtoth gefteift, 2blumig. 
14) P. inquinans, mit tuntsen am Ranbe beynabe 5lappis feidut eintgebogenten, ftumpf gezábnten Bláttern, und reiden Dolien mit Blumen, weldue in allen હd)attí rungen vom fleifobfarbnent bis ins Sorennendyod)rothe gefun= Den werben. Scierber gehoren: P. Bentinxianum, Blücher

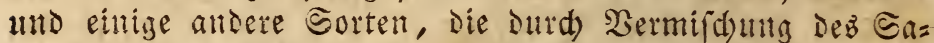
menftanbes you diefer 2trt und P. zonale entfanden zu renn (d)einen. Wisto $3-6$ fun hod).

15) P. la teripes, mit folloformigen, glatten, feicht Slappỉgen Bláttern, von Denen jeser sappen in cine frum= pfe Spise auslauft. Der faft fleifdige Etangel ift (d)wads, und wird frum jwey Fit hod). Die Dolden fitto mehrs

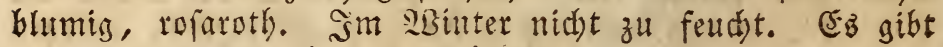
eine Epielart mit bunten Blattern.

16) P. l a c c e la tum. Die B̉atter find graugrín, lanzettformig, oft muldenfürmig gebogen, fpis, und bey jungen Erâftigen $\mathfrak{p}$ fianzen oft mit Seitenlappen verieken. Die B̉lumen find weişlid), Die obern Blátter etwab roth. 1-2blumig (P. glaucum). Sim Sỉinter nid)t zll feud)t.

17) P. peltatum. Die Blâtter gleidhen Denen yon Ne. 16., find aber weit fleifdiger, uno oft braun gegút= telt. Eir wird 2-3 Fun hod\%. Stectlinge morten mir nidjt gut wutreln, Daher zos id) Diefe 2 trt immer alls Samein. Nan gibt ihr im Winter fparfam Noaffer. Die Blumen fteken zu 2-5, und find fieifd farben. - Die

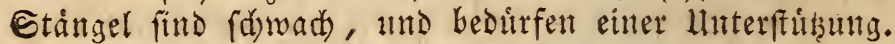

18) P. pencillatum. Die Blátter fino faft eyfỏr= mig, gezálynt, uno die Błtumen weí̄; die obern Blátter Der lestern find mit rothen verwajdenen Stridgen fobón ge= ziert, $1-2$ Fus bod), 2-4blumig.

19) P. Prinz Regent. Die Blátter find gró̈, runt,

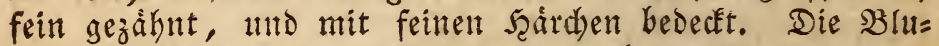
men gleiden denen yon Nr. 2., fino aber weit gróser nttro fdoiner, wird 4-6 Fuß bod), unt ift zumeilen etwas ei= genfintig im Blithen (P. Baringtoni major). - P. Regium

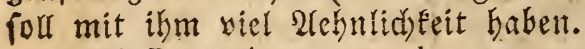

20) P. qu in qu e vulnerum. Die Blátter fitto áftíg,

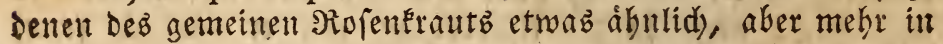
die \&änge gezogen, und in brey Fraupthappen vertheilt. Die Blumen find f(d)arzblutroth, iedes Blatt mit eintem weiss= lidben Rante verfehen. Der Etängel wird faum einen Fü bod), treibt wenis 2lefte, und diefe radben langfam;

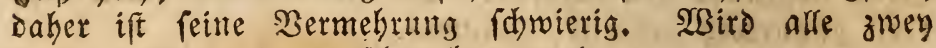
sabre verfent, da er dumads wurzelt. 
21) P. radula, mit yerfóicienenen Epielarten, yon Deneit radula roseum, Das Rofenfraut, Die befanntefe Eorte ift. Son lesterer bat man eine niediide buntblättrige Mlt: Die gewóbnlid P. molle fol. variegat. genant wird.

22) P. St. Hele num. Die Blátter finto beynage cis dentihnlid, 5-7lappig, tief eingefdnitten, flebrig uno

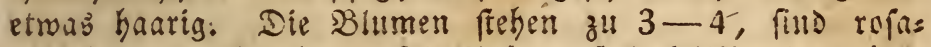
roth boer fleifdfarben. Die Blätter find feififúrmiz, abges ftubit, uno oben gezăhnt. Die beiden oberfen baben einige ounfertrothe Stridje, die oben in einen ounfeltrothen flete zufammentaufen, jedes untere Blatt bat einen beulern eint:

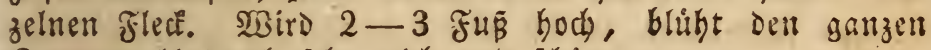
Eommer bindurd) Fegre reidy und (d)ón.

23) P. sanguineu m. Die BBătter find lang, glatt, ounkelgrún, fiederipaltig, in fúnf Şalpttheile getheilt, mit bennabe lanzettformigen Ráppoten. Der Etảngel if fale

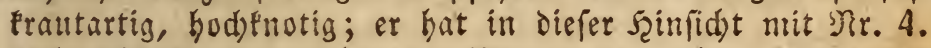
Ylehnlid) keit, ood) fethen die Blumen in nidyt ganz fo reis den Dolden, und find einfarbig, blutroth. SBill mait isa vermehren, fo fanneidet man, wie bey Nir. 4., Die Etánts.

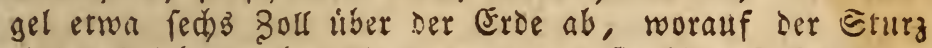
einige Iriebe mánt, die man, wenn fie $1 \frac{x}{2}-2$ zorf hing

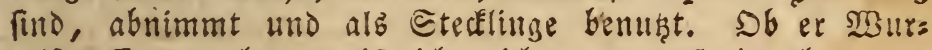

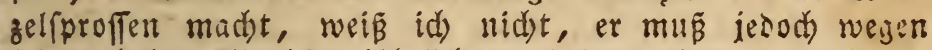

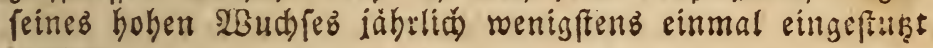
merden.

24) P. speciosum. Die Biátter finto faft herzfơr: mig, Faum gelappt, gezáfnt und ziemlid) groß́. Die Blus ment gró, faft wie die yon $\mathfrak{N r}$. 1., zumeilen aud mit blaşrothem (s) runde. - (P. formosum ift in alfen Iheilen etwab Eleiner, und Die Stridse wie ein wenig berlanfent. Edjeint nur cine Spielart yon deefem, und vielleidht yon

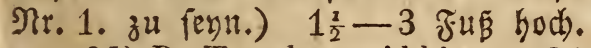

25) P. Tankervilliae. Die Blâtter find lanzetts

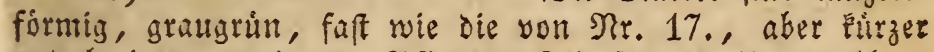
uno breiter; all itungen \$flanzen find fie zumeilen gezảhnt: Die Bzlumen faft gelblidsweí, wenn fie allfblühen, hers nad) teill meis, slânzent, und die obern mit idjonen vers:

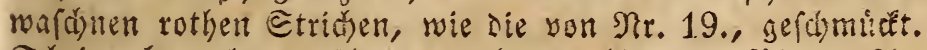
Edheint beynahe zwifden den beiden hier angefihteren 20 ten Dab Mittel zul balten. 2-36lumig. 1-1 $\frac{1}{2}$ fon boch.

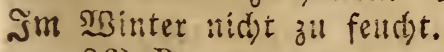

26) P. ternatum. Die Eleinen ffeifen Blátser bes 
fekten aus srey einjelnen Feilformigen sappoben, die an Dell Spisen gezáhnt uno gépalten find. Die Blumenblats ter find feifhforben, oben wentg Dunfler geftritgelt. 23 iro $3-5$ Fuf hoci), $1-26$ lumig.

27) P. tetrago num. Die gratiguinen, fieifdigen Stångel werben 2-5 ơn bod, who fehen ftare aufrecht;

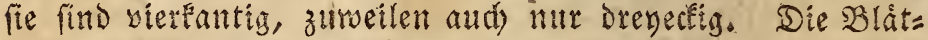
ter find fáfloformig, feicht, 5 lappig, etwas fumpf gezâknt, in Der Sugend mit einzelmen Fatrden befest, uno of uraut gegurtelt. Die rotblid)en, oben dunfel geaserten \$Blumen baben gewinnlidh nut yier Blitter, ifre Staubfioden find in eine febr lange, in ber snitte rtiefurmig gebogene Robre

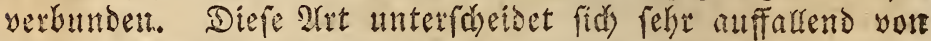
allen anden Plelargonten. Sie mirft zuneilen bie meiften

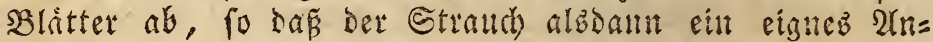
fehn berommt; blitgt faft ben ganzen Sommer uber, 2 -

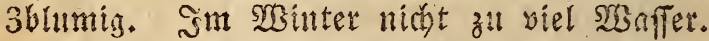

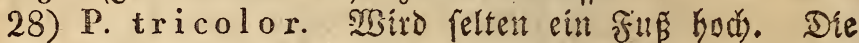

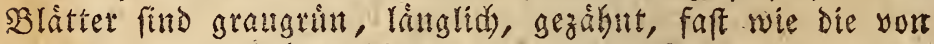
Nir. 7., und baken offers am untern Ende zwey Eletne linienformige Seitenlappden. 2-36lumig. Die obern

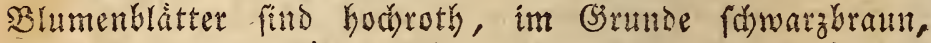

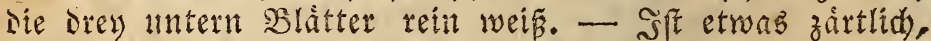
umo will des SBinterb einen guten, belfen uno fonnigent Stand. Die Sinospen exicheinen (d)on in Den Mintermo= naten, bie'Bhmen offtien fich jeoodi erft im May, uno

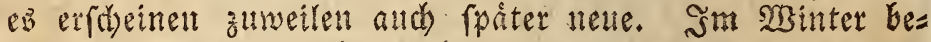
fonder mú man ibn mekt troden, als feud balten; reichte sanberbe, uno with etroa alle zwey sabre einmal umgefest. arte Stidfe treiben zuweilen Mutrzelfproffen.

29) P. iripartitum. Die Blâttdyent fino drevfpals

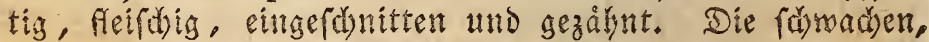

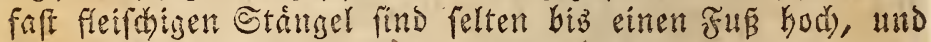
tragen 2-3 (d)marblattrige bleid)gelbe sBlumdyen, beren obere Blitter am (5runde mit cinem yerwafdnen fleinen purputrothen Fled verfeken fitto (Daker P. flavescens); if etruab zårtličs, und im Sisinter befonders gegen Nåffe em= pfitiliti.

30) P. triste. Die WHuzer beftet aus vielen Dun=

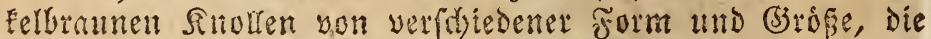
unter fich dutrib) fadenformige \$erlingermngen verbunden find. Faft ftängelíb. Die yielfach gefpaltenen, rauben,

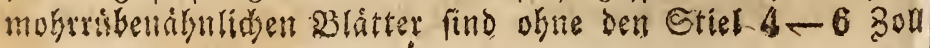


latis, und entipringen grobtentheils ats Den Murge'n un=

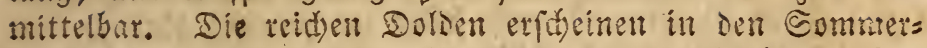

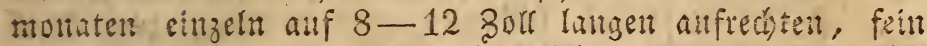

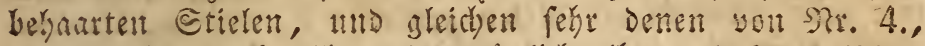

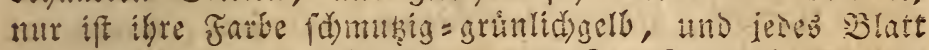
mit einem beetten forwargvioletten @treif verforen. Des

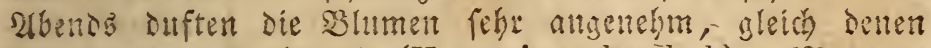
Der trautrigen Nadjtyiole (Hesperis melanchol.). Nan ge=

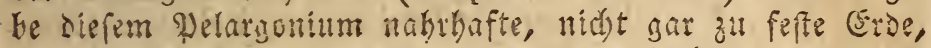
fesce ifn jesods mut alfe zwey oder drey sabre cimmal atm,

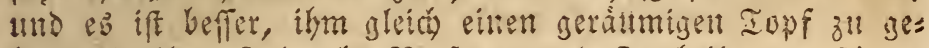

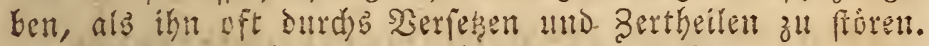
- Sm Sistuter balte man tha etwas trocen, umo went aud) viele Blatter abwelen, fo falabet diefes nicht yel.

31) P. unicolorum, hat viel geternlicheet mit P. Willdenowii, mut if er wentiger haarig, Die slument find Fleiner, rothyio!ett, und Die Flecte nida)t fo Deutlid). Die Blätter duften, wenn man fie reibt, wie die deb gemeis nen PopenErauts.

32) P. Willden owii (Link, enum. 2. p. 190), fonft aud gersibnlid) P. pulchellum genant, ood forl Der wirliche P. palchell. faft ftaingellos fern. Diefer affige Etraud wird etwa zwey Fus hod, hat fleine, fteife, faft

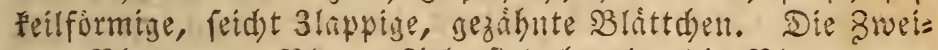
ge, Blatt= und şlumenfitele find haaris, die Blument an: ferblich, 2-4hfumis, reinweis, und bie obern mit vios Iettrothen, ápigut, thrzen Etrichen verpeben, die oben als

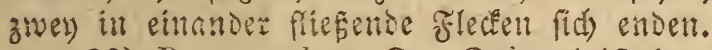

33) P. zonale. Der Stangel ift Frantartis, 3-5

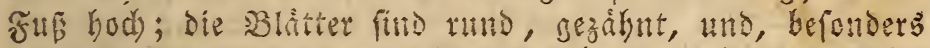
all Deft Eamenpflanzen, mit einem breiten, braunent (S) irs: tel verfehen. Dodh yerfiert fich serfelbe bey vielen tåten biefer Zutt. Die Blumen fehen in teichen Dorden allf 6-8 3oll langen Stångeln, und blukgen Den ganzen

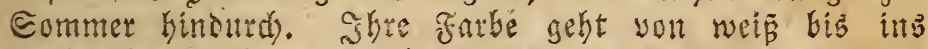

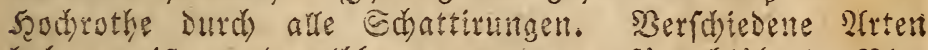
Gaben weis = und getbrunte, andere gefüt blubende sBht= men, D. h. Foldije, weldye fatt funf Balätter deren 6-10

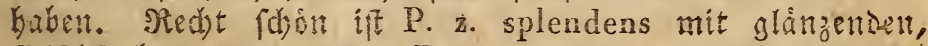
fleifa)farbenen şliment.-P: monstrosum, mit furzfies ligen am Rande getranfem Balt und bod)rothen Blumen balte ich auti) nur firr citie હpiefart yon zonale. - Nod)

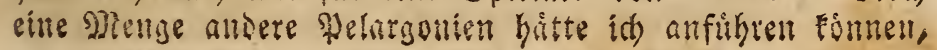




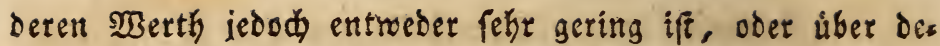
ren ridtige Namen id) nod felbft im zroifel bin.

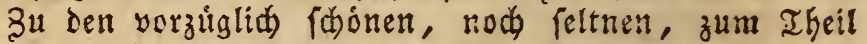
mir ganz unbofannten 2(rten gefóren: P. ardens, Daveyanum (blutroth, groß́), Denissianum eximium, fastuosum, gardnera glabrum, holosericeum, Huscianum, involucratum, intermedium Joungii, Leopoldinae, Lobatum, macranthum (Die Blumen follen bis brey Boll im Durd)mes: (er balten), Mathildinum, Oxfordianum, Princess Charlotte, recurvatum, Rowania, spectabile, splendens, versicolor, villosum u. f. w.

\section{Pentastemon (Pensthemon), Bartfaben, XIV. 2.}

P. campanulatus, glodenformiger $\mathfrak{D a r t f a}=$ Den (Chelone camp.), h. Mexico. - Die fámalen zu=

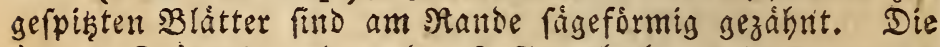

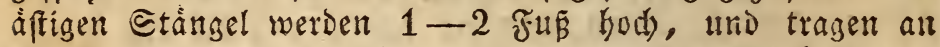
Der Epise eine Eurze Rispe mit Eleinen, Dunfelrothen, glos afenförmigen Blumen, ähnlid) Denen beg Digitalis purpurea, befekt. Er blifht dent gróbten Iheil deb Sommerz hindurd, liebt nabufafte fdumere Erde, und Deb Sommerz viel Fendidigkeit, uno vermebrt fidh leidt Durdi Stectinge und zertbeilung. U(ud) Samen beemmt man oft. Sm Minter 4-6 Girab.

Philadelphus, Pfeifenftraud, XII. 1.

P. coronarius, woblriedender pfeifen= ftra min genannt, $\mathfrak{h}$. Staleen. - Diefe bekannte Solzart, weld)e wegen ifrer weifen woblrietidenden Blumen faft in allen (3) arten neben dem gemeinen Flieder gezogen wird,

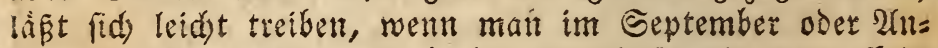
fang Detober paffende Stráut)er in Iipfe mit guter Eroe

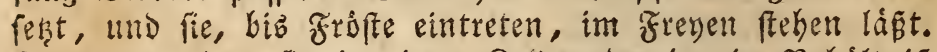
Eodann werden fie in einen Fetter oder in ein Bebátni

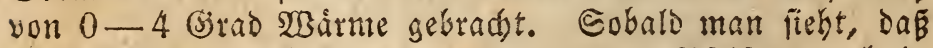

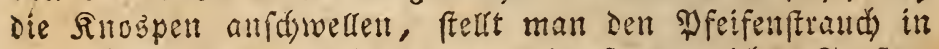

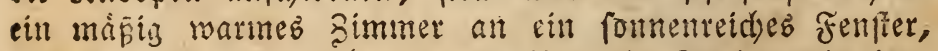
und befprengt des síbcrids jumeilen die slweige mit lau= warmen Maffer, worailf fich die Blumen balo entwideltr.

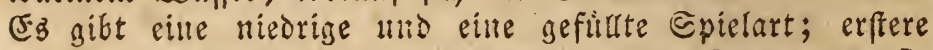
eignet fidh bejoniers für ien Iopf, lektere foll, wenn lie

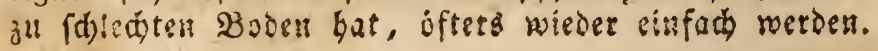


Phlomis, Phlomis, XIV. 1.

1) P. fruticosa, ftraudartiger phlomid, $h$. Epanien.-Die weisgrallen, filzigen, faft falbeyartigen, dod breitern Blátter ftehen paarweife an den weiß̈liden Zmei= gen diefes 2-3 Fus bohen હtraudes. Șm Frubling uno Eommer erfdeinen an den Spishen ier zrweige in 2 Birteln radsenfurmige, goldgelbe Blumen. Nan gibt igm ftare mit Rehm yerfegte tahrhafte sauberde, hbermintert ihn bey 4

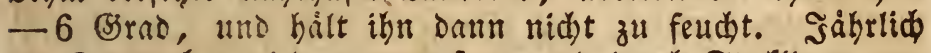
im ভeptember wird er umgefest, und durd Stcallinge ver= mehrt.

2) P. leonurus, rolftrappartiger phlos mis, h. Cap. - Die parweife fiehenden Blătter find 3-4 3oll lang, Dunfelgrin uno gezăhnt. Der Strauф

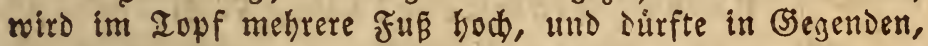
no er bie \$inter im Freben altb̧ălt, zu ben fdomften Bierpflatzen zu záblen feyn. Die rachenfórmigen, $2-3$ Solf langen Blumen fteken in 3-4 2 Birteln bidst zufam: mengedrängt lubereinander an ben Spihell Ier Zmeige, uno zwifhen jevem Mirtel ein Blátterpar. Sie find orange= roth, uno erfdeinen yon ber lesten frálfte dez Septem= ber $a b$, und dautern bis in ben 2 sinter binein. Man zieht biefe Pflamze im 2lpril oder sRay aแz Eteclingen, die man wo móglith ins Miftbeet bringt, oder bodi) mit (Stas bededit was warm bailt. S\$Benn fie jwen zoll lang getries ben baben, fo pfianzt man fie cinzeln in nidbt zu kleine Topfe mit nahthafter Erde, und bălt fie im Freyen, bey weld̈)er Gelegenbeit man es nie an $\mathfrak{B a f f e r}$ fehlen lást. Ehe es falt wirb, Eringt man die Stammden in ein 3im: mer yon 4-6 Grad Måme in die Nábe des Fenfters,

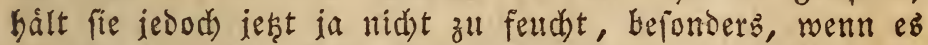
an Sonne fehlt. Sim nád)ften 2rpril werden fie wieberum in grispere Iopfe gefeht, und in Man in bie suft an ei= nen gefdisten Sort gebrad). SNunmebr erfdeinen im Ferbft die erften SBlumen, und jeşt eignen fid Die Båumdhen ganz befonders fur die Etube, Denn fpáter find fie, wenn man thren binlänglid Nabrung gibt, zu gró̈ gerworden, und erhalten fie zu Eleine Iopfe, fo bliken fie fparfam uno (d)led)t. Sm Dritten Fribling fest man ciefelben, wenn man Selegenbeit hat, inb freye sand. Sit der Boden nahrbaft, ullo fehlt es Dem Phlomis nie an Feuthtigeit, fo roidg et febr ftare, bringt im Seerbft mebrere Gundert 
Blumentifder, uth gewabet in der That cinen peidtigen

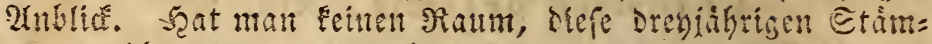

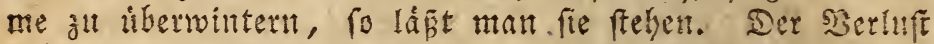

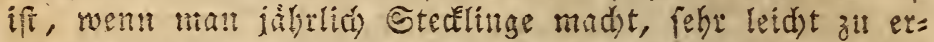

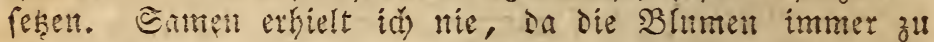
fout erfideinen. Jim Minteratartier werden nidst feltent

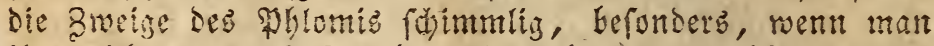

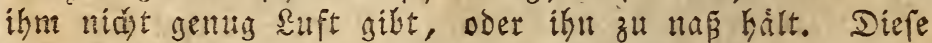
id)aigaften 3meize miffen gled entfernt, po roie ald, wenn mon furdotet, die: MBurzeln Fonnten gelitten baben, ren Baum in einen Eleinern Iopf mit fansiger Erse ums

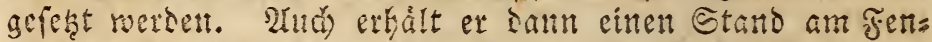

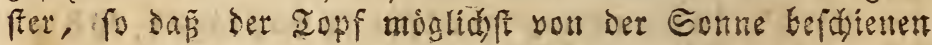
witr.

\section{Phlox, FlammenbItme, v. 1.}

Die meifen Flammentlumen ftammen aแt Norbames rica, eflitige alb Eibirien; fie fino Etausengewádspe, Die unfer Elima im sente gut vertragen; Daber ift ihre Eul= tur leidt, und es mad) feine smithe, fie alld im Iopf jut Blthe jubringen, wenn man zeitig im fribling et=

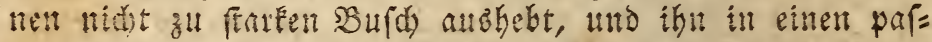

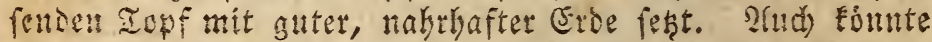
man fie wie dis Eampantilata bejandeln. Eie vermefren fich ungemein burdy die sourzelforoffen, uns fino daber bey

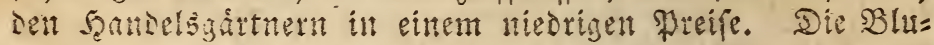
menfeingel mefrerer 2frten werden zwar in gittem 130 sen

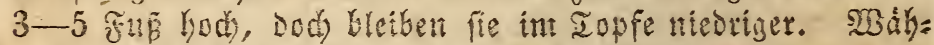
rend ser Begetation besurfent fie viel SMaffer.

1) P. a cuminata, mit edfigem 4-5 FH Goben

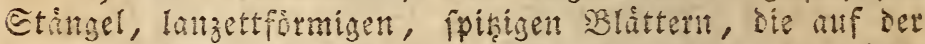
untern Eetie ctras filzis und faft geffielt fino, die obern Etingelbiatter fino bernabe betzformig. Die Blument bils Ien eine sieibe plapurrother Erovispe.

2) P. carolina, mit 2-3 FH: bokem Etaingel, glatten, lanzettónigen, ungeffelten Blatern, uno vio=

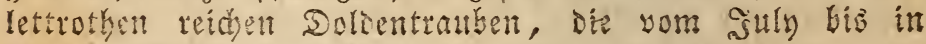
Det Serbit fid entwideltr.

3) P. divaricata. Die feinen miebergeduditen Ctáns

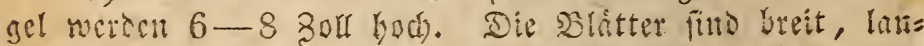
zettformis, mit einem founetsenden Mande verfeben. Die jungen Iricbe etwab banuig. Gehr zeitig, oft mit ien

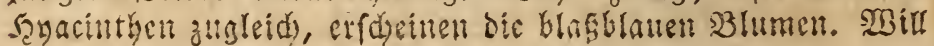




\section{N $\quad 183$

man ifn im Iopf jlefen, po wito er im September einges (est, bey) $0-4$ (sins liberwintert, und brift, an ein fons nigeb fenfer geftedt, fajon im Nårz. Nan vermefnt ifn am beften sutd) 2rofenfer Der jungent Iriebe, welde fdued

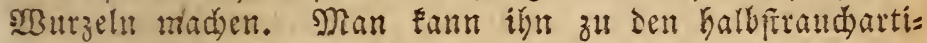
gen Sorten jifflen.

4) P. glaberrima. 1-2 Fú hod, bie Blátter find fámal, linientanzettformig, gefpis̨t, etwas glánzent, 3-4 3oll lang. Die Blumen blaf̧purpurtoth. Shatht

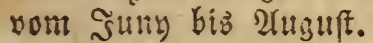

5) P. maculata. Die runben 3-4 Fü bohen Stångel fitto purpurroth uno grun geflect. Die Blátter långlid langettformig, gefpişt, am Ranse etroas fdarf uno ungeftie!t. Die Blumen find erft roth, Dann violett, und bilden eine Condraube, die aus entigegengepesten flas den Stringen beftegt. Die Etaubfaden fino orangeroth. Blithzeit: 2uguft mul September.

6) P. nitida. Sehr áhnlid গ̊r. 2. Die Stangel werben jebod nid)t fo hod), und bie grósern längern Blu= men find glánzcno = purpurreth. Die SBlátter gläuzento Dแnfe!gruำ.

7) P. ovata. Der Etangel wirb 6-12 3oll hod),

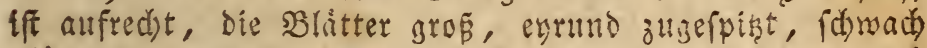

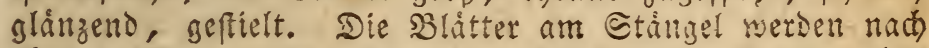

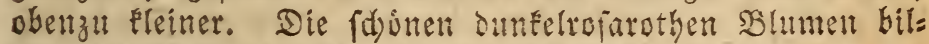
den eine Doloentraube. Eignet fid fit Den Iopf.

8) P. paniculata. Mird $3-4$ Fñ boch. Die

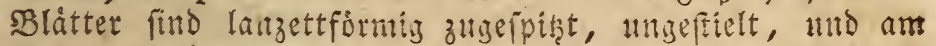

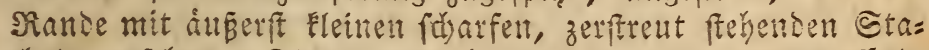

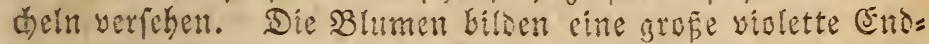

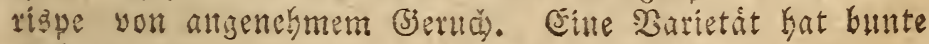
SBlitter.

9) P. reptans. Die Eproffen find Eriedend mit entregenuefisten, vertefrt eyformigen, ganzrandigen Blit=

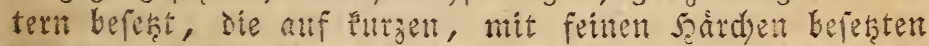
Etielen fehen. Die Blumenfangel wetden 6-8 Boll hod, mit 5-10 yiolettblatuen $B$ limdisen.

10) P. setacea. Die Ctångel fint Dinn, Gódtftelts einen $\mathfrak{F} u \tilde{B}$ lang, niebergeftecet, die feinen, limienfömigelt, phisigen Blitter faft gebifidyelt. Die Blumen erideinent im SRay, find violettrothlid) am Sield), mit iunflert zle= chen. Eignet fid firt ben Iopf, beennmt lefmige grobfan=

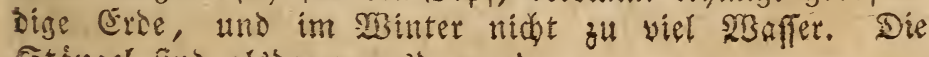

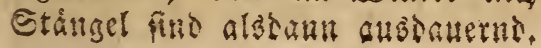


11) P. speciosa, h. - Die Etangel fint freauds= artỉ, alfred)t, glatt, fehr áftig, mit linienfórmigen Bláts tern Eefept, Dayon die obern roedjelsweife, lino an ber Bafis ausigebreitet fechen. Die Blumen bilden gipfelfåll: Dise, rispenartige Doldentrauben. Die frone tit weis mit rofurother Sd)eibe, âhnlid Den Blumen ber Vinca rosea

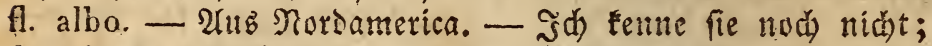
fie fonte feod wohl wie Ni. 14 behandelt, und-waht=

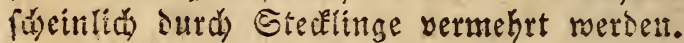

12) P. suaveolens. Die Pfanze ift glatt, bie

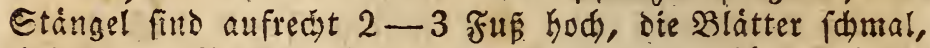
linientanzettformig, fpis und einander gegenúber ftebend. Die slumen ftefien in Endtrauben, find reinmeif, unt bas

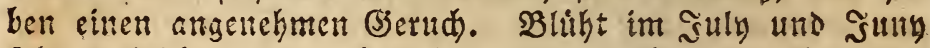
feber reidlid, uno wudgert in gutem, nidjt zu naffem $\mathfrak{B O}=$ Den faté.

13) P. subulata, febr áhnlidi Nit. 10., wirb wie diefe behandelt, und verbient aud einen Plah im Bimmer.

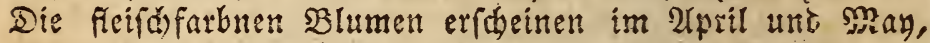

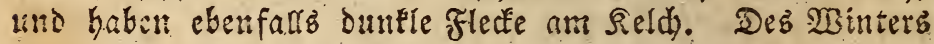
leibct fie in freyen Rande oft yom froft und yon ber Náffe.

14) P. suffenticosa. Die Stángel find Galbftratud = artig, Dod fdineide idj fie jeden Serbfi ab, indem bie WJurjelfproffen am idjonften blüben; fie merden $6-8$ zod hod); Die Blîtter find lanjettiöormig, ftumpf gefpisht, Dult=

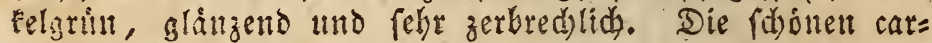

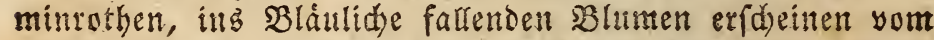
2 Ingut bis in Den Detober, andid zuroeilen fdon friber. Diefe Frammenbrume liebt lehmige Erbe, Eelommt Topfe

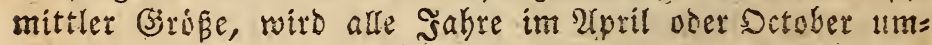

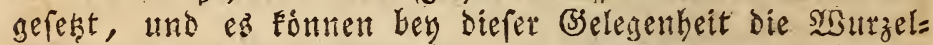
fproffen leid)t abgenommen roetden. Sie foll zwat in sats

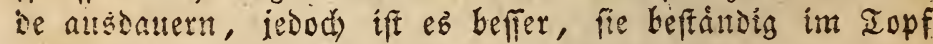

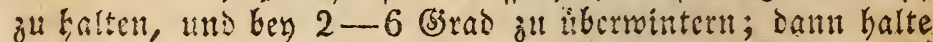
man fie nid)t zu feuddt. Eignet fid wegen ifres niedrigen

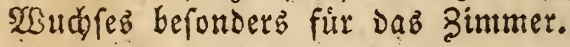

\section{Phoenix, Dattelpalme, XXII. 3.}

P. dactilifera, wabre Dattelpalme, $\zeta$. Sn ien heigen Begenden von 2rfitea und rrfen; wirs him sins mieser auti) in stalien uno Sicilien gezogen. - Die Ferne der befannten, im frandel băfig yortommenten 
Dutteln geben leid)t auf, weth man fie máfig feudbt und red)t roarm bålt, uno es madjt vielen Pfianzenliebhabern Nerghtigent, fie jul jieker, lim altd attb Der Elaffe Der

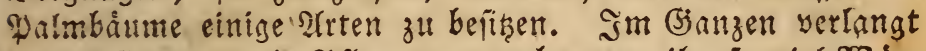

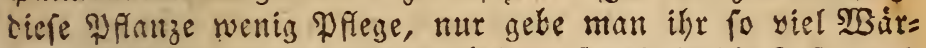
me und sid)t, alb man Fann, bringe fie nie in Die $\cong$ ft, und balte fie másig feud)t. Cie wád)ft polangfam, sas man faum furdten Darf, fie mersen fur bas Fenfter zul groß werden. Salle brey sabre etwa gibt man ifr eillen etwab zrókern, befonderz tiefern Iopf.

\section{Phormium, $\mathfrak{F l a d b l i l i e , ~ V I . ~} 1$.}

P. tenax, zábe Flad blilie, 24. Neufeetand. - Nur der umftand, dáj de wilden Einmohner yon Neus

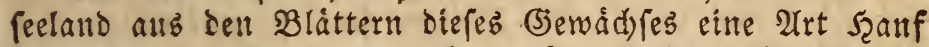
bereiten, und bá man aud) in Europa Berfude gemad)t

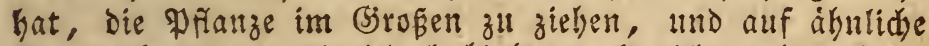

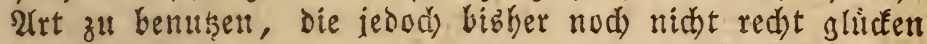
wouten, fant ber Flachslitie einen Plath im Bimmer yer= fdaffen, benn außer ben 2-4 Fuß laugen, glänzenigri= nen fdwertformigen Blättern, die altz eitner Enoltigen $\mathfrak{X}_{3}$ ur= zel, nad) Xrt Der gemeinen Edwertlilie, emporfeigen, ems p fieklt fie nidutz, ba fie bey uns hoddit felten blubt, unb

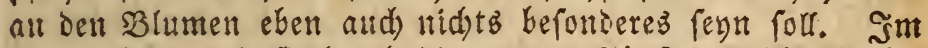
(Segentheil wird fie durd) die grosen Iapfe, welde fie be= Darf, bald láftig. Sie nimmt tibrigens mit iedem Boden yorlieb, verlangt viel Waffer, uno wird surd Bertheilung sermekrt. SRau Eann bie Yehr záben Blåtter, ftatt Baft, jum Inbinden set Báume uno Strảuder benthen, wie

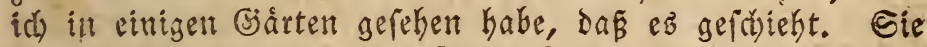
sautert zwar juweilen im freyen sande aus, jedod leibet fie in unfern nobrliden (segenden gemeiniglid fefre yom Froft; Daber gibt man iff in Winter $0-4$ Sirad.

$$
\text { Phylica, \$hylica, V. } 1 .
$$

P. ericoides, Mutenbeide, h. Arfica. Die furzen, beynake natelartigen Blätter find am Rande

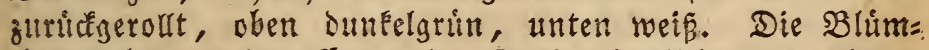
d)en ftehen an ben Enden der Żweige in Fleinen, weisen, filzigen Fopfden, uno eridseinen im Jrihling, oft ald im

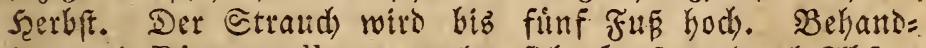
lung wie Diosma alba, vermehrt fid ebenfatab butd) 2tb fen= Fer unto Stectinge. 2estere mathte id im Suly, bedecte 
fie mit eitrem (3)afe, und bielt fie bey mäpiger fettotig= feit 4-6 Moduen in der Sonne. Sm Minter ift die \$hy= lica gegen in viele Feuditigeit empfindlich.

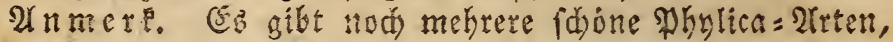
von benen io jeboth Eeine genau enne. P. a ce r os a

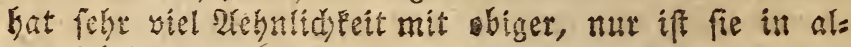
len Ihoilen Eleinet.

\section{Physalis, Sulutie, V. 1.}

1) P. alkekenge, sudenEirfde, 24. Deutid: lant. - Sogleid) biefe \$finnze eigentlid int freme sand gebort, fo foun man fie bod) and im Iopf Iakin bringen, Frithte fut tragen, went man im 2rpril einige feime itt einen nid) zu cugen Napf nit guter, Fieftger Erbe legt, und fie feudist bist. Die Blatter find fpisenformin, bie

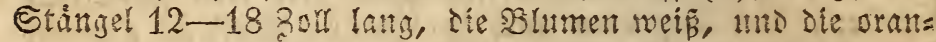
getothen rmisen Beeren auf einer aufgeblafenen fraut your

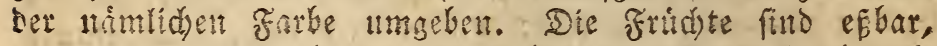
von eitem angenelynt! bittertid) = weinartigen (3) echmade,

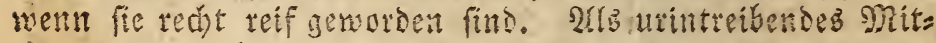

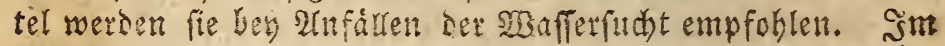
freyen sanbe gedeifen fie in leidyem eiefigen Boden am be= ften, und trägen reidylid Fridde.

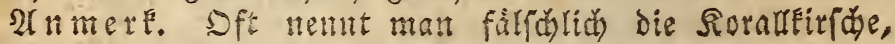
Solanum pseudocapsicum, șttontitrith.

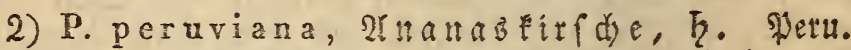

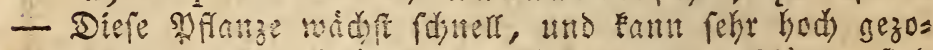
gen werben. Die Etăngel unt berzforntigen Bhläter find

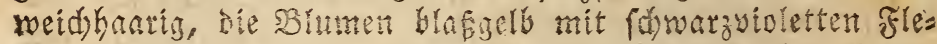

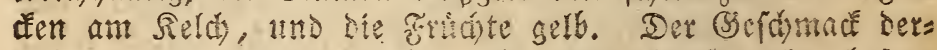

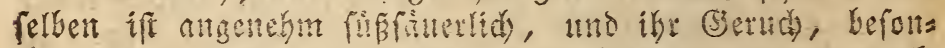
Ders, went man eimige in ener Sdublnde liegen und trots:

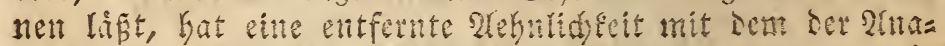
nas. Eie nimmt im simmer mit jesem Etante vorlies,

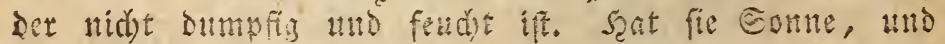

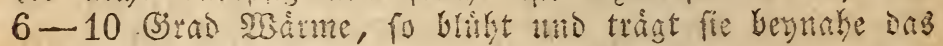

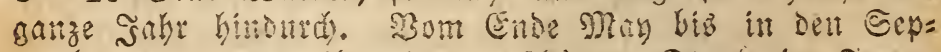

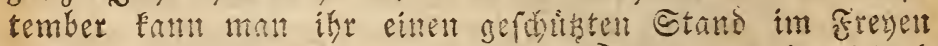

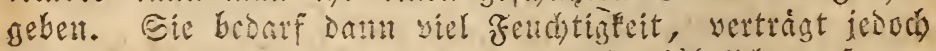

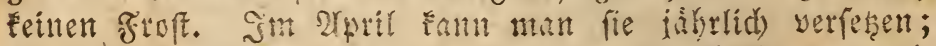
fie geseigt faft in jebem Booen gut. Durds Eamen und

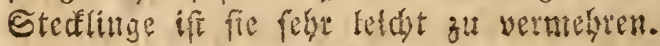




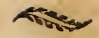

Plectranthus, fabluchfporn, XIV. 1 .

D. fruticosus, firatidatiger febnenfora,

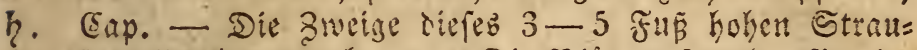
dec fino feiforis und bra!m. Die slatter foft beaformig, am Rambe jeferbt, und frar gendert. Sie babent eimen unnmenelymen (Sietud). Faft Das ganze Sabr bindurd ent= riclelt fids die blatter, allf ber untern sippe mit bumflen Pinctden verfek, ene Blumen, weldye in 4-6 3oll langen Iranben ftehen. - Eine wahre Stubenpfiange, welde wie Physalis peruviana betgandelt wird.

\section{Plumbago, Bleyturz, V. 1.}

P. capensis, blaublifende Bleytutz, দ. Cap. - Die Blåtter diefé fleinen હtrautdez fino länglid)= yerkehrt =ebformig. Die blä̈blauen Blumen erfdeinen ben ganzen Eommut difer in reidhen Dolden, uno gleidjen fehr Detten Dez Phlox divaricata. - Nan gibt diefer Nflanze

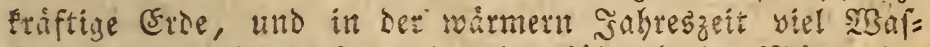
fer; fie murzelt fate, uno wird jabrlid im gratrz oder

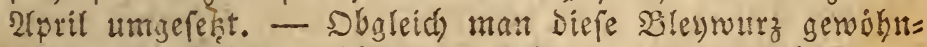

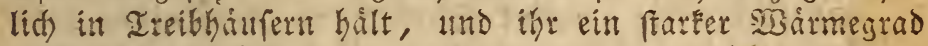
aud) fehr gut betommt, fo Eann mant fie dod in Erman=

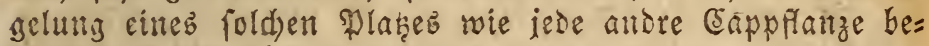

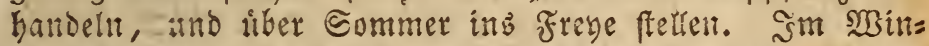
ter bringt man fie bey 4-8 (3rad gut Durd, wern man

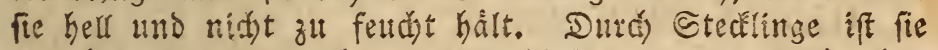
am beften zu vermegren, und biefe gezognen Nflanzdyen blikgen idoun bey 4-6 zoll frobe, dod miffen fie ân= fango warm gehalten roerden; juweilen lägt fié) aud bie \$2fianze zertheilen; Camen babe id nie erbalten. - Siede eillzelue Blume bliffe nitht viel lünger, alb eiment Iag, aber bey bittanglider Nafrumg und spliege blubt fie ben nod) faft ohne Unterbred)ung yom Nay ab bis zum Serbft;

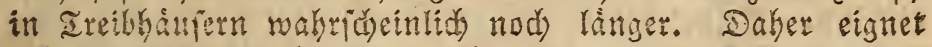
fid) Diefe Wfanze befonters fur fleine Eammlungen, 1tto

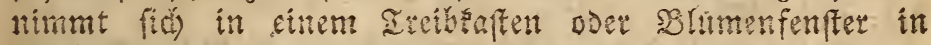

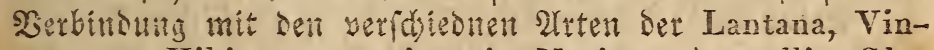
ca rosea, Hibiscus rosa sinensis, Terium, Anagallis, Gloxinia speciosa, Cyrilla pulchella, Caladium bicolor, Rosa thea um Tradescantia virginica, feht glt aub.

Polypodium, Ififelfarn, XXIV. 5.

P. vulgare, gemeluer 
befoniers in serggegenden, båufig wits wadafende Pfanze findet fich dafeibft zwifden Felsfpalten, auf alten Mautern, Batmmutrzeln 11 . F. ro. Die Murzel ift Fnollig, åfitg, unb

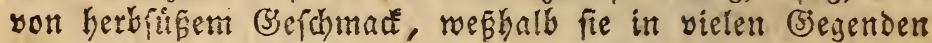
Eufrwurzel, aud Engelwurzel genannt wird. Die 4-8 Bud Iangen Blátter ftehen auf glatten Etielen, fino zu betoen Eeiten abrochlolno tief eingeidnitten, bie Samens băufden fehen auf jedem sáppden ill zmey. Feifen, uno find anfánglid gelblith = orangeroth, fpáter bráunlid. Man bebt die \$flanze an intem naturliden Standort aus, rekt fie in Sdutt mit sauberde vermengt, und bált fie fajattig und feudt. - Sie blúgt faft den ganzen Sommer bin outd), und verbient eher einen

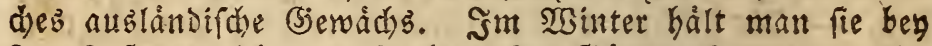

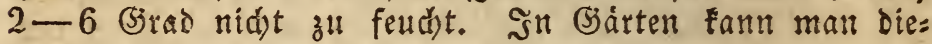
fen Iupfelfarm gut auf alten Mauern, zwifdsen Steinen unb $u m$ bie Wrurzeln alter $B$ áume anbringen.

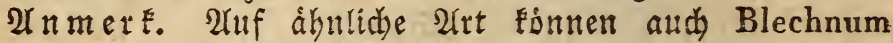
boreale, ber nordide sippenfarut, welder auf ho= ber Bebirgetr, z. B. Dem ærefeligebirge, gefunden wird, Defêgleid)en der gemeine Etridfartn, Asplenium scolopendrium (Scolopendrium officinarum), Den man im meftliden Deutidalano báufig zwifden Gelien an feudten Drten, aud zroifdan Den Eteinen augges maueter Brunter fittoet, und einige andere Farrn= fráute: befandelt werden.

Polianthes, Iubere, VI. 1.

P. tuberosa, gemeine Iuberofe. 21. Dft: indien, Beilon, Saya. - Man cultivitt gemóhntid in

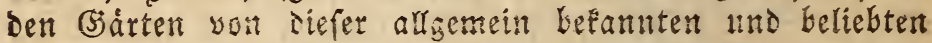
Pflanze Die einfactje lino gefurth blithende Corte; die Blu=

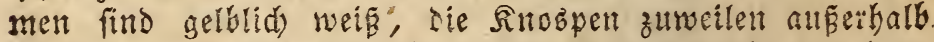

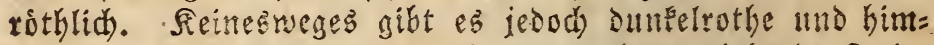
melblaue Iuberofen, mie nidst feiten berumziehende swies

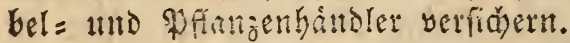

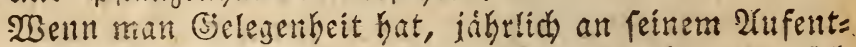

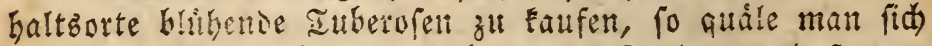

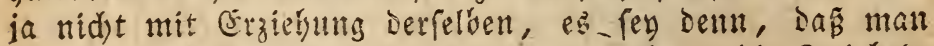

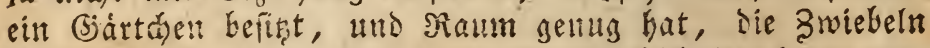

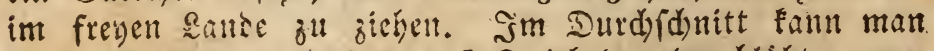

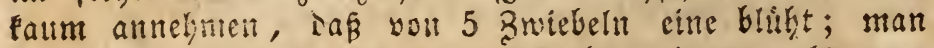
muß saber inamer weit mebe 3wiebelu legen, als man 


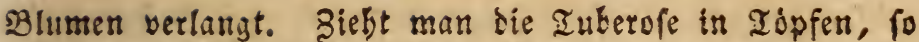
máblt man bierzu die ftárffen Brviebeln aแs. Die daran bangende 2 rut, die man obne Beidatigung ber Nutter= pfianze ablofen pann, nimmt man $a b$, und legt bie 3wie= beln einjeln, vom februar bis zum D(pril, je nudb)em man Die 33 lamen fruber oder pater ju erbalten winjat, in geráumige; tiefe ropfe. Cine gute ङromifdung entfebt, wenn man gleidse Ibeile gut gefaulte und gefiebte Mift: beet = Frde, sauberde, Flusfand und etwas weniges sebm mit einander mengt. Die Iopfe ftellt man an einen war=

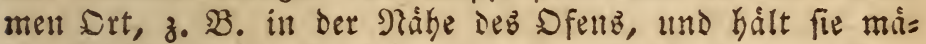

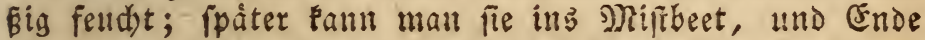
May in bie \&uft Eringell, wo ez gut ift, die Topfe an einer geiduthten fonnigen Stelle bis an Den Rand in bie Erde eingugraben. Se ftarter die Sroicbeln treiben, um fo mebr Waffer gibt man ihnen; am meiften, wenn bie Ctăn= gel ber Blumen fid erbeben, wab gewỏhnlid vom suly bis in Den Eeptember gefdieft. Dann lann man die Xopfe in die Stube nebmen, und binter ein fonniges Fen: fter ftellen, wo die Stängel jedod $4-5$ Fü bod werden, wábrend fie im sande felten mehr als 3 Fus Sobje erreidjen.

Bieht man die Iuberojen im frewen sande, wie idh gefeben babe, das diefes felbft in mordidaen Giegenden (z.

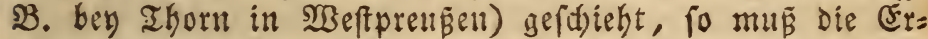
de zruar mabrbaft, jedod fandig feyn. Man weidst Mitte Nay die gereinigten 3roiebeln 24 Etunden lang in Baffer ein, lást fie hbertroḉnen, uto legt fie in Reiben $8-10$ Bolf aubeinander auf ein Beet. Bey trodnem Wetter be=

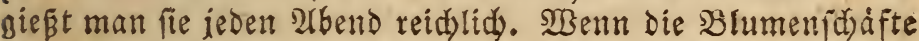
etwa $1 \frac{x}{2}$ Fú bod) gemorden find, Gebt man die Broiebeln mit Barrent aub ber Erde, pflangt fie in Iopfe, bålt fie einige Iage binter einer Secke in Echatten, mo bringt fie bieralf in die Sonne oder ins Simmer, wo die Blu= men fid) bald entwickelt. - Megen Des ftrren Duftes fer man im simmer vorfichtig, Eringe niemals zuviel blubente Iuberofen in daffelte, lind Dulde befonders Feine wber Nadit in einem Schlafcabinett.

Ment die Tuberoren abgeblubt baben, fo lảst man

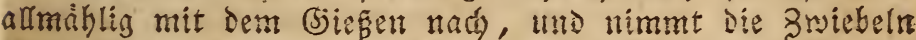

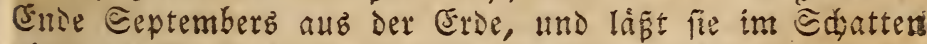
vollig trocknen. 2rub Diejenigen, weldbe noch im sande ftes ben, bebe man fruber aus, ehe Nadbtfrofte sintreten, dente diefe fud deu Swiebolu febr nachtheilig. Sind diejelben 
yodrig getroctnet, fo reiniget man fie von Blättern und Wurzeln, nto yerwabte fie in einem fladsen Faften oder in cinem Gat in Der warmen Stube, oder Dody an einem vollig froffregen Srte. Sat man eite farie Iuberojenzmiebel in einem Topf, und bat biefe nidy geblibt, fo fann man fie rubig in oer troctinen Eve liegen laffen, und Ende sas mat wieber anfangen zu treiben, worauf fie geroig zeitig bluben wird. - Die 2 Burgelbut Yese man jedenfallo den Commer liber ins sand, benn dort werden die 3miebeln meit beffer madjen und friber blibbar merden, als bey out befen sperge im Topf.

$$
\text { Primula, }
$$

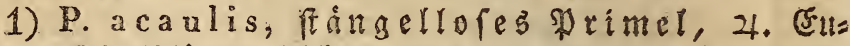
ropa. - Die Blâter gleiden benen deb gemeinen (3artenpri= mels. Die Blumen fehen jede auf einem einzelnen $3-5$ 3oll langen Ctangel, find gells, roth, lila und purpurroth in vielen. Conattungen. Befonders gefuaht werden bie gefituten Evielarten. SBebandung mie die folgende.

2) P. a uricula, 2lutifel, 21. 2tpen. - Sch will bier blof einige wenige Bemertungen ther diefe befannte Bfange mitheilen, benn cite boufandige Beforeibung und cintbilung igrer Gpielarten findet man in den meipten (S)artenbuabern. - SBéanntlid ziebt man bie 2rurife!n ent= reber burds Bertheirung det alten Grode, ober aub Gamen. 2uf.erferm Wege pfianzt man bereits als gut und fdon anertannte. Wfanzen fort, Durd) die Samenzudjt erbalt man nete zarben und Beidnungen. Die befte Beit, Die Xli= rifeln z" verpflanzen, iff bie leste Seifte bes Jionats

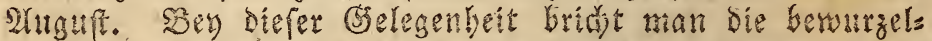
ten Geitenfprofien ab, uno fest fie in befonbere Topfe

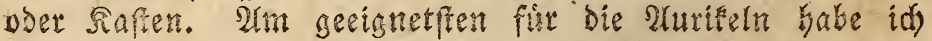
eine sifinung von gleiden abeilen sauberde, Iorf, sebm, Mifteeterde und grobem Sande gefunden, bod dat man

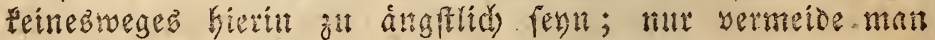
25, fio in zu feften, lehmigen szoden zu fesen. Sinn

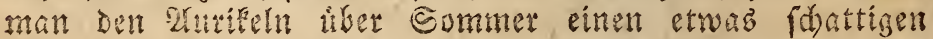
Ctand geber, fo if es gut; jebodi in frubling und in Serb隹 mifien fie ben ganzen aag bie volle Sonne gentefen,

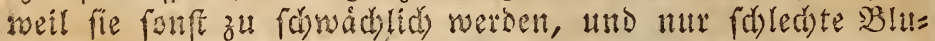

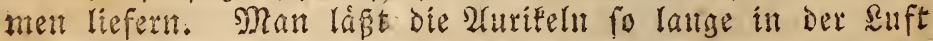
an einem yor Regen gefduthten Drte freben, bis anbaltende Frifte ou fommen orobell; bann bleiben fie bey $0-2$ Grad 
ruhts im Simmer, wo fie oftets Ruft ethalten; aber yout

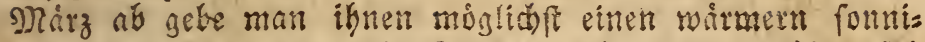

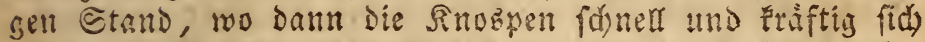
erhebelt. Ein plat in einein Wointerkaften ift isnen Daher

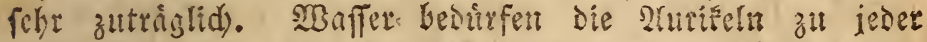
sabreşzeit viel, nur im 2 Binter mus man fiés mit Dem

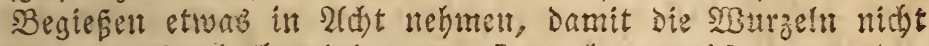
faulen. Des̆gals reinige man fie aud zum bften yon Den

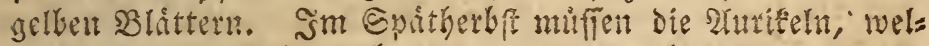
d)e in Iopfen freken, befonders gegen anthaltendes Regens

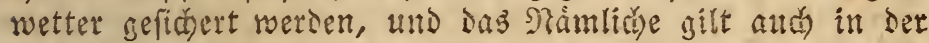
Blithzeit, bent nidht alfein verlieren sie sBlumen surd) Regen ajt ifrer Edjunbeit, fondern man befommt auth wes nis Samen. Biebt man die Slurifeln im freyen sande, fo rebe matt Daralif, dap ber Boden ia nidst zu feft ift, utto

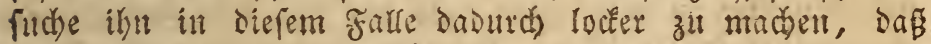
man red)t viel Sant, Saigefpảne und etwas Submift un=

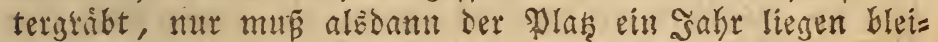
ben, uno wenigftens alle brey Monate einmal umgegraben

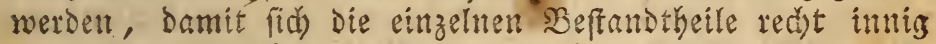

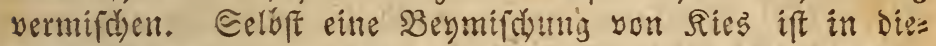

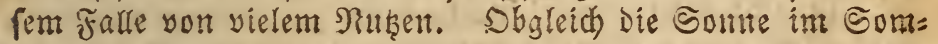
mer die 2turifelpflanzen oft fat verfengt, fo fähadet diefes

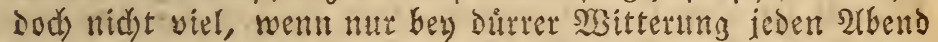

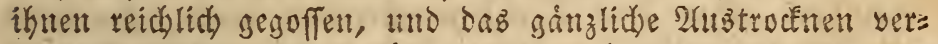
bindert roird. Sm Serbit merben fid bann bie \$frnzen

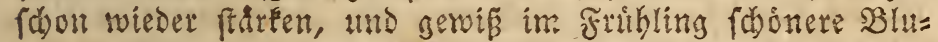
men bringen, als wenn mall fie it einen fdyattigen sibintel

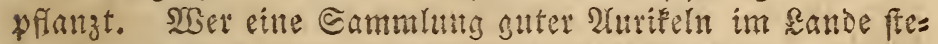
ben bat, und Eant isnen keinen Plah anmeifen, wo fie nut bis ctrua gegen Mittag die Sonne erhalten, Dem wird eg nitht

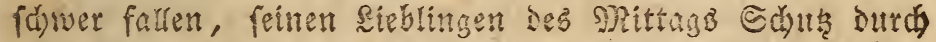
vorgefterte Decfen oder eine andere Bedacung zut geben.

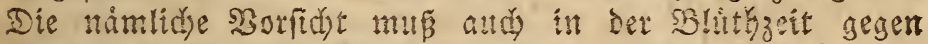

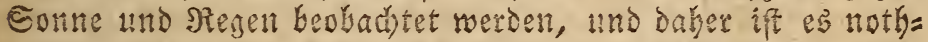
wendis, wenigften bie beffern Sorten auf ein Beer nebens

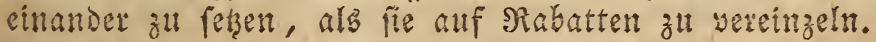

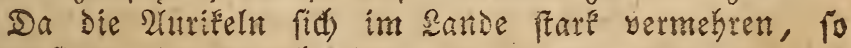
miffen fie wenigftens adyt zoll allsetnander gepflanzt, uno alfe sters Safre eitmal die Stỏce zertheit werden. Die befte Beit bierzu ift ebenfalts die leste Saalfte des anguft;

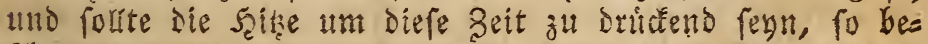
(d)atte man bie neu verpeţtem 24urifeln 8-14 Inge lang in 
Den Mittaggffunden, und balte fie feudt. Bey diefer (5es legenbeit roiro dab Beet mit verfaultem Mift etwas ges Düngt, uns nad) Bedarf Sand lutter Die Eroe gemengt.

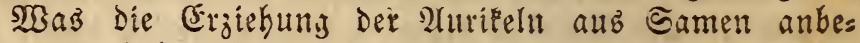
langt, fo Sabe id folgente Methode immer mit yielem

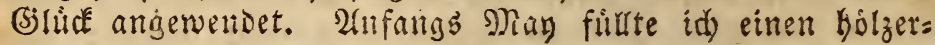

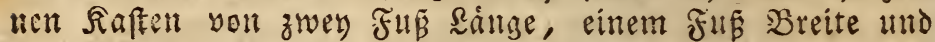
5-6 3oll Tiefe mit fo vis fein gefiebter Rauberde, Da nur etwa ein zoll bis an den Rand leer blieb. Die Eroe wurde mâsing feft gedruictet, und ber 2lurifelfame nid)t zu

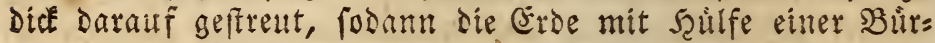
fte gut mit sigaffer angefprengt, ein Bretden, weld)eb ets was grỏfer, als der Saften fegn mus, darüter gedecit, und lesterer an einen plats geftert, wo ifn die Sonne yon bez Morizens bí gegen Mittaig befdien. Nunmehr war

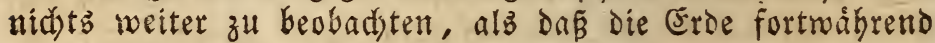

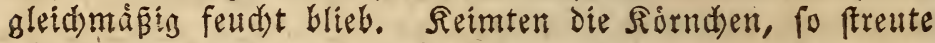
ich $D a$, two diefe gar zu bloź lagen, ein meniy Erde dars úber, und yon jebt $a b$ gab id) Der Dberfládse etwaz suft,

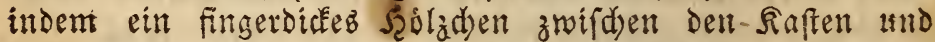
Den Decfel gelest wurde. - Cind die Pflanzen aufgezan= gen, to gibt man ihnen, inden man Das Bretchen aboelt, anfäinglidi) $\frac{x}{2}-1$ Stunde Des Morgens frủh, Sonne, bes fchatter fie aber fogleich wieder, Dod) fo, da bie suft uns gehindert unter Dem Dectel wegftreid)en fam. Se mebr die iungen 2lurifeln madjen, defto mehr gewognt man fie all bie Eonne, bis endlid man Die Decke ganz fort lás̆t, fie Goddfens in ser Mittaggftunde, wenn es nid)t móglid fenn folte, Derfelben bie pflanjen zul entzieken, nody an

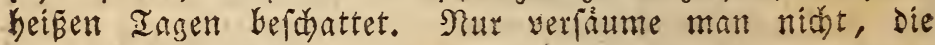
Erde immer gegorig feudt zu erhaltent. Sm 2luguft pflanat

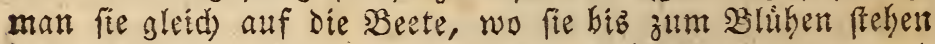
bleiben folfen. Daf man fie 2rnfangs befdattet, und and offers yom luteraut reinigt, bedarf wohl Eeiner Ermánumg. Selten blihen als Samen gezojene 2lurifelpfanjen yor Dem oritten Frithling, einzelne wohl nod) (pater, und inge ei= gentlichent Farben beftimmen fid' oft enft in Der Folge: Bes fommt man zeitis reifen alurielfamen, fo fantu er fogloids auggefäet werden. Sind die \$ranzen mtr einigermañen groß gem!g, fo verpflanzt man fie in der Mitte Eeptem= ber, uno gewinnt allf Diefe Meife oft ein ganzes Șith:

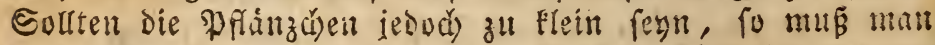

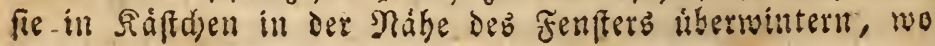


fie Sonte befommen, uno suft erbalten fỏnten, Damit

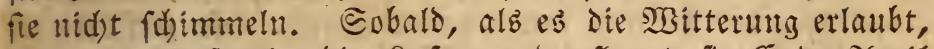
bringt man fie in bie suft, und pflanzt fie Ende 2fprif alf cill Beet.

Die sefutrten Shtrifeln werben in ber Regel weniger geadtet, alb Die einfaden, und unter diefen erbalten die

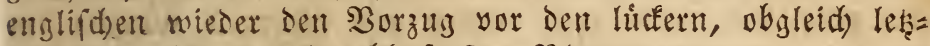
tere Die grósten und lebbafteften Blumen tragen.

3) P. cortusoides, fibirifde Primel, 4 . Cibiriet, - Nat geftielten, rutzliden, ein wentis gelapp= ten und geferbten Blittern, summem, $8-12$ 3oll hoben Etangel, an Deffen Epise bie fdonen lilafarbigen Blument

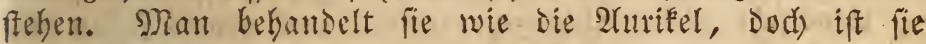
etwas zártlider, uno sezen Náffe befonders empfindlid).

4) P. elatior, (s) arten= $\mathfrak{d} \mathfrak{i m e l}, 24$. Dentf(t)= lant. - SBird faft ebent fo háunig gebaut, als bie 2luríel, mit ber fie aud im Betreff Der Bebandlung hereinftimmt, nut ift fie im SBinter etwab zadrtider, und letset in feftem oder fefr fdlammigem Boben oft surd) Den Froft. - Dft fommt ans sen Bhmen nod) eine zweyte 3 lume, P. elatior prolifera. Dods adjtet man bergleid)en Epielarten wenig.

5) P. minima, Eleinftez primel, 24. Europa,

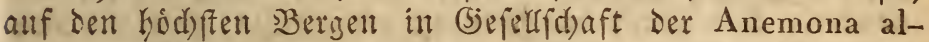
pina. - Soll bort mus man es bolen, benn feiten be=

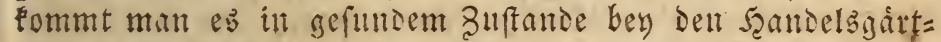
nern. Es wadje zwifden Felfen, rafenartig, Eaum einent Bou hod), die lebhaft grinen Blättd)en find Eeilfòrmig, an Der Eptige gezihnt; Die einzelnen Blumen ftehen alf Flt:

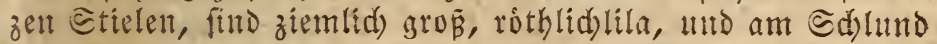
mit feinen weif́n Şârd)en verfehen. Sie eriddeinen im Friblitty, nuf Dell Bjebirgen einzełt bis in Den Eeptem=

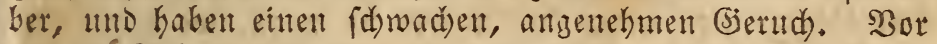
einigen Sabren fand id́) alf Dem Riefengebirige, am Ranto

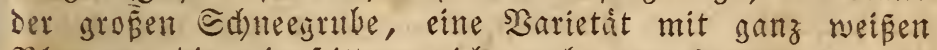
Blumen, die mir feitoem nidjt mehr yorigetommen ift. (5: bält fdumer, siefe Pflanze an bent Iapf zal gewohnen. Man fted) ganze Rafenfticfe, wortu biefe Primeln fitsen, fo wie man fie findet, tief genug aub, um die ftattent

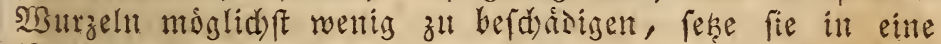
Mifdung yon sauberde, Iorf, Cand und Ries, und ftelle

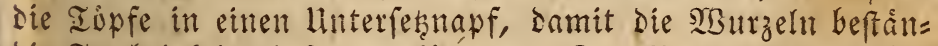

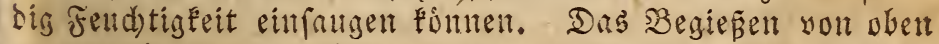

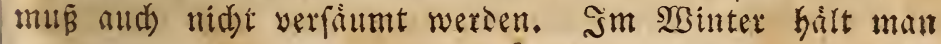




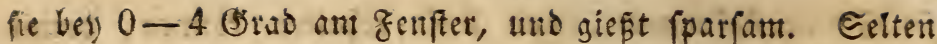
gluckt es, die Pflanze lange zu erbalten. Sft zetforte fie

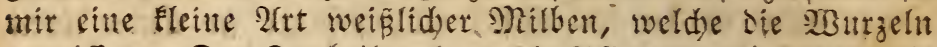
angriffen. Dab zertheilen fann Die Pflanze aud nid)t woht leiben. Sangere geit hielt fie bey mir im freyen sante alas, wenn id) fie in etwas fandige (Frde pehte. Der @as noe ging nit zwar allf, allein die Pflatzen moltten im

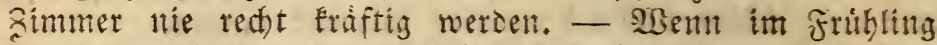
bie Sonne biejes Primel red)t surd)wirmt bat, fo Duften Die Blátter (d)wad) nad) Şeild)en; im Eommer babe id Diepe (Eigenthumlid)Eeit nie bemerft.

6) P. sinensis, dillefifdes Primel, 24. CHina. (Finte fehr (d) ìne siranze, die zwar nod) etwas felten ift, jecod) in jefre Eurzer Zeit diberalf zu crhalten fenn wird. Die Blátter fteben alf 2-4 zoll langen rithlidjen Êtielen, fint balb fiebenlappig, gezabint, unt gleidjen etwas sen Blattern dé Pelargon. radula; fie fins, fo wie die ganze

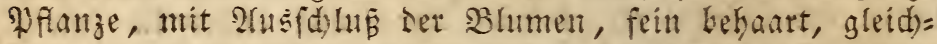
fam fettig anzufuhlen, und ried)en bernake wie sie Blu= nent ber gemeinent ed) liffelblume. Die Blumentátngel wer= ien 6-10 zoll hod), uno an ibnen feben die (oft gegen

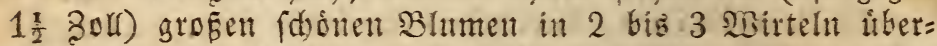

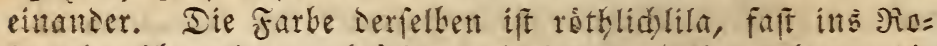
farothe ubergehend, blatfer uno sunfler, je nadeem bie

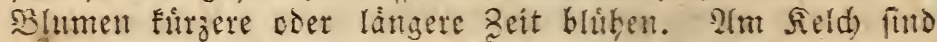
fie gelb mit funf gelterothen glecten, auf ser Buctecte faft weis. Die Blumenteld)e fehent wie anfgeblajen aus. Falt

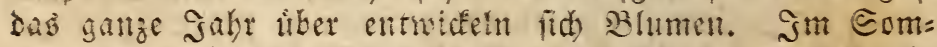
mer uno Sereft fint fie zwar Elein uno oft fo tuanjebnlid),

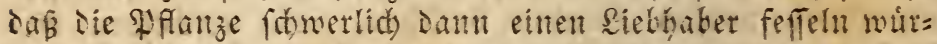

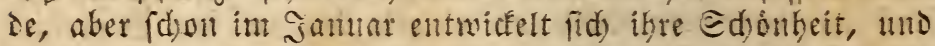
nimme bey guter Pflege bis in Dent Man zu, wo Dann bie Pflanze oft ganz mit Blumen úbersecft iff. - Eefr leidst ift bie Eultur diefer Bierpfanze. - Minn gete Dem dines

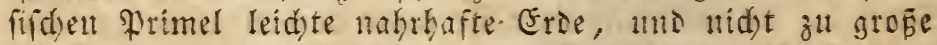
Iopfe; mahrend Der Begetationzzeit hinlanglid) Waffer, im Eommer einen fdattigen Etand, im sointer bey 4-S (jirad einem Plat zwifden Doptelfenfern, und suft, fo lange es nid)t friett, obgleidy die tiltern Planzen einen leidente froft vertragen folfen. Eomne witft feht wohl=

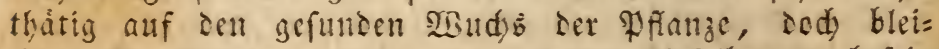
d)en die Blumen etwas alts, wenth Dicjelbe zul beftig

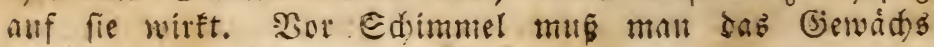


in 2tdyt nebmen. - Man vermegrt bas dillefirdae Primel surd) Bertheilung beum unfesen im 2utguft, oder nod) bef (er Durd) Samen, Dent man gleid) nad) Der Reife fảet, uno

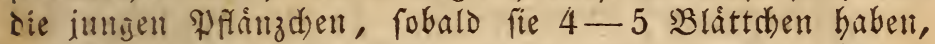
cinzeln in etwa vier Polf meite Iopfe fest. Sie bluken

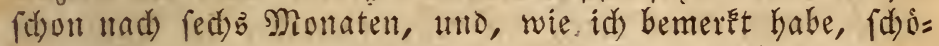
ner, alb̉ Die alten હtóce. Llm mit Beftimmtheit હamen zu erbalten, muß man sen Samenftaub mittelft einer fei= nen Feder auf bie (5riffel zu bringen fud)en, renigftenz feste bey mir jede to behandelte Blume eine ftarke Sapfel an, withrend bie dibrigen Blument abwelften, ofne da bies fes Der Fall mar. - Sb Diefe fdóne \$flanze aud) im sande über 2 inter auboauert, weís id) nid)t; weigen ifrer fribent Błumen eightet fie fid) aber auf jeden fall mehr für Den Iopf.

7) P. veris, Fithling b = ธd) luffelblume, Jatmelsfdluffel, 24. Dent (d)=

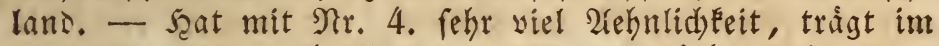
wilden Buftante gelbe Blumen, und biefe fárben fid), went man die Pranze in ben Garten bringt, und gut behandelt,

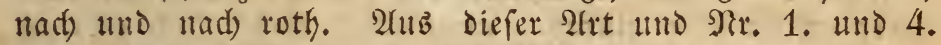

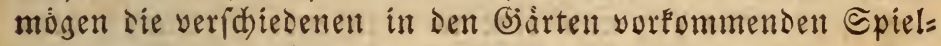
arten entfanden fern.

An mere. Bon den vielen úbrigen primeln, meift $2 \mathfrak{l}=$ penaemádje, Fenne id Feine.

Prunus, 疋irdie, XII. 1.

P. laurocerasus, ber $\mathfrak{i} \mathfrak{i}\{$ d d lorbeer, Sleinafient. - Ein ziemlith boker Baum mit lorbeerartigent, gláuzendgritnen, am Rande gezábuten, aub̉duernden Blát= tern. Die Blumen find weif́, und bilden eine lange hån= gentie Iraube; Die Rirfden find 价warg. Sm Iopf blügt er felten, reneng(tenb fann id) mid) nie erinnern, Blumen gefehen zu haben. N)an gibt ifm frảftige sauberde, eit

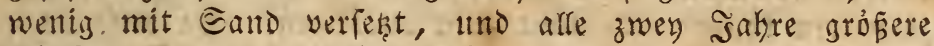
Iủpfe. Bey $0-4$ (j)rad lás̆t er fid gut liberwintern, und nimmt aud mit einem $\mathfrak{p l a b}$ im Feller yorlieb, wenn biefer nidjt zu warm ift. Ş(ud) im sande bålt

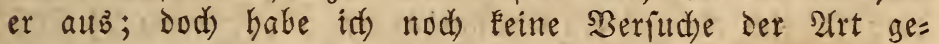
mad)t, obgleid) idj sen Sirfidlorbeer nidht felten in einem Bimmer $\mathfrak{i b e r w i n t e r t e , ~ i n ~ w e l d ) e m ~ d a s ~ I h e r m o m e t e r ~} 10$ 12 (Sirad unter 0 ftand. - Die Bzlätter, in Mild gefodbt, theilen lebsterex einen bittermancelartigen Befdhmaft mit; sod) (en man mit 2 (nwwendung derfelben yorichtig, insem 


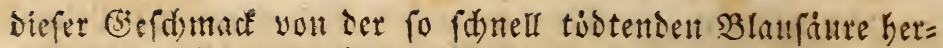
rithit, welde alle Iheile diefer Pfanze in Nenge enthal=

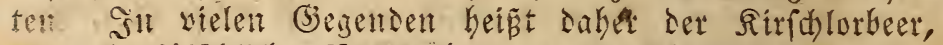

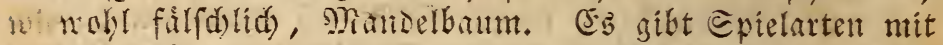

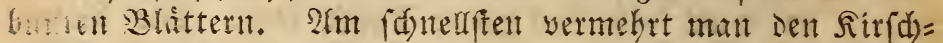

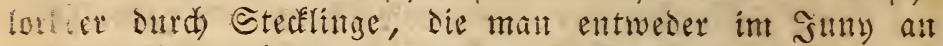

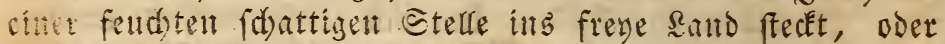
in inen Topf feht, und diefen zmen Monate lants im

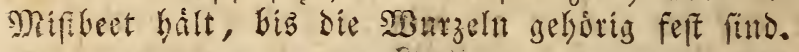

\section{Punica, (j) ז́tuatbant, XII. 1 .}

1) P. granatum, gemétuer (j) ranatbanm,

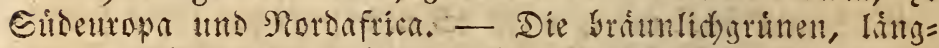

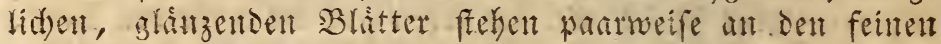
eckigen sweigen. Sie baben, menn man fie funt, einten

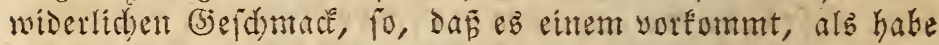

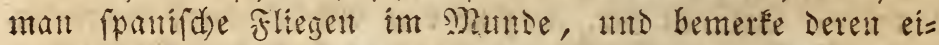
genthimlidjen (Sientd). Die priddtigen hod)rothen Bhtumen fthen zoll 3-4 an Dent Episient Der Zweige. Sie fint ent=

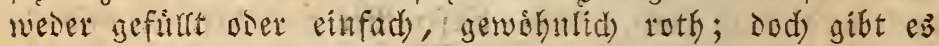

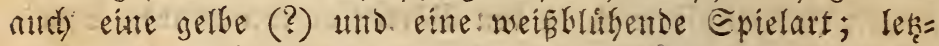

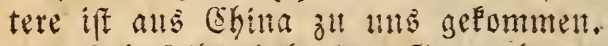

Sid) felbft habe Denl (Siranatbaum nod) nie in Bimmer gezogen, da es mir ftets an Raum fehlte. Llebrizents fí)eint aber and) derfelbe für unfer nordiddes (clima nid)t

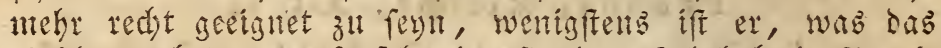
Bliken anbelangt, oft febreigenimnig. Sd babe in Frante

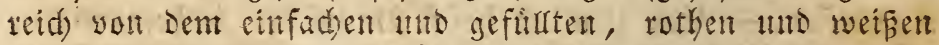

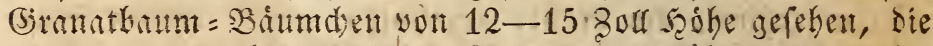
mit ien niedlidyfen Blumen prangten, wabreno man im

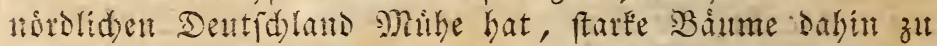
bringen, Sap fie thre Sinospen entfalten, bent nidist felten werfen fie biejerten alfe auf énmal ab, und bie fpiter fid)

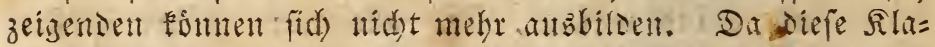
ge fait alfgemein iff, fo min id die Serantaffung Dem Clima-zuddreiben, weniger Dev Behantumg. Hebrigens

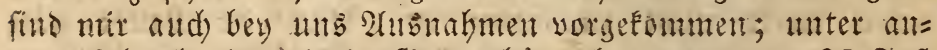

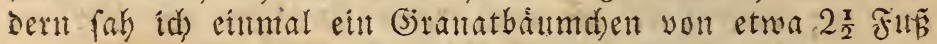

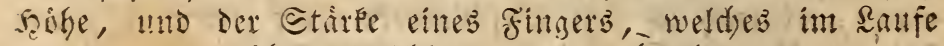

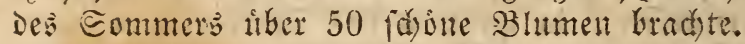

Raub = nuo Mitfbeet = Sroe mit etwa balb fo viel ge= meiner (s)artenerde unt etmas Flusfant gemifd)t, for bem (3)rindthanm am befen befommen, welder fid dibrigens 
leidst surd) Eteclinge vermebrt, bie man im Plpril matht, unter (S)las und warm bält; feltne Sorten vervielfilltigt man burd) 2rblatiren oder 2(bjaugen anf ben genteineit. (5't

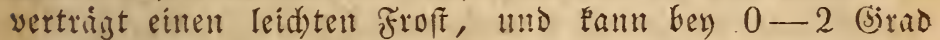
fehr gut im Feller liberwintert werden. Nan yerfest ifn alfe zwey sabre im fribling; im Eommer frelit mant ihn

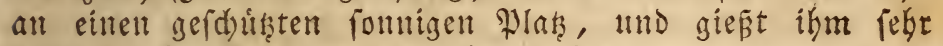
reid)lid), befonterb, went fid sie fnospen zeigen.

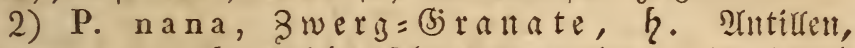
Enidamerica. - Er gleidst fehr Dem yorigen, ift jedods viel Eleiner, uno blift fehr willig. Bisher fentue ids mur

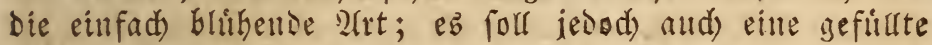
geben. Nod) babe id) siefen Strand) nid)t gezozen, uno er ift riberbaupt felten ed)t zubetomment. - Der szandels= girtuer Jlohnhaupt in Breslan vertauft yon Dem edsten eillfad)en bas Ettick mit 1 Iffle.

\section{Pyrola, SBintergitin, X. 1.}

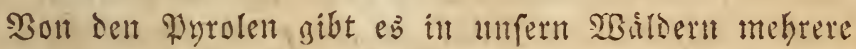
fehr niedidje Strten, welde wohl serdienten, unter Die 3 ahl Der Iopfpflanzent mit anfgenommen zu wersen. Sdh habe

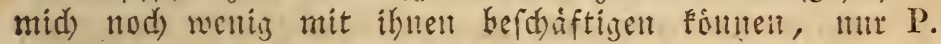
umbellata feste id) eimmal in ben Iopf, wo fie ant di) fort= wud) s, aber im erfen Sommer nidyt blihte; meine $\mathfrak{B e r}=$

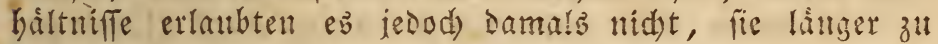

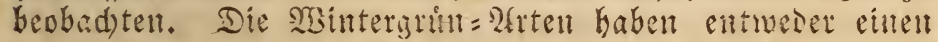
3-6 zoll langen holzigen Etăngel mit leserartigen, im= mergruinen, glänzenien Blittem, veer es find Etallsenge=

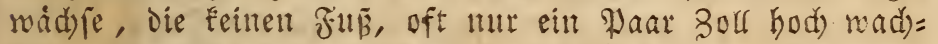
fen. Die Blumen eintiger arten baben 2(ehnlid)Eeit nit Denen Der Convallaria majalis. - S trit) nettne Gier nttr:

1) P. rotundifolia, 24. - MBideft in idattigen saubholzwáldern oft fehr háufis, und blift vom Mnary biz Sutty.

2) P. umbellata, দ. - Mit fali feilformizen,

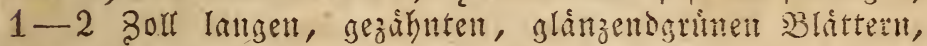
Sie an Den Spizen Der Sweige fait it einem sBirtel znam= mengervingt ftehen. Sizan fintet fie in trockent Riefermit= Dern cillzelt , oft in (Seferlfchaft Der Vaccinium vitisidea, Gat niedidye glockenformige, weipe Blumen, welde int

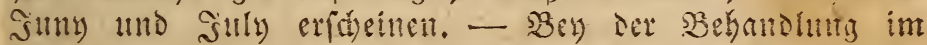

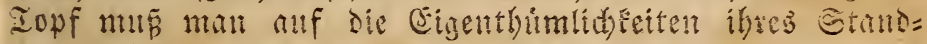

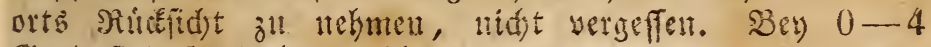
(3) rad fint fie feidst zat berwintern. 


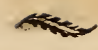 \\ Quercus, (Eide, XXI. 7.}

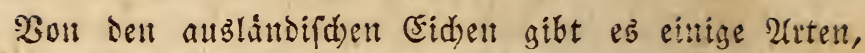
welde nur wentige fun hod wad) fen, uno weigen ifres

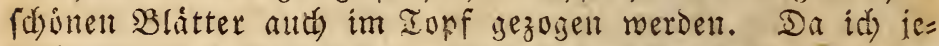
Dod) nod) Eeinen einzigent Betfud Der 2(rt gemad)t habe,

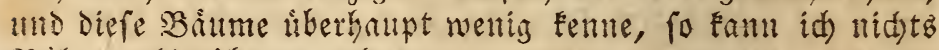
Shäheres berriber angebert.

\section{Rhapis, $\mathfrak{F} \mathfrak{a} d \mathfrak{d} \mathfrak{e} \mathfrak{p a l m e , ~ X X I I . ~} 1$.}

R. flabelliformis, hobe fád erparme, $冖$. Ehina, Sftindien. - Der Stanm wiro felbft im Iopf fünf und mehr Fun bod), widsft jedod jegr langfam. Die

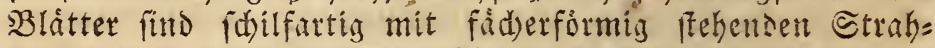
len, Die mit zunehmentem 2flter Des̉ Baltmes immer gró= Eer uno mehrftrahliger erfdeillen. Der હtamm if yon Dent Blattfielen und beren braumem baarartigen Semebe ganz umbüat. - Die Behandung. Siejer fononen Wfanze if jefr letd)t. 2ate 2-3 sabre im May gibt man ibr grófere nno tiefere Iopfe, wobey man fid hitet, die ftarken, faden=

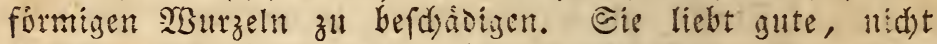

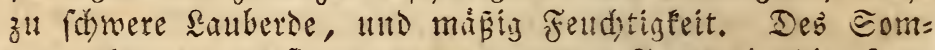
mers fann man fie zwat all warmen Iagen in die frene suft ferfen; allein fie gedeifyt beffer, went fie nabe am Gen: fiter in eitten fonnenteichen Bimmer in eitrer Iemperatur

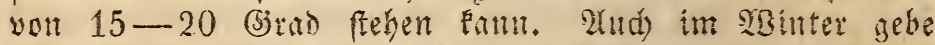
man igr wentiffens 8-12 (sirad Marme. Eonne ift ifr zwat inmer fefr wohlthátig, tod) babe idi) fie viele sabre an einem Sort tiberwintert, wohin niemals bie Somne fidin. Nur laffe man fie alsoann nidyt zu warm fteben, bamit

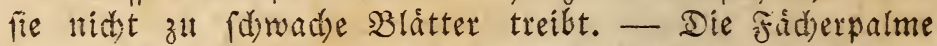

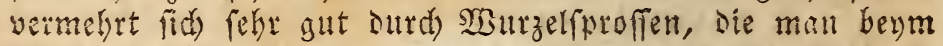
Sorfesien abnimmt, went man ant ifuen einzelne Murzeln bemert. Die jungen Eproffen haben anfünglide) mut ein einfaches Blatt, Dann betommen fie seren mit zwey Etrab= lent, und fo in ber Folge intmer grósere. Blumen bringt

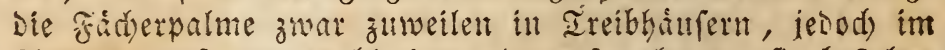
Simmer barf nan wohl faum sarauf rednen; fie beftehen

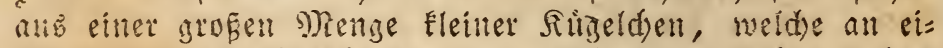
ner langen Ilaube hangen, Die zwifden Den Blattern her:

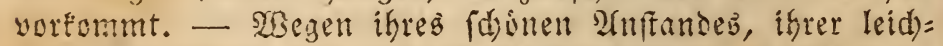
ten Bekatulung Int langfamen Wadsthumb verdent sie

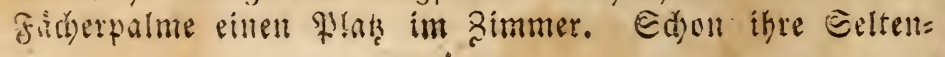


beit emprieflt jie, obgleid) fie in ben meiften Girten jicm= lidi) billing ift.

Rhododendron, Xrpentufe, x. 1 .

R. ponticum, poutifde Ilpentoje, l. ie yante, an fdattigen fenditen Drten. - Merdient eine Pplange die Uufnerffamfeit ier Blumenfreunde, fo ift es diefe art penrofe, weld)e nod lange nidht fo alfgentein befannt ift, als fie es fern follte. Der Straud) mas ziemlid) bod) wer= Den, dent id babe ifn felkf im Iopf funf Fus nud stuber sefeben. Die granbrame Rince ift rifitig, mut an sen 1 - 2ibrigen Bweigen glatt, rotbbraun. Die Blitter futro lorbeerablid), Duntel und immerigrun. Naan bat in Diefer Finfid)t verfdiedene Barietâten mit breiten und idjmalen, alld) wellenförmig gebogenen B!ătterll, die jufäring alts $\widehat{\Xi}=$ men entfeben, und feiner befonsern Bead)tung verdienen; fdoner find die Epielarten mit gelb= આno weifbunten Bzlat tern. Die Blumenfnospen erfá)einen bereits in Serbit,

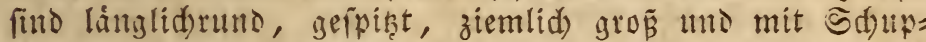

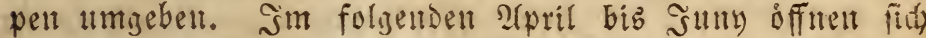
biefelbett, und es tretent aแs ifnen $12-25$ bernabe glo= denforming geftaltete shlumen auf etioa eillen soll langen Etielen hervor, weld)e einen balbEuge!forminen Ballen, gleid)

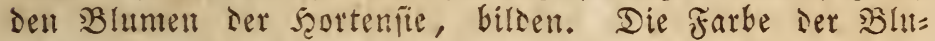
ment ift bellviolett, das unterfe Blatt jeder Blume gelb= braun getupfelt; fie baben eillen fdwadjen angenebmen (ij: rud). Nod) fdoner ift eine Epielart mit fleifd farbenen gro= Eern Blumen; aud) will man eine 2 trt mit gefiuten $\mathfrak{B} l u=$ mell baben.

Die 2lpenrofen lieben sauberse mit yerwittertem Iorf und Eand gemifdt. Da fie wenig wurzeln, fo ifi es bin= lánglid), fie alle zwey Sabre im rugut oder Septem= ber, wentl fie ien. zwerten Irieb gemadst, ooer beffer,

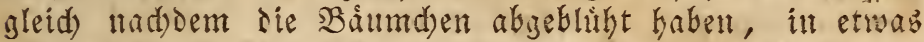
gröвere Iopfe z"l pflanzen, bey weldser (3) begentheit man

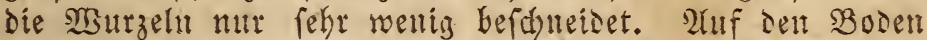
Des Iopfes legt mant einen zol bod Eleine Eteine, um

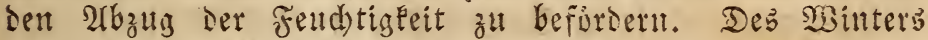
gibt man der 2lpentofe nid)t zu viel खbaffer, uto balt fie

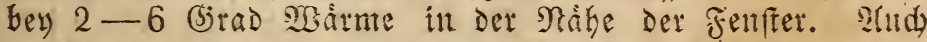
im freven sanie foll man diefe Alpentofe liberwintern fon= nen; bod) will idy es in unfern (segendent nidjt ratgen, ba sie Etcmmdren jegr oft, trots affer Eorbfalt, besuteno 
letsen. Sm Sommer bringt man bie 2llpenrofe all cinell etwab

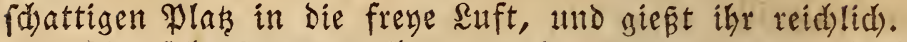

$2(m$ fid)erften vermebrt man bie 2llpentofen butd) $2(b=$ Fenfer, Die man im Frithling in befondere fleine Iopfe

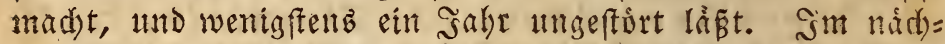
ften 2uguft lofit man fie ab, und fidsert fie 8-14 Iage gesen die Sonne, bis man úberzengt ift, daf́ fie hinlảng=

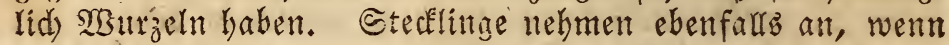
mall fie im 2(pril mad)t, else bie Iriebe fid) entwickeln, in leidjte crove pflanzt, mit eiltem geraumigen silafe zubedt, anto in ein warntes Miffueet ftert, wo fie die Sonne er=

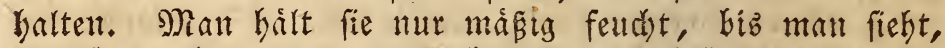
bá fie wadjen wolfen. Nidht immer glieft diefe 2(rt der Sermehrung. - 2tud) ber Same geft ziemlid leid)t auf, wenn man flarbe Iopfe mit Iorferde füth, den Sament alf Diefelbe ftreut, und mit einer (Slasplatte bedectet. Fend)= tigkeet und Sonnenwárme darf nie fehlen, und es if gut, Den Sopf in einen Unterfeb̧apf zu ftellen, Den man zu=

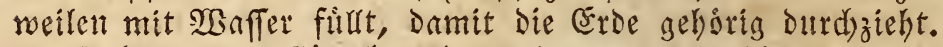
- Cobald die pflánzden Geryorkommen, gewobnt man fie atmablig an die suft, gibt ihnen jedoci) ntt die Norigen= fonme eit Daar Stunden lang.

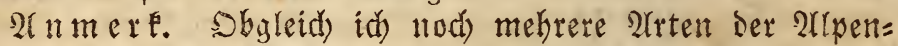
rofen befise, fo babe id biefelben bod) nod) nidyt ge= nutgam fenten gelernt. Nein Exemplar yon Rhododendron azaleoides ift etwa (ed) zo zolf bod, hat yier Błluthenetrospen, und diefer wirb wie R. ponticum befantelt. Seine Blumen babe ids nod) nidyt gereket.

Rochea (Larochea), Rodjea, V. 5.

Die Rodyen wurben frifher zu ben Crassula $=$ Yrten gezáblt, mit Denen fie gleides Baterland baben, mits fie wollen aud eben fo bebandelt fentr.

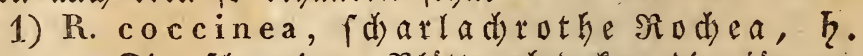
2rfica. - Die fduppigen Blátter bedecen die jüngerit 3weige faft ganz, tho diefe lestern tragen in ben Eom= mermonaten an ibren Epişen mehrere faft robrige, fülf=

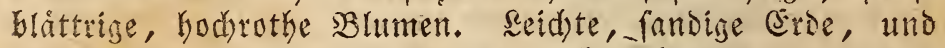

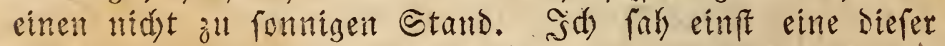

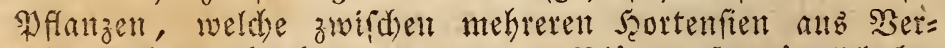
fehen fteken geblieben war, Deren Blatter fie gånzlid be iecten, uno im Eduatten Derfelben Gatte fie eine budjt 

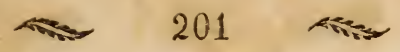

yourommene Blume entwiffelt, wie id midy nid)t allf eine

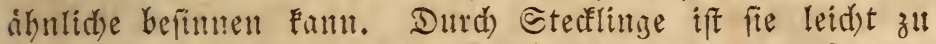
vermekren, und dieje nebmen anfünglid̆ mit einem Etante

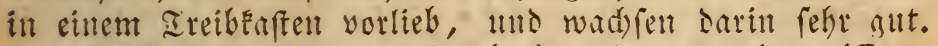

2) R. falcata, fidjelblattitige Rod)ea (Cras. decussata), ط. Cap. - Der Etamm wadthit graie auf=

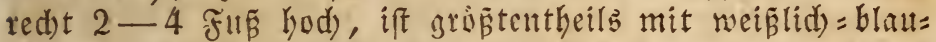

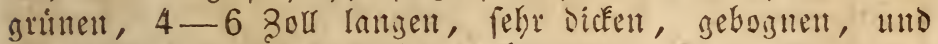
hiber Sirelz ftehenoen Blittern befletiet. (ser mad)t in ser Reizel meniz Eeitenafte. Die ganze Pflanze bat ein fon=

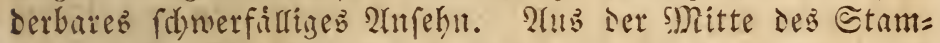
mez erfebt fid) allf kurzent Etängel eille grof̈e gedraingte Dolde, welde eine unzáflige Menge Eletimer orangerother Blimden enthilt. Diefe fdoune \$flanze ift oft fidsuer zum

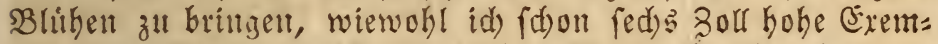
plate mit Blumen gefdmild fah. Man gebe ifr lefmigen, fandigen Boden, und verfesce fie, on fie wellis wurgelt, alfe

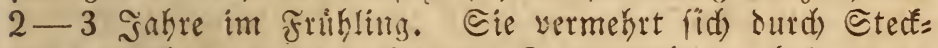
linge, Dod) mun man oft, um Eeitentriebe zแ befommen, Die Espise des Mutterftocks abutesmen. R(ud) loft man eines Der untern Blatter red)t befutfam yom Etamme ab, lápt

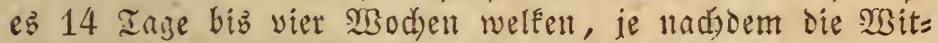
terung ift, pflanzt es in einen fleinen Iopf mit fansiger

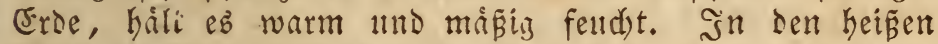

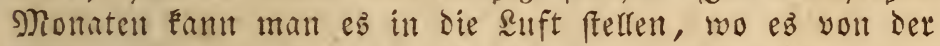
Mittagbfonne, Dod) nidjt vom Regen getroffen wiro. Dies

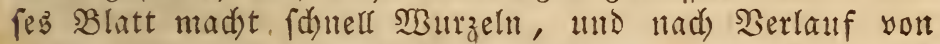
einigen Nonaten zeigen fid um saffelbe junge Sweige, bie man 1-2 Sabr ungeftort wad) fen lást, worallf man fie abuimmt, unt als einzelne Pflanzen befandelt. - Bon Mitte Nay bis gegen Den Eeptember ferft man diefe No= d)ea in bie freye suft, wo fie bie volfe Sonme geniest; junise Pflanzen lingt man ald wohl die erfen sabre in einem Treibeaften, samit fie fdneller in die Şdhe gehen. șm Winter 4-8 Girad, und in Den shonaten December bis Februar betommt fie God fit felten gegoffen.

\section{Rosa, Rofe, XII. 13.}

Sbgleid) bie Zahl ber Rofenarten fdon zur zeit un= glaublid) groṕs iff, uno Durd) Cultur uno arufintoen neuter

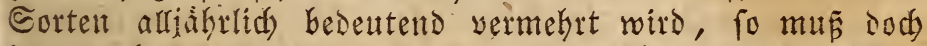

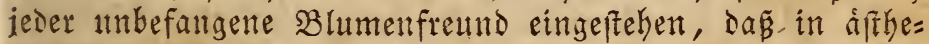

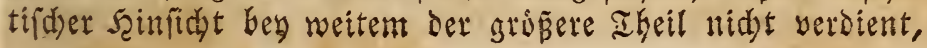


gezogen zu nerden, entweder weil ifre slumen zu fajledt find, ober weil fie fid zu wenig yon eiltander unterfdei= Den. Fü den Botanifer ift Dab̉ Gsebiet Der Rofen eitt Felo

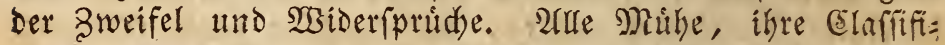
cation und Beftimmung ins Flare zu bringen, ift bís iest gropentheils frudstlos gewejen, uno (ja) artner, fo wie Błh= miffen, arbeiten fortwáhrend daran, nod) mehr Dunel úber Diefen Zweig Der: Botanif zu verbreiten.

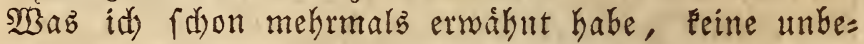
Fannte Corte eittez reid)en Dflanzengefd)led)t wenn man fid) nidjt durd) eignez $2(n$ fd)auen yon sem $\mathfrak{S e r t h}$ Derfelben diberzengt hat, diefe sefre findet bey den Rofen

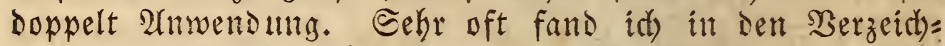

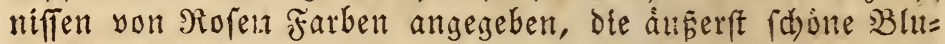
men verfpradent ; fah idh aber sie Rofe blihen, fo entoedete id) mit aller sixnftrengung meiner Einne feine હpur yon Den geprefefent Farben und Beidumngen.

Man Fontte die Rofen in zmen Srauptclaffen theilen:

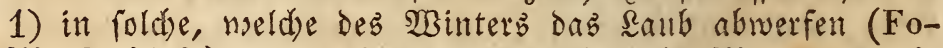
liis deciduis), unt 2) int immergrime (Foliis sempervirentibus), witewofl aud bier bie (j)renze nid)t genau ge= ntitg gezogen iff.

马u Den fhónen fommergrunen Rofen, bie id Fente, geforen folgende 2 retelt:

1) R. centifolia, bie huntertblattrige Rofe mit ifren veridhienenen Epielarten, yon ienen $\mathrm{R}$. c. maxima, minor, alba (R. unica) und dijonensis befonters (d) in fints.

2) R. muscosa, die Noosiofe. Die Noosto= fen gefioren wobl eigentlid) zu bent Centifolien, dent fie baben ien númlid)en Ball Der Stad)eln, Blatter uno Blu= men, nur fino Die Fnos̄pen uno Blumenfángel mit moos= artigen fáferchen bewadjen, was eben diefe Rofe fo be= liebt madit. Faft alle oben altgefihtten (Eentifolien findet man and als Moobrofen, tho auserdem alld eine einfa= d)e Sorte, Into einine antere.

3) R. lutea, bie gelbe Rofe, einfad) tmo ge= fultt. R. austriaca nit eisfadyen, intwentig brauturothen, altswendis gelben Blumen. Sie eignen fid) mehr fürb sant, als für ien Iopf.

4) R. alba, weíse Rofe. Mefred ober wentiger ge=

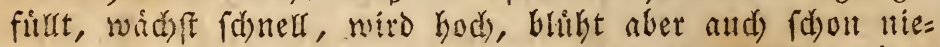

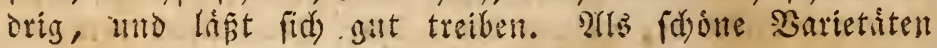




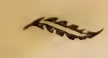

\section{3}

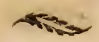

neune id): R. a. incarnata mit fleifd)farbuen Blumen, und R. a. puella erubens mit weifen Blumen แแto blaß̧rotber

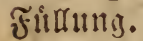

5) R. pomponia minor. Unter biefem Namen erbielt id) (yon den (sjebridern Boud)é in Berlin) eine

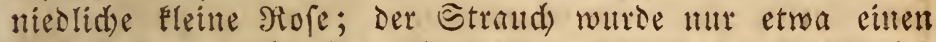

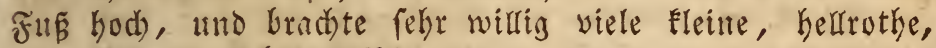
ranunfelartig gebaute Blumen.

6) R. vi o la ce a nova (ebentalts you Dent (Sebritbern Boud)é), halbgefutut, Dunfelroth mit violett fdattirt. Die Farbe war fehr verfdieden, ie nadjoem die Rofe fids in frewer suft entwidfelt batte, ooer im Miftbeet getrieben worden war. Sekterer Blumen hatten ein bremtendez Roth mit wenig violett, wákrend die der erfern beynabe ins 2lidblaue fielen.

R. burgundica (parvifolia), champaniensis, Vilmorina (transparens), coronata, lucida plena babe id) zwar frubler felbft gezogen, und niedlidje Blumen you denfelben

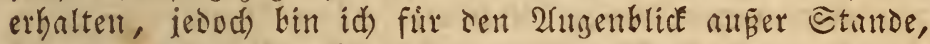
fie genau zu befdreiben, da eb zum Iheil fdit mehrere Sahre her ift. - Unter den samen Ranunel= und Sam= metrojen geben mebrere fehr fdodne Sorten. Erftere baben meiftens Dunkle, inb Biolette falfende Bhtmen, mit Fur= jell runten Bláttern, die fid etwas zurickéchlagen. Die Eammetrofen find mekr oder weniger gefull, ifre Blatter fammetartig, die Farbe meiftens fehr Dunfelroth mit \$ios= lett oder Edwwarzroth itberfogett. Die Neléprofe Eenne id) nodí) nidyt; fie foll rothe Blumen mit altsgezadéten Blát= tern baberr.

Bon den immergrinen, grós̄tentbeilż aud) immerbli: benden Rofen find mir foligense 2(rten beéant:

1) R. Banksia mit vielblumigen meiken Bäfd)eln.

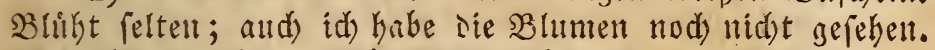
Die lebhaft grủten. Blátter fino långlid) zugefpibt.

2) R. chinensis hybrida, mit balbgefütten dun= Felviolettrothen, in Bufdein ftehenden Blumen. Sidh befine fie erft feit Furzer zeit, uno kaun noch nicht nit (jeswisheit

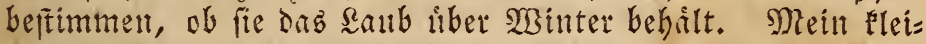
nes (Exemplar hatte die Blítter abgeworfen, iedod) gleiden Defelben fehi Denten der úbrigen inmergrinen soofen; alld nebment die Stećlinge ebent fo leid)t zu ieder sabreszeit an.

3) R. indica (R. semperfiorens minima), 3werg= toje, thesrig uno Elein, rofaroth, boppelt bribent. 
4) R. l a u rentia, nohl Eleiller, als bie vorige, ifr feht áhnlid. Man bat fie cinfach uno gefutut.

5) R. moschata. Die Blument biefer 2fut fins grof́,

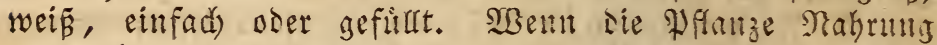
gentrg bat, fo ftehen Die Blumen in (ehr reidjen Bufdeln.

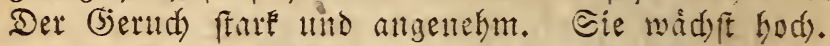

6) R. multiflora. Sefhr reid) blibento; Dods bilt es fdrwer, Mutterftóce Dahin zul bringen. Die BInment find mittler (jorife, Gerreth, aud foll es eine Irt mit Dunflen Bltmen gefent. Die Blâtter find ein wentis ranth, uno nid)t fo glänzendgrtit, als Die Der vibrizen Gier genann= tent Corten. SMaddft fefr fduell, uno wiro hod); Die fei= net ameige bedurfen jesod einer unteritusung.

7) R. noisette. Die Rofen fint ziemlidi grof, Glaproth, und an ben jungen Exaftigen Trieben finsen fid́ oft 20-30 Sinospent. - Eine Fehr fdonte 2lrt, yon wel=

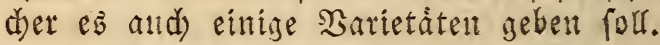

8) R. persicifolia (longifolia). Hnterfdeitet fid) yon afen andern Rofen burd die langen fdmalen Blátter. Die Blumen find irregulitr und (d)led)t. Slan bitt fie fur eine Epielart von R. semperfl.

9) R. semperflorens. - Ran muterfdeiset zmen Seanptarten yon ben immerblifenden Rofen, eine belt= mub

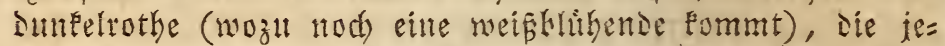
Do (b) Eeinesweges als Barietáten zu betrad ten find; Dem wate diefes anbigmadt, fo fonnte man wohl antegmen,

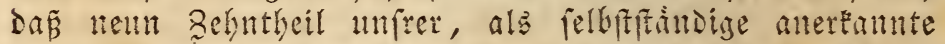

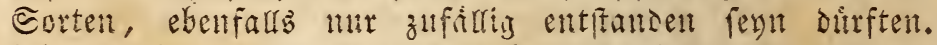
Shne nida weiter auf unterfudungen cinzulaffen, nemé id) biet:

a) R. s. alb a, meishtithent, einige Sुatietáten, you Denen die eine mit groṕent regeimásigen Blumen

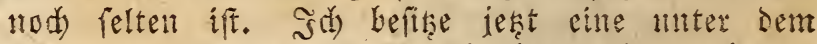
Namen albida, bie jesod fehr blaprothe, mbeden= tente Blumen triagt.

b) R. s. a nemonaeflora, sunfelroth. Die imtert Blátter fino fleiner, als die áusetn, tumo Etatb. Beid)utet fid nidit befonders ants.

c) R. s. Bischonii, Imteltoth, niesrig, felyr feime 3lueige, Der folgensen ăbulid).

d) R. s. ca rmesina, carminroth, ziemlidy gefiut, gut gebant, unt fleising blibento.

e) R. s. pallida, sie gemeine blafrotge, yon ter sie= 
jentige, weldye nod) ben Beynament centifolia firhth, oft Fehr fdionte Bhtumen liefert. - Beibe wad) fent, weut fie Nahrung getutg haben, $3-5$ Fur hodj.

f) R. s. atropurpurea, sie gemeine sunfetrothe;

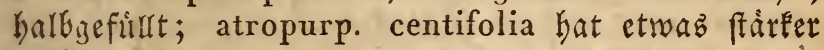
Befútute Bhumen.

g) R. s. de mons, Rafe yout Mionz. Die Brument fint ziemlid) grof́, sunfeltroth und fara sefülut. Der (Serud) ábulid') Dem der Eentifolientrofe. Die Bhtument variiten fehr binfidsts Der Farbe, Denti fie find oft faft blutroth, bald bláffer, bald mit vio=

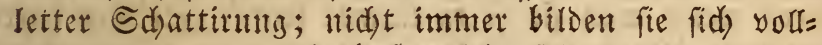
Fommen alls, weşalb fie nidjt pelten etwas șrres gullátés haben.

h) R. s. speciosa, prád)tige Monatỏrofe, ebenfartz

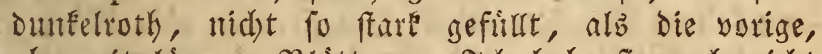
aber mit lángern $\mathfrak{B}$ låttern. Șd) babe fie nod) nidjt gezogent.

10) R. Thea, bie Igeerofe;

a) carnea, dieblaffe Ifeerofe, mit fegr grobent, sefúdten und farte ried)endent Bhument. Sie liebt Die 233 irme, wenn fie gut bluben forf. Eine yot= ziglid fdoune Pore;

b) rubra, mit Eleinern rofarothen Blumen; Fommt

- an Edyunheit Der vorigen nidat gleid. - Eş gibt icht ald) eitre getbe Theerufe, Dod) babe idd) Diefelbe nod) nidst gepeher.

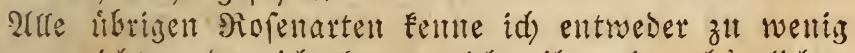

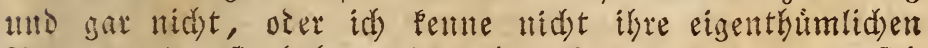
Namest, oder fie baben mir nidjt gefalten, und eignen fich

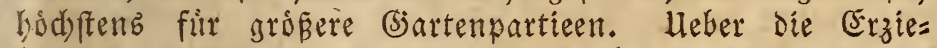
hun! Der Rojen Jolgentoes: Die Rofen befomment Drangeries

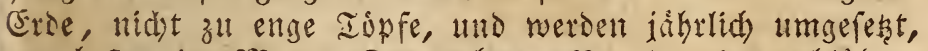
am beffer im Monat September. Ber Dent immerbluhben=

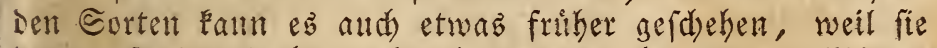

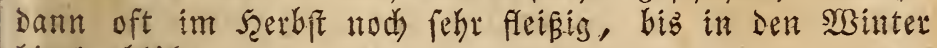
binein blithen.

WBitl man sandrofen treiben, fo bebt man fordde fdyon

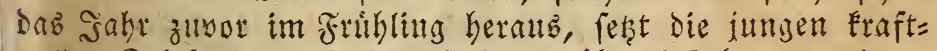
volfent Etócke in paffente Ioppe, grabt Diefelben an einem etwas bejchatteten Sorte int die Grode, bălt fie Dent Sommer

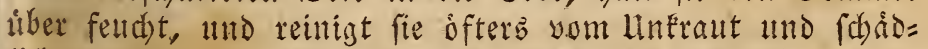

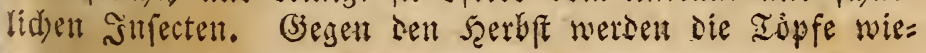




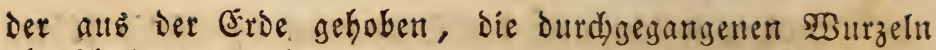
abgefdnitten, utto, wenn man fürdstet, es middte den Etòken an Nahrung fehlen, fo feht man fie in grófere

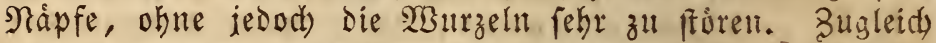

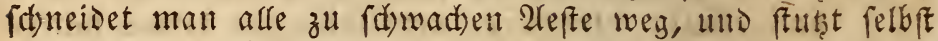

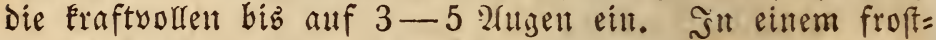

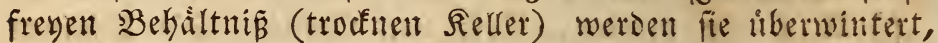
Dod) ia nid)t fo warm, dá fie ill Den 23 intermonatent ftark zu treiben anfangen. Sbgleid) Die Rofen unfern 2 Bin= ter ziemlid) gut im sande altshalten, fo fino Dod) Die in Iopfen gezogenen oft empfindlid) gegen große Rálte, befon= Derb wenn man fie fdon einmal friber getrieben batte, und id) Gabe ofters Eentifolien ganz verloren, went id) es wag= te, fie eitter Rálte yon 12-15 (5irab allz̧zueken, wáhreno

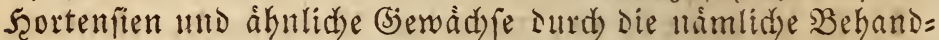

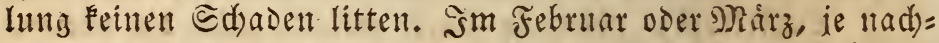
Dem die Mitterung und die sage des Binmers ift, bringt

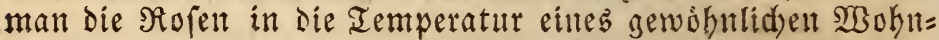

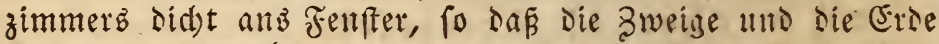
Sonne erbalten, befprenst fie zuweilen mit laumarmem $\mathfrak{B a}=$

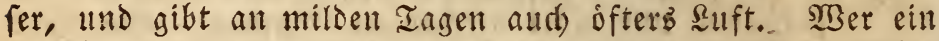
Mifteet zu feiner Dispofition hat, Der ftelle fie Mitte Marz in baffelbe. So mie die 3 weige fid) entwideln, mú man

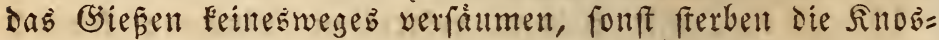
pen leidht ab, ald gefhiegt diefeb nid)t felten, went man Den Stand Der Rofen offers verindert. Die zeit, welde er= forberlid) ift, eine Rofe zum Blihen zubringen, ift rebr

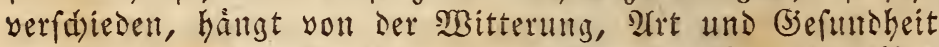
Des હtockeb ab. 2lm fritheften blithen R. alba, R. dijonensis und Centifol. minor, sod) fuche man niemals die

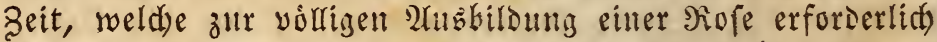
ift, ju úbereilen. Se langfamer man fie treibt, um fo (d) olnere Blumen erbălt mail.

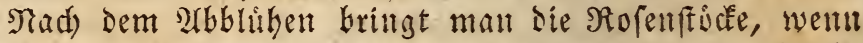

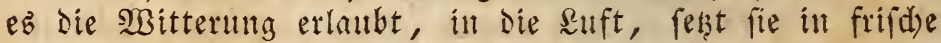

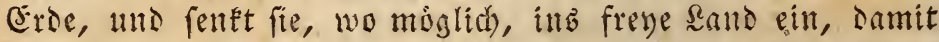

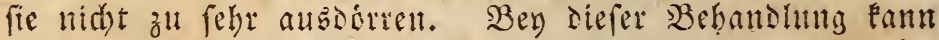
mant fie yiele Sabre int Bimmer zur Blithe bringen, be= fonders, wenn man nad) Dem Blihen Die absetragenen

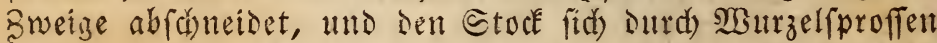

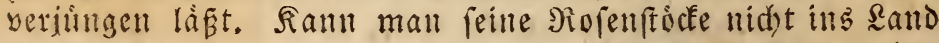
fesell, fo mur man fie an eillem fá)attigen Plat freben affen, wo fie die Morienfome erbalten. 

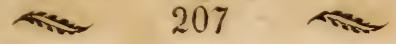

Die immergrunen Corten werdelt alf áhnlide ât

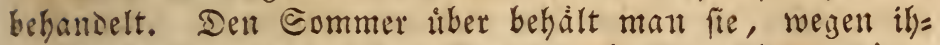
ver Blumen, gern allf dem Fenfer, obwohl nid)t zul lättg= nen ift, dás fie weit beffer blúken, went ald fie inb Rand gefenlt oder gepflanzt wúrden. Nutr wenige, z. B. Die Iheerofe, lieben einen rarmen Etand, und die genannte blubt am fdouften, wenn man fie in einem Ireibeaften bált. - Heber Winter läjt man fie bey 2-4 (3)rad fies hen, uno gibt ihnen ofters suft, bejonders im Seerbft, wo= jelbft ein leid)ter froft ifuen nidhts (d)adet. Woarme vertra= gen fie im $2 B i n t e r$ nidht gut, wentigftent merden fie fdwadd)=

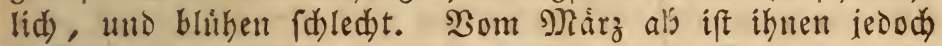
Eonne unentbehrlid), und fie muiffen Dant nabe anz $F e n=$

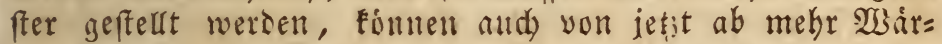
me befommen. Wenn man die alten 3 weige im herbft voer zeitig im grubling einftust, fo treiben die meiften

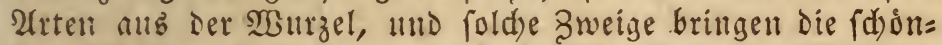
fen Blumen, befonterb wenn man sie Riofen im Miftbeet antreibt. Sinige Corten id)einen daz şeiduneisen nidyt gut zu vertragen, indem fie eine gewiffe (5róse erreiden milfen, ehe fie blithen, z. B. R. multiflora, moschata,

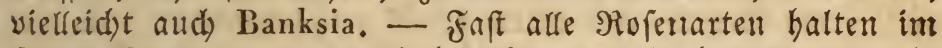
freyen sante aub. Dod) bedect man bie beffern, um fie gegen heftigen froft zu vermahren, indem mant die sweige nieierlegt, uno @trol, uno (Erie Dariber ausbreitet. Die Gohern હtimme muffen mit હtroh nno Baftmatten ver= hullt werden, cod) Fann man aud) fie, fis lange fie nidit

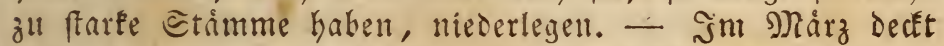
mant fie wieber allf, tulto bincet die zweinge an. Die áls tern zaweige werben nad) Umftănden aแbgefdnitten, uno felbft die iungen, fräftigen Iriebe, wenn man nidjt zur 2(bfid)t hat, fie bod zu ziehen, eingeftubt. Die immerblu= hende Rofe ift Befibern Fleiner Blumenftocte befonders zu empfehlen, sa fie ohme befondere Mrube den ganzen Gom= mer uber, bis tief in sen Saerb/t Ginein, fortwábreno blúbt. Erft, weitn es ernftlid) Ealt zu werdell broht, bedecht man

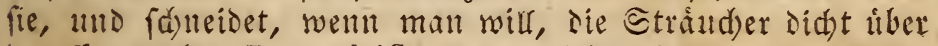
ber Erove ab. R. multiflora foll fid fehr gut firrs sallo eignen, Dort $8-12$ Fuf hod werden, und fehr reidhlids blühen. Die fálanéen Stúnme erlauben daš Niederlegen jebr gut.

Man vermebrt bie Rofen auf verfdiedene Art. Die

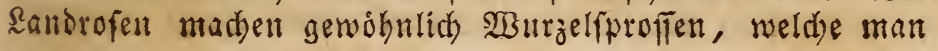




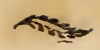

im Eeptember, fobalo fie tur einige $\mathfrak{N}_{3} n t z e l t$ baben, $a b=$ ffict)t. Da man aber bey mandien Sorten oft lange yer=

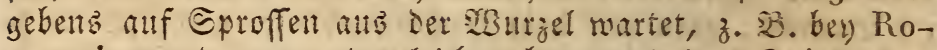
sa unica, oder man Dergleidjen bey vereselten @tămmden nidat erfalten fann, fo mad)t man vou dicfeli ins sano oder in santeben gefterte Iopfe im grtibling Pibfenfer, die man ein wenig eimfdneiset. (5s verfteht fich, Bns mant

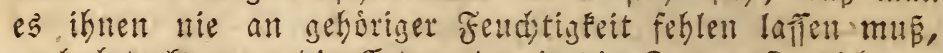
andi) bedecft man Die Erde, Damit die Eonne fie nidit zu

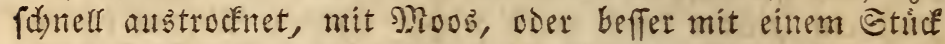
verkegrt Darauf gelegten Rajen. Sn Der Regel find diefe

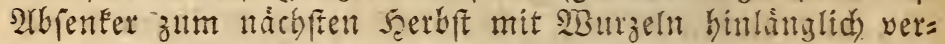
fehen. Die Stecklinge von Den fommergrunen Rofen habent mir nie einvurzeln wolfen, obgleids es zumeiten glicfen forf. - Dietrid) fabt in feinem sexicon beteriber Foligendes: ,Durd) Steclinge babe id die Eentifolia, sie Mroosrofe und einige altsere Urte'n vermebrt. Shierzu bentste id die jut= gen Iriebe yon Den Eträudyern, weld)e im sBinter it Ireibgatient und Bimmern geblift batten, und in ber

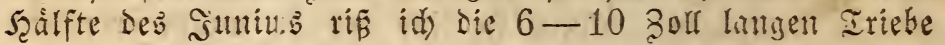

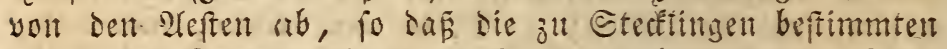
Bweige am (Sinnte mit einem $M$ u!f verfehen waren. Diefe

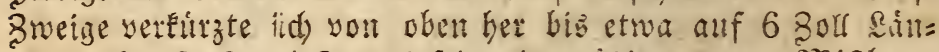

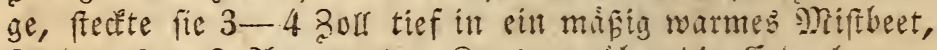

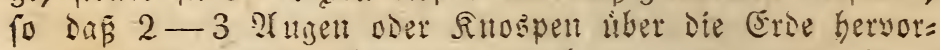
raigtent. Sin Eeptember und Detoher, Ia an Dent einges

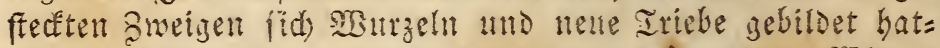
ten, wurben fir in Iopfe gepfonzt, Dent erften seinter

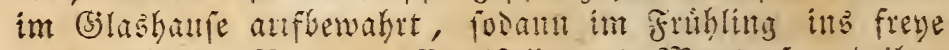

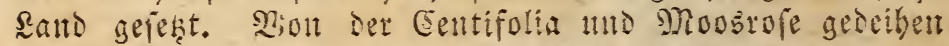
die Etedintige nad) beffer, ment man bie âweige yon sent

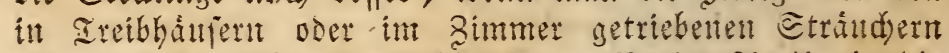

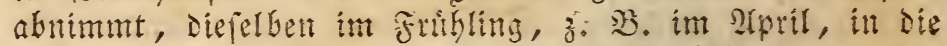
Erde feckt, tind nad obiger 3orfdrift bebanolt; Diefe

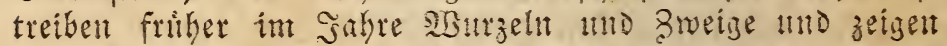

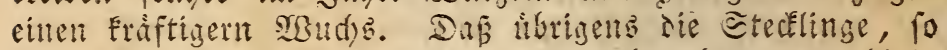
lange fid) nidht jumbe irtebe an Denfethen bifsen, gehöris befeudist und im Edatten gehalten werden miffen, ver=

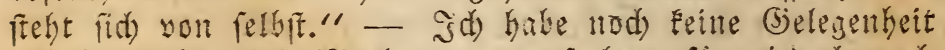

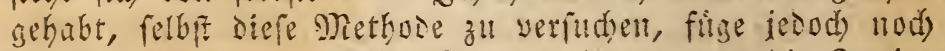

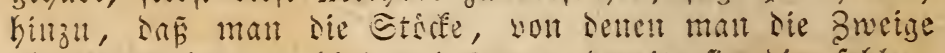
abgerifen bat, gehoris cinfubt, damit fie tie fehlen=

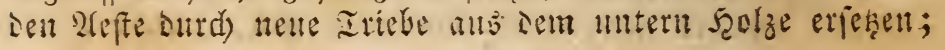




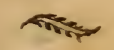

gewis wirs man fie algbant aud) oft juingen, Murzel= fleptell zu mad

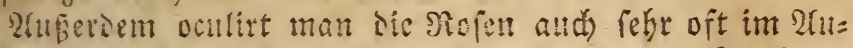
glife, vor copulirt fie im s) May uno grpril. Diejer Mnanieren

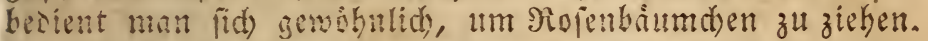
Da aber niat icser Blumenfrumb mit bergleidsen futt:

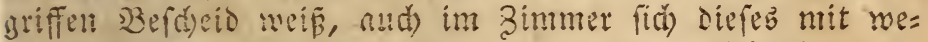

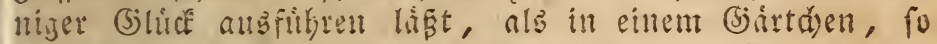

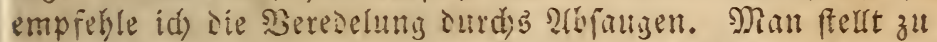

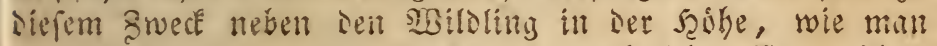

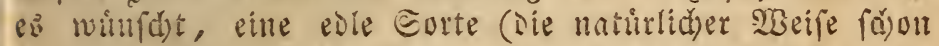

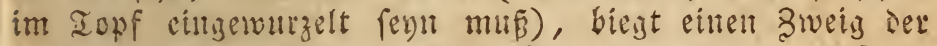

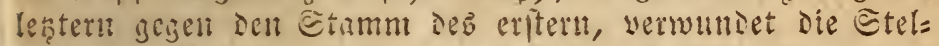
lent, wo fid beise betubien, etwa $1-2$ 3oll laniz surdib

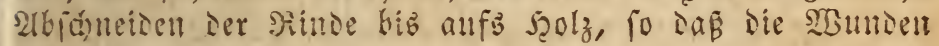
ziemlid) anf einander paffen, orudt fie gegen einander und verbincet fie gut mit Balt, liber weld)en man nod) Banm= swad)s recten fant. Eo bleiben beide fteben, uno man

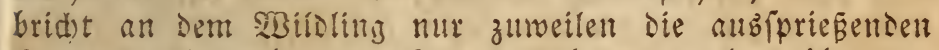

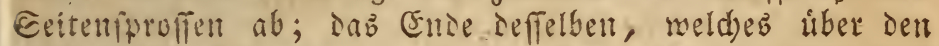
edlen 3rueig bervortagt, wird fo abzeftust, caf nur nodi)

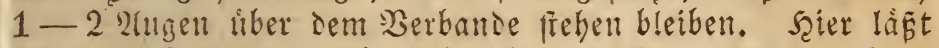
man die Rnosipen anfinglid) fid entwickeln, um ben Caft

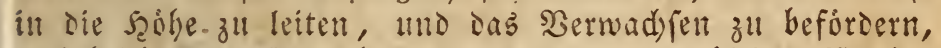

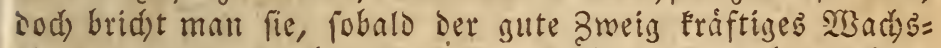
thum zeigt, aud) $a b$. Nad) $2-3$. Nonaten baben fid in Der Regel beive Ibeile fo vereinigt, san man ien angeleg=

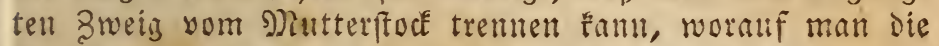

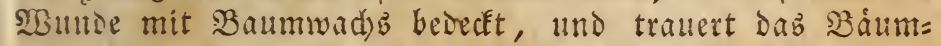
d)en, foldes nod) einige Iage im Edatten bîlt. Das úber= ftebente Cnoe Des Millolings fáneidet man einige Modben pater behutfam ab, nnd uberlebt die Eterle ebenfals mit 23ad)5. Diefes Derfabren fam man Den ganzen Sommer liber bey allell 2rten anwenten, und man erlangt babey ien grofen Bortheil, feimen Rofenboumdien gleid eime tleine Sro=

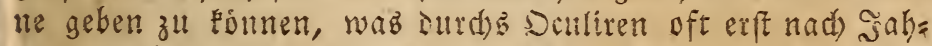
ren erreidst wirs. Nod) zroedmápiger fouent sas \$fropfen

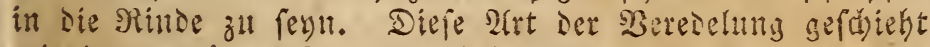
mit immergrunen Eorten auf folgende 2at: Bur 8eit, wo ber 2 Bilding eben am fråftigften zul treiben anfünt, fdnei= det man Den Stamm in beliebiger Sgibe quer Durch, wie beym geroofnliduen Pfropfen, madyt an einer Eeite you oben bermter einen, etwa einen Bod langen (sindduitt surdy 
Die Rinbe, loft biefelbe auf beiden Seiten, wie beym Dcu= liren, ein renis, tino fdiebt nun von oben einen edlen 3roeig binein. Derfelbe befált etwa 2-3 Slugen oder flei= ne Rebenfproffen, wird mit einem Edinitt Feilformig zuge=

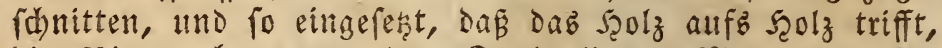
bie Rinte aber unter Dem Epalt liegt. Man umwidelt den obern Theil Des @tammd)enz zur Bereinigung beider

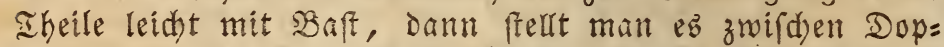
pelfenfter, oder in einen Ireibeaften; Damit aber die Son= ne Das eole Reis nidjt zu fehr allztrocinet, ehe es an= maddft, fo- Gefdattet man diefes, wab am einfadjefen ges fdieht, indem man ein Blatt Papier ummicfelt, und bies fez oben uber bem sweige und unten um sen Etanm fo zubindet, da und iungen aeftaen nirgend gesritat werden. Sif Der WBilding bey Siaften, fo entícheiset es fid gemeiniglid) (d)on binnen 14 Iagen, ob bie Sweige anwad) fen mersen. 2luf siefe $2 \mathfrak{t i t}$ habe id) $R$. thea, noisette, chinensis hybrida, de mons uno carmesina (obgleid) lehtere rehre fdoro=

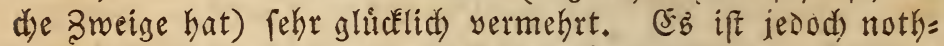

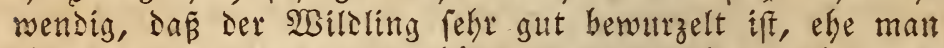
ín vereselt, denn fonft bålt er bas areiben nidst alts, unb bie fdonften Stámmden gehen leid)t wieder ein, went gleid) fie mefrere Boul lange Iriebe gemad)t haben. Dat= um balie man fie yorker etwa ein șiht lang im Iopf, und gerobine fie volfommen an diefen Stand. Sind fie erft mit hinlångliduen Wurzeln verfeken, fo san man bie immergrinen $\Re$ ofen faft den ganzen Eommer tiber auf diefe

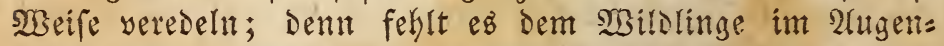
blife and an Ireiberaft, fo wird fie badurd erregt, Daß

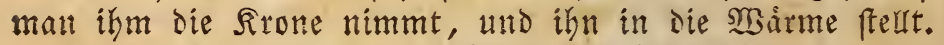
- R. noiseite batte auf frảftigen Etámmen binnen drey Ronaten oft $1 \frac{1}{2}-2$ Fur lang getrieben, uto die sweige Gingen your Snob̉en. - Mit fommerintunen Sorten babe id) nod feinen Berfud) gemadt, fie auf diefe 2trt zu ver: mehren; es forl aber eben fo leidst glicefen, wenn man die guten Reifer (d)on im Februar, went fie noch borrtis ru= ben, bridyt, in einem Fhblen Felfer in feud)ten હand fdlagt, uno im 2(pril oder Nay nad) obiger 2lrt auffest. NBenn aldí) bie rinive etrag welk zu werden anfingt, fo fdadet eb nichts, im (jegentheil roadjen Die zweige um fo leidster an, da fie gefdickter find, Saft aufzunehmen, ofne bá man befurditen darf, die Itugen würden erfitiden. 


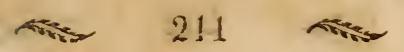

Beln jidem Bereseln binse man ben Baft, welther bie Theile zuammenhált, nidjt zul feft, und lufte ja ber zeis tell den Bund, fonft binbert deefes den freyen llmlanf sex Eifte, mo die gebuntene Etelfe wird bramsig.

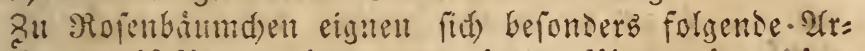
ten: $R$. centifolia, maxima tno minor, dijonensis, unica, muscosa, chinensis hybrida, de mons, noisette, carmesina, multiflora, semperflorens pallida centifolia, thea carnea, wbileids aud vicle andere fehr hubfdi find. Eebr nieslidf) für simmer find Batumd)en yon etwa bren fü fribe, welde auf jedem fenfer bequem ftehen foumen. Nod) ift zu bemerfen, Da fie veredelt, weit wetliger und teider bliken, als bie Muts terftocke. So foll biefes namentlid) bey multiflora Der Fall reyn, weldse oft hober, als bab simmer, wiro, ohne eille Fnošpe zul treiben; Dagegen habe id) vereselte Etámmat)en,

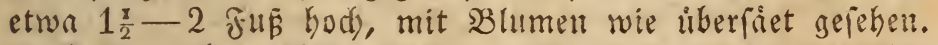
2lud) Die gelbe gefurdte Rofe, weldse leider fo felten eine yolfonmene Blume liefert, folf gut blihgen, wenn fie allf

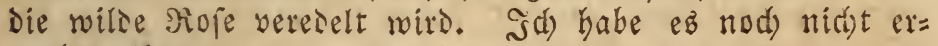
proben foumen.

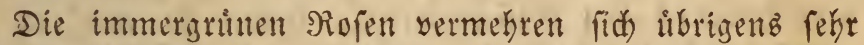

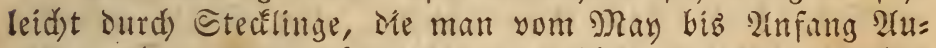

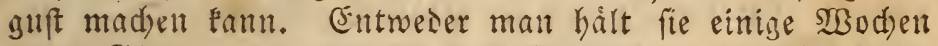
unter (3) las, und warm, oder fteckt fie an einen fid attigen, feud)ten Plats, im sumy ins freye salto in gltte (Erbe.

Sebrere santorofen blihent aud (d)on im erfen Frubs= ling, ment man fie zeitig in Iopfe pflanzt, und diefe ing sand gräbt. Eeht man fie dagegen erfí daun ein, wenn Die Snospen ddon eitten Boll getrieben haben, beidneiset man sie Bweige ftari, unt fenft fie ebenfall inz sand, fo bilden fid) im Suny neue Iriebe, die oft im 2luguft $\mathfrak{B}$ lu= men liefern.

Don affen Rofenatten zeidynet fid R. b e rb e rifolia durdy ihre eillfaden, ungefiederten, rehr furz gefielten Blatter und einfadjen gelben Blumen alls. Sith babe fie bisher nur in atbbildungen gefeben; fie foll etwab fdureris zu behandeln fenn, und ftammt aus dem morolidien per= fient.

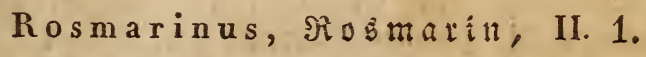

R. officinalis, gemeiner $\mathfrak{A}$ b $\mathfrak{z} \mathfrak{m} \mathfrak{i} \mathfrak{i}, \boldsymbol{Z}$.

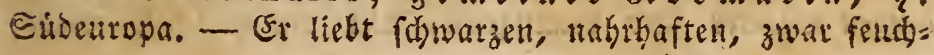




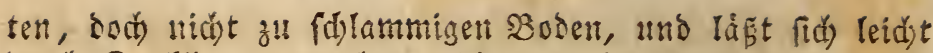
inrd) Etectinge vermekren, Die nan im 2rpril madit, und

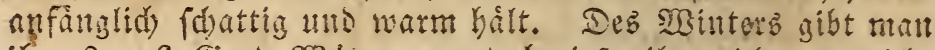

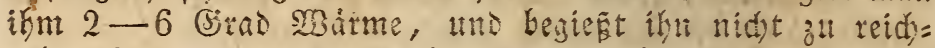
lid). Das zu farte Befdnetien, vertrigt siefer હtrand

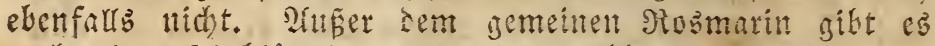
nod) einte feitblâtrige und eine gelbbunte Darietat. -

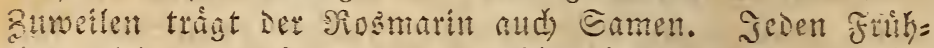

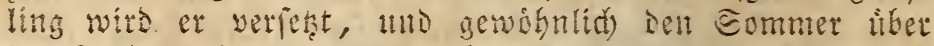

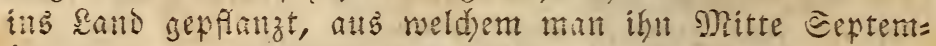
ber wieder zurienimmt, sa er unfre şinter nidjt yer=

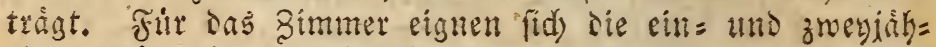

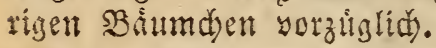

\section{Rubus, Brombeere, XII. 5.}

R. rosaefolius, tofenblattrige $B$ rombeere,

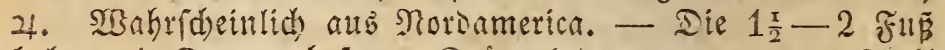
Goben mit Dornen befesten Etángel bauern zwar zum Ibeil liber Minter, jesod, fterben fie ab, fobald fie geblitht, oder eine gewifie (Grof́pe erreidut baben, und werten aub oer

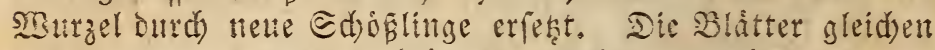
eimigermafen ien Rojenblattern, fteken z"l finf an ien Etialen, find jedod etwas faltig, ifre Etiele ebenfalls

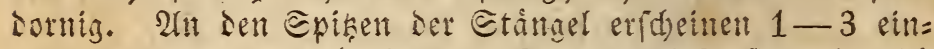
zelne Blumen zu unbeftimmten Beiten; Dieje find ziemlith

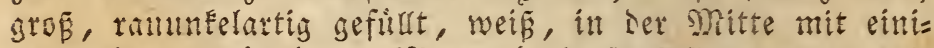
gen grunen Blattden. Nan gebe Diefer DFanze gute, mit sefm gemengte sauberde, balte fie feud)t uns warm. Unt befen ift $\mathrm{es}$, fie im Frubling in einen Ireibefafen oser it ein Mnifbeet zul bringen, Denn obne 2 airme blubt fie nur felten und lange nid)t fo fidon, wie id immer bemerfet

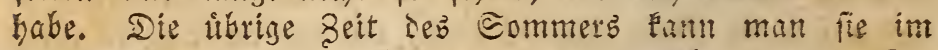
Freven fteben laffen, aud) folf fie im sande ibermintern. Eie wurzelt febr ftant, und madjt eine Nenge SBurzelproffen.

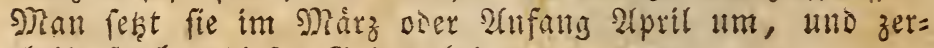
theilt fie bey diefer (jelegenbeit.

Rudbeckia, Rubbectia, XIX. 3.

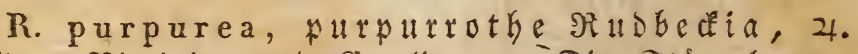
Florioa, Birginien mo Earolina. - Die Etangel wer= ie!t, je nadyem die Pflanze Tabrung Gat, $2-4$ Fun bod); die lanzettformigen, am Rande fein gezáknten, 4-6 Bou langen Blătter find braungrủn, und am Etångel etwas 
berablanfents. Die grofen, Geliputpurrothliden B̈lumen er=

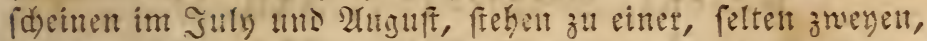
auf ien Etilen. Eie baben nut wentig Etrablen, und nte= mals babe ids solffomment Samen yon ihnen erhalten. Diefe phanze verlangt lehmigen Bosen, groǧe Topfe, im

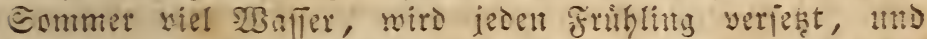

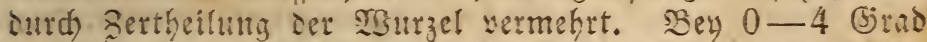
ift fie leid)t jul diberwintern, went mant fie nidjt ju felld Gult ; ald baltert fie sutt im sanse aus.

\section{Ruellia, Ruellia, XIV. 2.}

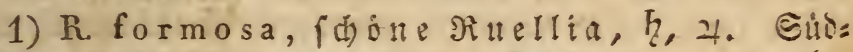
america. - Die Blitter fino länglichenformtis, etwas be= hart; die Etanjel werden 2-4 Fแร hod), แแto tragett sen janzen Eommer itber einjelne, faft trichterformige, blutrotbe Blumen auf fingerslanizen હtängeln, weldhe in

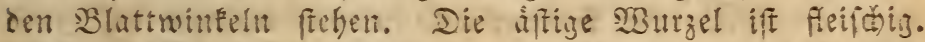
Eie verlangt leid)te Sanberde, im Eommer viel sBaffer,

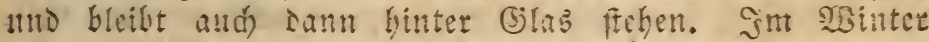

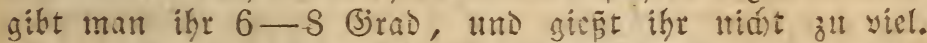
Mein Exemplar habe id $1 \frac{\pi}{2}$ Zall hed thber Der Murzel $a b=$

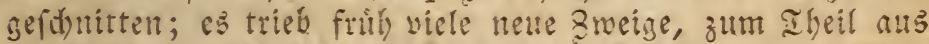

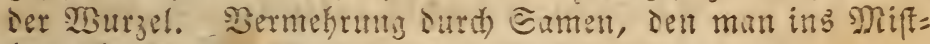
beet fảet, viefreid)t aud surd) Bertheilumis.

2) R. varians (Eranthemum pulchellum), ab vo $i=$ d)ende Ruellia, h. Dfimsien - Diejer Strandi wird $2-5$ Fuв hod), uno treibt viele fparrige 3weige. Die

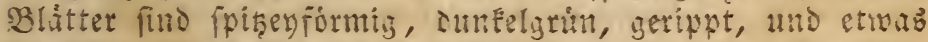

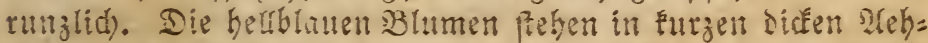
ren au den Episen ser 3weige. Eie bluht im હpantherbif

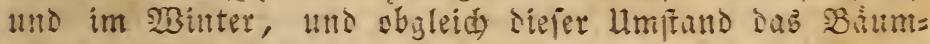
d)en emprieflt, fo ifit es ood fdwer, es im Bimmet zum Bliffen zut bringet, da um Diefe Beit oft Die Eomne fehtt, und die Etubenwirme Die Blument gewoihnlid) zerfobt. Dat= um balte idh eb fdon lingit nid)t nebr in meiner Eamm= lunj. - Nan gibt Diefer Rutlia fibrigens sauberse mit etwas Sefm nno Sand gemengt, diferwintert fie anf eitrem

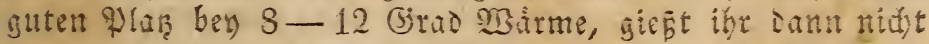

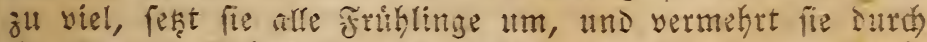
Steclinge. Heber Eommer frellt matt fie in einen Ireit= Eaften, oder behált fie menigftens Ginter fonnigen Fenţern. 2llstanu liebt fie gend)tigeteit. 


\section{Ruscus, Måufedorn, XXII. 13.}

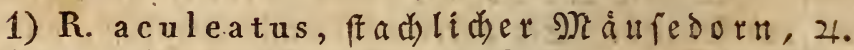

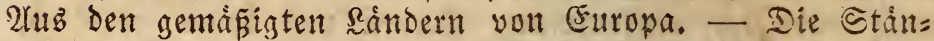

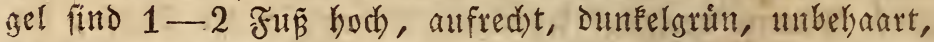
geftereift, theilen fid in megrese 2lefte, und trasen fleine fpiseyformige, fafí Golzartige, fduarzgrime Blatter, Die fid) in eine ftadylide Exise enden. Die f́leinen grunlidben

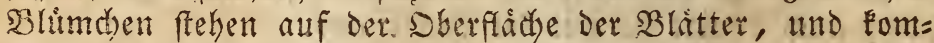
tmen int Fribling, alld oft (d)on im WBinter, Kersor. Nan gibt'inm guten lebmigen Boden, tiberwintert ihn ben $0-4$ (5rad, und yermehrt isn ourd Bertheilung. Man fann ifn zu jeder zeit umiegen; am paffendfen gefohieht

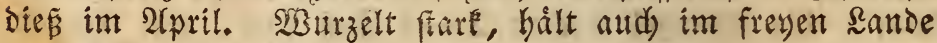
aแtoี.

2) R. hypoglossum, 8 ungenblatt, 24. $\mathfrak{u n}=$ gurn, Stalien. Die Blátter find gróser, meniger sice, uno nidte fourf an Der Spise, auf ihrer obern Flád)e feht ein Fleineres ähnlidieg Blatt, unter weldyen die flet= nen grinliden Blimd)en auf $\frac{\pi}{2}$ Boll langen Stielen ent= fpringen. Befandung wie bey der vorisen.

\section{Salvia, હaltey, II. 1.}

1) S. coccinea, fuarlatiothe Salbey, $h$. Florita. - Die Blatter find faft langlich herzformig, am Siande geferbt, und, mentgfens an den in frener seuft fte=

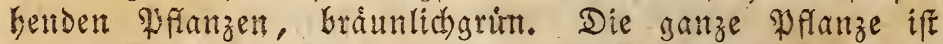
mit fehr feinen saadrden besedt; ihre Blumen fino bernake blutroth. Sie liebt gute sauberde, die man mit etrob sefm uno Sano vermengt, wird jẳatid im Urpril umges Fesct, und bringt viel Samen, burd welä)en fie fid' leidht

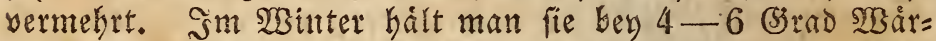
me, iesoch eher etwaz troden, alzz zu feud)t. Die obern Brweige fathe man im Fribling zuweilen ein, Damit fid bie Pflanze aub dem untern frolze wieder etwaz veriungt. 2tud) Glikgen die 2jäbrigen Exemplare in ber Regel am (b)onfert.

2) S. formosa (S. leonuroides), fdine salbey, h. \$eru. - Dieje 2tat hat cinen bolzigen

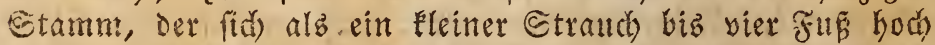
erbebt. Die Slatter fino fapt Gerzförmis, Dice lind glatt. Die Blumen zimnoberroth. Man gebe ifse leid)te, etraz mit Ganto gemifde, aber sod) nabrhafte Eroe, úberroin= 
tere lie bey 4-6 (sornd nake am Fenfer, bebandle fie ieood) mit 2lufmerffampeit, ba ihr die viele Felld)tigkeit

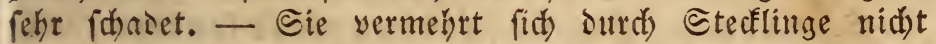

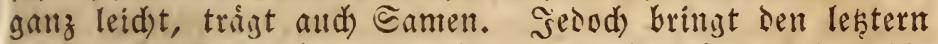
Felten die Pflanze bey unb obne Finftlide Frtilfe, indem die Rage des Stempels gezen Dte Der Staubfáden yon Der 2 Urt ift, Dak Feine Befrudtung Statt finden Fann, und die Sn= fecten, weld)e in ihrem S3aterlanie siefelbe mabred)einlid be= wirken, bey unb fehlen. Bringt man jedod) mit Şúlfe

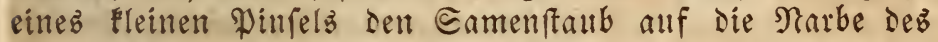
Stempelb, fo befommt man ieverzeit einige Rơrner, die im Gribling in Iopfe gefâet werien, welde man ing Miftbeet bringt. Sm Nay oder Suny verfeht man bie

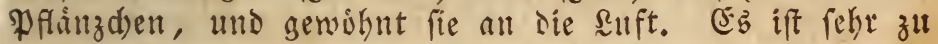
rathen, immer eine fleine Duantitát Samen vorráthis zu balten, da Diefe fdone Pfanze leidyt úber SBinter eingeht. Sb Die Samempflänzden fdyon im erften Sabre bluhen, wage id) nidtst zu beftimmen, $\delta a$ idf feit vielen sabren diefe Salbey nidgt mehr gezogen habe, indem ez mir an Raum fehlte.

3) S. splendens, glånacnde Salbey, দ. Wahrfdeinlid) Nordamerica. - Die Blitter find fpisenför mis, am Rande etwas gezáfnt uno hellgrutr. Die $3-6$ Boul langen Blumentrauben erfdeinen im May uno Suny, zumeilen aud) im Seerbft, tod) alşann gemeiniglid) zu (pát, um fid) nod) youltig zll entwickeln. Die Relde fino bren=

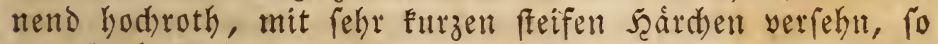
da fie bennabe fammetartis za fenn fdeinen. Sie fteben in Wirteln um Den 3weig. Die Blument find Fehr lang, utho yon eben ser Farbe. Cind gleid) Die Blumen fhon abgefalten, to fdeint die Pflanze wegen ses rothen Reldys Dennod) in yolfer Blithe zll ftehen. Maan gibt diefem (S)es

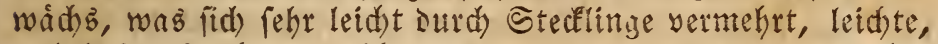
nahrbafte sauberse, uberwintert ez nake am Fenfer bey 2-4 (5rad $\mathfrak{3 a r m e , ~ f t e l l t ~} e$ b jerod) im Frubling in einen Ireiberften, oder zwifden Doppelfenfter, nady Dem Blúben aber in sie frene \&llft, wo es bib Ente September ftehen

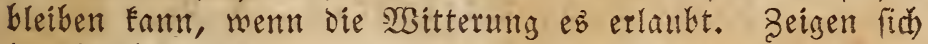
jedod) (d)on im 2luguft frnospen, fo ftellt man es wieser an einen wåmern \$las.

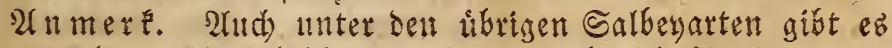

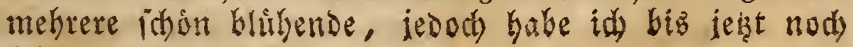
feine gezogen. 


\section{the \\ Sanseviera, હanfeyiere, VI. 1.}

S. carnea, fleifd fartene @anfeyicue, 24.

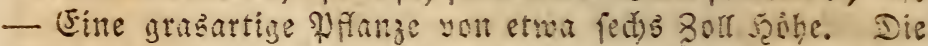
Brumen entipringen bide uber Der Sourzel, fethen anf énem 2-3 sor langen anfredten Etangel, find ficififarben,

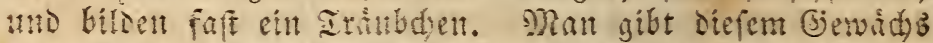
gewdhnlide (Etse, vermetst es suth zertheilung, uno ubers wintert es bey 2-6 (5rat. - Diefe, Sanjeviers if zu

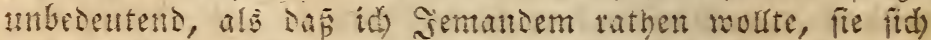

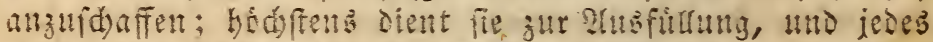

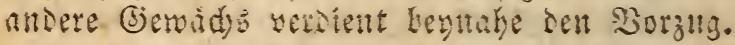

Santolina, Felligenplanze, XIX. 1.

1) S. chamaecyparissus, cypreffenartige beiligenpflanze, $h$. Elisuropa. - Die weï̈lid

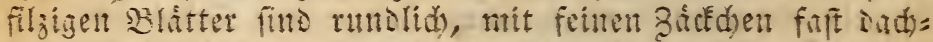
ziegelformis befest, fo baj ber Etrand), welder im Iopf

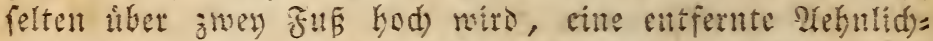
Feit mit Der Eypreffe bat. Die einzelten, an Der Epthe ier Zweige auf langen Etclen fefenten Bhmen fut gelb, und gleithen etwas ienen Der semeinen Cbamille, aud red)t Die ganze Dffanze domitrenartig. Der (jerud) Der Blátter foll Die Motten yertretben. - Man gibt Diefer Pranze Iefmige Croc, Des Sommers reid)lid) SBaffer, uno im SBits

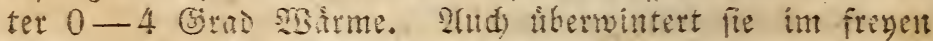
sanoe, wenn fie einen outen Etano bat, uno bey beftigem Froft mit maten 1. Dgl. zugedect wird. Bermefrumy surdi) Stectinge uno sertbetmin. Sie wurzelt frare, uno wiro oft itber Sommet ins samb gepfant, ofer bod ies ien Frtibling Imigefest.

2) S viridis, grune belligenflanze, Gat

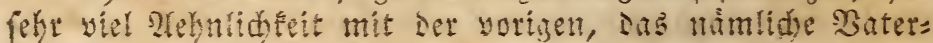
lano, und vertrudgt siefelbe Befondung, mte fino sie-Bblatt= den suntelgrin, und rieden faft unangentem. Sie wäd) geid) winter uns wiro boher.

$$
\text { Saxifraga, Steinbred), X. } 2 \text {. }
$$

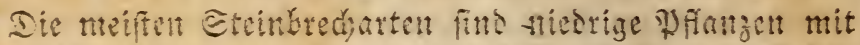

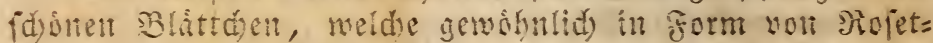
ten feken, uno niesliden szlimdien. Sie wadjen falt

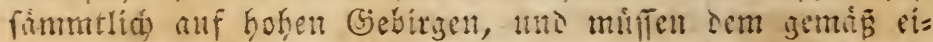
the vromifduthy ergalten, wie fie bey Primula minima ans 


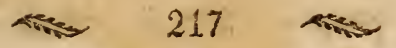

gegeben ift. Sm Eommer balte man fie etwas fidattis uno fencht, des SBinters bingegen megr troden, bey $0-4$ (siab dict) an einem founigen Fenfer, wo fie jeitis treis

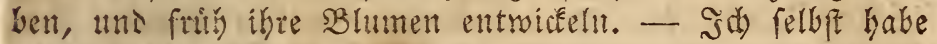
anfer ion folgenoen nod) Feine Eteinbred)art cultivirt, ies

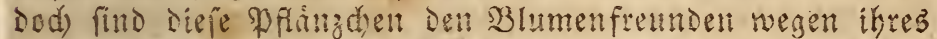

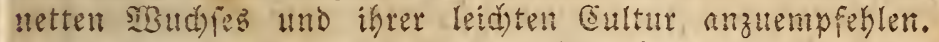

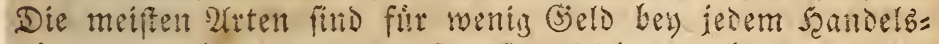

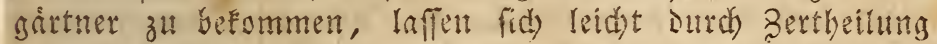

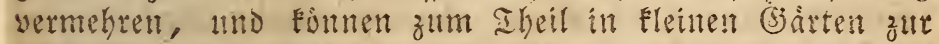
Cinfaffung yon Bhumenfticten sienten. Biebt man fie im

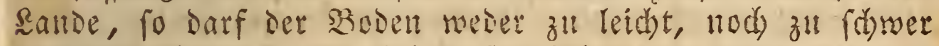
feyn; am befien eisutet fid kiefige sefynterse, und eist etwas rdattiger Etind.

1) S. punctata, punctirter steinfred, 21.

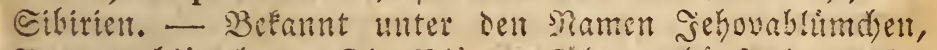

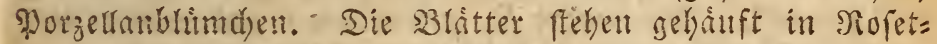
ten, auf $1-2$ zorr langen Etielen, find rundid', gezábut, oft rothlid). Die Blumenftangel werten $\frac{x}{2}-1$ Fus hod), theilen fich in einige Nebenziveige, uns tragen viele fieine, weise Blimd)en mit rothen unb gelben Dhinctden seziert. Sie fommt faft in jedem Boden fort, liberwintert im san= De, vermehrt fid) fehr ftan Durd) Sprofien, und wird, wenn man fie im Iopf traiben will, im Miàyz mit Bałfen aแb Dem sande gehoben.

2) S. sarmentosa, wudernder Gteinbed, 21. (Ehina. - Die Blátter find rmb, geferbt, 2-4 zod breit, oben dunfelgrin mit breiten weisen Etreifen Durd)= zogen, unten rỏthlid), mit Eleinen Dunflern MBarzchen punc= tirt. Sie fehen auf $3-5$ zolr langen rothliden Stielen, weldare, fo roie die Blátter, behaart find. Der Blumen=

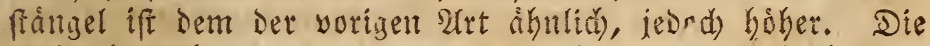
Blumd)en ebenfallz weis mit rothlichen Puncten, aber die beiden unterften Fronenbláter yiel lánger mo breiter, als Die ubrigen. Diefe Dfianze mad't, gleid' Der Erobeere, yiele lange Sanken, weldhe an mehrern Sterren MBurzeln ma= doett, mo junge Pflanzen treiben. NRan gibt the gute, fanoige sauberde mit Eteinden vermengt, und bibermintert fie bey 2-4 (5iras. Sie sautert zuweilen int sanse aus, roenn man fie bey trouftem froft mit saub oder Strof́ bedect. Sim Bimmer leidet diefe pffanje oft von ien Blattlälfen, deşalb gebe man ibr im frubling viel suft. 


\section{Kist

Scabiosa, Scabiofe, IV. 1.

S. c a u casica (S. elegans, grandiflora), aúa= fifde Scabiofe, 24. Kaukafus. - Die Blátter fino lanzettformin, die unterfren all Der Bafis federartig einge= (d) nitten, Die obern ungetbeilt. Die Blumen, weldje zu

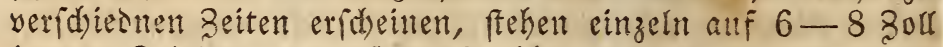
langen હtielen, mo fino oft tiber zwery solf grof́. Die Farbe Derfelben iff blä̆blatt. Sie gehout zul ien idjonen

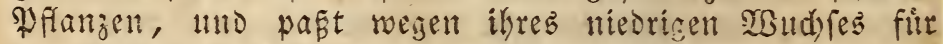
Den Iopf, obgleids fie gut im sande ausoautern, uno $\delta a=$

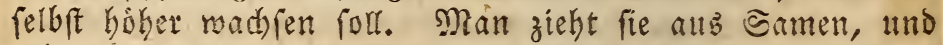
gibt ihr nabrbafte sauberie, fowie im Sommer hintáng=

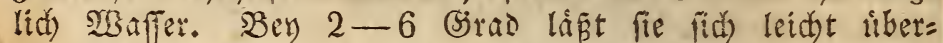
wintern, tub blübt yom Frubling ab faft Den ganzen Eommer.

An merk. Die wahre S. grandiflora hat weife oder

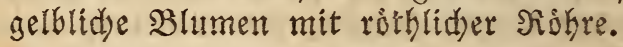

$$
\text { Schollia, ङbollic, V. } 2 .
$$

Schollia crassifolia (Hoyá carnosa, Asclepias carnosa), dickblattrige હchollie, h. Ebinn. - Diefes Ranéengewád)b bat an feinen âweigen paarweife fekente, fpişerformige, fleifdise Blitter, seren obere Flá

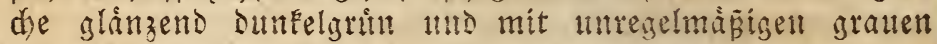
Flecten befprengt ift. Die Blumen erideinen neben dem Blatt auf Eurzen @ttelen, bisten eine bingende Dolde yon 12-20 uno mehr einzelnen Bramben. Sie find dick, fam= metartig, feifdfirben. Die Stempel uno Nectarien braun= roth und gelb. Sie fehen fehr făbon aus, zart, alỏ witren

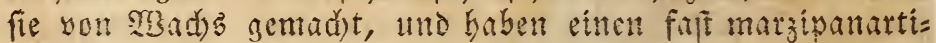

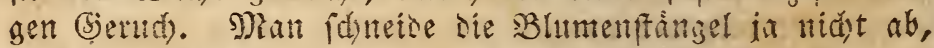
denn biefe bringen megrere Sahre hinter einander Bhtumen.

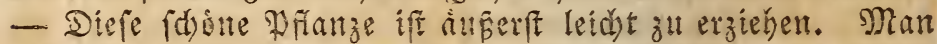
gebe ihr nahrhafte, mit etwas Sano gemifäte sauberse,

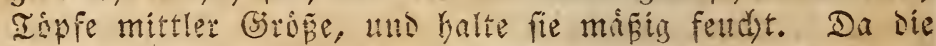
Raneell lang werten, fo thut man am beften, fie unt ei=

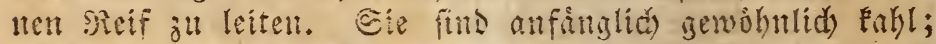
oft erit im folgenton Sommer eridheinen Die Bahtter, und

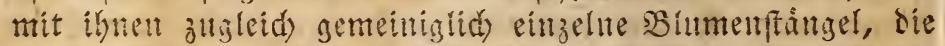
jecod) nidit immer im erfen Sabre Blumen tragen. Man britrgt die Seya nie in die Ruft, funtert lápt fie beftanoin hinter Fenfern feffen, wo fie Eonne befommt. 
Die freye Suft fterten; Dann begiest man es weit reid)liä)er, als im Sisinter. Seben fribling wird es verfent, uno leicht burab) Steclinge vermefrt, meld)e man zu jeser Sab= regzeit mind)en Fann. (5) gist folgende \$arietaten: 1) nit reis $=$; 2) mit gelbgeranderten Bráttern; und 3) eine mit Graumrsth gefärbten Blâttern.

3) S. globiferum, Fugernospiges faub= $\mathfrak{l}_{\mathfrak{a}} \mathfrak{b}, 2$. Finvet fich in Dentfhland und iem nurdichen Europa foft alfenthalben auf altent Mauern, zumeiten auf Felfer uno fondigen Setheln, die oft damit wie mit Rafen tiberzorsen fins. Die helfgrimen, zumeilen ruthliaben fleis

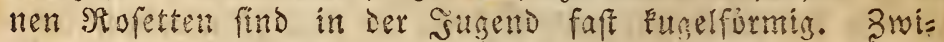
fiben ben anliegenden, feingefrangten Bliteduen entiprin= gen Eurz:, fasenformige Stiele, an benen eine furgelformige, f(c)uppige snozpe fist, weldbe entweder abfält, ober fonft, wenn fie sie Erde berihrt, Sgltrzeln treibt, und eille neue Pfanze bitoet. Die blapgelben Blumen frehen auf 6-12 Zort Goben Stielen, ie nadjoem der Boden it, auf weldbem

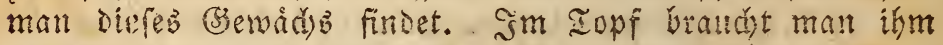

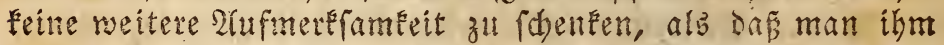

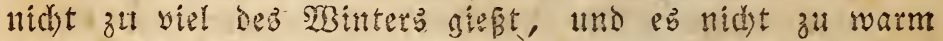

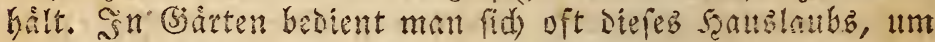

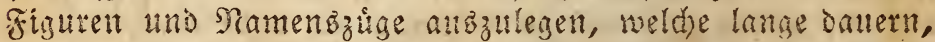
wenn man zuweilen mit einem reithen Befen bartiber fáfgrt, um Die junge Brut zu entfernen. 2Tud Fann Daffelbe zer= Dridet bey frifden $\mathfrak{B r a n o f d a d e n}$ umgefdalagen werden.

4) S. montanum, bergliebendes รูaแร่aแb, 2. Cefyr åhnlid bem yorigen, iesoćl find die Ropetten

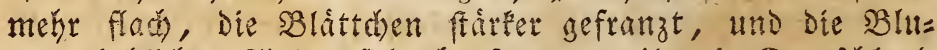

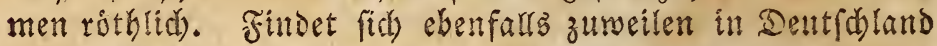
allf Bergen.

5) S. spinosum, bornigeb ร̧a Gibirien und Samichatea. - Die Blattben find blaugrim, und endigen fid̆ in eine lange, weiflithe, weidye Dornen= paise. Sie freken in Der Mitte ber $1-3$ goll breitent niedichen Rofetien fo bidis, daf man faft mur die હta=

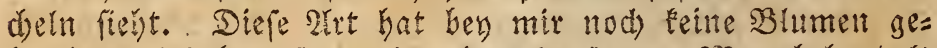
bratht, siefelben forten iedoch meip resn. Snan befandelt fie wie Sor. 1., allein fie ift im Wirter gersen bas Begie= B̈en nod) empfindlider uno yerlangt einen belfen fornigen Stand.

arnmer. Diefe Pflange geft aud unter den Namen

S. cuspidatum แlo Sedum spinosum. 


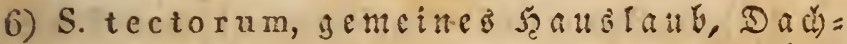

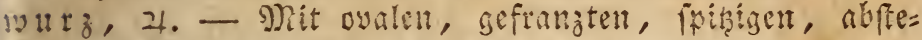
benten, rothlidien Blattern, grofen Rojerten und einem

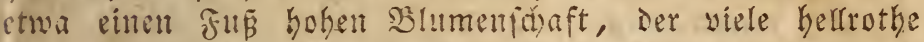

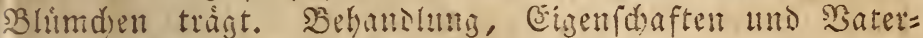
raiid wie গR: 3. - Sede Rofette blitht, wie ber allen

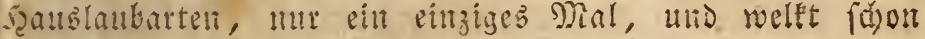
ab, wabrens ser Blumenfdaft fid erbebt.

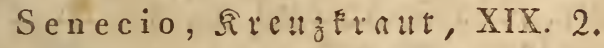

S. elegans, fdoneb freuzraut, $\odot$, 2luser Der gemeinen einfuben 2lut, weldye faft it allen

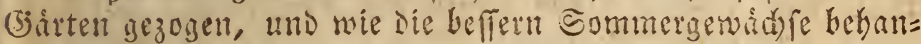
ielt miro, gibt es alth) eilte gefulte Spichit mit rothen, und cine mit faft weisen Blumen. Beeise, Dod befonders oft bie rothe, fallen baufiog aub Eamen. Da mant fid ie=

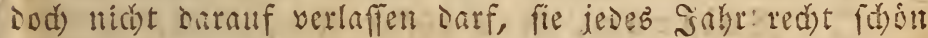

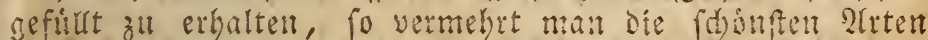

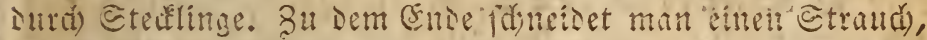

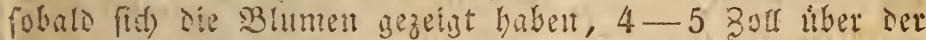
erie $a b ;$ es entwicteln fid mumebr junge Iriebe, weld be man, fobald alb fie fingerslang geworden find, abbridst, zu 3-6 in Iopfe mit guter sauberie fiect, (d)attig und feudit bilt, wo fie samn fonel wurzeln. Sim sointer bilt malt fie bey 4-6 (5iad Matme bitht am Fenfer, mo

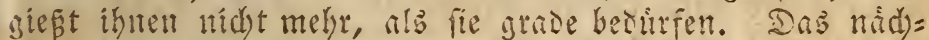

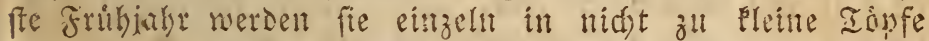
verfest. Dergledden Pfanzen Danern oft viele Sabre, und bcFommen etuen wirtid) bolzigen Etamm; jesod) blitben sie eill sabr alten Exemplare t!t bet Regel am fojnften.

Sisyrinchium, હducinsulffel, XVI. 1.

1) S, anceps, zweyfdneidiger 5 d weing ruffel, 21. Chiomerica. - 2rus der sidural fomment (d)male, fdwertformige, 4-6 Bolf lange SBlatter beryor, uno zwifitin ifnen anf einem platten, eben fo langent

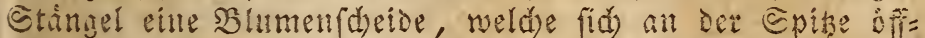
net, uno fleine berblatre, an ien fielden gelb gefirbro

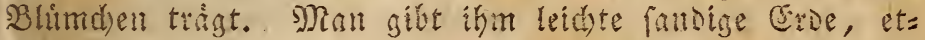
mab tiefe Iopfe, im Eommer jiemlía viel, ieb 13 inters meniger SBafler. Diefe Dffanze bait bey $2-4$ (jira Warme gut alib, folf fid fogat im freven biberwintern laffen, und vermethrt fich ourd Bertheilung und Samen Febr leidot. 


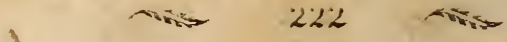

2) S. Bermudiana, Bermudifor Ed weins = iuffel, 2. Bermudifthe Snfeln. - Diefe orit hat mit

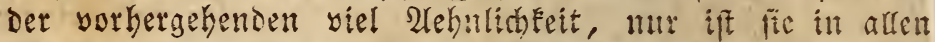
Theilen etwas groffer, und die 3 lument fito fdonet. Be 3

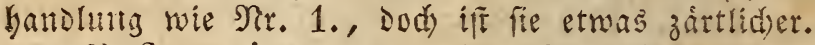

3) S. striatum, geftreifter Sdidweinstifiel, 2. SJtexico. - Die Blátter werten $1-1 \frac{1}{2}$ Jü lang, gleidten benen Der @dwertilien, und feben ebenfalls in zwey Sieihen. Bwifden ihnen fommt ser oft liber 2

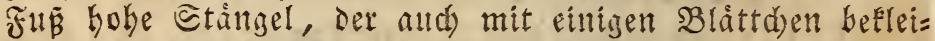

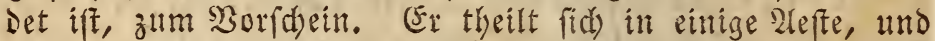
trågt eine lange, didate Yehre yon blaşgelben, aujerhalb Eraun geftrid)elten 2 lumen. Nan befandelt ifn mie $\mathfrak{N}$. 1., nut if er gegen Rălte empfindith. Die Blithzeit Dauert faft Den ganzen હommer bindurd

\section{Solanum, Nadid t d atten, V. 1.?}

1) S. dulcamarum, Bitterfú, $\mathfrak{B}$ aldnadt= (d) a t ten, 2. Diefe, in allen sanbbolzmâtiern, befonders am Ufer ser (Sierwaffer, auds auf હdutthaufen, uno relbft auf altem Jzauterwerk, băufig wilswad) fense flettentse Pflan=

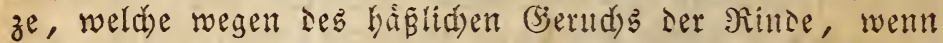
man dicfe fdabt oder reibt, unter dem Namen Náufebolz bekannt ift, babe id) bin und wieder in Etádten zur ßer= zierung Ier Fenfergitter angewentet gefelyen, wefhalb id fie hier mit aufnefmen witr. Die Blatter find meift dres= lappig, Die lockern Dolientrauben Gaben Glauviolette Blil= ment mit gelben Stanbbetteln, uto erfdeinen im Suny bis an Den Serbft. 2luf fie folgen weidhe länglid)e Beeren

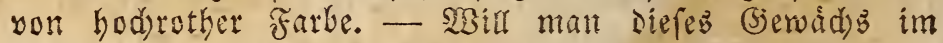
Iopfe zlehen, fo fudbe man eb im seerbft, roo moglids, an einam nidgt zu naffen Stansorte auf, pfinmze Die Buffice in gute, nabihafte, mit Touf gemengte Eroe, liberwintere fie bey $0-2$ (S)rnd, lmo bringe fie zeitig an ifsen $\mathfrak{B e}=$ fitmmungbort, wo man bie sumen sweige fogleid' befeftigt.

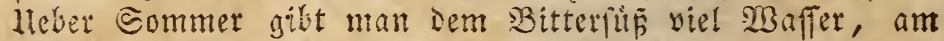
beften, mall felit ben Iopf in einen unterfę̧apf. Menn sie erfen ftarten froffe fommen, fo bringt man es itts misinteranartier. 2(ud) in (3)arten Eann man $e \mathfrak{s}$ zur $\mathfrak{B} e=$ Eleisung um sanben zwiflien antere bláttertedde હd)ling=

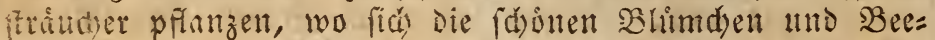

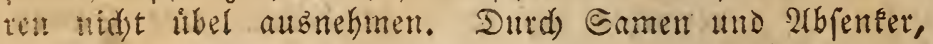

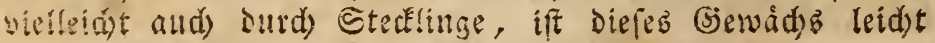


ju sermebten. - Es foll eine Euntbrattrige Barietat ge= bent.

2) S. melongena, eyertragender Nadytfdat= ten, ○. Er miro ganz wie Datura fastuosa behandelt.

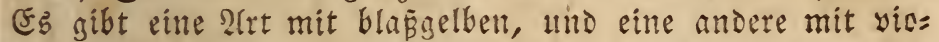

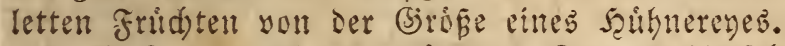

3) S. pseudocapsicum, Roralleirfd)e (fálfdo= lid) Fefre oft Subenfiridae genamnt). Marera. - Ein Băumden, meldes $3-4$ Fub bod mirs, lanzettformige Blatter, weise Blumen und runbe orangerothe Beeren trảgt. Snan zieft die Foralleirfdue fehr leid)t ans Eamen, gibt ifhr gute Srangerie= Crie, grofe Iopfe, viel S্Saffer, uno feţt fie jesen Frubling um. Sm Minter 4-6 Sirad. Befişt man viel Băumdyen, fo faun man fie Den Eommer

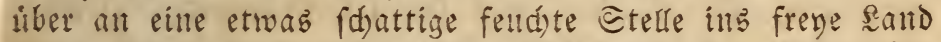
reşen, wo fie ftare treiben uno reid)lid) tragen. Dod frán= feln fie, went man fie im Scerbft wieder in die sopfe zu=

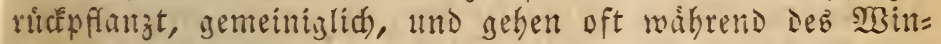
ters eill. Um diefes zu sermeiden, gebe man innen fo lan= ge sift, alb moglidy.

4) S. pyracanthas, rothftadiger Nad)t (d) atten, 2. Madagascar. - Die graugrunen, filzigen, allsgebogenen, faft gelappten Blatter find $3-4$ Boll lanz, wno in Der Mitte, oben unt unten, mit einer Reifse lan= ger Imatriorangerother Etadjeln befesct. Die Blumen glei= d)en senen ser Sattoffeln, und fins violett, sie frudide forwarzarith. Diefen Naditichatten vermefrt mant am be= ften Durd) Eamen; zhweilen befommt man aud) 9 Burzel= fproffen. glian gebe ifm leidite, tod nabrbafte (5rde, im

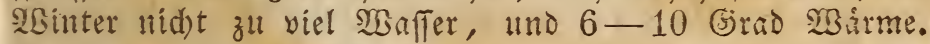
Iud) im Eommer fteht er gern rarm, und Eann immer binter Jenftern fteben bleiben. Die eill = und zweyiabrigen pflanzen feben am fónten aus.

\section{Soldanella, 2l lpenglófden, V. 1 .}

S. alpina, gemeines Ulpengló丶 Uuf ren wipen, nahe an ser Edyneregion. - Die tlei= nen, rundid) nierenformigen, leserartigen Blatter find im= mergrun, und ftehen auf Eurzen Etielen. Die Blumen=

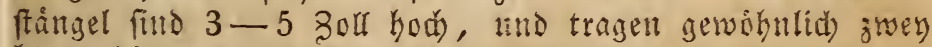

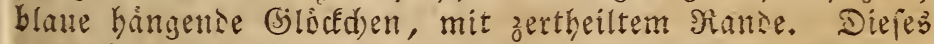

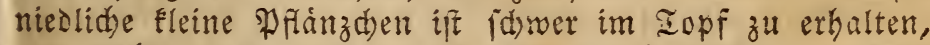
und fitbt, menn man es nid)t febr gut bebandelt, geroobus 


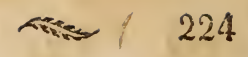

liib) nach einigen Şahren ab. (Ë erforiert die naimlitbe Befrandlung, wie Primula minima. Man nefme fid eben= falf in 2ldyt, Die Pffanze zul Tehr zul zertheilen, und balte fie in WBinter neder zu marm, nod) zu feldyt. Die Siluth=

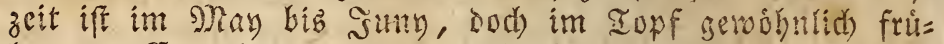

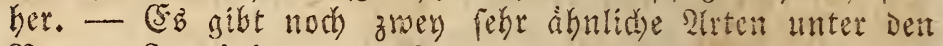
Ramen S: minima uno S, montana, Dodi) babe icí) nodi Feine Derfelben gefehen.

\section{Spartium, Befenfraut, XVII. 4.}

S. junceum, fanafdeb Befentratit, $\zeta$. Sibcuropa. - Die unden, giatten, binfenartigen Zrocige tragen in Der știgen lanzettformige, wentg behaarte Shatt=

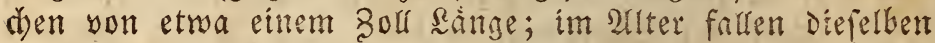
meif ab. Die Blumen fino fdumetterlingsatig, ziemlid grop , kurz geftelt, bod)gelb vout Farbe, who befisen einen

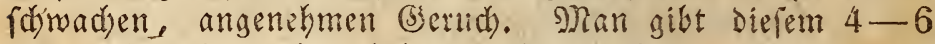
Fun buhen ভtrauch nahrhafte lehmige Erre, grop̉e Iopfe,

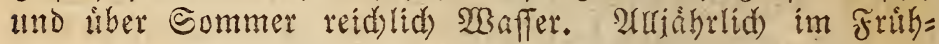
ling wird er verfest, uno bey $0-4$ (sind tiberwintert; nut in regr gelinden şintern bâl er gut in greven aus, fonft leidet er gemeiniglicí) Fehr Durd) Froft. Orm leichte fiten wird er burd) Enanen vermetrt; aud nelymen Eted= linge zuwetten an, wenn man fie zeitig inb פpifteet frectô. Nidet immer tragen die Btumen Samen, inoem Die Zett= gungsorgane unten im Ediffden Der Błume eingefáloffen find. Driteft man jedodi lesteres fanft mit Den Fingern,

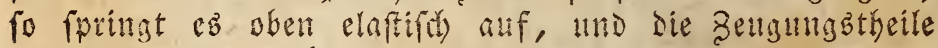
treten Gervor, mobey Die Befrudtung in Der Regel yor fid) geht. - (Ë gibt aud) eine gefultt blubende Epielart.

\section{Stapelia, Stapelie; V. 2.}

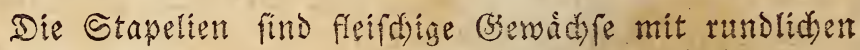
oder edfigen Zweigen, an Denten fid Fleitte Scátedent oder Erbabenbeiten befinden. Die meiften 2rtten bleiben niedrig,

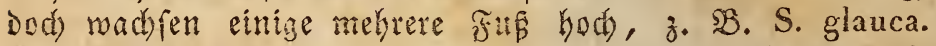
Die Bhlumen find entweder glueten=, oder feenformig, oft Flein, zumeilen 3-4 sorf gros, leberartig, bunt gezeichnet. Die gerobhulid yorfommenden Farben find: roth, braun, gelb, fidwarz, violett uno orau, in faft alten Edattirun= gen; zumeilen find fie behaart, oft runzlid. Der (Serud)

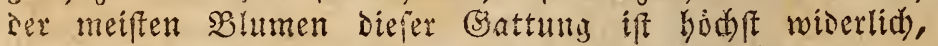

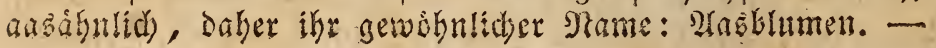


Des Sommers ftellt man fie an einen gefhlisten fonnen: reidjen Sort, uno fidjert fie gergen anbaltendez Regenmetter;

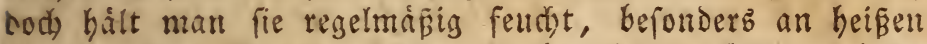
Iagen. Des Winters balten fie bey 4-8 Girad Wärme ridyt am fonfter fehr gut aus, wenn fie etwas Eonne ba= ben, uno nidjt zul warm ftehen; nur giese man iknen im December bis Ende Februar hodaft felten, wenn altá) bie 8reige anfaugen jolten; fehr zu welken. Nan vermehre fie int Suny und Suly Durd) Etecklinge, dic iedoch, wie

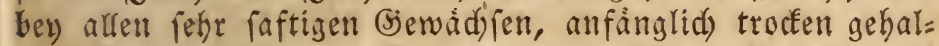
ten werden miffen, nad)dem man bie frifden $\mathfrak{W}$ unden mit Foblenftaub beftreut, und sie 3reige 8-14 Iage hat wel= fen laffen. 2(ud) Durd) Bertheilung fönnen viele 2lrten ver= megrt merien; eben ro babe id) sefehen, das mandhe Stape= lien Samen tragen, jebod) nod) Eeinen Beriud) gemad)t, wie er aufgeht. - Diefe sesemádyfe erhalten sauberde, ftar mit Rebm und groben હano vermengt; im Frihling, etwa alle zroen Sabre einmal, feţt man fie um, und legt dabers auf ten Booen Der Iopfe einen Boll hod) Fleine Eteine oder groben fïes. Die Blumen erfd)einen Den Sommer Ĺber. Jaft alfe હtapelien find anf Dem Borgebirge Der guten Sroffnung z" Şaufe. - Bon ben vielen 2 trten nente id nur: S. asterias, caespitosa, campanulata, elegans, geminata, glauca, grandiflora, hirsuta, humilis, incarnata, maculata, paniculata, picta, pulchella, pulvinata, quadrangula, reticulata, variegata, tubulosa, venusta $\mathfrak{u} . \tilde{j}$. $\mathfrak{w}$.

\section{Statice, (5) $\mathfrak{x}$ a zuelfe, V. 5.}

S. mucronata, Fraubblátirige (5) rabuelfe, 2. 2lub Dem nơrolid)en Ufrica. - Der gegliederte હtån= gel wird felten uber 1 Fuß Kodb, trågt mut einzelue Blát= ter, ift aber ber sånge nad) mit fraub herablaufenden hૃả: ten berectet, Die ifm ein fonderbares $2 \mathfrak{n}$ fehn geben. Die WBurzelflátter ftehen auf $1-2$ zoll langen Stielen, ino lecerartis, elliptifh, glatt uno mit einer Etadbelfpişe (mucrona) verfeben. Die rotblidsen sBlumen ftehen in Endáb)= ren, faft roie die des Heliotropium peruvianum. - Man gibt ihr leidate Eroe, verpflangt fie im Fribling, und bălt

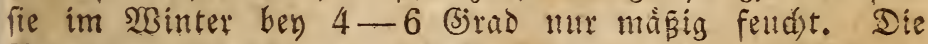
Dermehrang seddieft iurd Eamen; id bin jerod) nodh

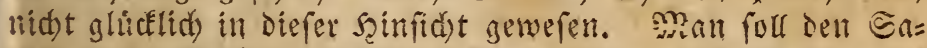
men in Iopfe fán, leidht beocét, an cinem temperirten orte

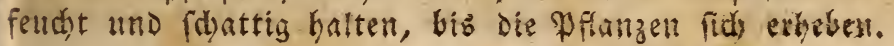




\section{6 \\ Syringa, flieder, II. 1.}

1) S. persica, perfifcer flieder, h, Per= fien. - Diefer fleine Etrmud mit bla $\bar{B}=$ róthlid $=$ lilafarbi= gen, aud weifen Blumen, iff alfgemein befannt und ges fdánt. - Man behandelt ihn, wenn er im Iopf blihen for, wie den If feifenftraud); Dods iff es beffer, wenn man bie Ctratucher fobn int frubling ozuyor, ehe man fie trei= ben will, in geráumige Iopfe mit guter nabrbafter Erde reşt, und Diefe uber Eomner ins sano pente Jd) babe bemertt, Dafis es gut iff, Den Flieser alsdann Der Eonne et= wab altşzupen, ihn aber nid)t zlt (d)attig gul halten, weil im lehtern zalle bie Bweige zwar lang mad) fen, aber wentig Blumen bringen. Beym Iteiben ift nod) zu merten, dá̧

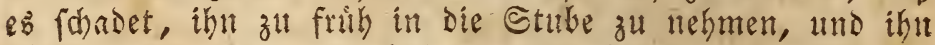

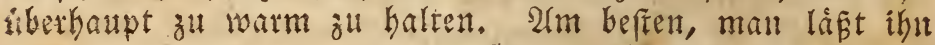
in einem 3immer yon 2-6 (3rad Måme did)t am Jen=

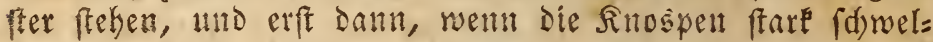
len, ihm mehr SBatme zu geben. (Sorrognlid) bluht er

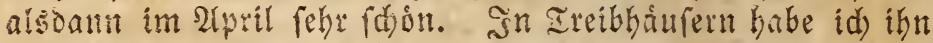
freilid) (d)on Mitte Gebruar mit Blumen gepeben, iedod)

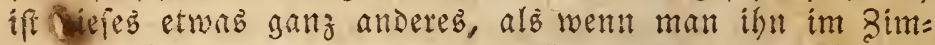

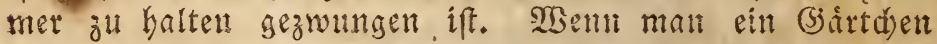
bejist, fo fann man fehi nedlid)e fliederbáumden fur ben Ionf ziehen, wenn man die jungen Sproffen, die man

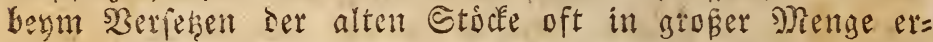

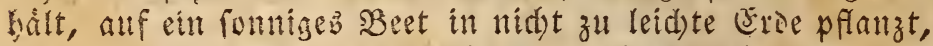

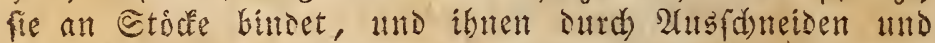

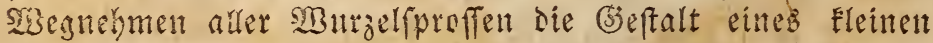
Baumes gibt. - Die abgeblibten Etófe merden tiber Som= net wie sie Centifolien behantelt. - Nan fennt alld ei= ne Enidart mit gefiecerten Bláttern.

2) S. vulgaris, genteiner frieder, h. Cuts= eltropa, und and faft fdyon in ganz Deutidylano einkei=

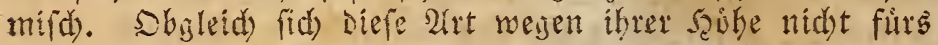
Bimmer cignet, fo for man rodi leidst Swergbanmdsen zie= ben fontuen, wenn man Die obern Sweige eines tragbaren

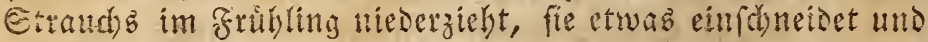
mit fonfen 2-3 sud tief in Die Erise befelingt. Damit

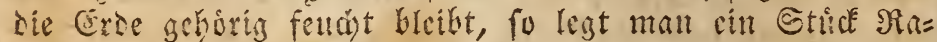

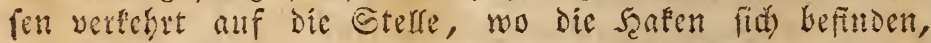

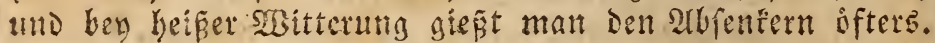

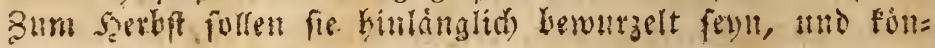




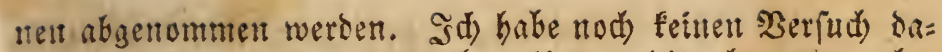
mit gemadt. - Benn Ireiben fünnen bie oben a algegebe= nen Bergeln ebenfalls befolgt werdent. Esa gibt mehrere Epielartert.

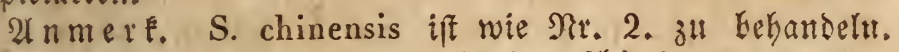
ere bliht fegr reid), und ift der fdo onfe yon den ge= nannten 2laten.

Tagetes, Sammetblume, XIX. 2.

T. lucida, gläjende Sammetblume, 2 . Mexico. - Die Etángel werten etwa $1-2 \mathfrak{F} u \tilde{p}$ hoă, die Blâtter find $1-1 \frac{1}{2}$ Boll lang, lanzettfơrmig, glån= zent, uno am Siande fein gezúfnt; gerieben riedsen fie wie 2 unis. Die fleinen hod)gelben $\mathfrak{B}$ lumen erfdeinen in Doldentrauben an Den Epiken Der Zweige vom Suly bis zum September. - Man gibt ifr rriftige Rauberde, und

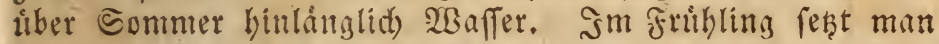
fie um, umo zertheilt die Etaude bey defer (j)elegengeit. Bey 2-4 (birad ift er leid)t zul ủberwintern.

Te ucrium, (s) amander, XIV. 1.

1) T. betonicum, betonenblattriger (b) a = mander, 乌. Elideuropa. - (T. fruticans) Der Etraud wito 3-5 Fub hod, bat élle graue Rinde, meergrune, 2-3 8oll lange, 1 zoll breite, am Ranie geterbte Blát= ter mit einem eigenthumlident (Berud) nad) Elepfeln. Die blap̧violetten Blumen erfoeinen an Den Epizen ier Bweige. Nan gebe inm lebmigen Bosen, Geudtigkeit, serfege ihn jâbrlid) in 2(pril, lino liberwintere ibn bey $4-6$ (stras. Durd) Stedfinge und SBurzelfproffen ift er febr leidyt zu vermefren.

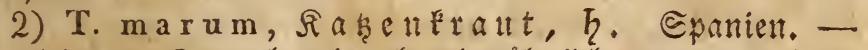

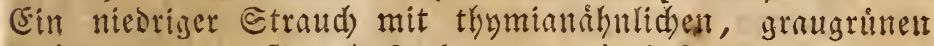
Blattert, Deren (Seruch ftare und gewurzhaft ift, wešmegen Der Ctraud) bailfitis gezogen wird, und im gemeinen seben unter Dem Namen Marumberum befannt ift. Die Blim= d)en, weldhe zu 2nfang des Commerz an Den Enven ber

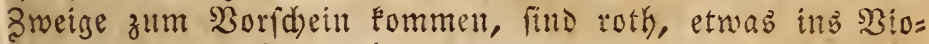
lette fallend. Mtan gibt Diefer Nfanze leidite, Mabrbafte Eroe, yerfebit fie jesen fruhling, uno liberwintert fie bey

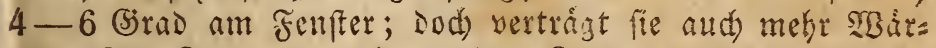
me. Sm Commer verlangt Dab Rakentrant viel $\mathfrak{B a f f e r}_{\text {, }}$

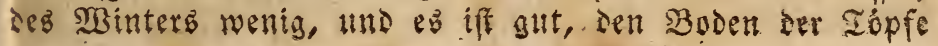


mit grobem sies zu belegen, Samit bie liberfuffige zend)= tigfeit leidyt abziehen fann. Die Bermehrung gefdielyt

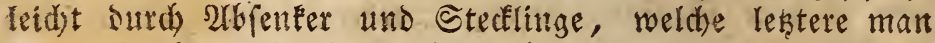
einige $230 d$ en mit einem (Slafe bebedf́t. Die alten Ståm= me fehen nidbt fo gut aub, als bie jungen, baher mur man fie oft erneuern. Da die Faken fo grofé Freundin= nen von defer Pflanze fino, fo mus mant fid yor denfel= ben fehr in 3idht nebmen, Denn fie yernidyten nitht alrein

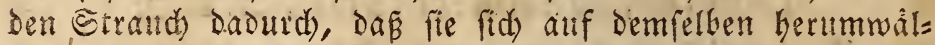
zen und ifn berabwerfen, fondern gemeiniglid trifft ein

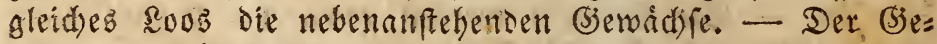
rud) Der Blátter if fo ftark, Daß̃ er Niefen erregt, und cben debgalb wito er gegen Fopfidmerzen empfohlen.

Thea, İeebaum, XIII. 1.

Den Iheebaum habe id, nod nid)t felbft gezogen, uno pr gefort in Dentfdaland nod) immer zit den feltnen (bje= wathfen. Sein Baterland ift Ehina und Sapan, und im

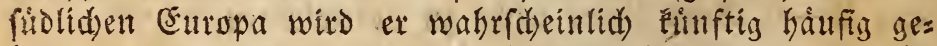
baut werden; denn er foll, nach Dietrichs 2 Uts fage, felbit in England fiber SBinter im sande nub̆halten. Die Blats tev find rederartig, glẩzend, oben dunfel=, unten belrgriu,

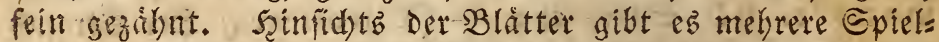
arten, die wohl wahrfdeinlid) nur Durel). Cultur entfanoen fitro, wenn gleidi) Die meifen Botanifer fie unter befondern Namen führen (T. Bohea, laxa, viridis, t. f. w.). Die Bhmen erfdeinen vom September bis zum Santar zth 2 -3 atif furzen Etielen in Den Blattwinfeln; fie fino weís mit gelben Etanbfáben, tho gleiden einigermaß̧en sen Blu= men Der Sirfdhent. Gameit trigt et in (Etropa nid)t. Eve

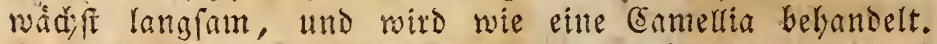
Zlm fiderften vermegrt man ign durdi) arbenfer, bie nad) zwey sabren gewobulitio bimlanglich bewurzelt find.

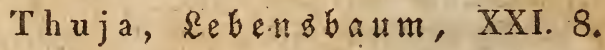

1) T. occidentalis, ber americantide $\Omega e=$ benbba:t

2) T. orientalis, der fáderblattige see bะกระ aแ m.

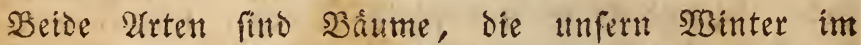
Freyen alsbalten, uno eine ziemlide sebhe erteichen, wenn Thuen der Etand zufagt. Die immergrinen nadelartigen

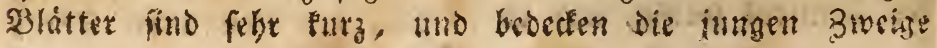




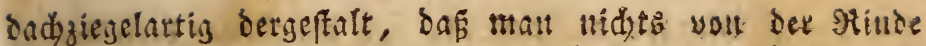
fiebt. Die Şlumen erfdeinen im Mårz, futo unbedeutend, und die Eamen liegen in Eleinen Bapfduen. - Nan zieht

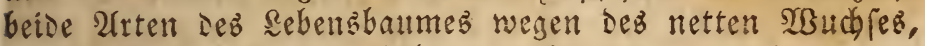
bell fie in der sugeno baben, nidjt felten in Sopfen. Mant (d)afft fich sie Pflanzen ant beften yon Szandelszårt= nern; Denn fie felbif aus Samen zieben zu mollen, foftet viel Beit, da er oft lange in ber Eroe liegt, uno die Pflanz= d)en langram wadjent. Hebrigens laffen fid) beide Eorten Durd) abjenter uno Etecklinge vermefren, nur múfen lę̧= tere in leid)ten Boden geftedt, 1110 (d)attig und feuds ges balten wersen. Sm Iopf gibt mant iften lebmige saub=

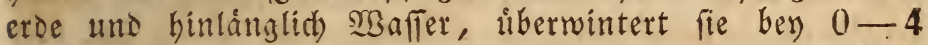
Srad, und reşt fie iâbrlid) entroeder im September oder im Nårz um. - Die erfte Urt wåd) in Eanara, die zroente in China will.

Tradescantie, Itabescalltie, VI. 1.

1) T. discolor, bunte Iradescantie, 24.

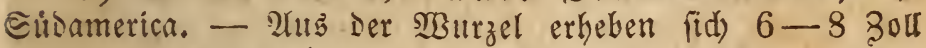
lange, $1-2$ 8oll breite, lanzettformige, glatte, gairzran= Dige, etwas freifdige Blátter, weldse obell dunfelgritn, uns ten fidin rothliduviolett gefábt find. Sie umfaffen fich an ber Bajis, und bilien, forwie fie abwelken, nad) uno nad) einen Eurzen, bicfen, flrotigen Etängel. Bwifden

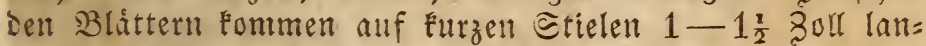
ge, zmenflappige, gefábte હdueisen bervor, in melden Eleine, weiflidbe Blumdsen fteben, die zwat you furger Dauer fint, Dod) faft tab ganze Jabr über fid entwictelt. - Man gift diefer Pfanze sauberse mit etwas Tonf แnd Sano gentengt, balt fie im Eommer bey 15-20 Grao binter Fenftern feuds, weniger jesod) im SBitter, wo fie aud) biel WBarme und einen berfen Etand liebt. Eic ver= mehrt fić) Durd) SMUrzeliptoffen.

2) T. virginica, yitgintide Irabebcatie

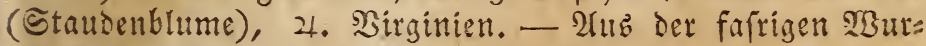
zel entipringen id)male grasartige slátter, oft hiber einent

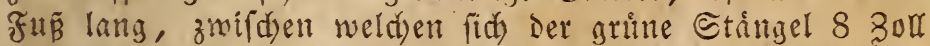
bis $1 \frac{1}{2}$ Fuß hod erhebt, ie nad)oem die \$flanze Nabrutg hat. 2tn dem Etangel fteben mebrere Blitter; Das oberfte Blatt ift fdeidenartig, tuto aub ihm entipringen eine Menge dres=

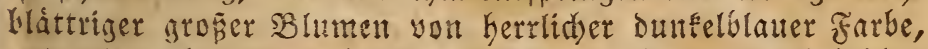
wne mit gelben Etanbbenteln setferen. (Semeiniglito bliben 


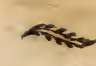

nut $1-3$ Blument auf einmal, uno bie Dauer berlelben ift Faum einen solen Iag. Da aber jeben Moryen fid neue Blumen entfalten, Die etaube audi siele Etanisel treift, uno gewohnlid aus jesent fid nod ettre imente, aud wohl oritte Blumeniácise entwicfelt, fo bat man bas Bersulgen, foif

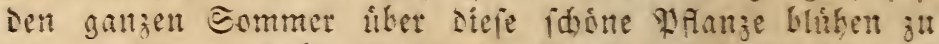
fohen. Eamen babe id nie erbulten, who Diejes if and

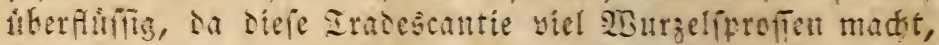
bejonter

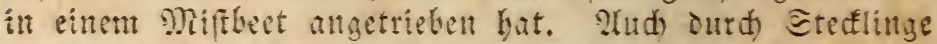
faum man fie leidit vermetiren, went man sie abgefliften Etangel in to siel Etitufe, als serjelbe finoten bat, zer= fduneiset, fo sas unter lestent immer nod) $1-2$ solr som

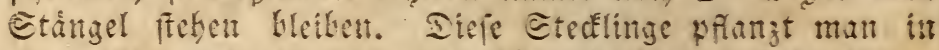
cinen Blumentopf mit leid)ter Eric fo tief, Dap Der Futo=

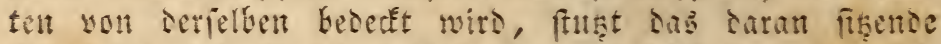
Blatt his auf etwa trey soll ab, thto balt fie warm uno misen feudst, worauf fie in Der Bezel bals austreibent. Die virginifde Etaudenblume liest gute nahrfafte Eroe,

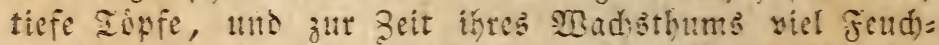
tigfeit. Se mekt Mabrum fie bat, sefto fonduer blikt fie;

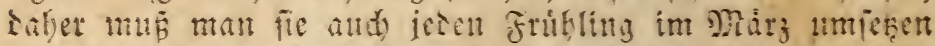

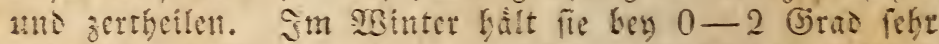

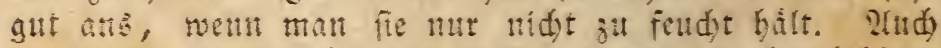
im freyen Rande liferwintert fie. 2uner ser blaublifen= Den gift es aud nod eine Rert mit purpurrotben, uno eis ne andere nit weisen Blumen. Bumeilen baben eingelne Etandent 2rnlage zum (jefiutmerien; idd felbit babe oft Blument mit 4-6 Blittern erfalten. Begen ifret leid)=

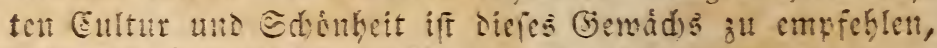
nino nod) bomerte id, Ea (d)usten Etano gefen mur , intem der şind sie langen fdmalen shatter oft leke beidstigt, woourd tie Yyange

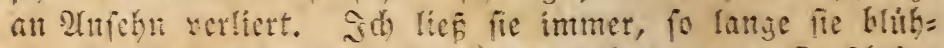
te, im simmer alf einem Fenfter frefen, senn fie fusint bie sistrme im Eommer zu liefen.

Tropaeolum, Fapuzituerefife, VIII. 1.

T. majus fl. pl., h. - Die gemeine einfa:

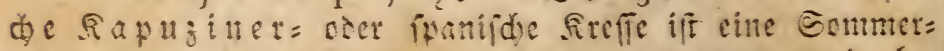
bfange, weld)e aus sifinden ftammt, und affgemein be: fant ift. Dutch Eultur bat man cine sefifftblibente

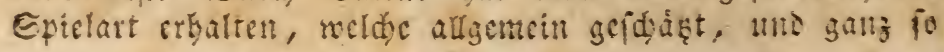


wie Senecio elegans fl. pl. behandelt witb. Sie geft úber Winter, went man fid mit dem Begiesen nid)t fefe it 2(d)t nimmt, leid)t ein, befonters alte Pfanzen; baber muв man immer Stecklinge mad)en, um Den Serluft fo= gleid) erfesien zu founen. Diefelben nefmen yom Man

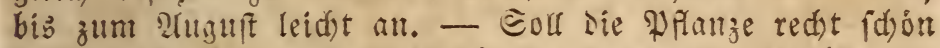
bliben, fo ftelfe mant fie im Eommer in einen Ireibfaften, oier zmifden Doppelfenfter; Denn int frever suft bleiben die Blumen viel fleiner, uno entwidfeln fid) oft nid)t vols=

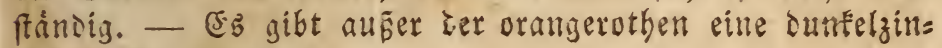
noberrothe Epielart.

\section{Tussilago," "Huflattio, XIX.}

T. fragrans, wohletedender 5ुuflattig, 24. Stalien. - Die rumblid)=herzfỏrmisen, etmab firzigen Bláts ter werten ziemlid) grof́. Der Blumenjängel erhebt fith

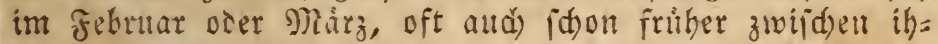
uen, etwa $6-8$ zoll hod), uno trảzt mehtere bräunlid $=$ violette Blumen, weldie mandelartis ried)er. Die Pllanze whd)ert fark. Nan yerpflanzt fie im September, gibe

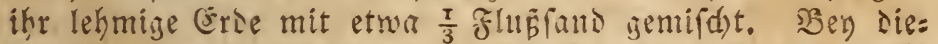

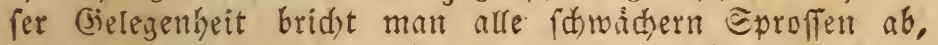
und fegt nur 3-4 ier ftíréften in eitten gerüumigen Iopf. Eo lange nod) Eein froft zu furd)ten ift, liagt mall bie

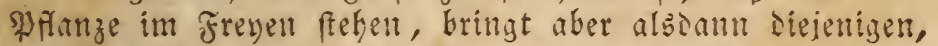

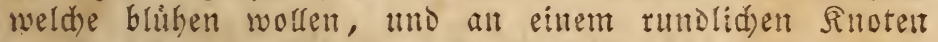
zwifden ten umfaffenten Blattfielen leidut zu erEennen fint, Did)t ant eitt fonniges Fenfer in eine Iemperatur yout 0-4 (3rats. - Frat man binteidseno Eonne, fo entroi=

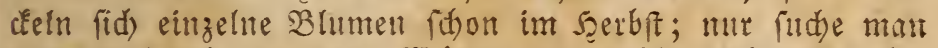

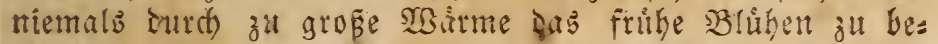
fortern. Nan laffe die Natur umgeftort wirken, mo yes= fefe sie Pflanzen mur mit Dem nöthigen SGafier. Heber Eommer feell man die Iopfe mit dem Sguflattig entweder

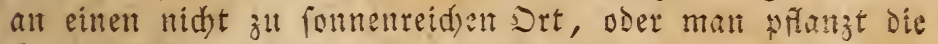
Etauden 6-8 3ou weit yon éthander all ein Beet, aus

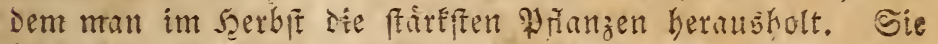

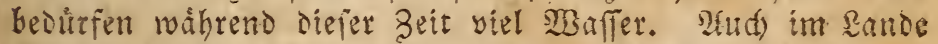
liberwintert diefe Pflatze zumeilen, jedod leiden die Rnob= pen gewobulid) wihrent Ieb Minter.

Ulex, 厄tedginfer, Xvil. 4.

1) U. curopaeus, geneincr etedginfer, F, 
Diefer im furdidyen Deutidland, in Fraufreid und Eug= lano bánfig roilomadhfende, fehr fadblidge Straud wiro $5-8$ Fuß hody. Die Zrweige find grill, geftreift, in Der sugend mit feinen weidhen Staaren belleidet, und mit gru= nen, langen, fararfen Dornen be est. Die Eleinen fdima= len Bláttchen fino unbedeuteno. Die Blumen erfdeinen an Den Bweigen ben ganzen હommer hindurd), find (d)met: terlingsăhnlid), flein, gelb, und binterlaffen fleime હbjot= d)en. - Diefer Stedginfter wird zumeilen im Iopf gezo= gen, befonders in nordichen (Siegenden, wo er die Minter nicht gut im Freyen vertrigt, yorzinglid dann, wenn ber

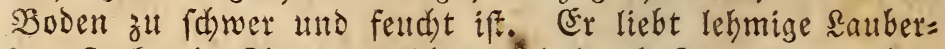
De, ftare mit Ries yermifd), wiro Duth Eamen vermelgrt, und bey $0-4$ (j) ad uberwintert. Sar das Bimmer ift er *einesweges zu empfeklen.

2) U. nanus, Eleiner Stedginfer, そ. Dem yorigen ábultad, jebodj bleiben die an ber Eroe lies genden Sroeige nut furg. Şd babe diefe 2art mehrmals

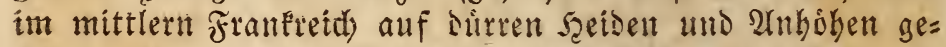
funten, ledoch waren die friedenden, sidjt mit feinen grus= men Dornen befesten Bweige nie tiber eine Epanne lang. Er blifhte im suly uno Yruguft gelb, fleiner, alö die yo=

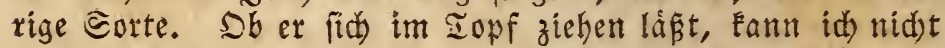
fagen; Dod) mitroe er eher einen plah in der Etube yers bienen, als jener, megen feiner Dornen oft láftige Straudd.

Vaccinium, feidelbeere, VIII. 1.

Die beisen gemeinen Seidelbecarten V. myrtillus (Blaubere) und V. vitis ide (Preugelbeere) babe ith noch nidt im Iopfe zu ziehen verfudt; Dod) gelingt dies jeś geviós, menn man paffende Ctráuder, die ia in jedem

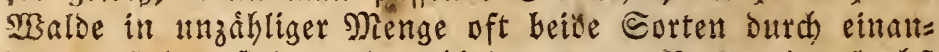
Der zu finden fint, mit geforig gropen Ballen im Serbft ober red)t zeitig in Frubling aubhebt, und fie in nidit zit enge Iopfe pflangt. STm beften ift $e$ bs, iften die namlide Eroe zu geben, rotin man fie gefuncen bat, und diefe mit etwag sauberse zu vermifden. Daß man fie im Soms

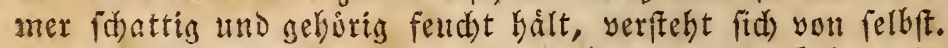
sit Fribling sagegen bedurfen fie Sonne. Eo befandelt,

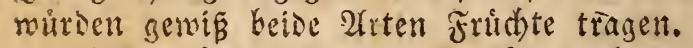

V. oxicoccos, die Moosbere, 万. - Eitre niestiche Iflauze, die in ganz Deutichlano auf fumpfigem Iorfbooel all finden iff, wo die fadenformigen Etängel 
Jwifhen Dem Roofe berumfriedent. Die fleiten myrten: ábulichen Blätter find oben glánzend dunkel=, unten weís= grin, und baben einen zurufligerouten Rand. Die Blumen: ftángel fint etwa $1-1 \frac{1}{2}$ Boll lang, ftehen aufredt, und tragen oben eine Eleine Blume, Deren vier Blâtter gẳzlić) zurictgebogen fino (gleid) senen Des Lilium martagon); ibre Farbe if rofaroth, in ber Mitte jeses Blattes eill etwab cunflerer Streif. Die Beeren find faft fo grof, alb Die Blaut= beeren, weids, fdòn roth, und onthalten cinen febr fauern Gaft. Sצill man biefe fdone feine \$flanze im Iopf zies ben, fo pflangt man fie mit Ballen in eine NRifdung yon Rauberde, Gano, verwittertem Iorf und fein gefduittenent \$Noob, uno bålt fie beftandis. feudbt; Daber ift es gut, Den Topf Des Commers in einen Lnterfentupf mit 2 Baffer zu ferren. Sie blüt im May uno Sulny, uno wito bey 0 -4 Ģrad neben atlpengewådajen (z. B. Primula minima) úberwintert.

Veltheimia, Beltheimia, VI.

1) V. uvaria, traubenblibende Seltheimie, 24. Eap. - (Seht: Tritomantha uvaria.) 2 Ub einer faft fnodigen SWurzel mad) len die 3 FuE langen, alld wohl lángern Blátter; fie fino f(bilfartib, orenfeitig, id)arfrandig, und Eniden gemeiniglid) mit ibrem obern Drittheil Bwifden ihnen erbebt fid im sulty der $3-4$ Fus bobe Blumenfd)aft, weldher an feiner Spise eine 6-8 3oll lange, gedrángte Iraube yon herabhàngenden, rỏhrigen, orangerothen Blumen trảgt. Diefe Pflanze verlangt Frảf= tige, nabrhafte sauberse, ráfrend ber Begetation viel

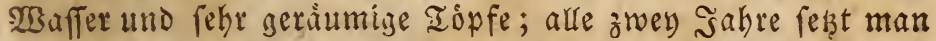
fie um. So fobon Diefe Seltheimie in Der Blutthe ift, fo wenig eignet fie fid) fur einen Blumenfreund, Der Eeinel (5)arten befist, wohin ex fie Den Sommer úber ftelfen kann, Dent die grof́n Napfe, welche fie verlangt, paffen Feines: megez auf ein Blumentret, auds ift fie oft fo eigenfinnis, Da mehrt fie fid) am beften; zumeilen erbålt man ald Neben= fprofien. Bey 4-6 (Sirad S3atrme ift fie leid)t zu úber= wintern, wenn man fie alsdann nidst zu fend thált.

2) V. viridifolia, gratiblattrige Belthei= mie, 21. Eap. - Eine Swiebelpflanze, weldye mehrere dut= Eelgrume, flad)e, glatte Blátter_yolr 8-12 zoll Ránge treibt, beren Rano wellenformin gebogen ift. Der Blu= 


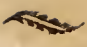

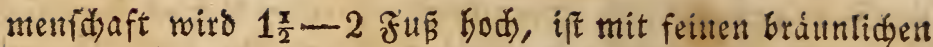
Puncten bedeckt, und trågt ofen eine gedringte 2 ehre yon bängenden, róthlithen Blumen. Diefe Pflatze verlangt leid)te, nahrbafte (Erte, und will iábrlid' verjeşt fenn, bey weldater (j)elegenheit man die Zwiebelbrut, wem fie fidh Leid)t trennen lägt, abuimmt. Die eigentlid) Bluthzeit Diefer Beltheimie find die Nonate Noyember tund December, alid) Samtar. Man mu范 ifr Dann einen warmen, mog= Iich) fommenreidyen Etano geben, indem die Blumen fonft

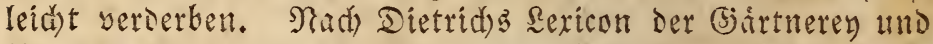
Botant Fann man jesod) Diefés (d)one (5ewaths aud) im Eommer zur Blithe bringen, wenn man ez folgenderma=

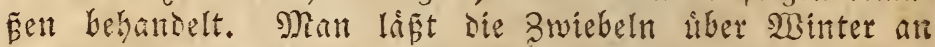
einem temperirten Drte in Ber Erse liegen uno gieft innen gar nidyt. Șm Márz oder 2rpril reinigt man fie you Den trocenen 20 urzeln uno Der ausern Edale, prianzt fie in

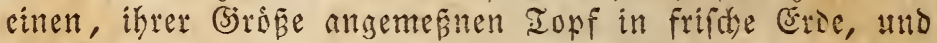
ftelit fie in cin warmes zimmer an das fenfter. Sn wars men Sommertagen bringt man fie ins Jreye an einen yor Regen gefduhten Sort. Sobalo der Blumenfidaft fid aus Der SButzel heryorbebt, bringt man die Pflatize wieder inz Bimmer hinter Genter, bis fid die Blumen vorlig entwis felt haben, weld)es alsonn yom suly bis zum Eiptember gefthieht. Sobalo der Same reif ift, und bie Dilåtter

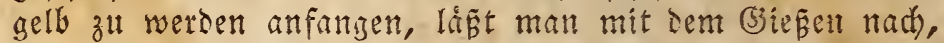

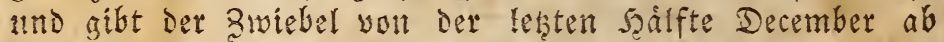

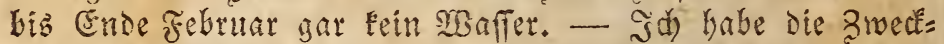

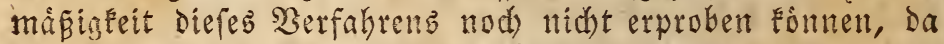

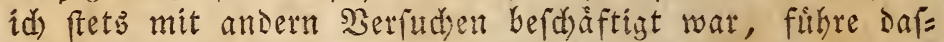
relbe jesoch an, da manthen Blumenfremen, -bie fich viels leid)t feit langer zeit vergebens bemiht haben, defe Selt= heimie zum $\mathfrak{B l i k g e n}$ zu bringen, Diefe Mittheilung nid)t unwirfommen fenn dirfte. - Die grinblattrige Selthet= mie trågt zwar Samen; jeood), Da fie binlánglid Nebent=

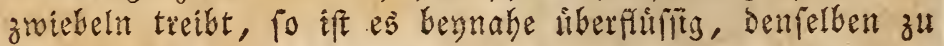
fammelnt.

If nmere. V. sarmentosa babe id nod nid)t gebaut.

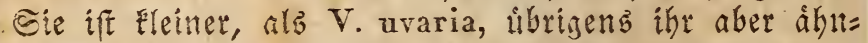
lidb; fie blihl weit williger, als lebtere, ift aber, wie id) mir babe fagent laffen, im $\mathfrak{B i n t e r}$ gegen Feuds= tigeete rehr empfindlids, vermebrt fidi) rehr gut surd

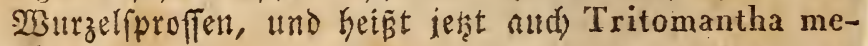
diat. 
Verbena, Eifenhart, II.

1) v: aubletia, fđidublifender Eifentart, ๑, 21. Carolina. - Der âfrige Etángel trígt enfórmige,

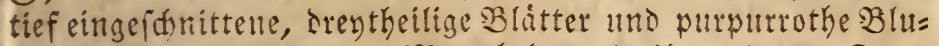

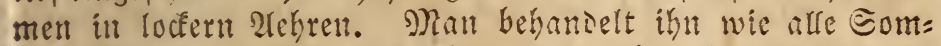
merpflanzen, uno ex ifit ebent nicht zartlids. Nahe ans

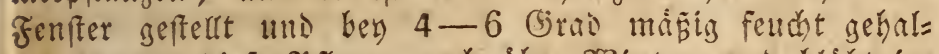

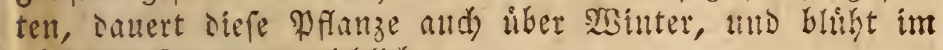
nåd)fent Sommer reidslidó.

2) V, triphylla, oreyblittriger Eifenfart,

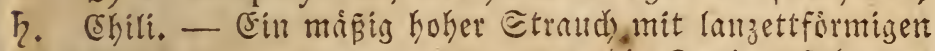
$2-3$ zoll langen, zul Drenen um oie sweige ferbenten Bláttern. Diefe fint fớarf, glattrandig, Dod) zumeiten

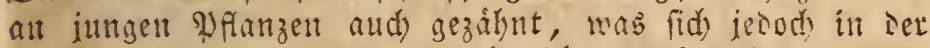
Folge wieder yerliert, vielfeidst aber audi Daher fomment maz, Dá

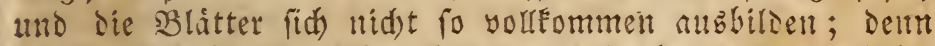

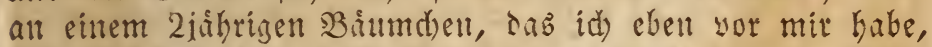

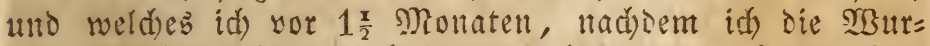
zeln Deffelben ferge fture beidnitten Gatte, in Fráftige Erde pfranzte, zeigen fidh iest and) wieser zâhnden an ben

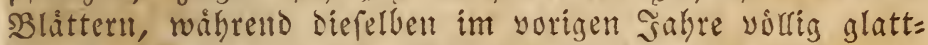

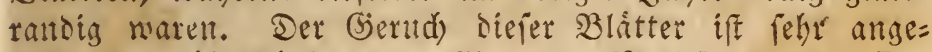
nehm und ábullid) Dem Der Eitrone, Meliffe unto Dez Pelargonium citriodorum. Die Blument erf(d)einen im șuny

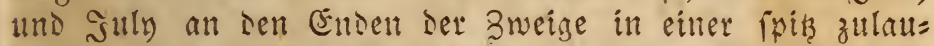
fentern, aufredsten, locelern, áftigen Iranbe. Eic fint Elein,

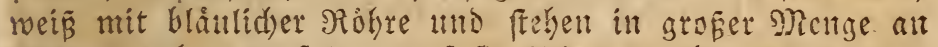

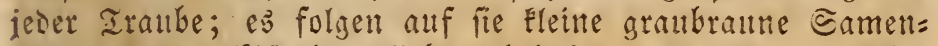
Poirner. Der Eifenhart liebt nahrbafte, mit Sefhm gemifdete

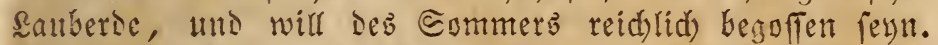

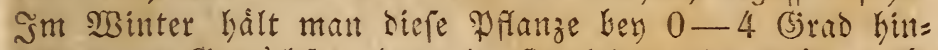

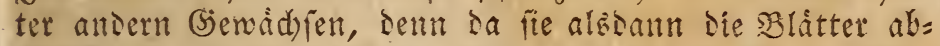

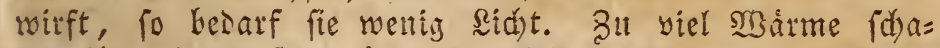
set ifye, seun fie grint zul zeitis wieser altb, und sie

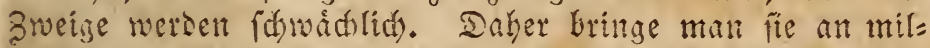

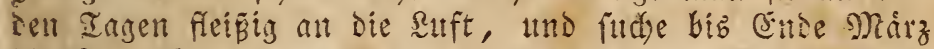

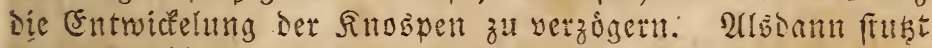

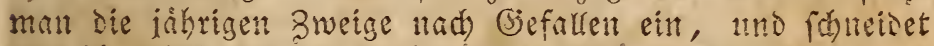

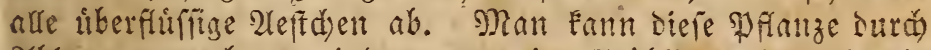
2rbleger vermehren, insem man im gruifhling einen sweig

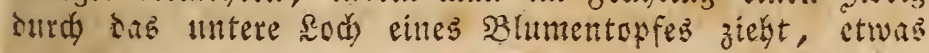


eillerbt, mit guter Erod umfüttert, unb beftänbig feudbt

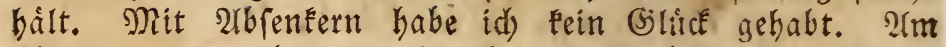
(d) nerfen vermehrt man jedod) diefe Berbena Durd) EtedE linge, indem man $2-3$ zoll lange sweige im grifhling yor Dem 2(ustreiben Der Blatter didst unter eiltem Snoten abidneiset, $5-10$ serfelben in einen Eleinen Iopf ftect, ein geráumiges (s)las Daruber bedt, uno fie in ein recht warmes Mipteet ftellt. Shat man einen ginfitigen 2ugen= blick getroffen, fo bleibt felten ein Stedling zurudf. Eind die jungen Breeige an senfelben etwa 3 zolf lants geworden, fo verpflanzt man fie einzeln in Iopfe, gibt innen einen nidjt zu ronnigen Etand uno viel geud)tigéeit, wo fie Dam im erfen Sommer oft 2 Fus bod) wad) fie bey Beiten an Etócke, wenn man grade Etámmden ers Galten will. Da man mur einen Blattfnoten in Der Eroe, und einen uber berfelben an den Stedlingen fehen zu laf: fen braltd), fo Eamt man oft einen Zrein in $5-8$ Studfe zerfdueisen. 2uth im Sommer nefmen junge Seitenfprof: fen oft an, wenn man fie mit (s)lab bedeckt, tuth marm bålt. Samen woute felten aufgehen. - Sadh neuern Beobadtungen Kilt die Berbena im Frenen alts, Dod) muF fie wahricheinlid) niedergelegt und mit Etroh und Erde be=

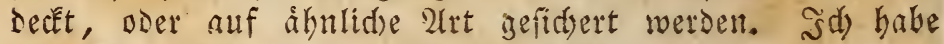

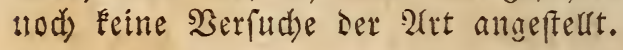

\section{Veronica, Eেgenpreib, II. 1.}

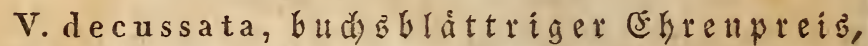
7. Faleland $=$ Stnel uno an der Magelfanif den Meerenge. - Diefer niestige Straud wird nut $1 \frac{\pi}{2}-2$ Fuß hod), und feine 3weige fino mit paarweife iber Jirellz ftehenden ellip:

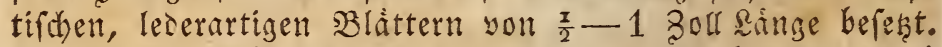
Die Blumen habe id) nodí nie gefehen, wejer ber mir, nod) in andern (sjárten; fie jollen jeood) eine rispenturtige, wes

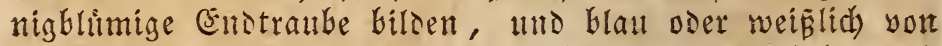
Farbe feyn. Nan gibt Diejem Ehrenpreis mit sebm und etwas Sand gemifde sauberie, iberwintert ifli bey 2 4 (sirao, uno vermehrt inn leidit burds Etedilinge. Er ift eben nidyt fehr zu empfeglen. Soll audi im sande nus= bauern.

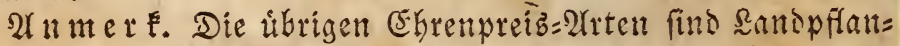

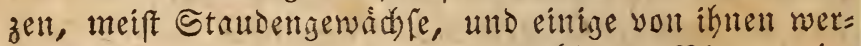
den wegen igrer fdosten, meift blauen Blumen im (5jarten gezogen. SBill man fie aud im 3immer bli: 

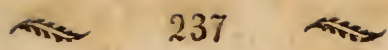

bend baben, fo behanielt man fie wie bie (s)locken= blumen. Feier nenne id): V. complicata, crenulata,

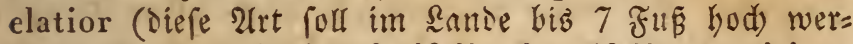
(cn), elegans, incisa, latifolia, longifolia, maritima, multifida, racemosa, sibirica, spicata. - Einige 2irten Davon find ftraudartig.

Viburnum, Sdneeballen, V. 3.

1) V. opulus roseum, gemeiner હdneebal= len, h. Fine veredelte Epielart des gemeinen wilden Edueebalten (V. opulus). Diejer befannte હtraud) lǟ̧t fid) leid)t im Iopf ziehen. Dir die aub $\$ 3$ urzelfproffen ge= zogenen Stoffe oft fibr hod) wercen, ehe fie blikgen, fo thut man am beften, im Nirirz einen im Sorten freben= ten blibbaren હdneeballen nieverlegen zu la ten, uno ei= nige paffenie obere Bweige mit seaken 2-4 3oll tief in

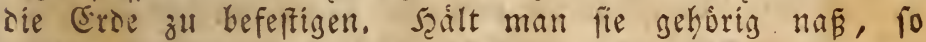
maden fie bis zum frerbft viel $2 B$ urzeln, fonnen fodann abgenommen, in Iopfe mit Drangerie= Cride gefest, und wie Syringa persica behandelt werden. Man treibe den

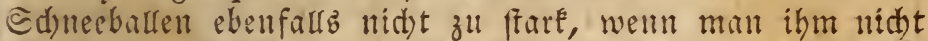
einen yorzuglida guten Etano geben Fann, foncen laffe ifm seit, fid) zu entwidfeln; Fend)tigeeit uno suft find

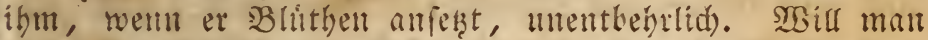
jeond) 3-4 Fub bohe Bitumden fur den Topf ziehen, fo madse man you ben unterften Eproffen eilles fraftyorren Etrauds 2ablenter, nebme diefe im Serbft ab, und pese fie im Gjarten auf einen etwas trocknen fonnigen Plas in lebmige Erse. Surd) 2rusfdneiden und 2rnbinsen gibt man ihm Die gehorige form, thto ftuht Die 3weige, weld)e z"l hod) gehen, ein. So last mant ifn fteken, bis fid die erfen sBlumen zeigen, worauf er im naddfen Serbft, mois= lideft mit sem Ballen aubgehoben uno in einen geráumi= gen Iopf ooer fleinen Fibel gefest wiro. Die im Iopf ftehenten werden iafrrid im Detober umgerest.

2) V. tinus, lorbeerblattriger Ed neeballen, (Laurus tinus), h. Eidoenropa. - Die Biatter fino lorbeet= artis, am Rame mit fleinen Scitiden gefrangt. Die

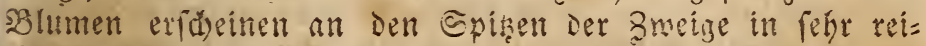
chen fiadien Doloen. Sie fino weip, die Sinospen, che fie aufgehen, rithlid. Man gibt siefem @d neebalfen Drange= rie = Croe, yerfist ifn im aruguft, uno diberwintert ifn bey

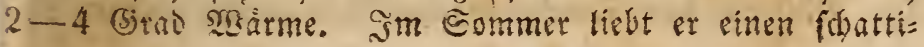




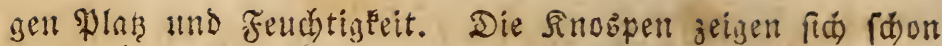
im Serbft; bodh bringe man den Etraulh ia nidist zu fruth ins Bimmer, fonft fallen fie meift ab, obne alfzubliken. Sá̧̧ man fie dedod in einer niesrigen Iemperatur all eitnem font nigen Fenfer ftehen, fo entwickeln fie fid im april und May. Som Srovember bis Ende Febrtar fann man Diefen Viburnum binter andere 2flanzen, die des sidfts mehr bea Durfen, ftellen, mur yom Màrz ab ift ifm Sontme surdid)=

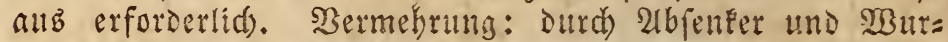
zelfproffen leidjt. (es gibt aud eine Epielart mit bunten Blàttern.

Vinca, Sinugrun, V. 1.

1) V. herbacea, pautartiges ธinnbutu, 4.

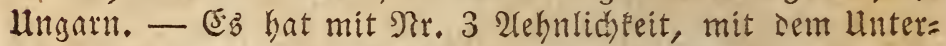

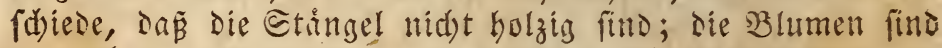
violettblau. Bebandung mit $\mathfrak{N}$. 2. Lbereinftimment.

2) V. major, grofes Sinngrin, h. Sudell= ropa. - Die liegenten 3weige werden oft $4-6$ Fus lang,

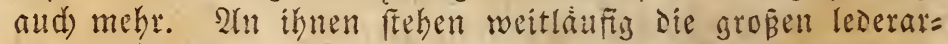

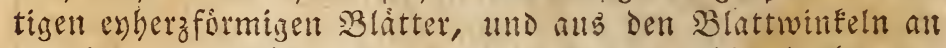
Den jungen Irieben, fommen Die grofen, fdoun herbinuen, fünfblättrigen flad)en Błlumen im 2(pril uno Nan, cinzeln aud) nod) (páter zum হ̧orfdeill. Diefe Planze bảlt zwar alld im freyen sande ans, leidet jeodd, befonders im naf: fen, lehmigen Boden, oft yom froft. Darum zieht man fie gewohnlid) in Iopfen. Eie liebt gute, nahrbafte, fdimere

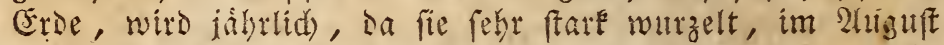

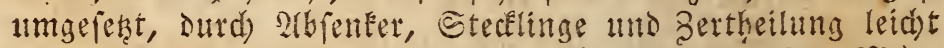
vermebrt, uno bey $0-4$ (Srad tiberwintert. Sm Mntrz (i) netset man gewibnlid die langen, alten Bweige ab, Da die jungen Iriebe fleisiger bliken, uno ftert Die Pfanze nabe ans Fenfer in sie Eonte. - Cis foll eine Barietát mit weisen und eine mit rothen (wahrid)einlid) violetten) Blu=

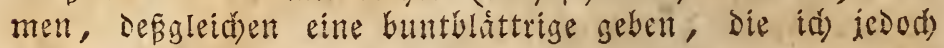
nod) nidet Eenne.

3) V. minor, fleines sinngrun, h. Șth Dentidano bin uno weteder in (Sebrifden, befonders in Berggenenden. Die feinen ameige Eried)en an Der Eroe, uno

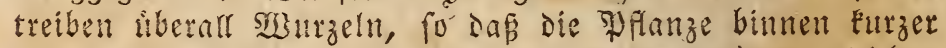

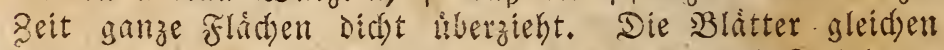

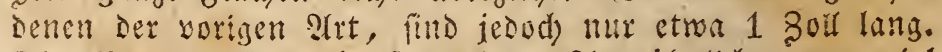

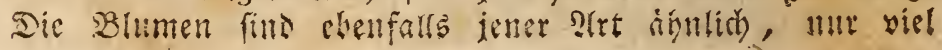




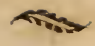

Pleiner. Mau fintet jie blau, violett (einfad) uno gefüut),

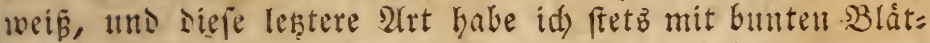
tern gefehn. Sann im Iopf wie গRr. 2 behandelt wer= sen. - 2(ud) befá id) eine Pflanze, weld)e id) als V. minor mit weif́bunten Blittern erhielt; siefe glid) Derfel= ben fehr, nur waren die Sweige mebr aufredit, alä friedjent, uno die Blitter etwas bieiter; fie bat nie Blumen ges brad)t. Die namlid)e planze fand id) fpater mit ganz grimen Blâttern bey oer Edonie (j) nadenfrey in Edblefien auf einer waloigen seibe in Brofer Nenge. Sth nabm etwab sayon mit, aber aud) fie hat im sallfe won mebres ren Sabren feine Blumen gezeigt, nno bie namliabe Flage borte id) in eiltem (sarten, wo eill ganzes Beet yolf bon Diefem (Sewridjs ftand, mit ieffen Broeigen Die sandelute Die Sirge verzierten, uno fie şintergrum namnten. Mit= gend bin fid) auf eine Epur geleitet worden, Den Shamen

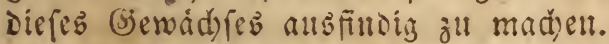

4) V. rosea, rorarothes ênugrin, h. Masa= gabcar. - Diefer fehr fdjone etrauch wird 3-4 Fuß hod),

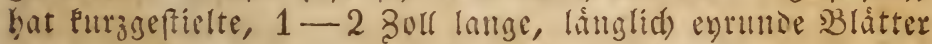
und rofarothe Blumen mit blutrotbem Edhlunte. Eie fte: ben zu zweyen in sen Blattwinleln, mo eine entfaltet fid nad) Ier andern. (E) gibt nod) foligenise Darietaten: mit ganz weifen B̉lumen, mit weisen BHmen und blutrotbem Ed)lunde, uno mit gelbem Edslunde. Die Blumen erfidei= then Den ganzen Sommer finturdh. Diefe Dinca verlangt

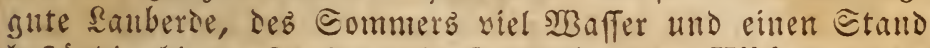

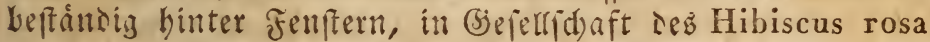
sinensis. Nan fann fie in warmtern Nonaten zwar aud alt Die suft ftellen, jeiod) blibt fie baun lange nicht fo (d)on. Eie tringt aber alsisann reid)lid) Eamen; felten, wenu man ihr die suft gallz entzieft. Durd) Êtectlinge,

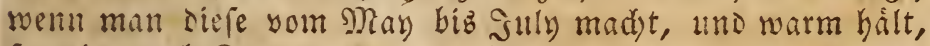
fo nie surd) Eamen ift fie leid)t zu vermehren, bey 6-12 (G) gut Durd). Sft fterben zwar die Episen Der Bweige ab, ierod) erholt fie fid im Eommer wieder, befonders, went

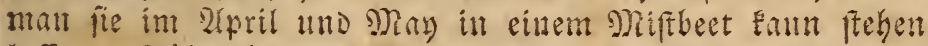
laffer. Sáfrlid im 2(pril wird fie umgejeşt.

st nmere. V. lutea Fenne id nodil nid)t. Der Stangel folf fid) winden, Die Blitter länglid), uno Die Blumen ziemlid) grof und flach fern; als (Earolina, uno fonth= te tafier moh! wie Mr. 2, bihnthelt meroen. 
Westringia, Meftrillgia, II. 1.

W. rosmariniformis, tosmarinartige NGeftringia, $h$. Nentyolland. - Diefer Etrand mádylt brynate pyramisenformig $3-5$ fus bod). Die 8weige fte: ben gemeiniglidh quirlformig. Hebrigens bat ser Sisnd) deb Etraudyes, fo wie peine Biảter uno Blimen, siel 2lehn= lidefeit mit Dem gemeinen Rosmarin. Die Blumen find

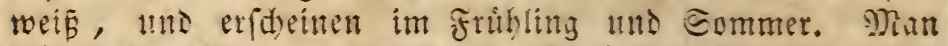
gibt Diefem Baume gute fandige Rauberde, feşt is,n jeden Fribing um, who yermehrt ihll am beften surd) Samen;

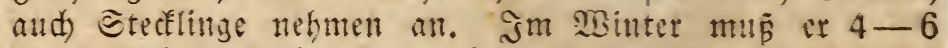
(5irad SBarme baben, vertrigt jesod alsbann nidit viel

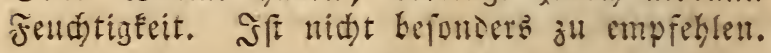

\section{Yucca, Y)usfe, VI. 1.}

1) Y. draconis, Drad)enbaum= Yate Citonmerica. - Der graubraume Etamm widdit aufred)

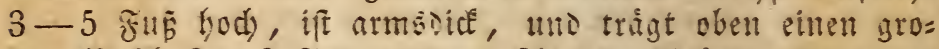
fen Bufd $2-3$ fun langer, (d)maler Blitter, Die einen (d) arfen Rano kaben, wno fid) in eine bornartige, fidarfe Epise endigen. Der Blumenfängel entipringt im suln

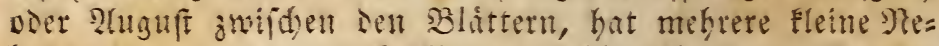
benjtweige an ien gloctenformigen hibergebogenen Blumen an ungleid) longen Eticlen ftehen, nno eine lange, didte,

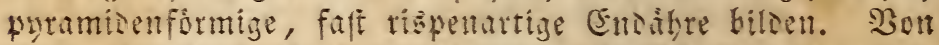

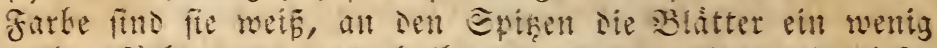
roth gefurbt, uno auserbalb mebe oder mentger mit seper Farbe idattirt.

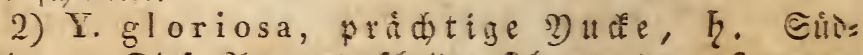

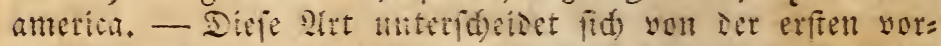
juglid) Durd) Die etwas Furzern, jedod) viel breitern Blit= ter, welde feinen fdurfen æano baben. Der Etamm wito oft 4-6 fus bod), aud wohl bober. Der Blumenftan= get if gegen 3 gup lang, uno die weiphliden Bhmen senen jenter Qut âfnlid).

Brite Pfanjen eigutn fid nidgt fir bie Etubencultur, incen fie zu gros, uno wegen ifter langen frad)ligen Blit= ter lifitig merden. Da fle langlam wadjen, fo mus mant

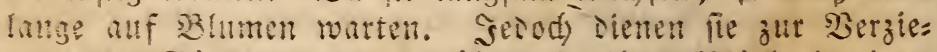
raing ser Gatten, uno gewabren in ifter Blithzeit einen

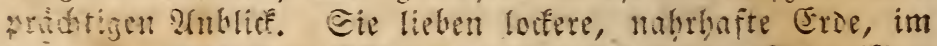

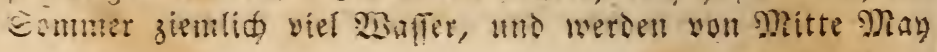




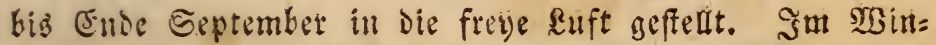
ter nefmen fie, gleich Den alloearten, bey 2-6 (5iad mit irocm હtantort yorlieb, mur mas mant fie nicht zu feuld balten. Seden fribling, ehe man fie in die Suft bringt, wersen fie umigefst, mo ber Diefer (Selegentheit die sheben=

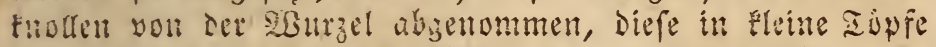
gelest, fo dor ser ofere abeil, wo der Jeim fid entwi= celt, etwa 1 solf bibe bor Erde hervorragt; fie treiben, went man fie warm bitt, bals als. Nr. 1. bringt wentig Sindfen, tho man mur oft, tm fie zu vermegren, die

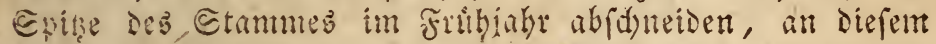

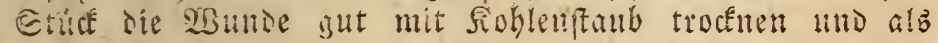

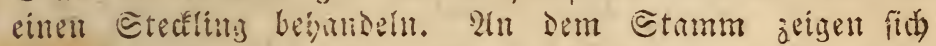
andi) balo Eeitenfroffen, bie man, fobalo fie bie geho=

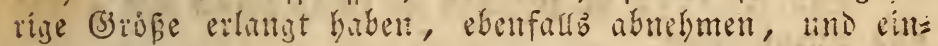

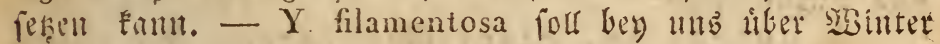

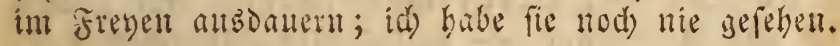

\section{Zinnia, Zimnie, XIX. 2.}

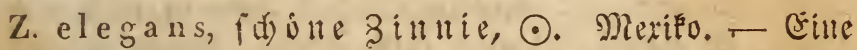
bifante Bierpflange mit fdouth, róthlid) violetten Błtumen. Die pfanzen werden gewohnlid) ins sand gefest, oder bleiben in sem Mifteet fteben. Dod erhilt man alsoann felten reifen Eamen; Daber iff ez gut, ftets ein \$aar \$flanzen in geriamige Iopfe mit nabibafter sauberde zat Fekeil, mito fie wie Datura fastuosa zil bebanielm. - Die

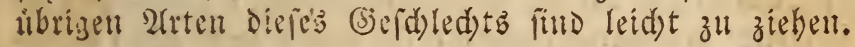

\section{Zigophyllum, Doppelblatt, X. 1.}

Z. sessilifolium, ungefteltes Doppelblatt; 7. Cap. - Die Etinngel jind vierfantis, 1-2 Fup hod), Die gepaarten Bhlitter feifdis, ungeftielt, verfebrt, läng= lid $=$ enformig. Die flid)en-Blumen baben etwa 1 Bofl im Duthmeffer, find gels, an Dem Jeld) mit einigen roth=

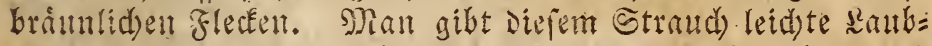
erde, fest ihn jeden fruhling um, uns vermegrt ihn ourdy Eteuflinge. Sm Eommer wird er inb Freye gefterf, uno bey $4-6$ (j)rad iberwintert. 

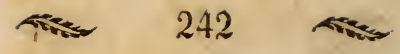

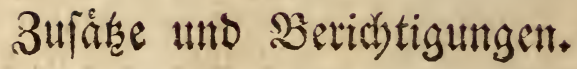

Bey A cacia iff zwifden A. Iophanta uno stricta einjufdiebent:

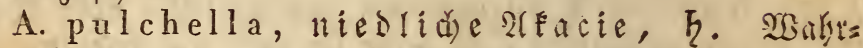

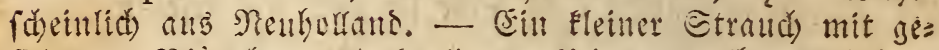
fiederten Bratted)en tho fugligen, Eletnen, gelben Blitthen,

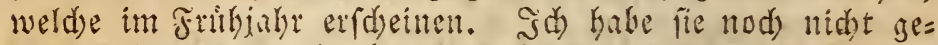

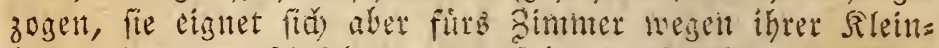

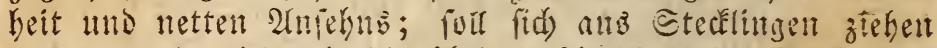

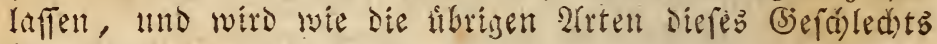
bekantielt.

Zit Alo ë brevifolia if ber Eentide Name: $\mathfrak{T} x$

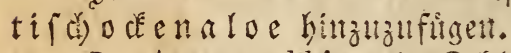

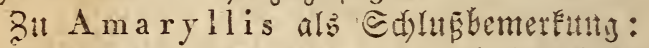

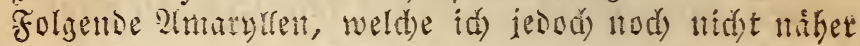
fente, wersen als ebenfalts feht fón empfoklen: A. purpurea (and) A. speciosa, Crinum speciosum) mit grop̃en butétrothen Bhtmen, gewofultid zu jweyen auf eittem Etängel; fie witr migefing wie A. belladonna bebandelt, mo folf milling blikent. - A. crocata lino ornata; beise fund vielbrumig, liebent die şarme, und befaltent bas ganze

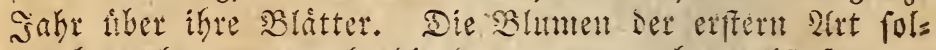
len beynthe orangeroth, sie der zweyten aber wein fern.

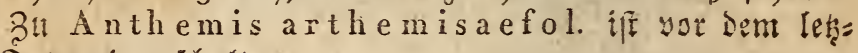
ten Enthe eimzuidalten:

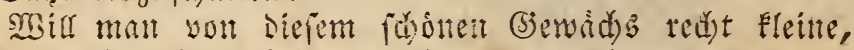

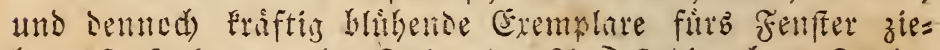

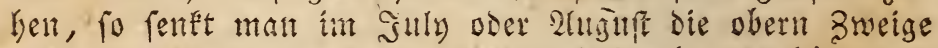

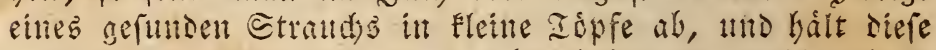
red)t feud)t. Sic Ense September baben fie gewóhnlid' fo

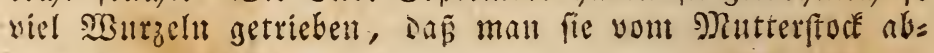


lófen fantr. Shan bült fie fobant cintige Inge int Edyat=

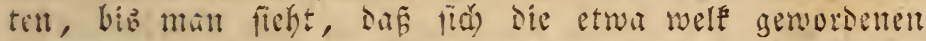

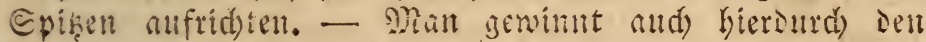
Sortheif, Daß beym lleburmintern Der Eorten die Eleinen Iopfe Wentis Raum einnefmen.

Antirrhium, sónenmanl, XIV. 2.

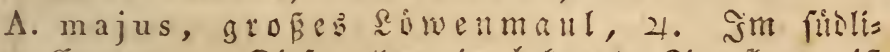
d)en Entrosa. - Dieje allyemein befannte Bierpfinmze if

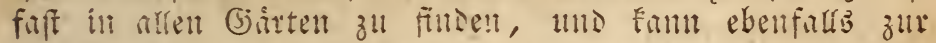

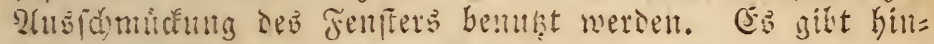

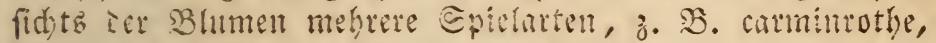

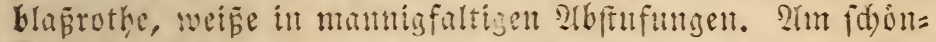
ften ift jerod) eine gand weipe alt mit sunfelcarmintothent sippen (gewóhulid) A. bicolor genanut), welde etft feit ein Panr Sahren bef́annt geworben ift. - 2tue Diefe Epiel= aitelt zitht man am beftell ahs Samen, Den man zeitig inz Miffueet oder in Iopfe fäet. Die Dplanzen feŗt man ins freje sand, wo fie jumeilen nod) im erfen Sommer

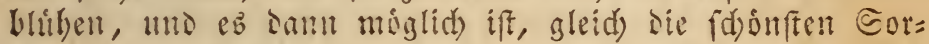

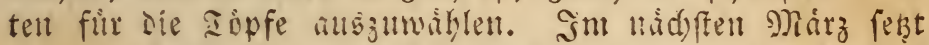
man fie in shipfe, mo befandelt fie wie die Bjlockenblu= metr. Pflangt mall fie gleid) 2tufangs in Topfe, fo sitr= fen siefe nidts zu flein fenn. Den Eommer über liebt bas

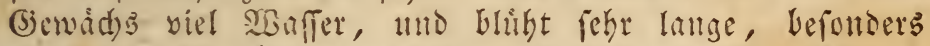
ment man bie abgetrainen હtangel cinftust. (5) gibt aud)

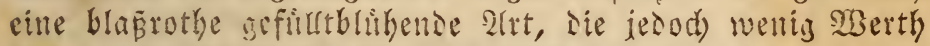
fat, leid)t Iurd) Etecklinge yermefyet wird, aber, Da fie zartlider ift, als die einfadse, im zimner liberwintert werben muг.

รu Arum:

2) A. dracunculus, Dradienarum, 24. Cuis=

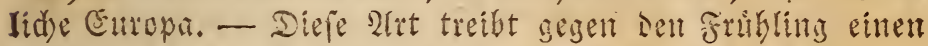

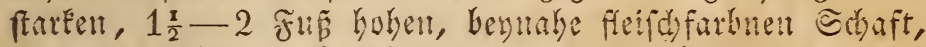

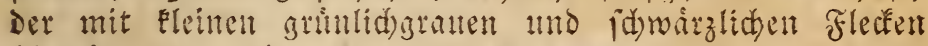
diberfiet ift. Sben fist eine firone von mebreren $5-7$

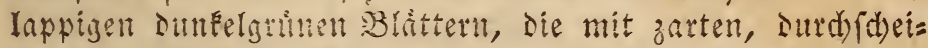
nenien weifen Etrísen geziert fins. Bwifden ifnen er:

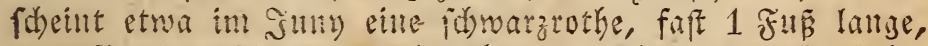
tutenformige Blume, welde aber fo hodff roiderlid ried t, sa gesulset wersen Eam. - Hebrigens ift der 2tmftand ber \$flalze (man fann fie faft mit einem \$almbaum verslei d)en) fess fobon, uns fie aus diefem (5innde z empfeh= 


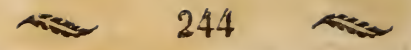

len. Nady Der Blithzeit fterben Blätter uno Eda aft all= máblis ab. Die Fnolfe bleibt mun bis zum Detober balb= trocken in Der Errè liergen, morauf man fie herausnimmt, fábert mo yon nettem in eillen geranmigen Iopf mit

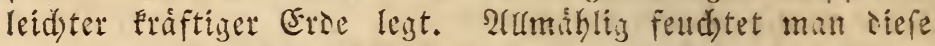
กI, und gieft in Serthiltnié wie die Triebe emporfdite=

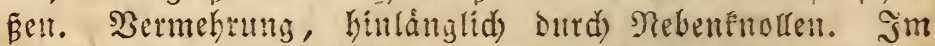

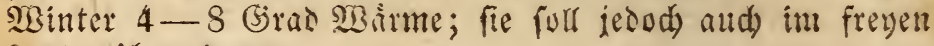
sante liberwintern. 


\section{Xllgemeines Regifter.}

Einleitung

2. Sion ien நlumentipfen ? • . . . 12

3. 2on Den Eriarten . . $\quad 14$

4. Neber Das Segiēen, Den Ëebraud Der Unterfeç=

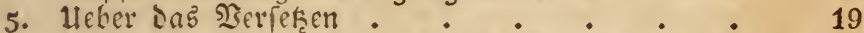

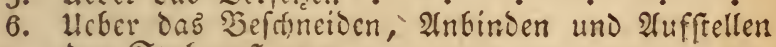
Der Stubenpflanjen. . . . 19

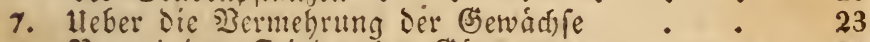

8. 23on einigen Jeinoen Der (5iartncrey . $\quad 25$

8. Erflarung ciniger Ṡejeid)nungen, uno furge $\mathfrak{U}_{e}=$ berfidt ies Simneifoen Pfanzeniufrems . . 27

Acacia, $\{$ facie . . . . . . 33

1) A. todonacifolia, boboncentattrige iffacie : 34

2) A. longifolia, langfláttrige 2Afacie • • . -

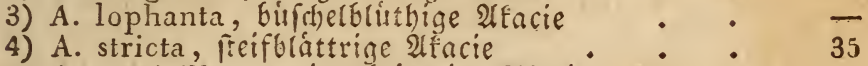

5) A. verticillata, wirtelblattrige afacie -

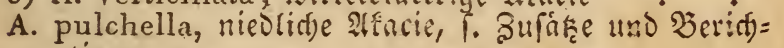
tigungen.

Acanthus, sionfiaue.

A. mollis, effte Birentiaue . . . . . \& 35

Achania, Sctiampappel.

A. malvaviscus, fortladrothe. St)ampappel . 36

Achillea, Garbe.

A. tormentosa, Die fingige C5arbe . . . . 37

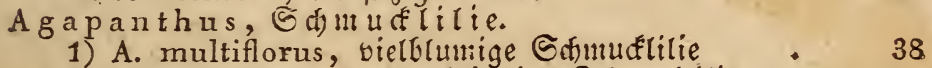

2) A. umbellatus, Dolbentíthige Єd)mudflitie : A gave, $\mathfrak{A} g a \mathfrak{g}$.

A. americana, antericaniffe Agave . . . 38

Agrostema, ia a e.

A. coronaria, Bartenrabe, Eammetnelfe . . 39 


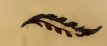

Alo ë, Atoe

1) A. arborescens, bammiartige $\mathfrak{A}$

2) A. atrovirens, fdmarsarime alloe

3) A. brevifolia, turzutattrige aloe

4) A. cymbaefolia, fabnfonige artoe

5) A. echinata, fradjlige utce

6) A. lingua, Bungenalor

7) A. maculata, geffecte Arre

8) A. margaritifera, Werlalce

9) A. mitracformis, Eifoofieminenformige siloe:

10) A. pentagona, funfedfige 2 loe

11) A. retusa, afocituste aloe

12) A. succotorina, gefréteflitie 2irce.

13) A. spiralis, gctumoene 2rtoe

14) A. variegata, bunte stoe, Tigernloe

15) A. verrucosa, manige Iflce

16) A. viscosa, flekrige 2iloe

17) A. vulgaris, gemteine 2 loe

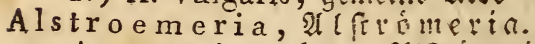

A. peregrina, Eunte भfercomeria

Amaryllis, $\mathfrak{A}$ ittar $y[$ ! $i \mathfrak{b}$

1) A. belladonna, sie foone frau, grofe weftino fre Anmarnllis

2) A. crispa, froufe smarurlio

3) A. formosissima, frónfte 2t:unculfis

4) A. sarniensis, jovaniffe 2luninlliš .

5) A. undulata, ivellentisttrige 2umarylits

6) A. vittata, banoirte 2tmaty!lis

A momum, Sngwe:.

1) A. zerumbet, Silcóng!ne:

2) A. zingiber, edfer Ingmer

Amygdalus, Mandel.

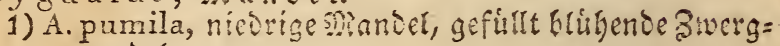
insmét

2) A. nana, sivergmantel . . . . . Anagallis, (ह) a dilleit.

1) A. collina, Suge! = Saudífeil

2) A. monelli, aufreuteb Eaudbeil : : :

Andromeda, MnD romese.

A. polifolia, polentattrige Pinoromete . . 52

Anemona, Ancmonc.

1) A. hepatica, Ecter Eraut

2) A. pulsatilla, grofe futhenifirle : : : 53

3) A. vernalis, fube fiudjendyefle

4) A. alpina, arpenancmone

Anthem is, Ehamilte.

A. artemisaefolium, bufufblattuige EGamille.

Antirrhium, "óvenmaul.

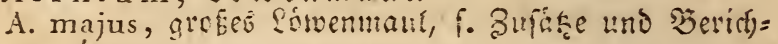
tigungen

Arctolis, is a renolir.

1) A. cincraria, afofontiges Djarencht

2) A. grandiflora, grof́blumiges sósenobs.

3) A. rosea, rofenrotbes Sitrutgr 


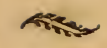

Arostolochia, Oftertugel.

A. sempervirens, immergine Dfteringey . Seite 57

A rum, ลิย hเ

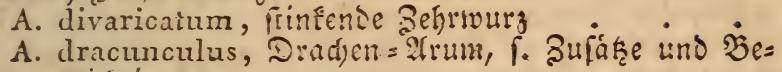
rititigungen

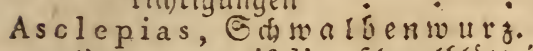

1) A, angustifolia, fthmalflattrige @atoalbentours 57

2) A. curassavica, orangerothe Edrtoalbenrourg a 58

3) A. fruticosa, friaudiartige Eatralfentvurg

4) A. carnosa, i. Schollia crassifol.

Aster, Eterniblume.

A. angustifolius, ifmalblattrige Sternblume - 68

A tragene, $\mathfrak{A}$ tragene.

A. balearica, Falearifine \&tragene . . $\quad 69$

A ucuba, $\mathscr{A} u c u \in$ a.

A. japonica, japariffte 2 lucuba . . . $\quad$. 59

Azalea, Felienftauch.

A. alba, aurantia, coccinea, pontica . . 60

Begonia, $\subseteq$ d) i efblatt.

B. discolor, funtes Edjiefulatt . . . . 60

Budleja, $j$ u d leja.

B. globosa, fugeltrigente $\mathfrak{B u b l e j a ~ . ~ . ~ . ~} 61$

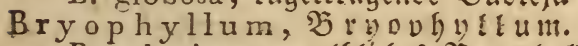

B. calycinum, gelblidbeb $\mathfrak{b r y o p h y l l u m ~ - ~ a ~}$

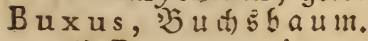

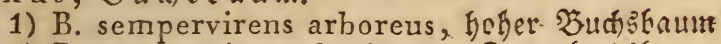

2) B. sempervirens fruticosus, 3tvergbudjbaum

Cacalia, 円e frum.

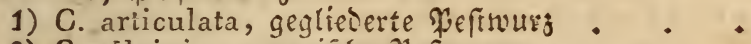

2) C. Kleinia, canarifde Peftwur;

Cactus, FadelDiftel

1) C. alatus, gefligelte Fanceloifrer : •

2) C. brasiliensis, Erafilianifite Faderbifict:

3) C. compressus, zufammengeirióte Fodersiftel

4) C. flagelliformis, peitfinentormige Focsetiftel

5) C. grandiflorus, grofiftumige sadeldiftel

6) C. hexagonus, fed)ŝfeitige Fadelifift

7) C. mamillaris, warzentragenie Facferdiftei

8) C. mamillaris spinis flavis, waizentragente $\dot{\vec{z}}=$ celoiftel mit gelben Etadieln

9) C. melocactus, melonenartige gacécioifrel :

10) C. opuntia, gemeine Facferoifrel : : 70

11) C. pendulus, hángende fadfelotifer: : :

12) C. peruvianus, peruvianifale Faćeldiftel : -

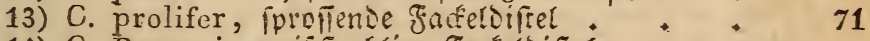

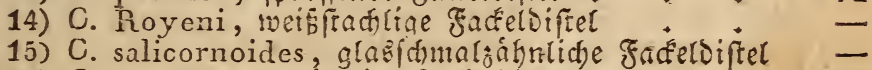

15) C. salicornoides, glaşdumalóântiche facteldiftel -

17) C. stellatus, gefrernte facfertoifrel

18) C. truncatus, aGgeftuţte Fadeloifiel : : Caladium, Ealabium.

C. bicolor, oweyfarbiges Ealaoiunt 


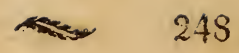

Caila, cdi ? angentrat.

1) C. aethiopica, atbiopif́des Edilangensiaut Eeite 74

2) (. palustris, (Eumpfid) (angentiaut

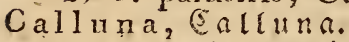

(:. vulgaris, gemcine Salluna

75

Calycanthus, Seld blume.

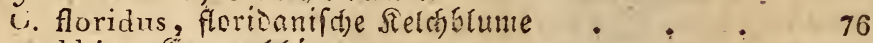

Camellia, Eamellia.

L. japonica, japanifde Enmelíi . . . . 76

C. japonica alba plena, anemonaeflora, coronata,

flavescens, paeoniflora, pomponica alba, rubra

pl., rubricaulis, variegata

$77 \% 8$

C. sansangua alba, rosea $\quad . \quad \cdot \quad \cdot \quad \cdot \quad 80$

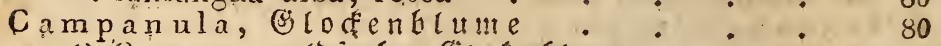

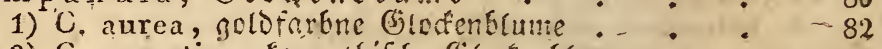

2) C. carpatica, farpatbiffe Gícfentlume . . -

3) C. mcdium, itif sinavienglode

4) C. pendula, Gángende Blodenbrume

5) C. persicifolia, pfinfitblattige (SIodenfrime

6) C. pyramidalis, p:!amibenformige Stofenblume

7) C. speculum, Senuspipiegel

8) C. versicolor, Gunte (sictenblume

Canna,

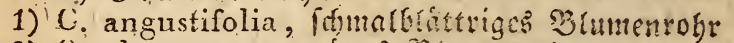

2) C. glauca, meergrunes Bulmutuobr

3) C. indica, intifobes Bo(men:ob!"

Capsicum, Beifbeere.

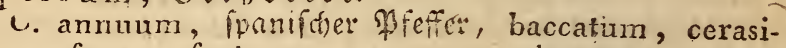
forme, frutescens, grossum, sinense . .

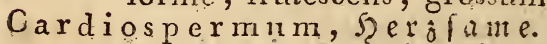

C. halicacabum, genteiner jetôfane . . . 86

Carduus, Difiel.

L. casabonae, Eafabonsbiffel $\quad$ - . . 86

Carlina, E 6 etw $4: 5$.

C. acaulis, frongellofe focrumis . . . . . 86

Ceratonia, Sobannisb:0o.

C. siliqua, gcisteines Gohannisbroo . . . 87

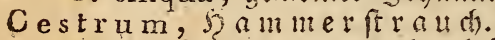

1) C. laurifolium, iorbeethiatriger Sganmenfinaud

2) C. Parqui, dififfor Sammerfrauch

Chelone, St filoblunte.

C. barbata, bártige Ehitobrume . . . . 88

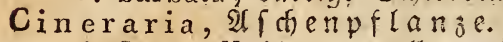

1) C. amelloides, antllenarige sffitenpflange . 88

Cistus, sifentofe.

2) C. maritima, sdeerftronos $=2$ fdenpfange

87

1) C. crispus, frouzblattriger Eiftus . . 88

2) C. ladaniferus, Inubanumtragento Eiffen:ofe $\quad 89$

3) C. purpureus, purpurrothe Eiftentofe . .

Citru s, Drange
C. aurantium, C. a. dulce, plenum, salicifolium,

striatum, C. decumana, japonica, myrtifolia 91

CI e rodendron, ?D

C. fragrans, wohtrichenocr scosbaum 


\section{Cneoram, 3citans.}

C. tricoceon, sreylamiger Scifand . Ecite 94

Cobbea, (5obbea.

C. scandens, fdilinnenie Eobeer . . . . 95

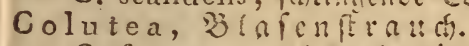

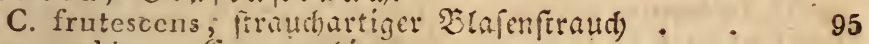

Commelina, (Eomme! ine.

1) C. coelcstis, himmtifite Commetine

2) C. tuberosa, ite fnofige Eommeline

Convallaria, Mi a blume.

1) L. japonica, japanifde Manflume . . . 97

Convolvulus, minoe.

C. cneorum, iip Eilfervinde

Corchorus, है o chorus.

C. japonicus, jayanifdiel Eorforus . . . 98

Catyledon, îa elfiat.

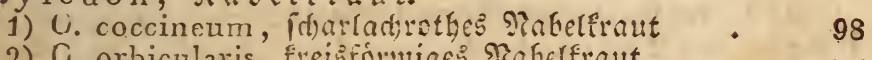

2) C. orbicularis, freisformiges Mabelfraut

Crassula, Didelatt.

C. coccinea, decussata, imbricata, lactea . . 99

Cucurbita, î́t r bis.

C. lagenaria, ick fraft)enfurtiz . . . , 99

C. anrantium, ier plommenonenfirtig $\quad 100$

Gupressus, Evpreffe.

C. sempervirens, immergrine Euprefle . . 100

Cyclamen, (E: D f d e ibe.

1) C. coum, runoblattrige (Erefácibe . . . 100

2) G. europaeum, eurcpeifife Erofiteibe

3) C. hederacfolium, ephaublittrige (Eofideife - 102

4) G. Farsicum, perfiffye erofdacibe . . . 103

Cydonia, $\mathcal{Q}$ uitie.

C. japonica, japanifáe Suitte . . . . 104

Gy noglossum, Sunosaunge.

C. omphaloides, frublings = 5̨unosgunge . . 104

Cyrilla, Enille.

C. pulchella, fidone Enrifle . . . . . 104

Daphne, Eeidelbait.

1) D. cneorum, bas Stein:ózden . . . 105

2) U. odora, wohtriertenter Eeioctbaft: -

3) D. laureola, lorbcerólattriger Eeioclbaft : $\quad 106$

Datura, ๔ted apfer.

1) D. fastuosa, fobrer Gtedapfer

2) D. suaveolens, wohtrichende: Eterbapfel

107

Delphinimm, Niterfporn.

1. grandiflorum, grofftumiger Sitterfporn

Dianthus; Sitelfe.

3. japonicus, japanifige sRelfe . . . . 109

Digitalis, fingerbut.

1) D. lutea, Der getbe fingethut . . . . 110

2) D. purpurea, Der rothe Fingergut : . : -

Dillenia, sillenia.

D. scandens, ftettembe Dillenia 


\section{सike 250 אike}

Diosma, Gitter $u f t$.

D. alba, weif́er Gotterouft . . . Ecite 110

Dodecatheon, Es ot teit blume.

D. meadia, virginiffie Eotterblume . . . 111

Dolichos, $\mathfrak{B}$ obne.

D. lignosus, holjartige bohne

Dracocephalus, Dradienfopf.

D. virginicus, virginifder Dradjentopf

112

112

Erica, Seide

E. gracilis, phylicoides, carnea, cinerea, multiflo. ra, stricta, tetralix, aitonia, cerinthoides, conspicua, grandiffora, jasminiftora, mammosa, margaritacea, speciosa, taxifolia, translucens, tubiflora, ventricosa, verticillata, vestita .

Eucomis, $\Xi_{\text {d opf }}=5$ yas inthe.

1) E. punctata, puncti:te Sifopf = j̧yajinthe, Siro= nen = Joyazintbe

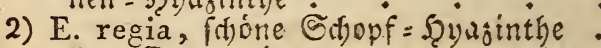

Eugenia, Eugenie.

E. australis, fiislicte engenie . . . . 115

Euphorbia, $\mathfrak{B}$ o! f $\mathfrak{s}$ mi $\mathfrak{l}$ d).

E. caput medusae, ins Nicoufentgaupt । . 116

Ficus, Feigenbaut

F. carica, gemeiner feigentaum . . . . 117

Fragraria, 5obeere.

1) F. indica, inoifice Erofecre . . 119

2) F. vesca, gemcine Eribere, জ̇alserobere

114

Fuchsia, ซuch fia.

F. coccinea, forarlacirotbe Juchfia . . . 120

Gardenia, Eিrienie.

G. florida, vieltibenoe

121

Georgina, Beorgine.

G. variabilis, veranoertide Georgine . . .

121

Geranium, Gtor difnatel.

1) G. anemonaefolium, anemonenbláttriger (ctorti)= (í) mabel

2) G. macrorhizum, srof̂tou:

Gladiolus, Ġeglvu:

1) G. cardinalis, Eartimals = Eiegmul;

2) G. communis, gemeine Etrgrvuto

Gloxinia, Glorinte.

G. speciosa, ftione Elorinie

123

Glycine, ङ̈Incine.

G. rubicunda, Ounferrotge Eturine . . . 125

Gorteria, (5) orterie.

G. rigens, frarre Eorterie . . . . . 125

Grevia, $\mathbb{G}$ revie.

G. occidentalis, africanifíse Grevie • • . 126

Helianthemum, Sonnentitinden.

H. laevipes, alatteg Eonnenbrimben- . • 126

Helianthus, Sonnenolume.

H. multiflorus, vielblumige Eontenblume • . 126

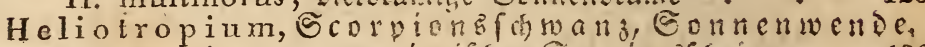

H. peruyianum, perubinnifge: Ecorpionsfthtwant 


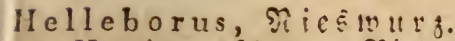

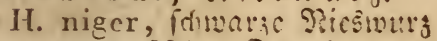

II e merocallis, Tagt tume.

1) H. alba, meif́e Tagélume, Saglitie . • 127

2) H. coerulea, linnc Ingflune . . . . 128

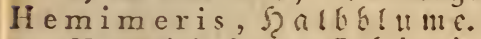

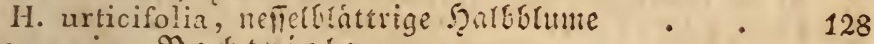

Hesperis, Na ditiole.

1) H. matronalis, cemeine 9indituio!e. . . 129

Hibiscus, (Eibif t).

1) H. manhiot, fitwefelgetoc: (5ifijá) - . 129

2) H. rusa sinensis, dinefifdie ßofe : $\quad$ : 130

3) H. syriacus, furifder (E) Eifif

Hieracium, 5 a 6 i d) $t 5 f: a u t$.

H. aurantiacum, onangerothes Şatidftefrnut . 131

Hortensia, \$? o: tenfia.

H. mutabilis, veranertidje Şortenfia • • • 131

Honstonia, Sqa fifonia.

H. coccinea, fdarlatfothe Şaufronie • • . 134

Hy pericum, Jobannisfiaut.

1) H. balearicum, Galearifdes Tobannigfraut . 134

2) H. calycinum, grofflumiges Tohannistraut:

3) H. monogynum, indifd)es Jobanništiat

Jasminum, Sasmin.

1) J. azoricum, aforifithet Saริmin

2) J. fruticans, fraudiartiger Jaŝnin

3) J. gracile, fftanfer Iasmin

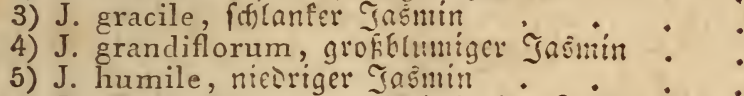

6) J. odoratissimum, wohlrict)enter Tramin - 136

7) J. officinale, gemeincr Insmin . . $\quad \overline{137}$

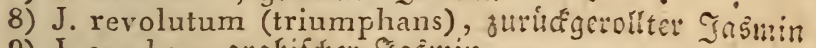

9) J. sambac, arobifite: Sastuin

10) J. trifurcatum, D:eygatiget J̈asmin

Ilex, Stectpalme.

I. aquifolium, genteine Etedjpalme . . . 138

Ipomea, Tritterivinoe.

1) I. coccinea, fharladrothe Triáterminie. . 138

Iris, ङdi) wertlitie.

1) I. persica, perfiffie Editwertel . . . . 139

2) I. sussiana, prádftige Edwwertlitic . . . 140

3) I. xiphium, fpanifrie Edyluettel . . . 141

4) I. xiphioides, engrifale Eaburter : : :

Ixia, Ixie.

Ixia crocata • • • • • • • • 171

Ixora, Irote.

I. coccinea, fdiarladtothe Trore . . . . 141

$\mathrm{Kalmia}$, fi a mie.

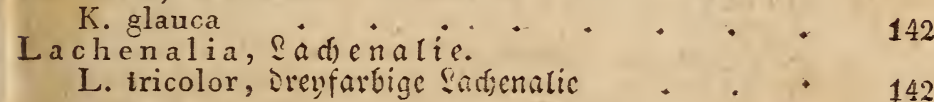




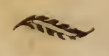

Lantana, ?antann.

1) L. aculeata, fradilige ?antana

2) L. camara, veranderlidje Santana

3) L. nivea, tweifie Inntana

4) L. rosea, rofenrothe Eantana

5) L. trifoliata, Drenflattrige fantana

Laurus, Rorbeer.

L. nobilis, Der gemeine Sorber

Lavendula, Laventel.

I. multifida, vieltheilige Insenoel

L. pinnata, gefieierte Enbenoel

Le dum,

L. palustre, wifoer Siosmatin

Le p tospermum, Sis ofeemyrte.

L. flavescens, juniperinum, obliqua, scoparium, thea , ilie.

Lilium, Pilie.
1) L. bulbiferum, feucritie

2) L. candidum, die qenteine weife fitie

3) Li. tigridum, Die Sigerlitie

Labelia, Iobelie.

1) L. cardinalis, Enisinatêtume

2) L. fulgens, feurige fobctie

3) L. erinus, fleine Soścíte.

Lonicera, eferolat

L. sempervirens, immetgrimeš Eeisblatt. .

Lotus, Gototentee.

L. jacobaens, fitwarier Ëbotenflee, Jacobistee

Lychnis, form a r a d b d en.

1) L. chalcedonica, brennende ?tebe . • . 149

2) L. coronata, gefiontes fínmentóstjen : • 150

3) L. viscosa fl. pl., gefirite pedtnelfe

Lycium,

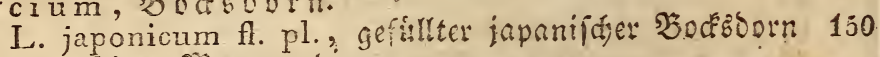
Magnolia, Nagnolic.

IM. grandiflora, grotsurmige seagnotic

Mahernia, slahernic:

1) I. odorata, tosblrietsnie magernie

2) M. pinmata, geficosute snobsernie

M. annua, jábriges Gemfenform.

1) M. artirrhiniflora, tóvenmautántidie matrandie

2) IVI. semperflorens, immetbtugende פinurandie

Medicago, Stherenter.

M, arborea, Saumartiaer Swnedenilee . . 153

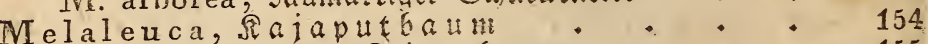

1) M. alba, ucifer Sajoputraut . • • 155

2) IV. coronata, fronentragenoer Sajaputbaum

3) M. hypericifolia, johannistratbtattriger $\Omega a j a=$

4) M. pulchella, néblidicr Siąoputbaum

5) IVT. splendens, glomsence Rajoputbaum

6) IV. tetragona, vierfeitigc: \$ajaputEaum 
Mesembrianthemum, Boferfitume

1) JI. crystallinum, Eistiaut, Diamantpflange - 157

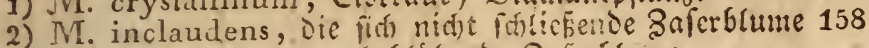

3) M. noctiflorum, nat)iblúbente 3aferblume

4) M. pinnatifidum, geficostc 3aferblume IM espilus, s? ispel.

Mt. pyracantha, Det Jeuerónf(t) . . . .

I I e trosideros, Cifenma n fi.

1) M. citrina, orangentifttrigco Eifenmaa $\quad 159$

2) IM. lanceolata, lamettelótriges Eifenmans - -

3) M. lophanta, Euffoclolintbiges Eifermaná .

4) M. pinifolium, tieferetátriges Eifemmańs.

5) M. salinga, weidentítattrtges Eifenmaás -

6) M. semperflorens, imucrhtubendez Eifenuaá Mimosa, Sinnpflanje.

M. pudica, fdambafte Simnifanje . . . 160

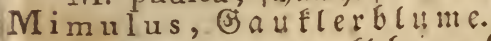

1) M. glutinosus, flebrigcr (S) anflet. . . 161

2) M. guttatus, getípfelte Baufletblume . . IMusa, ओaradebreige.

M. paradisiaca, getuobnlide paradesfeige, Pifang

161 Myositis, R a deold.

M. scorpioides, Nergin̈meinniđt . . 162

IV y ric.a, $5 B$ a d b b $a$ แm.

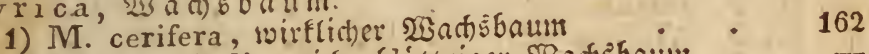

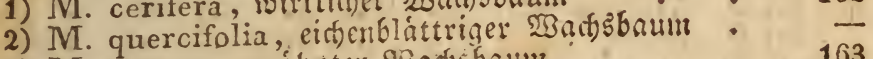

3) M. serrata, gegábnter şndjbaum • • • 163

Myrtus, SR y re.

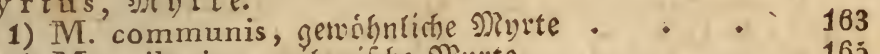

2) M. zeilanica, zcilanifide झintte • • • $16 j$

Nerium, Dleander.

1) N. odorum, tooftriedenter Deander . . 165

2) N. oleander, genteiner Dleanier - • -

3) N. splendens, práditiger Dlcander • • 167

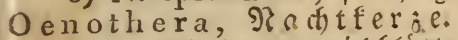

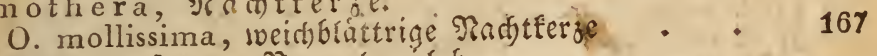

Ornithogalum, $\mathfrak{L}$ ogel it ild).

Oxalis, 厄auef flec.

O. versicolor, purpurea • . . . 168

Paeonia, \$áñe.

P. arhorea, Eamartige Tronie • . . 168

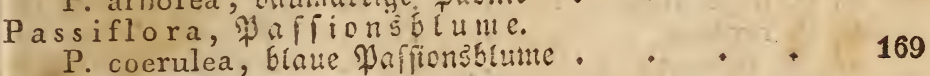

Pavonia, Mavonie.

P. praemorsa, abgebiliene \$avonie . • 171

Pelargonium, franiáfinabel. 173

1) P. amplissimum $\quad: \quad: \quad: \quad$ : 174

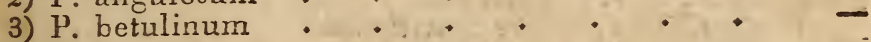

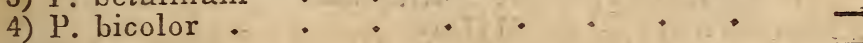

5) P. candidum

6) P. citriodorum

7) P. coronopifolium 


\section{सis 254 The}

8) Pelargonium daucifolium

9) P. echinatum

10) P. fulgidum

11) P. gibbosum

12) P. grandiflorum

13) P. hermanifolium

14) P. inquinans

15) P. lateripes

16) P. lanceolatum

17) P. peltatum

18) P. pencillatum

19) P. Prinz Regent

20) P. quinquevulnerum

21) P. radula .

22) P. St. Helenum

23) Panguipeunt $^{2}$

24) P. speciosum

25) P. Tankervilliae

26) P. ternatum

27) P. tetragonum

28) P. tricolor

29) P. iripartitum

30) P. triste

31) P. unicolorum

32) P. Willdenowii

33) P. zonale

Pentastemon, $\mathfrak{B} a r i f a b e r$.

P: campanulatus, glofenformiger Sartfaden

Phil adelphus, Pfeifenfrautió.

P. côronarius, wohlriechenoer Mféfenftraucá

180 Phlomis, Phiomi

1) P. fruticosa, frnutfortiger \$glomiz

180 Phlox, glammenblume.

1) P. acuminata

2) P: carolina

3) P. divaricata

4) P. glaberrima

5) P. maculata

6) P. nitida

7) P. ovata

8) P. paiiculata

9) P. reptans

10) P. setacea

11) P. speciosa

32) P. suaveolens

13) P. subulata

14) $P$. suffenticosa

Phoenix, Dattelpalme.

P. dactilifera, wabre Dattelpatme +. . + 184 Phormium, glad) \&lilite.

P. tenax, zabe Fladistilie 
Pliysalis, Sditutte.

1) $P$. alkekenge, Truontirjitie

2) P. pertuviana, 2̂(nanasfirfdre

Plectranthus, babnenfporn.

P. fruticosus, frraudartiger Şabnenfporn - . 187 Plumbago,

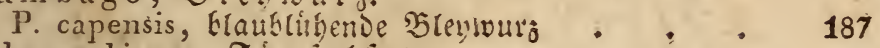

Polypodium, Titpelfarn.

P. vulgare, nemeinet Tupfelfarn - • . 187

Polianthes, $\mathfrak{T}$ uberofe.

P. tuberosa, gemeine Tuferofe • • • . 188

Primula, primel.

1) P. acaulis, fríngellofes Printel • . . 190

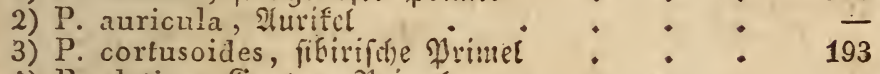

4) P. elatior, Garten = Drimel

5) P. minima, fleinfes Đrimel

6) P. sinensis, doinefifdes Primel Slume

Prunns, Sirfine.

P. laurocerasus, Dei Sirifflorbeer • • . 195 Punica, (5) ranatiaum.

1) P. granatum, geneiner (5ranatbaum • • 196

2) P. nana, Stwerggranate • . : : 197

Pyrola, $ֶ$ intergiun.

1) P. rotundifolia

2) P. umbellata

Quercus, एi ide

hhapis, Fád derpatme.

R. flabelliformis, hohe fadfervatme • • • 198

Rhododendron, 2 tpenrofe.

R. ponticum, vontiflde 2l(penrofe $\quad$ • . . 199

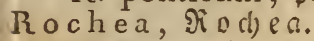

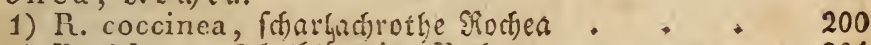

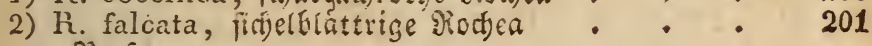
Rosa, Piofe.

Eommergrune æofen.

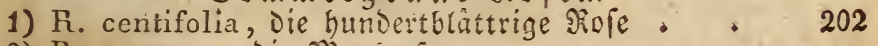

2) R. muscosa, sie M200srofe : • . . -

3) R. lutea, Die gelte Sidfe.

4) R. alba, meis̆e Siofe

5) R. pomponia minor

6) R. violacea nova .

203

R. burgundica, champaniensis, Vilmorina, coronata, lucida plena.

1) R. Banksia Intergitne soren.

2) R. chinensis hybrida

3) R. indica

4) R. laurentia

5) R. moschata

6) R. multiflora

7) R. noisette 


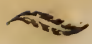

8) Rosa persicifolia

9) R. semperflorens
a) alba, toveturtucho
b) ariemonefora, iutfelroth
c) Bischonii, Oluteltoth, nieorig
d) carmesina
e) pallida, Díe gemeine hrof́rothe
f) atropurpurea, Dic scmeine sunfelrothe
g) de mons, Jito pen Jions
h) speciosa, prácitige Nicnatsiofe

10) R. Thea, Die Therrofe
a) carnea, bie blofie Egerdie
b) rubra, mit flemern rofarothen solumen

Rosmarinus, Go

R. officinalis, gementer Sosmarin

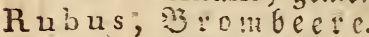

R. rosaefolius, rofentiaturige Bronbeere . . 212

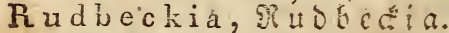

R. purpurea, nutpurotbe Subberia

Ruellia; íclita.

1) R. formosa, forne Suellia

213

2) R. varians, abveitionie Siuclia

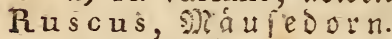

1) R. aculeatus, frad)liger siáufeoorn

2) R: hypoglossum; 3ungentlatt

Salvia, Saibey.

1) S. coccinea, forthturotbe Eatbey . . . 214

2) S. formosa, fitone Ealbels

3) S: splendens, glánzenie Gatbey

Sanseviera, Sanfevierc.

S. carnea, flctiffartene Eanfeviete

205

211

Santolina, seiligenpflanze.

1) S. chamaecyparissus, cupreflenartige Şciligenpflange 216

2) S. viridis, grine jeiligenpflange

Saxifraga, eteinbreb.

1) S: punctata, purctiter (Exeinstech)

2) S. sarmentosa, wubermiet Etcinbred

Scabiosa, Ecátiole.

S. caucasica, fautaffide Ecabiofe Schollia, (E) ditie.

S. crassifolia, Didfolattrige Edollie

Sempervivum, 5 a

1) S. arachnoideum, t.

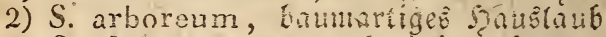

3) S. globiferum, tugeffnospiges jaukhub.

4) S. montanum, Eerghtiefiece Sauvitaitb

5) S. spinosum, dorniges Şauล

6) S, tectorum, gemeines Jุaนริเลแ

Senecio, Steugitut.

S. elegans, fibneg fiveutrout . • . 221

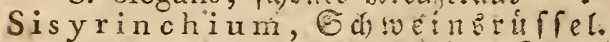

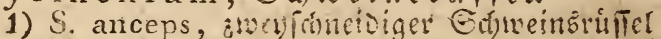

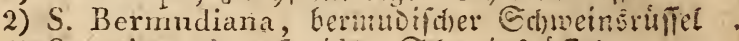

3) S. striatum, geftretftet Eitweinbrifiel 


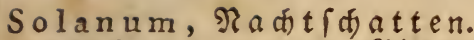

1) S. dulcamarum, Sitterfḯ, $\mathfrak{3 a l o n a d t h i f a t t e n ~ S e i t e ~} 222$

2) S. melongena, ewertragenoer Radjiffatten . 223

3) S. pseudocapsicum, Forallfirfore . . - -

4) S. pyracanthas, rothfractiger Nachtidjatten

Sol d a nella, $\mathfrak{A}$ lpengló df d) en.

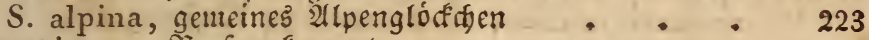

Spartium, bुefenfia $\mathfrak{a}$ t.

S. junceum, fpanifdes 'bejenfraut . . . 224

Stapelia, Etapelie.

S. asterias, caespitosa, campanulata, elegans, geminata, glauca, grandiffora, hirsuta, humilis, incarnata, maculata, paniculata, picta, pulchella, pulvinata, quadrangula, reticulata, variegata, tubulosa, venusta

Statice, कै r a s nelfe.

S. mucronata, fraแถ์lattrige Erasnelfe - . 225 Syringa, flieder.

1) S. persica, perfiffier Fliever . • . . 226

2) S. vulgaris, gemeiner Jlicier . . . . -

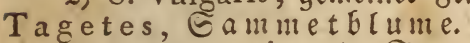

T. lucida, glanjende Eammetblume . . . 227

Teucrium, हs a ma ner.

1) T. betonicum, betonentlattriger B̋nmander . 227

2) T. marum, Jiaf̨enfraut . . . . . -

Thea, Theеbaum • • • • • • • 228

Thuja, Ee

1) T. occidentalis, Der americaniffe Sebenşaum 228

2) T. orientalis, Der fácbertáttrige £ebenşbaum

Tradescantia, Trabescantie.

1) T. discolor, Eunte Trabešcantie : : • 229

2) T. virginica, virginiffe Tiadercantie :

Tropaeolum, fipuzinetfieffe.

T. majus fl. pl., Die gefúllt Elubende నapuginerfrelle 230

Tussilago, Şuflattig.

T. fragrans, twohlried)ender Jृuflattig . . . 231

Ulex, Stediginfter.

1) U. europaeus, gemeinet Stedginfter . . 231

2) U. manus, fleiner Eted)ginfter . : : 232

Vaccinium, Şeidelbeere.

V. myrtillus, ఏlaubeere

$\mathrm{V}$. vitis idea, 叉reú̄elbeere

V. oxicoccos, Nooşbeere

Veltheimia, פ্Seltheimia.

1) V. uvaria, traubenbluhenie Seltheimie . . 233

2) V. viridifolia, grunbláttrige Neltbeinte:

Verbena, (Eifentart.

1) V. aubletia, foonfluhenoer Eirenfart . . 235

2) V. triphylla, Dreybláttriger Eifenbart . . -

Veronica, Érenpreis.

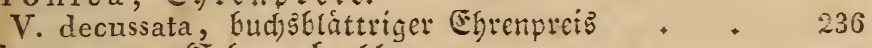

Viburnum, Én neeballen.

1) V. opulus roseum, gemeiner @dfneeballen • 237

2) V. tinus, lorberbláttriger Ednceballen 


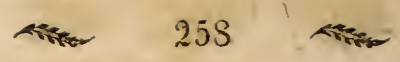

Vinca, Sinngrün.

1) V. herbacea, frautartiges Einngrun . Eeite 238

2) V. major, arø̄́es Einngrun

3) V. minor, fleines Einngrun

4) V. rosea, rofarothes Einngrin : : : 239

Westringia, 20 fr ringia.

W. rosmariniformis, rošmarinartige 23 efringia 240

Yucca, II u de.

1) Y' draconis, Drachentaum = Y)ude . . . 240

2) Y. gloriosa, príditige Duffe

Zinnia, Binnic.

Z. elegans, fúche Sinnie

Zy gophyllum, DoppelElatt.

Z. sessilifolium, ungefricltes Depperblatt 


\section{Deutides Megifter.}

Ia:onstume, f. Calla aethiop. 2faร̧blume, f. Stapelia. ADamenndef, F. Yucca. Af facie, f. Acacia. Iroe, f. Aloë.

- bunocrtjágrige, f. Agave americana.

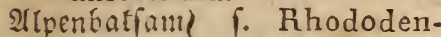
Alpenrofe $\}$ dron.

2lipenglóffden, f. Soldanella. Alfromerie, f. Alstroemeria. Itmaryllis, f. Amaryllis.

antromede, $i$. Andromeda.

Inemone, f. Anemona.

$\mathfrak{A}$ tif dyofen $^{2}$ loe, f. Aloë brevifolia in ben 3 ufáçen. 2(fd)enpflanjo, f. Cineraria. Atragene, $\Gamma_{0}$. Atragena. 2lucube, f. Aucuba.

Bátenflaue, f. Acanthus. bárenoh: r. Arctotis.

Bartfaden, r. Pentastemon. Beifbeere, i. Capsicum. כecienfraut, r. Spartium. כiberkaum, Magnolia. bitterfífi, f. Solanum dulcamarum.

Slofienfiraud), f. Colutea. Strubeere, f. Vaccinium myrtillus.

כ̇levtwurg, f. Plumbago. Slumenrohr, f. Canna.

Bobne, f. Dolichos.

\$odfsoorn, r. Lycium. Brennende Liefe, f. Lychnis chalcedon.
Srombere, f. Rubus.

Bryophyllum, f. Bryophyl. lum.

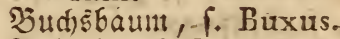

शुudleja, f. Budleja.

Ealadiun, f. Caladium.

Ealluna, f. Galluna.

Eamellia, 1. Camellia.

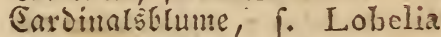
cardinalis.

Eiftenrofe, f. Gistus.

इobbea, f. Cobbea.

Sommetine, f. Commelina.

Soudorus, f. Corchorus.

Eyprelle, r. Cupressus unis Santolina.

Enrille , f. Cyrilla.

Dad)tours, f. Sempervivum.

Dattelpalme, r. Phoenix.

Diamantpflanje, f. Mesem. brianth. crystall.

Dićfblatt, f. Crassula uno Rochea.

Dillenic, f. Dillenia.

Diftel, f. Carduns.

Sopperbiatt, i. Zygophyllum. Drafenóaum, 1. Yucca draconis.

Dradientopf, f. Dracocepha. lus.

Eธertvur, r. Carlina.

Efrenpleis, 1. Veronica.

(Eibifi, r. Hibiscus.

Eid)e, f. Quercus.

Eyerpflanje, f. Solanum me. longena. 
Eifenbart, f. Verbena.

Eifenmañ , f. Metrosideros. Eisfraut r. Mesembrianth. Eizpflanje) crystall.

Engetivurz, f. Polypodium vulgare.

Erobeere, f. Fragaria.

Erofdeibe, f. Cyclamen.

Eugenie, 1. Eugenia.

Fadeloiftel, r. Cactus.

Jadyerpalme, f. Rhapis.

feige, gemeine, f. Ficus carica.

- indianiffel f. Cactus

- fpanifde opuntia.

Felfenftrauch, f. Azalea.

Ferrarie, f. Ferraria.

Fingerhut, f. Digitalis.

Jlacbilitie, f. Phormitum.

Slanmenblume, f. Phlox.

flammenrósúen, f. Lychnis.

Fitiever, r. Syringa.

Zuchfte, f. Fuchsia.

Bamander, f. Teucrium.

Gartie, f. Achillea.

Gardenie, f. Gardenia.

(5)artenrabe, f. Agrostema coronar.

Braudbeil, f. Anagallis.

(3) auflerblume, f. Mimulus.

(j)eibblatt, r. Lonicera.

Semfenborn, r. Martynia.

Georgine, r. Georgina.

Gincenblume, f. Campanula.

Grorinie, f. Gloxinia.

(S) rycine, f. Glycina.

Sotterblume, f. Dodecatheon.

(5) itterouft, f: Diosma.

Giorterie, f. Gorteria.

Granndilla, f. Passiflora.

Eiranate, f. Punica.

Bronnelfe, r. Statice.

(S) revie, f. Grevia.

Sabichtatraut, f. Hieracium.

5) abnenfporn, f. Plectranthus.

Salfblame, f. Hemimeris.

Sommerfit:aud , f. Cestrum.

Sous!nub)

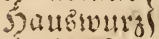

jeffirfole, r. Lonicera tartarica.

Scide, f. Erica uno Calluna.

Seiserbere, f. Vaccinium.

Sceiligenpflanze, f. Santolina.
Sherzfame, f. Cardiospermum.

Simbeere, f. Rubus.

Scimmelfdiflifel, f. Primula veris.

Soja, f. Schollia (Hoya).

Şollunier, perfifder, f. Syringa persica.

- turtifder) f. Syr.

- panifder vulgaris.

Sortenfie, f. Hortensia.

Soustonie, r. Houstonia.

Scutre, Şitbráf, f. Ilex aquifolium.

Squflattig, f. Tussilago.

Şunoşunge, f. Cynoglossum.

Jacobsitilie, f. Amaryll. for. mosiss.

Sașmin, f. Jasminum uno Philadelphus.

Jebovablumeben, f. Saxifraga punct.

Selangerietieber, f. Lonicera caprifolium.

Sgelditrel, f. Cactus mamillaris.

Tingtver, f. Amomum.

Tohanniśbroo, 1. Ceratonia.

Iubenfinftie, f. Physalis alkekenge, u. Solanum pseudocaps.

Srie, f. Ixia.

Grota, r. Ixora.

Sajaputbaum, Metrosideros uno Leptospermum.

Salbefratenbaum, f. Cestrum parqui.

Saltuie, r. Kalmia.

Ramille, f. Anthemis.

Rapusinerfrelle, f. Tropaenlum.

Natcenfraut, $f$. Teucrium marum.

fieldiblume, f. Calycanthus.

Sellerbals, f. Daphne mezereum.

Sitrfblorber, f. Prunus laurocerasus.

Nonigin Det Slactet, f. Gactus grandiflór.

Sornllfiridie, f. Solanum pseudocaps.

Itranidgfdnntel, f. Pelargon. 


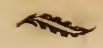

261
Strelte, fpanifde, f. Tropaeolum.

Ireusfrnut, f. Senecio.

Rúd)enficlle, f. Anemona pulsatilla uno verna.

Sîrbiहै, 1. Cucurbita.

Eartenatie, f. Lachenalia.

Eantane, f. Lantana.

Raveniel, f. Lavendula.

\&ebensfaum, f. Thuja.

Rebensblatt, f. Bryophyllum.

Qeberblumel 1 . Anemona he-

Seberfraut patica.

Qiebeรืblume, f. Agapanthus.

Qilie, f. Lilium.

Sobelie, f. Lobelia.

fórenmaul, f. Antirrhium.

\&óvenfatwang, f. Phlomis leonurus.

Eooş6aum, f. Clerodendron.

Sorbeer, f. Laurus.

Máufeoorn, f. Ruscus.

Náurefol \} T. Solanum dul-

Náufefrat camarum.

Sáufeobr, f. Hieracium.

Singnolie, r. Magnolia.

Siabernie, f. Mahernia.

Ranblume, $f$. Convallaria.

Sandel, $\Gamma$. Amygdalus.

Rarienglode, f. Campanula medium.

פลarumverum, f. Teucrium marum.

)iauranoia, f. MTaurandia.

Neerglviefel, f. Ornithogalum (Scylla).

Selonendiftel, f. Cactus melocactus.

Mifpel, f. Mespilus.

2) Rostecere, f. Vaccinium oxycoccos.

Minrte, f. Myrtus.

Murtentyeide, f. Phylica ericoides.

Rabelfraut, f. Cotyledon.

Nadfitferse, f. Oenothera.

sachtidyatten, f. Solanum.

शradituiole, f. Hesperis.

Nelfe, f. Dianthus.

9Riestwurg, f. Helleborus.

Dleander, f. Nerium.

Drange, f. Citrus.

Dfterlugev, f. Aristolochia.
Páonie, f. Paeonia.

Maradiesfeige, f. Musa.

Paffions6lume, f. Passiflora.

Wasonie, 1 . Pavonia.

\$ectnelfe, $\{$. Lychnis viscosa.

Deftrutr: r. Ciacalia:

P̂feifenftrautb, $f$. Philadelphus.

Yhlomis, r. Phlomis.

Phulica, f. Phylica.

Wifang, f. Musa.

Someranje, f. Citrus aurantium.

Pompelmuz, f. Gitrus decumanus.

Po:f(f), f. Ledum.

Porzellanblumden, f. Saxifrag. punct.

Preufelbeere, f. Vaccin. vitis idea.

Printel, f. Primula.

Tunfdfraut, f. Verbena triphylla.

Quitte, f. Cydonia.

Pabe, $r$. Agrostema.

Fitterfporn, f. Delphinium.

Focter, f. Rochea, Crassula.

Siofe, r. Rosa.

- cjinefifd)e, f. Hibiscus ro. sa sinens.

- japaniffie, r. Camellia.

- von Seriffo, f. Lonicera.

Pofenfraut, f. Pelargonium radula.

Rosmarin, f. Rosmarinus.

$$
\text { - witser, f. Ledum. }
$$

Siubbectie, r. Rudbeckia.

Sibbr midf nicht an, f. Mimosa pudica.

Puellie, f. Ruellia.

Galbey, r. Salvia.

Enmmetblume, fi. Tagetes.

Eammetnelfe)

Sammetrabe Agrostema.

Eanfeviere, f. Sanseviera.

Gaubrod, f. Cyclamen europ.

Eauterflee, f. Oxalis.

Ediafgarbe, r. Achillea.

Edimpappel, f. Achania.

Edjefblatt, f. Begonia.

Ediloblume, f. Chelone.

Edi)langenfraut, f. Galla.

Ed)liffelblume, f. Primula. 
Erflutte, f. Physalis.

Erfimudflitie, fo Agapanthus.

Edinedfenflee, r. Medicago.

Edineeballen, f. Viburnum.

Sodollia, f. Schollia.

Edopfhiazunthel

Soropfitie

f. Eucomis.

Edotenflee, f. Lotus.

E(f)warbenwur;, 1. Asclepias.

Éfweingaroo, f. Cyclamen curop:

Equbeinbrififer, f. Sisyrinchium.

edinertilie

Edivertel f. Tris.

Eeloethaf , f. Daphne mezcreum.

Scitenpflange, f. Asclepias, Schollia.

Eiegmur' , f. Gladiolns.

Einngrin, f. Vinca.

Sinmplanae, f. Mimosa. Ecabiofe, f. Scabiosa.

Ecurpionefritwanz, f. Heliotropium.

Connenbtumben, f. 'Helianthemmm.

Connentume, f. Helianthus. Eonnenmente, f. Heliotropium.

Eprimaui, Convallaria majalis.

Stapelie, f. Stapelia.

Stemopret, f. Datura.

Etedigntifer
Stechp frieme \%. Ulex.

Stectpalme, f. Ilex.

Eternblunie, f. Aster.

Eternenbiazunthe, f. Eucomis.

Steinbred, , r. Saxifraga.

Eerordidunabel, f. Geranium uns Pelargonium.
Stunicnblume, f. Tradescant. virzin.

Guifeeminte, f. Leptosperm. Einfivurzel, f. Polypodium vulg.

Tagblume, f. Hemerocallis.

Theetaun, f. Thea.

Tigerblume, f. Ferraria.

Ztabescantie, f. Tradescantia. Iricjterivinde, f. Ipomea.

Tuberofe, r. Polyanthes.

Tupfelpfarm, f. Polypodium. Sutenmalve, f. Achania.

Şanillientiaut, i. Heliotropium.

Sireltheimie, f. Veltheimia.

Senusfpiegel, f. Campanula speculum.

Siola matronatis, r. Hesperis. Sুogefmild), f. Ornithogalum. Bolfamerie \} f. Clerodendron. Moifmannies

23arorebe, f. (Clematis) Atragene.

\$gefringie, f. Westringia.

Winie, $f$. Convolvulus und Ipomea.

swintergrin, f. Pyrola uno Vinca minor.

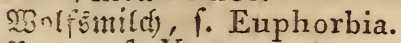

Oucca, f. Yucca.

zaferbfume, f. IMesembrianthemum.

Befrrourg, f. Arum.

Beiland, f. Cneorum.

Sinnie, f. Zinnia.

Bitrone, r. Citrus media.

Stoolfgotterblume, f. Dodecatheon.

3ungenblatt, f. Ruscus hypoglossum. 


\section{Şeržcichniz mefrerer Synomymen,}

weldye sie in Sen yortefonden Blittern beforiebenen (s)ewids fe fuhren.

Achimenes coccinea, f. Cyrilla pulchella. Adenandra, $r$. Diosma, 2Inmerf́. Adhadota zeyloniensium, . Justitia adhadota. Aletris capensis, $f$. Veltheimia viridifolia. - uvaria, f. Veltheimia u.

Aloë, Die hunbertjábrige, f. Agave americana.

- imbricata, $\Gamma_{\text {. A A spiralis. }}$

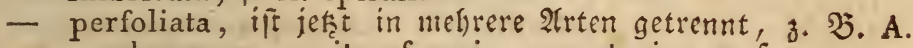
arborescens, mitraeformis, succotorina $u$. $\uparrow$. w. Alsisia citriodora, $r$. Verbena triphylla. Alstroemeria pelegrina, $f_{\text {. A . peregrina. }}$ Amica nocturna, - . Polyanthes tuberosa. Anagallis fruticosa, $\mathfrak{f}_{\text {. A. collina. }}$

Antirrhium bicolor, f. A. majus. coerulea, f. A. morrelli.

Apicra, Г. Aloë.

Arbre à feu, f. IMespilus pyracantha. Arum bicolor ?

- pictum f. Galadium bicolor.

Asclepias carnosa, f. Schollia crassifolia.

Asphodelus comosus, f. Eucomis punctata. Basilea punctata, f. Eucomis punctata. Begonia Evansiana, f. Begonia discolor. Bouvardia coccinea, $f$. Houstonia $c$.

Buddleja capitata, 1. B. globosa.

Calonchoe crenata, f. Bryophyllum calycinum.

Cassyta baccifera, $f$. Cactus pendulus.

Celsia urticifolia, ケ. Hemimeris u.

Cereus, Der alte Fame fur mefrete Eactubarten.

Cestrum grandifolium, $f$. C. laurifolium.

- virgatum, f. C. parqui. 
Chamerodaphne, f. Kalmia.

Chamerops excelsa, f. Rhapis flabelliformis.

Chelone campanulata, $f_{\text {. Pentastemon campan. }}$

- formosa, f. C. barbata.

- ruellioides, f. C. barbata

Chrysanthemum indicum, $r$. Anthemis artemisaefol.

Cistus laevipes, f. Helianthemum 1.

Clematis balearica)

- calycina f. Atragene balearica.

Cnicus casabonnae, f. Carduus c.

Colocassia, wird oft fáffeflich Die Calla aethiopica genannt.

Convolvulus argenteus, f. C. cneorum.

Cotyledon crenatum, 1 . Bryophyllum calycinum.

Crassula coccinea, $f_{\text {. Fochea coccinea. }}$

\section{- $\begin{gathered}\text { decussata } \\ \text { falcata }\end{gathered}$. Rochea falcata.}

Crinum africanum, 1 . Agapanthus umbellatus.

Cunilla fruticosa, f. Westringia rosmarinifol.

Dalea, f. Georgina.

Datura arborea, f. D. suaveolens.

Diosma ericoides, $f$. D. alba.

Eranthemum nervosum $\{$ pulchellum $\}$. Ruellia varians.

Erica herbacea, f. E. carnea.

- vulgaris, $f$. Calluna v.

Gelseminum catalonicum, f. Jasminum grandiflorum.

Geranium, geben jeşt grêstentbeilg unter Dem Ta!nen Pelargonium.

Gesneria pulchella, f. Cyrilla $p$.

Gladiolus crocatus, f. Ixia crocata.

Gomphocarpus angustifolius, f. Asclepias a.

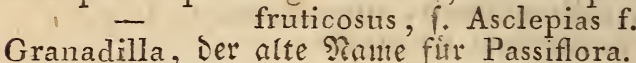

Hemerocallis japonica, $\hat{i}$. H. alba uno coerulea.

Hemerocallis plataginea, $f$. H. alba.

Hepatica triloba, f. Anemona hepatica.

Hermannia odorata, f. Mahernia o.

Hibbertia volubilis, f. Dillenia scandens.

Hibiscus malvaviscus, f. Achania m.

- praemorsus, f. Pavonia praemorsa.

Hosta coerulea und alba, f. Hemerocallis c. und a.

Hoya carnosa, f. Schollia crassifolia.

Hydrangea hortensis, $f$. Hortensia mutabilis.

Jasminum arabicum, $f$. J. Sambac.

- capense, f. Gardenia florida.

- catalonicum ?

- hispanicum f. J. grandiflorum.

- $\begin{aligned} & \text { geniculatum } \\ & \text { volubile }\end{aligned}$ f. J. gracile.

- $\quad$ triumphans, f. J. revolutum.

Iris anglica, f. I. xiphioides.

- hispanica, f. I. xiphium.

- suetia, f. Amaryllis formosissima.

Ixora americana, f. Houstonia coccinea.' 
Kerria japonica, f. Corchorus jap.

Ketmia syriaca, $f$. Hibiscus syriacus.

Larochea, . Rochea.

Laurus tinus, $f$. Viburnum tinus.

vulgaris, f. L. nobilis.

Leonurus africants, $i$. Phlomis leonurus.

Lilium cordatum, f. Hem $\circ$ rocallis alba.'

Limonium crispum, $f$. Statice mucronata.

Lychnis coccinea $\{$ r. L. coronata.

Lycopersicum esculentum, f. Solanum lycopersicum.

Malvaviscus arboreus, $f$. Achania malvaviscus.

Martynia speciosa, f. Gloxinia speciosa.

Marum verum, f. Teucrium marum.

Melaleuca armillaris

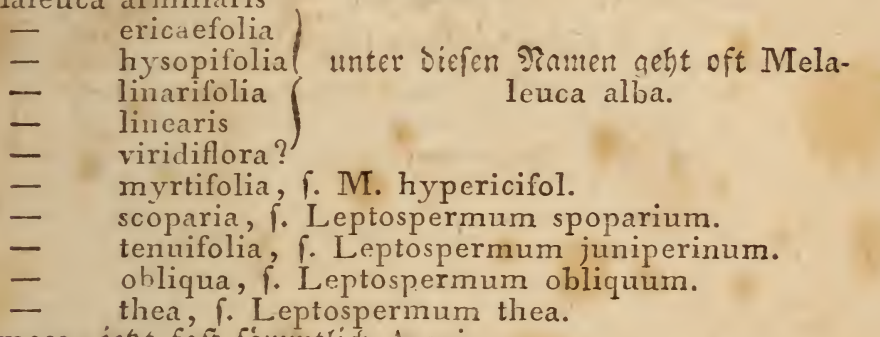

Mimosa, jef̨t fât fámuntlidi Acacia.

Mimulus aurantiacıs, f. M. glutinosus.

Mogorium sambac, f. Jasminum sambac.

Noli me tangere, f. Mimosa pudica.

Nyctanthes sambac, f. Jasminum s.

Ophiopogon japonicus, $f$. Convallaria $j$.

Opuntia, hiefen fonft Die fladblattrigen Eactusarten.

Ornithogalum punctatum, f. Eucomis punct.

Oxycoccos palustris, f. Vaccinium oxycoccos.

Pavonia cunaeifolia, f. P. praemorsa.

Pensthemon, f. Pentastemon.

Phorminm aloides, f. Lachenalia tricolor.

Pyrus japonica, f. Cydonia japon.

Rhipidodendron, f. Aloë, 2Ammert.

Ripsalis cassutha, f. Cactus pendulus.

Rosmarinus sylvestris, i. Ledum pulustre.

Salvia leonuroides, . S. S. formosa.

Scabiosa elegans $\{$ f. S. caucasica.

Scylla (Squilla) maritima, oft Nertvedffelung mit Ornithogalum caudatum.

Sedum spinosum, f. Sempervivum spinosum.

- tectorum, f. Sempervivum tectorum.

Sempervivum cuspidatum, f. S. spinosum.

- mutabilis, f. S. arbor. mit Eraunem פlatt.

Sisyrinchium gramineum, f. S. anceps.

Statice crispa, f. S. mucronata.

Tahernemontana coronaria, f. Nerium, Anmerf. 

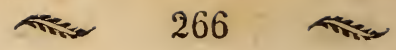

Tagetes integrifolia, f. T. lucida.

Teucrium canescens? fruticans $\{$ f. $T$. betonicum.

Thee Camellie, f. Camellia sansangua alba.

Tigridia pavonia, f. Ferraria $p$.

Trevirana coccinea, f. Cyrilla pulchella.

Tritoma

Tritomantha f. Veltheimia.

Tritoma media, f. Veltheimia sarmentosa.

Vaccinium punctatum, $f$. V. vitis idea.

Veltheimia capensis, f. V. viridifolia.

Verea crenata, $r$. Bryophyllum calycinum.

Viburnum serratum, $\Gamma$. Hortensia mutabilis.

Vieuseuxia, f. Ferraria.

Viola matronalis, f. Hesperus m.

Volkameria
Volkmannia f. Clerodendron fragrans. 


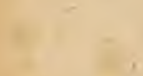<smiles>C[As]</smiles>

$+2+2$

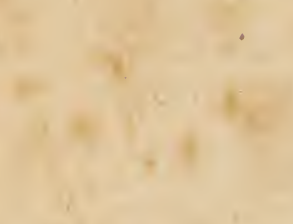

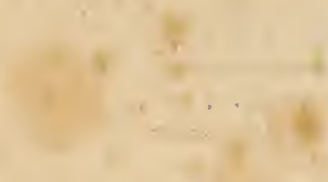

$+$

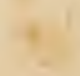

a

$$
1+x+2
$$

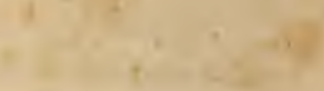

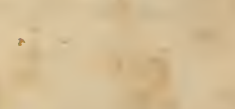
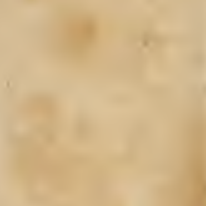







\section{LIBRARY OF CONGRESS \\ (III)

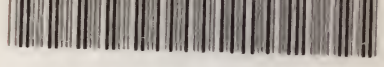 \\ 00009246137}

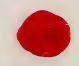

